\title{
DECLAMTR
}

LA-9328-MS

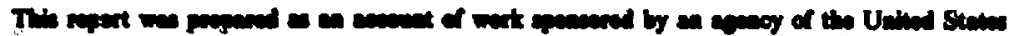

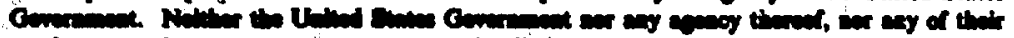

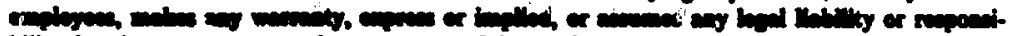

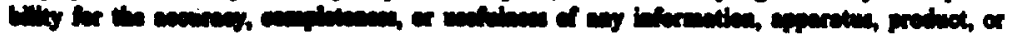

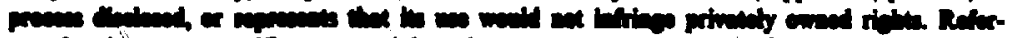

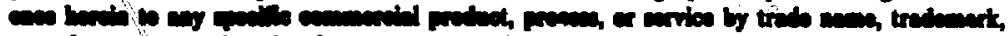

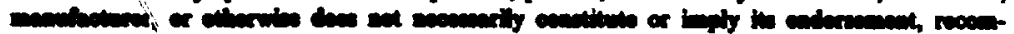

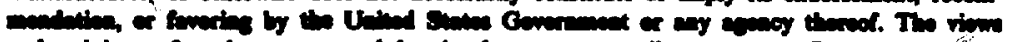

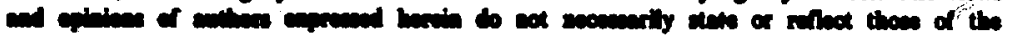

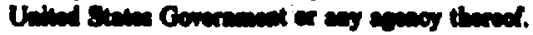

UC-70

lasuad: Decomber 1962

$L A--9328-M S$

DE83 013357

\section{Summary Report on the Geochemistry of Yucea Mountain and Environs}

\author{
W. R. Daniels \\ F. O. Lawrence \\ K. Wolfsberg \\ V. L. Rundberg \\ R. S. Rundberg \\ M. L. Skyes \\ A. E. Ogard \\ G. M. Thompson** \\ J. F. Korrisk \\ B. J. Travis \\ C. J. Duffy \\ E. N. Treher \\ T. W. Nowton \\ R. J. Vidale \\ J. L. Thompson* \\ G. R. Walter** \\ B. P. Bayhurst \\ R. D. Aguilar \\ D. L. Bish \\ M. R. Cisneros \\ J. D. Blacic \\ S. Maestas \\ B. M. Crowe \\ A. J. Mitchell \\ B. R. Erdal \\ P. Q. Oliver \\ J. F. Grifflth \\ N. A. Raybold \\ S. D. Knight \\ P. L. Wanek
}

\section{notice}

Pentrews of this chent anf lueciele.

it has been repreduced from the best swellethe bepr to pormit the hroadest poseine ovellawimy.

Depertment of Cheminiry, Who swie Uniwersity, Poceivino ID s8200.

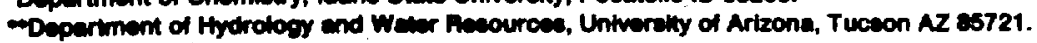

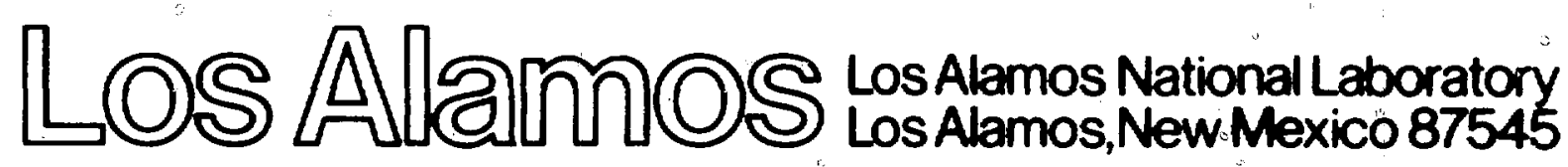


iv 
ABSTRACT . . . . . . . . . . . . . . . . . . . . . . . . . . . 1

EXECUTIVE SUMMARY. . . . . . . . . . . . . . . . . . . . . . . . 2

Chapter 1: INVESTIGATIONS OF THE GEOCHEMISTRY OF

YUCCA MOUNTAIN AND ENVIRONS. . . . . . . . . . . . . . . 16

I. INTRODUCTION . . . . . . . . . . . . . . . . . . . . . . . . . 17

II. GROUNDWATER GEOCHEMISTRY . . . . . . . . . . . . . . . . . . . . . . 21

A. Groundwater Chemistry . . . . . . . . . . . . . . . . . . 21

B. Actinide Behavior . . . . . . . . . . . . . . . . . . . 42

III. NEAR-FIELD ENVIRONMENT . . . . . . . . . . . . . . . . . . . . . . . 68

IV. GEOCHEMICAL RETARDATION. . . . . . . . . . . . . . . . . . . . 71

A. Sorptive Behavior of Tuff . . . . . . . . . . . . . . . 71

B. Permeability, Storage Capacity, and Porosity. . . . . . . . . . 139

C. Diffusion Measurements . . . . . . . . . . . . . . . . . . 160

D. Flow Studies. . . . . . . . . . . . . . . . . . 176

V. NATURAL ANALOGUES. . . . . . . . . . . . . . . . . . . . . . . . . 202

VI. GEOCHEMICAL AND TRANSPORT MODELING . . . . . . . . . . . . . . . . . 203

A. Geochemical Modeling of Groundwater Interactions. . . . . . . . 204

B. Calculated Solubilities of Uranium and Plutonium in

Well J-13 Water. . . . . . . . . . . . . . . . 224

C. Transport Modeling. . . . . . . . . . . . . . . . . . . . 231

VII. SHAFT AND BOREHOLE SEALING . . . . . . . . . . . . . . . . . . . . . 234

Chapter 2: PLANRED STUDIES OF THE GEOCHEMISTRY OF YUCCA MOUNTAIN. • • . 238

I. GROUNDWATER CHEMISTRY. . . . . . . . . . . . . . . . . . . . . . . . 239

A. Water Chemistry . . . . . . . . . . . . . . . . . 239

B. Behavior of Actinides and Other Multivalent Elements. . . . . . 243

C. Isotope Measurements. . . . . . . . . . . . . . . . . . . . . . 247

II. NEAR-FIELD ENVIRONMENT AND PERMEABILITY. . . . . . . . . . . . . . . 249

III. GEOCHEMICAL RETARDATION. . . . . . . . . . . . . . . . . . . . . . . 252

A. Sorptive Behavior of Tuff . . . . . . . . . . . . . . . . . 252

B. Diffusion . . . . . . . . . . . . . . . . . . . 255

C. Flow Studies. . . . . . . . . . . . . . . . . . . . . 257

D. Hazard Rank . . . . . . . . . . . . . . . . . . . . 261 
CONTENTS (Cont)

IV. NatURAL ANAlogUes. . . . . . . . . . . . . . . . . . . 262

V. GEOCHEMICAL AND TRANSPORT MODELING . . . . . . . . . . . . 263

VI. SHAFT AND BOREHOLE SEALING . . . . . . . . . . . . . . . 264

A. Laboratory Experiments in Agitated Vessels. . . . . . . . . 265

B. Laboratory Experiments in Temperature Gradient Circulating systems . . . . . . . . . . . . . . . . . 266

c. Field Tests.................... 266

D. Survey of Relevant Thermodynamic Data . . . . . . . . . 266 ACKNOWLEDGEMENTS . . . . . . . . . . . . . . . . . . . . . . 266 REFERENCES . . . . . . . . . . . . . . . . . . . . . . . . 267 APPENDIX A. SORPTION RATIO DATA FOR TUFFS OF THE YUCCA MOUNTAIN AREA. . 275 APPENDIX B. MINERALOGIC AND PETROLOGIC STUDIES OF TUFFS FROM YUCCA MOUNTAIN. . . . . . . . . . . . . . . 345 


\title{
SUMMARY REPORT ON THE GEOCHEMISTRY OF \\ YUCCA MOUNTAIN AND ENVIRONS
}

\section{by}

\author{
W. R. Daniels \\ K. Wolfsberg \\ R. S. Rundberg \\ A. E. Ogard \\ J. F. Kerrisk \\ C. J. Duffy \\ T. W. Newton \\ J. L. Thompson \\ B. P. Bayhurst \\ D. L. Bish \\ J. D. Blacic \\ B. M. Crowe \\ B. R. Erdal \\ J. F. Griffith \\ S. D. Knight
}
F. O. Lawrence
V. L. Rundberg
M. L. Sykes
G. M. Thompson
B. J. Travis
E. N. Treher
R. J. Vidale
G. R. Walter
R. D. Aguilar
M. R. Cisneros
S. Maestas
A. J. Mitchell
P. Q. Oliver
N. A. Raybold
P. L. Wanek

\begin{abstract}
This report gives a detailed description of work at Los Alamos that will help resolve geochemical issues pertinent to siting a high-level nuclear waste repository in tuff at Yucca Mountain, Nevada. It is necessary to understand the properties and setting of the host tuff because this rock provides the first natural barrier to migration of waste elements from a repository. The geochemistry of tuff is being investigated with particular emphasis on retardation processes. This report addresses the various aspects of sorption by tuff, physical and chemical makeup of tuff, diffusion processes, tuff/groundwater chemistry, waste element chemistry under expected repository conditions, transport processes involved in porous and fracture flow, and geochemical and transport modeling.
\end{abstract}


Executive Summary

This report gives a detailed description of technical contributions of the Los Alamos National Laboratory to the Nevada Nuclear Waste Storage Investigations (NNWSI) managed by the Nevada Operations Office of the US Department of Energy from the time of the Laboratory's first involvement with the project in FY 1977 until March of 1982. Efforts have been primarily devoted to resolving geochemistry issues pertinent to siting a nuclear waste repository in tuff at the Nevada Test Site (NTS), with emphasis on the Yucca Mountain area.

Water from the producing well $(\mathrm{J}-13)$ nearest the potential repository site at Yucca Mountain has been selected as the reference groundwater for laboratory experiments. For use in experiments on sorption of waste elements on tuff, the well J-13 reference groundwater was pretreated with tuff from individual strata of Yucca Mountain. Before being used in sorption experiments, the water was also filtered through 0.05- $\mu \mathrm{m}$ Nuclepore membranes to remove solid material. The well J-13 water composition is altered by contact with tuff of different strata; the sodium content decreases as much as $50 \%$, and there is a variable, but slight, decrease in the potassium, calcium, and magnesium contents of the water. An additional significant point is the importance of filtration on the analyzed composition of the water. The results of experiments show that filtration through a $0.45-\mu \mathrm{m}$ crossed-fiber membrane yields erroneously high results for the iron content; the filtration should be done through a $0.05-\mu \mathrm{m}$ membrane having uniform pore sizes. This is especially important because the iron concentration in solution is a part of the measure of the oxidation-reduction potential (Eh) of the solution.

To determine or estimate the effect of the groundwater composition on the waste package, on the waste itself or its compounds, and on retardation mechanisms, it is necessary to determine the composition of the formation water for each particular stratum under consideration for a repository or a transport path. The groundwater composition is also important in its effect on forming or dissolving the newly formed minerals brought about by the temperature gradient exerted on the repository by the waste package. Wells in the vicinity of Yucca Mountain and Pahute Mesa have been sampled in various ways in an attempt to determine the composition of the groundwaters as a function of location. Results from analysis of groundwaters from hydrology wells at Yucca Mountain indicate that deep waters may be oxygen deficient compared to water 
at the standing waste level. The presence of Fe $2+$ and Fe $3+$ influences the Eh of groundwater in geologic systems. Analysis of the concentration of these ions in groundwater may be used as part of the measure of the Eh. An alternative approach is to measure the concentration of $\mathrm{Fe} 2+$ and Fe $3+$ in different tuff strata and then to estimate the total oxidation-reduction capacity of the tuff mass. Attempts are being made to develop both alternatives as complementary information.

Short-term experiments have been done in which three tuffs from Yucca Mountain were contacted with groundwater at $152 \pm 1^{\circ} \mathrm{C}$ to study possible reactions between the solid and solution phases. Three tuff samples of different lithologies were used, Topopah Spring, tuffaceous beds of Calico Hills, and Bullfrog II. Before contact with groundwater, the samples were exanined with a scanning electron microscope (SEM) to observe general surface features and mineral phases. After contact with well J-13 water for 3 weeks at $152^{\circ} \mathrm{C}$, the tuff samples were examined again by SEM and the waters analyzed. The Bullfrog tuff showed little reaction other than some rounding of surfaces and precipitation of clays. The Topopah Spring tuff showed greatly increased amounts of clays or other fine-grained sheet silicates, which had formed on glass edges. The tuffaceous beds of Calico Hills showed marked dissolution of clinoptilolite crystals. The main changes in the composition of the contacting water were large increases in sodium, potassium, and silicon concentrations and large decreases in the magnesium and calcium concentrations.

Prediction of the hazards caused by actinides escaping from a repository depends upon knowledge of the chemical processes that can take place in the repository and along the routes to the accessible environment. Information is needed concerning solubility and speciation of actinides under repository conditions and under conditions encountered by mobile species along flow paths toward the accessible environment. Factors that affect the solubility of actinides and the chemical form of the dissolved species and thus mobility include (1) the chemical and physical state of the waste form; (2) the Eh, $\mathrm{pH}$, and concentration of dissolved material in the groundwater; (3) the temperature and flow rate of the groundwater; and (4) the physical and chemical state of the tuff encountered along the flow path. Each of these factors may vary with time and distance from the repository.

A number of studies that are relevant to understanding actinide solubility and speciation have been carried out: a method has been developed for preparing 
groundwater solutions traced with actinides for sorption studies with tuff, including methods of separating solid and aqueous phases after contact; plutonium chemistry in near-neutral solutions, including development of systems for controlling the Eh through use of osmium complexes or other Eh buffers, is being investigated; and particulate transport, which includes preparation of plutonium polymers, is being studied. One difficulty encountered in laboratory experiments with plutonium is control of Eh, and thus, control of the oxidation state in the $\mathrm{pH}$ range of interest. Osmium complexes are potentially useful as Eh buffers because (1) the redox potentials of the Os(II)-Os (III) couples are independent of $\mathrm{pH}$ in near-neutral solutions, (2) the standard potential of the couple can be changed by varying the coordinated ligands, (3) the complexes are unlikely to complex actinide species, and (4) Os(II) complexes are highly colored and thus optically measurable at low concentrations. Nine osmium complexes, most of them synthesized at this laboratory, were examined for their suitability as Eh buffers. Equivalent weights were determined, absorption spectra recorded, extinction coefficients obtained, and formal oxidation potentials determined. Many of these complexes were found to be stable in solution for periods of days or weeks, even when held at elevated temperatures and exposed to light.

A number of reactions between osmium complexes and the various oxidation states of plutonium were investigated, and apparent second-order rate constants measured. By use of an osmium bipyridine complex, the standard potential for the $\mathrm{Pu}(\mathrm{V})-\mathrm{Pu}(\mathrm{VI})$ couple was determined.

A number of Eh indicator systems that might be used as Eh buffers have been studied. Both Indigo Carmine and thionine show promise, although there is some evidence of slow attainment of chemical equilibrium. Equipment that enables us to conduct these studies in air or under a rigorously controlled inert atmosphere has been assembled.

Plutonium (IV) forms a polymer under certain conditions, so experiments have been initiated to characterize this polymer under conditions likely to exist in a repository. A technique is being investigated to measure the solubility of the polymer, that is, the extent to which the polymer converts to $\mathrm{Pu}(\mathrm{IV})$ ions. Measurements of this solubility, using ${ }^{239} \mathrm{Pu}$, were somewhat tentative because of radiolysis caused by the intense alpha activity of this nuclide. These measurements are being repeated using ${ }^{242} \mathrm{Pu}$, which has a lower specific activity. Experiments have been conducted in which the polymer appears to form over a broad range of concentration levels and $\mathrm{pH}$ values. 
One mechanism by which radionuclides may move through geologic media is particulate transport. Particulates may include radioactive elements aggregated as colloids or colloids formed by sorption of radioactive species on microscopic particles. Such material may form from radionuclides initially in solution or as a consequence of leaching of solid waste forms. Microautoradiographic procedures have been used to identify particulates containing americium and plutonium in the effluents of crushed-rock columns. Colloids of Pu(IV) in a controlled size range are being prepared so that this material may be used in future transport studies. Plastic spheres incorporating fluorescent dye or radioactive material to aid in their detection are also being used to study particulate transport.

Possible changes in the solid phases at Yucca Mountain caused by the presence of a repository are of concern because they could affect rock properties, especially sorption, strength, permeability, and porosity. This is especially true in the near field where temperature will rise as a result. of the repository emplacement. The phases most likely to change are the clays, zeolites, and glasses, all of which may be expected to alter to less hydrous phases of smaller volume. There may also be hydration of anhydrous phase assemblages, such as feldspar and silica, to zeolites or clays. Experiments to examine the phase changes in tuffs of varying mineral composition at known values of pressure and temperature have been started. The samples are ground and enclosed in gold capsules with water present. The capsules are then placed in standard cold-seal pressure vessels, which are pressurized and heated to the desired conditions. Preliminary hydrothermal experiments have provided evidence for the upper thermal stabilities of clinoptilolite and mordenite at 400 bars water pressure. The upper stability of mordenite is probably between 300 and $400^{\circ} \mathrm{C}$; that for clinoptilolite appears to be below $300^{\circ} \mathrm{C}$.

A study has been initiated to evaluate the geochemical stability of potential shaft and borehole sealing materials in the felsic volcanic-tuff environment of Yucca Mountain. The investigation deals with the chemical compatibility of potential sealing materials and felsic tuff.

The term "sorption" has generally been uised to describe processes by which elements are removed from solution by rock, such as ion-exchange phenomena, chemisorption, and diffusion into the rock matrix. Precipitation or coprecipitation can also occur and remove elements. Los Alamos has used batch and 
several types of column techniques in the laboratory to provide information on sorption processes, speciation, kinetics, diffusion, and surface effects. Sorption data have been obtained for samples from drill holes J-13, UE25a-1, and USW-G1 under different conditions (contact time, temperature, atmosphere, and particle size). Detailed data are given in the body and appendixes of this report.

The variation in the abundance of clinoptilolite, as well as that of other minerals in tuff, is related to the mode of emplacement and to alteration processes during cooling and by interaction with groundwater. Strontium, cesium, and barium are thought to sorb mainly by ion-exchange reactions. Their lowest sorption ratios or $R_{d}$ values (defined in the introduction) are associated with devitrified tuffs, which are generally welded to some degree and contain principally quartz, cristobalite, and alkali feldspar (plus some clays). The maximum sorption ratios correspond to nonwelded tuffs that contain the zeolite clinoptilolite. The variations of sorption of cerium, europium, plutonium, and americium with stratigraphy are not as regular as those for strontium, cesium, and barium. The chemistry of these elements is more complex in the near-neutral groundwater. The sorption ratios for plutonium cover a fairly narrow range, independent of sample location or mineralogy. In comparing americium's sorption ratios with its mineralogy, there is a rough correlation of high sorption with samples containing clinoptilolite or smectite and a correlation of low sorption with samples containing devitrification minerals. Although sorption of technetium, uranium, and neptunium has not been measured for many samples, the sorption ratios are relatively low; correlations with stratigraphic position cannot be made from the available data.

Sorption ratios for each element have been plotted as a function of clinoptilolite abundance for all the samples studied. The samples containing no clinoptilolite have significantly lower sorption ratios than those containing more than a few per cent of the zeolite. If the abundance of this zeolite is the only factor influencing sorption ratios, with no differences in sorptive properties caused by the exact composition of the clinoptilolite (or heulandite), then there should be a linear relationship between the distribution coefficient $K_{d}$ and clinoptilolite abundance. Least squares fits to our data points, for which the abundance of clinoptilolite is $\geq 10 \%$, give sorption ratios of $6.9 \times$ $10^{4}$ and $4.3 \times 10^{4} \mathrm{ml} / \mathrm{g}$ for $100 \%$ of the pure minerals. These values are compared with those calculated using simplifying assumptions from available thermodynamic 
data for different inineral samples of $1.8 \times 10^{5}$ and $3.8 \times 10^{4} \mathrm{~m} / \mathrm{g}$ for strontium and cesium, respectively.

Sorption ratios for technetium, cerium, europium, and americium show no obvious correlations or trends with abundance of the zeolite clinoptilolite. Sorption ratios of samples with no clinoptilolite scatter among those for samples with the zeolite. Sorption ratios for neptunium, uranium, and plutonium are higher for the zeolitized tuffs than for the nonzeolitized ones, although there are no trends with degree of zeolitization. There is an absence of any obvious trend for cesium sorption when considering smectite alone in nonzeolitized samples. Possible explanations are that trace quantities of clinoptilolite, not detected by $x$-ray diffraction, may mask any influence of smectites; that other minerals such as illite also contribute to sorption; and that sorption on clays involves other factors such as their texture or their availability to the groundwater. Sorption ratios for samples containing the zeolite analcime are not as lárge as those ratios expected for samples containing clinoptilolite, an effect related.to the structures of the zeolites.

A model based on a sorptive mineral content (SMC) concept has been shown to agree well with experimental values for sorption of cesium on a variety of tuffs. Because tuff may be composed of more than one sorbing mineral, the SMC concept is used to predict sorption by combining the effects of several minerals. The combined effect is defined as a weighted sum $S M C=\Sigma W_{i} X_{i}$, where $W_{i}$ is the weighting factor for each mineral phase relative to that of clinoptilolite and $x_{i}$ is the abundance $(\%) c$ each phase. The weighting factors are relative to clinoptilolite, and they are calculated from published thermodynamic data, using some simplifying assumptions. Clinoptilolite, mortmorillonite, mordenite, analcime, and glass were considered. For cesium, sorption ratios plotted as a function of SMC normally follow the same trend as if clinoptilolite were the only sorbing mineral in a sample. The SMC concept will be extended to other elements.

In general, $R_{d}$ values from desorption experiments are slightly higher than those from sorption experiments. The results for strontium and cesium from the two methods agree within $\sim 20 \%$ for most measurements. For barium there is reasonable agreement when sorption ratios are low (devitrified tuffs). For some of the zeolitized tuffs, values for barium from desorption experiments are greater than those from sorption experiments by factors of $\sim 2$ for most samples but are as great as $\sim 10$ for a few. It appears that barium sorbs on 
clinoptilolite somewhat more irreversibly than do strontium and cesium. For cerium, europium, and americium, the differences in sorption ratios (which are reasonably high) obtained by the sorption and desorption methods are greater. A large fraction of these elements are sorbed irreversibly, although in most cases the trends from the sorption measurements with stratigraphic position are qualitatively retained in the desorption results. The differences for plutonium, which do not show discernible trends, are also approximately a factor of 10. It should be neted that sorption ratios for technetium, uranium, and neptunium, which are low, are nevertheless significantly greater for desorption than for sorption. Sorption ratios for plutonium cover a fairly narrow range (less than a factor of 10), independent of sample location or mineralogy. Based on the limited data available, neptunium exhibits similar behavior with a range of less than a factor of 5 , although the $R_{d}$ values are about an order of magnitude less than those for plutonium. Americium sorption ratios show a much wider variation, from just over $100 \mathrm{~m} / \mathrm{g}$ to nearly 30000 $\mathrm{ml} / \mathrm{g}$, but again with essentially no correlation to mineralogy. Sorption ratios for technetium and uranium are low; correlations with stratigraphic position or mineralogy cannot be made from the limited data available.

Results from three batcb sorption experiments performed at $70^{\circ} \mathrm{C}$ have been compared with those performed at room temperature. Values are similar, with those for $70^{\circ}$ being generally higher by factors up to 5 than those for room temperature.

The presence of very fine particles $(<38 \mu \mathrm{m})$ in rock fractions of larger particle size apparently can change the observed sorption ratio of an element by a factor of 2 to 5 , especially for devitrified tuffs. It is, therefore, advisable that larger size fractions be wet-sieved to avoid the presence of fine particles, which may increase the observed sorption in an irreproducible manner. The removal of small particles may result in measurements being made on material that is not completely representative of the tuff; however, the observed results are useful for comparison purposes and are probably not far from the "true" values. Any eirors should be in the conservative direction, that is, too low $R_{d}$ values for samples that do not contain very fine particles.

Because reducing conditions are expected for some groundwater/rock systems, it may be anticipated that the sorptive behavior of some elements in such systems will be different from that under normal atmospheric conditions. These effects were investigated by comparing the results of batch studies 
performed in a nitrogen atmosphere $(<0.2 \mathrm{ppm}$ oxygen and $<20 \mathrm{ppm}$ carbon dioxide) with similar measurements made under normal atmospheric conditions on the same geologic materials. Depending upon the element, sorption was observed to be lorer, higher, or unchanged for different elements in the nonoxidizing atmosphere. Technetium, in particular, showed the effects of the atmosphere, giving $R_{d}$ values in the nitrogen atmosphere greater than 10 times those in air.

The circulating system used at Los Alamos for sorption measurements incorporates features of both batch and column methodologies. The batch and circulating-system procedures are similar in some ways, but the solid phase remains stationary in the circulating system and is not subject to the possible self-grinding of the batch measurements. The presence of smaller particles could result in greater sorption as a result of greater surface area or differences in mineralogy. In most cases, the results from the two methods fall within the spread of individual experimental values. Devitritied tuffs tend to give slightly higher sorption ratios by the batch method than by the circulating-system method. The observed difference could well be the result of the presence of smaller particles in the batch measurements.

The study of sorption isotherms is important for several reasons; it will be used to (a) determine the influence of groundwater/tuff interactions on the sorptive properties of tuff, (b) accurately model the retardation of waste elements under various source-term andgroundwater conditions, (c) detect irreversible sorption processes, which would be a potentially highly positive property if present in tuff, (d) correctly interpret and model diffusion into the tuff matrix as would occur in fracture flow, and (e) explain the observed dependence of the sorption ratio (distribution coefficient) on the solution-tosolid ratio. The Langmuir and Freundlich isotherms have been used for these applications as well as to determine a relationship from mass-action equilibrium. Experimental fits to the Freundlich isotherms for strontium, cesium, barium, cerium, and europium generally indicate nonlinear behavior for nonzeolitized, welded tuffs and linear behavior for zeolitized tuffs. For plutonium, there appears to be little correlation between sorption ratio and the element concentration; the sorption isotherm for the zeolitized tuff is linear, whereas that of the devitrified tuff deviates. The effects of nonlinear isotherms on sorption phenomena have also been studied, and equations and computer programs to solve the diffusion equations with nonlinear isotherms have been developed. 
Some simple relations have been derived that can explain the dependence of $K_{d}$ on the solution-to-solid ratio. Experimental results in which the solution-tosolid ratio was varied can be directly compared with the results of measurements in which the element concentration was varied (isotherm determinations). These relationships are important because of the difference in solution-to-solid ratios between laboratory batch determinations and real situations.

Laboratory measurement of permeability and storage capacity has been accomplished by a transient pressure pulse method. Porosity has been obtained by measuring the wet and dry weights of the samples, by grain density measurements, and by mercury poresimetry. These measurements are basic to understanding the transport of waste elements in groundwater through tuff. Data for both permeability and storage capacity are necessary for comparison with field tests and to predict flow through unfractured tuff in response to a pressure gradient; this gradient might be the regional hydrologic gradient or the result of heating by the repository. Porosity is a necessary parameter in calculating the effects of diffusion. The combined results for permeability, storage capacity, and porosity can be used to gain insight into the pore structure of the tuff. This understanding of pore structure is important to the understanding of diffusion because it will help estimate constrictivity and tortuosity parameters. The permeability of tuff is quite low and contrasts with the relatively high porosity ( 7 to $40 \%$ ) of the samples tested. Porosity clearly does not determine permeability because there is no correlation between the two. Mercury porosimetry indicates the permeability is more closely related to pore size. In tuffs, low permeabilities are probably caused by small connections between larger pores, which suggests that the constrictivity of tuff may be relatively large. Permeability shows only a slight variation with effective confining pressure. The storage capacity and porosity values taken together indicate that the pore compressibility of tuff is quite small, and as a consequence the porosity of tuff also varies little with effective confining pressure. Because the permeability does not show a marked decrease with increasing effective confining pressure, it seems likely that the stiffness of the pores indicated by the pore compressibility applies to the small connections as well as to the larger pores that make up most of the porosity. Therefore, studies of the pore struc'ure of tuff made on unpressurized samples should ipply well to the rock at depth. Permeability measurements on tuff samples before and after heating wet at $120^{\circ} \mathrm{C}$ for 5.5 months show no significant change in permeability in samples composed predominantly of zeolites. However, 
some increase in permeability was observed in samples with quartz, cristobalite, and potassium feldspar as predominant phases.

Diffusion into the rock matrix is an important mechanism for retarding the transport of radionuclides through fractures in tuff. It is of particular importance for nonsorbing soluble species. The diffusion coefficient for a given radionuclide in tuff matrix depends on properties that are intrinsic to the chemical species, such as ionic mobility, and properties of the tuff, such as porosity, tortuosity, and $R_{d}$. It is, therefore, necessary to measure the diffusion coefficients of waste element species in various tuff units. An experimental program to accomplish this has been initiated using several cechniques.

In general, the equations that have been used to describe fracture flow with matrix diffusion and simple diffusion into tuffaceous rock treated sorption as iinear with concentration. This approach clearly has a serious deficiency because sorption on nonzeolitized tuff has already been shown to be nonlinear. Isotherm measurements on tuff YM-22 show that sorption of simple cations of strontium, cesium, and barium gives a Freundlich isotherm exponent <1.0. A nonlinear isotherm complicates the equations for matrix diffusion by giving the diffusion coefficient a concentration dependence, rendering the differential equations nonlinear. A computer program, using the finite difference method, is being developed to apply some of the mechanisms to matrix diffusion. Eventually, the program will be incorporated into a transport model so that a more realistic model can be developed.

Experiments specifically designed to examine transport in a single fracture provide information about the effectiveness of diffusion in retarding radionuclides and the effectiveness of sorption processes in rapidly flowing systems such as occur in a single fracture. The results of these experiments have been compared with transport model predictions to validate or demonstrate deficiencies in the models. The shape of the elution curve calculated for fracture flow in tuffs is not in agreement with the observed elution. The activity desorbs more slowly than would be expecked for reversible, diffusion-controlled sorption. This observation is also consistent with previous measurements of sorption on tuff. In general, the $R_{d}$ values determined by desorbing activity from tuff are considerably larger than those determined from the sorption process. The lack of agreement between the experimental and theoretical elution curves suggests a more complex sorption mechanism than simple linear sorption; sorption and matrix diffusion in tuff (especially welded tuff) appear to be more complex than expected. 
Imporiant conclusions resulting from laboratory fracture-flow studies are that (1) matrix diffusion is an important mechanism contributing to the retardation of radionuclides in fracture flow; however, simple analytic models do not appear to be adequate to predict accurately the transport of waste elements in tuff fractures; (2) the high porosity of tuff makes matrix diffusion much more effective in retarding the movement of soluble species than does the low porosity of crystalline rock such as granite; and (3) undisplaced, induced Bullfrog- and Tram-Member tuff fractures subjected to a simulated lithostatic st.ress of 3000 psi sealed to cause a fracture permeability comparable to that of the undisturbed matrix.

Experiments examining the transport of radionuclides through porous media have been conducted in an effort to determine the radionuclide retardation that will be provided by geochemical processes along flow paths. These experiments, using both crushed-tuff and solid-tuff columns, provide intermediate steps in the laboratory-to-field link. Chrcimatographic columns packed with crushed rock have been used for most of the studies. The following radionucides have been used: ${ }^{131} \mathrm{I},{ }^{85} \mathrm{Sr},{ }^{137} \mathrm{Cs},{ }^{133} \mathrm{Ba},{ }^{141} \mathrm{Ce},{ }^{152} \mathrm{Eu},{ }^{95} \mathrm{Tc}$, and ${ }^{3} \mathrm{H}$. Some of the columns have been run at two flow rates. The crushed-rock columns have begun to provide information on dynamic effects in radionuclide transport through porous media. In addition to the crushed-rock columns, some solid-core columins have been run and more solid-core column experiments are being run. These columns are providirg data to establish whether minerals are made available by crushing that are not naturally available and also to examine dynamic effects. General ronclusions from these studies are as follows. (1) The sorption ratios determined by using column methods agree with those determined by the batch techniques within a factor of 10 . Recent studies indicate that much of the previously reported disparity between batch and column results may be raused by particle fractionation. When the same particle-size distributions are used, the results are in reasonable agreement. (2) At water velocities comparable to regional flow velocities $\left(\sim 10^{-5} \mathrm{~cm} /\right.$ second), the shapes of peak elutions for some simple ions are comparable to what would be expected from diffusional broadening alone. This may indicate that at these velocities, kinetics is not an important factor. (3) The anion exclusion effect may have been observed in a highly zeolitized tuff. (4) Plutonium particulate matter was filtered out by flow through a solid-core column.

Kinetic sorption experiments (sorption as a function of time) have been performed on thin tablets of tuft. The uptake of activity has been measured 
as a function of time for a number of elements on several tuffs. These data should fit the solution for diffusion into a plane sheet if one ignores any edge effect. When the analytic solution to a one-dimensional plane sheet is applied to the data, a good fit cannot be achieved. This is typical of the nonsteady state diffusion experiments that have been performed with tuff samples.

Some new fluorobenzoate tracers, with very low detection limits, have been developed to use in field experiments. The diffusion coefficients of these tracers have been measured so that their usefulness as diffusing tracers in characterizing fracture-flow systems can be evaluated. The ionic diffusion coefficients are generally close to $8.0 \times 10^{-6} \mathrm{~cm}^{2} /$ second. This is considerably lower than the diffusivity of tritiated water $\left(2.4 \times 10^{-5} \mathrm{~cm}^{2} /\right.$ second $)$ and has potential application to field experiments.

The study of natural analogues to waste repository environments can give important information on long-term chemical reaction and transport. Such analogues can be used to extrapolate experimental data from laboratory time, days and months, to "geologic time," the hundreds to hundreds of thousands of years that may be required for isolation of waste in a repository. A potentially important source of information on the long-term behavior of Yucca Mountain tuffs in a hydrothermal gradient is the study of hot-spring environments in felsic tuffs in Nevada. The mineral alteration in these localities can give information about (1) the response of the near-field repository environment to the thermal pulse that is expected after the initial emplacement of the waste and (2) the response to the normal geothermal gradient, the natural increase of temperature with depth. Another useful analog study may be the investigation of rock matrix diffusion of elements near ore bodies. Matrix diffusion has been proposed as a process that would retard the transport of elements in media in which the hydrology is dominated by fracture flow. Recent measurements of lead isotope abundance in a rock core from the Oklo uranium mines suggest that lead may have diffused into a crystalline rock matrix from a fracture that was an aqueous transport channel in past geologic times.

Field experiments are performed to collect radionuclide migration data under conditions that approximate those of a nuclear waste repository more closely than can be obtained in laboratory measurements. Data from field experiments will be used for two primary purposes: to verify the accuracy of 
models used for repository performance assessment and to determine the extent to which laboratory measurements can be scaled to give results valid for field conditions.

The techniques used in field migration studies are of two types: one addresses aqueous flow and transport through a fracture, and the other addresses diffusion into the rock matrix from water but without aqueous flow. Three fracture-flow experiments performed by others have been reported in some detail; all are in granitic rock. A nuclide migration field experiment in tuff was begun, but geochemical and hydraulic conductivity measurements showed that a more suitable tuff and location than the one originally chosen must be selected for this work.

Geochemical and transport models have been developed and tested both in support of the nuclear waste management programs at Los Alamos and to contribute to development of an overall performance model. The efforts in geochemical modeling have concentrated on testing the available codes and improving the thermodynamic data base. Geochemical models can be used to predict the chemical species that should occur in a groundwater system and also the mineral solubility and solubility limits for the waste element species. These predictions influence the expected retardation of the waste elements. For instance, an anionic species would not be sorbed by zeolite minerals, whereas most cations are strongly sorbed. Currently the data base for these geochemical models is being updated to include thermodynamic data for the minerals composing Yucca Mountain tuff. The geochemical model EQ3 has been used to calculate uranium and plutonium solubilities in water from well $\mathrm{J}-13$.

Several transport codes available at Los Alamos can be used to model a variety of problems, including multiphase flow, unsaturated flow, the inverse problem, fracture flow, and three-dimensional systems. Transport models are being updated to include appropriate sorption mechanisms and to account for the dependence of sorption on concentration, that is, nonlinear isotherms. These codes will provide a means of modeling field and laboratory experiments. In the near future it is hoped that some of these codes can be validated by designing and executing appropriate experiments in the laboratory and field. Code TRACR3D simulates transient air, water, and tracer flow in permeable media for a three-dimensional geometry. Tracer motion can occur in either the air phase or the liquid phase. Transport mechanisms include advection; molecular diffusion, mechanical dispersion, and capillary action. Several equili- 
brium sorption models as well as nonequilibrium sorption model are included. Material properties such as permeability can vary spatially. The code also has the capability of simulating flow in a fracture system with transport into or out of a porous material surrounding the fractures. This code has been used to model a field experiment with a single fracture to predict the effect of partial saturation and to aid in the design of such experiments.

The second part of this report delineates the proposed experimental program that will be necessary to resolve those geochemistry issues of importance to the use of the Yucca Mountain area of the NTS as a nuclear waste repository site. For the most part, these studies will be natural extensions of those already in progress and described above, with the addition of some complementary experiments. In particular, Los Alamos has not been using isotopic techniques to obtain information about Yucca Mountain, and believes such techniques used by the US Geological Survey for Yucca Mountain investigations have been limited to ${ }^{14} \mathrm{C}$ and ${ }^{18} \mathrm{O}$ analyses. The information that can potentially be obtained from isotopic analyses includes (1) origins of the groundwaters and pore waters, (2) flow paths and mixing of aquifers, (3) age and age gradients of the water, (4) paleoclimate information, and (5) natural water/rock interactions and geothermometry.

Work is planned that will use cosmogenically produced ${ }^{36} \mathrm{Cl}$ (half-life $3 \times 10^{5}$ years) to measure the ages of old groundwaters and "bomb pulse" ${ }^{36} \mathrm{Cl}$ to measure the ages of old groundwaters and "bomb pulse" ${ }^{36} \mathrm{Cl}$ to measure recent rates of water movement in the unsaturated zone. Other nuclides being considered are ${ }^{2} \mathrm{H},{ }^{3} \mathrm{H},{ }^{4} \mathrm{He},{ }^{13} \mathrm{C},{ }^{14} \mathrm{C},{ }^{18} \mathrm{O},{ }^{34} \mathrm{~S},{ }^{39} \mathrm{Ar},{ }^{81} \mathrm{Kr},{ }^{129} \mathrm{I}$, and uranium and radium isotopes and their daughters.

Obviously, isotope techniques can yield useful information about how the natural system has behaved over recent and geologic time. Interpretation requires wise selection of the proper models. 
Chapter 1:

INVESTIGATIONS OF THE GEOCHEMISTRY OF YUCCA MOUNTAIN AND ENVIRONS 


\section{INTRODUCTION}

This report details the technical contributions of Los Alamos National Laboratcry to the Nevada Nuclear Waste Storage Investigations (NNWSI) project since the time of its inception in FY 1977 (as the NTS Terminal Waste Storage project) until March of 1982. The NNWSI project is managed by the Nevada Operations Orfice of the Department of Energy. Efforts have been devoted primarily to resolving geochemistry issues pertinent to siting a nuclear waste repository in tuff at the Nevada Test Site (NTS).

The Los Alamos National Laboratory studies of the sorptive behavior of tuff and transport of radionuclides through tuff have been partly generic in nature (to understand the sorptive behavior of tuff as a function of many variables) and partly site specific (to obtain data for a possible repository site in tuff). It is necessary to have an understanding of the mechanisms of radionuclide transport and retardation in tuff, as well as to have a data base of sorptive behavior, to perform the required safety assessment dealing with possible releases from a repository in tuff. Previous reports in this series are Refs. 1,2 , and 3 .

When the work was initiated, the only appropriate tuff samples for this study were from drill hole (later well) J-13 (Ref. 4) in western Jackass Flats at the NTS. When the NNWSI identified Yucca Mountain as a possible repository location and undertook an exploratory drilling program, samples from additional drill holes became available.

Tuffaceous groundwater used in these studies has been obtained from well J-13 in Jackass Flats except for studies of the Yucca Mountain groundwaters themselves. To better simulate water in contact with the rocks under investigation, the water from well $\mathrm{J}-13$ is pretreated with the particular rock of interest. Analyses of the water before and after such treatment have indicated only minor changes in composition.

Perhaps a more critical problem is simulation of the redox conditions that exist in the actual rock/groundwater systems. If the underground conditions are reducing, as is postulated for many deep geologic systems, then the sorptive behavior of elements such as technetium, uranium, neptunium, and plutonium will be different from that under the normal, mildly oxidizing conditions in air. In their lower oxidation states, these elements are generally more insoluble or sorb better on geologic media and, consequently, should be retarded more than in the higher states. However, at the present time, there is no 
definitive description of the actual redox conditions in the tuff formations under investigation or of conditions that might be in possible release scenarios. Preliminary observations of the alteration features of the mafic minerals in zones of the bedded tuff of Calico Hills and Prow Pass Member of the Crater Flat Tuff suggest possible oxidizing conditions, at least at the time when alteration was occurring. Observations of the dissolved oxygen content of water pumped from the $\mathrm{J}-13$ well and drill holes at Yucca Mountain also suggest oxidizing conditions; however, results from the analysis of groundwaters from hydrology wells at Yucca Mountain indicate that deeper waters may be oxygen deficient when compared to water at the standing water level. Independent work by the US Geological Survey (USGS) has shown that there are significant concentrations of dissolved oxygen in water from tuffaceous wells ( $J-12$ and $\mathrm{J}-13$ ), as well as in some other deep water. ${ }^{5}$ However, contact of crushed tuff with groundwater under a controlled atmosphere $(\leq 0.2 \mathrm{ppm}$ oxygen, $\leqq 20 \mathrm{ppm}$ carbon dioxide) gave apparently negative Eh values. ${ }^{3}$ the crushing may have exposed previously unexposed minerals. Investigations have been conducted under both atmospheric and near-oxygen-free conditions and there are plans to do experiments under controlled Eh conditions.

Buffers are being developed to control the oxidation potential during labolatory experiments. Formation and transport of particles containing actinides (and presumably other radionuclides) have been found to be important in waste element mobility. Because $\mathrm{Pu}(4+)$ polymer may be mobile, its behavior and rate of formation are being studied. Waste element sorption has been found to correlate with stratigraphy and mineralogy. The capability has been developed to predict retardation properties, based on thermodynamics and the sorptive mineral content. A strategy for identifying and obtaining geochemical thermodynamic data was developed and implemented. Matrix diffusion and hydraulic properties were found to correlate with pore structure. Predictions of diffusion of nonsorbing tracers agree with experiments, but problems have been encountered in predicting the behavior of sorbing elements, presumably because of slow kinetics and nonequilibrium. A field test program has been defined and will be used to validate geochemical transport models.

The sorption ratio, designated by $R_{d}$, is used as a measure of sorption as a function of many parameters. It is defined as

$$
R_{d}=\frac{\text { activity in solid phase per unit mass of solid }}{\text { activity in solution per unit volume of solution }}
$$


Many authors refer to this ratio as the distribution coefficient $\mathrm{K}_{\mathrm{d}}$. Los Alamos prefers not to use this term, which implies equilibrium, knowing that reversible equilibrium is usually not attained. If equilibrium is attained, then $K_{d}$ is related to a retardation factor, $R_{f}$, in a uniform flowing system by

$$
\mathbf{R}_{\mathbf{f}}=\mathbf{K}_{\mathbf{d}}(\rho / \varepsilon)+1
$$

where $\rho$ is the bulk density and $\varepsilon$ is the porosity.

A detailed listing of the measured sorption ratios is given in App. A. The origin of the tuff samples studied and their mineralogic composition have been discussed in an earlier report ${ }^{6}$ or are given in this report. Petrographic descriptions of tuff thin sections are given in App. B. The prefix JA- indicates the sample was obtained from hole J-13; the prefix YM- from hole UE25a-1. The prefix G⿰三丨⿰丨三 indicates the sample was obtained from drill hole USW-G非 the four numbers following the prefix give the depth in feet from which the sample was obtained.

These investigations were performed under the Los flamos quality assurance program for the NNWSI, which is designed to ensure that the data and interpretive reports produced are consistent with formally specified procedures and reviews.

Responsibility for the planning and implementation of the Los Alamos quality assurance program rests with the Materials Science and Technology Division (MST) quality assurance organization. Reference 7 contains a complete account of the Los Alamos NNWSI quality assurance program, including the Quality Assurance Program Plan and the detailed procedures developed for the project.

The quality assurance program developed for the NNWSI at Los Alamos is outlined in the Quality Assurance Program Plan (QAPP), which is updated to meet changing requirements. It is structured to meet the requirements of 10-CFR-50, App. $\mathrm{B}^{8}$ as applied to the evaluation of major geologic formations with regard to their suitability as locations of permanent repositories for high-level radioactive wastes. The Los Alamos Quality Assurance Manual ${ }^{9}$ (now being revised) is used as a primary compliance document. The procedures described therein are applicable unless otherwise stated in the QAPP or specific procedure documents issued for the NWWSI.

Work Plans are the primary planning documents covering the Los Alamos technical activities for the NNWSI. These Work Plans are written to provide 
an adequate description of the scope and purpose of the task. They include, directly or by reference, the quality assurance requirements with regard to data validity and documentation. Review boards are used for the quality assurance program acceptance of the Work Plans, as well as for review and acceptance of design and acceptance of final documents. The review board consists of, at a minimum, a management member, a quality assurance member, and an independent technical reviewer who is experienced and competent in the field under review but has no direct program responsibility.

In addition to the QAPP, quality assurance procedures specific to the Los Alamos effort in the NNWSI have been established for document control and procurement. These procedures supplement the guidelines in the Quality Assurance Manual; they are given in full in Ref. 7. Detailed quality assurance procedures have also been prepared for most of the technical areas of the project, including all facets of obtaining, handling, and shipping geologic samples; mineralogical and petrological tests; geophysical and geochemical measurements; and radionuclide interactions with geologic materials in both laboratory and in situ experiments. Revisions of these technical procedures are prepared as necessary as more experience is gained or better techniques are developed. Reference 7 contains the current versions of these quality assurance procedures. A one-time research effort may be documented in a Los Alamos notebook with, as a minimum, technical approval at defined intervals.

Periodic surveillance in accordance with pre-established check lists is used to maintain quality assurance standards in technical efforts. Measuring and testing equipment that require calibration is controlled in accordance with the applicable sections of the Quality Assurance Manual. Corrective action for signjficant conditions adverse to quality is provided in accordance with Sec. QMR 12 of the manual. Compilance with the quality assurance program is verified by periodic audits that are planned, documented, and carried out in accordance with Sec. QMR 15 of the Quality Assurance Manual. 


\section{GROUNDWATER GEOCHEMISTRY}

\section{A. Groundwater Chemistry}

1. Reference Groundwater for Laboratory Experiments. In a laboratory sorption experiment that duplicates field conditions for a specific location, one problem is the selection of the reference composition of the groundwater. Ideally, water from individual tuff layers in Yucca Mountain would be used, but at this time there is no producing well at Yucca Mountain and no way of obtaining formation water from particular tuff layers. Therefore, the water from well J-13, the nearest producing well, was chosen as reference water, and it his been used for several years in all sorption experiments. At the end of 1980, this reference-water composition was as shown in Table I, based on multiple analyses of well J-13 water by the USGS. Because the composition of the water directly from the well may change slightly over time depending on well usage, a standard composition for well J-13 water composition was established.

\section{TABLE I}

REFERENCE GROUNDWATER COMPOSITION FOR TUFF

FILTERED THROUGH $0.45-\mu \mathrm{m}$ MILLIPORE FILTER

\begin{tabular}{lc} 
Emission Spectroscopy & Concentration $(\mathrm{mg} / \mathrm{l})$ \\
\cline { 2 - 2 } Magnesium & 2.1 \\
Silicon & 31 \\
Iron & 0.04 \\
Strontium & 0.05 \\
Barium & 0.003 \\
Calcium & 14 \\
Lithium & 0.05 \\
Potassium & 4.9 \\
Aluminum & 0.03 \\
Sodium & 51
\end{tabular}

Anion Chromatography

Fluoride 2.2

Chloride

7.5

Phosphate

0.12

Nitrate 5.6

Sulfate 22

Carbonate 0.0

Bicarbonate 120

Other Conditions

$\mathrm{pH}$ - slightly basic $(7.1)$ 
Examples of well J-13 water composition now and the extent of change during specific time periods are shown in Table II. The first six analyses are of water collected in plastic-lined barrels in June 1981 over several hours. The last analysis is of water collected in January 1982. Before analysis the waters were filtered through $0.05-\mu \mathrm{m}$ Nuclepore membranes and then acidified with ultrapure $\mathrm{HNO}_{3}$ for cation analysis. This table shows that with time there are minor variations in composition beyond the standard deviation and limits of detection of the elements; however, these variations are minor when compared to other variables in experiments in which the water is used.

The techniques to determine the cation and anion concentrations listed in the tables of this section are state-of-the-art techniques. Cations are analyzed on acid-stabilized solutions with a Spectrometrics, Inc., 20-channel, direct-current, plasma-source, emission spectrometer. Analyses for anion concentrations are performed on a Dionex Ion Chromatograph; alkalinity and $\mathrm{pH}$ are measured on a Brinkmann Metrohm Dosimat and Titroprocessor.

The analytical techniques for $\mathrm{NO}_{2}^{-}$and $\mathrm{NO}_{3}^{-}$, arsenic at $<0.1 \mathrm{mg} / \mathrm{l}$, and $\mathrm{Fe}^{2+}$ at $<0.02 \mathrm{mg} / \ell$ need further development. These analyses are especially important because the ions may be the major oxidizing-reducing species in Yucca Mountain waters.

As pumped From the well, J-13 water contains $\sim 5.5 \mathrm{ppm}$ oxygen and exhibits a $\mathrm{pH}$ of $\sim 7.1$. As the water stands, the $\mathrm{pH}$ slowly increases--presumably the result of a loss of $\mathrm{CO}_{2}$ from the water.

2. Water for Sorption Experiments. In preparation for experiments to determine the sorption of waste elements on tuff, well J-13 reference groundwater was precontacted with tuff from individual strata of Yucca Mountain and then was filtered through $0.05-\mu \mathrm{m}$ Nuclepore membranes. The three parts of Table III illustrate changes in the composition of wel1 J-13 water that occur when it is contacted with tuff for $\sim 3$ weeks. The sample number in the table reflects the depth in feet in the drill hole from which the sample was taken (for example, sample G1-1854 was taken from well USW-G1 at 1854-ft depth) and the sample sequence number in the $J-13$ drill hole (JA-18).

The tuff sample JA-18 water results are derived from contacting well J-13 water with tuff from the lower Topapah Spring Member, which was sampled during the drilling of the $J-13$ well and is from the approximate region of main water production in the well. It was expected that this tuff would be already in equilibrium with the water, but, as shown in Table III, the magnesium concen- 
TABLE I I

COMPOSITION OF WELL J-13 GROUNDWATER

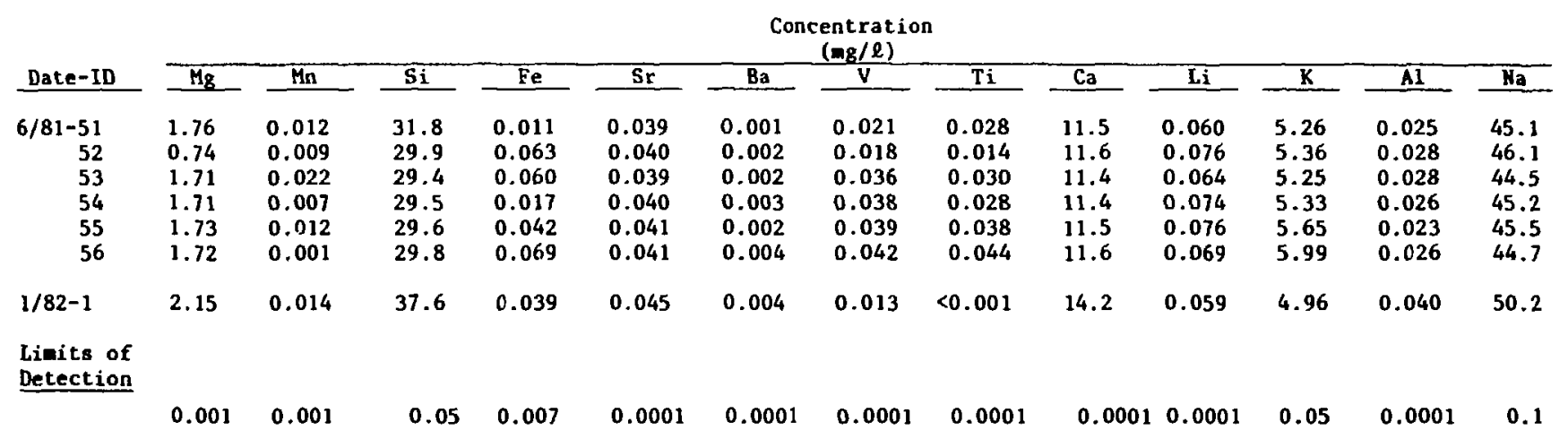

Typical Standard

Deviation of

Instrupental A.talysis

\begin{tabular}{|c|c|c|c|c|c|c|c|c|c|c|c|}
\hline \\
\hline & 0.016 & 0.001 & 0.007 & 0.005 & 0.001 & 0.005 & 0.13 & 0.005 & 0.09 & 0.005 & 0.9 \\
\hline Date-ID & $\mathbf{F}^{-}$ & $\mathrm{Cl}^{-}$ & $\mathrm{PO}_{\mathrm{a}}^{3-}$ & $\mathrm{NO}_{3}^{-}$ & $\mathrm{SO}_{3}^{2-}$ & Alk & & pH & & & \\
\hline $6 / 81-51$ & $2.1^{3}$ & $6.4^{\mathrm{a}}$ & $0.1^{\mathrm{a}}$ & $10.1^{\mathrm{a}}$ & $18.1^{\mathrm{a}}$ & $2.339^{b}$ & & 6.9 & & & \\
\hline $1 / 82-1$ & 1.8 & 6.3 & $<0.1$ & 9.1 & 18.3 & 2.089 & & 8.3 & & & \\
\hline \multicolumn{12}{|c|}{$\begin{array}{l}\text { Typical } \\
\text { Standard } \\
\text { Deviation }\end{array}$} \\
\hline & 0.1 & 0.4 & 0.01 & 0.4 & 0.1 & 0.04 & & & & & \\
\hline
\end{tabular}


TABLE III

COMPOSITION OF WELI. J-13 GROUNDWATER

AFTER CONTACT WITH USW-GI AND J-13 TUFFS

Concent ration

(mg/l)

Original groundwater ${ }^{a}$

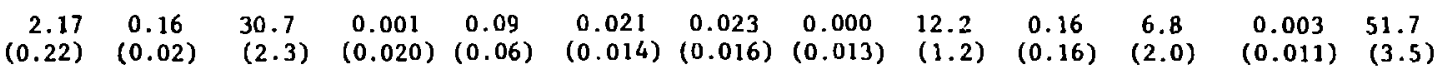

Tuff filtered through $0.45-\mu \mathrm{m}$ membrane

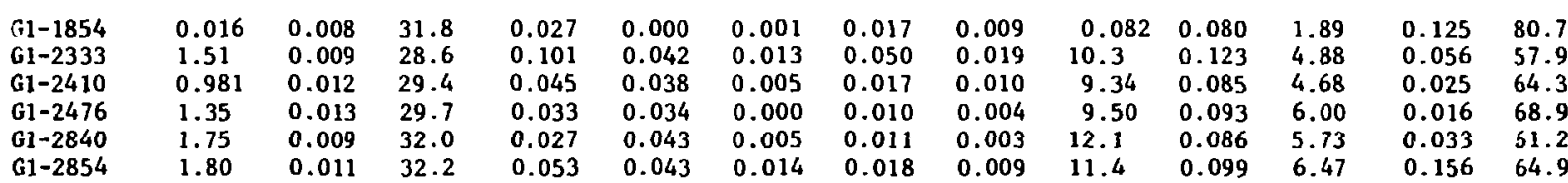

Tuff filtered through $0.05-\mu m$ membrane

\begin{tabular}{|c|c|c|c|c|c|c|c|c|c|c|c|c|c|}
\hline $\begin{array}{l}G 1-2289 \\
G 1-2363 \\
G 1-2476 \\
G 1-2539 \\
G 1-2840\end{array}$ & $\begin{array}{l}0.121 \\
1.04 \\
1.03 \\
0.848 \\
1.66\end{array}$ & $\begin{array}{l}0.004 \\
0.008 \\
0.050 \\
0.012 \\
0.009\end{array}$ & $\begin{array}{l}32.3 \\
31.0 \\
26.6 \\
25.5 \\
33.2\end{array}$ & $\begin{array}{c}<0.007 \\
<0.007 \\
<0.007 \\
0.0316 \\
<0.007\end{array}$ & $\begin{array}{l}0.001 \\
0.036 \\
0.040 \\
0.012 \\
0.037\end{array}$ & $\begin{array}{c}0.006 \\
0.034 \\
0.149 \\
<0.0001 \\
0.002\end{array}$ & $\begin{array}{l}0.007 \\
0.007 \\
0.002 \\
0.011 \\
0.010\end{array}$ & $\begin{array}{c}<0.0001 \\
<0.0001 \\
<0.0001 \\
<0.0001 \\
0.001\end{array}$ & $\begin{array}{c}0.414 \\
10.3 \\
10.4 \\
4.66 \\
11.9\end{array}$ & $\begin{array}{l}0.048 \\
0.058 \\
0.066 \\
0.067 \\
0.057\end{array}$ & $\begin{array}{c}1.29 \\
4.81 \\
8.71 \\
10.1 \\
5.07\end{array}$ & $\begin{array}{l}0.008 \\
0.011 \\
0.009 \\
0.062 \\
0.010\end{array}$ & $\begin{array}{l}75.5 \\
53.5 \\
67.3 \\
69.7 \\
48.3\end{array}$ \\
\hline$J A-18$ & 0.949 & .007 & 3.4 & 0.004 & .002 & 0.001 & 0.011 & $<0.005$ & 11.0 & .054 & 6.4 & 0.015 & 54.1 \\
\hline
\end{tabular}

Well J-13 water; no contact with solid. Mean of seven measurements made over preceding 6-month period.

Value in parentheses is the standard deviation of the mean for the well $\mathrm{J}-13$ water. 
tration of the water does change on further equilibration. No conclusive explanation can be given at this time; the carbon dioxide content of the water in the laboratory is probably less than at depth, the temperature is lower in the laboratory by $4^{\circ} \mathrm{C}$, and the groundwater may actually not be in equilibrium with the tuff.

Table III also illustrates changes observed in well $\mathrm{J}-13$ water that is contacted with tuffs from various depths of the USW-G1 drill hole. The main differences are a decrease in sodium content and an increase in the magnesium content of the water with depth in the drill hole. There is also a difference in waters filtered through $0.05-\mu \mathrm{m}$ Nuclepore membranes rather than $0.45-\mu \mathrm{m}$ Millipore filters. For example, the iron content is drastically reduced when filtered through the finer membrane. Because accurate iron contents may be very important for estimating the oxidation-reduction capabilities of solutions, the Los Alamos procedure recommends filtering all solutions through $0.05-\mu \mathrm{m}$ Nuclepore membranes. With state-of-the-art equipment, filtration through membranes with smaller pores is too difficult and time consuming.

An additional example of the importance of filtration is given in Table IV. This well J-13 water was contacted with tuffs from the NTS G tunnel (outside the proposed repository site) and then was filtered in various ways. The tuff from the G-tunnel beds exhibited a much greater tendency to produce small particles and colloids than any of the tuffs studied in the NNWSI program.

3. Temperature Effects on Groundwater Composition. A major question to be answered by this study is whether or not a temperature increase such as that caused by the heat from the waste package will change the water composition enough to affect the transport of waste elements in the groundwater. In short-term experiments three tuffs from Yucca Mountain were contacted with groundwater at $152 \pm 1^{\circ} \mathrm{C}$ to study possible reactions between the solid and solution phases.

Three tuff samples of different lithologies were used (Tables $V$ and VI). Before contact with groundwater, the samples ( 2 $\mathrm{mm}$ thick by $19 \mathrm{~mm}$ in diameter) were examined with a scanning electron microscope (SEM) to observe general surface features and mineral phases. The tuff wafers were then contacted with water from well J-13 in Teflon-lined Parr bombs. A Teflon screen separated the wafer and solution, and contact between the two was made by inverting the Parr bomb during the experiment. In future experiments the waters will be filtered at temperature before analysis because the increases in silicon and 
TABLE IV

EFFECT OF FILTRATION ON APPARENT COMPOSITION

OF WELL J-13 WATER $^{a}$

\begin{tabular}{|c|c|c|c|}
\hline \multirow[b]{2}{*}{ Element } & \multicolumn{3}{|c|}{$\begin{array}{c}\text { Concentration } \\
(\mathrm{mg} / \mathrm{l})\end{array}$} \\
\hline & $0.45 \mu \mathrm{m}^{\mathrm{b}}$ & $0.40 \mu \mathrm{m}^{\mathrm{c}}$ & $0.05 \mu \mathrm{m}^{\mathrm{c}}$ \\
\hline $\mathrm{Mg}$ & 4.0 & 0.11 & 0.08 \\
\hline Mn & 7.4 & 0.058 & 0.021 \\
\hline $\mathrm{Si}$ & 219 & 28.3 & 27.1 \\
\hline $\mathrm{Fe}$ & 82.0 & 1.5 & 0.6 \\
\hline $\mathrm{Sr}$ & 0.045 & 0.000 & 0.001 \\
\hline $\mathrm{Ba}$ & 9.0 & 0.05 & 0.12 \\
\hline v & 0.17 & 0.005 & 0.008 \\
\hline $\mathrm{Ti}$ & 14 & 0.09 & 0.00 \\
\hline $\mathrm{Ca}$ & 5.4 & 0.70 & 0.56 \\
\hline$L \mathbf{i}$ & 80 & 0.059 & 0.050 \\
\hline K & 30 & 4.5 & 4.9 \\
\hline $\mathrm{Al}$ & 39 & 0.7 & 0.009 \\
\hline $\mathrm{Na}$ & 102.5 & 94.1 & 89.0 \\
\hline
\end{tabular}

TABLE $\mathrm{V}$

TUFF SAMPLES FOR INITIAL ALTERATION STUDY

Sample

G1-1292

G1-1436

G1-2476
Unit

Topopah Spring

Tuffaceous beds of Calico Hills

Bullfrog II
Rock Type

vitrophyre

zeolitized nonwelded tuff devitrified welded tuff 


\begin{tabular}{|c|c|c|c|c|c|}
\hline Sample & $\begin{array}{c}\text { Glass } \\
(\%)\end{array}$ & $\begin{array}{c}\mathrm{Clay}^{\mathrm{a}} \\
(\%) \\
\end{array}$ & $\begin{array}{c}\text { Zeolite } \\
(\%)\end{array}$ & $\begin{array}{l}\text { Alkali } \\
\text { Feldspar } \\
(\%) \\
\end{array}$ & $\begin{array}{c}\mathrm{SiO}_{2}{ }^{\mathrm{C}} \\
(\%)\end{array}$ \\
\hline G1-1292 & $80-90$ & $\operatorname{tr} \mathrm{mnt}$ & 0 & $5-10$ & $5-10 \mathrm{cr}$ \\
\hline \multirow[t]{2}{*}{ G1-1436 } & 0 & $\operatorname{tr} i / m$ & $65-85$ & $5-10$ & $10-20 \mathrm{qz}$ \\
\hline & & & & & $2-5 \quad$ cr \\
\hline \multirow[t]{2}{*}{ G1-2476 } & 0 & $\operatorname{tr} \mathrm{mnt}$ & 0 & $30-50$ & $25-40 \mathrm{qz}$ \\
\hline & & $2-5 \mathrm{i} / \mathrm{m}$ & & & $5-10 \mathrm{cr}$ \\
\hline
\end{tabular}

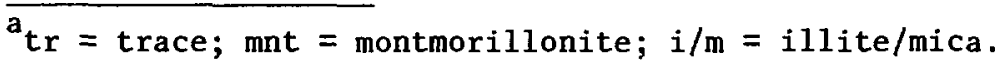

${ }^{b}$ clinoptilolite.

$\mathrm{c}_{\mathrm{cr}}=$ cristobalite; $\mathrm{qz}=$ quartz.
}

iron may be in the form of suspended colloids or polymers that will be filtered out of solution.

After a contact time of 3 weeks at elevated temperature, the bombs were reinverted to separate the phases and were allowed to cool. The tuff samples were again examined by SEM to determine if reaction had occurred. Sample G1-2476 containing cristobalite, alkali feldspar, and silica showed little reaction other than some rounding of surfaces and precipitation of clays. The vitrophyre, sample G1-1292, showed greatly increased amounts of clays or other fine-grained sheet silicates, which had formed on glass edges. Globules, analyzed as pure $\mathrm{SiO}_{2}$, also were observed. An unusual surface fracture network developed (Fig. 1), and in some cases these fractures were filled with a phase of the same composition as that of the glass. Sample G1-1436 showed marked dissolution of clinoptilolite crystals, and the latest formed phases, mordenite and cristobalite, which were observed by SEM before reaction, apparently dissolved. The SEM photographs (Figs. 2 and 3) show sample G1-1436 before and after contact with well $\mathrm{J}-13$ water. Figure 2 shows clumps of fresh cristobalite crystals over clinoptilolite before the experiment. After soaking at elevated temperature, the cristobalite is no longer present and the clinoptilolite is distinctly etched (Fig. 3).

In Table VII cation concentrations in the solutions after contact at $152^{\circ} \mathrm{C}$ are compared with cation concentrations in we1l J-13 water treated with 


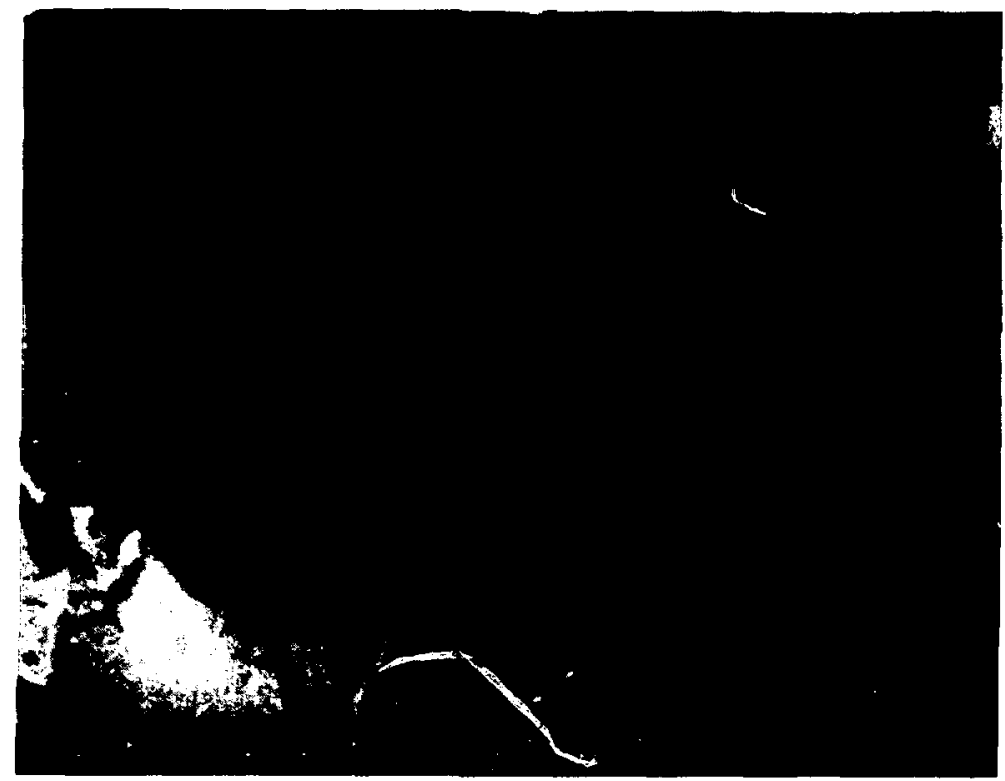

Fig. 1. SEM photograph of sample G1-1292 after contact with water from well $\mathrm{J}-13$ at $152^{\circ} \mathrm{C}$ for 3 weeks .

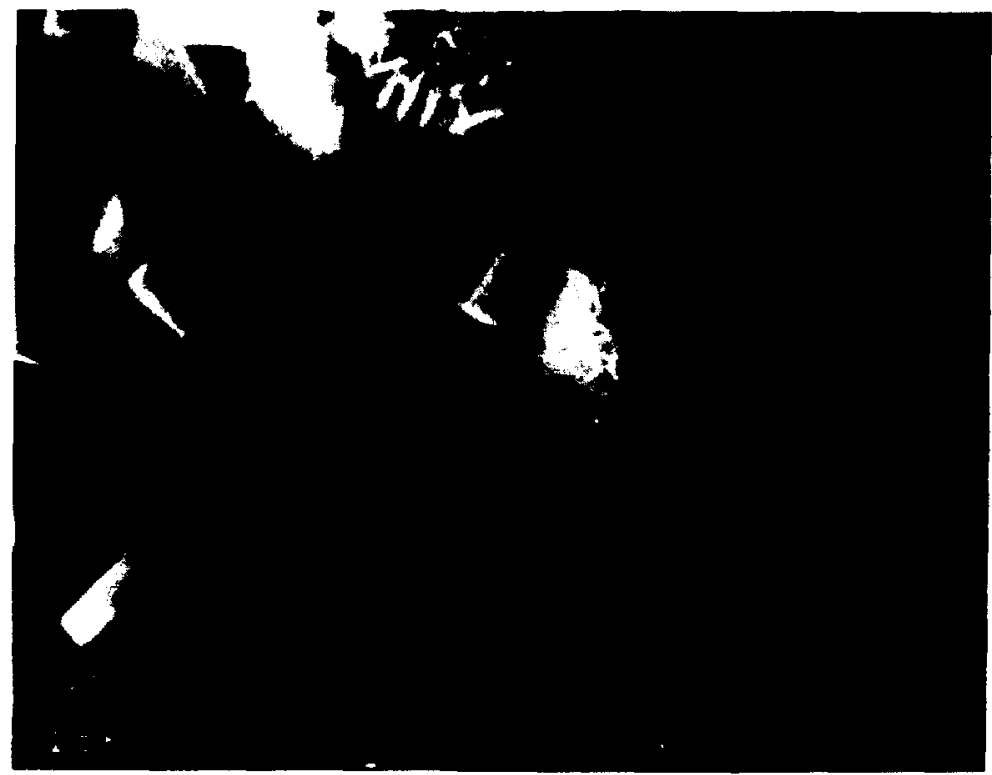

Fig. 2. SEM photograph of sample G1-1436 before contact with well J-13 water. 


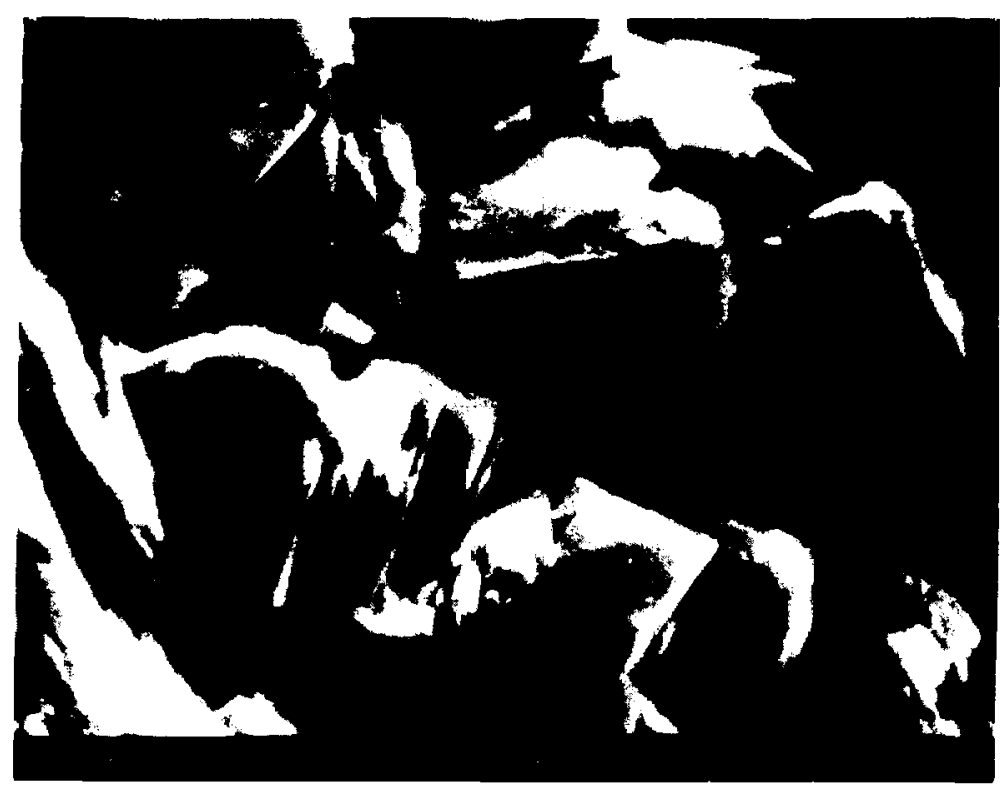

Fig. 3. SEM photograph of sample G1-1436 after contact with well J-13 water.

the same tuffs at $22^{\circ} \mathrm{C}$. Significant increases in the concentrations of silicon, iron, potassium, and sodium and decreases in the concentration of magnesium were observed after reaction of samples G1-2476 and G1-1292 at the higher temperature; these changes represent dissolution and precipitation, respectively.

4. Oxidation-Reduction Potential of Groundwater. The oxidation-reduction potentials (Eh) of groundwaters at Yucca Mountain cannot currently be measured without ambiguity because they do not contain sufficiently high concentrations $\left(>10^{-5} \mathrm{M}\right)$ of oxidizable or reducible species. Therefore, oxygen and sulfide poisoning of the Eh electrode are very real problems. 10 If the water as sampled contains measurable oxygen of $>0.1 \mathrm{ppm}$, redox potential measurements will be meaningless because the electrode is poisoned. A reading of $\sim 350 \mathrm{mV}$ at $\mathrm{pH} 7$ will be obtained. In the presence of large amounts of sulfide, the electrode may also be poisoned so that measurements below $-200 \mathrm{mV}$ are probably in error.

Although most waters at the NTS do not contain measurable quantities of sulfide ions, the sulfide electrode can help estimate when the Eh electrode would be poisoned by oxygen. Theoretically, the Eh electrode and sulfide 
TABLE VII

COMPOSITION OF WELL $J-13$ GROUNDWATER AFTER

CONTACT WITH USW-G1 TUFFS AT $22^{\circ}$ AND $152^{\circ} \mathrm{C}$

Concentration

\begin{tabular}{|c|c|c|c|c|c|c|}
\hline \multirow[b]{3}{*}{ Cation } & \multicolumn{6}{|c|}{$(\mathrm{mg} / \mathrm{l})$} \\
\hline & \multicolumn{2}{|c|}{ G1-2476 } & \multicolumn{2}{|c|}{ G1-1436 } & \multicolumn{2}{|c|}{$\mathrm{G} 1-1292$} \\
\hline & $22^{\circ} \mathrm{C}$ & $152^{\circ} \mathrm{C}$ & $22^{\circ} \mathrm{C}$ & $152^{\circ} \mathrm{C}$ & $22^{\circ} \mathrm{C}$ & $152^{\circ} \mathrm{C}$ \\
\hline $\mathrm{Mg}$ & 1.35 & 0.042 & 0.009 & 0.015 & 1.79 & 0.006 \\
\hline Mn & 0.013 & 0.044 & 0.020 & 0.022 & 0.010 & 0.018 \\
\hline $\mathrm{Si}$ & 29.7 & $>60$ & 30.8 & $>60$ & 32.3 & $>60$ \\
\hline $\mathrm{Fe}$ & 0.033 & 0.120 & 0.064 & 0.285 & 0.020 & 0.063 \\
\hline $\mathrm{Sr}$ & 0.034 & 0.013 & 0.000 & 0.019 & 0.090 & 0.011 \\
\hline $\mathrm{Ba}$ & 0.000 & 0.000 & 0.002 & 0.000 & 0.000 & 0.000 \\
\hline V & 0.010 & 0.000 & 0.019 & 0.000 & 0.000 & 0.021 \\
\hline $\mathrm{Ti}$ & 0.004 & 1.02 & 0.000 & 0.549 & 1.72 & 0.000 \\
\hline $\mathrm{Ca}$ & 9.50 & 0.657 & 0.176 & 0.031 & 13.1 & 0.534 \\
\hline $\mathrm{Li}$ & 0.093 & 0.223 & 0.074 & 0.099 & 0.084 & 0.086 \\
\hline K & 6.00 & 7.68 & 3.51 & $>10$ & 5.02 & $>10$ \\
\hline $\mathrm{Al}$ & 0.016 & 0.418 & 0.042 & 0.000 & 0.000 & 3.26 \\
\hline $\mathrm{Na}$ & 68.9 & $122 \pm 4$ & $78 \pm 5$ & $134 \pm 6$ & $57 \pm 4$ & $128 \pm 2$ \\
\hline
\end{tabular}

electrode readings should be $\sim 180 \mathrm{mV}$ apart for the same solution, with the Eh electrode showing the more positive measurement. ${ }^{11}$ For this reason the conditions where sulfide electrodes are usable have been investigated.

The sulfide-ion electrode contains a silver sulfide membrane. The voltage across this membrane is a function of the $\mathrm{Ag}^{+}$concentration and can be expressed in terms of the $\mathrm{s}^{2-}$ concentration by using the solubility product of $\mathrm{Ag}_{2} \mathrm{~S}$. The potential of the electrode can then be expressed by the Nernst equation

$$
\mathrm{E}_{\mathrm{S}^{2}}{ }^{-}=\left(\mathrm{E}_{\mathrm{S}^{2}}^{\mathrm{o}}\right)-\frac{\mathrm{RT}}{2 \mathrm{~F}} \ln \left(\mathrm{S}^{2-}\right)
$$

A plot of measured $\mathrm{E}_{\mathrm{S}^{2-}}$ vs $\log \left(\mathrm{S}^{2-}\right)$ should be a straight line of slope $-29.58 \mathrm{mV}$ for each decade change of concentration. Figure 4 shows the results for a 
change in the sulfide-electrode potential as the $\mathrm{s}^{2-}$ concentration is varied in the range of $4 \times 10^{-3}$ to $4 \times 10^{-9} \mathrm{M}$. The four sets of experiments were either done at $\mathrm{pH} 8$, or the measurements were corrected to $\mathrm{pH} 8$. The lower limit of accurate detection of $\mathrm{s}^{2-}$ from this graph is $\sim 4 \times 10^{-5} \mathrm{M}( \pm 20 \%)$. However, the sulfide electrode is qualitatively useful to concentrations as Low as $10^{-9} \mathrm{M}$.

Figure 5 shows the effect of ionic strength on the electrode potential of $\mathrm{S}^{2-}$ solutions. Sulfide solutions were prepared over the concentration range of $4 \times 10^{-3}$ to $4 \times 10^{-9} \mathrm{M}$ and adjusted to $\mathrm{pH} 8 \pm 1$; then the $\mathrm{s}^{2-}$ potentials were measured. Sufficient KCl was added to each solution to make it $0.1 \mathrm{M}$ in $\mathrm{KCl}$, and the $\mathrm{S}^{2-}$ potentials of the solutions were again measured with the sulfide-ion electrode. From Fig. 5 it can be seen that at $\mathrm{S}^{2-}$ concentrations below $4 \times 10^{-6} \mathrm{M}$, the ionic strength of the solution has a large effect.

Complexes of $\mathrm{H}^{+}$and $\mathrm{s}^{2-}$ form as the $\mathrm{pH}$ of a solution is increased. At $\mathrm{pH}$ 11 the major species in solution is $\mathrm{s}^{2-}$, whereas at $\mathrm{pH} 7$ and $\mathrm{pH} 4$ the major species are $\mathrm{HS}^{-}$and $\mathrm{H}_{2} \mathrm{~S}$, respectively. Because th sulfide-ion electrode responds only to $\mathrm{s}^{2-}$, all electrode manufacturers have recommended that the $\mathrm{pH}$ of a solution be adjusted to $\mathrm{pH} 11$ before measurement. Boulègue ${ }^{11}$ has shown that this is not necessary. The $\mathrm{s}^{2-}$ concentration and electrode potential can be calculated for any $\mathrm{pH}$ and total $\mathrm{H}_{2} \mathrm{~S}$ concentration and can be used to form calibration curves as $\mathrm{E}_{\mathrm{S}^{2}}{ }^{-}$vs $\mathrm{pH}$. Figure 6 also shows that the electrodemeasured $\mathrm{S}^{2-}$ potentials agree quite well with the calculated values. Eight sets of experiments in which the $\mathrm{s}^{2-}$ concentration was $10^{-3} \mathrm{M}$ are shown on the graph. Also included are calculated $\mathrm{E}_{\mathrm{S}^{2}}$ vs $\mathrm{pH}$ lines.

The electrode manufacturers also recommend using an antioxidant such as ascorbic acid in the solution so that the $\mathrm{s}^{2-}$ will not oxidize. The reaction of $\mathrm{s}^{2-}$ with air is reasonably slow, and the antioxidant is not necessary if the measurements are carried out within a reasonable time (a few hours) after sampling (Fig. 6). At most, the measured potential increases by $20 \mathrm{mV}$ when air is present in the solution.

A platinum Eh electrode can be used in a sulfide system to measure the potential of the half-cell $\mathrm{s}^{2-}=\mathrm{s}+2 \mathrm{e}^{-}$. Using Eq. (1), Eh $=\mathrm{E}^{\mathbf{0}}-0.029510 \mathrm{~g}$ $\left(\mathrm{S}^{2-}\right)$. When this equation is combined with that for the potential of a sulfideion electrode, $\mathrm{Eh}=\mathrm{E}_{\mathrm{S}^{2}}+\left(\mathrm{E}^{\circ}-\mathrm{E}_{\mathrm{S}^{2}}^{\circ}\right)$. Boulègue and Michard ${ }^{12}$ estimated from thermodynamic data that $\left(E^{\circ}-E_{S^{2}}^{o}\right)$ is $180 \mathrm{mV}$. Therefore, $E \mathrm{~h}=\left(E_{S^{2}}{ }^{-}\right)+180 \mathrm{mV}$. This equation is plotted in Fig. 7 with measurements (shown as $x$ and + ) of Eh 


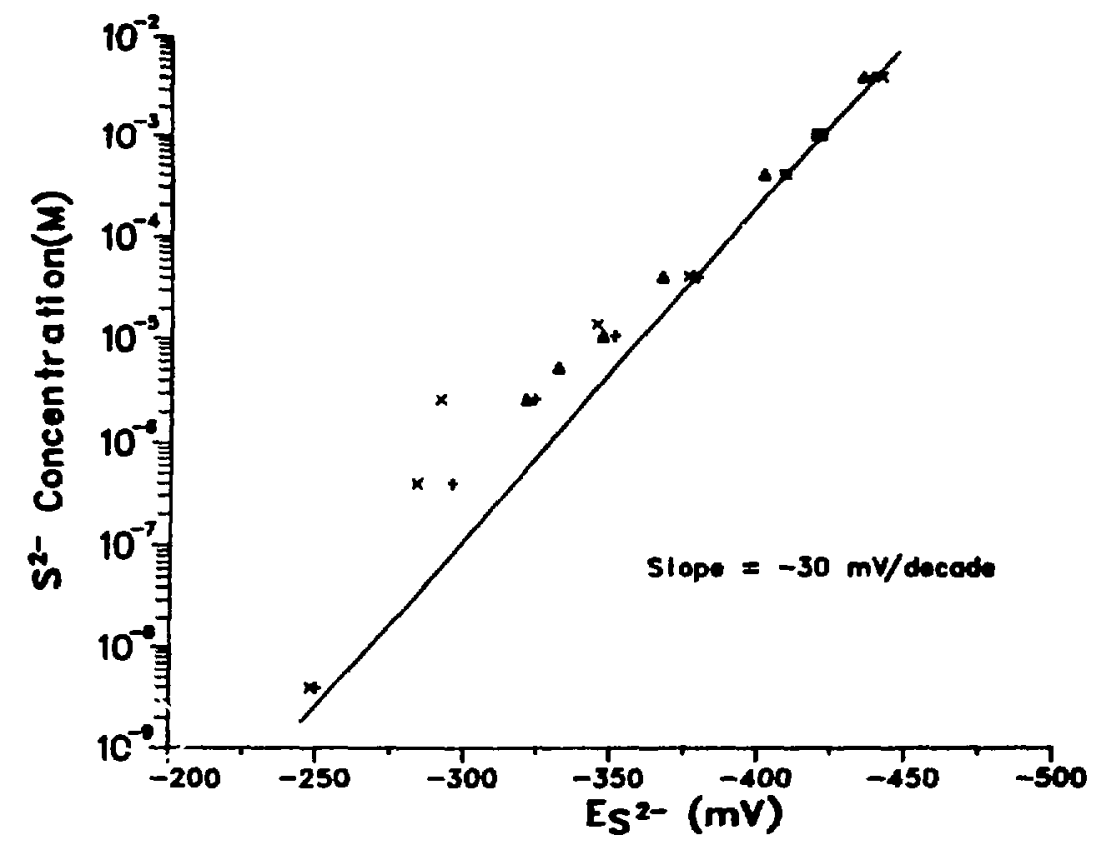

Fig. 4. Variation of sulfide-electrode potential with sulfide concentration.

and $\mathrm{E}_{\mathrm{S}^{2}}{ }^{-}$carried out in the oxygen-free nitrogen atmosphere of a Vacuum-Atmosphere glove box. In an oxygen-free atmosphere the $\mathrm{Eh}_{-\mathrm{E}} \mathrm{S}^{2}$ - relationship is very good.

In addition, Eh and $\mathrm{E}_{\mathrm{S}^{2}}$ measurements were plotted for solutions of constant $\mathrm{S}^{2-}$ concentration but with different oxygen contents. The vertical lines (Fig. 7) connect experiments of equal $\mathrm{pH}$ and, supposedly, the same Eh and $E_{\mathrm{S}^{2}}{ }^{-}$. The uppermost measurements on each line represent solutions either in air or in a poor glove box that had air leaks. The middle points represent these same solutions after ascorbic acid was added to remove the oxygen in the solution. Three conclusions can be drawn from this figure: (1) the Eh electrode measurements are influenced by oxygen contamination, even in a sulfide environment; (2) the sulfide electrode is not affected to a large extent by the oxygen contamination; and (3) ascorbic acid either does not eliminate all the oxygen in a solution or does not clean the Eh electrode enough to give a reading that represents the absence of oxygen. 


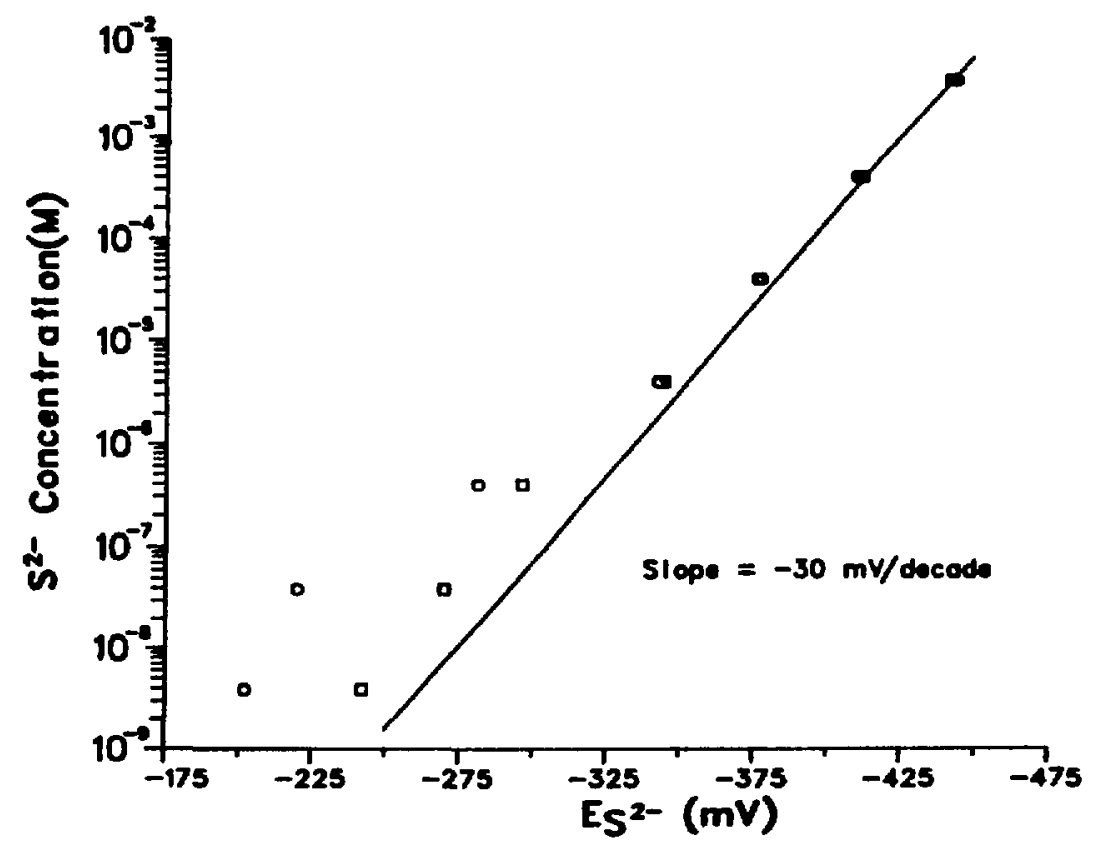

Fig. 5. Effect of ionic strength on sulfide-electrode potentials.

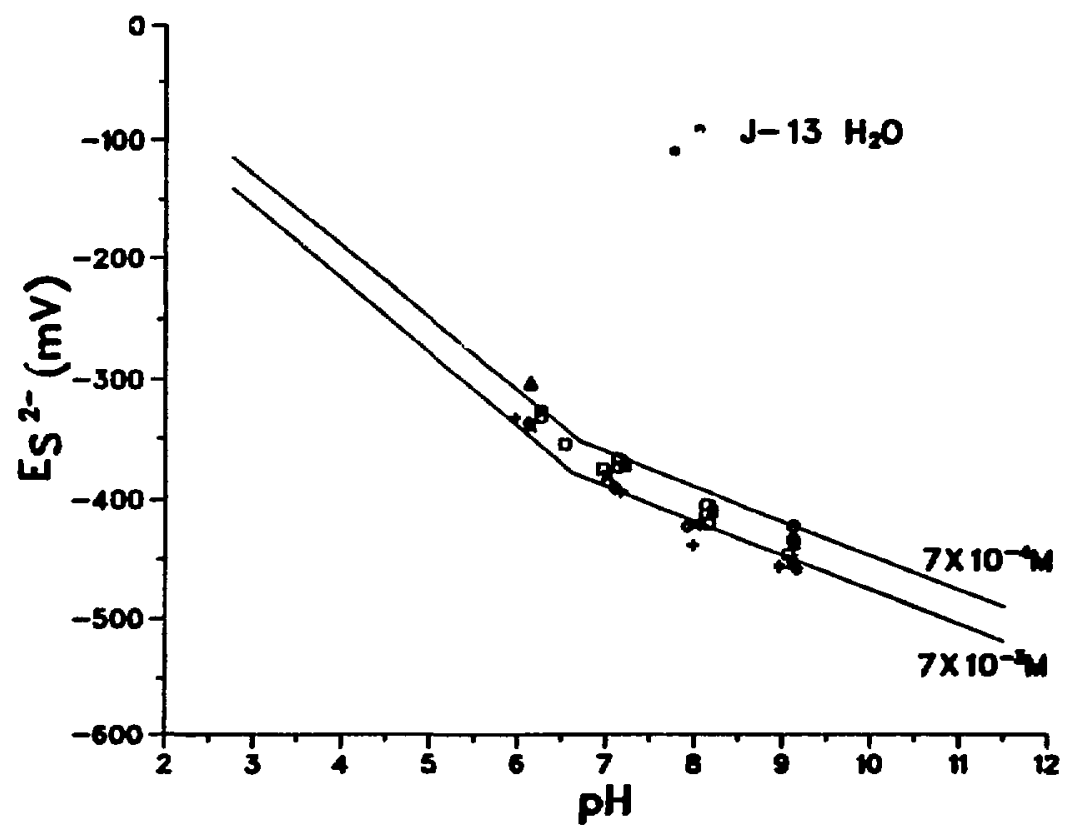

Fig. 6. Variation of sulfide-electrode potential with pH. 


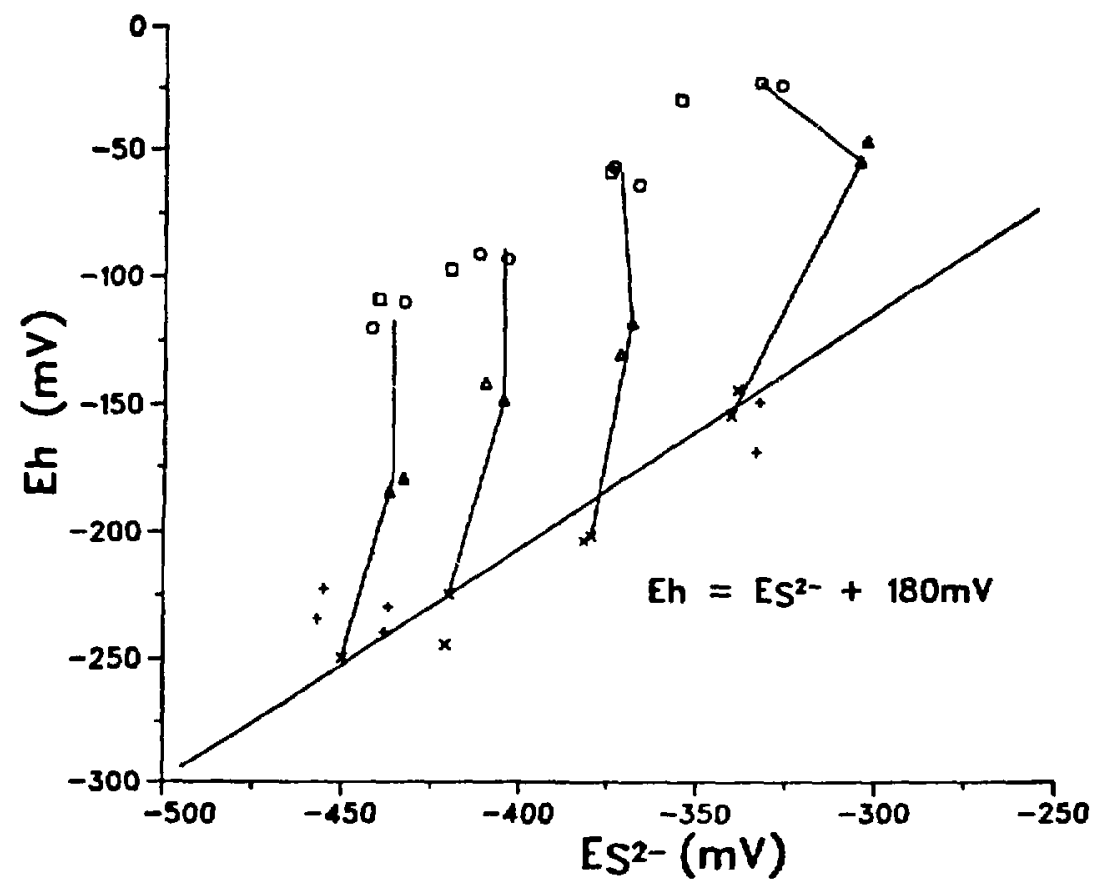

Fig. 7. Relationship of Eh and $\mathrm{E}_{\mathrm{S}^{2}}$ for sulfide solutions with varying oxygen contents.

It would be interesting to pursue this study further to test whether the divergence of the Eh measurement from the $\mathrm{Eh}=\left(\mathrm{E}_{\mathrm{S}^{2}}{ }^{-}\right)+180-\mathrm{mV}$ relationship can be used as a measure of the oxygen content of the solution.

The Eh and $\mathrm{E}_{\mathrm{S}^{2}}$ - values were measured for well J-13 water inside the inert-atmosphere glove box. This water had been kept sealed in a container inside the glove box for most of the time since it was collected at the wellhead. An Eh of $+100 \mathrm{mV}$ vs the $\mathrm{H}_{2}$ electrode and an $\mathrm{E}_{\mathrm{S}^{2}}$ - of $-100 \mathrm{mV}$ were measured. The results agree quite well with the expression $\mathrm{Eh}=\left(\mathrm{E}_{\mathrm{S}^{2}}{ }^{-}\right)+180 \mathrm{mV}$; however, the real significance of these measurements is still unclear because the $\mathrm{s}^{2-}$ concentration is below the limits of accurate detection (Figs. 4 and 6). Unfortunately, there are no known redox couples of sufficient concentration in well $\mathrm{J}-13$ water to give a meaningful Eh measurement. 
The Eh anu sulfide electrodes have been tested on a producing well in which the dissolved iron concentration was known to be high enough, and therefore, the Eh reading is probably valid. An oxygen electrode was also included to measure any dissolved oxygen in the groundwater. iable VIII lists the chemical analysis and the electrode readings taken anaerobically on water from the Barmon-1 well at Chimayo, New Mexico.

It is possible to calculate from the chemical composition what could be expected for a measured $E h$. Using a value of $K_{s p}=10^{38}$ for the solubility product of $\mathrm{Fe}(\mathrm{OH})_{3}, \mathrm{pH}=7.2$, standard $\mathrm{Fe}^{2+} \rightarrow \mathrm{Fe}^{3+}+\mathrm{e}^{-}$potential $=771 \mathrm{mV}$, and the measured $\mathrm{Fe}^{2+}$ concentration of $2.40 \pm 0.02 \mathrm{mg} / \mathrm{Q}$, an $\mathrm{Eh}$ between +25 and $+100 \mathrm{mV}$ can be calculated. Measurements of $+75 \mathrm{mV}$ were found in this case.

5. Composition of Yucca Mountain Groundwater. To determine or estimate the effect of the groundwater composition on the waste package, on the waste itself or its compounds, and on retardation mechanisms, it is necessary to determine the composition of the formation water in each particular stratum being considered for a repository or a transport path. The groundwater composition is also important in its effect on forming or dissolving the newly formed minerals brought about by the temperature gradient exerted on the repository by the waste package.

Wells in the vicinity of Yucca Mountain and Pahute Mesa have been sampled in various ways to determine the groundwater composition as a function of location. Wells 8 , UE25b-1, and UE29a-2 were sampled while they were being pumped at a high rate. These samples were integral ones; the main contribution was made by the water from the permeable region (aquifer) nearest the pump intake. The water's $\mathrm{Eh}, \mathrm{pH}$, temperature, and oxygen were measured under anaerobic conditions for wells UE25b-1 and UE29a-2. Well USW-H1 was sampled under different conditions. Evacuated sample bottles were lowered by wire line to selected depths of the well. An electric valve on the bottle was opened to admit water, after which the valve was closed and the bottle was retrieved. These water samples were opened inside an inert-atmosphere glove box at Los Alamos, where Eh, sulfide and oxygen contents, and $\mathrm{pH}$ were measured before the samples were passed through 0.05 - fim Nuclepore membranes and chemically analysized. Tables $I X$ and $X$ list the cation and anion compositions and electrode measurements for these samples. The nomenclature, or the sample number, for samples taken from well USW-H1 again indicates the depth from which the sample was taken. 
TABLE VIII

COMPOSITION OF BARMON-I WELL (NEW MEXICO) GROUNDWATER ${ }^{a}$

Concentration (mg/l)

\begin{tabular}{|c|c|c|c|c|c|c|c|c|c|c|c|c|}
\hline $\mathrm{Mg}$ & $\mathrm{Mn}$ & $\mathbf{S i}$ & $\mathrm{Fe}$ & $S r$ & $\mathrm{Ba}$ & $\mathrm{V}$ & $\mathrm{Ti}$ & $\mathrm{Ca}$ & $\mathrm{Li}$ & $\mathrm{K}$ & $\mathrm{Al}$ & $\mathrm{Na}$ \\
\hline 10.5 & 0.29 & 5.9 & $3.1^{b}$ & 1.00 & 0.15 & N.D. & N.D. & 69.7 & 0.12 & 5.5 & 0.08 & 7.7 \\
\hline
\end{tabular}

\section{Concentration}

$$
(\mathrm{mg} / \ell)
$$

$\frac{\mathrm{F}^{-}}{0.8} \quad \frac{\mathrm{C1}^{-}}{6.8} \quad \frac{\mathrm{NO}^{-}}{\text {N.D. }} \quad \frac{\mathrm{PO}_{4}^{3}}{\text { N.D. }} \quad \frac{\mathrm{NO}_{3}^{-}}{\text {N.D. }} \quad \frac{\mathrm{SO}_{4}^{2}}{12.0} \quad \frac{\mathrm{S}^{2-}}{0.032} \quad \frac{\mathrm{O}_{2}}{<1.2} \quad \frac{\mathrm{Alk}^{\mathrm{d}}}{5.904} \frac{\mathrm{pH}^{-}}{7.16}$

Each sample was passed through $0.05-\mu \mathrm{m}$ Nuclepore membrane.

$\mathrm{b}_{\mathrm{Fe}}{ }^{2+}$ content is $2.4 \mathrm{mg} / \mathrm{l}$.

$\mathrm{c}_{\mathrm{N} . \mathrm{D} .}$ = not detected.

dAlkalinity in meg/ $\ell$.

${ }^{\mathrm{e}} \mathrm{mV}$ vs a hydrogen electrode. 
TABLE IX

CATION COMPOSITION OF GROUNDWATERS ${ }^{\mathrm{a}}$

\begin{tabular}{|c|c|c|c|c|c|c|c|c|c|c|c|c|c|}
\hline \multirow[b]{2}{*}{ Well } & & \multicolumn{12}{|c|}{$\begin{array}{c}\text { Concentration } \\
(\mathrm{mg} / \mathrm{l})\end{array}$} \\
\hline & $M_{8}$ & Mn & $\mathbf{S i}$ & $\mathrm{Fe}$ & $\underline{S}$ & $\mathrm{Ba}$ & V & $\mathrm{Ti}$ & $\mathrm{Ca}$ & $\underline{\mathrm{Li}}$ & $\mathbf{K}$ & $\mathrm{Al}$ & $\mathrm{Na}$ \\
\hline 8 & 1.377 & 0.005 & 25.6 & 0.009 & 0.007 & $<0.0001$ & 0.003 & $<0.0001$ & 8.2 & 0.045 & 4.13 & 0.035 & 36.5 \\
\hline \multicolumn{14}{|l|}{ UE25b- 1} \\
\hline $8 / 7 / 81$ & $(0.740)$ & $(0.192)$ & $(30.1)$ & $(0.154)$ & $(0.051)$ & $(0.005)$ & $(0.010)$ & $(0.023)$ & $(20.4)$ & $(0.834)$ & $(3.62)$ & $(0.044)$ & $(59.8)$ \\
\hline $8 / 7 / 81$ & 0.833 & 0.193 & 31.6 & 0.047 & 0.055 & 0.005 & 0.011 & 0.015 & 22.5 & C. 873 & 3.69 & 0.032 & 63.0 \\
\hline $9 / 11 / 81$ & $(0.616)$ & $(0.004)$ & $(28.1)$ & $(0.34)$ & $(0.041)$ & $(0.006)$ & $(0.006)$ & $(0.014)$ & $(17.3)$ & $(0.262)$ & $(3.19)$ & $(0.015)$ & $(53.6)$ \\
\hline $9 / 11 / 81$ & 0.677 & 0.004 & 31.5 & 0.035 & 0.046 & 0.007 & 0.015 & 0.026 & 19.7 & 0.283 & 3.28 & 0.028 & 55.8 \\
\hline \multirow[t]{2}{*}{ UE29a-2 } & $(0.303)$ & $(<0.002)$ & $(25.9)$ & $(0.008)$ & $(0.037)$ & $(0.001)$ & $(0.002)$ & $(<0.0001)$ & $(10.3)$ & $(0.094)$ & (1.13) & $(0.016)$ & $(50.1)$ \\
\hline & 0.343 & 0.034 & 25.8 & 0.048 & 0.041 & 0.03 & 0.003 & $<0.0001$ & 11.1 & 0.105 & 1.17 & 0.041 & 50.8 \\
\hline H $1-2000$ & 0.196 & 0.112 & 3.55 & 0.019 & 0.027 & 0.38 & 0.001 & 0.004 & 3.45 & 0.074 & 6.38 & 0.017 & 106.0 \\
\hline 3000 & 0.087 & 0.08 & 11.5 & 0.143 & 0.035 & 0.007 & 0.005 & 0.004 & 5.23 & 0.092 & 1.37 & 0.028 & 153.0 \\
\hline 4000 & 0.074 & 0.036 & 12.9 & 0.026 & 0.043 & 0.008 & 0.007 & 0.003 & 1.68 & 0.112 & 1.45 & 0.018 & 166.0 \\
\hline 5900 & 0.149 & 0.146 & 16.1 & 0.214 & 0.106 & 0.013 & 0.006 & $<0.001$ & 6.18 & 0.143 & 2.19 & 0.022 & 120.0 \\
\hline
\end{tabular}

\footnotetext{
${ }^{a}$ Values in parentheses for waters that were not filtered through $0.05-\mu \mathrm{m}$ Nuclepore membranes.
} 
Concentration

\begin{tabular}{|c|c|c|c|c|c|c|c|c|c|c|}
\hline \multirow[b]{2}{*}{ We11 } & \multicolumn{7}{|c|}{$(\mathrm{mg} / \mathrm{l})$} & \multirow[b]{2}{*}{$A 1 k^{b}$} & \multirow[b]{2}{*}{$\mathrm{pH}$} & \multirow[b]{2}{*}{$\mathrm{Eh}^{\mathrm{d}}$} \\
\hline & $\mathrm{F}^{-}$ & $\mathrm{Cl}^{-}$ & $\mathrm{PO}_{4}^{3^{-}}$ & $\mathrm{NO}_{3}^{-}$ & $\mathrm{SO}_{4}^{2}$ & $0_{2}^{c}$ & $\mathrm{~s}^{2^{-}}$ & & & \\
\hline 8 & 0.5 & 6.9 & N.D. ${ }^{a}$ & 5.4 & 14.6 & & & 1.407 & 6.6 & \\
\hline $\begin{array}{l}\text { UE } 25 b-1 \\
8 / 7 / 81 \\
9 / 11 / 81\end{array}$ & $\begin{array}{l}1.07 \\
1.2\end{array}$ & $\begin{array}{r}11.4 \\
7.1\end{array}$ & $\begin{array}{l}\text { N.D. } \\
\text { N.D. }\end{array}$ & $\begin{array}{l}\text { N.D. } \\
0.6\end{array}$ & $\begin{array}{l}21.1 \\
20.6\end{array}$ & 1.8 & N.D. & $\begin{array}{l}3.421 \\
2.213\end{array}$ & $\begin{array}{l}8.7 \\
7.7\end{array}$ & 220 \\
\hline UE29a-2 & 0.56 & 8.3 & $<0.2$ & 18.7 & 22.7 & 5.7 & N.D. & 1.769 & 7.0 & 305 \\
\hline $\begin{array}{r}H 1-2000 \\
3000 \\
4000 \\
5900\end{array}$ & $\begin{array}{r}2.7 \\
17.7 \\
13.1 \\
16.8\end{array}$ & $\begin{array}{r}24.6 \\
8.3 \\
8.4 \\
9.5\end{array}$ & $\begin{array}{l}\text { N.D. } \\
\text { N.D. } \\
\text { N.D. } \\
\text { N.D. }\end{array}$ & $\begin{array}{l}\text { N.D. } \\
\text { N.D. } \\
\text { N.D. } \\
\text { N.D. }\end{array}$ & $\begin{array}{l}13.9 \\
34.4 \\
60.9 \\
50.0\end{array}$ & $\begin{array}{r}3.4 \\
1.3 \\
1.3 \\
<1.2\end{array}$ & $\begin{array}{l}\text { N.D. } \\
0.064 \\
0.000064 \\
0.032\end{array}$ & $\begin{array}{l}3.494 \\
5.861 \\
5.898 \\
4.253\end{array}$ & $\begin{array}{l}7.2 \\
7.0 \\
8.0 \\
7.6\end{array}$ & $\begin{array}{r}270 \\
-40 \\
-25 \\
-105\end{array}$ \\
\hline
\end{tabular}

\footnotetext{
${ }^{a}$ N.D. = not detected.

b Alkalinity in meq/e.

Measured after return to Los Alamos.

${ }_{\mathrm{e}} \mathrm{mV}$ vs a hydrogen electrode.

The 1.2 was the minimum detectable.
} 
When making comparisons among the results for the wells, the pumping history of each well must be kept in mind. Wells 8 and J-13 are producing wells that are still in use. Well UE25b-1 was pumped for more than $1.3 \times 10^{7} \mathrm{gal}$., but the water still contained detergent from the drilling of the well. Well UE29a-2 was pumped for a relatively short time and also contained detergent, and well USW-H1 was a stagnant hole that had not been pumped for over 6 months.

Despite the differences in pumping histories, several generalizations can be made.

(1) Wel1 8 water composition is very similar to that of well $\mathrm{J}-13$. The concentrations of cations and anions in well 8 are all lower but are at approximately the same ratios to each other as those for well J-13 water.

(2) Well UE25b-1 water, however, contains higher sodium and calcium and lower magnesium and potassium concentrations. There is a large drop in manganese concentration in well UE25b-1 with time. The difference in results for unfiltered and filtered water from well UE25b-1 again shows the importance of filtration through 0.05- $\mu \mathrm{m}$ Nuclepore membranes when measuring iron concentration. An Eh of $220 \mathrm{mV}$ was measured for this water, representing a solution without measurable quantities of oxygen present ( $<0.1 \mathrm{ppm})$. Oxygen analysis on the water after it was shipped to Los Alamos gave the high value of $1.8 \mathrm{ppm}$, which must be considered as a maximum because of the handling involved. Using the same constants and procedure that were used to calculate the measured iron concentration for well UE25b-1 (Table IX), the calculated Eh would be $+110 \pm 30 \mathrm{mV}$. The measured $\mathrm{Eh}$ of $+220 \mathrm{mV}$ (Table X) is too positive and may indicate poisoning by some oxygen in the system or during sample handling. The difference between the Eh and sulfide-electrode measurements $(290 \mathrm{mV})$ is also greater than $180 \mathrm{mV}, 9$ indicating that the Eh electrode was poisoned by oxygen and was giving too positive a reading. The higher measurement may also be a result of mixing the water from two aquifers, one of which contains oxygen, during pumping.

(3) Well UE29a-2 water is very similar to well J-13 water except for a lower magnesium concentration and lower alkalinity. This water, like well J-13 water, contained $\sim 5.7$ ppm dissolved oxygen. Both well UE29a-2 and well J-13 produce water in a permeable zone at or near the standing water level; therefore, it is not surprising to see high oxygen contents. Well UE25b-1, however, produces water from several zones.

(4) The results on the at-depth samples from well USW-H1 are very different from those of pumped wells. The sodium is very high, whereas magnesium, 
calcium, and potassium are low. At several depths the sulfide and iron concentrations are very high; these depths approximate the strata in which pyrite is found. The fluoride, chloride, and sulfate concentrations and the alkalinity are very high in these same samples, and a negative Eh is measured. At the time these measurements were taken, $1.2 \mathrm{mg} / \ell$ was the minimum detectable oxygen using the Yellow Springs Instrument electrode. These results probably represent reactions that can take place with time after drilling rather than conditions that existed before drilling. The difference in $\mathrm{NO}_{3}^{-}$results between wells may be an indication that wells USW-H1 and UE25b-1 are not fed by the same aquifer as wells $8, \mathrm{~J}-13$, and UE29a-1 and that well UE29a-1 may be unique.

These results are important because there is now some evidence that all groundwaters at NTS are not highly oxidizing with measurable amounts of dissolved oxygen. Therefore, samples of formation water from particular strata must be obtained.

6. Determination of $\mathrm{Fe}^{2+}$ in Water. The presence of $\mathrm{Fe}^{2+}$ and $\mathrm{Fe}^{3+}$ in groundwaters has important effects on the oxidation potential in geologic systems. Total iron can easily be determined, but measuring the $\mathrm{Fe}^{2+}$ content is usually difficult in the presence of $\mathrm{Fe}^{3+}$. However, using a procedure adopted originally from Lee and Stumm, ${ }^{13}$ there seems to be very little interference from $\mathrm{Fe}^{3+}$. The reagent, 4,7 dipheny1-1,10 phenanthroline, commonly called bathophenanthroline, is more sensitive and the color developed is more stable than other phenanthrolines. Ferric iron does form a slightly colored complex with the bathophenanthroline, but unless large quantities of ferric iron are present its contribution to the absorbance can be considered negligible. If needed, correction for large amounts of ferric iron can be made by establishing a calibration curve for ferric iron; however, the solubility of $\mathrm{Fe}^{3+}$ at $\mathrm{pH} 7$ should be very low.

Reagents are prepared iron free by extractions to reduce the blank. The present lower limit for the procedure is $\sim 1 \mu \mathrm{g} \mathrm{Fe}{ }^{2+}$, which is $0.0125 \mathrm{mg} / \ell$ for an 80-me sample.

None of the common anions chloride, nitrate, acetate, or sulfate interfere in the determination of iron with bathophenanthroline, nor do the alkali and alkaline earth cations. Results for several groundwater samples are presented in Table XI. 
TABLE XI

$\mathrm{Fe}^{2+}$ IN GROUNDWATER

Filter

\begin{tabular}{|c|c|}
\hline Water & $(\mu \mathrm{m})$ \\
\hline G Tunnel ${ }^{b}$ & none ${ }^{c}$ \\
\hline G Tunnel ${ }^{b}$ & 0.05 \\
\hline$Y M-46^{b}$ & 0.45 \\
\hline$Y M-54^{b}$ & 0.45 \\
\hline Barmon No. $1^{e}$ & 0.40 \\
\hline Barmon No. $1^{e}$ & none \\
\hline
\end{tabular}

\begin{tabular}{|c|c|}
\hline $\mathrm{Fe}^{2+}$ & $\begin{array}{l}\text { Concentration } \\
(\mathrm{ppm} \leq)^{\mathrm{a}}\end{array}$ \\
\hline & 0.057 \\
\hline & 0.0125 \\
\hline & 0.037 \\
\hline & $0.023^{d}$ \\
\hline & 3.0 \\
\hline & 3.0 \\
\hline
\end{tabular}

a Reported as $\leq$ because the small contribution of Fe(III) has not been subtracted. $b_{\text {Well }} \mathrm{J}-13$ water contacted with indicated tuff cores.

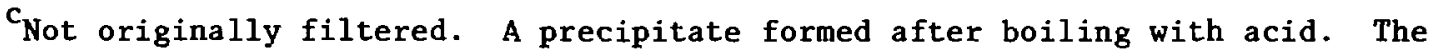
solids were filtered out on No. 541 Whatman filter paper before extraction. Iron results may be low if the precipitate trapped iron.

${ }^{d}$ Limit is high because only a small sample was available.

ew Mexico well used to test equipment.

7. Determination of $\mathrm{Fe}^{2+}$ in Silicate Rocks. The presence of $\mathrm{Fe}^{2+}$ and $\mathrm{Fe}^{3+}$ influences the oxidation potential in geologic systems. An estimate of the total quantity of $\mathrm{Fe}^{2+}$ and $\mathrm{Fe}^{3+}$ in tuffs could be an indication of the total oxidation-reduction capacity of the tuff in Yucca Mountain. Total iron can easily be determined; however, a good method for determinating $\mathrm{Fe}^{2+}$ in silicate rocks must involve special techniques to prevent $\mathrm{Fe}^{2+}$ from oxidizing during separation or at other times when it is not being measured. Using a modification of Banerjee's technique, ${ }^{14}$ in which the silicate rock is dissolved, the Los Alamos method includes a simultaneous oxidation of $\mathrm{Fe}^{2+}$ by ICl to form $\mathrm{I}_{2}$. Iodine monochloride has a lower oxidation potential than either permanganate or dichromate, other oxidizing agents that might have been used; however, the use of ICl as oxidant precludes interference by Mn(II) or Cr(III). Pertinent half-cell reactions are

$$
\begin{array}{cr}
\mathrm{MnO}_{4}^{-}+8 \mathrm{H}^{+}+5 \mathrm{e}^{-}=\mathrm{Mn}^{2+}+4 \mathrm{H}_{2} \mathrm{O} & \mathrm{E}^{\circ}=1.52 \mathrm{~V} \\
\mathrm{Cr}_{2} \mathrm{O}_{7}^{2-}+14 \mathrm{H}^{+}+6 \mathrm{e}^{-}=2 \mathrm{Cr}^{3+}+7 \mathrm{H}_{2} \mathrm{O} & \mathrm{E}^{\circ}=1.33 \mathrm{~V}
\end{array}
$$




$$
\begin{array}{cl}
\mathrm{MnO}_{2}+4 \mathrm{H}^{+}+2 \mathrm{e}^{-}=\mathrm{Mn}^{2+}+2 \mathrm{H}_{2} \mathrm{O} & \mathrm{E}^{\mathrm{O}}=1.23 \mathrm{~V} \\
2\left(\mathrm{I}^{+} \mathrm{Cl}^{-}\right)+2 \mathrm{e}^{-}=\mathrm{I}_{2}+2 \mathrm{Cl} 1^{-} & \mathrm{E}^{\mathrm{O}}=1.19 \mathrm{~V} \\
\mathrm{Fe}^{3+}+\mathrm{e}^{-}=\mathrm{Fe}^{2+} & \mathrm{E}^{\circ}=0.77 \mathrm{~V}
\end{array}
$$

The Los Alamos procedure uses ${ }^{131} \mathrm{I}$-tagged ICl. The ${ }^{131_{1}} \mathrm{I}_{2}$ is reduced with $\mathrm{NaHSO}_{3}$, extracted from an organic into an aqueous phase, and counted on a Ge(Li) detector. Standards are run at the same time for calibration. The method uses hydrofluoric acid in the dissolution, which limits the vessels that can be used. The FEP Oak Ridge-type centrifuge cones $(50 \mathrm{~m} \ell)$ and fluoropolymer screw caps (Nalgene 3114 ) are suitable; most other plastics cannot be used because $\mathrm{I}_{2}$ sorbs or diffuses into them. Several USGS "standard" rocks were analyzed, with a $2.0 \%$ average deviation from the published results.

In previous analyses, the $\mathrm{Fe}^{2+}$ was detectable in $\mathrm{YM}-22, \mathrm{YM}-38$, and $\mathrm{YM}-46$ tuffs. These analyses will be repeated, however, because a less sensitive analysis of $\mathrm{I}_{2}$ than the ${ }^{131} \mathrm{I}$ tracer method was used. Two analyses using the radioactive method were completed on G-Tunnel core U12G-RNM9. The Fe ${ }^{2+}$ content was $<0.1 \%$.

The procedures must be improved before they can be used for routine rock analyses.

\section{B. Actinide Behavior}

To assess the radiochemical hazards that would be associated with dissolved actinides in the repository, the geochemical processes along possible flow paths from the repository to the accessible environment must be known. The principal issues concerning actinide chemistry are:

\section{(1) Speciation}
(a) oxidation state
(b) hydrolyzed forms
(c) complex ions
(d) polymeric forms

(2) Solubility of oxides and hydrous oxides
(a) effect of Eh and $\mathrm{pH}$
(b) effect of temperature
(c) effect of complexing anions [closely related to (1c) above]
(d) rates of precipitation and dissolution. 
These issues must to be resolved before reasonable predictions of the behavior of plutonium and other actinides in the environment can be made. These issues are not strictly site specific; many are dependent upon the composition of the groundwater and rock.

The behavior of an element in solution under various conditions will depend on the species present. Thus, speciation is an important underlying issue in any discussion of the effect of groundwater chemistry on the behavior of waste elements. For elements that can exist in more than one oxidation state, the species present will depend on the $\mathrm{Eh}$, the $\mathrm{pH}$, and the concentration of complexing anions.

For plutonium and the other lighter actinides (An), the species present in acid solutions are well understood: $\mathrm{An}^{3+}, \mathrm{An}^{4+}, \mathrm{AnO}_{2}^{+}$, and $\mathrm{AnO}_{2}^{2+}$ for the III, IV, V, and VI oxidation states, respectively, in the absence of significant concentrations of complexing ions. Plutonium (VII) is a very strong oxidizing agent and is not stable in acid solutions. At lower acidities, hydrolysis becomes important, and for plutonium significant concentrations of $\mathrm{PuOH}^{2+}$, $\mathrm{PuOH}^{3+}, \mathrm{PuO}_{2} \mathrm{CH}$, and $\mathrm{PuO}_{2} \mathrm{OH}^{+}$appear at $\mathrm{pH}$ values of $\sim 8.0,0.5,9.7$, and 5.6, respectively. i5 More highly hydrolyzed species are known for $\mathrm{PuO}_{2}^{2+}$ and are almost certainly important for $\mathrm{Pu}^{4+}$ as well. However, $\mathrm{Pu}($ IV) forms polymers in the $\mathrm{pH}$ region where wore highly hydrolyzed species are expected, and no data exist.

The species present in acid solutions of many complexing agents are known, and a number of complexing constants have been determined. ${ }^{16}$ Complex ions are expected to hydrolyze, but very little has been reported on these mixed complexes. 17

The very low solubilities of the An(IV) oxides and hydrous oxides often limit the available concentrations in the environment and in the laboratory. Direct spectrophotometric methods for studying speciation usually require concentrations of $\sim 10^{-4} \mathrm{M}$, and determination of the oxidation state usually requires $\sim 10^{-6} \mathrm{M}$, much higher concentrations than those expected under environmental conditions. To determine an average charge (a step toward understanding speciation) by electrophoretic methods requires concentrations that are high enough that sorption on the medium is not important. Species present at low concentrations can sometimes be inferred by the effects that $\mathrm{pH}$, Eh, and complexing ion concentrations have on the solubility equilibria and various distribution equilibria (for example, ion exchange and solvent extraction). 
Quantitative consideration of any plausible set of solubility products, hydrolysis constants, and oxidation potentials for plutonium, uranium, and neptunium shows that the net solubility in noncomplexing media is a complicated function of both Eh and $\mathrm{pH}$. The most stable solid phase is Pu(IV) oxide or hydrous oxide for most of the accessible Eh-pH region, but there are large regions where $\mathrm{Pu}(\mathrm{III})$ and $\mathrm{Pu}(\mathrm{V})$ are the principal solution species. ${ }^{18}$

A number of studies that are relevant to actinide solubility and speciation have been carried out. Los Alamos' experience in determining sorption ratios for actinides in tuff is outlined below, including the methods of preparation of the feed solutions and techniques for separating the solid and aqueous phases after contact. Studies of plutonium chemistry in near-neutral solutions are reviewed, including work with osmium complexes and observations concerning Pu(IV) polymeric material. Finally, the preliminary work related to particulate transport is reported, including production of actinides in the form of polymers or colloids.

\section{Preparing Actinide Feed Solutions and Separating Solid and Aqueous} Phases After Contact in Batch Sorption Measurements. Introducing plutonium or americium into groundwater is difficult because both elements tend to behave unpredictably in near-neutral solutions. Precipitation, polymerization, and/or colloid formation frequently result if great care is not taken in the preparation of the traced feed solutions and separation of the final aqueous and solid phases. Most traced feed solutions were prepared by evaporation of the tracer, violent agitation with groundwater, and finally, filtration through two different size filters. Sample separation problems were caused primarily by actinide sorption on very fine particles and filters and also by the the difficulty in removing such particles from the contacted groundwater. To determine, as far as possible, what species might be present in both plutonium and americium "solutions," a number of sample and traced feed solutions were characterized by microautoradiographs of the filters used in their preparation.

The traced solutions were normally prepared using rock-pretreated groundwaters, isotopically pure ${ }^{241}$ Am tracer from Oak Ridge National Laboratory, ${ }^{239} \mathrm{Pu}$ tracer (weapons grade) from Los Alamos, and ${ }^{237} \mathrm{Pu}$ tracer from Argonne National Laboratory. Tracer purities were checked by both alpha and gamma spectroscopy. Feed solutions were usually prepared to contain $\sim 2 \times 10^{6} \mathrm{dpm} / \mathrm{ml}$ of ${ }^{241} \mathrm{Am}$, and/or $\sim 3 \times 10^{5} \mathrm{dpm} / \mathrm{ml}$ of ${ }^{239} \mathrm{Pu}$, or $\sim 2.4 \times 10^{3} \mathrm{dpm} / \mathrm{m} \ell{ }^{237} \mathrm{Pu}$ at $100 \%$ 
yield of tracer, which was generally not obtained. These correspond to mass concentrations of $\sim 1 \times 10^{-6} \mathrm{M}$ americium, $\sim 1 \times 10^{-5} \mathrm{M}$ plutonium when ${ }^{239} \mathrm{Pu}$ was used, and $\sim 4 \times 10^{-13} \mathrm{M}$ plutonium when ${ }^{237} \mathrm{Pu}$ was used. The ${ }^{237} \mathrm{Pu}$ tracer was treated with $\mathrm{NaNO}_{2}$ so that the plutonium was in the IV oxidation state at the beginning, which resulted in the addition of $\sim 10^{-4} \mathrm{M}$ sodium ion to the feed solutions containing ${ }^{237} \mathrm{Pu}$. Batches of traced feed solution were prepared in sufficient quantity to contact a predetermined number of crushed-rock samples, to aliquot for concentration determination, and to measure the $\mathrm{pH}$ value.

The "pH-adjusted" feed solutions were prepared by adding tracer solution directly to the groundwater and then adjusting the $\mathrm{pH}$ to the original value by adding $\mathrm{NaOH}$ solution, which resulted in the addition of $\sim 10^{-2}$ sodium ion. "Dried" feed solutions were prepared by first evaporating an aliquot of tracer solution in air at room temperature in a polypropylene tube. The dried activity was then contacted several times with $20-\mathrm{ml}$ volumes of groundwater for periods of from a few minutes to overnight. After each contact the tube was centrifuged for 1 hour at $12000 \mathrm{rpm}(\sim 28000 \mathrm{~g})$, and the aqueous phase was added to a large polyethylene bottle. These two contact processes were continued until no significant decrease in $\gamma$-ray activity was observed in the tube; generally, this required three contacts. Groundwater was added then to the bulk of the feed solution in the bottle to give a volume $\sim 20 \mathrm{ml}$ less than the desired final volume, and this bulk solution was shaken overnight. At the same time, the final contact was being made in the original tube. The next day the tube was centrifuged, and the solution was added to the bottle. Water was added to give the desired final volume, and the bottle was shaken for at least 1 hour. The solution was then centrifuged for 1 hour at $\geq 6000 \mathrm{rpm}$ and transferred to a new bottle.

In early experiments, feed solutions were not filtered. The yields of the traced feed solutions generally varied from $\sim 5$ to $70 \%$. The procedure currently in use to prepare traced feed solutions is described in Sec. IV.A.2. Within 1 hour after preparation of a feed solution, an aliquot was taken and acidified for later assay for the initial concentration of each tracer, and 20-in 2 portions were added to crushed-rock samples in polypropylene tubes and to empty tubes for use as "controls." The pH value of the remaining solution was then measured.

The results of experiments using the $\mathrm{pH}$-adjusted feed solution differed from measurements using feed solution prepared from dried tracer. Individual sample sorption ratio values are presented in Ref. 2; average values are 
presented here in Table XII. Differences are presumably the result of differences in speciation that were caused by the alternate methods of feed solution preparation.

Feed solutions for early actinide sorption experiments were prepared in a manner to optimize the actinide concentration of the feed solution, and ensure, as far as possible, the exclusion of any polymeric or colloidal particles. However, the centrifugation described in Ref. I probably did not eliminate any particles because the feed solution was transferred from the centrifugation bottle by decanting. For all later experiments, the centrifugation was omitted, and the feed solutions were passed directly through a double filter $(0.4$ and $0.05 \mu \mathrm{m})$, thus eliminating two container changes. The method removing activity from the tube in which it is dried was changed to include a 2- or 3-minute contact with rock-treated water using a vibrator or

TABLE XII

SORPTION RATIOS ${ }^{a}(\mathrm{ml} / \mathrm{g})$ FOR AMERICIUM AND PLUTONIUM DETERMINED USING TWO METHODS FOR PREPARING FEED SOLUTIONS

\begin{tabular}{|c|c|c|c|c|c|c|}
\hline \multirow[b]{3}{*}{ Element } & \multirow[b]{3}{*}{ Core } & \multirow{3}{*}{$\begin{array}{l}\text { Temp } \\
\left({ }^{\circ} \mathrm{C}\right)\end{array}$} & \multicolumn{4}{|c|}{ Feed Solutions ${ }^{a}$} \\
\hline & & & \multicolumn{2}{|c|}{ Dried } & \multicolumn{2}{|c|}{ pH Adjusted } \\
\hline & & & Sorption & Desorption & Sorption & Desorption \\
\hline \multirow[t]{6}{*}{$\mathrm{Am}$} & $\mathrm{JA}-18$ & 22 & $180(30)$ & $1100(260)$ & $435(6)$ & $960(15)$ \\
\hline & & 70 & $230(30)$ & $3400(300)$ & & \\
\hline & $\mathrm{JA}-32$ & 22 & $130(30)$ & $2200(650)$ & $1100(120)$ & $2300(310)$ \\
\hline & & 70 & $110(30)$ & & & \\
\hline & $\mathrm{JA}-37$ & 22 & $670(210)$ & $17000(3500)$ & $8800(1100)$ & $12000(2000)$ \\
\hline & & 70 & $970(240)$ & & $34000(6000)$ & $5300(720)$ \\
\hline \multirow[t]{4}{*}{$\mathrm{Pu}$} & JA-18 & 22 & $140(30)$ & $350(140)$ & & \\
\hline & $\mathrm{JA}-32$ & 22 & $\sim 110$ & & $1200(210)$ & $750(170)$ \\
\hline & $\mathrm{JA}-37$ & 22 & $280(100)$ & & $3300(1200)$ & $3800(950)$ \\
\hline & & 70 & $\sim 240$ & & & \\
\hline
\end{tabular}

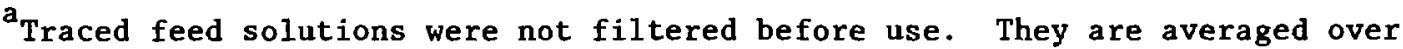

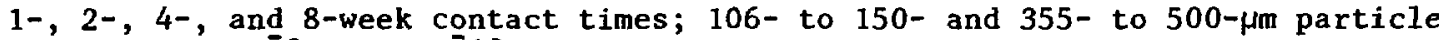
sizes, and $\sim 10^{-6}$ and $\sim 10^{-13} \mathrm{M}$ plutonium concentrations. Values in parentheses are the absolute-value standard deviation of the means. 
ultrasonic bath and then a second 2- or 3-minute contact with a fresh portion of rock-treated water, again using an ultrasonic bath. Subsequent contacts removed successively smaller portions of the remaining dried tracer; in general, 90 to $99 \%$ of the activity that can be removed is removed in the first two contacts.

Investigations detected the presence of centrifugable or filterable actinide species generated during feed preparation and sorption procedure. Improvements were made in the method used to separate solid and liquid phases after contact, presumably reducing the amount of particulates present. in the aqueous phase and, thereby, giving more accurate results. A few operations were added to examine (1) container sorption for samples and controls, (2) the fraction of centrifugable species in the controls, (3) the effect of filtering successive portions of the same "postcontact" solution through the same filter membrane, (4) the effects of filtering postcontact solutions through filter membranes with different pore sizes, and (5) the effects of centrifuging postcontact solutions a different number of times and for different durations. The procedure to separate phases after contact was usually three centrifugings, for 1,1 , and 2 hours, respectively, at $12000 \mathrm{rpm}(28000 \mathrm{~g}$ ). Extreme care was taken to avoid transferring any particulates from the bottom of the tube or in surface films. The centrifuged solutions were then filtered in various ways. The relative difference in sorption values obtained from pH-adjusted feed solutions but different postcontact phase-separation procedures is shown in Table XIII. The higher $R_{d}$ values observed with the improved phase-separation procedure indicate a more efficient removal of solid particles (with their associated high count rates) from the aqueous phase before counting. In some cases, aliquots were taken for counting after each centrifuging and each filtering.

Sorption on container walls during tuff contacts was measured by transferring the contents after contact, without centrifuging, to new tubes and counting the original tubes. Sorption on the container walls when solids were present was measured for twenty-four ${ }^{241} \mathrm{Am}$ and seven ${ }^{237} \mathrm{Pu}$ samples. The amount of activity sorbed on the container, with the possibility of retention of a small amount of solid even after transfer, averaged $2.1 \%$ for americium and $2.5 \%$ for plutonium. For controls (no solids present), container sorption averaged $24 \%$ for nine americium solutions at room temperature, $74 \%$ for four americium solutions at $70^{\circ} \mathrm{C}$, and $16 \%$ for two plutonium solutions at room temperature. 
TABLE XIII

SORPTION RATIOS ${ }^{a}(\mathrm{ml} / \mathrm{g})$ FOR AMERICIUM

AND PLUTONIUM DETERMINED USING DIFFERENT METHODS OF SEPARATING SOLID AND AQUEOUS PHASES ${ }^{b}$

Type of Phase

Separation

Original procedure

JA-32

JA-37

Improved procedure

$\mathrm{JA}-32$

JA-37
Americium

$\begin{array}{cc}\text { Sorption } & \text { Desorption } \\ 950(160)^{d} & 920 \\ 5000(800) & 4000(1300) \\ 1400(110) & 2700(430) \\ 12000(410) & 14000(2100)\end{array}$

Plutonium $^{c}$

\begin{tabular}{lr} 
Sorption & Desorption \\
\cline { 2 - 2 } $1200(200)$ & $750(170)$ \\
$8000(1400)$ & $1100(300)$
\end{tabular}

${ }^{a}$ Sorption ratios are averaged values for two particle sizes (106 to 150 and 355 to $500 \mu \mathrm{m}$ ).

$\mathrm{b}$

Experiments run in air at ambient temperature. Fecd solutions prepared by addition of acid solution of tracer and readjustment of $\mathrm{pH}$. Data given for postcontact solutions after centrifugation but before filtration.

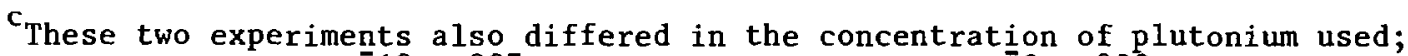
the first used $\sim 10^{-13} \mathrm{M}^{237} \mathrm{Pu}$ and the second used $\sim 10^{-6} \mathrm{M} 2{ }^{26} \mathrm{Pu}$. See

Sec. IV.C. 3 for a discussion of concentration effects.

${ }^{d}$ Values in parentheses are the absolute-value standard deviation of the means.

The amount of plutonium or americium activity remaining on the containers is obviously much lower for the samples than for the controls.

For comparison, container sorption during experiments with argillite samples from the Eleana formation at NTS was also investigated. The measurement methods were essentially the same as those used on the tuff except that the control samples were centrifuged and the aqueous phase was transferred to another tube. The activity observed in a control tube, therefore, represented the sum of wall sorption and centrifugable species. Sorption on the container walls when solids were present was measured for thirteen ${ }^{241} \mathrm{Am}$ and nine ${ }^{237} \mathrm{Pu}$ samples. The amount of activity sorbed on the container averaged $1.2 \%$ for americium and $0.6 \%$ for plutonium, with the possibility of retaining a small amount of solid even after transfer. For the controls, the americium activity remaining in the container averaged $13 \%$ for 13 solutions at ambient temperature and $90 \%$ for 4 solutions at $70^{\circ} \mathrm{C}$. 
For two plutonium controls at each temperature, the values were $43 \%$ at ambient and $88 \%$ at elevated temperature. Again, container sorption. was much higher for the controls than for samples containing crushed rock.

To determine the distribution of activity sorbed on the tuff control tubes, the bottoms of 6 tubes were washed twice with 2.5 -m $\ell$ portions of $3 \mathrm{M}$ $\mathrm{HCl}$; the activity removed was assumed to represent that which sorbed on the bottom of the tube. The tubes were then completely washed with two $2.5-\mathrm{m} \ell$ portions of $3 \mathrm{M} \mathrm{HCl}$, and the activity removed from each tube was again assumed to represent that which sorbed on the walls. The final "clean" tubes were checked by gamma counting. The activity was calculated per unit area; the ratio of the activity on the bottom to the activity on the walls varied from 0.3 to 1.1 with an average of 0.7 . The activity on control tubes appears to have been sorbed fairly evenly on the surface. The above results for container sorption suggest that sorption is dependent on available surface area, so wheri crushed cuff is present, container sorption is negligible.

For the tuff controls described above, the fraction of centrifugable species was measured by centrifuging the contents of the new tubes after the first transfer, again transferring the liquid, and counting the activity left in the tubes. The average fraction of the activity in the solution after contact that was removed by one 1 -hour centrifuging was $17 \%$ for nine americium controls at room temperature, $37 \%$ for four americium controls at $70^{\circ} \mathrm{C}$, and $13 \%$ for two plutonium controls at room temperature. Because there was a significant amount of centrifugable species in the control solutions, presumably a similar fraction of the activity was also present with the samples; there $j t$ would have been combined with the crushed rock and counted with the sorbed activity. Each of three centrifugings (1, 1, and 2 hours) removed additional activity from americium solutions after contact and, therefore, would appear to be necessary. (These measurements were not repeated with plutonium solutions.)

In an experiment to see if effects related to the filtering process would result in a change in the radionuclide concentration of solutions after contact, three $\sim 3-m \ell$ portions of a solution were passed through the same $0.4-\mu m$ polycarbonate membrane. Each portion was aliquoted and counted after passing through the membrane. Filtering successive portions of the same postcontact solution through the same filter membrane did not appear to result in a significant difference in the concentration of plutonium or americium in the solution.

The effects of centrifuging the postcontact solutions and passing them through filters with various pore sizes were examined by counting aliquots of 
the solution after each step. Data for a number of samples and controls are given in Table XIV. The filter sequences were $1.0 / 0.4 / 0.05 \mu \mathrm{m}, 0.4 / 0.4 \mu \mathrm{m}$, and $0.05 / 0.05 \mu \mathrm{m}$. The most recent procedure for postcontact centrifuging and transferring produced plutonium solutions from whish no additional plutonium was removed by filtering. This was not true for americium, where a factor of 2 or more of the activity was sometimes removed by filtering the centrifuged solutions, the resuits varied with the contact temperature. These results suggest that plutonium does not significantly sorb on polycarbonate filter membranes, at. least in the time required for filtration. The activity present in a piutonium solution after it has been centrifuged three times is, therefore, probably the correct value for calculating sorption ratios. For americium, it appears that centrifuging the solution afier contact establishes a lower limit to the sorption ratio because crushed-rock particles and particulates remaining with the solution tend to lower the calculated $R_{d}$.

In an attempt to better understand the mechanism by which americium is retained on filter membranes, 38 membranes from the sequential filterings listed in Table XIV were examined by a microautoradiographic technique. Approximately one-half to one-third of each filter membrane was mounted on a glass slide by coating j.t with a thin layer of parlodion (a $2 \%$ solution in isopentyl acetate). The slide was then alpha counted to determine the length of exposure time needed (calculated to give $\sim 10^{7}$ total disintegrations) and clamped together with a second slide on which a Kodak AR.10 strippable emulsion had been mounted. Mounting the emulsion directly onto the coated filter made it difficult to see single tracks. To ensure that the emulsion adhered well to blank glass slides, the slides were first etched with a dilute solution of $H \bar{r}$, then dried and coated with a solution of $2 \%$ parlodion in isopentyl acetate, dried again, and finally coated with a thin gelatin layer by dipping the slide in a water solution containing $0.5 \%$ gelatin and $0.06 \% \operatorname{KCr}\left(\mathrm{SO}_{4}\right)_{2} \cdot 12 \mathrm{H}_{2} \mathrm{O}$.

Microautoradiography of the "sorption" membranes (those from solutions after initial sorption experiments) showed not only single-alpha tracks but also clusters and stars, indicating the presence of large complex species, polymers, or colloids in the so-called solutions, even after multiple centrilugings. Some sorption solutions were filtered successively through several membranes with the same or derreasing pore sizes (Table XIV); essentially all these membranes were found to retain americium in clusters, stars, and single tracks. Americium species in these solutions probably exist in a broad range of particle sizes. In contrast, the "desorption" membranes (those from 
TABLE XIV

AVERAGE ACTIVITY ${ }^{a}$ REMOVED FROM SOLUTIONS BY CENTRIFUGING AND FILTERING

Samples

Treatment

2nd Centrifuge ${ }^{c}$

1 hour

3rd Centrifuge

2 hours

Filter

$0.1 \mu \mathrm{m}$

$0.4 \mu \mathrm{m}$

$0.05 \mu \mathrm{m}$

or

$0.4 \mu \mathrm{m}$

$0.4 \mu \mathrm{m}$

22

11

$\frac{(\%)}{\frac{237_{\mathrm{Pu}}}{28}} \frac{{ }_{\mathrm{Am}}}{\frac{241 \mathrm{Am}^{\mathrm{b}}}{51}}$

$24 \quad 26$

10

13

27
Controls

\begin{tabular}{|c|c|c|}
\hline & (\%) & \\
\hline$\overline{237 \mathrm{Pu}}$ & $241 \mathrm{Am}$ & $241 \mathrm{Am}^{\mathrm{b}}$ \\
\hline & 14 & 8 \\
\hline
\end{tabular}

6

8

3

28

0

$2 \quad 26$

28

0
48

or
$0.05 \mu \mathrm{m}$
0
39
21
70
40
$0.05 \mu \mathrm{m}$
0
6
32

${ }^{a}$ Decreases in activity are in per cent of input activity for a given operation removed by that operation.

${ }^{b}$ For $70^{\circ} \mathrm{C}$ solutions.

${ }^{\mathrm{All}}$ centrifugings were at $12000 \mathrm{rpm}$.

solutions after desorption experiments) generally showed only single-alpha tracks, suggesting that some americium sorbed on the membranes and that large particulates were neither removed from the solids during desorption nor formed in the aqueous phase during contact.

Some caution must be exercised in interpreting these autoradiograms because radiocolloids and particles coated with radioactive material of ten produce a similar pattern of tracks. It is sometimes possible to distinguish between then by viewing the original particle or colloid with reflected as well as transmitted light. In related work, Allard ${ }^{*}$ has observed sorption

\footnotetext{
From information received from B. M. Allard, Chalmers University, Göteborg,
} Sweden. 
ratios for Millipore filter paper that are comparable to ratios for rockforming minerals during batch sorption experiments with americium in simulated groundwater.

Considering the above results, a conservative approach was taken in calculating americium sorption ratios; the results from the solutions after they been centrifuged but not filtered were used. After the early postcontact experiments described here, all feed solutions were prepared by drying the tracer and redissolving it in groundwater (Sec. IV.2). ${ }^{7}$

It must be emphasized that the measured sorption ratios for plutonium and americium include effects other than sorption. There may well be differences in the behavior of plutonium or americium even between supposedly identical solutions at $\mathrm{pH} 8$ to 8.5 ; for example, the degree of polymerization and radiocolloid formation and hydrolysis result in variations in species (including charge) and particle size. Grebenshchikova and Davydov ${ }^{19}$ reported that the charge on colloidal Pu(IV) species may be either positive (at low pH values) or negative (at high values) and that the isoelectric $\mathrm{pH}$, or point of zero charge, is in the $\mathrm{pH}$ region 8.0 to 8.5 . Polzer and Miner ${ }^{20}$ presented a plot of effective charge (caused by hydrolysis) of the americium species vs $\mathrm{pH}$ for a $0.1 \mathrm{M} \mathrm{LiClO}_{4}$ solution. Between $\mathrm{pH} 8.0$ and 8.5 the average effective positive charge per atom of americium varied from $\sim 1.3$ to $\sim 0.0$. Therefore, large variations in the behavior of both plutonium and americium can be expected in this pH range.

2. Plutonium Chemistry in Near-Neutral Solutions. In an effort to control the Eh in laboratory experiments involving plutonium and other multivalent elements, several systems are being investigated. A number of osmium complexes that could be used for this purpose have also been prepared and tested. Because of the importance of the $\mathrm{Pu}$ (IV) polymer as a possible migrating species from a repository (as described in Sec. II.B.2.e), its rate of formation at very low concentrations and its ionic dissociation are being studied. Because the $\mathrm{Pu}(\mathrm{V})-\mathrm{Pu}(\mathrm{VI})$ potential in speciation and solubility at Eh greater than $\sim 0.9 \mathrm{~V}$ and $\mathrm{pH}$ less than $\sim 7$ is important, measurements of the equilibrium in selected $\mathrm{Pu}(\mathrm{VI})-\mathrm{Os}$ (II) reactions are being used for an independent determination of the $\mathrm{Pu}(\mathrm{V})-\mathrm{Pu}(\mathrm{VI})$ potential at low acid concentrations and ionic strengths.

a. Eh Control. Measurements of Eh in the laboratory have been made with either a platinum or a gold electrode referenced to a standard calomel 
electrode. Standards of +430 and $-388 \mathrm{mV}$, relative to the normal hydrogen electrode, have been used for calibration.

Measurements of Eh on unpoised or complex systems are difficult to

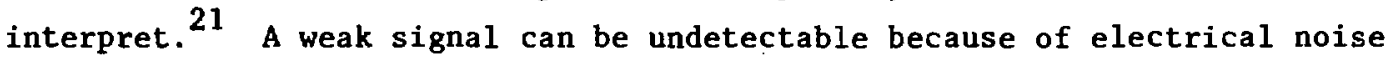
in the system. Laboratory measurements of both aerated and oxygen-free groundwaters in the absence or presence of many rock samples studied (tuff, Climax Stock granite, and Eleana argillite) have generally shown positive potentials. However, mixtures of tuffs YM-54 (divitrified) or tuff YM-38 (zeolitized) and water from well J-13 that have been shaken in a controlled atmosphere for more than a year show slightly lower Eh values, -20 to $-140 \mathrm{mV}$, compared to values of +300 to $+350 \mathrm{mV}$ for aerated solutions with $\mathrm{pH}$ values of $\sim 8$.

Next, $\mathrm{NH}_{2} \mathrm{OH} \cdot \mathrm{HCl}$ was examined as a reducing agent. Solutions were sparged for several hours with argon gas to remove some of the oxygen, $\mathrm{NH}_{2} \mathrm{OH} \cdot \mathrm{HCl}$ was added, and the $\mathrm{pH}$ was adjusted with dilute $\mathrm{NaOH}$ to neutral or slightly basic. The Eh was measured as a function of $\mathrm{pH}$, and an approximately linear correlation was found. At $\mathrm{pH} 7, \mathrm{Eh} \cong 120 \mathrm{mV}$; at $\mathrm{pH} 10.8$, Eh $\cong-130 \mathrm{mV}$.

Before a potentiostat was obtained and set up, a preliminary experiment was run with $10^{-6} \mathrm{M}$ Nile Blue in sodium carbonate solution; ferrous iron was used as reductant to decrease the Eh to the Nile Blue theoretical value of $-160 \mathrm{mV}$ at $\mathrm{pH} 8.3$. This reading was maintained for several hours with the addition of ferrous ammonium sulfate. It is unclear which couple was actually measured: $\operatorname{Ottaway}^{22}$ states that concentrations of $\leq 10^{-7} \mathrm{M}$ are needed for true solution behavior of Nile Blue.

A potentiostat cell similar to those of Harrar ${ }^{23}$ and Rai et al. ${ }^{24}$ was built with a $3.6-\mathrm{cm}$ diam and $7.5-\mathrm{cm}$ height. The bottom has a convex indentation to hold a 5/8-in. Spinfin for magnetic stirring, which allows the very vigorous mixing needed to deoxygenate any solution used under controlled Eh conditions. ${ }^{23}$ The working electrode is a $3.3-\mathrm{cm}-\mathrm{diam}$, platinum-gauze cylinder. The counter electrode is a folded 5- by $2-\mathrm{cm}$ platinum gauze isolated in a glass cell with a porous Vycor frit making contact with the solution. A salt bridge contains a calomel reference electrode. Inlet and outlet tubes for inert-gas sparging and a sample port with a stopper are included.

Control of $\mathrm{pH}$ is necessary when working with Eh buffer-potentiostat systems. Although tris-(hydroxymethyl)-amino-methane makes usable buffers in the desired (underground systems) $\mathrm{pH}$ range, the borate-boric acid systens have been the most useful and probably are less likely to interfere in the rockgroundwater systens. 
Work at Eh values below that of the $\mathrm{H}_{2} \mathrm{O}_{2}-\mathrm{O}_{2}$ couple $(0.68-0.059 \times \mathrm{pH}) \mathrm{V}$ is difficult because of oxidation by oxygen in the air. It is necessary to work in an inert-atmosphere glove box when studying sy csins with Eh values $\lesssim 0.1$ V. A Vacuum Atmospheres controlled-inert-atmosphere glove box with a Dri-Train purification system is used. The oxygen content is $\leq 0.2$ ppm and the carbon dioxide content is $\leq 20 \mathrm{ppm}$ in the atmosphere of the box. Argon gas that has been through a chromous-perchlorate, zinc-amalgam scrubber and an acid scrubber is piped into the box so that various solutions under study can be sparged in the box.

The first investigations of possible redox buffers have focused on redox indicators because a wide range of potentials is available. ${ }^{22}$ However, in addition to having an appropriate potential, a satisfactory Eh buffer system must be relatively stable in both oxidized and reduced forms; it must also show a relatively rapid redox reaction rate with the species of interest, but must not react in other ways.

The first work in Los Alamos involved the organic redox indicator Indigo Carmine. The formal potential (that given by equimolar concentrations of oxidized and reduced forms) for Indigo Carmine is reported ${ }^{22}$ to be $(0.291-0.059 \cdot \mathrm{pH}) \mathrm{V}$. Using this dye, Los Alamos scientists have maintained stable Eh values of $(-0.19 \pm 0.02) \mathrm{V}$ at $\mathrm{pH} 7$ for several days. Indigo Carmine, however, has some drawbacks; unstable Eh values are observed if attempts are made to reduce the last $0.01 \%$ using a potentiostat. Also, Los Alamos has been unable to reproduce the reported $\mathrm{Eh}-\mathrm{pH}$ function--the values at $\mathrm{pH}=1.1$ and 7.6 are $0.1 \mathrm{~V}$ lower and $0.04 \mathrm{~V}$ higher, respectively, than published values. 22

Preliminary experiments with thionine show that it may be useful at Eh values somewhat higher than those provided by Indigo Carmine. The range might be useful for experiments with technetium. The formal potentials are reported to be 0.563 and $0.064 \mathrm{~V}$ at $\mathrm{pH}=0$ and 7 , respectively. Reasonably satisfactory behavior was observed at pH 5.6 after stirring the solution and sparging it with argon for several days and then reducing with a potentiostat. After this treatment the measured formal potential came to within $0.003 \mathrm{~V}$ of the theoretical value in 3 days. This time may indicate slow attainment of equilibrium at the electrodes.

b. Osmium Complexes. Couples involving selected osmium complexes will probably be useful as Eh buffers or moderators. H. Taube, who has had conFrom information provided by H. Taube, Stanford University, Stanford, CA 94305 (1980). 
siderable experience with a wide variety of inorganic complexes, suggested that although many ruthenium complex couples have desirable potentials, they are probably not stable enough for these purposes. Certain osmium complexes are much more stable, and Taube suggested that the II-III couples such as Os(bipyridyl) $)_{3}$ and Os(bipyridyl) ${ }_{2}(\mathrm{CN})_{2}$ should be tried. This section describes the preparation and some of the properties of these complexes. Osmium complexes have the advantages that (a) the redox potentials of the Os(II)-Os(III) couples are essentially independent of $\mathrm{pH}$ in the range of interest, (b) the standard potential of the couple can be changed by varying the coordinated ligands, (c) the complexes are neutral or positively charged so they are not expected to complex plutonium or other actinide species, and (d) the Os(II) complexes are highly colored so that reactions can be studied at low concentrations. Long-term stability with respect to decomposition in solution and rapidity of redox reaction with plutonium or other actinide species are additional properties required for suitability of complexes as buffers or moderators. If the complexes were to be used as moderators in conjunction with a potentiostat, rapid electrochemical reversibility would also be required.

Preparation. The tris complexes [Os(bipy) $\left.{ }_{3}\right]_{\mathrm{Cl}_{2}}$ (identified as complex A), [Os (dimebipy) $\left.{ }_{3}\right] \mathrm{Cl}_{2}(\mathrm{~B}),[\mathrm{Os} \text { (diphenbipy) }]_{3} \mathrm{Cl}_{2}(\mathrm{C})$, and $\left[\mathrm{Os}\right.$ (nitrophen) $\left.{ }_{3}\right] \mathrm{Cl}_{2}(\mathrm{D})^{*}$ were prepared by refluxing an excess of ligand with $\left(\mathrm{NH}_{4}\right)_{2} \mathrm{OsCl}_{6}$ in ethylene glycol according to the procedure described by Fabian et al. ${ }^{\circ}$ Excess solvent was removed under vacuum, methanol was added, and the product was recovered using a column of Sephadex LH-20 with methanol as eluant. Complexes A, B, and $D$ were recrystallized from methyl ethyl ketone-methanol mixtures under vacuum using a rotating flask. Complex $C$ was precipitated from methanol by an excess of ethyl ether.

The other complexes, $\left[\mathrm{Os}(\mathrm{en})\left(\mathrm{bipy}_{2}\right] \mathrm{I}_{2}(\mathrm{E}),\left[\mathrm{OsCl}(\mathrm{py})(\mathrm{bipy})_{2}\right] \mathrm{ClO} \mathrm{C}_{4}(\mathrm{~F})\right.$, and $\left[\mathrm{OsCl}(\mathrm{py})\left(\mathrm{dimebipy}_{2}\right] \mathrm{Cl}(\mathrm{G})\right.$, were prepared from the appropriate dichlorocomplexes using modifications of the procedures described by Buckingham et al. 26 Complex $\mathrm{F}$ was precipitated from the reaction mixture using $\mathrm{NaClO}_{4}$, separated from a large amount of by-product using the Sephadex column, and finally precipitated from the methanol solution using ethyl ether. The preparation of complex $G$ was similar to that of complex $F$ except that the reaction

Abbreviations for the ligands are bipy $=2,2$ 'bipyridine; dimebipy $=$ 4,4'-dimethyl-2 $2^{\prime}$-bipyridine; diphenbipy $=4,4^{\prime}$-diphenyl-2,2 $2^{\prime}$-bipyridine; nitrophen = 5-nitro-orthophenanthroline; en = ethylenediamine; $p y=$ pyridine; and acac $=$ acetonylacetonate . 
mixture was taken to small volume under vacuum, methanol was added, and the solution was transferred directly to the Sephadex LH-20 column. The product was repurified by a second passage through the column and recovered by removing the methanol at $\sim 45^{\circ} \mathrm{C}$.

Solutions of the Os(II) complexes were assayed by spectrophotometric titrations based on the colors of the complexes. Standard solutions of Ce(IV) or Mn(VII) were used as oxidants. The equivalent weights and peak extinction coefficients determined for some of the complexes are listed in Table XV. The absorption spectra between 300 and $600 \mathrm{~nm}$ have been used to identify complexes to indicate chemical change.

Properties. Formal oxidation potentials were determined in dilute sulfuric acid by titration with $\mathrm{Ce}$ (IV) or Mn(VII) in cells equipped with bright platinum and silver-silver chloride or mercury-calomel electrodes. Because the oxidation potential for 0 s(bipy) ${ }_{3}^{2+}$ is accurately known, 27 it was used as a standard for the other complexes, Tritrations of this complex were run consecutively with the other complexes to calibrate the electrodes and the procedure. The titration data, that is, cell potential vs volume of oxidant added, were fit to the Nernst equation, and the formal cell potential was determined by a least squares procedure. The standard potentials were determined by comparing the formal cell potentials with the measurements taken with os(bipy) ${ }_{3}^{2+}$ made at approximately the same time. Standard potentials determined in this way are included in Table XV.

The stabilities of the complexes in solution were determined by observing changes in the spectra as a function of time.

Solutions of 0 s(bipy) ${ }_{3}^{2+}$ were stored in water for several months at room temperature without significant changes in the spectra. A sample of the material was refluxed in $6 \mathrm{M} \mathrm{NaCl}$ for 16 hours, again with no significant changes. A solution of 0 (bipy) $3_{3}^{3+}$ was prepared from the Os(II) complex by oxidizing it with chlorine, drying it on a vacuum line, and redissolving it in water. After 72 days at room temperature in the dark, this solution showed large amounts of the Os(II) complex. The potentials are such that the Os(III) complex can oxidize water in near-neutral solutions.

Long-term experiments have not been done on solutions of Os (dimebipy) ${ }_{3}^{2+}$, but it is expected to be as stable as the corresponding bipy complex. The spectrum of $O$ (dimebipy) ${ }_{3}^{3+}$ in water was found to be unchanged after 90 hours at room temperature or 15 minutes at $90^{\circ} \mathrm{C}$. However, a 200 -minute exposure to daylight caused a $20 \%$ decrease in the absorption peak at $488 \mathrm{~nm}$. 
TABLE XV

PROPERTIES OF SELECTED OSMIUM COMPLEXES

\begin{tabular}{|c|c|c|c|c|}
\hline Complex & $\begin{array}{c}\text { Equivalent } \\
\text { Weight } \\
\text { (g/equiv) }\end{array}$ & 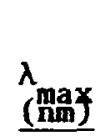 & $\begin{array}{c}\text { Extinction } \\
\text { Coefficient } \\
\left(M^{-1} \mathrm{~cm}^{-1}\right)\end{array}$ & $\begin{array}{c}\text { Oxidation } \\
\text { Potential } \\
\text { (V) } \\
\end{array}$ \\
\hline A. $\left[0 \mathrm{~s}\right.$ (bipy) $\left.{ }_{3}\right] \mathrm{Cl}_{2}$ & $\begin{array}{c}782^{a} \\
(784,3)^{c}\end{array}$ & 480 & $1.36 \times 10^{4}$ & $0.8846^{b}$ \\
\hline B. $\left[0\right.$ s (dimebipy) $\left.{ }_{3}\right] \mathrm{Cl}_{2}$ & $\begin{array}{l}837 \pm 6^{a} \\
(832,1)^{c}\end{array}$ & 488 & $1.42 \times 10^{4}$ & $0.694 \pm 0.002$ \\
\hline c. $\left[0\right.$ s(diphenbipy) $\left.{ }_{3}\right] \mathrm{Cl}_{2}$ & $\begin{array}{l}1155 \pm 38 \\
(1186,0)^{c}\end{array}$ & 502 & $2.45 \times 10^{4}$ & $0.849 \pm 0.004$ \\
\hline D. $\left[0 s\right.$ (nitrophen) $\left.{ }_{3}\right] \mathrm{Cl}_{2}$ & & 470 & & $\sim 1.05^{\mathrm{e}}$ \\
\hline E. $\left[0 \mathrm{~s}(\mathrm{en})\left(\right.\right.$ bipy) $\left.{ }_{2}\right] \mathrm{I}_{2}$ & & 505 & & \\
\hline F. $\left[0 s C 1(p y)(b i p y)_{2} \mathrm{IClO}_{4}\right.$ & $\begin{array}{c}741 \\
(735,1)^{c}\end{array}$ & 345 & $1.17 \times 10^{4}$ & $\begin{array}{c}0.476 \pm 0.005 \\
0.4836^{b}\end{array}$ \\
\hline G. $\left[0 \mathrm{scl}\right.$ (py)(dimebipy) $\left.{ }_{2}\right] \mathrm{Cl}$ & & 473 & & 0.421 \\
\hline H. $\left[0 \mathrm{~s}(\mathrm{acac})(\mathrm{bipy})_{2}\right] \mathrm{Cl}{ }^{(\mathrm{d})}$ & & 515 & & $0.153^{b}$ \\
\hline $\begin{array}{l}\text { I. }\left[0 \mathrm{~s}\left(2-\left(2^{\prime}-\text { pyridyl)quinoline }\right)_{3}\right] \mathrm{Cl}_{2}{ }^{(d)}\right. \\
\text { J. }\left[0 \mathrm{os}(6-\text { methyl-2,2'bipyridine })_{3}\right] \mathrm{Cl}_{2}{ }^{(d)}\end{array}$ & & 503 & & $\begin{array}{c}0.990 \pm 0.003 \\
\sim 0.90^{\mathrm{e}}\end{array}$ \\
\hline
\end{tabular}

a Heated to $\sim 50^{\circ} \mathrm{C}$ under vacuum.

${ }^{b}$ Reference 25 .

${ }^{C}$ Calculated equivalent weight for indicated hydrate.

Material furnished by D. M. Klassen, Department of Chemistry, McMurry College, Abilene, TX 79605.

Potential is only approximate because of serious drifts. 
The stabilities of the bis-(bipy) and bis-(dimebipy) complexes were examined in experiments in which the complexes were dissolved in argon-swept water and sealed into 13-mm-o.d. Pyrex tubes. The +3 oxidation states were prepared using iodine as the oxidizing agent. The spectra were determined, and the absorbances at characteristic peaks and valleys were recorded for periods of a week or more; the results are summarized in Table XVI. Except for two of the oxidized forms, [Os(acac)(bipy) $\left.{ }_{2}\right]_{C l}$ and $\left[\mathrm{OsCl}(\mathrm{py})\left(\right.\right.$ dimebipy) $\left.{ }_{2}\right] \mathrm{Cl}_{2}$, the spectral changes are quite small. Also, $\left[0 \mathrm{SCl}(\mathrm{py})(\mathrm{bipy})_{2}\right] \mathrm{ClO}_{4}$ was found to be relatively unstable in solution at $\mathrm{pH} 3$.

After the room temperature experiments were completed, the sealed samples were subjected to a temperature of $\sim 90^{\circ} \mathrm{C}$ for 2.5 hours and then room temperature under daylight for $\sim 50$ hours. Neither of these treatments caused drastic changes; in all cases the spectra of the final solutions were similar to the original ones. [Os(acac)(bipy) $)_{2} \mathrm{Cl}_{2}$ and $\left[\mathrm{OsCl}\right.$ (py)(bipy) $\left.{ }_{2}\right]\left(\mathrm{ClO}_{4}\right)_{2}$ appeared to be the least stable, showing absorbance changes up to 12 and $43 \%$, respectively.

c. Reaction Rates Between Plutonium Species and Osmium Complexes. The rates of a number of reactions between osmium complexes and the various oxidation states of plutonium were investigated because such data are required when evaluating the complexes for possible use as Eh buffers and moderators.

TABLE XVI

STABILITY OF VARIOUS OSMIUM COMPLEXES IN WATER AT ROOM TEMPERATURE

\begin{tabular}{|c|c|c|}
\hline Complex & $\begin{array}{c}\text { Observation } \\
\text { Period } \\
\text { (days) } \\
\end{array}$ & $\begin{array}{l}\text { Spectral_Changes } \\
\left(\% \text { day }{ }^{1}\right) \\
\end{array}$ \\
\hline$\therefore\left[\mathrm{Os}(\mathrm{en})(\mathrm{bipy})_{2}\right] \mathrm{I}_{2}$ & 73 & +0.01 to +0.03 \\
\hline$\left[\mathrm{Os}(\mathrm{en})\left(\right.\right.$ bipy $\left._{2}\right] \mathrm{I}_{3}$ & 11 & 0 to -0.23 \\
\hline F. $\left[\operatorname{OsCl}(p y)(b i p y)_{2}\right] \operatorname{clo}_{4}^{a}$ & 3 & -0.10 to $+0.40^{b}$ \\
\hline$\left[0 \mathrm{Cl} 1\right.$ (py)(bipy) $\left.{ }_{2}\right]\left(\mathrm{ClO}_{4}\right)_{2}$ & 11 & -0.30 to -0.50 \\
\hline G. $\left[0 \mathrm{sCl}(\mathrm{py})\left(\right.\right.$ dimebipy)$\left._{2}\right] \mathrm{Cl}$ & 13 & -0.08 to -0.23 \\
\hline$\left[\mathrm{OsCl}(\mathrm{py})\left(\right.\right.$ dimebipy) $\left.{ }_{2}\right] \mathrm{Cl}_{2}$ & 13 & -0.11 to -1.44 \\
\hline H. $\left[O s(a c a c)(b i p y)_{2}\right] \mathrm{cl}$ & 20 & 0 to -0.05 \\
\hline$\left[\mathrm{Os}(\mathrm{acac})(\mathrm{bipy})_{2}\right] \mathrm{Cl}_{2}$ & 12 & -1.0 to -4.0 \\
\hline
\end{tabular}

${ }^{\mathrm{a}}$ Run in ordinary $1.00-\mathrm{cm}$ cell.

${ }^{b}$ Values were -1.8 to $-3.3 \%$ day $^{-1}$ at $\mathrm{pH} 3$. 
Plutonium(III) solutions were prepared by dissolving weighed pieces of electrorefined ${ }^{239} \mathrm{Pu}$ metal in concentrated $\mathrm{HClO}_{4}$ and diluting to the desired concentration or by reducing other oxidation states on zinc-amalgam. Plutonium(VI) was made from $\mathrm{Pu}(\mathrm{III})$ by oxidation in strongly fuming $\mathrm{HClO}_{4}$. Plutonium(IV) solutions were made by mixing appropriate amounts of $\mathrm{Pu}(\mathrm{III})$ and $\mathrm{Pu}(\mathrm{VI})$ in $3 \mathrm{M}$ acid.

The reactions were studied spectrophotometrically at room temperature, and the results are summarized in Table XVII. Rapidly stirred $10-\mathrm{cm}$ absorption cells were used for the faster reactions. Except for the reduction of $\mathrm{Pu}(\mathrm{V})$, the rates are sufficiently rapid for satisfactory Eh buffer behavior.

The reaction

$$
\mathrm{Pu}(\mathrm{III})+20 \mathrm{~s}(\mathrm{bipy})_{3}^{3+}=\mathrm{Pu}(\mathrm{V})+20 \text { s(bipy) }{ }_{3}^{2+}
$$

was studied at $\mathrm{pH}$ values of 3.0 and 3.8 , using a great excess of the Os(III) complex. The concentration-vs-time curves at $\mathrm{pH} 3$ were consistent with consecutive pseudo first-order reactions with the second-order rate constants listed in Table XVII. The results obtained at a $\mathrm{pH}$ of 3.8 are difficult to interpret quantitatively because the overall change in absorbance corresponded to only $\sim 82 \%$ conversion of $\mathrm{Pu}($ III) to $\mathrm{Pu}(\mathrm{V})$. However, the data indicate that the oxidation of $\mathrm{Pu}(\mathrm{IV})$ to $\mathrm{Pu}(\mathrm{V})$ is about five times faster, and the oxidation of $\mathrm{Pu}$ (III) to $\mathrm{Pu}$ (IV) is about two times faster, than at the lower $\mathrm{pH}$ value.

d. The $\mathrm{Pu}(\mathrm{V})-\mathrm{Pu}(\mathrm{VI})$ Potential. The reaction

$$
\mathrm{PuO}_{2}^{2+}+\mathrm{Os}(\text { bipy })_{3}^{2+}=\mathrm{PuO}_{2}^{+}+\mathrm{Os}(\text { bipy })_{3}^{3+}
$$

deserves further comment because it was found not to go to completion at pH 2 . Net absorbance change was measured at the Os(II) peak in experiments in which the initial $\mathrm{Pu}(\mathrm{VI})$ concentrations were in the range $(1.7$ to 8.4$) \times 10^{-6} \mathrm{M}$, and the initial Os(II) concentrations were $(1.9$ to 9.4$) \times 10^{-6} \mathrm{M}$. In each of seven runs, values for the equilibrium quotient $Q$ and the extinction coefficient difference for the reaction were found that minimize the sum of the squares of the differences between the observed and calculated absorbance values. Values for $Q$ were found to depend very strongly on the initial $\mathrm{Pu}(\mathrm{VI}) / \mathrm{Os}$ (II) ratios, so the analytical determination of the reactants must be very accurate. The average of the seven separate determinations of $Q$ is 14 with a mean deviation 
TABLE XVII

REACTION RATES BETWEEN OSMIUM COMPLEXES AND THE VARIOUS OXIDATION STATES OF PLUTONIUM IN AQUEOUS SOLUTIONS AT ROOM TEMPERATURE

\begin{tabular}{|c|c|c|c|c|c|}
\hline \multirow[b]{2}{*}{ Reaction } & \multicolumn{3}{|c|}{ Concentration } & \multirow{2}{*}{$\begin{array}{l}\text { Ionic } \\
\text { Strength } \\
(M) \\
\end{array}$} & \multirow{2}{*}{$\begin{array}{l}\text { Apparent Second-Order } \\
\text { Rate }{ }_{1} \text { Constant } \\
\left(\mathrm{M}^{-1} \mathrm{~S}^{-1}\right)\end{array}$} \\
\hline & $\left(M \times 10^{6}\right)$ & $\stackrel{0 s}{\left(M \times 10^{6}\right)}$ & $\underset{\mathrm{H}}{\mathrm{H}^{+}}$ & & \\
\hline $\begin{array}{c}P u(V I)+0 s\left(\text { bipy) } 3_{3}^{2+}=\right. \\
P u(V)+0 s(\text { bipy) } \\
3+\end{array}$ & 1.7 to 8.4 & 1.9 to 9.4 & 0.01 & 0.01 & $\left(4.45 \pm 0.17 \times 10^{4}\right.$ \\
\hline $\begin{array}{l}P u(V I)+0 s(d i m e b i p y))_{3}^{2+}= \\
P u(V)+0 s(d i m e b i p y) \\
3\end{array}$ & 2.0 & 2.0 & 0.001 & 0.001 & $(3.7 \pm 0.02) \times 10^{5}$ \\
\hline $\begin{array}{l}\mathrm{Pu}(V)+0 \text { s (dimebipy) }{ }_{3}^{2+}= \\
\mathrm{Pu}(\mathrm{IV})+\text { Os (dimebipy) }{ }_{3}^{3+}\end{array}$ & 12 & 34.5 & 0.006 & 0.006 & $3.2 \times 10^{-3}$ \\
\hline $\begin{array}{c}P u(v)+0 s(a c a c)(\text { bipy })_{2}^{+}= \\
P u(I V)+O s(\text { acac })(b i p y)_{2}^{2+}\end{array}$ & 21 & 130 & 0.002 & 0.0022 & $0.013^{\mathrm{a}}$ \\
\hline $\begin{array}{l}\mathrm{Pu}(\mathrm{IV})+0 \text { os(bipy) }{ }_{3}^{2+}= \\
\quad \mathrm{Pu}(\mathrm{III})+0 \text { os(bipy) } \\
3\end{array}$ & $\begin{array}{c}1.5 \text { to } 2.0 \\
1.5 \\
1.9 \text { to } 3.8\end{array}$ & $\begin{array}{c}6.5 \\
6.5 \\
1.8 \text { to } 9.1\end{array}$ & $\begin{array}{c}0.01 \text { to } 0.82 \\
0.10 \\
0.01\end{array}$ & $\begin{array}{l}0.82 \\
0.10 \\
0.01\end{array}$ & $\begin{array}{r}(6.2 \pm 0.2) \times 10^{3 b} \\
1.1 \times 10^{3} \\
(6.8 \pm 0.2) \times 10^{2}\end{array}$ \\
\hline $\begin{array}{l}\mathrm{Pu}(\mathrm{IV})+0 \mathrm{~s}\left(\text { dimebipy) }{ }_{3}^{2+}=\right. \\
\mathrm{Pu}(\mathrm{III})+\text { os (dimebipy) }{ }_{3}^{3+}\end{array}$ & 2.1 & 6.2 & $(0.17$ to 10$) \times 10^{-3}$ & 0.01 & $2 \times 10^{4} /\left[1+3.56 \times 10^{-4} /\left(H^{+}\right)\right]$ \\
\hline $\begin{array}{c}\mathrm{Pu}(\mathrm{III})+0 \mathrm{~s}(\mathrm{bipy})_{3}^{3+}= \\
\mathrm{Pu}(\mathrm{IV})+\mathrm{Os}_{3}(\text { bipy })_{3}^{2+}\end{array}$ & 1.9 & 73 & 0.001 & 0.0015 & 46 \\
\hline $\begin{array}{l}\operatorname{Pu}(I V)+0 s(\text { bipy })_{3}^{3+}= \\
\quad P u(V)+0 s(\text { bipy })_{3}^{2+}\end{array}$ & 1.9 & 73 & 0.001 & 0.0015 & 4 \\
\hline
\end{tabular}

\footnotetext{
aery approximate because the stoichiometry is not understood.
}

bo significant $\mathrm{H}^{+}$dependence. 
of 2.4. This average value is readily extrapolated from $0.01 \mathrm{M}$ to zero ionic strength giving $11.7 \pm 2.4$ for the equilibrium constant. Combining this with $0.8846 \mathrm{~V}$, the accurately determined value for the 0 (II) $-0 s$ (III) couple, ${ }^{27}$ gives $0.948 \pm 0.005 \mathrm{~V}$ for the $\mathrm{Pu}(\mathrm{V})-\mathrm{Pu}(\mathrm{VI})$ standard potential. This result is compared to $1.016 \pm 0.050 \mathrm{~V}$ recommended by Fuger and 0 etting ${ }^{28}$ and $0.933 \mathrm{~V}$ estimated by Allard et al. 18

e. Ionic Dissociation of the Pu(IV) Polymer. The existence of bright green $\mathrm{Pu}$ (IV) polymer suspensions is common knowledge. ${ }^{29}$ This polymer is of interest as a possible migrating species from a repository and also because it is probably related to the nonreactive Pu(IV) that is discussed in Sec. II.B.2.f.

Suspensions of the polymer have been prepared by diluting $\mathrm{Pu}$ (IV) in $\mathrm{HCl}-\mathrm{HClO}_{4}$ mixtures, rather than by using $\mathrm{HNO}_{3}$ as previously described. ${ }^{30,31}$ The formation of polymer was observed to be much slower than reported for $\mathrm{HNO}_{3}$ solutions, ${ }^{32}$ but after ionic species were removed with a cation exchange resin, the spectra of the preparations were essentially the same as those reported earlier. ${ }^{32}$ In a second method of preparing the polymer, Pu(IV) in $\mathrm{HClO}_{4}$ was partially neutralized with $\mathrm{NaOH}$ solution and then heated to $90^{\circ} \mathrm{C}$ for 30 minutes. The spectrum of the suspension prepared in this way and treated with a cation exchange resin agreed with published spectra.

The solubility of the polymer $\left[\left(\mathrm{PuO}_{2} \cdot \mathrm{mH}_{2} \mathrm{O}\right) \mathrm{n}\right]$ may be defined in terms of the equilibrium

$$
\begin{aligned}
& (1 / \mathrm{n})\left(\mathrm{PuO}_{2} \cdot \mathrm{mH}_{2} \mathrm{O}\right) \mathrm{n}+4 \mathrm{H}^{+}=\mathrm{Pu}^{4+}+\left(2+\mathrm{m}_{2} \mathrm{H}_{2} \mathrm{O}\right. \text {. } \\
& \text { polymer }
\end{aligned}
$$

In a previous attempt to measure the solubility, ${ }^{31}$ polymer was equilibrated in solutions with $3 \leq \mathrm{pH} \leq 7$. Soluble plutonium was defined as all plutonium species that pass through a Centriflo filter with 2-nm pore size. These species were shown to be predominantly $\mathrm{Pu}(\mathrm{V})$, although the oxidizing agent was not identified.

A preliminary experiment tested the feasibility of determining the solubility of the Pu(IV) polymer in a way that should avoid the difficulties associated with the above method. In this experiment $\mathrm{PuO}_{2}^{2+}$ was mixed with polymer at $\mathrm{pH} 3$, where the equilibrium reaction is probably

$$
(1 / \mathrm{n})\left(\mathrm{PuO}_{2} \cdot \mathrm{mH}_{2} \mathrm{O}\right)_{\mathrm{n}}+\mathrm{PuO}_{2}^{2+}=2 \mathrm{PuO}_{2}^{+}+\mathrm{mH}_{2} \mathrm{O} .
$$


The concentration of $\mathrm{PuO}_{2}^{2+}$ was determined spectrophotometrically as a function of time. The question is whether concentrations can be identified where the rates of the forward and reverse reactions are equal. If so, the equilibrium quotient for reaction (4) can be determined and that of reaction (3) calculated from it by using the $\mathrm{Pu}^{4+}-\mathrm{PuO}_{2}^{+}$and the $\mathrm{PuO}_{2}^{+}-\mathrm{PuO}_{2}^{2+}$ potentials.

Three mixtures were prepared in $10^{-3} \mathrm{M} \mathrm{HClO}_{4}:$ (A) $10^{-4} \mathrm{~N}$ polymer, (B) $5 \times 10^{-5} \mathrm{M} \mathrm{PuO}_{2}^{2+}$, and (C) $10^{-4} \mathrm{~N}$ polymer and $5 \times 10^{-5} \mathrm{M} \mathrm{PuO}_{2}^{2+}$. The mixtures were placed in $10-\mathrm{cm}$ absorption cells, and the concentrations of $\mathrm{PuO}_{2}^{2+}$ were determined spectrophotometrically at $830 \mathrm{~nm}$ for 30 days.

Mixture (A) showed an increase in $\mathrm{PuO}_{2}^{2+}$ concentration corresponding to $<0.01 \%$ per day based on the total plutonium present. In an experiment in much higher acid, a mixture $\sim 5 \times 10^{-3} \mathrm{~N}$ in polymer and $0.04 \mathrm{M}$ in $\mathrm{HClO}_{4}$ was followed spectrophotometrically for 18 days. During this period the Pu(VI) concentration increased at a rate of $\sim 0.11 \%$ of the total plutonium per day.

Mixture (B) showed a linear decrease in $\mathrm{PuO}_{2}^{2+}$ concentration because of the net reaction

$$
2 \mathrm{PuO}_{2}^{2+}+\mathrm{H}_{2} \mathrm{O}=2 \mathrm{PuO}_{2}^{+}+2 \mathrm{H}^{+}+\frac{1}{2} \mathrm{O}_{2},
$$

caused by alpha particle self-irradiation from the decay of the ${ }^{239} \mathrm{Pu}$. The observed rate of this reaction was $1.25 \%$ of the total plutonium per day. This is in satisfactory agreement with the value of $1.5 \%$ per day reported for solutions in $1 \mathrm{M} \mathrm{HClO}_{4} 33$

The mixture of $\mathrm{PuO}_{2}^{2+}$ and polymer, mixture $(C)$, showed a nonlinear decrease in $\mathrm{PuO}_{2}^{2+}$ concentration. The absorbance values were found to agree within 0.002 with the empirical equation

$$
A=0.325 \exp (-0.0299 t)-0.050
$$

where $A$ is the absorbance and $t$ is the time in days. The initial decrease in absorbance, from this equation, is 0.0097 per day or $3.5 \%$ of the original Pu(VI) per day, or $1.16 \%$ per day based on the total plutonium present.

At the end of the 30-day period, excess Ce(IV) was added to estimate the ionic plutonium species present. Sufficient $\mathrm{Ce}\left(\mathrm{ClO}_{4}\right)_{4}$ was added to produce final mixtures that were $3 \times 10^{-4} \mathrm{M}$ in $\mathrm{Ce}$ (IV) and $0.079 \mathrm{M}$ in $\mathrm{HClO}_{4}$.

Mixture (A), polymer with no added $\mathrm{PuO}_{2}^{2+}$, showed a rapid absorbance increase of 0.03 during the first minute after the Ce(IV) was added. This was 
followed by a much slower increase with a rate of $\sim 2.5 \times 10^{-4}$ per day for the next 150 minutes. These results indicate that Ce(IV) reacts relatively slowly with the polymer, but $\sim 5 \%$ of the plutonium present reacted rapidly, suggesting that small amounts of ionic $\mathrm{Pu}(\mathrm{III}), \mathrm{Pu}(\mathrm{IV})$, or $\mathrm{Pu}(\mathrm{V})$ had formed.

Mixture (B), $\mathrm{PuO}_{2}{ }^{2+}$ with no polymer, reacted rapidly with $\mathrm{Ce}$ (IV). The final absorbance value was reached within 1 minute and remained constant for the next 163 hours. The $\mathrm{PuO}_{2}^{+}$formed in reaction (5) was apparently reoxidized to $\mathrm{PuO}_{2}^{2+}$.

Mixture (C), polymer plus $\mathrm{PuO}_{2}{ }^{2+}$, showed a rapid increase in absorbance when the $\mathrm{Ce}(\mathrm{IV})$ was added, followed by a slower increase similar to that observed for mixture (A). The absorbance, extrapolated to the time the Ce(IV) was added, was greater than that of the original mixture. The concentrations of $\mathrm{PuO}_{2}^{2+}$ in mixture (C) before and after adding the Ce(IV) were calculated from the absorbance values and are given in Table XVIII; two derived quantities are also given.

Entry (d) in Table XVIII shows that reaction (4) occurred at a slow but measurable rate. From entry (e) it can be calculated that the average rate of reaction (5) was $0.67 \%$ per day, based on the total plutonium present. This is about one-half the rate observed for mixture (B), where no polymer was present. This smaller valu indicates that some of the alpha particles from the decay of the ${ }^{2.39} \mathrm{Pu}$ are assorbed in the colloidal particles and do not lead to chemical reaction.

Because the results in Table XVIII show that most of the reduction of $\mathrm{PuO}_{2}^{2+}$ was by reaction (5), an accurate estimation of the equilibrium quotient for reaction (4) cannot be expected. In addition, an assumption abou- the rate of reaction (5) must be made. However, the following calculation will demonstrate the process. Equation (6) can be used to show that the observed rate of reduction of $\mathrm{PuO}_{2}^{2+}$ equaled the average rate of reaction (5) at $\sim 18.5$ days. If the rate of reaction (5) was essentially constant in the mixture containing the polymer as well as in mixture (B), then the net rate of reaction (4) must have been zero at $\sim 18.5$ days. At this time the rates of the forward and reverse reactions would have been equal, and the equilibriur quotient, $Q=$ $\left[\mathrm{PuO}_{2}^{+}\right]^{2} /\left[\mathrm{PuO}_{2}^{2+}\right]$, can be calculated from the concentrations for that time. The value obtalned is $\sim 4 \times 10^{-5} \mathrm{M}$, with an experimental uncertainty of $20 \%$.

The potentials for the $\mathrm{Pu}^{4+}-\mathrm{PuO}_{2}^{+}$and the $\mathrm{PuO}_{2}^{+}-\mathrm{PuO}_{2}^{2+}$ have been estimated as 1.115 and $0.933 \mathrm{~V}$, respectively, at an ionic strength of zero, ${ }^{18}$ which 
TABLE XVIII

$\mathrm{PuO}_{2}^{2+}$ CONCENTRATIONS IN MIXTURE C

(a) Original mixture

Description

Concentration

(b) After 30 days; room temperature

$\frac{\left(M \times 10^{5}\right)}{4.86}$

(c) After adding Ce(IV); extrapolated to time of mixing

1.40

(d) Consimed by reaction (4), (c)-(a)

5.34

0.48

(e) Consumed by reaction (5), (a)-(b)-(d)

2.98

leads to $\left.\left[\mathrm{Pu}^{4+}\right]\left[\mathrm{PuO}_{2}^{2+}\right] / \mathrm{PuO}_{2}^{+}\right]^{2}\left[\mathrm{H}^{+}\right]^{4}=1.2 \times 10^{3} \mathrm{M}^{-4}$. Combining this with our provisional estimate of $4 \times 10^{-5} \mathrm{M}$ for $\left[\mathrm{PuO}_{2}^{+}\right]^{2} /\left[\mathrm{PuO}_{2}^{2+}\right]$ gives $5 \times 10^{-2} \mathrm{M}^{-3}$ for reaction ( 3 ). This result is reasonable, so the experiment is being repeated using ${ }^{242}$ Pu to minimize the conplexities caused by alpha particle self-irradiation, reaction (5).

f. Polymer Formed in Dilute Solutions. The rate of Pu(IV) polymer formation has been studied in $\mathrm{HNO}_{3}$ solutions with plutonium concentrations in the range from 0.009 to $0.05 \mathrm{M}$ and the acid concentration from 0.02 to $0.4 \mathrm{M}$ (Refs. 32, 34). Preliminary experiments at much lower concentrations of both plutonium and acid strongly suggest the formation of polymer under these conditions also.

Studies of the reaction between $\mathrm{Pu}(\mathrm{IV})$ and $\mathrm{Os}(\mathrm{dimebipy}){ }_{3}^{2+}$ revealed that the amount of Os(II) oxidized depended on the history of the Pu(IV) solution. Experiments were performed in which a stock solution $\sim 0.04 \mathrm{M}$ in $\mathrm{Pu}(\mathrm{IV})$ and $3 \mathrm{M}$ in $\mathrm{HClO}_{4}$ was diluted to a $\mathrm{pH}$ of 3 . Aliquots were removed periodically and added to excess os(1I), and the absorbance change was determined. The amount of plutonium capable of rapid reaction with Os(II) decreased with time in approximately second-order fashion. Possible reactions to account for this loss include the following.

$$
\begin{array}{lrl}
\mathrm{O}_{2} \text { oxidation: } & 2 \mathrm{Pu}(\mathrm{IV})+\frac{1}{2} \mathrm{O}_{2} & =2 \mathrm{Pu}(\mathrm{V})+\mathrm{H}_{2} \mathrm{O} \\
\text { disproportionation: } & 2 \mathrm{Pu}(\mathrm{IV}) & =\mathrm{Pu}(\mathrm{III})+\mathrm{Pu}(\mathrm{V}) \\
\text { polymerization: } & \mathrm{nPu}(\mathrm{IV}) & =[\mathrm{Fu}(\mathrm{IV})] \mathrm{n}
\end{array}
$$

The effect of oxygen on the disappearance of reactive Pu(IV) was determined by adding concentrated plutonium stock to either oxygen- or argou-saturated 
solutions. The concentrations in the diluted solutions were $4.4 \times 10^{-6} \mathrm{M}$ $\mathrm{Pu}(\mathrm{IV}), 1 \times 10^{-3} \mathrm{M} \mathrm{HClO}_{4}$, and $9 \times 10^{-3} \mathrm{M} \mathrm{LiClO}_{4}$. After 30 minutes, during which the appropriate gas was passed over the surface of the solution, a $33 \%$ excess of Os(dimebipy) ${ }_{3}^{2+}$ was added. Fractions of reactive $\mathrm{Pu}$ (IV) remaining were determined by measuring absorbance changes caused by oxidation of the Os (II); they were $0.304 \pm 0.037$ for the oxygen saturated solution and $0.299 \pm$ 0.009 for the argon-saturated solution. These data lead to the conclusion that no more than $\sim 6 \%$ of the disappearance of the Pu(IV) is the result of oxygen oxidation.

The fact that $\mathrm{Ce}$ (IV) in $0.5 \mathrm{M} \mathrm{H}_{2} \mathrm{SO}_{4}$ reacts rapidly with $\mathrm{Pu(III)}$ and $\mathrm{Pu(V)}$ but only slowly with Pu(IV) shows that disproportionation is relatively unimportant. Separate solutions $2 \times 10^{-6} \mathrm{M}$ in $\mathrm{Pu}(\mathrm{IV}), 1 \times 10^{-3} \mathrm{M}$ in $\mathrm{HClO}_{4}$, and $9 \times 10^{-3} \mathrm{M}$ in $\mathrm{LiClO}_{4}$ were prepared from $\mathrm{Pu}(\mathrm{IV})$ in $3 \mathrm{M}$ acid and were allowed to stand for 2 and 84 minutes, respectively. A sevenfold excess of Ce(IV) was then added to react with any $\mathrm{Pu}(\mathrm{III})$ and $\mathrm{Pu}(\mathrm{V})$ that might have formed. Sulfuric acid was added with the $\mathrm{Ce}(\mathrm{IV})$, so the final solutions were $0.5 \mathrm{M}$ in $\mathrm{H}_{2} \mathrm{SO}_{4}$ and $1.4 \times 10^{-5} \mathrm{M}$ in $\mathrm{Ce}$ (IV). Unreacted Ce(IV) was determined from the absorbance at $320 \mathrm{~nm}$. The two solutions showed an absorbance difference of 0.009 , indicating that slightly more reducing agent was formed in the solution that stood for 84 minutes. These results indicate that $\sim 9 \pm 4 \%$ of the Pu(IV) may have reacted to produce $\mathrm{Pu}(\mathrm{III})$ and $\mathrm{Pu}(\mathrm{V})$ in the period between 2 and 84 minutes. Previous experiments under the same conditions showed that in the same time interval the amount of reactive $\mathrm{Pu}(\mathrm{IV})$ decreased by at least $54 \%$. This experiment should be repeated using a more sensitive reagent, but the tentative conclusion is that most of the disappearance of reactive Pu(IV) is not caused by disproportionation. This conclusion requires that the reverse of reaction (2) not be rapid in $0.5 \mathrm{M} \mathrm{H}_{2} \mathrm{SO}_{4}$ compared with the oxidation of Pu(III) and $\mathrm{Pu}(\mathrm{V})$ by $\mathrm{Ce}(\mathrm{IV})$. This was shown to be the case in a separate experiment. The discussion given above makes it appear highly likely, but does not prove, that disappearance of reactive $\mathrm{Pu}(\mathrm{IV})$ is the result of polymerization. The effect of reducing the hydrogen ion concentration from $1.0 \times 10^{-3}$ to $5.0 \times 10^{-4} \mathrm{M}$ was determined in experiments in which $0 \mathrm{sCl}(\mathrm{py})(\mathrm{bipy})_{2}^{+}$was used as the reducing agent. The second-order rate constants found at the two hydrogen ion concentrations indicate that the empirical rate law is

$$
-d[P u(I V)] / d t=6.2 \times 10^{-3}[P u(I V)]^{2}\left(H^{+}\right)^{-2.2} \mathrm{Mmin}^{-1}
$$


This rate law is written in terms of the plutonium species present in the solution in the $\mathrm{pH}$ range studied, 3.0 to 3.3 . If the hydrolysis constants published by Baes and Mesmer ${ }^{15}$ are accepted, the observed hydrogen ion dependence is consistent with a rate determining step in which $\mathrm{Pu}(\mathrm{OH})_{3}^{+}$reacts with $\mathrm{Pu}(\mathrm{OH})_{4}^{\circ}$.

3. Particulate Transport. One mechanism by which radionuclides may move through geologic media is particulate transport. Dissolved radionuclides can sorb onto particulates consisting of microscopic pieces of rock, dust, fibers, or other debris present in the liquid phase. If these particulates are less than a micrometer in diameter, they are often called radiocolloids. Dissolved radionuclides may also aggregate to form colloids or other stable associations. In addition, leaching of solids containing radioactive material may produce collodial material that is then transported with the aqueous leachant. The extent to which particulate transport may contribute to radionuclide migration in geologic media has not been widely studied. Presumably, such transport would depend on the aqueous flow rate, pore and fracture size of the rock media, ionic composition of the water, and the nature of the particulate, among other factors. Filterable aggregates containing americium and plutonium have been observed in the effluents of crushed-rock columns. Because the feed solutions that were used contained both ionic and aggregated species, it is not clear whether the aggregates in the effluent formed during passage of ions through the column, or whether the aggregates passed intact through the column. Better characterization and control of the feed solutions are necessary in order to do studies of this type.

Particulate size is likely to be a determining factor when particulates pass through connected rock pores or constricted fractures. The Pu(IV) polymers prepared by dilution of $\mathrm{Pu}(\mathrm{IV})$ ionic solutions under controlled $\mathrm{pH}$ conditions are being studied. The characteristics of these polymers depend somewhat on the conditions of their formation. The size of these colloids varies considerably; $\sim 10 \%$ are sufficiently small that the material cannot be centrifuged at $12000 \mathrm{rpm}(28000 \mathrm{~g})$. Eventually, perhaps, the size of this polymer can be controlled by varying the conditions under which it is formed. To date, particulate transport research in the laboratory has relied upon centrifugation and filtration to separate particulates according to size and on microautoradiography to detect the presence of aggregated radioactive species on filters or other surfaces. Determining the presence of aggregates in the aqueous phase 
by microautoradiography has proved to be more difficult. With this technique, an aliquot of the solution is dried, usually on a glass pl ie, then microautoradiography is used to determine whether the radioactive material was present in dispersed or aggregated forms. Problems may arise because under some conditions dissolved material may form aggregates during the drying process. Experiments with freeze-drying techniques and substrates other than glass have not resolved all the problems associated with this method of identifying aggregates in the liquid phase.

In some preliminary work, fluorescent dyes were incorporated in microscopic plastic particles to trace particulate flow through crushed-rock columns or through solid-tuff disks. This seems to be a promising technique for tracing the transport of particles in the size range of a few micrometers or less.

4. Microautoradiography Studies. Microautoradiography, a technique in which autoradiographs are examined using optical microscopy, has been employed at Los Alamos in speciation studies of actinides (Sec. II.B.1, II.B.3.) and in sorption studies of actinides on specific minerals. Details concerning the application of this technique to sorption investigations are given in two reports and are summarized here. 35,36 Standard $30-\mu \mathrm{m}$ polished petrographic thin sections are contacted for periods up to several days with groundwater solutions of the actinide of interest. After this solution is rinsed off, a thin photographic emulsion is adhered to the thin section and exposed for a period determined by the level of sorbed activity. The emulsion is then developed in situ, and the alpha-particle tracks are readily visible with an optical microscope at 50 to $400 \mathrm{x}$. Because the emulsion remains adhered to the thin section, the tracks can be related to the mineral site at which the radionuclide was sorbed. Thus, one can distinguish, for example, between sorption on a bulk mineral phase or on the thin layer of alteration phase at the mineral boundary. 36 Also, it is possible to distinguish individual sorbed species from sorbed aggregates (for example, colloids) because the latter give rise to multiple tracks called stars. 35

The microautogradiography technique can be extended to trace the movement of radionuclides through fracture systems in rock cores and over rock surfaces that are not too rough. There may be difficulties in undercutting such rock samples so that they will transmit light. In some instances, the alpha-particle tracks may be observed using reflected light. Autoradiographs may be prepared using beta particles (for example, from ${ }^{63} \mathrm{Ni},{ }^{90} \mathrm{Sr}$, or ${ }^{233} \mathrm{~Pa}$ ) or Auger electrons (from ${ }^{85} \mathrm{Sr}$ ), but the spots left in the emulsion by the electrons are much 
more difficult to identify against the mottled background typical of thin sections than are alpha-particle tracks. In general, microautoradiography is a useful adjunct to other analytical techniques commonly used in geochemical investigations.

\section{I I NEAR-FIELD ENVIRONMENT}

Possible changes in the solid phases at Yucca Mountain are of concern because they could affect rock properties, especially. sorption, strength, permeability, and porosity. This is especially true in the near field where temperature will rise as a result of the repository emplacement. The phases most likely to change are the clays, zeolites, and glass, which may be expected to alter to less hydrous phases having a smaller volume. Auhydrous phase assemblages such as feldspar and silica phases may also be hydrated to zeolites or clays. To predict the mineral assemblages that will be present in Yucca Mouncain as a function of time and location, phase changes that may occur in the Yucca Mountain tuffs must be studied in the laboratory. These changes must be studied as a function of pressure, temperature, and bulk composition.

Phase changes in tuffs of varying mineral composition at known values of pressure and temperature are being examined using samples from drill holes in Yucca Mountain. The samples are ground and enclosed in gold capsules with water. The capsules are then placed in standard cold-seal pressure vessels that are pressurized and heated to the desired conditions. In these experiments water pressure is equal to the total pressure. Table XIX shows the mineral composition of the starting materials and the composition of the final products after 2 weeks at $400^{\circ} \mathrm{C}$ and 400 bars and after 4 weeks at $300^{\circ} \mathrm{C}$ and 400 bars.

The preliminary hydrothermal experiments illustrate several interesting points. The upper stability limit of mordenite is apparently below $400^{\circ} \mathrm{C}$ at 400 bars in most of these rocks; however, in sample BH, mordenite is apparently stable at $400^{\circ} \mathrm{C}$. Two explanations are possible. The mordenite may be metastable at $400^{\circ} \mathrm{C}$, which seems unlikely because clinoptilolite in the other runs at $400^{\circ} \mathrm{C}$ did not produce mordenite. It is more likely that the stability of mordenite in this sample is caused by a difference in composition. Such a difference might be in the ratio of potassium to sodium. There is also some indication that the mordenite in these samples is stable above $300^{\circ} \mathrm{C}$. In the samples that originally contained clinoptilolite and mordenite, mordenite has crystallized at the expense 
TABLE XIX

MINERAL COMPOSITTON OF STARTING MATERIALS AND

HYDROTHERMAL RUN PRODUCTS AT 400 BARS WATER PRESSURE ${ }^{a}$

\begin{tabular}{|c|c|c|}
\hline $\begin{array}{l}\text { Sample } \\
\text { Number }\end{array}$ & $\begin{array}{c}\text { Starting } \\
\text { Composition } \\
\end{array}$ & $\begin{array}{c}\text { Run at } 300^{\circ} \mathrm{C} \\
(4 \text { weeks })\end{array}$ \\
\hline $\mathrm{BH}$ & clinoptilolite & clinoptilolite \\
\hline G1- 1319 & $\begin{array}{l}\text { glass } \\
\text { feldspar } \\
\text { cristobalite? }\end{array}$ & $\begin{array}{l}\text { glass } \\
\text { feldspar } \\
\text { cristobalite? }\end{array}$ \\
\hline Gl-1639 & $\begin{array}{l}\text { clinoptilolite } \\
\text { minor mordenite }\end{array}$ & $\begin{array}{l}\text { clinoptilolite } \\
\text { mordenite } \\
\text { cristobalite } \\
\text { feldspar? }\end{array}$ \\
\hline G2-547 & $\begin{array}{l}\text { feldspar } \\
\text { montmorillonite }\end{array}$ & $\begin{array}{l}\text { feldspar } \\
\text { montmorillonite? }\end{array}$ \\
\hline$G 2-762$ & $\begin{array}{l}\text { clinoptilolite } \\
\text { minor cristobalite }\end{array}$ & $\begin{array}{l}\text { clinoptilolite } \\
\text { cristobalite }\end{array}$ \\
\hline G2-2001 & $\begin{array}{l}\text { mordenite } \\
\text { clinoptilolite }\end{array}$ & $\begin{array}{l}\text { mordenite } \\
\text { minor quartz } \\
\text { cristobalite? }\end{array}$ \\
\hline G2-2667 & $\begin{array}{l}\text { mordenite } \\
\text { quartz }\end{array}$ & $\begin{array}{l}\text { mordenite } \\
\text { quartz }\end{array}$ \\
\hline
\end{tabular}

Minerals are listed in approximate order of abundance.

of clinoptilolite. This is, however, not definitive evidence of mordenite stability; mordenite may well be a metastable product of clinoptilolite decomposition. Certainly this is the case for cristobalite, which is known to be metastable with respect to quartz under these conditions. The observation of mordenite growth at the expense of clinoptilolite also indicates that the upper temperature stability limit of clinoptilolite is $<300^{\circ} \mathrm{C}$ at 400 bars water pressure. Fesults for sample G2-547 indicate that this may also be true for montmorillonite. These conclusions are made somewhat uncertain by lack of knowledge of the exact compositions of the minerals and by questions of metastability.

Experiments have also been performed in which tuff was reacted with $\mathrm{J}-13$ water in Teflon lined Parr Bombs at $152^{\circ} \mathrm{C}$. These experiments are discussed in detail in the groundwater chemistry section (II.A.3). The major difference between these experiments and the gold capsule experiments is that the fluid-to- 
rock ratio is much greater here. In these experiments, the growth of clays and other fine-grained sheet silicates was observed on glass. This would certainly increase the sorptive capacity of the rock. The dissolution of cristobalite, mordenite, and clinoptilolite was also observed. This, however, should not be taken as proof that clinoptilolite and mordenite are unstable at this temperature. It may be that there was insufficient cristobalite available to the solution to raise the silica activity to an equilibrium value with cristobalite because of the large amount of water present in the experiment. Low silica activity, rather than the temperature may be the reason for mordenite and clinoptilolite instability.

Another study has been conducted on cylinders of intact tuff to examine changes in thermomechanical properties as well as changes in mineralogy. The details of this study are given in Ref. 37. Large changes in tensile strength, compressive strength, and permeability were observed in these experiments, but with a few exceptions, porosity, grain density, and thermal properties were observed to be unaffected. Mineralogic and petrologic examination of the test samples has established the operation of reactions involving the dissolution of silica and feldspar, formation of clays, and possible conversion of clinoptilolite to mordenite. However, it has not been possible to establish a one-to-one correlation of mineralogic and structural changes with physical properties changes. Changes in the volumes of minerals involved in these reactions were observed to be very small, reflecting their sluggish nature. This can explain, in a qualitative way, why some properties were unchanged. For example, thermal properties are dominantly determined by the inherent thermal properties of the constituent mineral phases. Unless there are substantial changes in the amounts of minerals with significantly different thermal properties, the thermal properties of the rock are not expected to change. It should be kept in mind, however, that the duration of this test was short relative to the operational time of a repository. The thermal pulse of a repository will last for hundreds of years. During this time slow processes of the type identified could cause significant changes in the thermomechanical properties of the host rock, and should be taken into consideration. Furthermore, it is believed that the strength changes observed are related to the subtle surface modifications of minerals observed, probably most actively along grain boundaries and fracture surfaces where the catalytic action of water is effective. It is expected that these same processes will be inportant in controlling the mechanics of discontinuities such as joints. Indeed, there is 
evidence that rock friction is time-dependent, reflecting viscoplastic processes at point contacts of the surfaces.

A quantitative inclusion of these time-dependent phenomena will require careful measurements on target-horizon tuff samples held at simulated repository conditions for long time periods. Detailed examination of tested samples should identify the physical-chemical mechanisms involved. In addition, the difficult task of determining the rates of the processes will be required. Once these are determined, they can be incorporated in design and performance models to predict the response of the host rock mass over the lifetime of the repository.

\section{GEOCHEMICAL RETARDATION}

\section{A. Sorptive Behavior of Tuff}

To predict the retardation possibilities in the Yucca Mountain area, a data base must be established from which models can be produced. Geochemical retardation processes and flow paths between the repository and the accessible environment must be identified. Geochemical retardation will depend on a number of factors, including (1) sorption processes, (2) the horizon and rock type of the repository, (3) temperature variations, (4) groundwater geochemistry, (5) fixation reactions, (6) diffusion processes, and (7) the effect of mineral precipitation. Information collected through studies of these processes will help predict the rates and concentrations in which radionuclides could be released into the environment and assess the hazards associated with such potential releases. The experimental program thus far has addressed various aspects of sorption by tuff, the physical makeup of tuff, the diffusion process, and various transport processes involved in porous and fracture flow. Planned extentions and additions to these retardation studies will be discussed in Chap. 2 of this report.

1. Introduction. The term sorption has generally been used to describe processes by which elements are removed from solution through their interaction with rock, such as ion exchange phenomena, chemisorption, and diffusion into the rock matrix; these processes may ultimately end in a variety of precipitation or coprecipitation reactions. A variety of experimental techniques have been used to gain an understanding of these processes, to study the importance of the parameters involved, and to build up a data base that will be used to model the sorption of nuclides by tuffs in the Yucca Mountain area. 
Much effort has been spent studying sorption by a simple batch technique for measuring the distribution of an element between groundwater solutions and crushed tuff. The method is valuable because it is simple and a large number of samples can be processed in a relatively short period (a few days to several months). Other methods may also provide information on speciation, kinetics, diffusion, and surface effects, but they require more elaborate equipment and fewer samples can be studied. They frequently require much more time, and some information cannot be obtained within the time limits of the NNWSI. One modification of this batch method uses machined wafers, or disks, of tuff instead of crushed-rock samples. The technique, discussed in Sec. IV.C of this report, gives similar results when the two methods can be compared.

Another modification of the batch technique involves a system in which the groundwater is circulated through a column of cruslied tuff. This method was studied to investigate whether the agitation of the rock in the batch studies caused effects, such as self-grinding, that might affect results. Also the ratio of rock and solution volume is closer to that in the field geologic setting, relative to the high solution-to-solid ratios that are required in the batch work. The effect of variation in solution-to-rock ratios is discussed in Sec. IV.A.13 of this report.

The behavior of radionuclides eluted through columns of crushed tuff, in which nuclides are eluted as if through ion-exchange resin columns, was studied ${ }^{38}$ as a step to a dynamic system. The results are summarized in this section. Another step toward understanding the behavior of transport involves studying elution of tuffs through solid sanples (cylinders and blocks) of tuff or samples containing real or artificial fractures. These techniques and results are discussed in Sec. IV.D.

2. Batch Measurements--Experimental Method. In batch measurements of sorptive properties, the distribution of a radionuclide between groundwater and crushed tuff is measured as a function of such parameters as contact time, concentration of sorbing element, particle size, temperature, atmosphere, and lithology.

Considerable time was spent developing a satisfactory procedure; the actinides were particularly difficult, both in preparation of traced solutions and in separation of aqueous and solid phases (Sec. II.B.1). Sorption data collected during procedure development have been included in this section when they are believed valid. 
In this batch sorption procedure, the crushed-tuff sample is pretreated by contact for at least 2 weeks with well J-13 groundwater. The groundwater that is used to prepare the solution containing radionuclides (the "traced feed" solution) is pretreated by at least 2 weeks contact with tuff from the same sample as the tuff being studied; it is then filtered through a $0.05-\mu \mathrm{m}$ Nuclepore membrane. There are slight variations in the procedure, depending on the element to be studied.

Preparation of the tuff sample is accomplished by weighing $\sim 1 \quad g$ of crushed solid material into a weighed and washed polyethylene or polycarbonate tube with a cap, adding $20 \mathrm{~m} \ell$ of groundwater to the tube, shaking the mixture well, and putting the tube in a shaker to be agitated at a speed of $\sim 200 \mathrm{rpm}$ for not less than 2 weeks. At the end of the 2-week period, the sample is removed from the shaker and centrifuged for 1 hour at $\sim 12000 \mathrm{rpm}$. The liquid phase is decanted and the sample is reweighed and then capped; contact with traced feed solutions is started within 2 to 24 hours.

Feed solutions containing barium, strontium, cesium, cerium, europium, iodine, nickel, cobalt, sodium, tin, iron, manganese, or selenium are prepared using commercially produced nuclides. The firal concentrations of the elements studied in these feed solutions generally range from $10^{-6}$ to $10^{-9} \mathrm{M}$. The tracer is evaporated at room temperature in a polyethylene or polycarbonate container. After a few drops of $\mathrm{HCl}$ are added, a second evaporation is carried out. The appropriate amount and type of pretreated groundwater is added, the container is capped, and the traced solution is agitated in a shaker for 1 or 2 days. The traced feed solution is then filtered through a $0.05-\mu m$ Nuclepore membrane just before use.

Exceptions to the above general procedure include technetium, uranium, and the actinides. Because technetium volatilizes when heated in acid solution and is more stable in base, the tracer is delivered in $0.1 \mathrm{M}$ amonium hydroxide solution. It is added in a small volume to the appropriate tuff-treated water in amounts to produce $10^{-3}$ to $10^{-9} \mathrm{M}$ feed solutions. Once the dilution has been made, the solution is equilibrated for a few days and then passed through a $0.05-\mu m$ filter just before use.

Uranium-traced feed solutions are prepared from a dilution of a stock solution prepared by dissolving a weighed amount of uranyl nitrate in water that has been purified with a Millipore de-ionizing system and filtering this solution through a $0.05-\mu \mathrm{m}$ membrane. The final preparation of a uranium-traced 
feed solution consists of adding an appropriate amount of the uranium stock solution to tuff-treated water, shaking the mixture for a period of up to a week, and then filtering the solution through a $0.05-\mu \mathrm{m}$ membrane just before use. The resulting solutions are approximately $10^{-7} \mathrm{M}$ in uranium.

The preparation of americium-, plutonium-, and neptunium-traced feed solutions is done in the following manner. Americium tracer obtained from Oak Ridge National Laboratory, ${ }^{237}$ Pu obtained from Argonne National Laboratory, or ${ }^{239} \mathrm{Pu}$ tracer (weapons grade) from Los Alamos is dried at room temperature in air in a polycarbonate or polypropylene container. The plutonium is treated with sodium nitrite before drying to ensure the (IV) oxidation state. The dried activity is removed from the container in two steps: (1) a 1- or 2-minute contact with tuff-treated groundwater using a vibrator or ultrasonic bath and (2) a second 1- or 2-minute contact with a fresh portion of tufftreated water, again using an ultrasonic bath. After each contact the aqueous phase is added to a large polyethylene bottle. The solution is shaken from 1 to 2 days and then passed through $0.4-$ and $0.05-\mu m$ filters serially just before use. Final solutions are approximately $1 \times 10^{-6} \mathrm{M}$ for americium solutions, $1 \times 10^{-6} \mathrm{M}$ for ${ }^{239} \mathrm{Pu}$ solutions, and $4 \times 10^{-13} \mathrm{M}$ for ${ }^{237} \mathrm{Pu}$ solutions. Contact starts when $20 \mathrm{ml}$ of traced feed solution are added to $1 \mathrm{~g}$ of groundwater-treated tuff in a polyethylene or polycarbonate tube (Tube 1 ), the two phases are mixed thoroughly, and the sample is placed in a shake to be agitated for a predetermined time. The time at which contact starts is noted. At the end of the sorption period, the time is noted again, and the sample is removed from the shaker and centrifuged for 1 hour at $\sim 12000 \mathrm{rpm}(28000 \mathrm{~g})$. A portion of the liquid phase (the top 15 to $18 \mathrm{~m} \ell$ ) is pipetted to a clean polyethylene or polycarbonate tube (Tube 2) and capped. The remaining liquid is carefully removed to another separate tube (Tube 3). The solid phase in Tube 1 is weighed and then prepared for counting. The liquid phase in Tube 2 is centrifuged at $\sim 12000 \mathrm{rpm}$ for 1 hour. A portion of the liquid (the top 12 to $16 \mathrm{~m} \ell$ ) is pipetted to a clean polyethylene or polycarbonate tube (Tube 4), capped, and centrifuged for 2 hours at $\sim 12000 \mathrm{rpm}$. The 2 to $3 \mathrm{~m} \ell$ remaining in Tube 2 is added to Tube 3 . When the 2-hour centrifugation is finished, a portion of the liquid (the top 9 to $10 \mathrm{~m}$ ) is pipetted to a clean polyethylene or polycarbonate tube (Tube 5) and prepared for counting (see below). Any liquid remaining ( 0 to $3 \mathrm{ml}$ ) in Tube 4 is combined with the previously saved liquid (Tube 3), and the combination is used to measure $\mathrm{pH}$. 
After the solid phase has been sampled for counting, or after counting is complete if the solid phase is counted, the desorption step of the procedure can be started. Twenty milliliters of tuff-treated water is added to the remaining solid phase in its tube. The tube is capped and weighed and the two phases are thoroughly mixed. The sample is placed in a shaker to agitate at $\sim 200 \mathrm{rpm}$ for a predetermined time. The time at the start of desorption is noted. At the end of the desorption period, the sample is treated and separated in exactly the same manner as were the solid and liquid phases of the sorption sample.

The tracer activity in the separated phases is determined in several ways. The gamma-emitting actinides, except for uranium, are counted in the following manner. The solid phase in its polyethylene or polycarbonate container is counted in a $\mathrm{NaI}(\mathrm{T} 1)$ well detector. Standards are prepared by using a known amount of the appropriate activity in geometry and conditions identical to those of the samples; these standards are counted whenever the samples are counted. Three milliliters of the liquid phase is transferred by automatic pipettor to a polyethylene counting vial and acidified by adding $1 \mathrm{~m} \ell$ of concentrated hydrochloric acid. The mixture is mixed well and the tube capped. These liquid samples are counted in both the $\mathrm{NaI}(\mathrm{T} 1$ ) well counter and in an automatic gamma scintillation counter. Alternative methods for counting plutonium samples when the tracer used is plutonium other than ${ }^{237} \mathrm{Pu}$ include radiochemical analysis of both the liquid and the dissolved solid sample or liquid scintillation counting of both fractions. The uranium sorption ratio is such that it is necessary to count only the liquid phase. When the tracer used is ${ }^{237} \mathrm{U}$, a portion of the liquid phase is placed in a vial and gamma counted with a $\mathrm{Ge}(\mathrm{Li})$ detector. At present, natural uranium is being used as tracer, and the liquid samples are being counted by a delayed neutron counting method. The remaining elements are prepared for gamma counting in the following manner. A fraction of the solid phase is dried and weighed, then transferred to a vial, sealed, and counted with a Ge(Li) detector. The liquid samples are prepared by acidifying $10 \mathrm{ml}$ of the aqueous phase in a vial with $1 \mathrm{ml}$ of concentrated $\mathrm{HCl}$ and sealing the vial. The liquid sample is also counted with a $\mathrm{Ge}(\mathrm{Li})$ detector.

A value of the sorption ratio $R_{d}$ is obtained from the batch measurements. It is defined by

$$
R_{d}=\frac{\text { activity on solid phase per unit mass of solid }}{\text { activity in solution per unit volume of solution }} .
$$


Many investigators refer to this quantity as the distribution coefficient $K_{d}$. Los Alamos prefers not to use this term except under equilibrium conditions. Los Alamos data indicate that equilibrium is not achieved in many instances, but the sorption ratio is a measurement of an element's distribution between phases under specified conditions, although not necessarily at equilibrium. Sorption from near-neutral groundwater onto a rock is complicated on tuff; it may involve many competing cations and complexed or hydrated species. Many equilibria would have to be described in equations leading to the thermodynamic quantities for sorption of an element. Later in this section some simplifying assumptions will be applied in relationships between an equilibrium constant and the distribution coefficient.

Los Alamos' previously published ${ }^{1-3}$ and recent unpublished data are consolidated in App. A, which gives data from individual batch sorption and desorption experiments on tuffs, including the (1) parameters of contact time, (2) concentration of sorbing element in the groundwater, (3) particle size of crushed tuff, (4) temperature, and (5) atmosphere in which the experiment was conducted. Tuff samples from drill holes J-13 (Ref. 4), UE25a-1 (Ref. 39), and USW-G1 (Refs. 40 and 41) have been assigned the prefixes $\mathrm{JA}^{-}$, YM-, and $\mathrm{G1-}$, respectively. At this time, in an attempt to put the results of these measurements on a common basis, any lateral variation of properties within tuff units in the Yucca Mountain region is ignored, and depth equivalents in drill hole USW-G1 are assigned to samples from the other two drill holes (Table XX). These depths are designated according to the sample's relative position within a given stratigraphic unit and compensate for vertical variations between holes. The thicknesses of the Bullfrog Member in drill hole UE25a-1 and the Tram Member in drill hole J-13 are not known at this time and are assumed to be the same as in drill hole USW-G1. Although this treatment is fairly successful for the three drill holes considered, it cannot be applied to all of Yucca Mountain because of lateral variation in other drill holes.

Previous discussions ${ }^{1-3}$ and examination of the data in App. A indicate that sorption ratios change only slowly with contact time $>1$ week or that there is no definite correlation with the length of contact. For devitrified tuffs, which contain some clays but not zeolites, sorption ratios are greater (usually by factors of 2 to 3 ) for the finest $(<38-\mu \mathrm{m})$ fractions of ground tuff than for the coarser fractions (Sec IV.A.9). Accordingly, in averaging the values in App. A, values for fractions containing $<75-\mu \mathrm{m}$ particles were not used except with cores for which only data for a $<500-\mu \mathrm{m}$ fraction was available. 
TABLE $X X$

\section{DEPTH INTERVALS OF MAJOR TUFF \\ STRATIGRAPHIC UNITS ${ }^{2}$}

\begin{tabular}{|c|c|c|c|c|}
\hline Tuff Stratum & Symbol ${ }^{b}$ & $\begin{array}{c}\text { Drill Hole } \\
\mathrm{J} 13 \\
\end{array}$ & $\begin{array}{c}\text { Drill Hole } \\
\text { UE } 25 a-1\end{array}$ & $\begin{array}{c}\text { Drill Hole } \\
\text { USW-Gl }\end{array}$ \\
\hline Yucca Mountain & Tpy & & & $60-235$ \\
\hline Tiva Canyon & Tpc & $426-1037$ & $30-270$ & \\
\hline Topopah Spring & Tpt & $1037-1476$ & $270-1362$ & $235-1426$ \\
\hline Calico Hills & Tht & $1476-1821$ & $1362-1834$ & $1426-1802$ \\
\hline Prow Pass & Tcp & $1821-1991$ & $1834-2333$ & $1802-2173$ \\
\hline Bullfrog & $\mathbf{T c b}$ & $1991-2851$ & 2333- & $2173-2640$ \\
\hline Tram & Tct & $2851-$ & & $2640-3558$ \\
\hline Dacite Flow-Breccia & $\mathrm{Tb}$ & & & $3558-3946$ \\
\hline
\end{tabular}

The average values are given in Table XXI for sorption and in Table XXII for desorption experiments. Samples are ordered according to depth equivalent in drill hole USW-G1. Some of the values in these tables are not the exact averages of those in App. A because the numbers in the appendix have been rounded off. Data ${ }^{1,2}$ for the cation exchange capacity and surface area are given in Table XXIII.

The uncertainties associated with averages of sorption data (such as in Table XXI) are the standard deviations of the means $\sigma_{m}$, defined by

$$
\sigma_{m}=\left(\frac{\sum d_{i}}{n(n-1)}\right)^{1 / 2},
$$

where

$d_{i}$ is the deviation from the mean of the ith experimental value, and $\mathrm{n}$ is the number of values.

The standard deviation of the mean is used rather than the standard deviation of the sample or the population so as to avoid including zero in the range of uncertainty when, for example, averaging a large range of 
TABLE XX1

AVERAGE SORPTION RATIOS FOR PULVERIZED TUFF FROH SORPTION EXPERIYENTS ${ }^{2}$

USW-G1

$R_{d}(m \ell / \varepsilon)$

\begin{tabular}{|c|c|c|c|c|c|c|c|c|c|c|c|c|}
\hline \multirow{2}{*}{ Saple } & \multirow{2}{*}{$\begin{array}{l}\text { Depth } \\
\text { (ft) }\end{array}$} & \multirow{2}{*}{$\begin{array}{l}\text { Deptt: } \\
\text { (ft) }\end{array}$} & \\
\hline & & & $s_{r}$ & Cs & Be & Ce & Eu & $\Lambda$ & Pu & $\underline{u}$ & Tc & $v_{0}$ \\
\hline $\begin{array}{l}\mathrm{JA}-8 \\
\mathrm{YH}-5 \\
\mathrm{YH}-22 \\
\mathrm{Gl}-1292 \\
\mathrm{YH}-30\end{array}$ & $\begin{array}{r}606 \\
251 \\
848 \\
1292 \\
1254\end{array}$ & $\begin{array}{r}172 \\
221 \\
868 \\
1292 \\
1318\end{array}$ & $\begin{array}{l}270(5) \\
280(80)^{c} \\
53(4) \\
200(6) \\
260(80)\end{array}$ & $\begin{array}{c}2700(400) \\
5800(800)^{c} \\
290(30) \\
430(26)^{e} \\
655(5)\end{array}$ & $\begin{array}{l}435(15) \\
1100(200) c \\
900(30) \\
2100(300) \\
3400(1500)\end{array}$ & $\begin{array}{c}450000(240000)^{c} \\
1270(40) \\
66(f)^{\mathrm{C}} \\
230000(? 00000)\end{array}$ & $\begin{array}{c}2100(300) \\
230000(40000)^{c} \\
1390(110) \\
140(14) \\
160000(50000)\end{array}$ & $1200(130)^{c, d}$ & $64(20)^{c}$ & $1.8(0.2)^{c}$ & $0.30(0.14)^{C}$ & $7.0(1.0)^{C}$ \\
\hline $\begin{array}{l}\text { JA-18 } \\
\text { G1-1436 } \\
\mathrm{YH}-38 \\
\mathrm{YH}-42 \\
\mathrm{G} 1-1854\end{array}$ & $\begin{array}{l}1420 \\
1636 \\
1504 \\
1624 \\
1854\end{array}$ & $\begin{array}{l}1339 \\
1436 \\
1538 \\
1802 \\
1854\end{array}$ & $\begin{array}{c}17000(3000) \\
36000(3000) \\
17000(2000) \\
3900(600) \\
60000(14000)\end{array}$ & $\begin{array}{c}16000(1000) \\
7800(500) \\
13000(2000) \\
17000(1000) \\
13000(2000)\end{array}$ & $\begin{array}{c}38000(18000) \\
150000(24000) \\
100000(10000) \\
94000(16000) \\
45000(7000)\end{array}$ & $\begin{array}{c}2800(1400)^{d} \\
59000(7000) \\
760(160) \\
49000(7000)\end{array}$ & $\begin{array}{l}1400(200)^{d} \\
30000(2000) \\
1600(200) \\
52000(4000) \\
>15000\end{array}$ & $\begin{array}{c}180(30) \\
4600(1100)\end{array}$ & $\begin{array}{l}120(20) \\
140(30)\end{array}$ & $\begin{array}{l}2.5(0.4) \\
5.3(02)\end{array}$ & & $11.0(0.7)$ \\
\hline $\begin{array}{l}Y H-45 \\
G 1-1883 \\
Y H-46 \\
G 1-1982 \\
Y I-48\end{array}$ & $\begin{array}{l}1930 \\
1683 \\
2002 \\
1982 \\
2114\end{array}$ & $\begin{array}{l}1873 \\
1863 \\
1926 \\
1982 \\
2019\end{array}$ & $\begin{array}{c}194(14) \\
22.0(0.2) \\
190(60) \\
55(4) \\
2100(400)\end{array}$ & $\begin{array}{l}520(90) \\
287(3) \\
840(6) \\
1120(110) \\
9000(4000)\end{array}$ & $\begin{array}{c}1200(100) \\
182(12) \\
14000(6000) \\
700(50) \\
18000(6000)\end{array}$ & $\begin{array}{c}730(100) \\
140(20) \\
310000(110000) \\
560(40) \\
1400(500)\end{array}$ & $\begin{array}{c}1600(200) \\
307000(110000) \\
970(150) \\
2200(500)\end{array}$ & $4700(300)$ & $77(11)$ & & $0.15(0.02)$ & $6.4(0.6)$ \\
\hline $\begin{array}{l}\mathrm{YH}-49 \\
\mathrm{JA}-26 \\
\mathrm{JA}-28 \\
\mathrm{GI}-2233 \\
\mathrm{GI}-2289\end{array}$ & $\begin{array}{l}2221 \\
1995 \\
2001 \\
2233 \\
2289\end{array}$ & $\begin{array}{l}2090 \\
2173 \\
2178 \\
2233 \\
2289\end{array}$ & $\begin{array}{c}3200(300) \\
95(35) \\
94(20) \\
48000(3000) \\
7300(500)\end{array}$ & $\begin{array}{c}36000(3000) \\
1500(600) \\
1640(210) \\
13500(800) \\
37000(13000)\end{array}$ & $\begin{array}{c}42000(8000) \\
800(300) \\
820(50) \\
250000(30000) \\
66000(9000)\end{array}$ & $1400(300)$ & $\begin{array}{l}1200(100) \\
2100(1000) \\
900(200) \\
797(10)\end{array}$ & $4300(1400)$ & $230(50)^{d}$ & & $0.21(0.02)$ & $9(3)$ \\
\hline $\begin{array}{l}\mathrm{M}-54 \\
G 1-2333 \\
G 1-2363 \\
G 1-2410 \\
\mathrm{JA}-32\end{array}$ & $\begin{array}{l}2491 \\
2333 \\
2363 \\
2410 \\
2533\end{array}$ & $\begin{array}{l}2330 \\
2333 \\
2363 \\
2410 \\
2467\end{array}$ & $\begin{array}{c}62(12) \\
180(20) \\
64(3) \\
169(1) \\
57(3)\end{array}$ & $\begin{array}{c}180(40) \\
1400(130) \\
470(40) \\
1250(50) \\
123(4)\end{array}$ & $\begin{array}{c}400(150) \\
1500(200) \\
235(9) \\
1780 \\
380(30)\end{array}$ & $150(40)$ & $\begin{array}{c}470(40) \\
2300(400) \\
730(50) \\
440(80) \\
90(20)\end{array}$ & $130(30)$ & $80(20)$ & $2.2(0.9)$ & $4.2(0.5)$ & \\
\hline $\begin{array}{l}G 1-2476 \\
G 1-2698 \\
G 1-2 \$ 40 \\
G 1-2854 \\
G 1-2901\end{array}$ & $\begin{array}{l}2476 \\
2698 \\
2840 \\
2854 \\
2901\end{array}$ & $\begin{array}{l}2476 \\
2698 \\
2840 \\
2854 \\
2901\end{array}$ & $\begin{array}{c}41(1) \\
42000(3000)^{e} \\
160(1) \\
94(1) \\
68(1)^{e}\end{array}$ & $\begin{array}{l}700(40) \\
7700(400)^{e} \\
2200(200) \\
1080(120) \\
1290(110)^{e}\end{array}$ & $\begin{array}{c}385(11) \\
63000(5000) \\
2070(70) \\
1000(50) \\
1600(200)^{e}\end{array}$ & $\begin{array}{c}240(30)^{e} \\
42000(3000)^{e}\end{array}$ & $\begin{array}{c}3200(100) \\
200(30)^{\ell} \\
4900(400) \\
1300(200) \\
160000(50000)^{r}\end{array}$ & & & & & \\
\hline $\begin{array}{l}G 1-3116 \\
J A-37 \\
G 1-3658\end{array}$ & $\begin{array}{l}3116 \\
3697 \\
3658\end{array}$ & $\begin{array}{l}3115 \\
3286 \\
3658\end{array}$ & $\begin{array}{c}2400(17)^{e} \\
287(14) \\
13000(0)\end{array}$ & $\begin{array}{l}6600(500)^{e} \\
610(40) \\
4950(50)\end{array}$ & $\begin{array}{c}12000(4000)^{e} \\
760(150) \\
13500(500)\end{array}$ & $\begin{array}{c}100(10)^{e} \\
1000(200)^{e}\end{array}$ & $\begin{array}{c}760(60)^{e} \\
6000(800) \\
530(40)\end{array}$ & $2800+(10000)^{d}$ & $400(70)^{d}$ & $4.6(0.3)$ & & $28(7)$ \\
\hline
\end{tabular}

Ambient conditions, air, $20 \pm 4^{\circ} \mathrm{C}$; fractions do not coatein <75-pa-dian particles except those designated by footnote $e$.

bepth equivalent in drill hole USW-G1 according to fosition in geolosic unit.

Conveighted nverage; values in parentheses are the absolute-value standard deviations of the means.

"some data vere rejected in averasing.

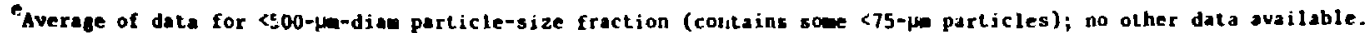


TABLE XXI1

AVERAGE SORPTICN RATIOS FOR PULVERIZED TUFE FROH DESORPTION EXPERIGENTS*

\begin{tabular}{|c|c|c|c|c|c|c|c|c|c|c|c|c|}
\hline \multirow[b]{2}{*}{ Snple } & \multirow{2}{*}{$\begin{array}{l}\text { Depth } \\
\text { (ft) }\end{array}$} & \multirow{2}{*}{$\begin{array}{l}\text { USW-Gl } \\
\text { Depthb } \\
\text { (ft) } \\
\end{array}$} & \multicolumn{10}{|c|}{$R_{d}(n / g)$} \\
\hline & & & Sr & $C_{3}$ & B. & Ce & Eu & A & Pü & u & Tc & $N_{p}$ \\
\hline $\begin{array}{l}J A-8 \\
Y H-5 \\
Y H-22 \\
G 1-1292 \\
Y-30\end{array}$ & $\begin{array}{r}606 \\
251 \\
848 \\
1292 \\
1264\end{array}$ & \begin{tabular}{r|}
172 \\
221 \\
868 \\
1292 \\
1318
\end{tabular} & $\begin{array}{l}311(3) \\
320(30) \mathrm{c} \\
59(2) \mathrm{e} \\
120(5)^{\mathrm{e}} \\
210(30)\end{array}$ & $\begin{array}{c}4600(400) \\
8900(600)^{c} \\
365(7) \\
510(20)^{e} \\
1500(100)\end{array}$ & $\begin{array}{l}480(50) \\
1200(120) c \\
830(100) \\
1500(100) d, e \\
3100(600)\end{array}$ & $\begin{array}{c}310000(30000)^{c} \\
6500(800)^{c} \\
600(200)^{e} \\
170000(: 5000)\end{array}$ & $\begin{array}{l}10000(3000) \\
36000(14000)^{c} \\
3500(200) \\
600(70) \\
11000(700)\end{array}$ & $2500(400)^{c}$ & $1330(140)^{C}$ & $5(2)^{c}$ & $1.2(0.3)^{c}$ & $33(5)^{c}$ \\
\hline $\begin{array}{l}J A-18 \\
G 1-1436 \\
Y H-38 \\
Y H-42 \\
G 1-1854\end{array}$ & $\begin{array}{l}1420 \\
1436 \\
1540 \\
1824 \\
1854\end{array}$ & $\begin{array}{l}1339 \\
1436 \\
1538 \\
1802 \\
1854\end{array}$ & $\begin{array}{l}15000(2000) \\
87000(12000) \\
22000 \\
4100(1000) \\
72000(13000)^{d}\end{array}$ & $\begin{array}{l}17500(700) \\
24000(2000) \\
13000 \\
21000(2000) \\
14000(2000)\end{array}$ & $\begin{array}{l}280000(50000) \\
340000(90000) \\
260000 \\
90000(30000) \\
150000(40000)\end{array}$ & $\begin{array}{l}1600(500)^{d} \\
6700(600) \\
2600 \\
44000(5000)\end{array}$ & $\begin{array}{c}2400(300)^{d} \\
5300(600) \\
7300 \\
64000(3000) \\
4800(700)\end{array}$ & $\begin{array}{l}1100(300) \\
7100(1200)\end{array}$ & $\begin{array}{r}350(140) \\
1600(300)\end{array}$ & $\begin{array}{r}9.4(1.4) \\
14.8(1.0)\end{array}$ & & $24(2)$ \\
\hline $\begin{array}{l}\eta M-45 \\
G 1-1883 \\
m-46 \\
G 1-1982 \\
n-48\end{array}$ & $\begin{array}{l}1930 \\
1883 \\
2002 \\
1982 \\
2114\end{array}$ & $\begin{array}{l}1873 \\
1883 \\
1926 \\
1982 \\
2019\end{array}$ & $\begin{array}{c}210(20) \\
59(1) \\
260(20) \\
322(8) \\
2700(200)\end{array}$ & $\begin{array}{c}620(118) \\
430(4) \\
1800(300) \\
2300(200)^{e} \\
27000(4000)\end{array}$ & $\begin{array}{c}1310(60) \\
440(10) \\
21000(3000) \\
2780(120) \\
36000(7000)\end{array}$ & $\begin{array}{c}5800(600) \\
2200(100)^{e} \\
300000(50000) \\
7000(900)^{e} \\
128000(300)\end{array}$ & $\begin{array}{c}7300(900) \\
1350(50)^{t} \\
31000(2000) \\
6370(130) \\
8100(1200)\end{array}$ & $7200(900)$ & $890(60)$ & & $1.6(0.2)$ & $36(10)$ \\
\hline $\begin{array}{l}Y 1-49 \\
J A-26 \\
J A-28 \\
G 1-2233 \\
G 1-2289\end{array}$ & $\begin{array}{l}2221 \\
1995 \\
2001 \\
2233 \\
2289\end{array}$ & $\begin{array}{l}2090 \\
2173 \\
2178 \\
2233 \\
2289\end{array}$ & $\begin{array}{c}4400(100) \\
39(3) \\
114(3) \\
90000(40000)^{2}\end{array}$ & $\begin{array}{c}39000(1000) \\
1580(90) \\
2400(100) \\
23000(6000)\end{array}$ & $\begin{array}{c}65000(7000) \\
450(13) \\
1160(20) \\
240000(80000)^{e}\end{array}$ & $\begin{array}{l}1040(40) \\
20000(13000)^{d}\end{array}$ & $\begin{array}{c}2100(500) \\
2900(200) \\
12300(500) \\
5000(2000)^{e}\end{array}$ & $3400(400)^{d}$ & $720(90)$ & & $2.0(0.3)$ & $12(4)$ \\
\hline $\begin{array}{l}Y H-54 \\
G 1-2333 \\
G 1-2363 \\
G 1-2610 \\
J A-32\end{array}$ & $\begin{array}{l}2491 \\
2333 \\
2363 \\
2410 \\
2533\end{array}$ & $\begin{array}{l}2330 \\
2333 \\
2363 \\
2410 \\
2467\end{array}$ & $\begin{array}{c}97(9) \\
140(13) \\
150(6) \\
140(14) \\
53(3)\end{array}$ & $\begin{array}{c}310(20) \\
1230(100) \\
1200(30) \\
1120(100) \\
175(11)\end{array}$ & $\begin{array}{c}660(20) \\
1460(130) \\
820(20) \\
1760(150) \\
490(40)\end{array}$ & $\begin{array}{c}1000(200) \\
130000(6000)^{e} \\
530(120)\end{array}$ & $\begin{array}{l}1840(110) \\
9900(1200) \\
6100(300) \\
6000(3000) \\
850(130)\end{array}$ & $2200(600)$ & $720(40)$ & $12(8)$ & $2.0(0.3)$ & \\
\hline $\begin{array}{l}G 1-2476 \\
G 1-2698 \\
G 1-2840 \\
G 1-2854 \\
G 1-2901\end{array}$ & $\begin{array}{l}2676 \\
2698 \\
2840 \\
2854 \\
2901\end{array}$ & $\begin{array}{l}2476 \\
2698 \\
2040 \\
2854 \\
2901\end{array}$ & $\begin{array}{c}200(4) \\
210000(50000)^{e} \\
150(4) \\
96(1) \\
67(1)^{d, e}\end{array}$ & $\begin{array}{c}1520(0) \\
17000(1100)^{e} \\
2300(130) \\
1160(20) \\
1380(30)^{e}\end{array}$ & $\begin{array}{l}190000(80000)^{e} \\
2500(200) \\
1330(0) \\
1980(30)^{e}\end{array}$ & $\begin{array}{c}2000(400)^{e} \\
39000(1000)^{e}\end{array}$ & $\begin{array}{c}9000(1100) \\
5000(200) \\
210000(50000)\end{array}$ & & & & & \\
\hline $\begin{array}{l}G 1-3116 \\
J A-37 \\
61-3658\end{array}$ & $\begin{array}{l}3116 \\
3497 \\
3658\end{array}$ & $\begin{array}{l}3116 \\
3286 \\
3658\end{array}$ & $\begin{array}{c}24000(13000)^{e} \\
312(9) \\
12000(3000)^{e}\end{array}$ & $\begin{array}{c}11000(3000)^{e} \\
850(50) \\
12000(2000)^{e}\end{array}$ & $\begin{array}{c}160000(80000)^{e} \\
920(40) \\
10000(4000)^{e}\end{array}$ & $\begin{array}{l}3000(1000)^{e} \\
9000(4000)^{e}\end{array}$ & $\begin{array}{c}8000(3000)^{e} \\
11000(2000) \\
9050(3000)^{e}\end{array}$ & $32000(10000)$ & $1400(300)$ & $9.9(0.4)$ & & $170(50)$ \\
\hline
\end{tabular}

ambient conditions, air, $20 \pm 4^{\circ} \mathrm{C}$; fractions do not contain $<75-\mu$ dia-particles except those designated by footnote e.

bepth equivelent in hole drill usw-Gl according to position in geologic unit.

"Monveighted averaze; valuex in parentheser are the standard deviations of the means.

dowe data were rejected in averoging.

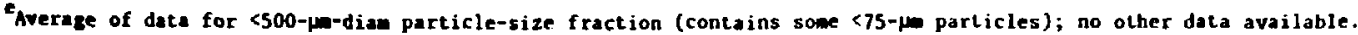


TABLE XXIII

CATION EXCHANGE CAPACITY AND SURFACE AREA

\begin{tabular}{|c|c|c|c|c|}
\hline \multirow[b]{2}{*}{ Sample } & \multirow{2}{*}{$\begin{array}{c}\text { Mesh Size } \\
(\mu \mathrm{m}) \\
\end{array}$} & \multicolumn{2}{|c|}{$\begin{array}{c}\text { Cation Exchange } \\
\text { Capacity } \\
(\text { meg } / 100 g)\end{array}$} & \multirow{2}{*}{$\begin{array}{c}\text { Surface Area } \\
\left(\mathrm{m}^{2} / \mathrm{g}\right)\end{array}$} \\
\hline & & Cs & $\mathrm{Sr}$ & \\
\hline$J A=18$ & $106-150$ & 75 & 48 & 31 \\
\hline$J A-18$ & $355-500$ & 80 & 44 & 46 \\
\hline$J A-32$ & $106-150$ & 2 & 2 & $8,8^{b}$ \\
\hline$J A-32$ & $355-500$ & 2 & 3 & 9 \\
\hline $\mathrm{JA}-37$ & $106-150$ & 17 & 63 & $94,115^{b}$ \\
\hline$J A-37$ & $355-500$ & 18 & 30 & 131 \\
\hline$Y M-22$ & $106-500$ & 2 & 3 & 22 \\
\hline YM- 38 & $106-500$ & 109 & 54 & 103 \\
\hline$Y M-45$ & $106-500$ & 6 & 6 & 43 \\
\hline$Y M-48$ & $106-500$ & 51 & 21 & 19 \\
\hline$Y M-49$ & $106-500$ & 107 & 47 & \\
\hline YM-54 & $106-500$ & 4 & 4 & \\
\hline
\end{tabular}

${ }^{\mathrm{a}}$ By the glycol method. 1

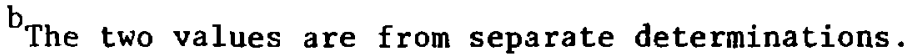

$\mathrm{R}_{\mathrm{d}}$ values from individual determinations, all of which indicate a high sorption ratio. The total spread of values averaged in the measurements is often a factor of 5 larger than $\sigma_{m}$. This is the result of the choice of $\sigma_{m}$ just discussed, the nonweighted averaging, and the simplifying assumption that the samples belong to the same population when averaging results for different times, particle sizes, etc. These uncertainties should not necessarily be used in assessment calcuiations as bounds for $K_{d}$ values within a given unit.

3. Lithology. The lithologies of the samples were determined by a number of analytical techniques, ${ }^{4,39,40}$ including $x$-ray diffraction, optical microscopy, and electron microprobe. Table XXIV lists the petrologic characterization of samples selected for this work in the various particle size ranges. The actual depths of the samples from the drill holes, the equivalent depth in drill hole USW-G1, and the stratigraphic unit are also listed. The 
TABLE XXIV

PETROLOGIC CHARACTERIZATION OF TUFF SAMPLES ${ }^{2}$

Abundance

\begin{tabular}{|c|c|c|c|c|c|c|c|c|c|c|c|c|c|c|c|c|c|}
\hline \multirow[b]{2}{*}{ Sucole } & \multirow[b]{2}{*}{$\begin{array}{l}\text { Depth } \\
\text { (ft) }\end{array}$} & \multirow{2}{*}{$\begin{array}{l}\text { Equiv. } \\
\text { usw-Gi } \\
\text { Depthb } \\
\text { (ft) } \\
\end{array}$} & \multicolumn{9}{|c|}{$\begin{array}{c}\text { Abundance } \\
(\mathrm{x})\end{array}$} & \multirow[b]{2}{*}{$\begin{array}{l}\text { Dry Bulk } \\
\text { Density } \\
\left.\text { (g/ca }{ }^{3}\right) \\
\end{array}$} & \multirow[b]{2}{*}{$\begin{array}{l}\text { Degree } \\
\text { of } \\
\text { Weldingd } \\
\end{array}$} & \multirow[b]{2}{*}{$\begin{array}{c}\text { Oxidation } \\
\text { State }\end{array}$} & \multirow[b]{2}{*}{$\begin{array}{c}\text { Crystals } \\
(\mathbf{z}) \\
\end{array}$} & \multirow[b]{2}{*}{$\begin{array}{l}\text { Eithics } \\
(\mathrm{z}) \\
\end{array}$} & \multirow[b]{2}{*}{ Unit ${ }^{\prime}$} \\
\hline & & & $\begin{array}{c}\text { Particle } \\
\text { Size } \\
(\mu \text { II) } \\
\end{array}$ & Smectite & $\begin{array}{c}\text { Illite } \\
\text { Muscovite }\end{array}$ & $\begin{array}{l}\text { Clinoptil- } \\
\text { olite }\end{array}$ & Quartz & $\begin{array}{l}\text { Cristob- } \\
\text { Ilite }\end{array}$ & $\begin{array}{c}\text { Alkali } \\
\text { Feldspar }\end{array}$ & Glass & $\underline{\text { Other }}^{\mathrm{c}}$ & & & & & & \\
\hline $\begin{array}{c}\mathrm{JA}-8 \\
\pi \\
11\end{array}$ & $\begin{array}{c}606 \\
" 1 \\
"\end{array}$ & $\begin{array}{c}172 \\
" 1\end{array}$ & $\begin{array}{c}211 \\
75-500 \\
<75\end{array}$ & $\begin{array}{l}25-50 \\
30-60 \\
30-60\end{array}$ & $\ddot{-}$ & $\begin{array}{l}-- \\
-\end{array}$ & 45 & $\begin{array}{l}10-20 \\
10-20 \\
10-20\end{array}$ & $\begin{array}{l}\text { tr } \\
-- \\
--\end{array}$ & $\begin{array}{l}25-50 \\
20-50 \\
30-60\end{array}$ & $\ddot{-}$ & & N & & 8.9 & 6.7 & $\begin{array}{l}\text { Tpc } \\
" 1\end{array}$ \\
\hline YH-5 & 251 & 221 & $.11^{8}$ & 10 & $--^{h}$ & -- & $<5$ & $<5$ & $10-20$ & $\sim 70$ & -- & & N & & 10.9 & 4.3 & Tpc \\
\hline $\begin{array}{l}\mathbf{M}-22 \\
" 1 \\
"\end{array}$ & $\begin{array}{c}848 \\
" 1 \\
" 1\end{array}$ & $\begin{array}{l}868 \\
" 1 \\
" 1\end{array}$ & $\begin{array}{c}-11 \\
106-500 \\
38-106 \\
<38\end{array}$ & $\begin{array}{c}5-10 \\
<5 \\
<2 \\
<5\end{array}$ & $\begin{array}{l}-- \\
<2 \\
\text { tr } \\
<2\end{array}$ & $\begin{array}{l}\ddot{-} \\
\ddot{-}\end{array}$ & $\begin{array}{l}40-60 \\
30-50 \\
30-50 \\
30-50\end{array}$ & $\begin{array}{l}-- \\
-- \\
--\end{array}$ & $\begin{array}{l}40-60 \\
30-50 \\
30-50 \\
30-50\end{array}$ & $\begin{array}{l}-- \\
-- \\
--\end{array}$ & $\begin{array}{l}- \\
\because \\
-\end{array}$ & 2.3 & D & $\cos (2-7)$ & 1.0 & 0.4 & $\begin{array}{c}\text { Tpt } \\
" \\
" \\
"\end{array}$ \\
\hline G1-1292 & 1292 & $\stackrel{1292}{1 "}$ & $75-500$ & tr & $\ddot{-}$ & 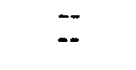 & $=-$ & $\begin{array}{r}5-10 \\
15-30\end{array}$ & $\begin{array}{l}10-20 \\
10-20\end{array}$ & $\begin{array}{l}80-90 \\
40-60\end{array}$ & $=$ & & $v$ & C1 & & & \\
\hline $\begin{array}{l}M 11-30 \\
" 1\end{array}$ & $\begin{array}{c}1264 \\
"\end{array}$ & $\begin{array}{c}1318 \\
"\end{array}$ & $\begin{array}{c}=11 \\
75-500 \\
<75\end{array}$ & $\begin{array}{c}5-10 \\
-- \\
--\end{array}$ & $\begin{array}{l}5 \\
\therefore- \\
--\end{array}$ & $\begin{array}{c}5-10 \\
15 \\
15\end{array}$ & $\begin{array}{c}40-60 \\
30 \\
30\end{array}$ & $\begin{array}{c}5-15 \\
20 \\
20\end{array}$ & $\begin{array}{c}30-50 \\
35 \\
35\end{array}$ & $\begin{array}{l}-- \\
-- \\
--\end{array}$ & $=$ & 2.1 & D & $\operatorname{cs}(2-7)$ & 2.1 & 21.6 & $"$ \\
\hline $\begin{array}{l}\text { JA-18 } \\
" \\
"\end{array}$ & $\begin{array}{c}1420 \\
" \\
"\end{array}$ & $\begin{array}{c}1339 \\
" \\
"\end{array}$ & $\begin{array}{c}=11 \\
355-500 \\
106-150\end{array}$ & $\begin{array}{r}5 \\
-5 \\
-5\end{array}$ & $\begin{array}{l}5 \\
-5\end{array}$ & $\begin{array}{r}5-10 \\
10-20 \\
10-20\end{array}$ & $\begin{array}{l}-- \\
--\end{array}$ & $\begin{array}{l}15=\vdots \\
35: 50 \\
3 j-j !\end{array}$ & $\begin{array}{l}15-25 \\
30-50 \\
30-50\end{array}$ & $\begin{array}{l}\sim 50 \\
\sim 40 \\
\sim 40\end{array}$ & $=$ & & N & $c 3(2-5)$ & 1.8 & 11.9 & " \\
\hline G1-1436 & 1436 & 1436 & $75-500$ & $<5$ & $<5$ & $75-90$ & $5-10$ & -- & $\sim 5$ & -- & - & 1.6 & & $c 6(5-7)$ & 5.2 & 3.2 & Tht \\
\hline $\begin{array}{l}\mathrm{YH}-3 \mathbf{B} \\
" 1\end{array}$ & $\begin{array}{c}1504 \\
" 1\end{array}$ & $\begin{array}{c}1538 \\
" \\
"\end{array}$ & $\begin{array}{c}106-500 \\
38-106 \\
<38\end{array}$ & $\begin{array}{l}5-10 \\
5-10 \\
5-15\end{array}$ & $\begin{array}{l}<2 \\
<5 \\
<5\end{array}$ & $\begin{array}{l}30-50 \\
40-60 \\
40-60\end{array}$ & $\begin{array}{r}15-30 \\
2-10 \\
2-10\end{array}$ & $\begin{array}{l}1:: 0 \\
10-20 \\
10-20\end{array}$ & $\begin{array}{r}5-15 \\
5-15 \\
10-20\end{array}$ & $\begin{array}{l}-- \\
--\end{array}$ & $\begin{array}{l}A, t r \\
A, t r\end{array}$ & 1.8 & N & $\operatorname{cs}(4-6)$ & 4.0 & 7.7 & " \\
\hline$M_{11}^{M-42}$ & 1824 & $\stackrel{1802}{" 1}$ & $\begin{array}{c}75-500 \\
<75\end{array}$ & $\begin{array}{l}\mathrm{tr} \\
\mathrm{tr}\end{array}$ & $\begin{array}{l}<5 \\
\text { tr }\end{array}$ & $\begin{array}{l}20 \\
20\end{array}$ & $\begin{array}{c}35-40 \\
40\end{array}$ & -- & $\begin{array}{l}40 \\
40\end{array}$ & -- & 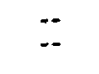 & 2.3 & & & 15.6 & 46.6 & $"$ \\
\hline G1-1854 & $\underset{" 1854}{" ~}$ & $\begin{array}{c}1854 \\
11\end{array}$ & $\begin{array}{c}75-500 \\
<75\end{array}$ & $\begin{array}{r}5-10 \\
5-10\end{array}$ & $\ddot{--}$ & $\begin{array}{l}30-50 \\
40-60\end{array}$ & $\begin{array}{r}5-15 \\
20-40\end{array}$ & $\begin{array}{l}15-30 \\
15-30\end{array}$ & $\begin{array}{l}20-40 \\
10-30\end{array}$ & $=$ & $\because$ & & & & & & $\underset{\|}{\text { Tcp }}$ \\
\hline Mi-4S & 1930 & 1873 & .11 & $1-5$ & -- & -- & $40-60$ & tr & $30-50$ & $\cdots$ & & & * & $C_{4}(3-5)$ & 13.5 & 0.6 & $"$ \\
\hline $\begin{array}{l}\text { G1-1883 } \\
" \\
" \\
"\end{array}$ & $\begin{array}{c}1883 \\
" \\
"\end{array}$ & $\begin{array}{c}1883 \\
" 1 \\
" 1\end{array}$ & $\begin{array}{r}75-500 \\
106-500 \\
38-106 \\
<38\end{array}$ & $\begin{array}{r}<2 \\
<2 \\
<2 \\
2-5\end{array}$ & $\begin{array}{l}<5 \\
<2 \\
<5 \\
<2\end{array}$ & $\begin{array}{l}-- \\
-- \\
--\end{array}$ & $\begin{array}{l}30-50 \\
20-40 \\
30-50 \\
20-40\end{array}$ & $\begin{array}{l}-- \\
0-10 \\
0-10 \\
0-10\end{array}$ & $\begin{array}{l}50-70 \\
40-60 \\
30-50 \\
40-60\end{array}$ & $\begin{array}{l}-- \\
-- \\
--\end{array}$ & $\begin{array}{l}= \\
\because- \\
--\end{array}$ & 1.7 & $\mathbf{p}$ & $C_{4}(3-5)$ & 16.6 & 1.0 & " \\
\hline $\mathrm{YH}_{11}-46$ & 2002 & $\begin{array}{c}1926 \\
"\end{array}$ & $\underset{75-500}{211}$ & $\begin{array}{l}<5 \\
<5\end{array}$ & $<5$ & 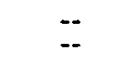 & $\begin{array}{c}40-60 \\
50\end{array}$ & $=$ & $\begin{array}{c}35-55 \\
45\end{array}$ & $=$ & $=$ & 2.1 & D & & 12.7 & 0.3 & " \\
\hline
\end{tabular}


Abundance

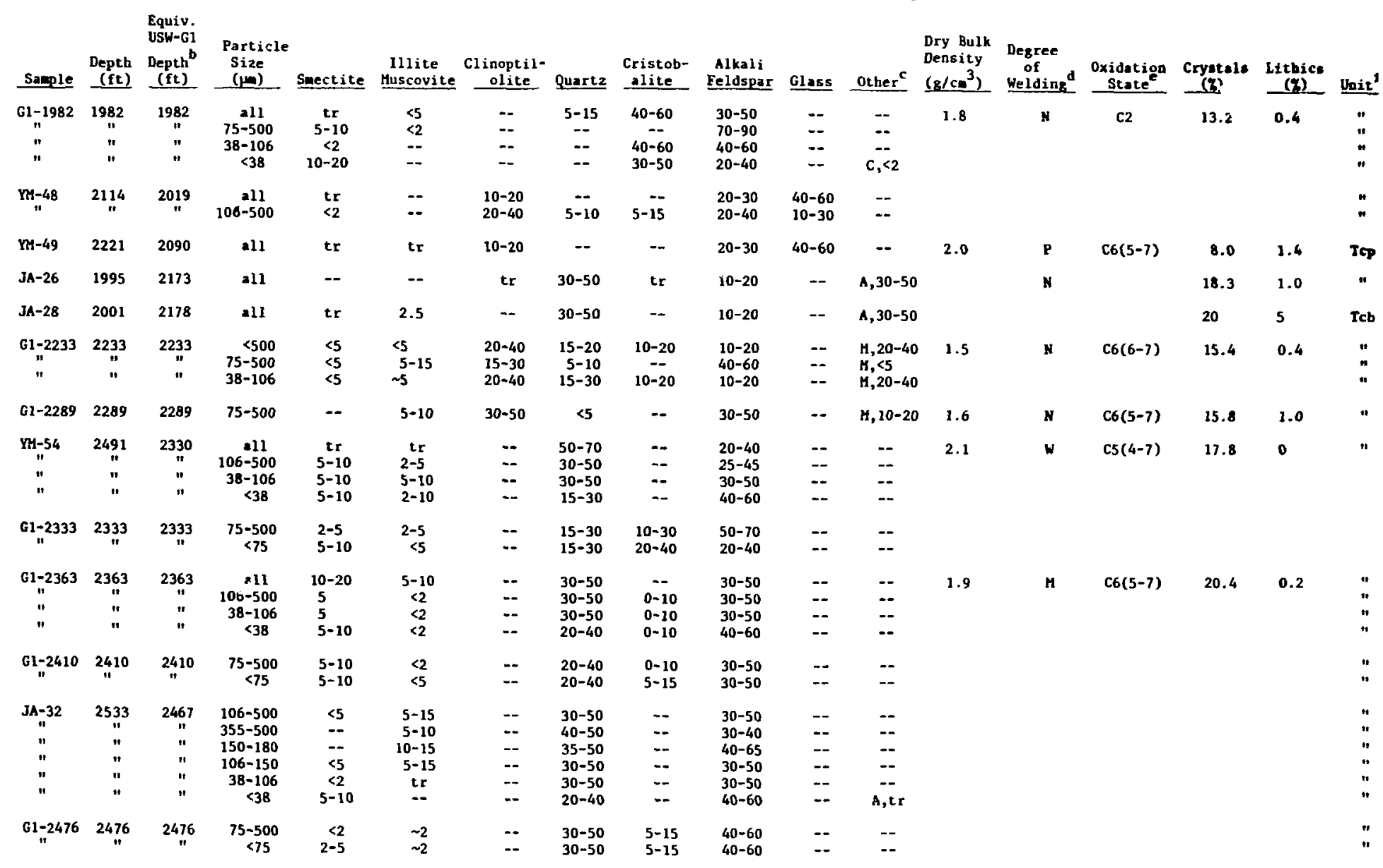


TABLE XXIV (cont)

\begin{tabular}{|c|c|c|c|c|c|c|c|c|c|c|c|c|c|c|c|c|c|}
\hline \multirow[b]{2}{*}{ Sanple } & \multirow[b]{2}{*}{$\begin{array}{l}\text { Depth } \\
\text { (ft) }\end{array}$} & \multirow{2}{*}{$\begin{array}{l}\text { Equiv. } \\
\text { USW-Gi } \\
\text { Depthb } \\
\text { (ft) } \\
\end{array}$} & \multirow[b]{2}{*}{$\begin{array}{c}\text { Particle } \\
\text { Size } \\
\text { (jw) } \\
\end{array}$} & \multicolumn{8}{|c|}{$\begin{array}{c}\text { Abundance } \\
\text { (z) }\end{array}$} & \multirow{2}{*}{$\begin{array}{l}\text { Dry Bulk } \\
\text { Density } \\
\left(8 / c^{3}\right) \\
\end{array}$} & \multirow[b]{2}{*}{$\begin{array}{l}\text { Degree } \\
\text { of } \\
\text { Welding } \\
\end{array}$} & \multirow[b]{2}{*}{$\begin{array}{c}\text { Oxidation } \\
\text { State }\end{array}$} & \multirow[b]{2}{*}{$\begin{array}{c}\text { Crystals } \\
(\mathrm{y})\end{array}$} & \multirow[b]{2}{*}{$\begin{array}{l}\text { Lithics } \\
(\boldsymbol{y}) \\
\end{array}$} & \multirow[b]{2}{*}{ Unit $^{1}$} \\
\hline & & & & Seectite & $\begin{array}{c}\text { 11lite } \\
\text { Muscovite }\end{array}$ & $\begin{array}{l}\text { Clinoptil- } \\
\text { olite }\end{array}$ & Quartz & $\begin{array}{l}\text { Cristob- } \\
\text { slite } \\
\end{array}$ & $\begin{array}{c}\text { Alkali } \\
\text { Feldspar }\end{array}$ & Gless & other ${ }^{\mathrm{C}}$ & & & & & & \\
\hline$G 1-2698$ & 2698 & 2698 & all & $<5$ & $10-15$ & $30-50$ & $<5$ & -- & $30-50$ & - & $\mathbf{n},<5$ & 1.8 & $\mathrm{~N}$ & C6(5-7) & 13.2 & 1 & Tct \\
\hline $\begin{array}{l}\text { G1-2840 } \\
"\end{array}$ & $\begin{array}{c}2840 \\
11\end{array}$ & $\begin{array}{c}2840 \\
"\end{array}$ & $\begin{array}{c}75-500 \\
<75\end{array}$ & $\begin{array}{l}2-5 \\
2-5\end{array}$ & $\begin{array}{l}2-5 \\
2-5\end{array}$ & 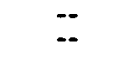 & $\begin{array}{l}40-6 j 0 \\
40-60\end{array}$ & $\begin{array}{l}0-10 \\
0-10\end{array}$ & $\begin{array}{l}30-50 \\
30-50\end{array}$ & -- & $\ddot{--}$ & & & & & & $"$ \\
\hline${ }^{\text {G1-2856 }}$ & $\underset{ }{2854}$ & 2854 & $\begin{array}{c}75-500 \\
<75\end{array}$ & $\begin{array}{l}<2 \\
<2\end{array}$ & $\begin{array}{l}5-10 \\
5-10\end{array}$ & $=$ & $\begin{array}{l}30-50 \\
30-50\end{array}$ & $\begin{array}{l}0-10 \\
0-10\end{array}$ & $\begin{array}{l}30-50 \\
30-50\end{array}$ & $=$ & $=$ & & & & & & $"$ \\
\hline G1-2901 & 2901 & 2901 & .11 & $5-10$ & $5-10$ & -- & $20-40$ & -- & $40-60$ & -- & -- & & $w$ & $c 6(6-7)$ & 16.5 & 2 & $"$ \\
\hline $61-3116$ & 3116 & 3116 & al1 & $5-10$ & $5-10$ & $5-15$ & $20-40$ & -- & $20-40$ & -- & $A, 10-30$ & 1.9 & & $\cos (6-7)$ & 4.0 & 21.4 & $"$ \\
\hline $\begin{array}{c}\text { JA-37 } \\
" 1 \\
"\end{array}$ & $\begin{array}{c}3497 \\
" 1\end{array}$ & $\begin{array}{c}3286 \\
" 1\end{array}$ & $\begin{array}{c}=11 \\
355-500 \\
106-150\end{array}$ & $\begin{array}{r}20-40 \\
10-15 \\
5-10\end{array}$ & $\begin{array}{l}5 \\
--\end{array}$ & $\begin{array}{l}\sim 5 \\
\text { tr } \\
\cdots\end{array}$ & $\begin{array}{l}30-60 \\
40-50 \\
40-50\end{array}$ & $=$ & $\begin{array}{l}15-30 \\
30-40 \\
30-40\end{array}$ & $\begin{array}{l}-- \\
--\end{array}$ & $\begin{array}{l}-- \\
\mathcal{c}, \operatorname{tr} \\
\mathcal{c}, t r\end{array}$ & & & & & & $\begin{array}{c}\text { Tct } \\
" \\
"\end{array}$ \\
\hline $\begin{array}{c}01-3658 \\
\text { " } \\
\text { " }\end{array}$ & $\begin{array}{c}3658 \\
" 1 \\
"\end{array}$ & $\begin{array}{c}3658 \\
" \\
"\end{array}$ & $\begin{array}{r}75-500 \\
106-500 \\
38-106 \\
<38\end{array}$ & $\begin{array}{l}40-60 \\
40-60 \\
30-50 \\
50-70\end{array}$ & $\begin{array}{l}-- \\
-- \\
--\end{array}$ & $\begin{array}{l}-- \\
\overline{--} \\
-\end{array}$ & $\begin{array}{l}-- \\
-- \\
--\end{array}$ & $\begin{array}{l}=- \\
\overline{-} \\
--\end{array}$ & $\begin{array}{l}40-60 \\
40-60 \\
50-70 \\
30-50\end{array}$ & & & 2.3 & & c3 & 23.4 & 0 & $\begin{array}{l}\text { T1 } \\
" 1 \\
"\end{array}$ \\
\hline
\end{tabular}

Analyses were performed by Los Alawor ESS Division; methods are discussed in Ref. 43.

Equivalent depth in hole USW-G1 according to relative position in stratigraphic unit. The thickness of the Bullfros and Tran units in drill holes Ue25a-1 and $J-13$, respectively, are astured to be of the same thickness as the corresponding units in drill hole USW-G1.

$C_{A}=$ analcine; $C=$ celcite; and $\mathrm{H}=$ mordenite.

$\mathrm{S}=$ nonvelded; $\mathrm{P}=$ partly welded; $\mathrm{H}=$ enderately welded; $\mathrm{D}=$ densely velded; $V=$ very densely walded (vitrophyre); and $\mathrm{W}=$ interwediate degree of welding.

The empirical tage of oxidation of iron-titaniu exsolution oxide phases; $C 1$ denotes unoxidized and $c 7$ denotes completely oxidized. See Ref. 44 for a discussion of oxide gineral alteration trendr.

$f_{\text {Tpc }}=$ Tive Conyon Hewber of the Paintbrush Tuff; Tpt $=$ Topopah Spring Member of the Paintbrush Tuff; Tht = tuffaceous beds of Calico Hills; Tcp $=$ Prow Pass Heaber of the Crater Flat Tuff; Tcb = Bullfros Hewber of the Crater Flat Tuff; Tet = Tran unit of the Crater Flat Tuff; and Tl = lava flow and flaw breccia.

Beginning with whole rock.

h blank indicates that no onalysis was perforwed, and a dash indicates that the mineral was not detected; $t r=t r a c e(\xi 1 \%)$. 
average composition of $>75-\mu r$-diam. particles is plotted as a function of depth in Fig. 8 for unaltered glass and for the secondary minerals smectite, illite or muscovite, and clinoptilolite. In Fig. 9 the values are plotted in Fig. 9 for the devitrification minerals quartz, cristobalite, and feldspar and for the sum of the three. In Fig. 10, the abundances are plotted for the sum of the silica minerals quartz and cristobalite and for the zeolites analcime and mordenite. Because the Tiva Canyon Hember does not occur in the USW-G1 hole, the plot includes the values measured for samples from this member in drill holes $\mathrm{J}-13$ and UE25a-1 instead of the Yucca Mountain Member in drill hole USW-G1. The compositions determined ${ }^{40}$ for a more complete suite of samples from the USW-GI drill hole are also plotted. The compositions of the samples used for sorptive studies are in reasonable agreement with those characterized in Ref. 40, and the compositions of the samples from the three drill holes show fairly consistent trends. In a search for a glass sample, the two samples from the UE25a-1 hole in the Prow Pass Member (Tcp), which contain high percentages of unaltered glass, were selected. The lithologies of samples from the three drill holes are detailed in Refs. 4, 6, 39, 41, and 42.

The plots indicate that additional samples from some regions of high clinoptilolite and smectite should be studied to complete the analysis and that more samples in the Topopah Member (Tpt) and bedded tuffs of Calico Hills (Tht) should be studied. Because the unsaturated zone has only recently been seriously considered for a repository site, these units had not been studied in detail earlier. The plots also indicate that more samples containing high percentages of smectite, mordenite, analcime, and glass should be studied.

4. Sorption as a Function of Stratigraphic Position. The data for sorption in Table XXII are plotted in Figs. 11 through 20 as a function of the drill hole USW-G1 depth for the elements studied. Figures 12-14 indicate that the data for strontium, cesium, and barium from the three drill holes follow approximately the same trends. Sorption ratios increase from a fairly low level (relative to the maximum values) near the base of the Topopah Spring Member (Tpt), stay high through the bedded tuffs of Calico Hills (Tht), and then decrease below the top of the Prow Pass Member (Tcp) in the upper third of the unit. The ratios increase again through the rest of the unit, but the two samples from the J-13 drill hole have lower sorption ratios for the three elements. Sorption ratios in the Bullfrog Member (Tcb) are high, whereas 


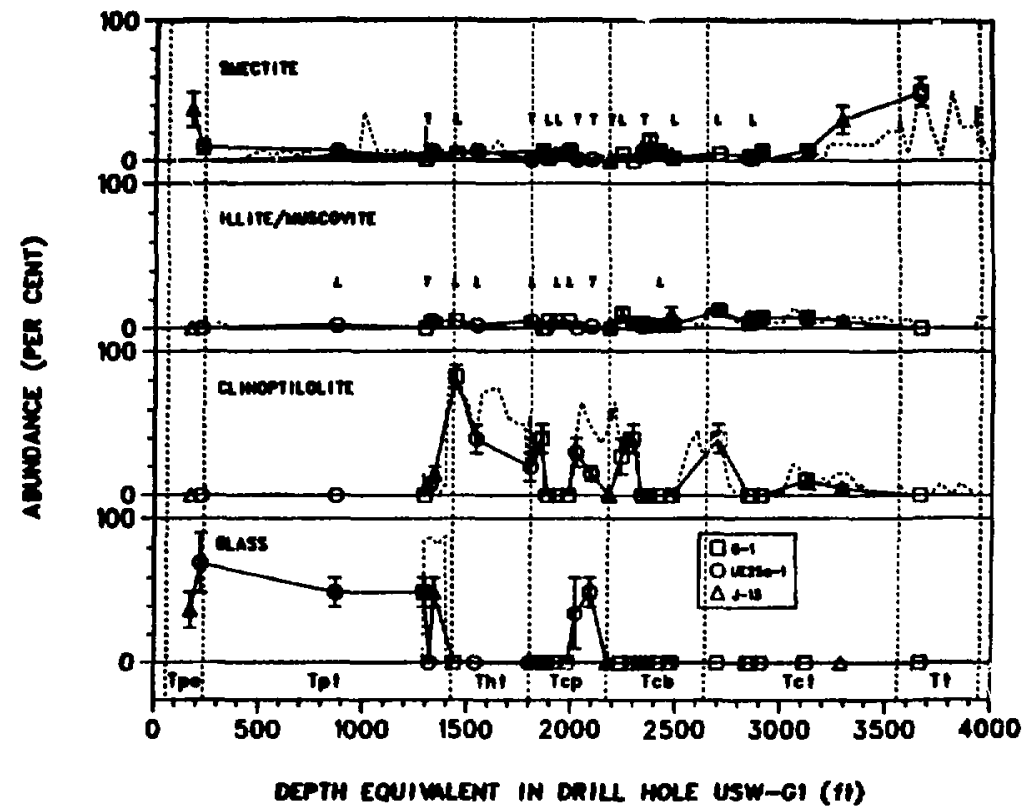

Fig. 8. Variation in mineralogy with depth for alteration minerals smectite, illite or muscovite, and clinoptilofite. Dashed lines are data for a more complete set of samples. ${ }^{2}$ The letters $T$ and $L$ indicate trace $(\leqslant 1 \%)$ amounts and upper limits, respectively.

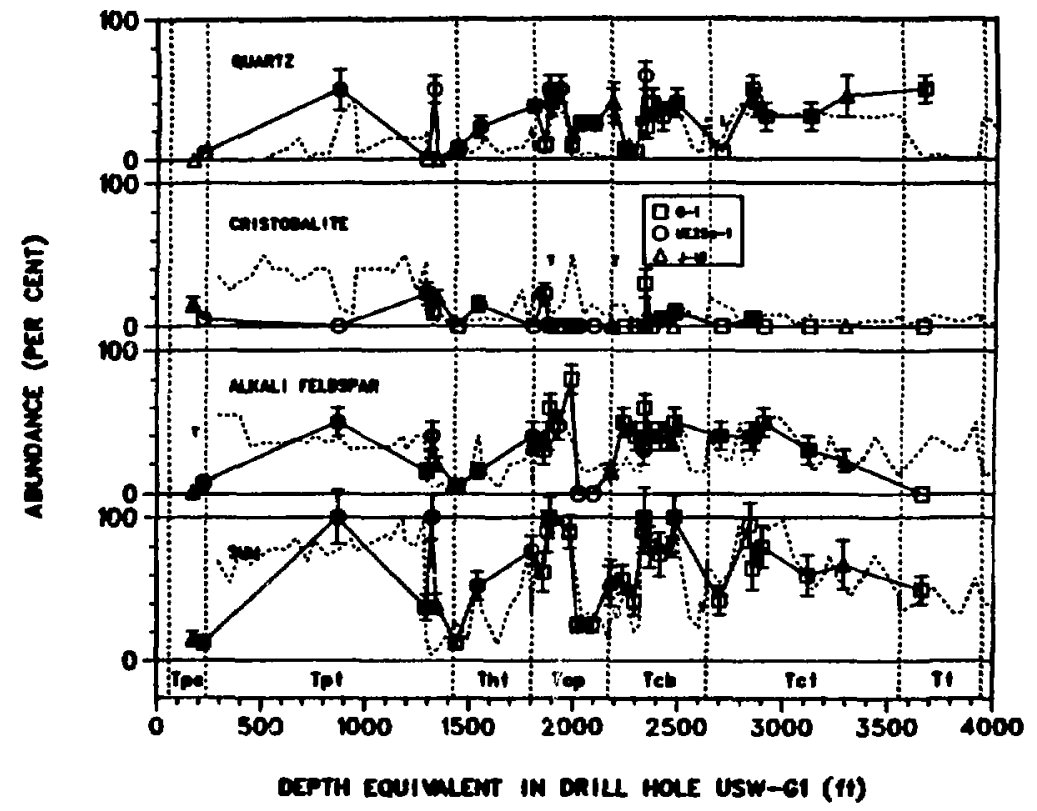

Fig. 9. Variation in mineralogy with depth for devitrification minerals quartz, cristobalite, and feldspgr. Dashed lines are data for a more complete set of samples. ${ }^{4}$ The letter $T$ indicates trace ( $<1 \%)$ amounts. 


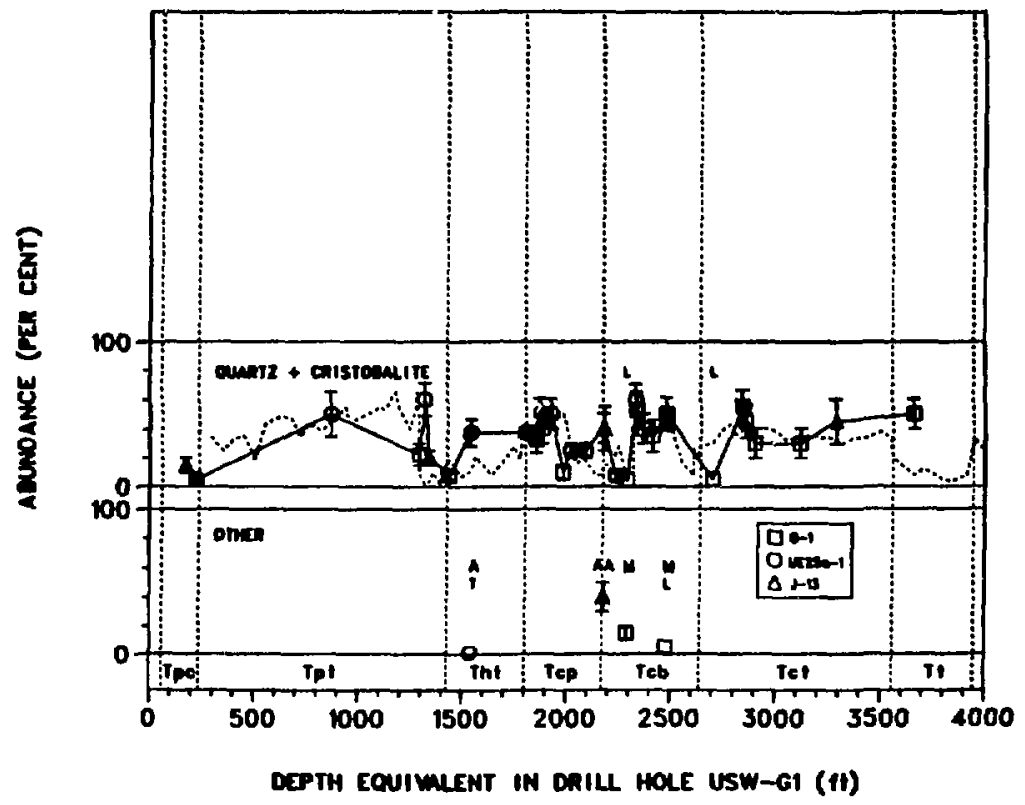

Fig. 10. Variation in mineralogy with depth (a) for the sum of silica minerals quartz and cristobalite and (b) for the zeolites analcime (A) and mordenite (M). The letters $T$ and $L$ indicate trace $(\leq 1 \%)$ amounts and upper limits, respectively.

those in the center of this unit are lower. In the Tram Member (Tct), sorption ratios are again high at the top of the unit and start to decrease with depth; however, one sample in the center of the Tram Member exhibits higher sorption ratios.

The physical and mineralogic variations within tuff units are related to the mode of emplacement and to alteration processes both during cooling and by interaction with groundwater (see, for example, Refs. 6 and 40). The lowest sorption ratios for strontium, cesium, and barium, which are thought to sorb mainly by ion-exchange reactions, are associated with devitrified tuffs. These tuffs are generally welded to some degree and contain principally quartz, cristobalite, and alkali feldspars (with some clays). The maximum sorption ratios correspond to nonwelded tuffs the contain the zeolite clinoptilolite.

The variations of sorption of cerium, europium, plutonium, and amexicium with stratigraphy (Figs. 15, 16, 19, and 20) are not as regular as those for strontium, cesium, and barium. The chemistry of these elements is more complex in the near-neutral groundwater (Sec. II.B). The sorption ratios for plutonium cover a fairly narrow range and are independent of sample position or mineralogy. 


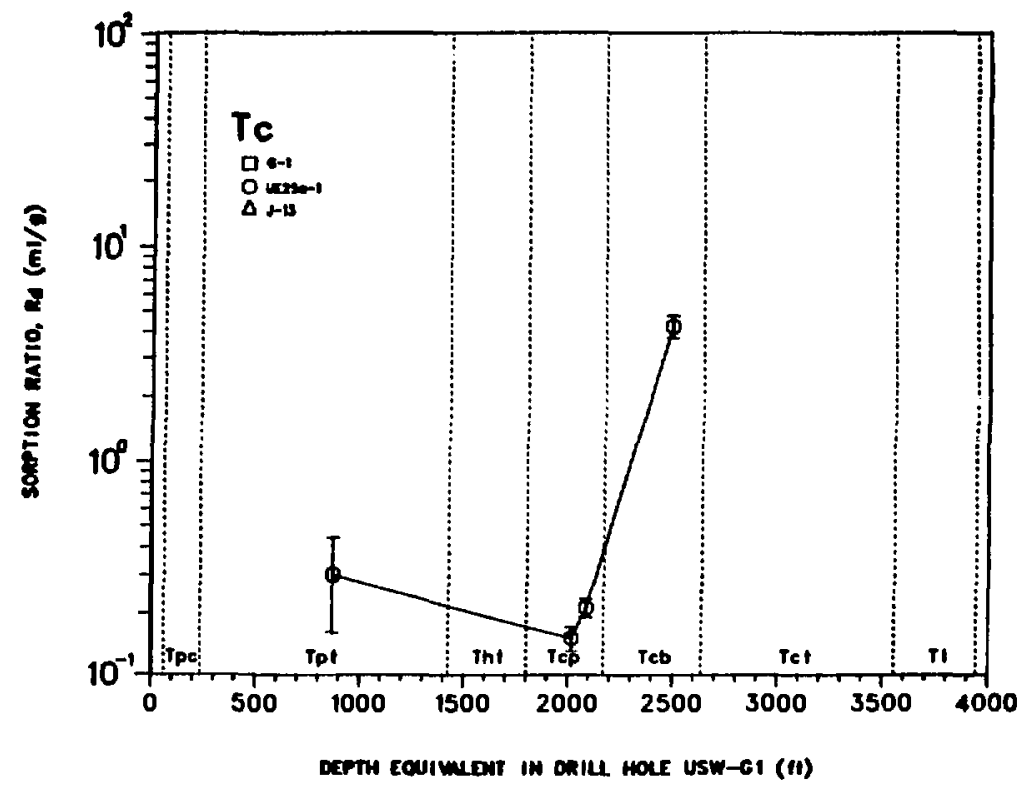

Fig. 11. Sorption ratio variation for technetium as a function of stratigraphic position. Samples are from drill hole UE25a-1.

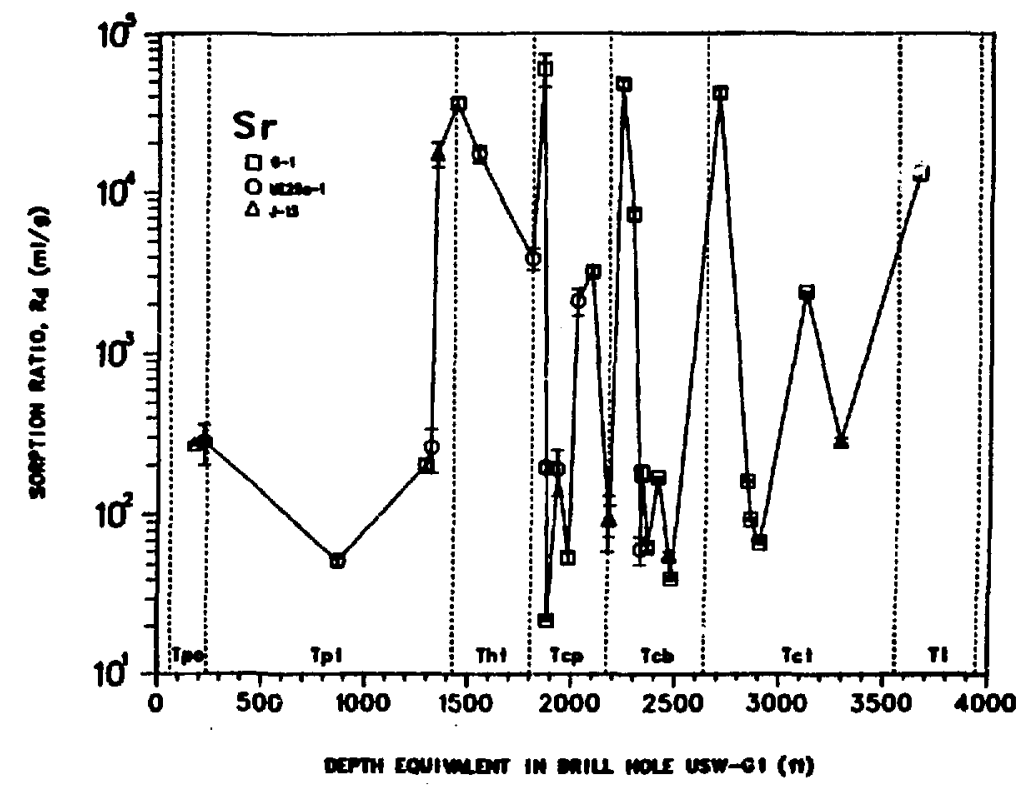

Fig, 12. Sorption ratio variation for strontium as a function of stratigraphic position. The drill holes from which each sample originated are indicated by symbols in this and subsequent figures. 


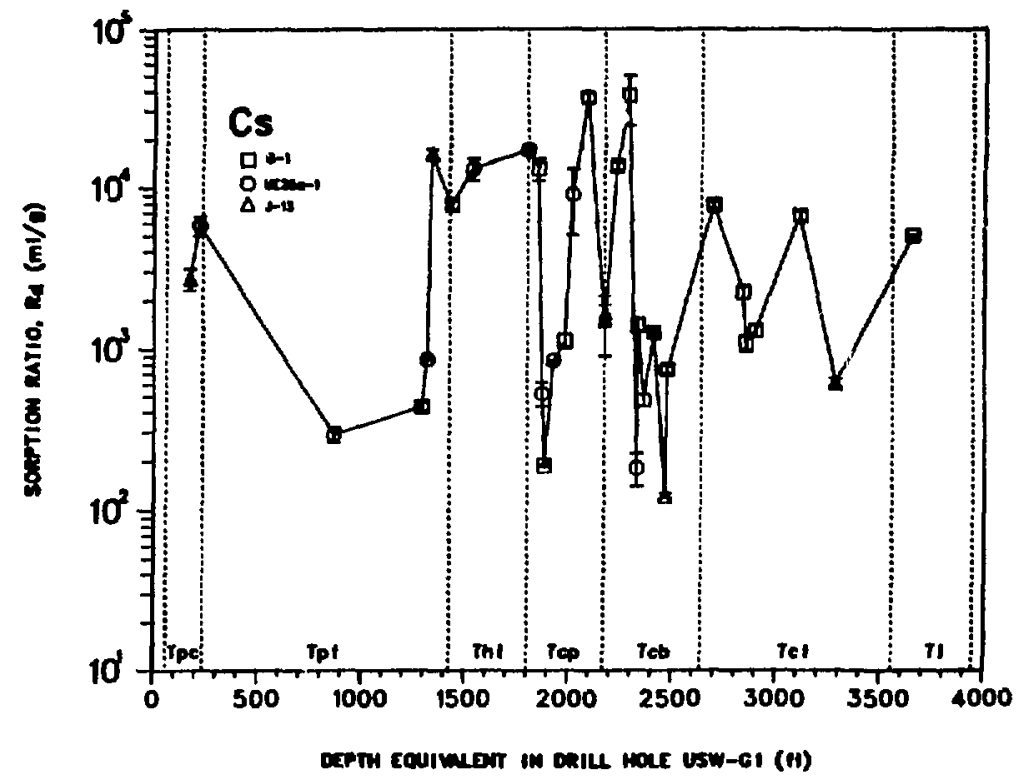

Fig. 13. Sorption ratio variation for cesium as a function of stratigraphic position.

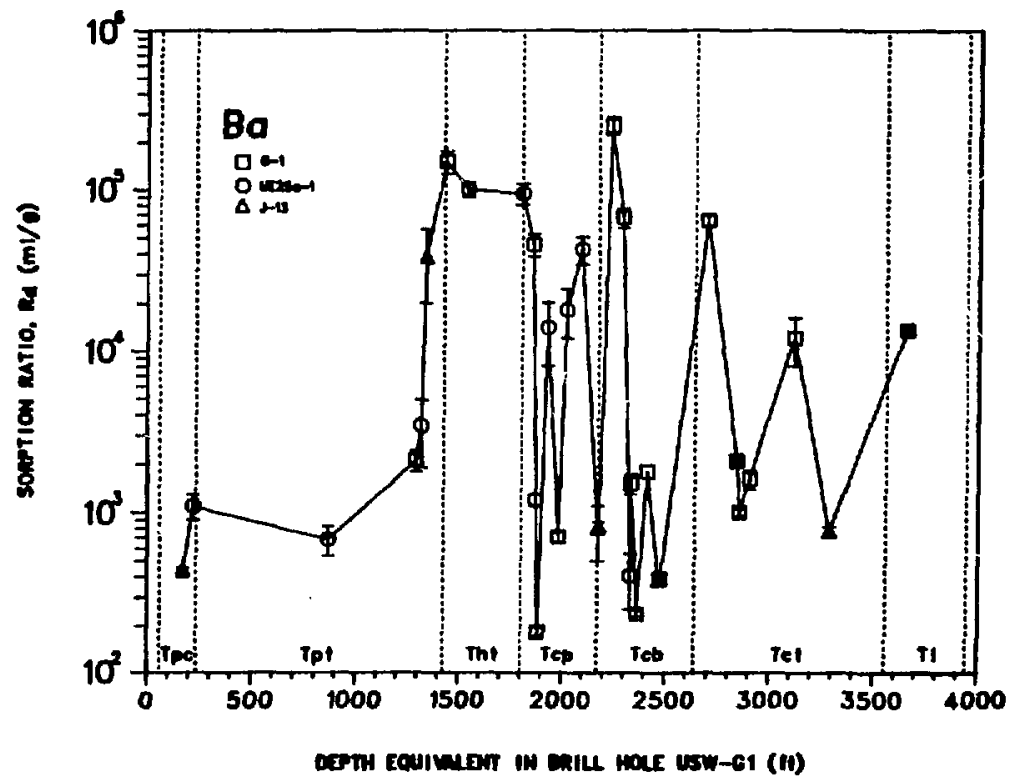

Fig. 14. Sorption ratio variation for barium as a function of stratigraphic position. 


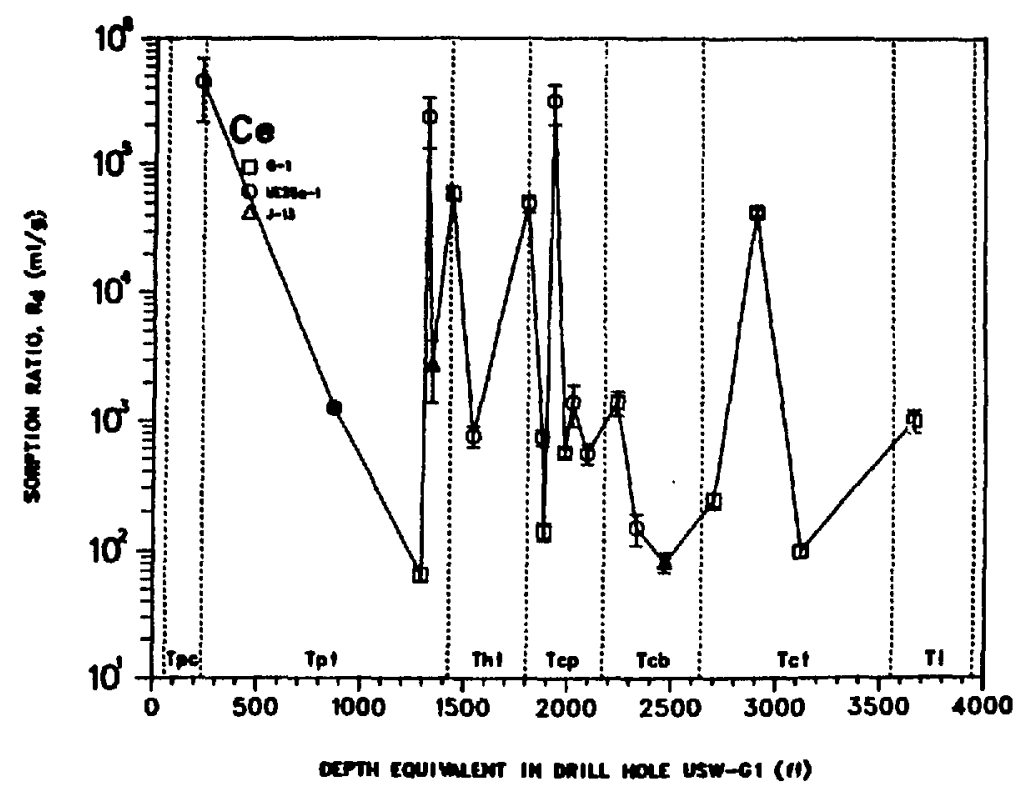

Fig. 15. Sorption ratio variation for cerium as a function of stratigraphic position.

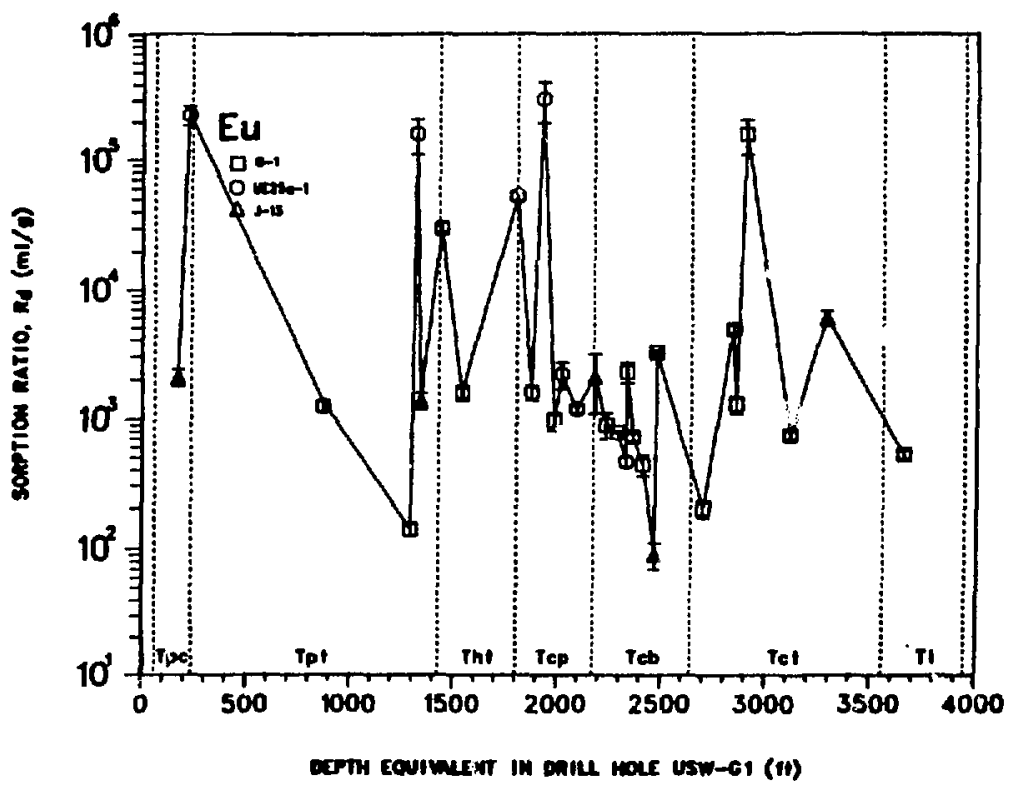

Fig. 16. Sorption ratio variation for europium as a function of stratigraphic position. 


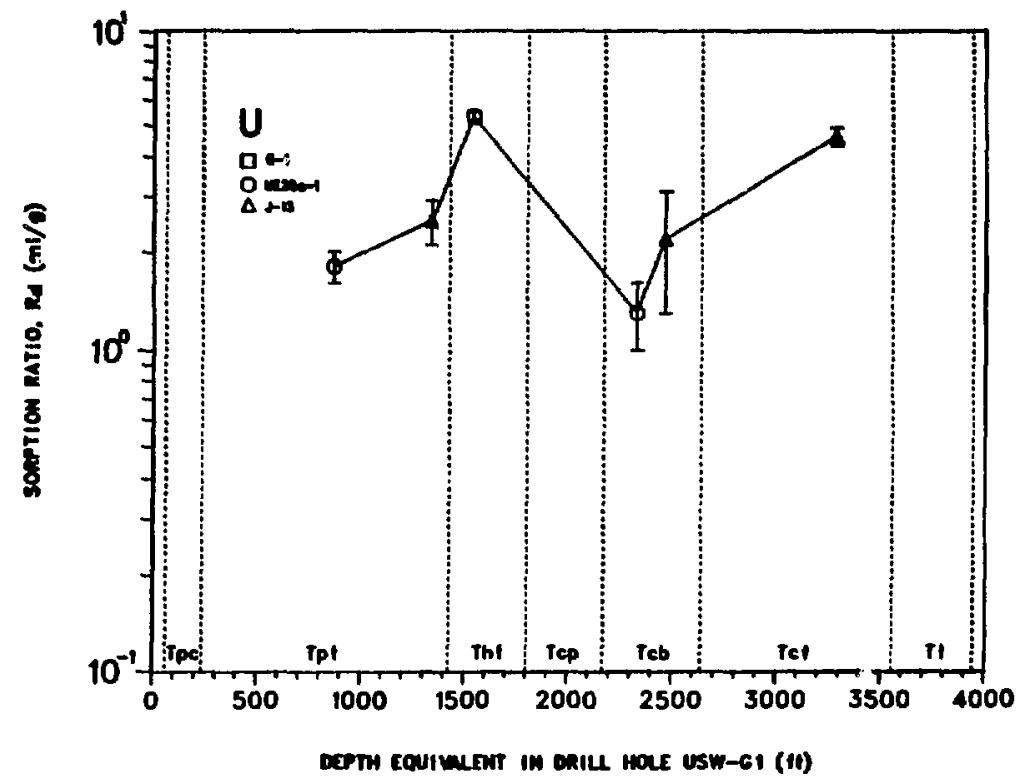

Fig. 17. Sorption ratio variation for uranium as a function of stratigraphic position.

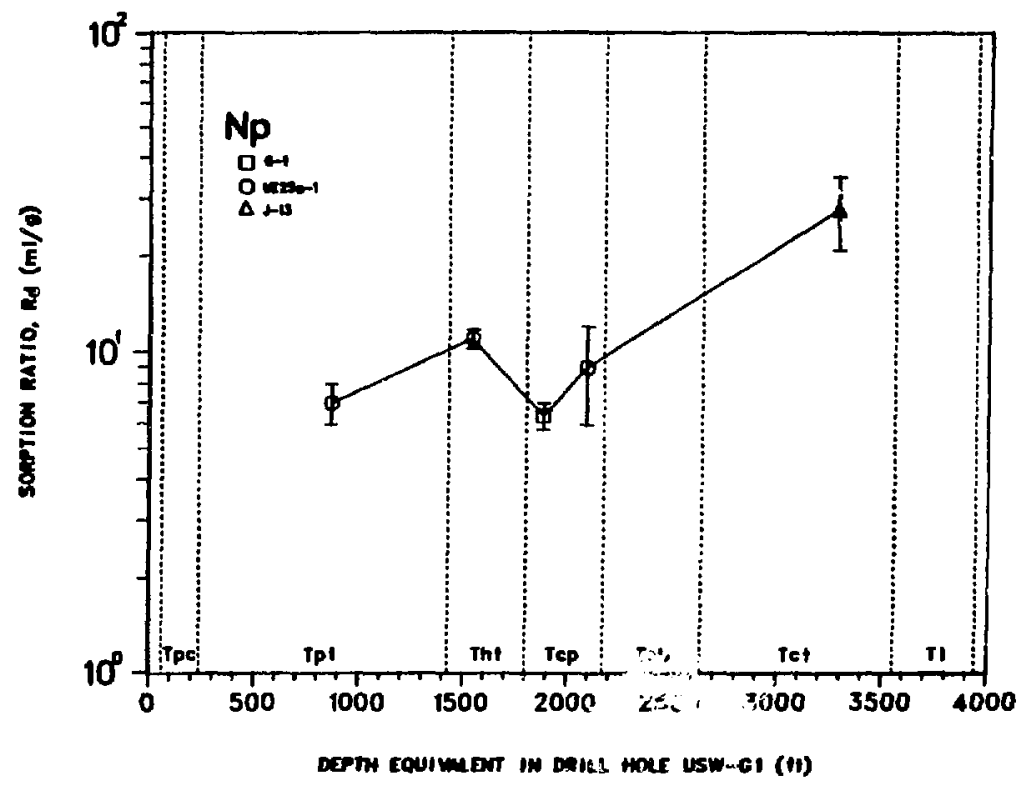

Fig. 18. Sorption ratio variation for neptunium as a function of stratigraphic position. 


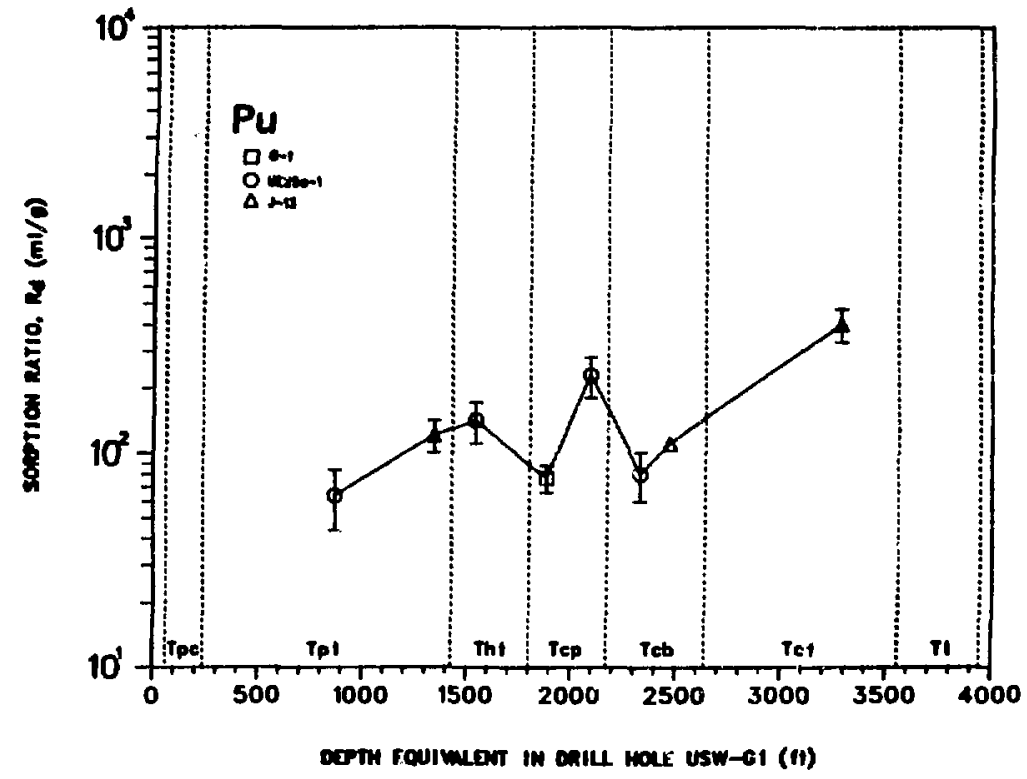

Fig. 19. Sorption ratio variation for plutonium as a function of stratigraphic position.

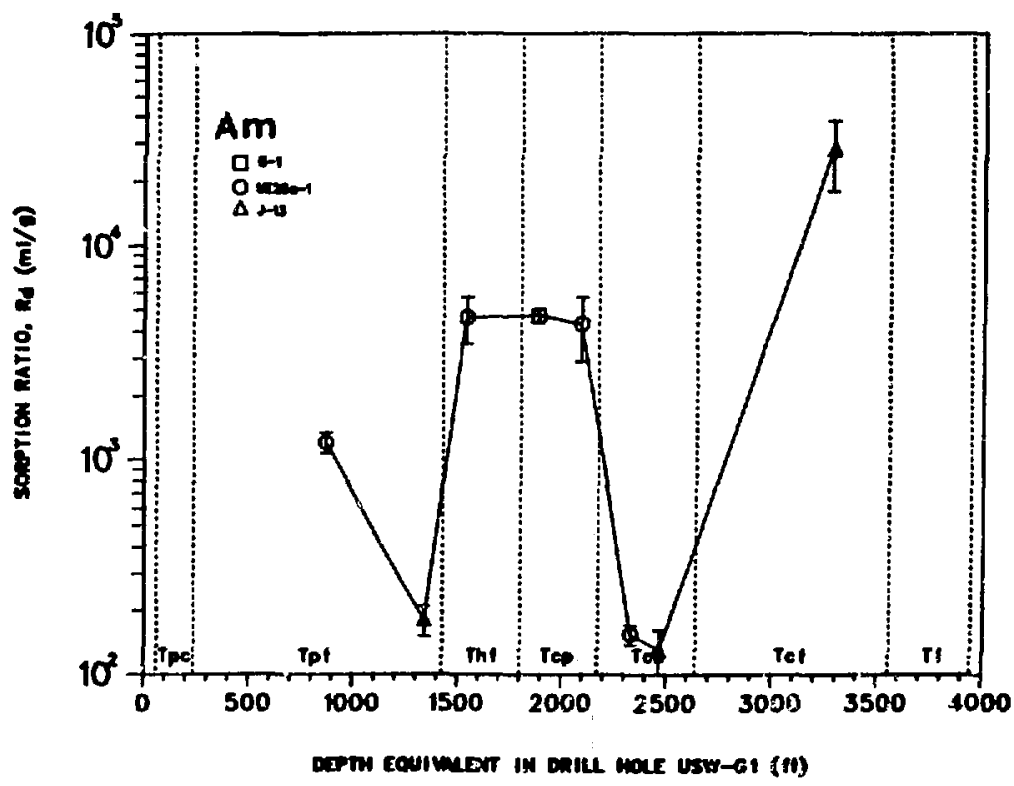

Fig. 20. Sorption ratio variation for americium as a function of stratigraphic position. 
In comparing the sorption ratios for anericium with sample mineralogy, there is a rough correlation of high sorption with samples containing clinoptilolite and swectite and low sorption with samples containing devitrification minerals.

Although sorption of technetium, uranium, and neptunium (Fig. 11, 17, and 18) has not been measured for many samples, the sorption ratios are relatively low; correlations with stratigraphic position cannot be made.

5. Sorption as a Function of Tuff Mineralogy. The variations of sorption of strontium and cesium (Figs. 12 and 13) and the abundance of the zeolite clinoptilolite (Fig. 8) are compared as a function of stratigraphic position in Figs. 21 and 22. The stratigraphic correspondences are quite striking, showing the correlation of increasing strontium and cesium sorption with increasing clinoptilolite abundance. A similar correlation can be made for sorption of barium by comparing the plot in Fig. 14 with the plot of clinoptilolite abundance in Fig. 8 .

Sorption ratios are plotted as a function of clinoptilolite abundance in Figs. 23-25, for strontium, cesium, and barium for all of the samples studied. Again, the samples containing no clinoptilolite have significantly lower sorption ratios than those containing more than a few per cent of the zeolite. If the abundance of this zeolite is the only factor influencing sorption ratios, with no differences in sorptive properties caused by changes in the composition of the clinoptilolite (or heulandite), then there should be a linear relationship of the form

$$
\mathrm{K}_{\mathrm{d}}=\mathrm{kc},
$$

where

$k$ is a constant, and

c is the clinoptilolite abundance (in per cent).

A nonweighted least squares $\mathrm{fit}^{45}$ to the data points, for which the abundance of clinoptilolite is $\geq 10 \%$, gave values of $690 \pm 170,430 \pm 150$, and $2300 \pm 700$ for strontium, cesium, and barium, respectively. The fits for strontium and cesium give $K_{d}$ values of $6.9 \times 10^{4}$ and $4.3 \times 10^{4} \mathrm{ml} / \mathrm{g}$ for $100 \%$ of the pure minerals. These values are compared with those calculated (see below) using simplifying assumptions from Ames' thermodynamic data, ${ }^{26,47}$ which used different 
mineral samples, of $1.8 \times 10^{5}$ and $3.8 \times 10^{4} \mathrm{ml} / \mathrm{g}$ for strontium and cesium, respectively.

The lines in Figs. 23-25 represent these fits with the dashed lines defining $\pm 2 \sigma$ envelopes. Although the least square fits are not particularly good, the points fall within the uncertainty envelopes fairly well. The dotted lines in Fig. 23 through 25 define an uncertainty of a factor of \pm 3 for later comparison. Most values for cesium with clinoptilolite abundances of $\geq 10 \%$ fall within this larger envelope.

One could equally well argue that all tuffs containing $>10 \%$ clinoptilolite fall into one grouping for cesium and two groupings ( 10 to $25 \%$ and $>25 \%$ ) for both strontiun and barium with the sorption ratio in each group known within one order of magnitude.

In Figs. 26-29, sorption ratios for technetium, cerium, europium, and americium are shown as a function of clinoptilolite abundance. There are no obvious correlations or trends with zeolite abundance; sorption ratios of samples with no clinoptilolite scatter among those for samples with the zeolite.

Sorption ratios for uranium, neptunium, and plutonium are plotted similarly in Figs. 30-32. Although there are no trends with increasing zeolitization, for each element, sorption ratios are higher for the zeolitized tuffs than for the nonzeolitized one.

Can simple correlations be made for other minerals in tuffs where clinoptilolite is absent? Consider the smectite clays: Fig. 33 shows the absence of any obvious trend for cesium when considering smectite alone in nonzeolitized samples. Similar plots for other nuclides, not shown, do not indicate any apparent correlations. Possible explanations are that (1) trace quantities of clinoptilolite, undetected by $x$-ray diffraction, may wask any influence of smectites, (2) combinations of all sorbing minerals contribute to the sorption ratio, and (3) sorption by clays invclves other factors, such as their texture or their availability to the groundwater. One of these possibilities is discussed later in this section.

Tuff samples JA-26 and JA-28 contain the zeolite analcine. The sorption ratios for these samples, shown as triangles at the botton of the Prow Pass Member in Figs. 8-16, do not exhibit the large values expected for clinoptilolite. Evidently, the nore randon structure of analciee, compared to the 


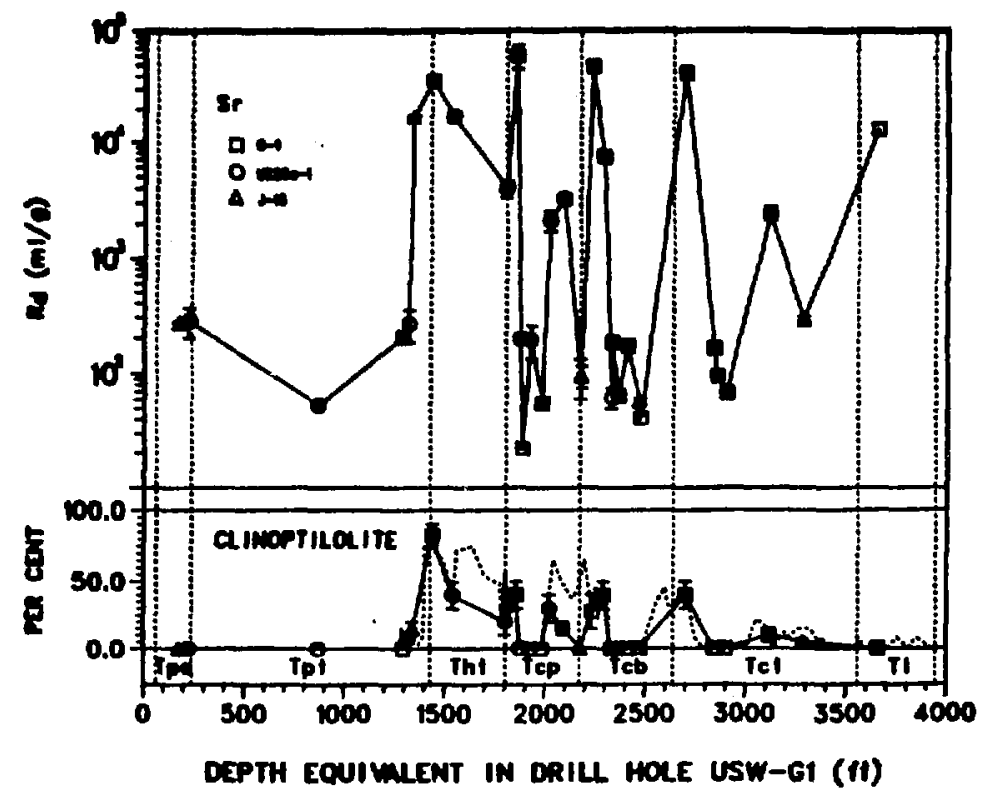

Fig. 21. Sorption ratio variation for strontium with abundance of clinoptilolite and stratigraphic position.

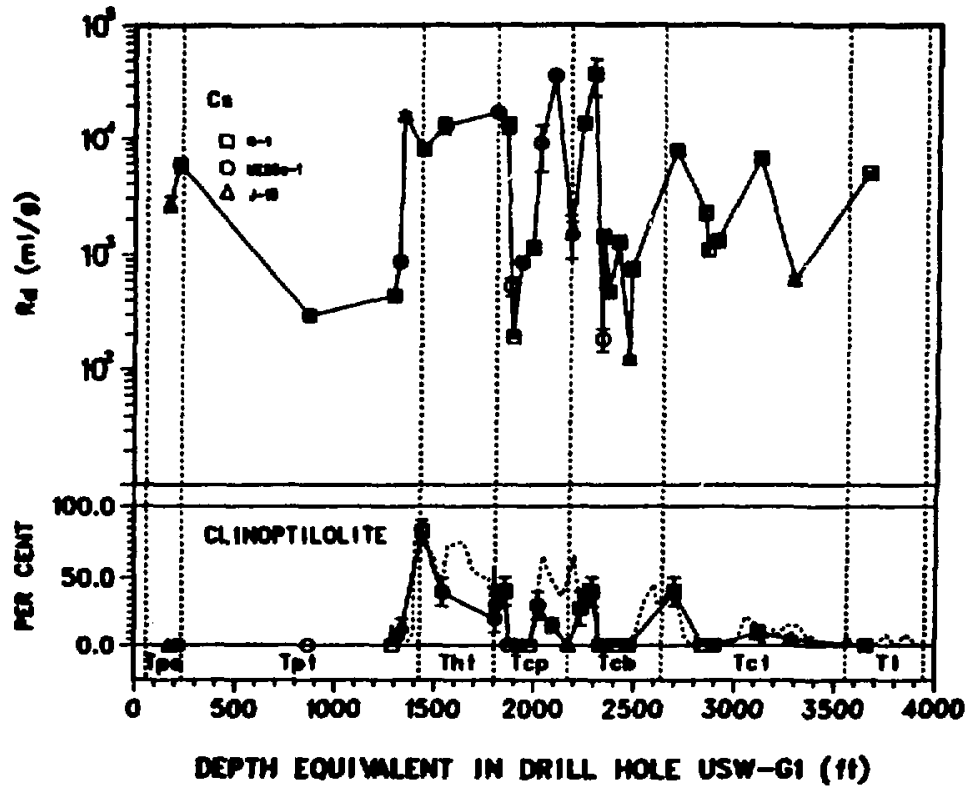

Fig. 22. Sorption ratio variation for cesium with abundance of clinoptilolite and stratigraphic position. 


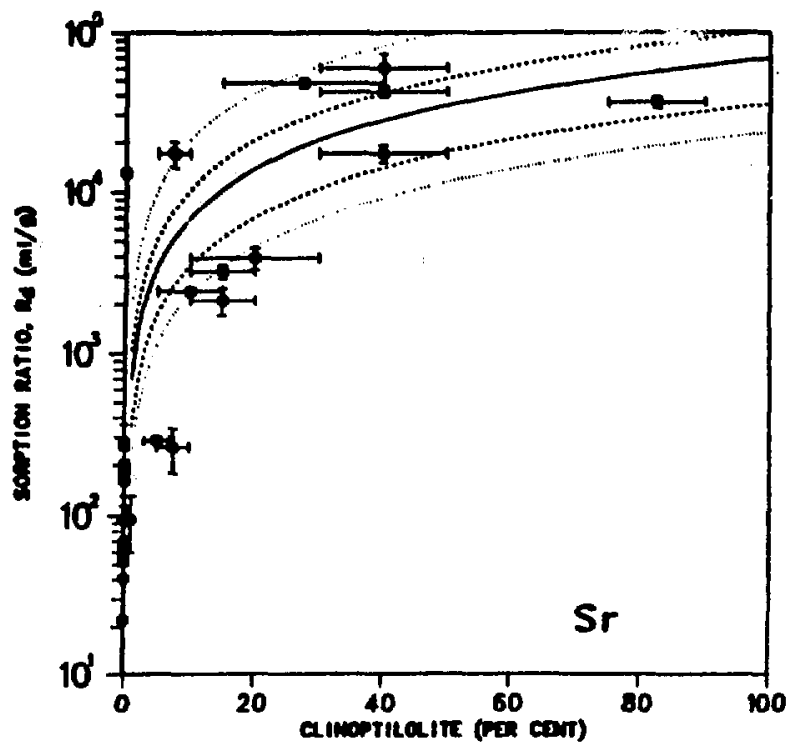

Fig. 23. Sorption ratio variation for strontium with clinoptilolite abundance. Solid line represents $K_{d}=6.9 \times 10^{4} \mathrm{ml} / 8$ for pure clinoptilolite. Dashed and dotted lines for Figs. 23, 24, and 25 indicate error bands for $2 \sigma$ and factor of \pm 3 , respectively.

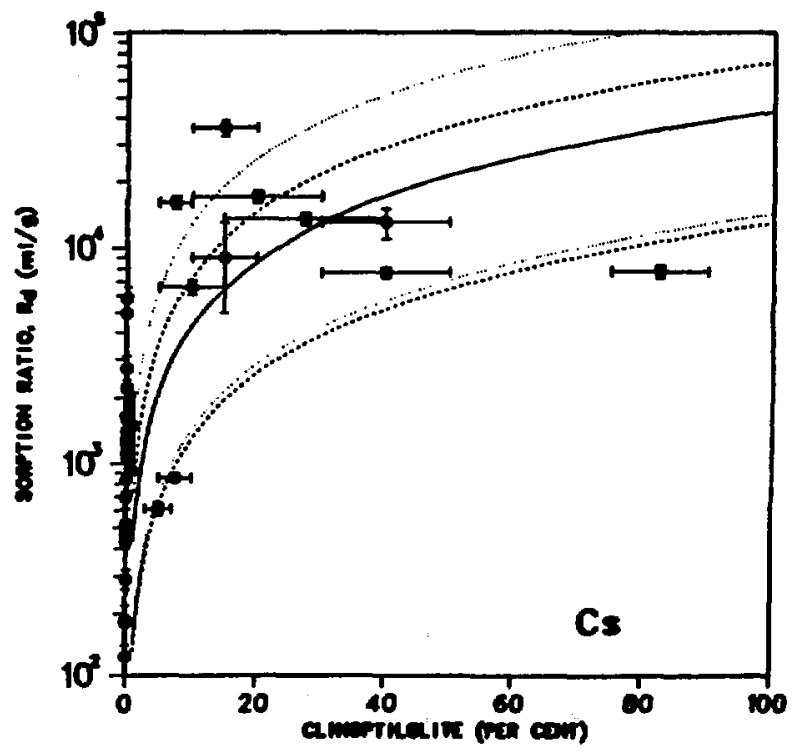

Fig. 24. Sorption ratio variation for cesium as a function of clinoptilolite abundance. Solid line represents $K_{d}=4.3 \times 10^{4} \mathrm{~m} / 8$ for pure clinoptilolite. 


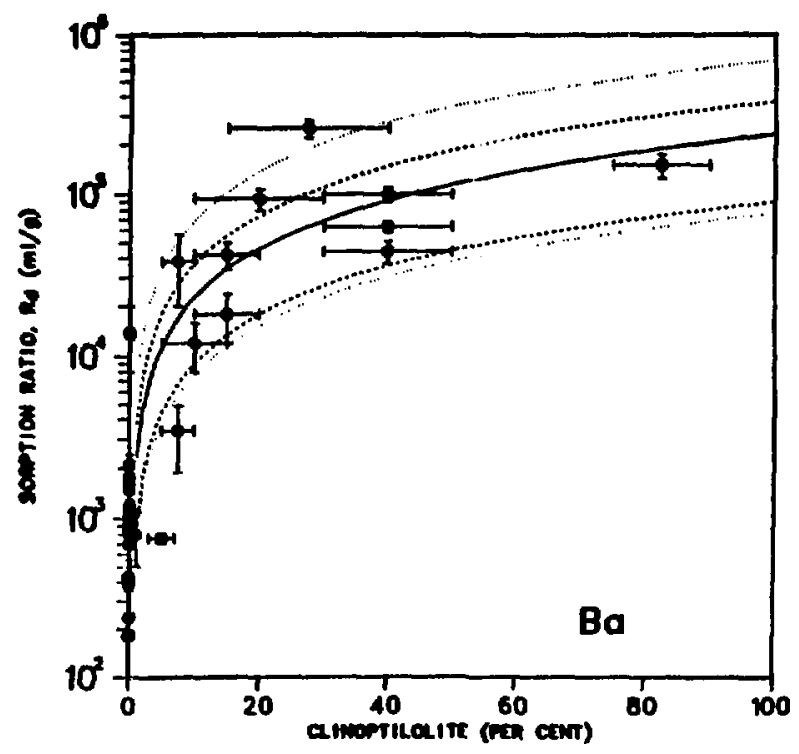
Fig. 25. Sorption ratio variation for barium. Solid line represents $\mathrm{K}_{\mathrm{d}}=$
$2.3 \times 10^{5} \mathrm{ml} / \mathrm{g}$ for the pure mineral.

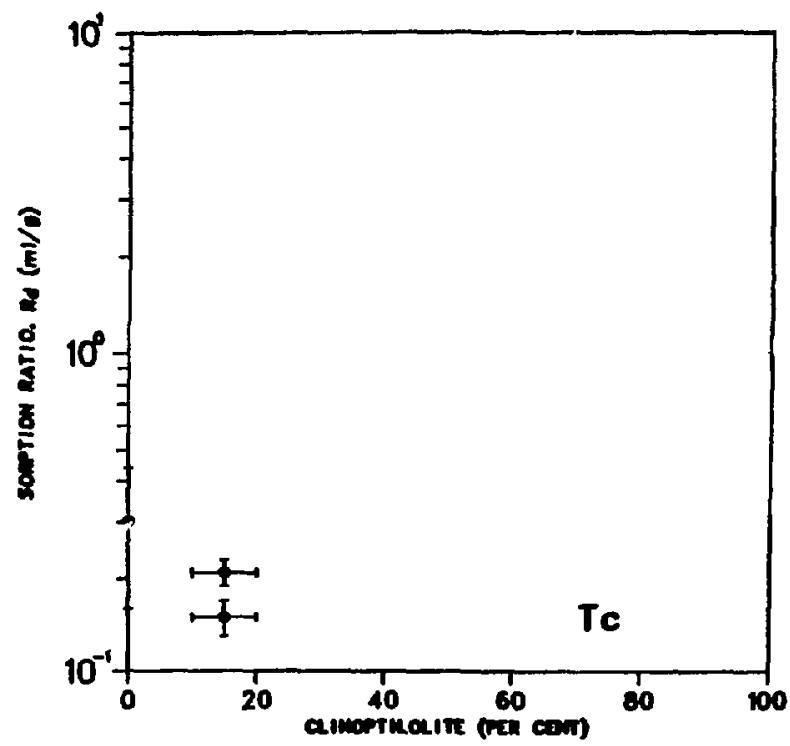

Fig. 26. Sorption ratio variation for technetium as function of clinoptilolite abundance. 


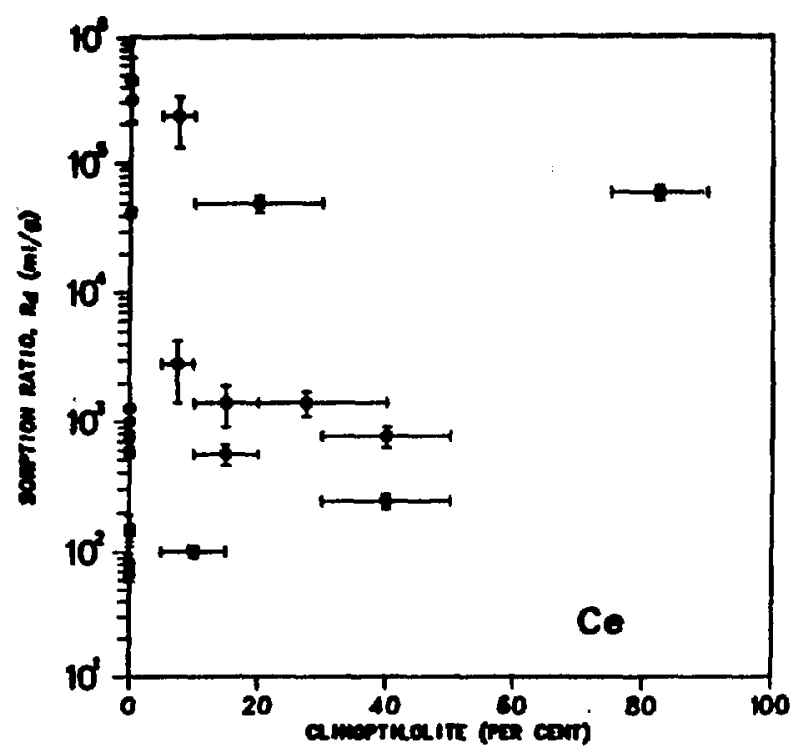

Fig. 27. Sorption ratio variation for cerium as a function of clinoptilolite abundance.

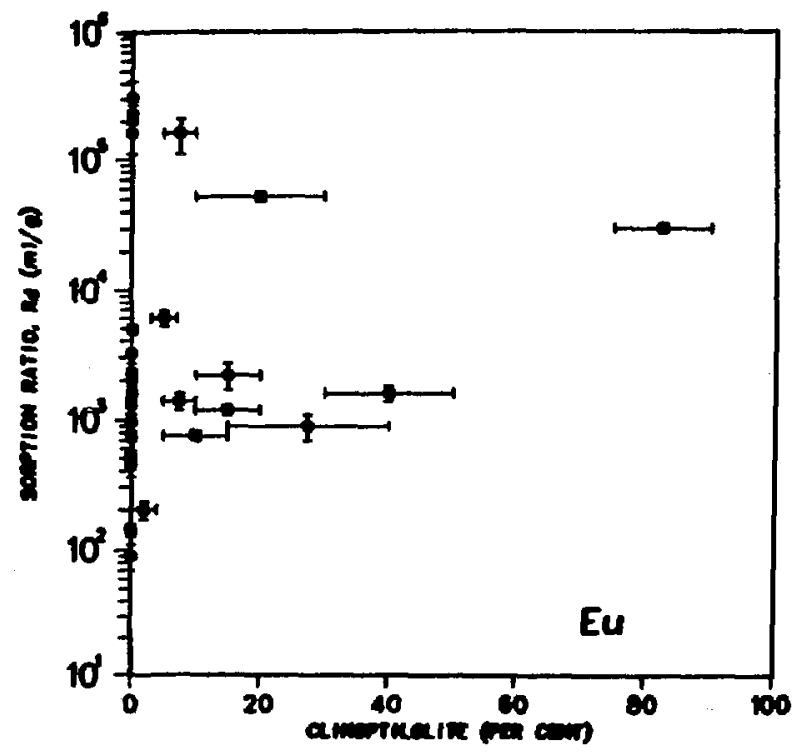

Fig. 28. Sorption ratio variation for europiu as a function of clinoptilolite abundance. 


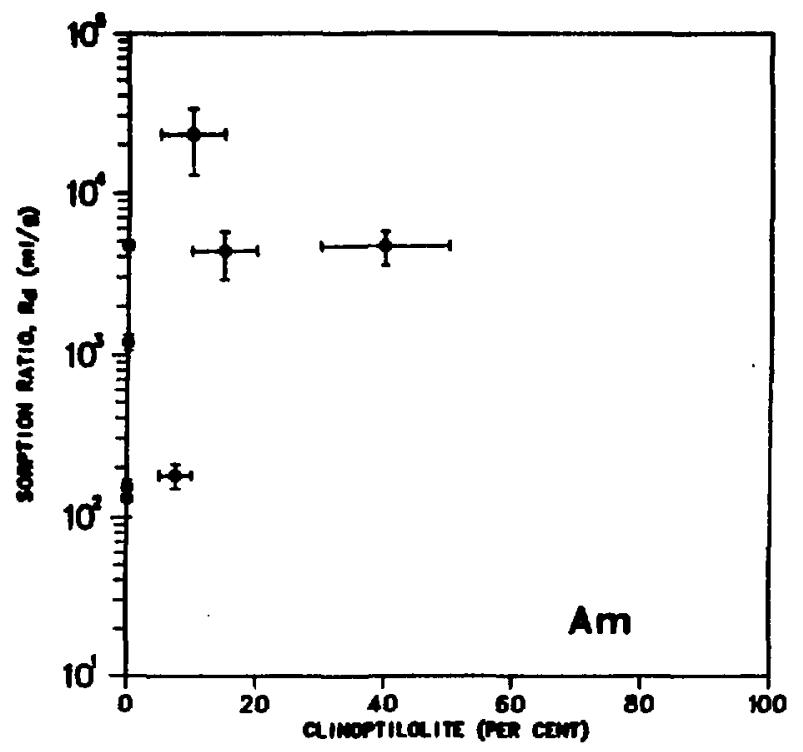

Fig. 29. Sorption ratio variation for americium as a function of clinoptilolite abundance.

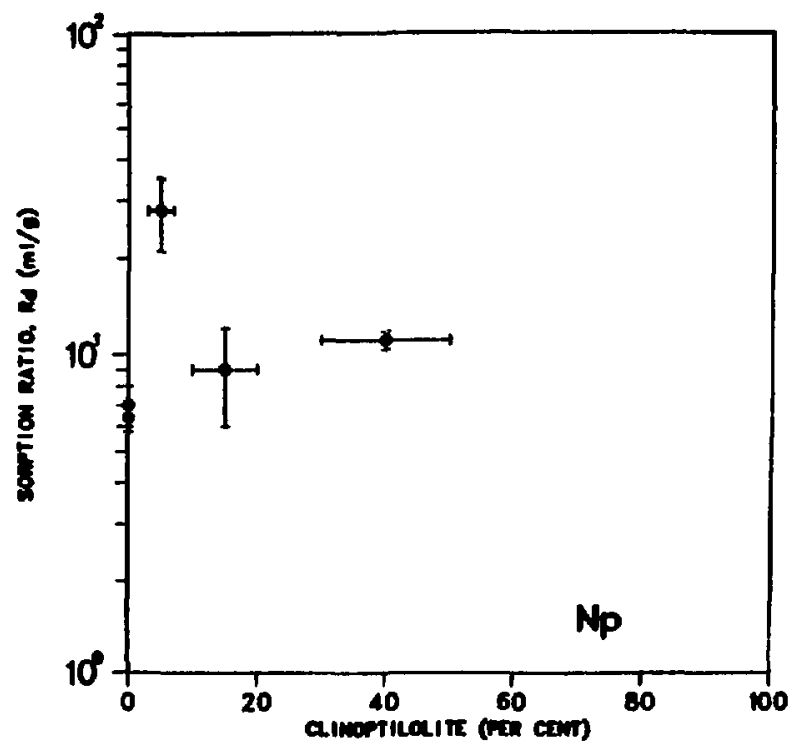

Fig. 30. Sorption ratio variation for neptunium as a function of clinoptilolite abundance. 


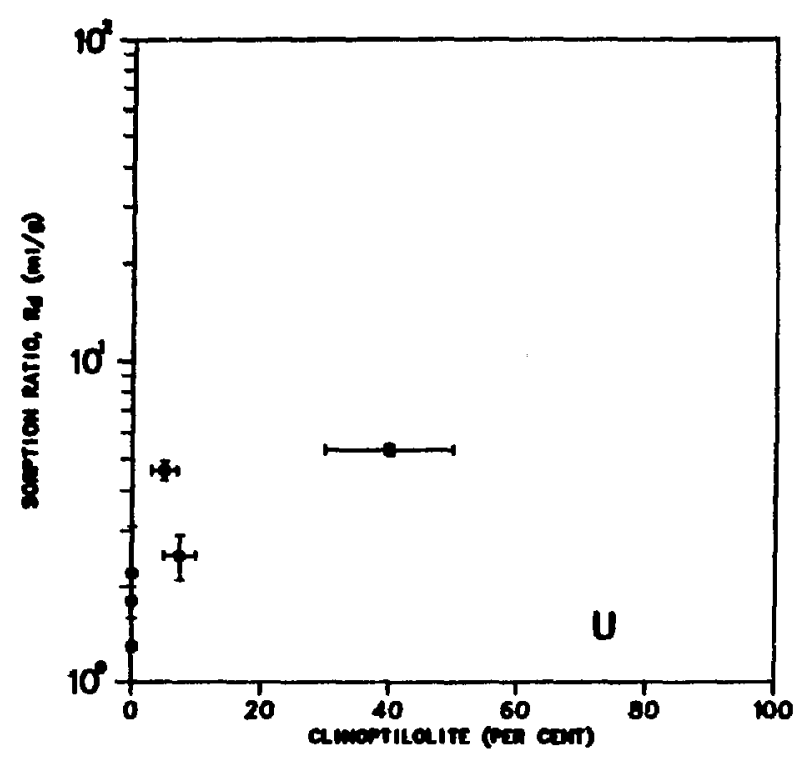

Fig. 31. Sorption ratio variation for uranium as a function of clinoptilolite abundance.

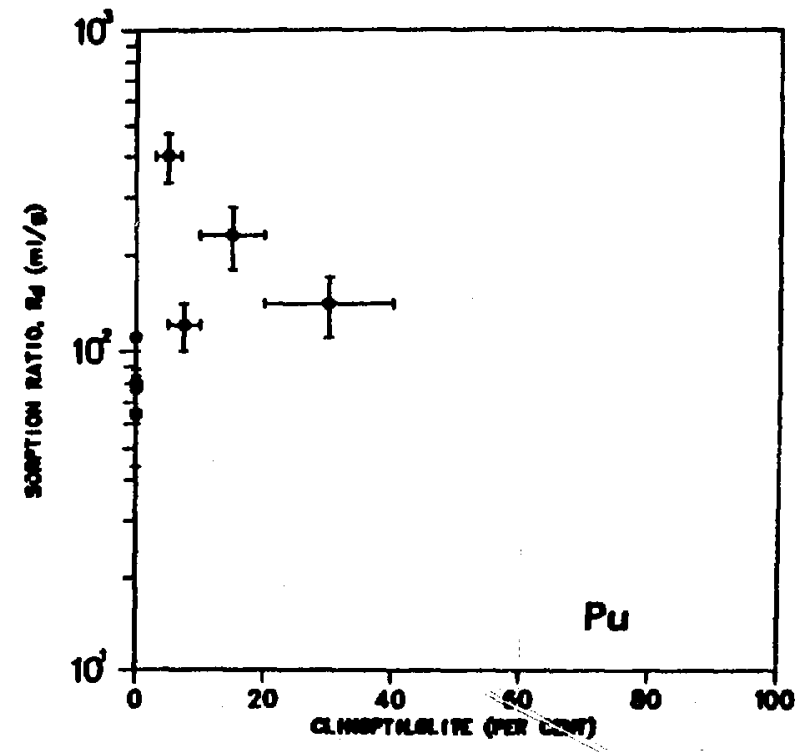

Fig. 32. Sorption ratio variation for plutoniun as function of clinoptilolite abundance. 


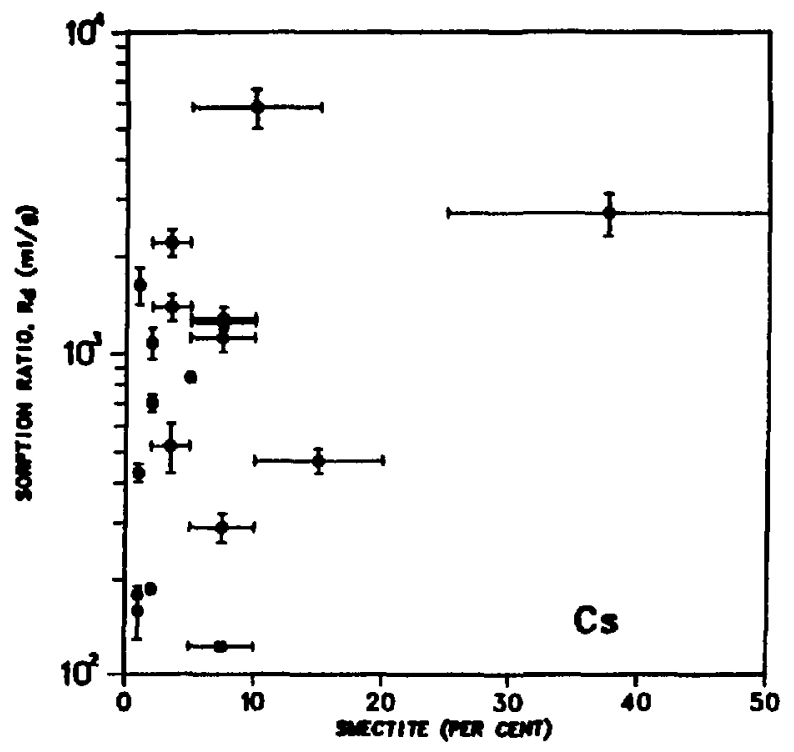

Fig. 33. Sorption ratio variation for cesium with smectite content for nonzeolitized samples.

open-cage structure of clinoptilolite, ${ }^{48}$ inhibits exchange of ions such as strontium, cesium, and barium. The ring sizes $49-51$ in analcime, $\sim 2.2 \AA$, are smaller than those in clinoptilolite, $3.5 \times 7.9$ and $3.0 \times 4.4 \AA$, which further inhibits in analcime an exchange of ions larger than $\mathrm{K}^{+}$. The two mordenitecontaining samples studied also contain clinoptilolite, which may have masked any effect of the mordenite.

The three tuffs containing fresh glass and no zeolites are samples YM-5, JA-8, and G1-1292. For cesium, which exchanges strongly on glass, the first two samples show sorption ratios approximately an order of magnitude higher than the third. Sample G1-1292, although very high in glass, is from the vitrophyre and probably has much less surface area, even in pulverized form, than the other two fine-grained samples. Trends for divalent strontium and barium are not as evident as for cesium.

Because tuff samples may be composed of more than one sorbing mineral, Los Alamos is currently researching ways to predict sorption ratios by combining the effects of several minerals. Tentatively, the combined effect is defined as a weighted sun, the "sorptive mineral content (SMC)," determined by 


$$
\text { SMC }=\Sigma w_{i} x_{i}
$$

where

$w_{i}$ is the weighting factor for each mineral phase and $x_{i}$ is the abundance $(\%)$ of each phase.

The weighting factors are determined relative to clinoptilolite, to which a value of 1.0 is assigned because it is the most strongly sorbing mineral in the group. A set of values for sorption of cesium on other minerals must be obtained from data for the pure minerals or inferred from mixtures. The $\bar{k}_{d}$ values for clinoptilolite, mordenite, and montmorillonite are calculated from published thermodynamic data. $46,47,49-51$ Some simplifying assumptions (mentioned earlier in this section) were made in applying the data to calculate $K_{d}$ values for the specific tuff/groundwater cases. The assumptions are that (1) all mineral phases observed in the $x$-ray diffraction analyses, which are performed $^{40}$ on samples ground to $<10 \mu \mathrm{m}$, are available for sorption, and the only competition for sorption sites is, in this case, between trace amounts of cesium and sodium; (2) the sodium concentration in the assumed groundwater is $3 \times 10^{-3} \mathrm{M}$ (the approximate ionic strength of the trace groundwater); and (3) one set of thermodynamic data applies to all samples of each mineral. The last assumption is not strictly true because samples of the same mineral from different localities, when prepared differently, gave different thermodynamic constants. 47

Consider the following equilibrium

$$
\mathrm{Cs}^{+}+\mathrm{Na}-\mathrm{R}=\mathrm{Cs}-\mathrm{R}+\mathrm{Na}^{+},
$$

where

Cs-R and Na-R represent the ion sorbed on a mineral.

Define

$K=$ the equilibrium constant for equilibrium (7), CEC = cation exchange capacity in meq/ 8 , and $\left[\mathrm{Na}^{+}\right]=$sodium concentration; 
then,

$$
\mathrm{K}_{\mathrm{d}}=(\mathrm{CEC}) \mathrm{K} /\left[\mathrm{Na}^{+}\right]
$$

Table XXV gives the values used for $K$ and CEC. If clinoptilolite is the only mineral available for exchange in the samples listed in Table XXIII, then CECs of 1.7 to 5.3 and 0.7 to $3.5 \mathrm{meq} / \mathrm{g}$ for cesium and strontium, respectively, are inferred for the pure zeolite. The values obtained by Ames ${ }^{46}$ for two clinoptilolites are 1.7 and $2.0 \mathrm{meq} / \mathrm{g}$, and Barrer 49 gives a value of 3.3 for heulandite. Thus, there are some differences in the properties of clinoptilolite, depending on the sample.

The weighting factor for illite was somewhat arbitrarily set at 0.05 because the CEC for this mineral ${ }^{52,53}$ is 10 times less than that for montmorillonite. The factor for analcime was set at 0.10 to correspond to the sorption ratio for sample JA-26, which contains $40 \%$ analcime as the only sorbing mineral. The factor for glass was likewise adjusted to give the observed results for samples G1-1292 and YM-48, which contain glass as the principal sorbing phase. Quartz, cristobalite, and feldspars are assumed to have negligible contributions to sorption. The $K_{d}$ values, calculated or assumed, and the corresponding weighting factors $W_{i}$ are given in Table XXVI.

The sorption ratios in Table XXI are plotted as a function of SMC in Fig. 34. Figure 35 is the same plot for low values of $R_{d}$. The solid line is the theoretical line for clinoptilolite with a $\mathrm{K}_{\mathrm{d}}$ value of $3.8 \times 10^{4} \mathrm{~m} / \mathrm{g}$. The dashed lines represent an error envelope for uncertainties of a factor of 3 ;

TABLE XXV

VALUES USED FOR CALCULATING $K_{d}$ FOR CESIUM

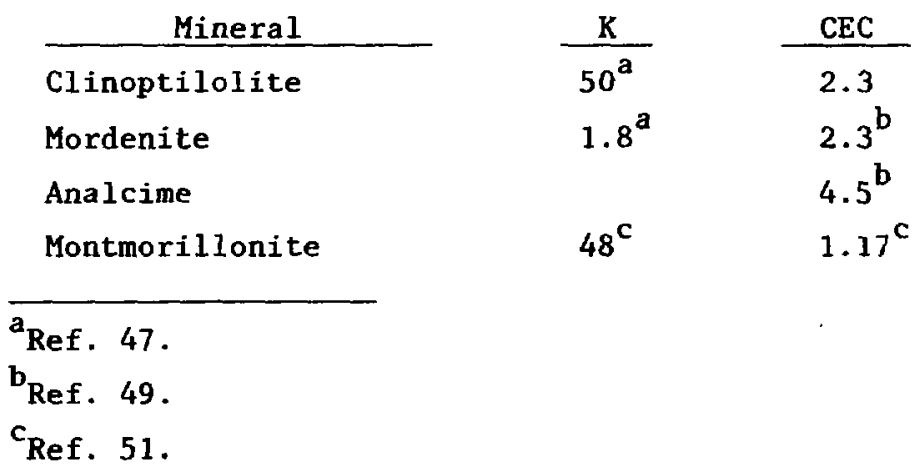


TABLE XXVI

$k_{d}$ AND $w_{i}$ VALUES USED IN SMC EVALUATION OF CESIUM

$\frac{\text { Mineral }}{\text { Clinoptilolité }}$
Montmorillonite
Mordenite
Analcime
Glass

$\begin{array}{cc}\frac{K_{d}(\mathrm{ml} / \mathrm{g})}{3.8 \times 10^{4}} & \frac{W_{i}}{1.00} \\ 1.9 \times 10^{4} & 0.50 \\ 1.4 \times 10^{3} & 0.04 \\ & 0.05 \\ & 0.016\end{array}$

most of the experimental $R_{d}$ values fall within the envelope. This treatment will help predict sorptive properties for nonzeolitized as well as zeolitized tuffs; sorption of cesium is probably the simplest test of this type of concept.

6. Desorption Experiments: Reversibility. If equilibrium were established and if observed $R_{d}$ values for tuffs were true $K_{d}$ values, the same $R_{d}$ values should be obtained from desorption and sorption experiments. The values from sorption and desorption experiments are compared as a function of stratigraphic position in Figs. 36-45. Some differences between the two types of measurement arise from large ranges in individual determinations (App. A); the averaging process, which assumed that the samples represent the same population, may not have been proper for such cases. The results for strontium and cesium from the two methods agree within $\sim 20 \%$ for most measurements. In general, values from desorption experiments are slightly higher than those from sorption experiments. For barium there is reasonable agreement when $R_{d}$ values are low (devitrified tuffs). For some of the zeolitized tuffs, however, values for barium from desorption experiments are greater than those from sorption experiments by factors of $\sim 2$ for most samples to $\sim 10$ for a few. It appears that barium sorbs on clinoptilolite somewhat more irreversibly than do strontium and cesium. For cerium, europium, and americium, the differences in $R_{d}$ values (which are reasonably high) by the sorption and desorption methods are greater. A large fraction of these elements is sorbed irreversibly, although in most cases the trends from the sorption measurements with stratigraphic position are qualitatively retained in the desorption results. The differences for plutonium, which do not show discernible trends, are also approximately a 


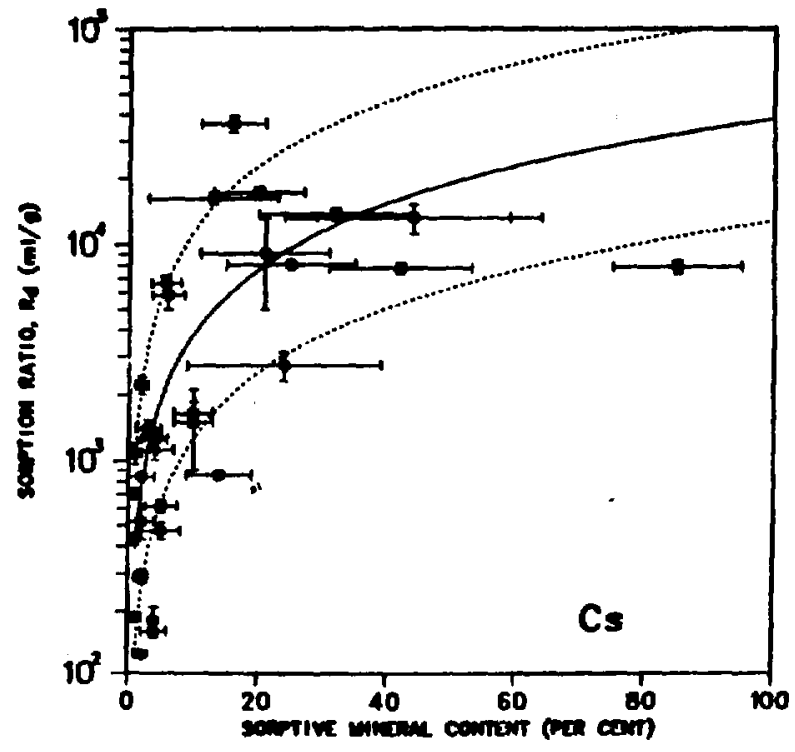

Fig. 34. Sorption ratios for cesium as a function of sorptive mineral content. Soljd line is theoretical line for clinoptilolite with $K_{d}=3.8 \times 10^{4} \mathrm{~m} \ell / \mathrm{g}$. Dashed lines give an uncertainty envelope of a factor of \pm 3 .

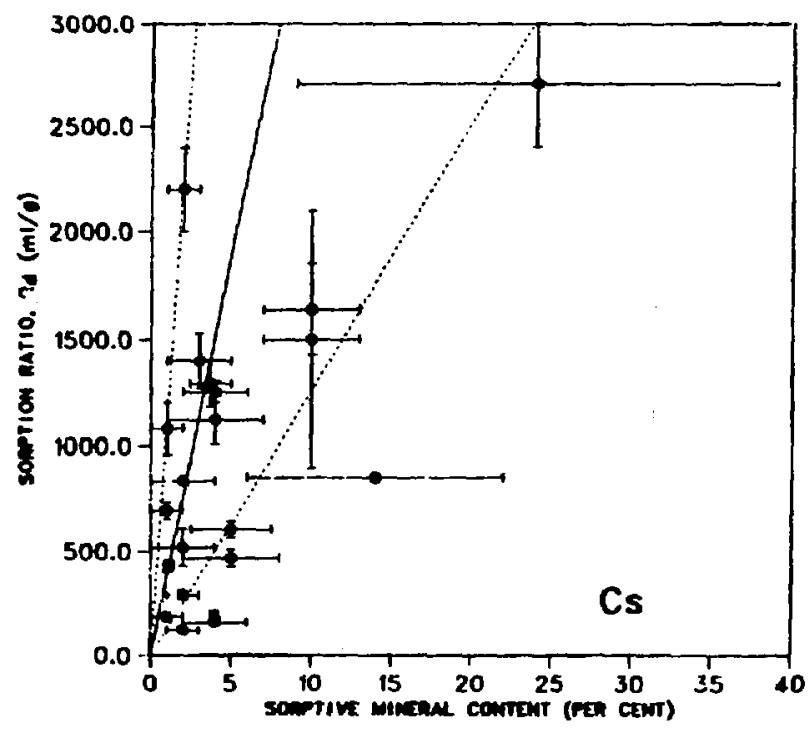

Fig. 35. Early part of Fig. 34 on linear scale. 


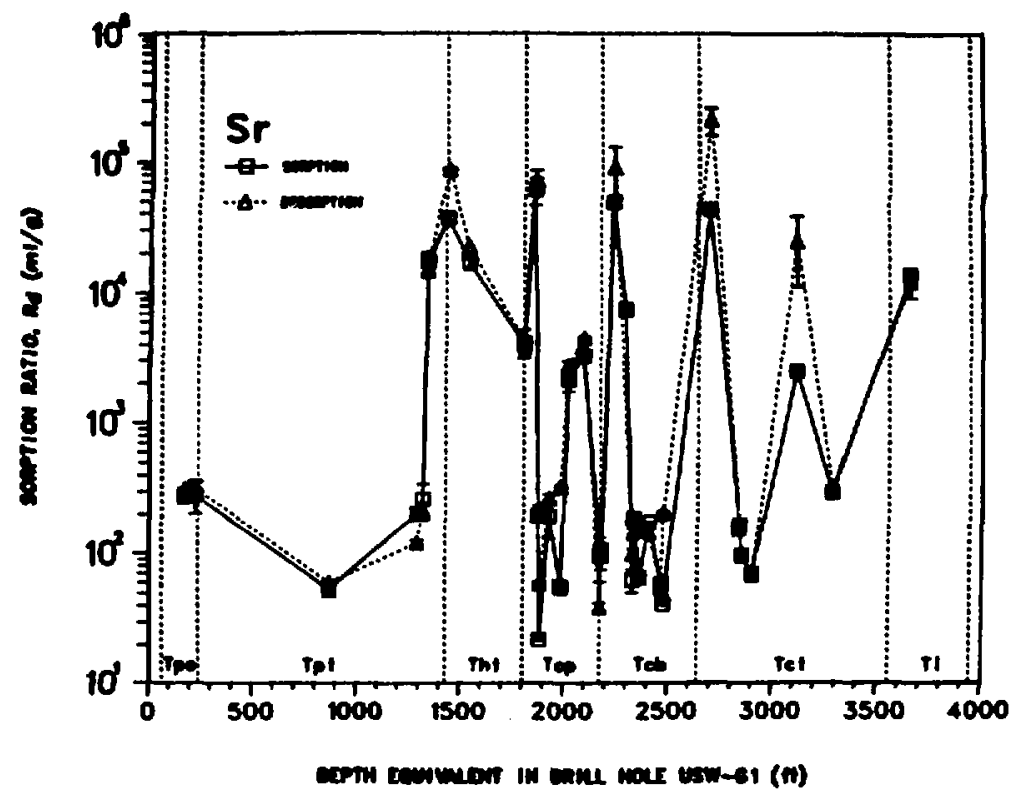

Fig. 36. Comparison of strontium ratios for sorption and desorption as a function of stratigraphic position.

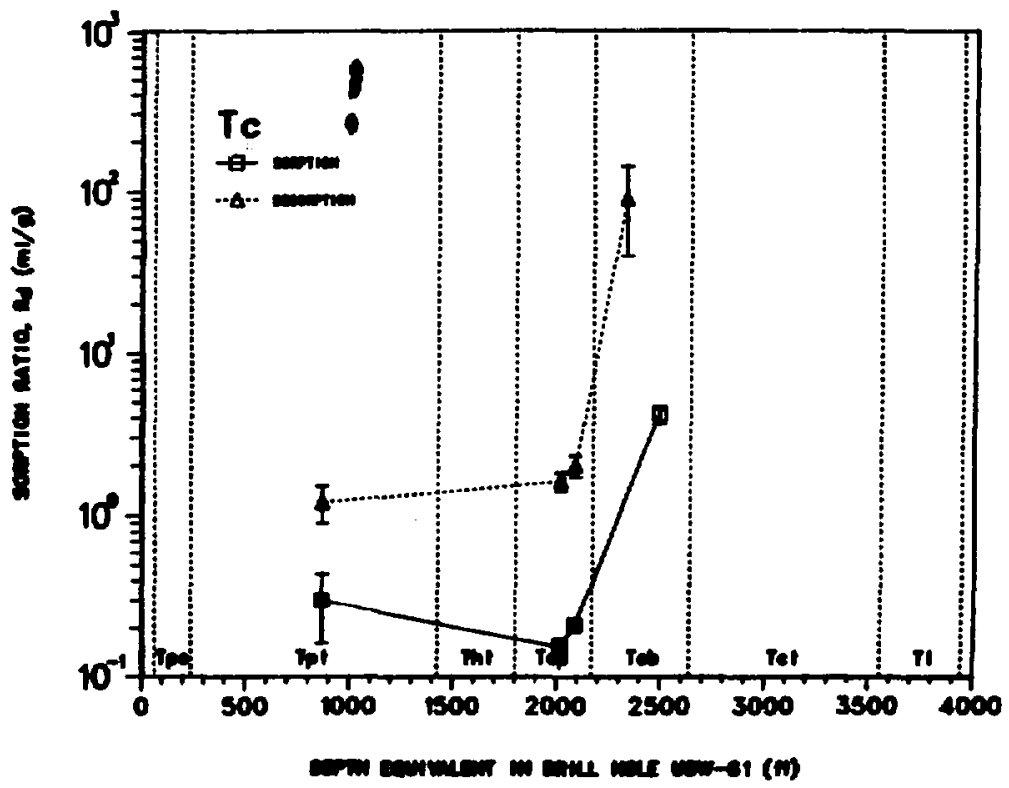

Fig. 37. Comparison of technetium sorption ratios for sorption and desorption as a function of stratigraphic position. 


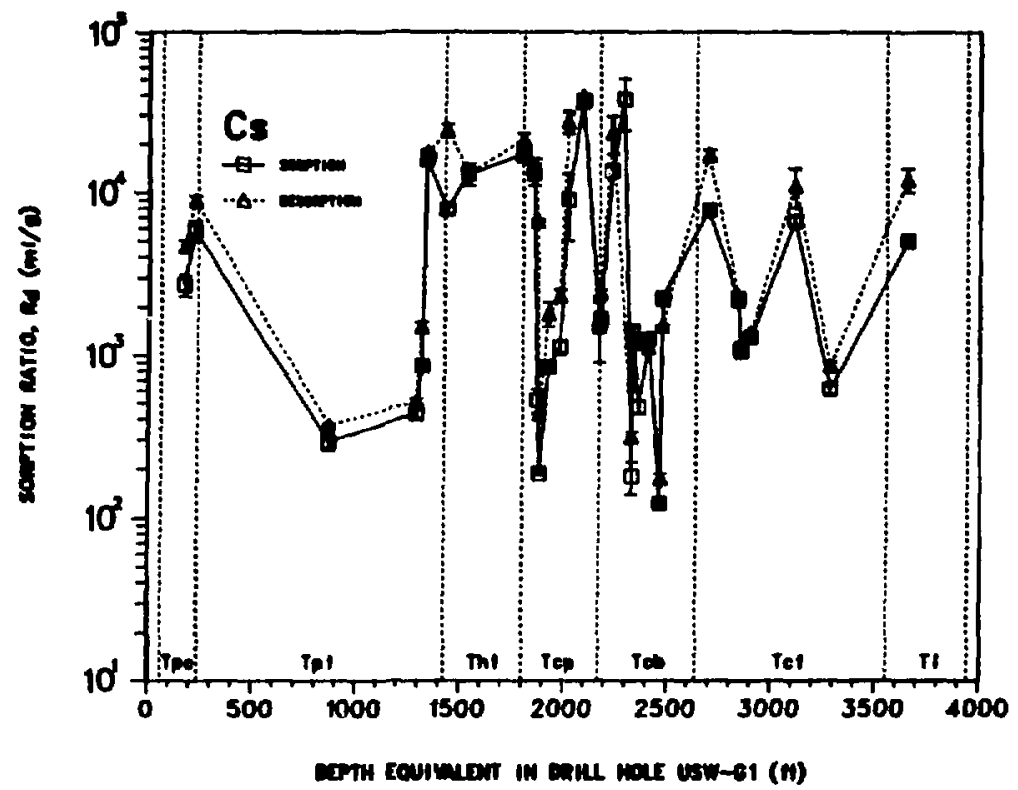

Fig. 38. Comparison of cesium sorption ratios for sorption and desorption as a function of stratigraphic position.

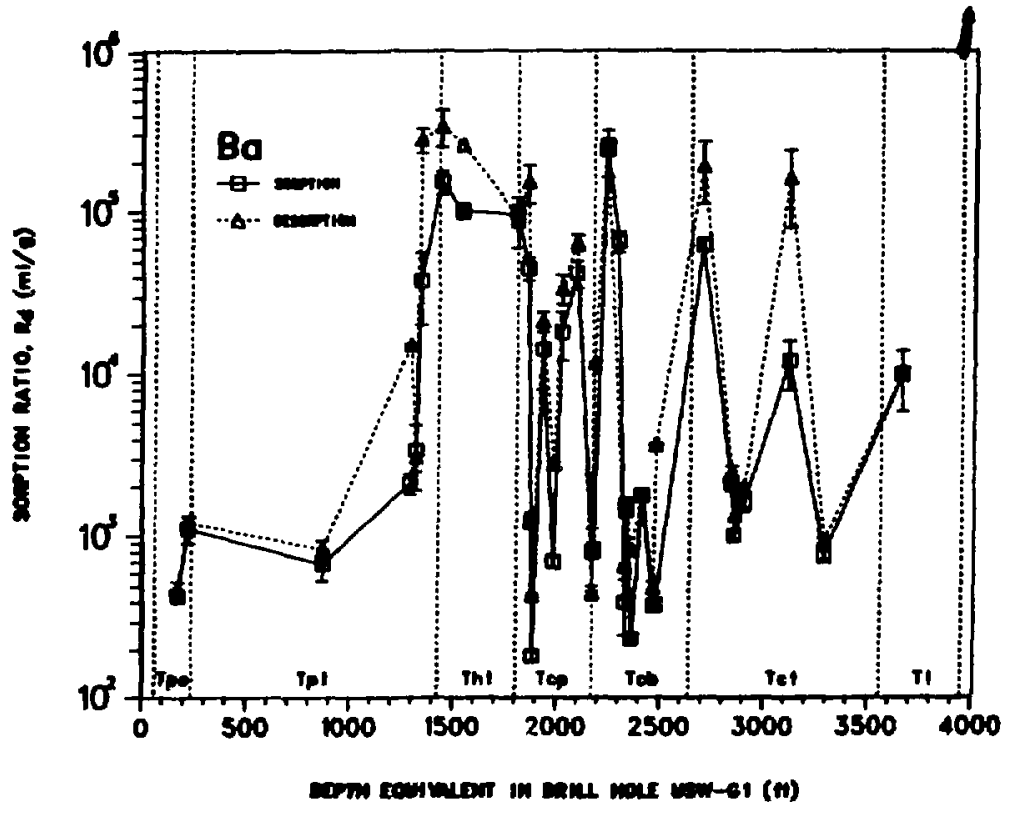

Fig. 39. Comparison of barium sorption ratios for sorption and desorption as a function of stratigraphic position. 


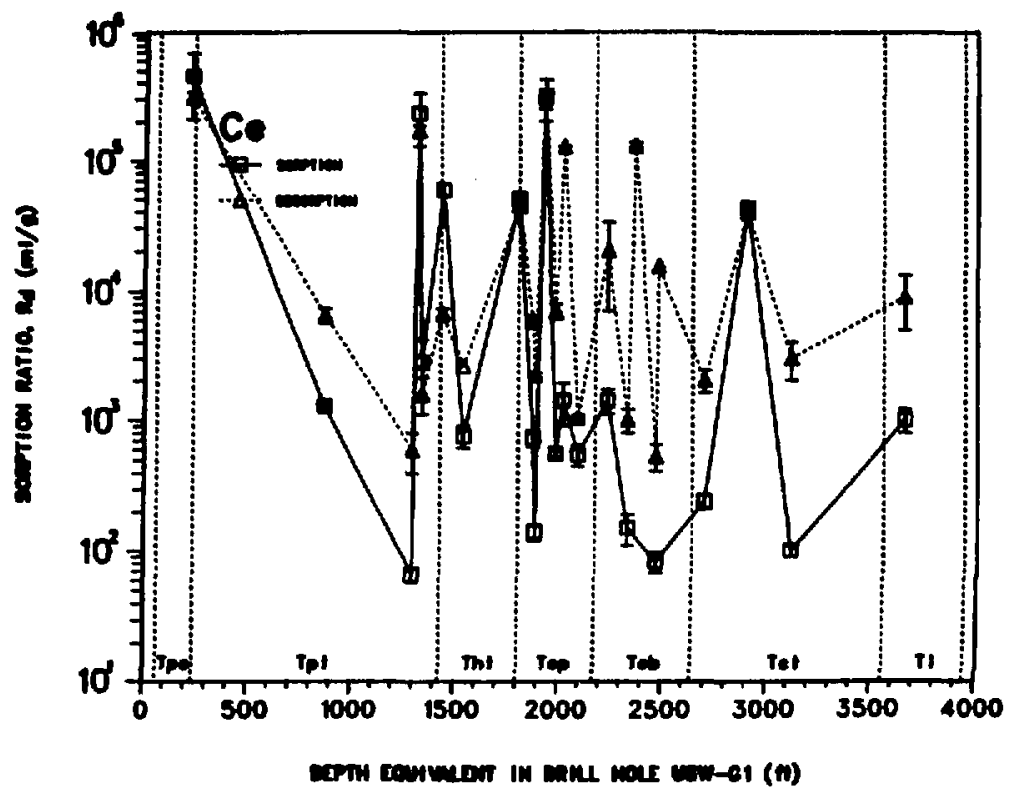

Fig. 40. Comparison of cerium sorption ratios for sorption and desorption as a function of stratigraphic position.

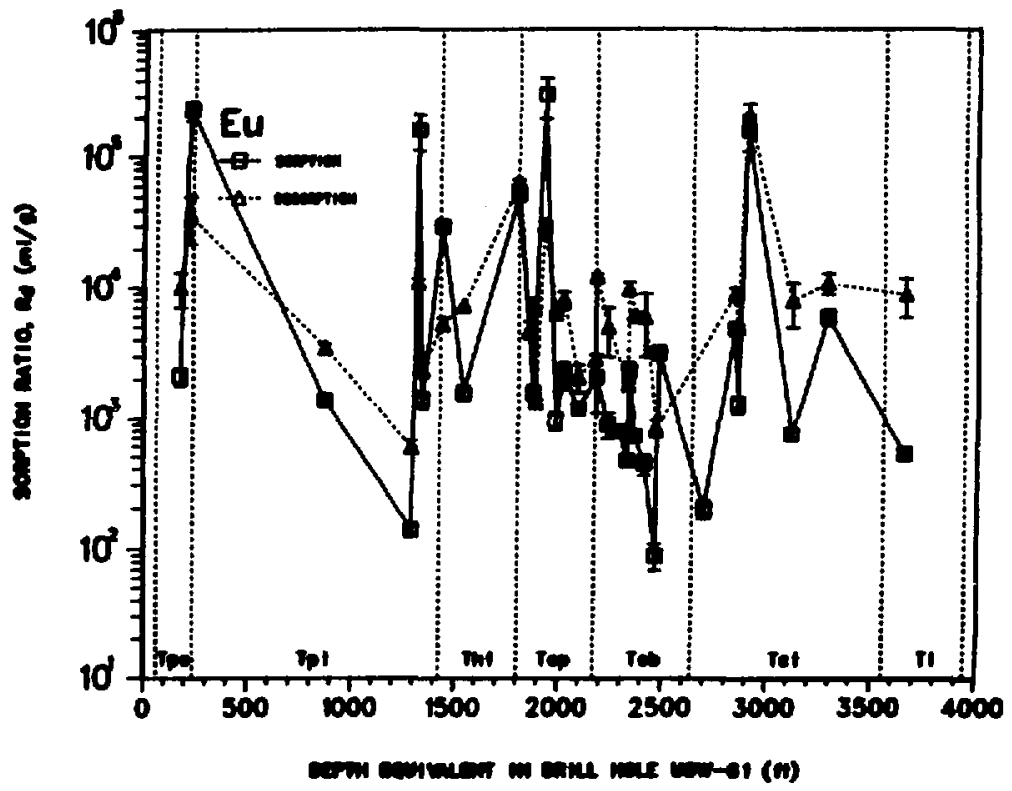

Fig. 41. Comparison of europium sorption ratios for sorption and desorption as a function of stratigraphic position. 


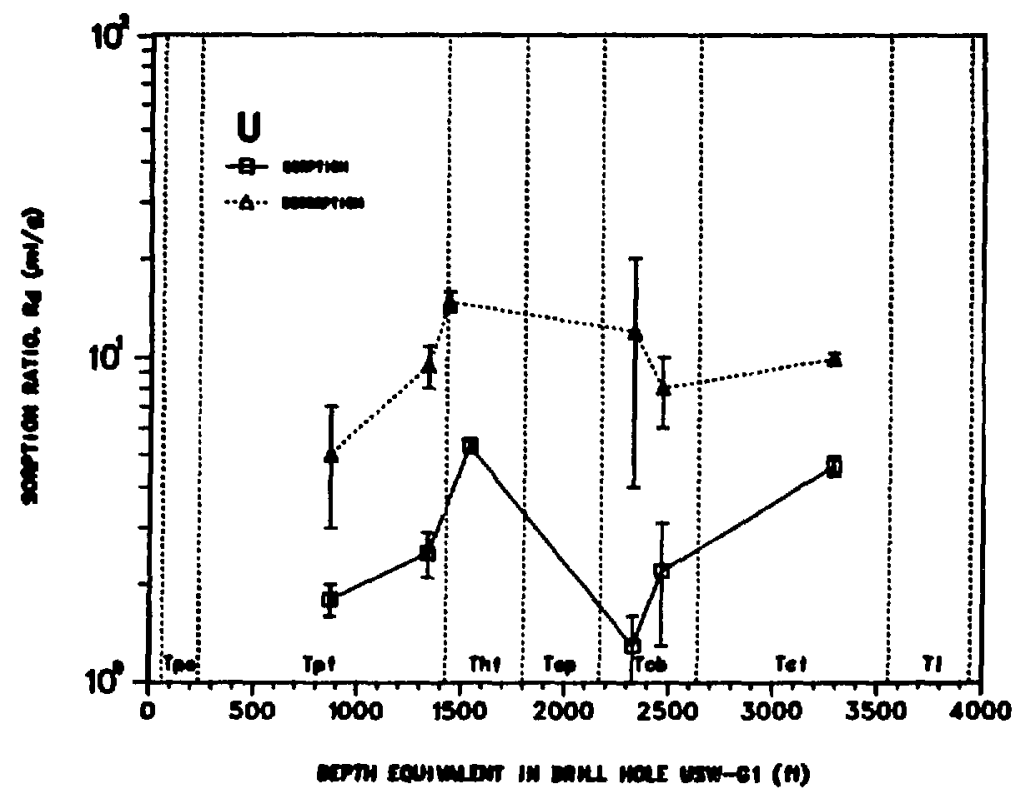

Fig. 42. Comparison of uranium sorption ratios for sorption and desorption as a function of stratigraphic position.

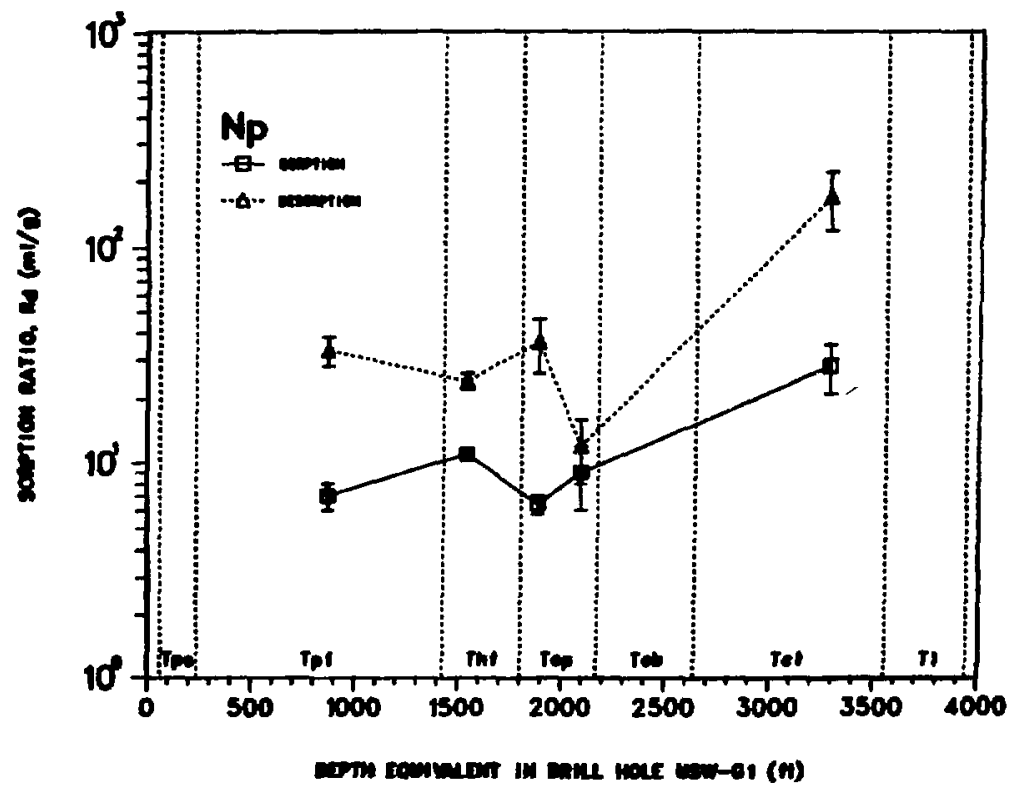

Fig. 43. Comparison of neptunium sorption ratios for sorption and desorptio as a function of stratigraphic position. 


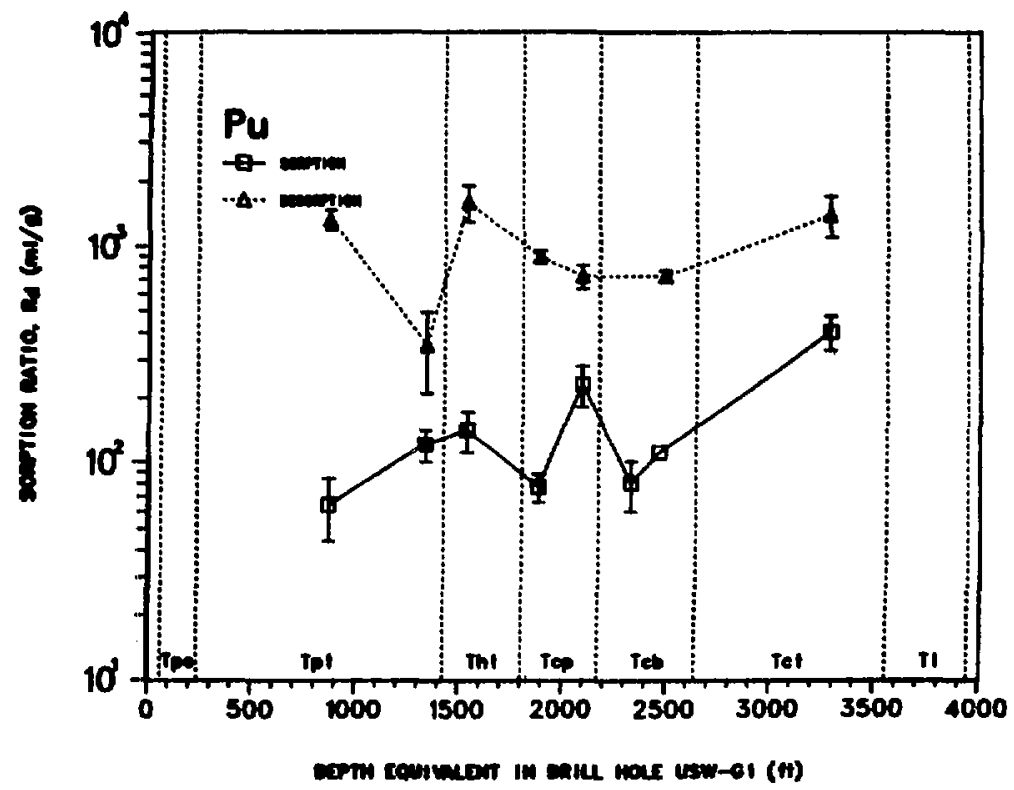

Fig. 44. Comparison of plutonium sorption ratios for sorption and desorption as a function of stratigraphic position.

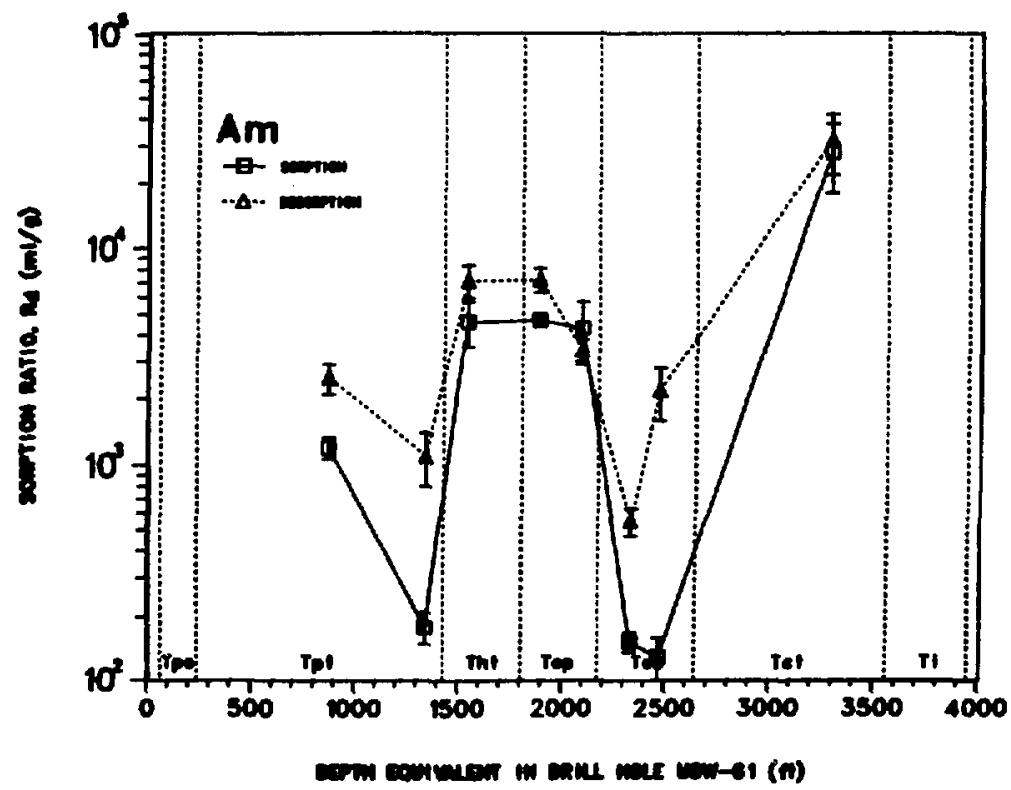

Fig. 45. Comparison of americiun sorption ratios for sorption and desorption as a function of stratigraphic position. 
factor of 10 . Sorption ratios for technetium, uranium, and neptunium, which are low, are nevertheless significantly greater for desorption than for sorption.

7. Sorption of Actinides and Technetium. The chemistry of these elements is complex in near-neutral groundwater (Secs. II and IV.A.11). Sorption ratios for plutonium cover a fairly narrow range (less than a factor of 10) that is independent of sample position or mineralogy. Based on the limited data available, neptunium exhibits similar behavior with a range of less than a factor of 5 , although the $R_{d}$ values are about an order of magnitude less than those for plutoniun. Americium sorption ratios show a much wider variation, from just over $100 \mathrm{ml} / \mathrm{g}$ to nearly $30000 \mathrm{ml} / \mathrm{g}$, but again with essentially no correlation to mineralogy. Sample JA-37 gave the highest $R_{d}$ values for all three actinides, which could be related to its relatively high clay content (Table XXIV). It is worth noting that this sample gave the most unusual results anong those whose sorptive behaviors were compared using both batch and circulating system techniques (Sec. IV.A.11). Obviously, additional sorption ratio measurements must be made for these elements, particularly for neptunium. Although sorption of technetium and uranium (Fig. 11-18) has not been measured for many samples, the sorption ratios are relatively low; correlations with stratigraphic position or mineralogy cannot be made.

It is interesting to compare the Los Alamos results for actinide sorption with those of Allard et al. ${ }^{54,55}$ who examined the sorption of americium and neptunium on common rock-forming minerals: quartz, biotite, bytownite, albite, microcline, olivine, kaolinite, hornblende, and augite. They used a synthetic groundwater and a somewhat different batch technique and observed a difference of about a factor of 10 between the low-sorbing quartz and high-sorbing biotite. The sorption ratios they report (for pure minerals) tend to be somewhat higher (factors of 10) than those reported here (for tuff) for neptunium, but they are quite similar for americium.

8. Effects of Elevated Temperature. The averages of results from three batch experiments performed at $70^{\circ} \mathrm{C}$ are given in Table XXVII. These values are compared with those for room temperature given in Tables XXI and XXII. The values are generally similar but higher than those for room temperature by factors up to 5. A limited number of experiments should be performed for other elements.

9. Effects of Particle Size on Sorptive Behavior. Sorption measurements were performed by the batch technique to determine whether the presence of 
TABLE XXVII

AVERAGE SORPTION AND DESORPTION RATIOS FOR PULVERIZED TUEF AT $70^{\circ} \mathrm{C}$

\begin{tabular}{|c|c|c|c|c|c|c|c|c|c|c|}
\hline \multirow[b]{2}{*}{ Sacple } & \multirow{2}{*}{$\begin{array}{l}\text { Depth } \\
\text { (ft) }\end{array}$} & \multirow{2}{*}{$\begin{array}{l}\text { Equiv. } \\
\text { USw-G1 } \\
\text { Depth }^{b} \\
\text { (ft) } \\
\end{array}$} & \multicolumn{8}{|c|}{$R_{d}(m / / 8)$} \\
\hline & & & Sr & $\mathrm{Cs}_{8}$ & $\mathbf{B a}$ & Ce & Eu & $A$ & Pu & $\mathbf{U}$ \\
\hline \multirow{3}{*}{$\begin{array}{l}J A-18 \\
J A-32 \\
J A-37\end{array}$} & & & & & Sorption & & & & & \\
\hline & $\begin{array}{l}1420 \\
2533 \\
3497\end{array}$ & $\begin{array}{l}1339 \\
2467 \\
3286\end{array}$ & $\begin{array}{c}18000(2000)^{c, d} \\
113(9) \\
1050(130)\end{array}$ & $\begin{array}{c}18000(1000) \\
97(6) \\
1360(85)\end{array}$ & $\begin{array}{c}49000(7000) \\
110(120) \\
3670(700)\end{array}$ & $80(20)$ & $\begin{array}{c}140(30) \\
4200(400)\end{array}$ & $\begin{array}{c}110(20) \\
1000(200)\end{array}$ & 240 & $\begin{array}{l}4.0(0.2) \\
11.7(1.7) \\
16(2)\end{array}$ \\
\hline & \multicolumn{10}{|c|}{ Desorption } \\
\hline $\begin{array}{l}J A-18 \\
J A-32 \\
J A-37\end{array}$ & $\begin{array}{l}1420 \\
2533 \\
3497\end{array}$ & $\begin{array}{l}1339 \\
2467 \\
3286\end{array}$ & $\begin{array}{c}21000(2000) \\
100(8) \\
1340(110)\end{array}$ & $\begin{array}{c}19300(1300) \\
108(4) \\
2700(500)\end{array}$ & $\begin{array}{c}108000(13000) \\
1160(100) \\
5900(900)\end{array}$ & $640(8)$ & $\begin{array}{c}1800(300) \\
14000(1000)\end{array}$ & & & $\begin{array}{l}14(3) \\
21.1(1) \\
47(6)\end{array}$ \\
\hline
\end{tabular}

\footnotetext{
Air; fractions do not contain $<75$ un-diam particles.

bepth equivalent in drill hole USW-Gl relative to position in geologic unit.

Conweighted average; values in parentheses are the absolute-value standard deviations of the neans.

dSoese data were rejected in averaging.
} 
very small $(<38-\mu \mathrm{m})$ particles in the samples would result in large differences in the ratios measured for tuff samples. This investigation was notivated by the observation that sorption ratios from crushed-rock column measurements are frequently 2 to 3 times lower than batch measurements for a $<500-\mu m$ fraction (Sec. IV.A.11). All material in the crushed-rock columns was $>35 \mu m$, the size of the end frits.

The experimental samples were the zeolitized tuffs YM-38, G1-2289, and G1-3658 and the devitrified tuffs YM-54, G1-1883, G1-1982, and G1-2363. For all samples except G1-1982, the fractions were <38, 38 to 106 , and 106 to $500 \mu \mathrm{m}$. For sample G1-1982 the fractions were $<38,38$ to 75,75 to 250 , and 250 to $500 \mu \mathrm{m}$. The larger fractions were wet-sieved to remove very fine particles. Rock samples were contacted with traced groundwater solutions at ambient temperature under atmospheric conditions for 3 weeks. (Contact periods for sample G1-1982 were both 2 and 3 weeks.)

Tables XXVIII and XXIX show the dependence of the sorption ratio on particle size. Samples YM-54, G1-1883, G1-1982, and G1-2363 (a11 of which are devitrified and do not contain zeolites) show higher sorption ratios (by factors of 2 to 5 for the <38- $\mu$ m fraction) for strontium, cesium, and barium. The $<38-\mu m$ fraction of the devitrified tuff samples contains a higher percentage of smectite clays than do the larger size fractions (Table XXIV). These clays may be responsible for the increased sorption by ion exchange of strontium, cesium, and barium.

For a different set of samples (Table XXIX), all of which are devitrified except G-1854 [zeolitized with some clinoptilolite (Table XXIV)], the <75- mm fraction gave $R_{d}$ values averaging a factor of only $\sim 1.4$ greater than those for the 75- to 500- $\mu \mathrm{m}$ fraction. Apparently, the large number of particles between 38 and $75 \mu \mathrm{m}$ in the $<75-\mu \mathrm{m}$ fraction significantly reduces the difference in sorption between the two fractions studied, and particles in the 38- to 75- $\mu \mathrm{m}$ range have sorption ratios similar to those in the 75- to 500- $\mu \mathrm{m}$ fraction for each tuff. Unfortunately, no data are available for direct comparison of $<38-\mu m$ and $<75-\mu m$ fractions of the same samples.

For the zeolitized tuff samples YM-38, G1-1854, G1-2289, and G1-3658, sorption ratios for strontium, cesium, and barium are very high. In general, the smaller fractions have $R_{d}$ values less than a factor of $\sim 2$ higlier than the coarser fractions. The increase in sorption ratios for the fine fractions of sample G1-2289, which exhibited the largest fractionation of minerals after sieving (Table XXIV), is no greater than would be expected from the increase in 
TABLE YXVIII

DEPENDENCE OF SORPTION RATIO ON PARTICLE SIZE

\begin{tabular}{|c|c|c|c|c|}
\hline \multirow[b]{2}{*}{ Sapple } & \multirow[b]{2}{*}{ Element } & \multicolumn{3}{|c|}{$R_{d}(n e / g)$} \\
\hline & & $<38 \mu$ & $38-106 \mu$ & $106-500 \mu$ \\
\hline $\mathrm{YH}-38$ & $\mathbf{S r}$ & $13900^{2}$ & $20300^{\circ}$ & $17600^{\circ}$ \\
\hline$"$ & " & $20500^{\circ}$ & $19600^{2}$ & $17600^{\circledR}$ \\
\hline$"$ & $"$ & $17000(3000)$ & $20000(400)$ & $17600(0)$ \\
\hline$"$ & CE & 11100 & 16600 & 14300 \\
\hline$"$ & $"$ & 19600 & 14900 & 14000 \\
\hline$"$ & $"$ & $15000(4000)$ & $16000(900)$ & $14200(200)$ \\
\hline$"$ & Ba & 69000 & 119000 & 56500 \\
\hline$n$ & $"$ & 187000 & 102000 & 103000 \\
\hline$"$ & $"$ & $130000(60000)$ & $110000(9000)$ & $80000(23000)$ \\
\hline " & Eu & 2250 & 1350 & 1330 \\
\hline " & $"$ & 2990 & 1340 & 1510 \\
\hline$"$ & $"$ & $2600(400)$ & $1340(10)$ & $1400(100)$ \\
\hline$M-54$ & $\mathbf{S r}$ & 277 & 56.8 & 37.1 \\
\hline$"$ & $"$ & 274 & 56.1 & 56.1 \\
\hline$"$ & $"$ & $276(2)$ & $56.5(0.4)$ & $47(10)$ \\
\hline$" 1$ & Cs & 937 & 188 & 114 \\
\hline$n$ & $"$ & 889 & 186 & 132 \\
\hline$"$ & 11 & $910(30)$ & $187(1)$ & $120(10)$ \\
\hline$"$ & $\mathrm{Ba}$ & 1720 & 476 & 134 \\
\hline$"$ & $"$ & 1610 & 471 & 148 \\
\hline$"$ & " & $1670(60)$ & $474(3)$ & $140(7)$ \\
\hline$"$ & Eu & 1610 & 255 & 489 \\
\hline$"$ & $"$ & 1590 & 420 & 444 \\
\hline$"$ & $"$ & $1600(10)$ & $340(80)$ & $470(20)$ \\
\hline G1-1883 & Sr & 26.2 & 22.2 & 22.4 \\
\hline$"$ & " & 80.2 & 21.7 & 21.8 \\
\hline$"$ & $"$ & $50(30)$ & $22(1)$ & $22(1)$ \\
\hline " & $\mathrm{Cs}$ & 306 & 198 & 186 \\
\hline$"$ & $"$ & 717 & 183 & 181 \\
\hline$"$ & $"$ & $500(200)$ & $190(10)$ & $184(3)$ \\
\hline
\end{tabular}


TABLE XXVIII (cont)

\begin{tabular}{|c|c|c|c|c|}
\hline \multirow[b]{2}{*}{ Sample } & \multirow[b]{2}{*}{ Elevent } & \multicolumn{3}{|c|}{$R_{d}(n / g)$} \\
\hline & & $\angle 36 \mu$ & $38-106 \mu$ & $106-500 \mu$ \\
\hline G1-1883 & $\mathbf{B a}$ & 234 & 208 & 161 \\
\hline$"$ & $"$ & 753 & 199 & 162 \\
\hline$"$ & $"$ & $500(300)$ & $204(5)$ & $162(1)$ \\
\hline 4 & Eu & 375 & 108 & 173 \\
\hline$"$ & $"$ & 653 & 119 & 153 \\
\hline$" 1$ & $"$ & $510(200)$ & $110(10)$ & $160(10)$ \\
\hline G1-2289 & $\mathbf{S r}$ & 16600 & 6340 & 7830 \\
\hline$"$ & " & 11700 & 6410 & 8450 \\
\hline$"$ & $"$ & $14000(3000)$ & $6380(40)$ & $8100(300)$ \\
\hline$"$ & $\mathrm{Cs}$ & 43400 & 33800 & 12100 \\
\hline$"$ & $"$ & 27100 & 29100 & 72100 \\
\hline$"$ & $"$ & $35000(8000)$ & $31000(3000)$ & $42000(30000)$ \\
\hline "t & $\mathbf{B a}$ & 173000 & 54000 & 90300 \\
\hline$"$ & $"$ & 114000 & 48500 & 69600 \\
\hline$"$ & $"$ & $140000(30000)$ & $51000(3000)$ & $80000(10000)$ \\
\hline$"$ & Eu & 1650 & 780 & 817 \\
\hline$"$ & $"$ & 1400 & 778 & 812 \\
\hline$"$ & $"$ & $1500(100)$ & $779(1)$ & $815(3)$ \\
\hline G1-2363 & $\mathbf{S r}$ & 179 & 73.9 & 58.2 \\
\hline$"$ & $"$ & 168 & 62.2 & 61.4 \\
\hline$"$ & $"$ & $170(10)$ & $70(10)$ & $60(2)$ \\
\hline$"$ & $\mathrm{Cs}$ & 1390 & 553 & 414 \\
\hline$"$ & $"$ & 1270 & 520 & 382 \\
\hline$"$ & $"$ & $1300(100)$ & $540(20)$ & $400(20)$ \\
\hline$"$ & Ba & 918 & 243 & 230 \\
\hline$"$ & $"$ & 865 & 255 & 212 \\
\hline$"$ & $"$ & $890(30)$ & $250(10)$ & $220(10)$ \\
\hline$"$ & Eu & 5650 & 778 & 780 \\
\hline$"$ & $"$ & 5440 & 794 & 578 \\
\hline$"$ & $"$ & $5500(100)$ & $786(8)$ & $680(100)$ \\
\hline
\end{tabular}


TABLE XXVIII (cont)

\begin{tabular}{|c|c|c|c|c|}
\hline \multirow[b]{2}{*}{ Sample } & \multirow[b]{2}{*}{ Element } & \multicolumn{3}{|c|}{$R_{d}(-2 / 8)$} \\
\hline & & 53810 & $38-106+4$ & $106-500 \mathrm{H}$ \\
\hline$G 1-3658$ & $S_{r}$ & 9830 & 11600 & 13100 \\
\hline$"$ & $"$ & 15700 & 13500 & 13400 \\
\hline$"$ & $"$ & $13000(3000)$ & $13000(1000)$ & $13200(200)$ \\
\hline$"$ & Cs & 13900 & 6140 & 4910 \\
\hline$"$ & $"$ & 18500 & 7080 & 5040 \\
\hline$"$ & $"$ & $16000(2000)$ & $6600(500)$ & $4980(70)$ \\
\hline$"$ & Ba & 11700 & 9020 & 12700 \\
\hline$"$ & $"$ & 18600 & 10200 & 14200 \\
\hline$"$ & $"$ & $15000(4000)$ & $9600(600)$ & $13000(1000)$ \\
\hline$"$ & $\mathbf{E u}$ & 14600 & 414 & 488 \\
\hline$"$ & $"$ & 15700 & 477 & 566 \\
\hline$"$ & $"$ & $15000(600)$ & $440(30)$ & $530(40)$ \\
\hline
\end{tabular}

\begin{tabular}{|c|c|c|c|c|c|}
\hline & & & & (8) & \\
\hline & & $\angle 38 \quad 1$ & $38-75+$ & $75-250 \quad 1$ & $250-500 \mu$ \\
\hline GI-1982 ${ }^{b}$ & Sx & 1200 & 59 & 49 & 51 \\
\hline$"$ & $"$ & 1200 & 66 & 53 & 66 \\
\hline$"$ & $"$ & $1200(0)$ & $63(4)$ & $51(2)$ & $59(7)$ \\
\hline$"$ & Cs & 3800 & 1200 & 960 & 1200 \\
\hline$"$ & $"$ & 3500 & 1300 & 1100 & 1200 \\
\hline$"$ & $"$ & $3650(200)$ & $1250(50)$ & $1000(70)$ & $1200(0)$ \\
\hline$"$ & Be & 10000 & 670 & 568 & 693 \\
\hline$"$ & $"$ & 10000 & 844 & 780 & 780 \\
\hline$"$ & $"$ & $10000(0)$ & $760(90)$ & $670(100)$ & $740(40)$ \\
\hline$"$ & $\mathbf{E u}$ & 1200 & 535 & 614 & 864 \\
\hline$"$ & $"$ & 2500 & B85 & 1300 & 1100 \\
\hline$"$ & $"$ & $1900(700)$ & $710(180)$ & $960(300)$ & $980(120)$ \\
\hline
\end{tabular}

\footnotetext{
aor all samples except G1-1982, the first two lines for each element give duplicate neasurements for 3-week contact periods. The third line sives the sverage of the duplicate messurements. Values in parentheses are atendard deviations of the mean. bFor sample 61-1982, the first two lines give seasurements for 2- and 3-week contect periods, respectively; the third line gives the average of the two. Values in parentheses are the atandard devistions of the rean.
} 
TABLE XXIX

DEPENDENCE OF SORPTION RATIO ON PARTICLE SIZE ${ }^{a}$

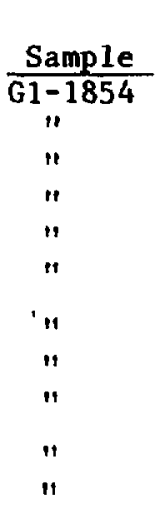

\begin{tabular}{c} 
Element \\
\hline "r \\
$" 1$ \\
$" 1$ \\
$" 1$
\end{tabular}

$R_{d}(m l / 8)$

G1-2333

11

$"$

"1

It

"I

11

11

n

II

II

11

$\mathrm{G1}_{\text {II }}-2410$

"

"

"

11

II

II

"I

"

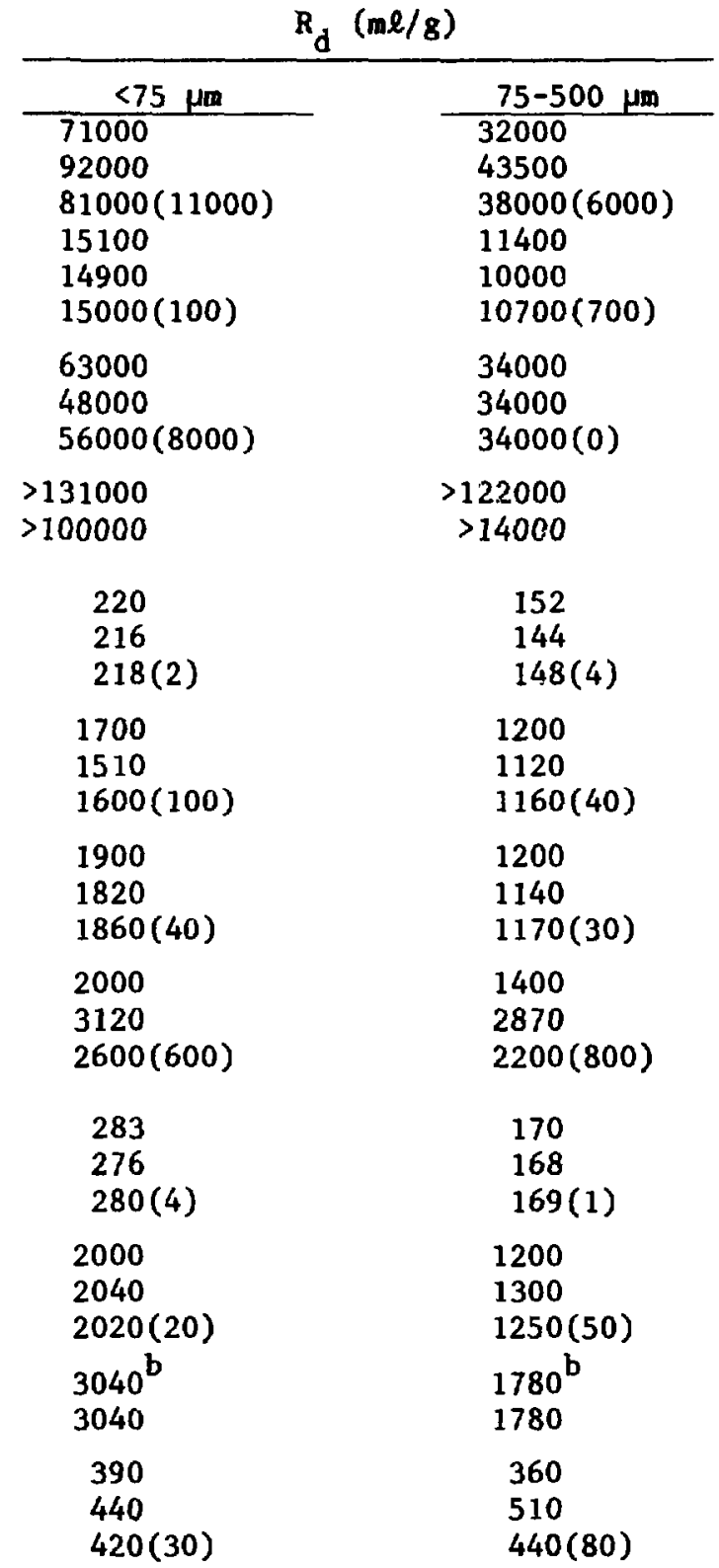


TABLE XXIX (cont)

\begin{tabular}{|c|c|c|c|}
\hline & & & \\
\hline Sample & Element & $<75 \mu \mathrm{m}$ & $75-500 \mu \mathrm{m}$ \\
\hline$\overline{G 1-2476}$ & $\mathbf{s r}$ & 49 & 41 \\
\hline " & $"$ & & 40 \\
\hline$"$ & $"$ & $50(1)$ & $41(1)$ \\
\hline$"$ & Cs & 815 & 660 \\
\hline$"$ & $"$ & 919 & 741 \\
\hline$"$ & $"$ & $870(50)$ & $700(40)$ \\
\hline$"$ & $\mathbf{B a}$ & 480 & 374 \\
\hline$"$ & $"$ & 518 & 396 \\
\hline$"$ & $"$ & $500(20)$ & $385(10)$ \\
\hline$"$ & Eu & 4600 & 3300 \\
\hline$"$ & $"$ & 5080 & 3110 \\
\hline$"$ & $"$ & $4800(300)$ & $3200(100)$ \\
\hline G1-2840 & Sr & 170 & 160 \\
\hline$"$ & $"$ & 171 & 159 \\
\hline$"$ & $"$ & $170(1)$ & $160(1)$ \\
\hline$"$ & Cs & 2800 & 2400 \\
\hline$"$ & $"$ & 2480 & 2020 \\
\hline " & $"$ & $2600(200)$ & $2200(200)$ \\
\hline$"$ & $\mathbf{B a}$ & 2300 & 2000 \\
\hline$"$ & $"$ & 2620 & 2140 \\
\hline$"$ & $"$ & $2500(200)$ & $2070(70)$ \\
\hline$"$ & Eu & 5000 & 4500 \\
\hline$"$ & $"$ & 6200 & 5330 \\
\hline$"$ & $"$ & $5600(600)$ & $4900(400)$ \\
\hline G1-2854 & $\mathbf{S r}$ & 120 & 94 \\
\hline " & $"$ & 59 & 93 \\
\hline$"$ & $"$ & $90(30)$ & $94(1)$ \\
\hline$"$ & Cs & 1700 & 1200 \\
\hline$"$ & $"$ & 510 & 952 \\
\hline$"$ & $"$ & $1100(600)$ & $1080(120)$ \\
\hline$"$ & $\mathbf{B a}$ & 1600 & 950 \\
\hline$"$ & $"$ & 6510 & 1040 \\
\hline$"$ & $"$ & $4000(2000)$ & $1000(50)$ \\
\hline$"$ & Eu & 1100 & 1100 \\
\hline$"$ & $"$ & 2560 & 1530 \\
\hline$"$ & $"$ & $1800(800)$ & $1300(200)$ \\
\hline
\end{tabular}

\footnotetext{
The first two lines are measureaents for 2- and 3 -week contact periods, respectively; the third line gives the average of the two. Values in parentheses are the standard deviations of the means.

bonly one weasurement available.
} 
zeolite content (Sec. IV.A). Sample G1-3658 exhibited similar variations without a corresponding variation in mineral abundances.

Sorption ratios are higher for europium for the $<38-\mu m$ fractions of both zeolitized and devitrified tuffs, which may be a result of sorption on the increased surface areas by processes other than ion exchange.

Preliminary results from whole-core column sifudies indicate that there is better agreement with the results of batch experiments when batch work is performed with samples from which the very fine particles have been removed, However, greater variation should be expected in whole-core samples because of the heterogeneity of tuff; as more whole-core experiments are completed, samples having $R_{d}$ values greater than those from the batch experiments may be found. Perhaps the fine clays are not generally available to fluids for sorption in real situations or they have been enriched in the samples studied. Of course, there is better agreement between batch results from sorption on larger particles and crushed-rock column measurements because the fine particles were deliberately removed for the crushed-rock column work.

The presence of very fine particles in larger fractions apparently can change the observed sorption ratio of an element by a factor of 2 to 5 , especially in the case of devitrified tuffs. Therefore, it is advisable to wetsieve larger fractions to avoid the presence of fine particles that might increase the observed sorption in an irreproducible manner. The removal of small particles may result in measurements on material that is not completely representative of the tuff; however, the results are useful for comparative purposes and are probably not far from the "true" values. Any errors should be in the conservative direction, that is, too low $R_{d}$ values for samples that do not contain very fine particles.

10. Comparison of Batch Studies Made Under Atmospheric and ControlledAtmosphere Conditions. Because reducing conditions are expected for some groundwater/rock systems, the sorptive behavior of some elements in such systems may be different from that under normal atmospheric conditions. Differences in the sorptive capacity of a rock type could be expected if the rock's surface were altered by exposure to air. These effects were investigated by comparing the results of batch studies performed on the same geologic materials in a nitrogen atmosphere ( $\leq 0.2 \mathrm{ppm}$ oxygen and $\leq 20 \mathrm{ppm}$ carbon dioxide) with similar measurements made under normal atmospheric conditions. However, the controlled- 
atmosphere studies were not truly representative of the conditions to be found in deep geologic systems because very little carbon dioxide was present.

The $\mathrm{pH}$ values of the groundwaters after the experiments in the controlled atmosphere were $\sim 0.5$ unit higher than for similar experiments in air. This might be a consequence of some loss of carbon dioxide (and total carbonate) from solution and might affect sorption of $U(V I)$, which is strongly complexed by carbonate. The changes in $\mathrm{pH}$ may decrease significantly the solubility of some multivalent ions and also might result in changes in ionic charge, degree of hydration, etc.

For most experiments, the radionuclides were ${ }^{85} \mathrm{Sr},{ }^{137} \mathrm{Cs},{ }^{133} \mathrm{Ba},{ }^{141} \mathrm{Ce}$, ${ }^{152} \mathrm{Eu},{ }^{95} \mathrm{Tc}^{\mathrm{m}},{ }^{237} \mathrm{U},{ }^{237} \mathrm{Pu},{ }^{241} \mathrm{Am}$, and ${ }^{235} \mathrm{~Np}$; the rock samples were from tuff cores $M-22, M-38$, and $M-54$. Experiments were also performed with ${ }^{22} \mathrm{Na}$, ${ }^{54} \mathrm{Mn},{ }^{75} \mathrm{Se}$, and ${ }^{113} \mathrm{Sn}$ and tuff core $\mathrm{G1-2233}$. The groundwaters used for the determinations in air and nitrogen atmospheres had the same initial composition. Fractions of $<75$ and 75 to $500 \mu \mathrm{m}$ were used for most studies under both conditions; fractions of $<106$ and 106 to $500 \mu \mathrm{m}$ were used in some experiments under atmospheric conditions.

The $\mathbf{R}_{\mathbf{d}}$ values from both sorption and desorption studies, given in Table XXX, are the averages of measurements for 3-, 6-, and 12-week contact periods. The effects of atmosphere on the sorptive behavior of the 14 elements studied are summarized in Table XXXI and are discussed below.

Strontium, cesium, and barium $R_{d}$ values are essentially the same for atmospheric and controlled-atmosphere conditions. The sorptive behavior of tuff for these elements appears to be independent of the atmosphere involved.

Cerium and europium $R_{d}$ values are similar for the two different atmospheres. The sorption of cerium and europium may be strongly dependent on the formation of insoluble forms of these elements, such as precipitates or colloids.

Americiun has lower $R_{d}$ values for both sorption and desorption in the YM-22 (devitrified) tuff under the controlled atmosphere. For samples M-54 (devitrified) and $M M-38$ (zeolitized), americium $R_{d}$ values for both sorption and desorption are either the sane or slightly greater under the controlled atwosphere. Anericium has exhibited a tendency toward large variations in behavior when experimental conditions are changed, which may be the result of speciation effects. 
TABLE XXX

SORPTION RATIOS $(\mathrm{m} / / \mathrm{g})$ FOR ATMOSPHERIC AND CONTROLLED-ATMOSPHERE CONDITIONS

\begin{tabular}{|c|c|c|c|c|c|}
\hline \multirow[b]{2}{*}{ Sample } & \multirow[b]{2}{*}{ Element } & \multicolumn{2}{|c|}{ Sorption } & \multicolumn{2}{|c|}{ Desorption } \\
\hline & & Atmospheric & $C A^{a}$ & Atmospheric & $C A^{a}$ \\
\hline YM-22 & Sr & $56(4)^{b}$ & $63(6)^{b}$ & $63(4)^{b}$ & $104(12)^{b}$ \\
\hline$Y M-38$ & $"$ & $11900(3200)$ & $10600(2600)$ & 21700 & $17000(2300)$ \\
\hline$Y M-54$ & $"$ & $90(4)$ & $110(13)$ & $94(9)$ & $126(7)$ \\
\hline YM-22 & Cs & $340(60)$ & $330(50)$ & $400(30)$ & $420(60)$ \\
\hline YM-38 & $"$ & $8600(1700)$ & $9300(1200)$ & 13000 & $12000(1600)$ \\
\hline$Y M-54$ & $"$ & $250(20)$ & $300(30)$ & $310(20)$ & $360(50)$ \\
\hline YM-22 & $\mathrm{Ba}$ & $980(80)$ & $550(130)$ & $1000(210)$ & $830(210)$ \\
\hline YM-38 & $"$ & $66000(13000)$ & $>62000$ & 260000 & $64000(9000)$ \\
\hline$Y M-54$ & $"$ & $620(80)$ & $560(70)$ & $660(20)$ & $600(40)$ \\
\hline YM-22 & $\mathrm{Ce}$ & $1300(100)$ & $920(170)$ & $6100(700)$ & $2300(100)$ \\
\hline YM-38 & $"$ & $820(100)$ & $570(90)$ & 2640 & $7000(5000)$ \\
\hline YM-54 & $"$ & $140(40)$ & $520(140)$ & $1000(200)$ & $1500(400)$ \\
\hline$Y M-22$ & Eu & $1400(100)$ & $970(110)$ & 3600 & $2400(300)$ \\
\hline $\mathrm{YM}-38$ & $"$ & $3000(1000)$ & $850(110)$ & 7300 & $7000(5000)$ \\
\hline YM-54 & $"$ & $510(80)$ & $900(200)$ & $1800(100)$ & $2000(200)$ \\
\hline G1-2233 & $\mathrm{Na}$ & $141(4)$ & $150(2)$ & $160(10)$ & $150(3)$ \\
\hline$"$ & Mn & $6000(400)$ & $1500(900)$ & $>9300$ & $2300(800)$ \\
\hline$"$ & Se & $11(2)$ & $14(0)$ & $46(5)$ & $99(31)$ \\
\hline$"$ & Sn & $460(130)$ & $210(2)$ & $580(70)$ & $740(240)$ \\
\hline$M M-22$ & Am & $4000(1200)$ & $1 \div 00(200)$ & $4700(1000)$ & $3700(800)$ \\
\hline$M-38$ & $"$ & $5500(1000)$ & $5600(1000)$ & $9500(1300)$ & $14000(2000)$ \\
\hline YM-54 & $"$ & $590(210)$ & $1000(400)$ & $600(50)$ & $2600(400)$ \\
\hline
\end{tabular}


TABLE $\mathrm{XXX}$ (cont)

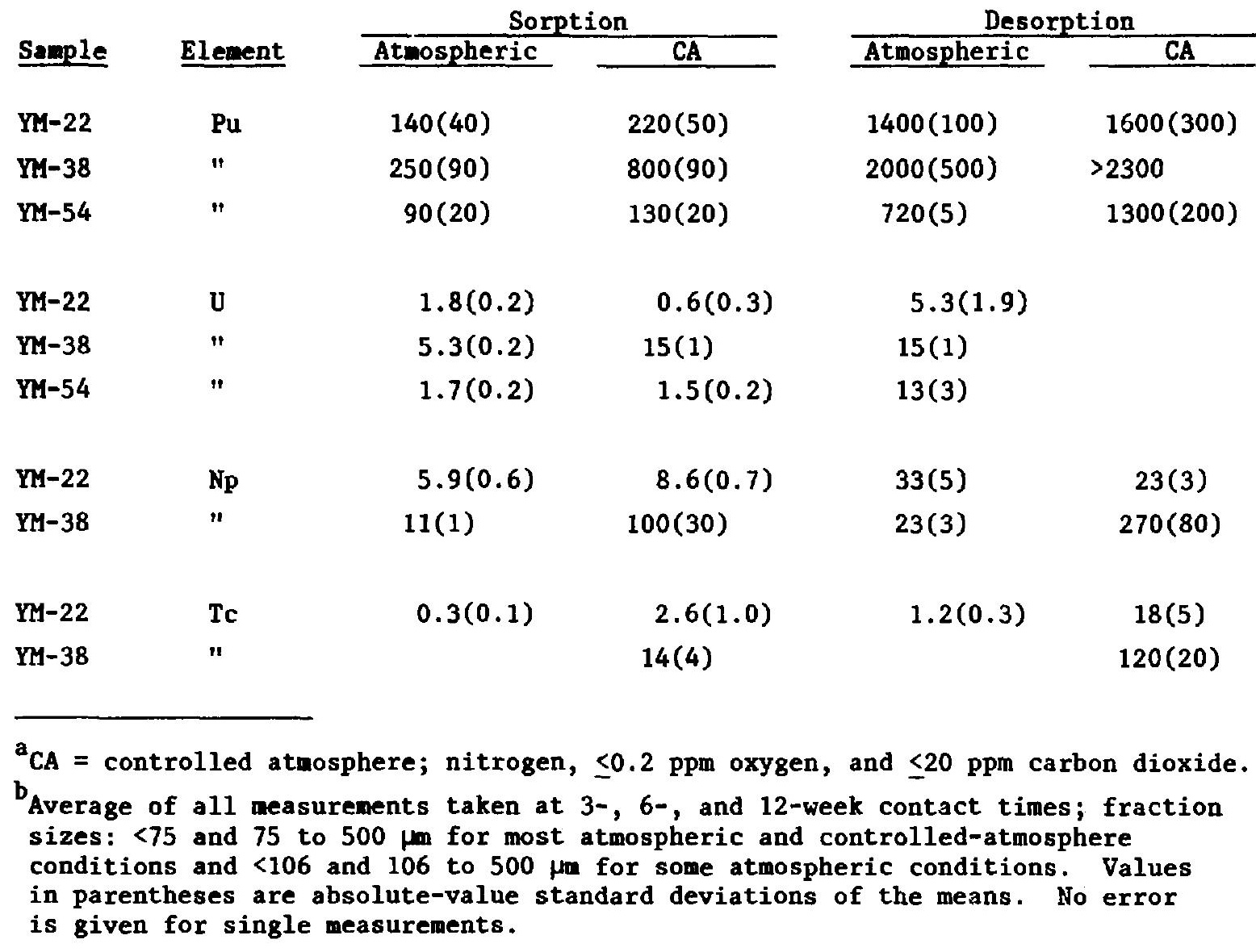

Plutonium $\mathbf{R}_{\mathrm{d}}$ values for both sorption and desorption for the zeolitized tuff YM-38 are significantly higher under controlled-atmosphere conditions than in air. This is perhaps consistent with the tendency of plutonium to exhibit variations in behavior when experimental conditions are changed, presumably the result of speciation effects (Sec. II). Technetium sorbs relatively more strongly in a controlled atmosphere where conditions are presumably more reducing. The sorption ratios on zeolitic tuffs are about 15 times larger under controlled-atmosphere conditions than in air. The values for devitrified tuffs are 10 to 25 times larger under the controlled-atmosphere conditions.

Uraniue sorption ratios under controlled-atmosphere conditions are similar to those in air for MM-22 and MM-54 samples (devitrified) but somewhat 
TABLE XXXI

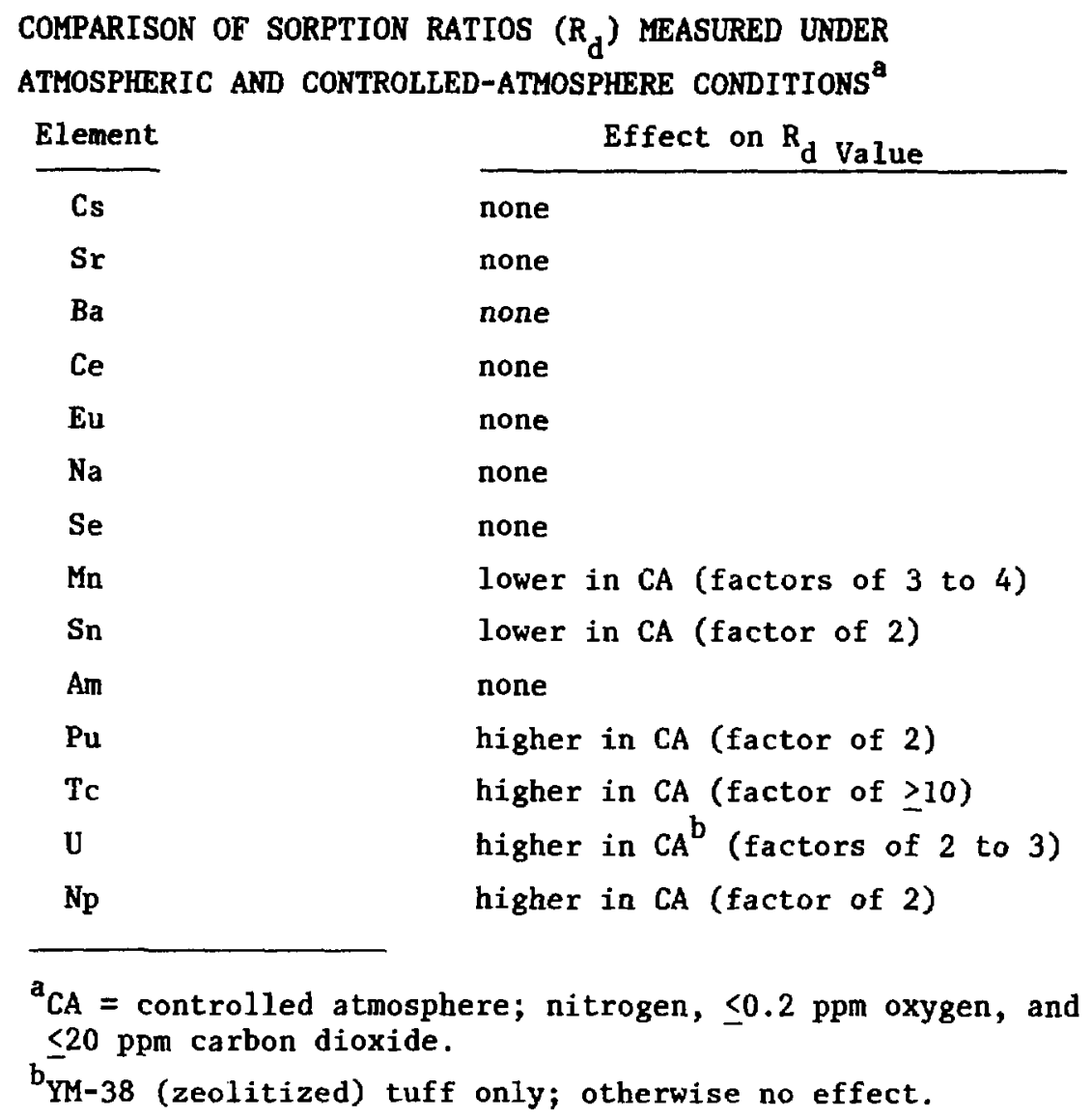

higher for YM-38 samples (zeolitized). The U(VI) apparently remains strongly complexed by carbonate in the groundwater even though the total carbonate concentration is reduced in the controlled atmosphere. Neptunium sorption and desorption ratios are higher in the controlledatmosphere than in air, especially for the zeolitized tuff. There is perhaps a change in the oxidation state of neptunium in the controlled atmosphere, which could favor increased sorption.

Sodium and selenium show no observable difference in $R_{d}$ values for sorption and desorption when measured in air or in the controlled atmosphere. Manganese has lower $\mathrm{R}_{\mathrm{d}}$ values for both sorption and desorption when measured in the controlled atmosphere; tin values for sorption are also lower in the controlled atmosphere, but values for desorption are about the same. It seems that there is less formation of insoluble 


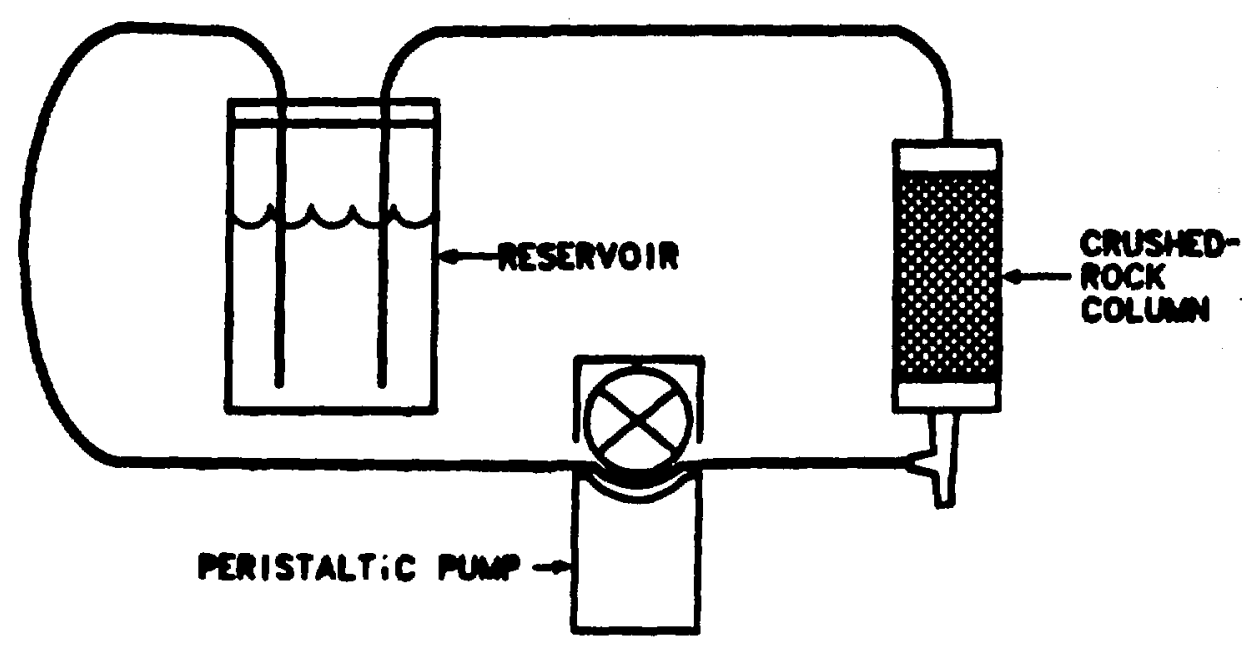

Fig. 46. Circulating system for crushed-rock system studies.

oxidized compounds of tin and manganese in the controlled atmosphere, resulting in lower sorption ratios.

If reducing conditions exist in any tuff/groundwater system, the effect would probably be enhanced sorption or precipitation for a number of elements. Furthermore, if $\mathrm{Fe}(\mathrm{II})$ is present in solution under reducing conditions, the precipitation of ferric hydroxide from such groundwaters under oxidizing conditions should result in scavenging other waste elements from solution.

11. Comparison of Sorption Ratios Measured by Batch and Circulating-System Methods. The circulating system (Ref. 3 and Fig. 46) is a hybrid that incorporates features of both batch and column methods. The batch and circulatingsystem procedures are similar in some ways, but the solid phase remains stationary in the circulating system and is not subject to the possible selfgrinding of the batch measurements. The presence of smaller particles could result in greater sorption as a result of greater surface area or differences in mineralogy.

Sorption ratios for strontium, cesium, and barium were determined using one feed solution for the circulating system and another for the batch measurements. The actinide series of comparisons was made using the same feed solution for both circulating-system and batch measurements for each actinide. Although the feed solutions for both the batch and circulating systens were prepared 
just before the start of batch sorptions, there was a delay of from 6 to 16 days before the feed solution could be introduced in the circulating system. The traced feed solutions were made in the standard manner by drying the tracer (Sec. IV.A.2). The concentrations of tracer and element added were approximately $5 \times 10^{-9} \mathrm{M}$ for cesium, $5 \times 10^{-8} \mathrm{M}$ for barium, and $5 \times 10^{-7} \mathrm{M}$ for strontium. Solutions traced with americium or plutonium varied with each preparation. The concentrations of plutonium were $3.1 \times 10^{-12} \mathrm{M}$ for experiments with YM-49 and $4.1 \times 10^{-12} \mathrm{M}$ for $\mathrm{JA}-37$ and G1-1883. Americium concentrations in feed solutions were $1.2 \times 10^{-7} \mathrm{M}$ for $\mathrm{YM}-49,1.3 \times 10^{-7} \mathrm{M}$ for $\mathrm{JA}-37$, and $1.2 \times 10^{-7} \mathrm{M}$ for G1-1883. Desorption experiments were performed with the circulating-system columns from the actinide measurements with fresh, untraced groundwater that had been pretreated with the appropriate tuff.

Table XXXII shows the results of individual sorption measurements for strontium, cesium, and barium that were taken using circulating systems. Results of batch sorption measurements for these same elements are summarized in Table XXI. Pertinent data from the batch and circulating-system sorption and desorption measurements are presented in Tables XXXIII and XXXIV.

The actinide $R_{d}$ values for desorption measurements with the circulating system are higher than for sorption, just as they are for the batch technique. This apparent irreversibility was discussed earlier (Sec. IV.A.6).

The average $R_{d}$ values for sorption by the two methods are given in Table XXXV, and the ratios of these results are given in Table XXXVI. Considering the spread of experimental values, the agreement between the two methods is good. In most cases, the results fall within the spread of individual experiment values (see the tables in this section and in App. A). The errors given in Table XXXVI arise from propagating the standard deviation of the mean, which is discussed in Sec. IV.A.2 above. The barium sorption ratio obtained for sample YM-22 by the column method ${ }^{38}(137 \mathrm{ml} / \mathrm{g})$ is in much better agreement with the results from the circulating-system method than is the batch data.

The devitrified tuffs tended to give slightly higher sorption ratios by the batch method than by the circulating-system method. The observed difference could well be the result of the presence of smaller particles arising from self-grinding in the batch measurements. Similar particle-size effects have been observed in other experiments (Sec. IV.A.9). The results are quite similar for the simple cations, which presumably sorb by ion exchange, and the 
TABLE XXXII

STRONTIUM, CESIUM, AND BARIUM SORPTION RATIOS FROM CIRCULATING-SYSTEM SORPTION MEASUREMENTS

\begin{tabular}{|c|c|c|c|c|}
\hline \multirow[b]{2}{*}{ Core } & \multirow{2}{*}{$\begin{array}{l}\text { Contact Time } \\
\text { (days) }\end{array}$} & \multicolumn{3}{|c|}{$R_{d}(m l / g)$} \\
\hline & & $\mathrm{Sr}$ & $\mathrm{Cs}$ & $\mathrm{Ba}$ \\
\hline \multirow[t]{5}{*}{ YNJ-22 } & 26 & $9^{a}$ & $99^{a}$ & $33^{a}$ \\
\hline & 49 & 29 & 405 & 129 \\
\hline & 63 & 28 & 441 & 129 \\
\hline & 84 & 30 & 616 & 133 \\
\hline & 112 & $\underline{21}$ & 494 & 102 \\
\hline Average & & $27(2)^{b}$ & $490(50)^{b}$ & $120(10)^{b}$ \\
\hline \multirow[t]{5}{*}{ YM-54 } & 26 & 39 & 105 & 111 \\
\hline & 49 & 53 & 158 & 153 \\
\hline & 63 & 49 & 131 & 146 \\
\hline & 84 & 44 & 112 & 132 \\
\hline & 112 & 41 & 99 & $\underline{116}$ \\
\hline Average & & $45(3)$ & $120(10)$ & $130(10)$ \\
\hline \multirow[t]{5}{*}{$J A-37$} & 26 & 401 & 1770 & 948 \\
\hline & 49 & 390 & 1890 & 819 \\
\hline & 63 & 398 & 1800 & 891 \\
\hline & 84 & 420 & 1920 & 729 \\
\hline & 112 & $\underline{365}$ & 1480 & 899 \\
\hline Average & & $390(10)$ & $1800(80)$ & $860(40)$ \\
\hline
\end{tabular}

\footnotetext{
${ }^{a}$ Value not included in average.

${ }^{b}$ values in parentheses are the absolute-value standard deviations of the means.
}

actinides, which probably sorb by a more complex process. The $R_{d}$ value ratios for zeolitized tuffs, which in general have higher $R_{d}$ values than the devitrified tuffs, scatter considerably and show no consistent pattern; the differences may be the result of experimental uncertainties. Results from other experiments (Sec. IV.A.9) indicate a much smaller effect for zeolitized tuffs than for devitrified tuffs as a result of small particles present in the samples. 
TABLE XXXIII

AMERICIUM SORPTION RATIOS FROM

BATCH AND CIRCULATING-SYSTEM MEASUREMENTS

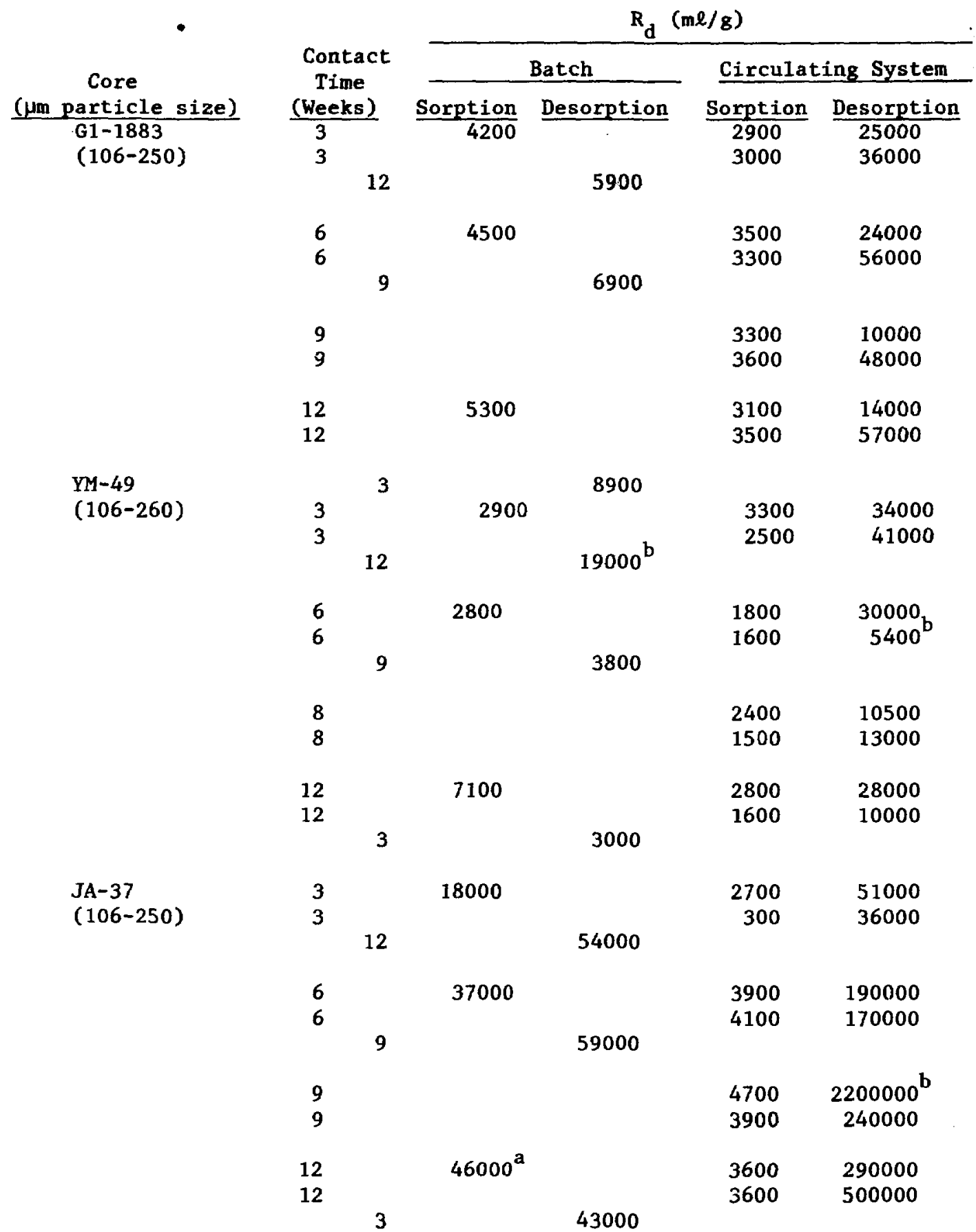

\footnotetext{
${ }^{a}$ Value not included in calculation. Value is from filtered sample; unfiltered sample analogous to those for all other data from these batch measurements was unavailable. $\mathrm{b}$ alue not included in calculations.
} 
TABIE XXXIV

PLUTONIUM SORPTION RATIOS FROM

BATCH AND CIRCULATING-SYSTEM MEASUREMENTS

\begin{tabular}{|c|c|c|c|c|c|}
\hline \multirow{3}{*}{$\begin{array}{c}\text { Core } \\
\text { (Hm particle size) }\end{array}$} & \multirow{3}{*}{$\begin{array}{c}\begin{array}{c}\text { Contact } \\
\text { Time } \\
\text { (Weeks) }\end{array} \\
\end{array}$} & \multicolumn{4}{|c|}{$R_{d}(m \ell / 8)$} \\
\hline & & \multicolumn{2}{|r|}{ Batch } & \multicolumn{2}{|c|}{ Circulating System } \\
\hline & & Sorption & Desorption & Sorption & Desorption \\
\hline \multirow[t]{5}{*}{$\begin{array}{l}G 1-1883 \\
(106-250)\end{array}$} & $\begin{array}{l}3 \\
4\end{array}$ & 51 & & 46 & \\
\hline & $\begin{array}{r}6 \\
12\end{array}$ & & 830 & & 465 \\
\hline & $\begin{array}{l}6 \\
6\end{array}$ & $\begin{array}{l}52^{\mathrm{a}} \\
91\end{array}$ & & 67 & \\
\hline & $\begin{array}{l}8 \\
8 \\
9\end{array}$ & & $\begin{array}{c}740^{\circ} \\
1100\end{array}$ & & 700 \\
\hline & $\begin{array}{l}13 \\
13\end{array}$ & $\begin{array}{c}82^{\mathrm{a}} \\
107\end{array}$ & $\begin{array}{l}960^{a} \\
850\end{array}$ & & \\
\hline
\end{tabular}

YYM-49

(106-250)

$\begin{array}{ll}3 & 140 \\ 4 & \end{array}$

12

$390^{\mathrm{a}}$

410

$6 \quad 160$

9

$200^{a}$

9

410

740

12

12

210

$820^{\mathrm{a}}$

3

660

3

$930^{a}$

JA-37

3

300

260

(106-250)

12

870

6

$420^{a}$

560

$\begin{array}{rr}9 & 890^{\circ} \\ 9 & 1700\end{array}$

12

$760^{a}$

1900

$\begin{array}{ll}3 & 1300^{\star} \\ 3 & 2400\end{array}$

aretreatment of crushed rock was for 4.5 months rather than the noral 2 weeks. 
TABLE XXXV

AVERAGE SORPTION RATIOS FROM BATCH AND

CIRCULATING-SYSTEM SORPTION MEASUREMENTS

\begin{tabular}{|c|c|c|c|}
\hline \multirow[b]{2}{*}{ Element } & \multirow[b]{2}{*}{ Tuff Core } & \multicolumn{2}{|c|}{$R_{d}(m \ell / g)$} \\
\hline & & Batch $^{\mathbf{a}}$ & Circulating System \\
\hline$s r^{a}$ & $\begin{array}{l}Y M-22 \\
Y M-54 \\
J A-37\end{array}$ & $\begin{array}{r}53(3)^{b} \\
62(12) \\
287(14)\end{array}$ & $\begin{array}{c}27(2)^{b} \\
45(3) \\
390(10)\end{array}$ \\
\hline $\mathrm{cs}^{\mathrm{a}}$ & $\begin{array}{l}\text { YM-22 } \\
\text { YM-54 } \\
\text { JA-37 }\end{array}$ & $\begin{array}{l}290(30) \\
180(40) \\
610(40)\end{array}$ & $\begin{array}{r}490(50) \\
120(10) \\
1800(80)\end{array}$ \\
\hline $\mathrm{Ba}^{\mathrm{a}}$ & $\begin{array}{l}\mathrm{YM}-22 \\
\mathrm{YM}-54 \\
\mathrm{JA}-37\end{array}$ & $\begin{array}{l}900(30) \\
400(150) \\
760(150)\end{array}$ & $\begin{array}{l}120(10) \\
130(10) \\
860(40)\end{array}$ \\
\hline Am & $\begin{array}{l}Y M-49 \\
\text { JA-37 } \\
\text { G1-1883 }\end{array}$ & $\begin{array}{c}4300(1400) \\
28000(10000) \\
4700(300)\end{array}$ & $\begin{array}{l}2200(300) \\
3400(600) \\
3300(100)\end{array}$ \\
\hline $\mathrm{Pu}$ & $\begin{array}{l}Y M-49 \\
J A-37 \\
G 1-1883\end{array}$ & $\begin{array}{r}230(50) \\
400(70) \\
77(11)\end{array}$ & $\begin{array}{c}570(170) \\
290(20) \\
56(11)\end{array}$ \\
\hline
\end{tabular}

TABLE XXXVI

COMPARISON OF AVERAGE SORPTION RATIOS FROM BATCH AND CIRCULATING-SYSTEM SORPTION MEASUREMENTS

Batch $\mathbf{R}_{\mathrm{d}}$-to-Circulating System $\mathbf{R}_{\mathrm{d}}$ Ratio

Zeolitized Tuffs

Batch $\mathbf{R}_{\mathrm{d}}$-to-Circulating System $\mathbf{R}_{\mathrm{d}}$ Ratio
$\mathrm{Sr}-\mathrm{Cs}-\mathrm{Ba}-\mathrm{Am}-\mathrm{Pu}$

$\mathrm{JA}-37^{\mathrm{a}}$

$0.74(0.04)^{\mathrm{b}} \quad 0.34(0.03) \quad 0.88(0.18)$

YM-49

Devitrified Tuffs

YM-22

YM-54

G1-1883
$2.0(0.2)$

$1.3(0.2)$

$$
0.59(0.09)
$$$$
1.3(0.3)
$$

$8.2(3.3)$

$2.0(0.7)$

$1.4(0.3)$

$0.40(0.15)$

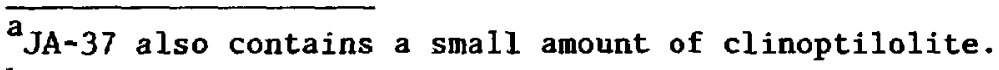

$\mathrm{b}_{\mathrm{Values}}$ in parentheses are the errors propagated from errors given in Table XXXV. 
The very large differences between $R_{d}$ values measured by the two methods for sorption of barium on tuff $\mathrm{YM}-22$ and americium on tuff JA-37 are currently unexplained. They do, however, indicate the complexity of sorption processes and sorption measurements and, further, the need for additional study. The americium result for sample JA-37 may be related to the relatively high concentration of montmorillonite in this tuff (Table XXIV). The presence of a large number of highly sorptive fine clay particles in the batch samples could possibly lead to the high $R_{d}$ value. Early attempts to measure the sorption ratio for americium on tuff JA-37 by using the original inadequate phaseseparation technique (Sec. II) resulted in much lower values. This might also be attributable to the presence of bighly sorptive, fine clay particles that might be particularly difficult to remove from the aqueous phase (thereby resulting in a low $R_{d}$ value). The situation is not clear because the plutonium results do not show the same behavior; there is much better agreement between circulating-system and batch measurements and between old and new separation techniques. However, the behavior of americium has consistently been more of a problem than that of plutonium. The extremely high $R_{d}$ values for americium desorption that were obtained in the circulating-system measurement (Table XXXIII) may in some way be related.

The possibility of transport on small particles may have a bearing on the results of the circulating systen measurements and is discussed in Sec. II.B.

12. Crushed-Rock Column Studies. A complete report on these studies is available $e^{38}$ and is only summarized here. Elutions of radionuclides from columns of crushed tuff, granite, and argillite have been used as a simple first step in trying to relate laboratory batch-type measurements to a flowing system. ${ }^{2,3,38}$ Although primarily tuffs were studied, granite and argillite were also included to obtain a more general data base. Because radionuclides are often sorbed quite strongly by these rock types, small columns $(\leq 0.5 \mathrm{~cm}$ in diameter by 2 to $5 \mathrm{~cm}$ long) were used to minimize the duration of an experiment. However, the elutions of nuclides from some of the columns still required 2 to 3 years. The columns were loaded with $\sim 5$ - to 10- $\mu \ell$ spikes of groundwater containing one or more radioisotopes. Groundwater was pushed upward through the colunn by syringe pumps at flow rates of 11 to 77 a/year, although faster flow rates were used in a few cases. The velocity of the radionuclide was measured directly and then compared to the groundwater velocity (measured using HTO or ${ }^{131}$ I, which does not sorb) to calculate the retardation factor 
$R_{f}$. In simple ion-exchange theory, the $R_{f}$ is related to the sorption ratio $R_{d}$ (or more precisely, the distribution coefficient $K_{d}$ ) by the expression

$$
R_{f}=1+(\rho / \varepsilon) R_{d},
$$

where $p$ is the density of the rock column and $\varepsilon$ is the porosity.

Several general observations can be made from the data of approximately 40 columns. ${ }^{38}$ (1) The sorption ratios of strontiun, cesium, and barium, measured with the colums, generally fall within the range of measured batch $R_{d}$ values when the batch measurements are made on fractions washed free of fine $(\Sigma 35-\mu m)$ particles. Previous reports ${ }^{2,3}$ indicated that $R_{d}$ values inferred from the column studies were 1 to 5 times smaller than from batch study $R_{d}$ values; however, these earlier comparisons were made with batch measurements using material containing fine particles, and they should not be considered valid. (2) Elution of strontium from a tuff from a vitrophyre was unusual, giving a broad, asymetric peak; strontium peaks were generally narrow and symetric. (3) Broad, asymmetric peaks were typical of cesiun elutions. In addition, cesium frequently seemed to be eluted either in two broad, partially overlapping peaks or in one major peak with a distinct shoulder. On granite columns, cesium seemed to be fixed at the load end, possibly as a result of irreversible sorption on biotite; such irreversible sorption was not observed on any of the tuffs.

In addition to ${ }^{85} \mathrm{Sr},{ }^{137} \mathrm{Cs}$, and ${ }^{133} \mathrm{Ba}$ behavior, that of the radionuclides ${ }^{95} \mathrm{Tc}^{\mathrm{m}},{ }^{152} \mathrm{Eu}$, and ${ }^{141} \mathrm{Ce}$ has been studied. Cerium was loaded on two columns but decayed before eluting. The ${ }^{95} \mathrm{Tc}^{\mathrm{m}_{0}} \mathrm{O}_{4}^{-}$was strongly affected by kinetics. This is not surprising because the retardation mechanism of technetium is probably by reduction of $\mathrm{TcO}_{4}^{-}$to $\mathrm{Tc}(\mathrm{IV})$, perhaps as $\mathrm{TcO}_{2}$, rather than by ion exchange. At flow rates of $\sim 2000 \mathrm{~m} /$ year, either in air or in a controlled atmosphere of nitrogen with $\leq 0.2 \mathrm{ppm} \mathrm{O}_{2}$ and $\leq 20 \mathrm{ppm} \mathrm{CO}_{2}$, argillite-column sorption ratios were 0.29 to $0.43 \mathrm{ml} / \mathrm{g}$. The corresponding batch sorption ratio in air was 18 to $222 \mathrm{ml} / \mathrm{g}$. When the flow rate was slowed to $20 \mathrm{~m} / \mathrm{year}$, allowing more time for reaction, the $R_{d}$ value for technetium increased to $72 \mathrm{ml} / \mathrm{g}$.

Five columns were loaded with ${ }^{152} \mathrm{Eu}$; for three of the columns, the measured batch sorption ratios fell between the $R_{d}$ values corresponding to a small amount of ${ }^{152} \mathrm{Eu}$, which was eluted initially, and the majority of the ${ }^{152} \mathrm{Eu}$, which remained on the columns. (The columns were sectioned, and the distribution of activity along each column was measured to estimate an $R_{d}$ value.) It 
is not clear whether the europium that eluted initially from the coluns was the result of colloidal species. Of the last two colunns loaded with ${ }^{152}$ Eu, one was contaminated with iron and the other gave a sorption ratio that overlapped with the large range of batch sorption ratios. Conclusions can not be drawn for ${ }^{152}$ Eu until additional measurements are made--ideally using a continuous feed "loading" technique, rather than a spike. The use of spikes can lead to problens because of the isotherm effects discussed in the next section.

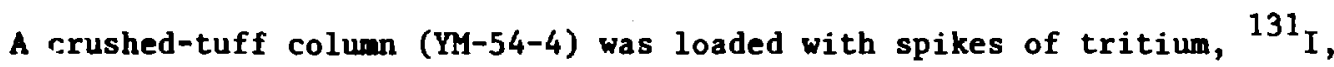
and ${ }^{237} \mathrm{U}$. Groundwater elution curves result in an $R_{d}$ value of $0.72 \mathrm{~m} \ell / \mathrm{g}$ for uranium, whereas the value obtained by batch measurements is $1.5 \mathrm{~m} \ell / \mathrm{g}$. The uranium peak was quite asywetric, and the activity per milliliter slowly decreased; by drop 23, when most of the iodine had been eluted, only $50 \%$ of the uranium had been removed from the column. The marked asymetry may be an effect of the complicated sorption illustrated by the large difference between sorption $(1.5 \mathrm{al} / \mathrm{g})$ and desorption $(11 \mathrm{ml} / \mathrm{g}) \mathrm{R}_{\mathrm{d}}$ values when measured by the usual batch method.

The general agreement between column and batch ieasurements for ${ }^{85} \mathrm{Sr}$, ${ }^{137} \mathrm{Cs}$, and ${ }^{133} \mathrm{Ba}$ on washed samples is encouraging, because the results of batch measurements are often used to show relative sorption under a variety of conditions, and their relevance to the migration of radionuclides under flowing conditions has been questioned. Whether sample-crushing influences the rock chemistry in both the batch and crushed-rock columns will be determined by studying radionuclide migration through colunns of intact rock, larger blocks of intact rock, and in the field.

13. Sorption Isotherms. The study of sorption isotherms is used to

(a) determine the influence of groundwater/tuff interactions on the sorptive properties of tuff,

(b) accurately model the retardation of waste elements under various source-tere and groundwater conditions,

(c) detect irreversible sorption processes that could be very positive properties if discovered in tuff,

(d) interpret and model diffusion into the tuff matrix as it would occur in fracture flow, and

(e) explain the observed dependence of the distribution coefficient on the solution-to-solid ratio and predict real conditions from laboratory measurenents. 
In most transport codes, sorption is normally treated as a linear isotherm; that is, the distribution coefficient $K_{d}$ does not depend on the cation concentration. This standard treatment should be valid for ion-exchange equilibrium at tracer-level concentrations with zeolitic tuffs; however, welded, nonzeolitized horizons have been proposed as repository hosts because of their thermomechanical properties.

The Langmuir isotherm is also commonly used. It is appropriate to any sorption mechanism that is first order and has a limited number of sorption sites. The form of the Langauir isotherm is

$$
q=\frac{Q K c}{c_{0}+(K-1) c} \text {, }
$$

where

$$
\begin{aligned}
& q=\text { the solute concentration in the solid phase, } \\
& c=\text { the solute concentration in the aqueous phase, } \\
& c_{0}=\text { the total cation concentration, } \\
& Q=\text { the CEC, and } \\
& K=\text { the equilibrium constant. }
\end{aligned}
$$

At small solute concentration, $\mathrm{Kc} \ll<$, the Langmuir isotherm becomes linear. The Freundlich isotherm is also commonly used; it is a purely empirical formula that can be used to summarize a large amount of data. The form of the Freundlich isotherm is

$$
\mathrm{q}=\mathrm{kc}^{\mathrm{n}},
$$

where $k$ and $n$ are constants. Unfortunately, this isotherm is unbounded and does not account for saturation of sites; in addition, it can yield infinite $k_{d}$ values at zero concentration.

Another approach, which seems most appropriate from a chemical standpoint, is the so-called mass-action equilibrium. This approach seems particularly appropriate for cations that have a charge greater than 1 . This approach for a given equilibrium takes the form

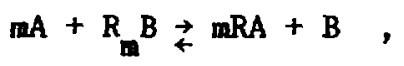


and the equilibrium relation is

$$
K_{A B}=\left(\frac{q_{A}}{c_{A}}\right)^{\prime} \frac{c_{B}}{q_{B}},
$$

where $m$ ' lies between $m$ and unity. This approach is more general than the Langmuir isotherm and still has the advantage of incorporating a limited number of sorption sites.

In addition to these sorption isotherms, which imply equilibrium, there is the possibility of an irreversible sorption process. In this case, a certain amount of the waste element would be sorbed independently of the initial concentration.

One of the important tasks of these sorption studies is to identify which of these isotherms is most appropriate to describe sorption on tuff. In addition, it may be possible to extract thermodynamic parameters such as equilibriun constants, thus providing a data base for correlating sorption ratios with mineralogy and groundwater composition.

a. Experimental results. Isotherms have been determined for three tuff samples from the UE25a-1 drill hole: YM-22, YM-38, and YM-49. Sample YM-22 is a densely welded tuff from the Topopah Spring Member, sample YM-38 is a highly zeolitized tuff from the bedded tuff of Calico Hills, and sample YM-49 is a partially zeolitized and partially welded tuff from the lower Prow Pass Member. Sorption isotherms for strontium, cesium, barium, and europium were determined for samples YM-22 and YM-38. A brief summary of the published report $^{3}$ is given here.

Measurements of strontium, cesium, barium, and europium sorption ratios were made at 4 to 5 different aqueous concentrations; however, there were not enough measurements to make a statistically meaningful distinction among the isotheras mentioned above. The data were all fit to a Freundlich isotherm; the results, shown in Table XXXVII, indicate nonlinear behavior for the welded tuff YM-22 and, with the exception of strontium, linear behavior for the zeolitized tuff $\mathrm{YM}-38$. However, there was a great deal of scatter in the results from sample $M M-3$, as has often been observed for samples with high $K_{d}$ values. This is to be expected because a zeolitized naterial should sorb by a predominantly ion-exchange mechanism and the high CEC should preclude a Langairtype site saturation. 
TABLE XXXVII

FREUNDLICH ISOTHERM PARAMETERS ${ }^{a}$

Fraction

Size

\begin{tabular}{|c|c|c|c|c|}
\hline Tuff & $(\mu \mathrm{m})$ & Element & $\mathbf{n}$ & $-\log k$ \\
\hline \multirow[t]{8}{*}{ YM-22 } & $<75$ & Sr & 0.83 & 2.23 \\
\hline & & Cs & 0.80 & 2.17 \\
\hline & & $\mathrm{Ba}$ & 0.83 & 1.34 \\
\hline & & Eu & 1.1 & -1.20 \\
\hline & $75-500$ & $\mathrm{Sr}$ & 0.71 & 2.85 \\
\hline & & Cs & 0.79 & 2.34 \\
\hline & & $\mathbf{B a}$ & 0.82 & 1.57 \\
\hline & & $\mathbf{E u}$ & 0.92 & 0.46 \\
\hline \multirow[t]{8}{*}{ YM-38 } & $<75$ & $\mathrm{Sr}$ & 0.85 & 0.38 \\
\hline & & Cs & 1.0 & -0.66 \\
\hline & & $\mathrm{Ba}$ & 1.0 & -2.41 \\
\hline & & Eu & 1.1 & -1.0 \\
\hline & $75-500$ & Sr & 0.87 & 0.41 \\
\hline & & Cs & 1.0 & -0.65 \\
\hline & & $\mathrm{Ba}$ & 1.1 & -2.50 \\
\hline & & Eu & 0.98 & -0.29 \\
\hline
\end{tabular}

${ }^{\mathrm{a}}$ From Eq. (8).

Sorption ratios were determined for plutonium on YM-22 and YM-49 tuffs for 10 concentrations from $2.9 \times 10^{-8}$ to $5.9 \times 10^{-13} \mathrm{M}$ (Tables XXXVIII and $\mathrm{XXXIX).}$

Table XXXVIII lists final results from batch desorption measurements of the dependence of the plutonius sorption ratio on element concentration. The desorption contacts were carried out for 3 weeks (equal to the sorption time).

The average $R_{\mathrm{d}}$ values for the desorptions are $780 \mathrm{ml} / \mathrm{g}$ for the $\mathrm{YM}-22$ tuff and $650 \mathrm{ml} / \mathrm{g}$ for the YM-49 tuff, with standard deviations of the mean of 110 and $48 \mathrm{ml} / \mathrm{g}$, respectively. The desorption $R_{d}$ values are higher than the sorption $R_{d}$ values by factors of 2 to 4 rather than 10 , as was found in earlier work. 3 
TABLE XXXVIII

DEPENDENCE OF PLUTONIUM SORPTION RATIO ON CONCENTRATION Initial Plutonium Concentration ${ }^{a}$ (M)

$\frac{\text { Tuff }}{\text { YMI-22 }}$

$\begin{array}{ll}\frac{\text { Added }}{1.2 \times 10^{-11}} & \frac{8.0 \times 10^{-12}}{8.0 \times 1} \\ 5.6 \times 10^{-11} & 3.0 \times 10^{-11} \\ 4.4 \times 10^{-10} & 2.1 \times 10^{-10} \\ 3.9 \times 10^{-9} & 1.8 \times 10^{-9} \\ 3.9 \times 10^{-8} & 1.0 \times 10^{-8}\end{array}$

YM-49

$1.2 \times 10^{-11}$

$$
1.6 \times 10^{-12}
$$

$5.6 \times 10^{-11}$

$7.7 \times 10^{-12}$

$4.4 \times 10^{-10}$

$1.3 \times 10^{-10}$

$3.9 \times 10^{-9}$

$4.3 \times 10^{-10}$

$5.4 \times 10^{-8}$

$2.9 \times 10^{-8}$

Initial Plutonium Concentration ${ }^{a}$ (M)

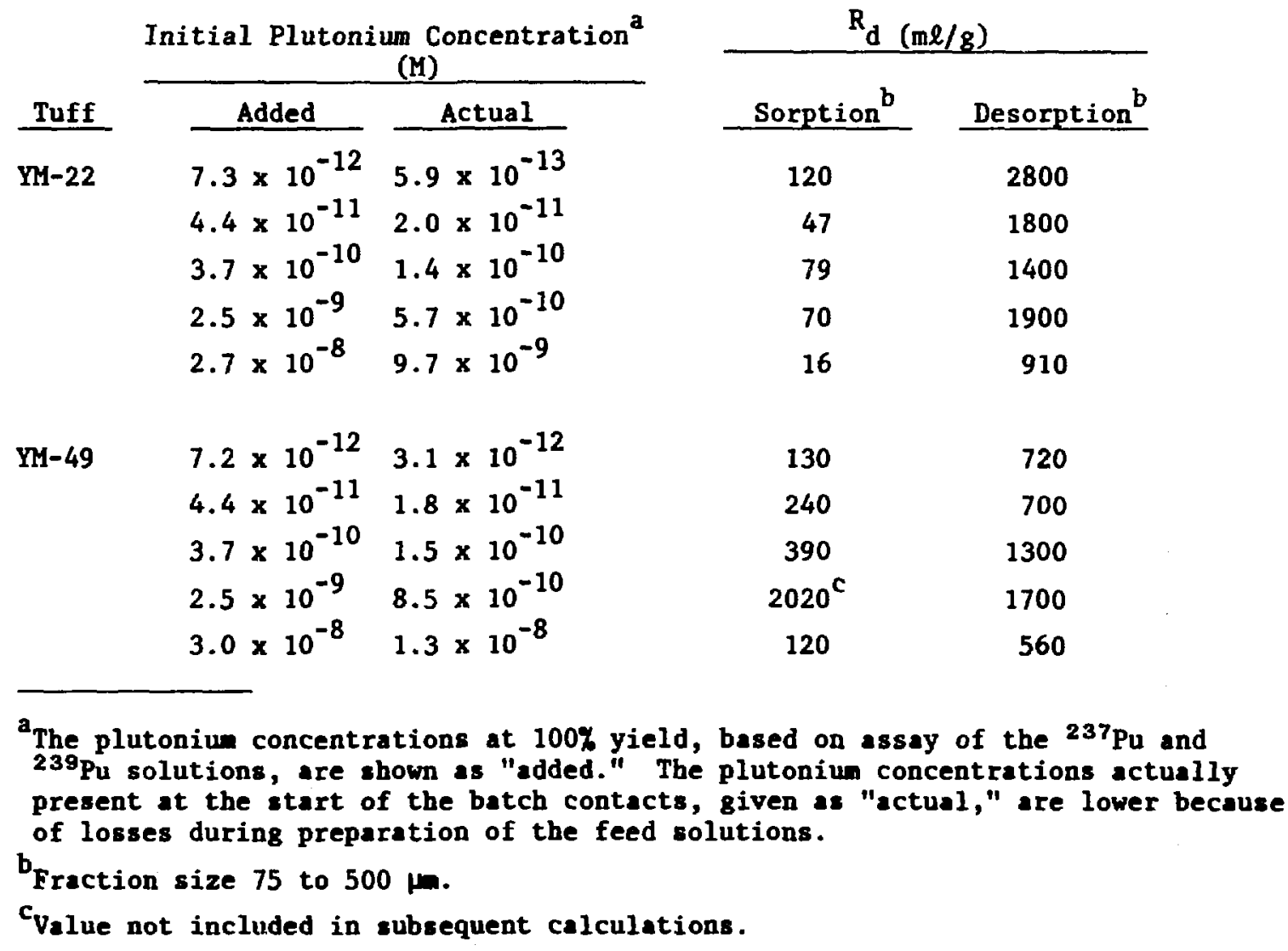

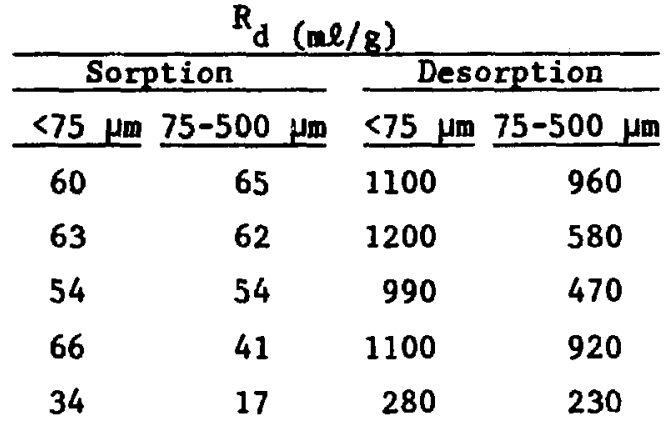

$R_{d}(m \ell / g)$

170

150

700

670

160

89

840

790

130

140

780

590

180

220

490

620

270

240

690

340 
TABLE XXXIX

SUMMARY OF DEPENDENCE OF THE PLUTONIUM SORPTION

RATIO ON CONCENTRATION

\begin{tabular}{|c|c|c|c|}
\hline \multirow[b]{2}{*}{ Tuff } & \multirow{2}{*}{$\begin{array}{c}\text { Initial Plutonium } \\
\text { Concentration } \\
\text { (M) }\end{array}$} & \multicolumn{2}{|c|}{$R_{d}(m \ell / g)$} \\
\hline & & Sorption ${ }^{a}$ & Desorption $^{a}$ \\
\hline \multirow[t]{10}{*}{$Y M-22$} & $5.9 \times 10^{-13}$ & 120 & 2800 \\
\hline & $8.0 \times 10^{-12}$ & 65 & 960 \\
\hline & $2.0 \times 10^{-11}$ & 47 & 1800 \\
\hline & $3.0 \times 10^{-11}$ & 62 & 580 \\
\hline & $1.4 \times 10^{-10}$ & 79 & 1400 \\
\hline & $2.1 \times 10^{-10}$ & 54 & 470 \\
\hline & $5.7 \times 10^{-10}$ & 70 & 1900 \\
\hline & $1.8 \times 10^{-9}$ & 41 & 920 \\
\hline & $9.7 \times 10^{-9}$ & 16 & 910 \\
\hline & $1.0 \times 10^{-8}$ & 34 & 230 \\
\hline \multirow[t]{10}{*}{$Y M-49$} & $1.6 \times 10^{-12}$ & 150 & 670 \\
\hline & $3.1 \times 10^{-12}$ & 130 & 720 \\
\hline & $7.7 \times 10^{-12}$ & 160 & 790 \\
\hline & $1.8 \times 10^{-11}$ & 240 & 700 \\
\hline & $1.3 \times 10^{-10}$ & 140 & 590 \\
\hline & $1.5 \times 10^{-10}$ & 390 & 1300 \\
\hline & $4.3 \times 10^{-10}$ & 220 & 620 \\
\hline & $8.5 \times 10^{-10}$ & $2020^{b}$ & 1700 \\
\hline & $1.3 \times 10^{-8}$ & 120 & 560 \\
\hline & $2.9 \times 10^{-8}$ & 240 & 340 \\
\hline
\end{tabular}

It is possible that the difference may be the result of the shorter total contact time for sorption plus desorption that was used for these isotherm studies (that is, 6 compared to 15 weeks). Except for the highest plutonium concentration, which exhibits the lowest $R_{d}$ value in three of the five groups, there 
appears to be little correlation between sorption ratio and element concentration in the range studied. There is some tendency toward lower $R_{d}$ values with the larger particle size, especially for sample YM-22. When the Freundlich equation is applied to the desorption data, the parameters given in Table XL are obtained.

The Freundlich isotherm parameters for tuff YM-22 appear to be significantly less than 1, whereas the parameters for tuff YM-49 are approximately linear. This is consistent with the results of the isotherm measurements using strontium, cesium, barium, and europium. Because the scatter in the plutonium data is large, it would not be possible to select between isotherms on the basis of these data.

b. Dependence of the Distribution Coefficient on the Freundlich Isotherm. The effects of nonlinear isotherms on sorption phenomena have also been studied, and equations and computer programs to solve the diffusion equations with nonlinear isotherms have been developed. In the course of this activity, some simple relations have been derived that can explain the dependence of the distribution coefficient $K_{d}$ on the solution-to-solid ratio. Experimental results in which the solution-to-solid ratio was varied can be directly compared with the results of measurements in which the element concentration was varied (isotherm determinations).

The Freundlich isotherm can be expressed as

$$
\frac{\mathrm{x}}{\mathrm{m}}=\mathbf{k} \mathbf{c}^{\mathrm{n}},
$$

where

$$
\begin{aligned}
& x=\text { the number of moles of tracer in the solid, } \\
& m=\text { the mass of the solid, } \\
& c=\text { the final concentration of tracer in solution, and } \\
& k \text { and } n=\text { constants. }
\end{aligned}
$$

Combining

$$
k_{d}=\frac{x / m}{c}=k c^{n-1}
$$


- TABLE XL

FREUNDLICH ISOTHERM PARAMETERS ${ }^{a}$ FOR SORPTION

AND DESORPTION OF PLUTONIUM

\section{$\underline{\text { Tuff }}$}

YM-22

YM-49

${ }^{\text {From Eq. } 8 .}$

${ }^{b}$ Coefficient of determination $r^{2}$.
Sorption

$\underline{\mathrm{k}} \quad \underline{\text { Fit }^{\mathbf{b}}}$

0.84

0.001

0.99

0.96

0.98

\begin{tabular}{|c|c|c|}
\hline & & \\
\hline $\mathrm{n}$ & $\mathbf{k}$ & Fit $^{b}$ \\
\hline
\end{tabular}

0.88

0.04

0.98

1.00

0.80

0.98

and

$$
c+\frac{m}{V} k c^{n}=c_{0},
$$

where

$$
\begin{aligned}
& c_{0}=\text { the initial concentration and } \\
& v=\text { the volume of the solution, }
\end{aligned}
$$

$$
K_{d}=k\left(\frac{c_{o}}{1+\frac{m}{V} K_{d}}\right)^{n-1}
$$

therefore,

$$
\mathrm{K}_{\mathrm{d}}^{1 / 1-\mathrm{n}} \propto 1+\frac{\mathrm{m}}{\mathrm{V}} \mathrm{K}_{\mathrm{d}}
$$

For $\frac{m}{V} K_{d} \gg 1$,

$$
K_{d} \propto\left(\frac{m}{V}\right)^{\frac{1-n}{n}}
$$


The distribution-coefficient dependence on the solid-to-solution ratio can be expressed in terms of the Freundlich isotherm parameter $n$. This simple relation seems adequate to explain the observed dependence of $R_{d}$ values on the solution-to-solid ratios. In batch experiments with crushed samples of YM-22 and YM-38 tuffs, $R_{d}$ values were determined using solution-to-solid ratios of 5, 10, and 30 (Tables XII and XLII). These values were fit to the power-law expression derived above, using a least squares program. The resultant Freundlich isotherm parameters were compared with those previously determined using the standard batch technique. ${ }^{3}$ The comparison for sample $\mathrm{YM}-22$ is given in Table XLIII; a similar comparison for sample YM-38 was not made because the experimental uncertainties associated with high $R_{d}$ values were too large to give a meaningful comparison. The agreement between the two experiments is good, the trends are consistent, and the values of $\mathrm{n}$ determined by the two methods are reasonably close.

14. Conclusions. A variety of techniques using crushed-tuff samples has been investigated to obtain information on the sorptive behavior of tuff and the possible application of the data to flowing systems and to real situations. The applicability of these experiments to solid samples is discussed in Sec. IV.D.

When material of the same particle-size distribution is used, the results from batch, column, and circulating-column methods are in reasonable agreement. The column method gives information on dispersion that cannot be obtained in batch systems. Batch methods, however, allow for processing a large number of samples with ease under a variety of conditions.

Sorption of strontium, cesium, and barium occurs mainly by ion exchange; the interaction of these elements with tuff can be explained on the basis of mineralogy: the zeolite clinoptilolite is the principal sorbing mineral in many samples. At this time, sorption of the lanthanides, americium, and plutonium does not appear to correlate well with mineralogy.

B. Permeability, Storage Capacity, and Porosity

Because moving groundwater provides by far the most probable means of transporting waste elenents from the repository, it is necessary to understand water's ability to move through the tuff formations. It is generally assuned that groundwater flow in the tuffs will be primarily through fractures; the permeability of a fracture is certainly much greater than that of the bulk rock. However, fractures must form a continuous connected network to provide 
VARIATION OF THE SORPTION RATIO FOR YM-22 TUFF WITH THE SOLUTION-TO-SOLID RATIO

\begin{tabular}{|c|c|c|c|c|c|c|c|}
\hline \multirow{2}{*}{$\begin{array}{c}\text { Fraction } \\
\text { Size } \\
(\mu \mathrm{m}) \\
\end{array}$} & \multirow{2}{*}{$\begin{array}{l}\text { Solution- } \\
\text { to-Solid } \\
\text { Ratio }\end{array}$} & \multirow{2}{*}{$\begin{array}{c}\text { Contact } \\
\text { Time } \\
\text { (weeks) }\end{array}$} & \multicolumn{5}{|c|}{$R_{d}(m / / g)$} \\
\hline & & & $\mathrm{Cs}$ & $\mathrm{Sr}$ & $\mathrm{Ba}$ & $\mathrm{Ce}$ & Eu \\
\hline \multirow[t]{6}{*}{$<75$} & $5: 1$ & 3 & $827(4.7)^{\mathrm{a}}$ & $122(2.0)$ & $1490(1.8)$ & $459(8.5)$ & 926 \\
\hline & $10: 1$ & 3 & $755(2.9)$ & $80.3(2.1)$ & $1360(1.4)$ & $500(5.7)$ & 1270 \\
\hline & $30: 1$ & 3 & $749(2.1)$ & $67.4(2.1)$ & $1010(1.1)$ & $1050(4.5)$ & 1640 \\
\hline & $5: 1$ & 6 & $740(4.6)$ & $129(2.0)$ & $1280(1.8)$ & $297(8.9)$ & $749(7.9)$ \\
\hline & $10: 1$ & 6 & $857(3.0)$ & $99.4(2.0)$ & $1820(1.5)$ & $748 \quad(7.2)$ & $2250(6.2)$ \\
\hline & $30: 1$ & 6 & $1100(2.2)$ & $97.9(2.3)$ & $1850(1.1)$ & & \\
\hline \multirow[t]{6}{*}{$75-500$} & $5: 1$ & 3 & $363(3.4)$ & $63.0(2.0)$ & $601(2.2)$ & $303(6.6)$ & 794 \\
\hline & $10: 1$ & 3 & $336(2.5)$ & $44.9(2.2)$ & $530(1.9)$ & $508 \quad(5.4)$ & 909 \\
\hline & $30: 1$ & 3 & $368(2.2)$ & $195(2.0)$ & $412(1.9)$ & $1000(4.6)$ & 1600 \\
\hline & $5: 1$ & 6 & $565(4.0)$ & $32.5(3.6)$ & $805(1.8)$ & $524(9.5)$ & $910(6.9)$ \\
\hline & $10: 1$ & 6 & $457(2.6)$ & $59.4(1.5)$ & $605(1.3)$ & $1040(9.2)$ & $1300(4.9)$ \\
\hline & $30: 1$ & 6 & $522(2.1)$ & & $568(1.2)$ & $1630(6.7)$ & $2010(3.5)$ \\
\hline
\end{tabular}


TABLE XIII

VARIATION OF THE SORPTION RATIO FOR YM-38 TUFF WITH THE SOLUTION-TO-SOLID RATIO

\begin{tabular}{|c|c|c|c|c|c|c|c|}
\hline \multirow{2}{*}{$\begin{array}{c}\text { Fraction } \\
\text { Size } \\
(\boldsymbol{\mu}) \\
\end{array}$} & \multirow{2}{*}{$\begin{array}{l}\text { Solution- } \\
\text { to-Solid } \\
\text { Ratio } \\
\end{array}$} & \multirow{2}{*}{$\begin{array}{l}\text { Contact } \\
\text { Time } \\
\text { (wंeeks) } \\
\end{array}$} & \multicolumn{5}{|c|}{$R_{d}(m \ell / g)$} \\
\hline & & & Cs & $\mathbf{S r}$ & $\mathbf{B a}$ & $\mathrm{Ce}$ & Eu \\
\hline \multirow[t]{6}{*}{$<75$} & $5: 1$ & 3 & $5970(11.7)^{a}$ & $2770(5.4)$ & $11000(5.1)$ & $1330(17.3)$ & $2700(17.7)$ \\
\hline & $10: 1$ & 3 & $5760(7.5)$ & $2790(3.4)$ & $60000(9.6)$ & $6390(13.1)$ & $9070(13.2)$ \\
\hline & $30: 1$ & 3 & $7980(3.4)$ & $3610(2.2)$ & $81500(3.6)$ & $9070(6.0)$ & $9700(5.4)$ \\
\hline & $5: 1$ & 6 & $5100(12.2)$ & $2370(6.4)$ & $9020(5.2)$ & $1140(20.4)$ & $2160(18.4)$ \\
\hline & $10: 1$ & 6 & $5120(6.1)$ & $2410(3.4)$ & $16000(3.4)$ & $5460(15.3)$ & $4330(9.4)$ \\
\hline & $30: 1$ & 6 & $8050(3.8)$ & $3700(2.4)$ & $78900(3.7)$ & $9190(8.3)$ & $6000(5.7)$ \\
\hline \multirow[t]{6}{*}{$75-500$} & $5: 1$ & 3 & $3660(10.6)$ & $2040(4.9)$ & $7410(5.1)$ & $2560(17.2)$ & $3060(1.63)$ \\
\hline & $10: 1$ & 3 & $5750(7.0)$ & $2840(3.3)$ & $48000(7.2)$ & $7230(13.0)$ & $6780(11.3)$ \\
\hline & $30: 1$ & 3 & $5540(3.2)$ & $2770(2.1)$ & $54200(3.1)$ & $16000(5.8)$ & $10400(4.9)$ \\
\hline & $5: 1$ & 6 & $5110(18.1)$ & $1760(5.5)$ & $6180(4.1)$ & $1600(19.3)$ & $2500(17.7)$ \\
\hline & $10: 1$ & 6 & $7280(7.0)$ & $3080(3.8)$ & $23200(3.9)$ & $6050(15.3)$ & $6330(11.2)$ \\
\hline & $30: 1$ & 6 & $6270(4.3)$ & $2050(2.6)$ & $49200(3.8)$ & $11600(11.3)$ & $8810(7.4)$ \\
\hline
\end{tabular}

\footnotetext{
${ }^{a}$ The values in parentheses are the standard deviations for a single measurement of the $R_{\text {falues }}$ expressed in per cent. They were obtained from the errors associated with activity measurements and estimated uncertainties for various parameters entering into the calculation. These estimated uncertainties were propagated using the rule of change of variables in a moment matrix, assuming independence of the variables.
} 
TABLE XLIII

FREUNDLICH ISOTHERM

PARAMETERS DETERMINED FROM BATCH ISOTHERM

AND SOLUTION/SOLID EXPERIMENTS

\begin{tabular}{|c|c|c|c|c|c|c|}
\hline \multirow[b]{2}{*}{ Sample } & \multirow{2}{*}{$\begin{array}{l}\text { Fraction } \\
\text { Size } \\
(\mu \mathrm{m}) \\
\end{array}$} & \multirow[b]{2}{*}{ Method } & \multicolumn{4}{|c|}{ Element } \\
\hline & & & Cs & Sr & $\mathrm{Ba}$ & Eu \\
\hline$Y M-22$ & $<75$ & isotherm & 0.95 & 0.76 & 0.82 & 1.45 \\
\hline $\mathrm{YM}-22$ & $<75$ & solution/solid & $1.01^{\mathrm{a}}$ & 0.83 & 0.83 & 1.1 \\
\hline$Y M-22$ & $75-500$ & isotherm & 1.01 & 0.60 & 0.83 & 1.67 \\
\hline YM-22 & $75-500$ & solution/solid & 0.88 & 0.71 & 0.82 & 0.9 \\
\hline
\end{tabular}

These $n$ values are based on measurements for a cesium concentration $<_{10}^{-8} M$
because the initial concentration used in the solution-to-solid experiments
was $<10^{-8} \mathrm{M}$. Values are different from those reported originally because the
cesium isotherm appears to change slope at $\sim 10^{-} \mathrm{M}$.

an effective pathway for fluid movement. The connectedness of fracture systems can be assessed by comparing laboratory and field measurements of bulk permeability. The issue of fracture connectedness is not only important when characterizing the volume of groundwater flow, but is also necessary to assess the volume of rock that will be contacted by flowing groundwater. If an appreciable amount of porous flow occurs through the bulk rock, the surface area available for sorption will be appreciably increased.

Laboratory measurements of permeability and storage capacity have been accomplished by means of a transient pressure pulse method. Porosity has been obtained by measuring the grain density and the wet and dry weights of the samples and by mercury porosimetry. These measurements are basic to the understanding of the transport of waste elements in groundwater through tuff. Both permeability and storage capacity are needed to compare with field tests and to predict flow through unfractured tuff in response to a pressure gradient. Such pressure gradients might occur where there is either a regional hydrologic gradient or gradients that result from heat caused by the repository. Porosity 
is a necessary parameter when calculating the effects of diffusion. The combined results of permeability, storage capacity, and porosity measurements can be used to gain insights into the pore structure of the tuff, which, in turn, will aid in estimating the diffusion parameters of constrictivity and tortuosity.

1. Porosity. A mercury infusion porosimeter was constructed for porosity and pore-size distribution studies. The mercury infusion apparatus (schematic, Fig. 47) was constructed by modifying an existing Ruska mercury pump and

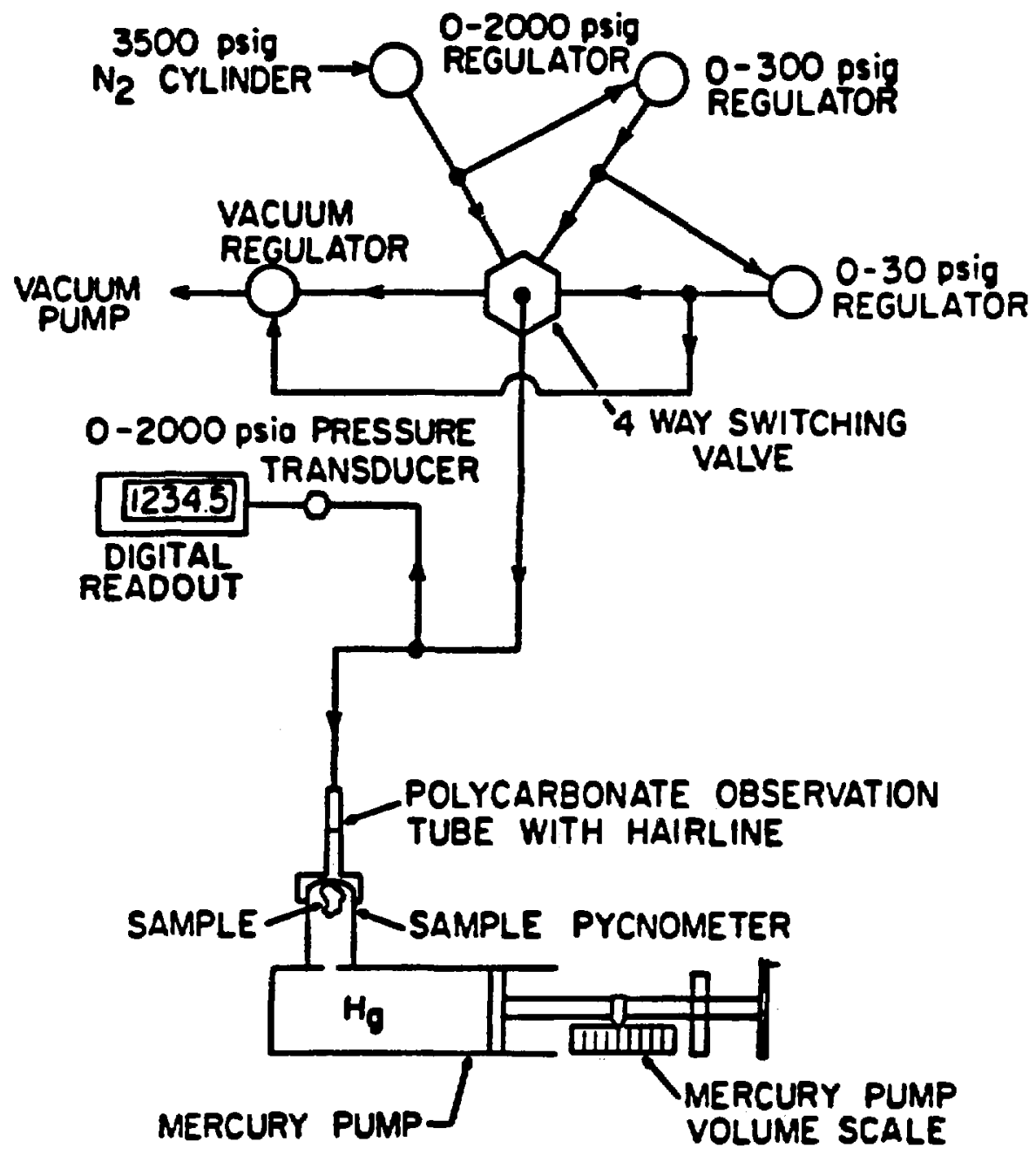

Fig. 47. Mercury infusion porosineter. 
pycnometer, adding a polycarbonate mercury-level-observation tube to the top of the pycnometer and the necessary pressure and vacuum regulation and measurement system. System pressures are measured using a Setra 0- to 2000-psig pressure transducer with a digital read-out, which also measures partial vacuums. The porosimeter is now capable of operating at pressures ranging from $\sim 13 \mathrm{~Pa}(1 \mathrm{~mm} \mathrm{Hg})$ to $1.4 \times 10^{6} \mathrm{~Pa}(2000 \mathrm{psia})$. Thus, the porosimeter is capable of measuring pore-size distributions for pores with theoretical diameters between $10^{-1}$ and $10^{-5} \mathrm{~cm}$. This porosimeter meets or exceeds the specifications of commercially available porosimeters and has a total system expansion correction of $<0.4 \mathrm{~cm}^{3}$ at maximum pressure.

Initially, daily temperature fluctuations of several degrees Celsius in the laboratory caused problems in making accurate volume measurements. This problem has been partially corrected by repairs to the laboratory cooling system and by application of an ambient room-temperature correction factor to the raw volume data. To measure more accurately the working temperature in the mercury reservoir, a thermistor temperature probe will be placed in the mercury reservoir.

Porosity and pore-size distribution measurements are made by placing a dried and weighed sample of tuff in the pycnometer and evacuating the system to $<1 \mathrm{~mm} \mathrm{Hg}$. The pump forces mercury into the pycnometer until the mercury level rises to a hairline in the observation tube. The displacement of the mercury pump piston is then read to $0.001 \mathrm{~cm}^{3}$. The total displacement from the zero position gives the bulk-sample volume because at $1-\mathrm{mm} \mathrm{Hg}$ pressure. virtually none of the mercury will infuse into the sample. In the past, total sample porosity has been calculated from the sample weight and bulk volume by using the average grain density of $2.301 \pm 0.04 \mathrm{~g} / \mathrm{cm}^{3}$ reported by Manger ${ }^{56}$ for tuffs from subunit $\mathrm{T}$ of the Paintbrush Tuff. Pycnometer measurements for grain density will be used to obtain more accurate total porosity measurements in the future.

When the bulk volume has been determined, the pressure in the pycnometer is increased stepwise by releasing vacuum and applying nitrogen gas pressure. After each step increase in pressure, the system is maintained at that pressure for several minutes to allow the mercury to infuse into the sample. The cumulative volume change is then measured by using the pump to bring the mercury level back to the hairline. Typically, 10 to 20 steps are used per order-ofmagnitude change in pressure. 
The pressure-volume (P-V) data are analyzed by subtracting the system volume expansion at each pressure from the cumulative volume change during the sample run and correcting for temperature changes, The system expansion correction is determined at low pressure using a semilog regression of the $P-V$ data from a blank run. Above $-3.5 \times 10^{4} \mathrm{~Pa}$, a linear regression equation is used for the system expansion correction. A P-V curve with the regression line for a typical blank run is shown in Fig. 48.

The theoretical pore diameters were calculated using the Washburn equation:

$$
d_{T}=\frac{4 \gamma \cos \theta}{P}
$$

where

$$
\begin{aligned}
& d_{T}=\text { the theoretical pore diameter, } \\
& P=\text { pressure, } \\
& \gamma=\text { the surface tension of mercury, and } \\
& \theta=\text { the contact angle. }
\end{aligned}
$$

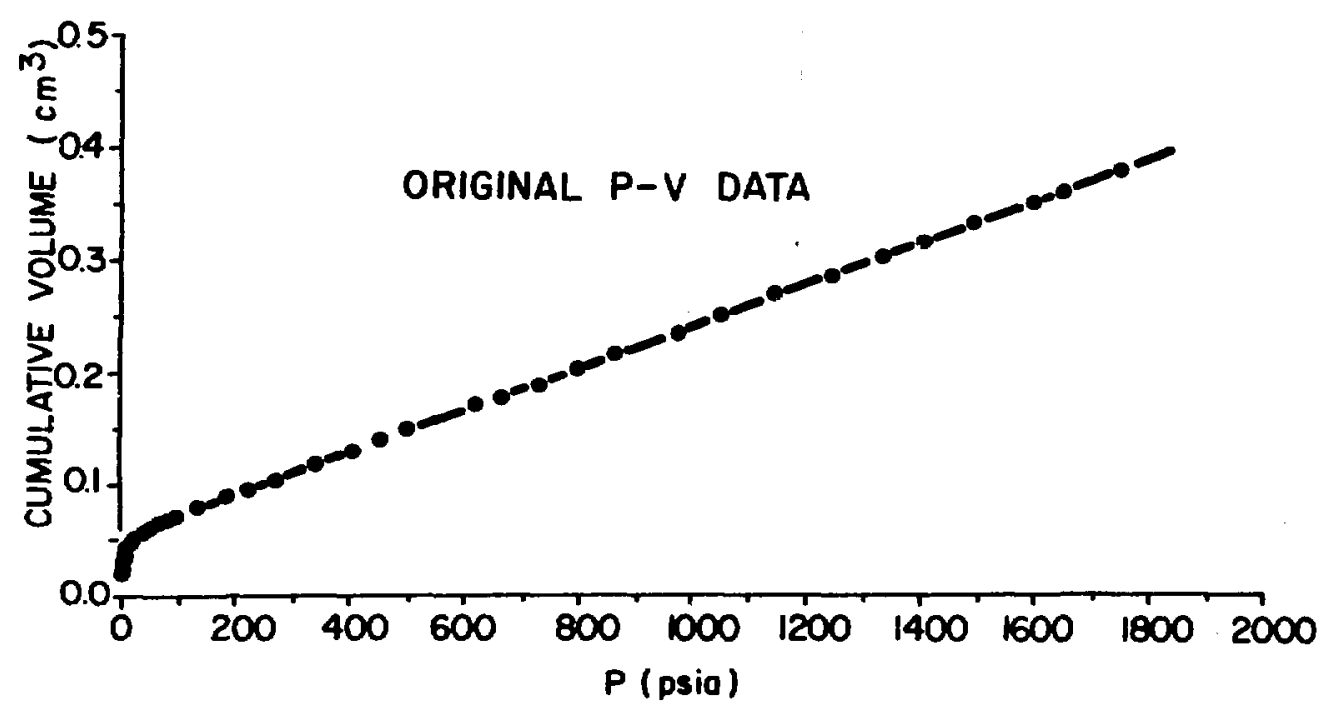

Fig. 48. Blank-run $P-V$ curve of the mercury infusion porosimeter. 
Figure 49 indicates several significant differences between these two samples. First, whereas the median pore diameter (determined from the $50 \%$ porusity line) for sample Gl-2290 is $3.9 \times 10^{-5} \mathrm{~cm}$, the median diameter for sample HF-23 cannot be determined because it is $<10^{-5} \mathrm{~cm}$. Extrapolating the line through the last six data points for sample HF-23 suggests a median diameter of $\sim 2.5 \times 10^{-6} \mathrm{~cm}(0.02 \mu \mathrm{m})$. Assuming the total porosity calculations are valid, $85 \%$ of the total porosity of sample G1-2290 was from pores $>10^{-5} \mathrm{~cm}$, but only $24 \%$ of the pores in sample $H F-23$ are $>10^{-5} \mathrm{~cm}$. Second, the roughly linear trend of the data on the probability graph for sample G1-2290 indicates that pore-size distribution is approximately log-normal. The distribution for sample HF-23 is very nonlinear, although the smallest pores may approach a log-normal distribution.

Pore-size distribution measurements have been completed on 10 tuff samples by using mercury infusion porosimetry. The pore-size measurements were made for pores with theoretical diameters ranging from $10^{-1}$ to $10^{-5} \mathrm{~cm}$, based on $\mathrm{Eq}$. (9).

To summarize the porosimetry data the volume of mercury intruded at each pressure was divided by the total volume intruded at the maximum porosimeter pressure ( $2000 \mathrm{psi})$. The fractional volume intruded for each sample was then multiplied by 100 to obtain the cumulative percentage of porosity for pores with diameters $>10^{-5} \mathrm{~cm}$. Equation (9) was then used to compute the theoretical

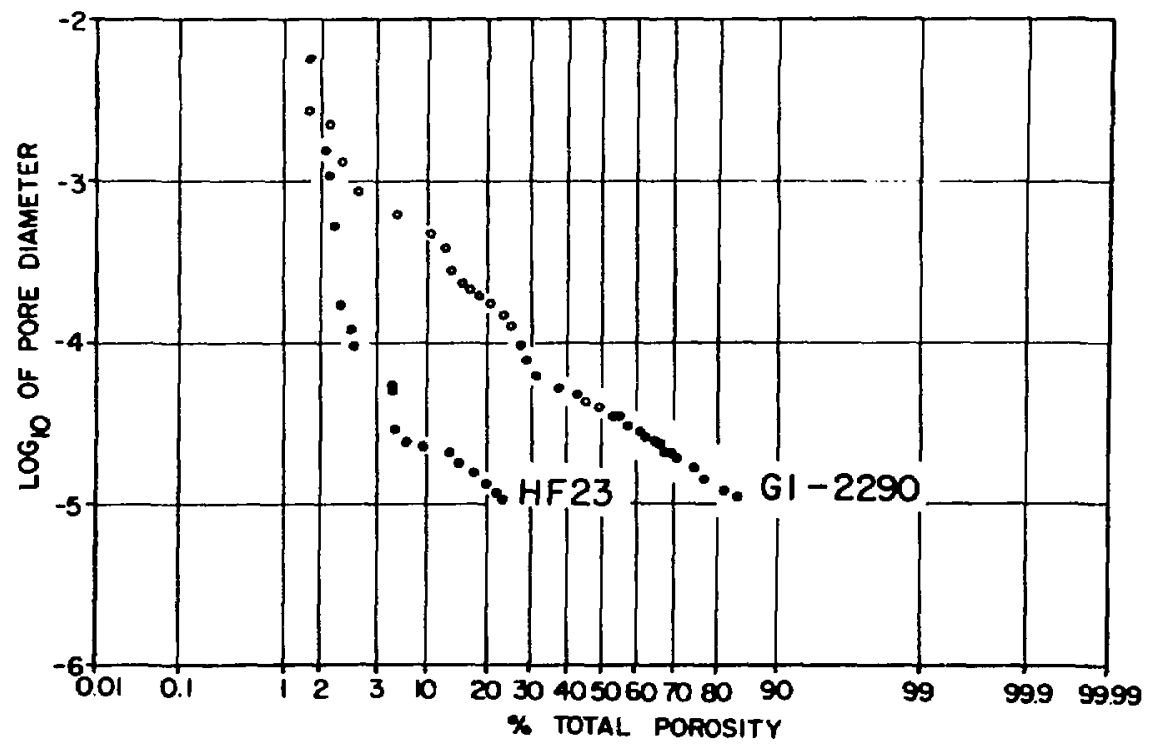

Fig. 49. Log of pore diameter vs per cent of total porosity determined by mercury infusion porosity for samples HF-23 and G1-2290. 
pore diameter corresponding to each measured pressure, and these data were used to construct the pore-size distribution curves shown in Fig. 50. As can be seen from Fig. 50, nearly all of the porosity in these samples is the result of pores $<10^{-2} \mathrm{~cm}$ in dianeter. In most of the samples, over $50 \%$ of the porosity is attributable to pores $<10^{-4} \mathrm{~cm}(1 \mu \mathrm{m})$ in diameter. Possibly half of the samples contain a large fraction of their porosity in pores $<10^{-5} \mathrm{~cm}$ in dianeter.

2. Permeability and Storage Capacity. Permeability and porosity have been neasured for a number of samples from the NTS. For 10 samples from USW-G1, 5 from G Tunnel, and 1 from the UE25a-1 drill hole, the permeabilities range from $1 \times 10^{-19}$ to $>2.5 \times 10^{-17} \mathrm{~m}^{2}$. Individual permeability measurements for those samples within the measurement range of the apparatus $\left(<2.5 \times 10^{-17} \mathrm{~m}^{2}\right)$ are shown in Figs. 51-64. Mercury porosimetry and grain density measurements have also been made on a number of these samples. These data, with permeability and total porosity calculated from wet and dry weight measurements, are shown in Table XLIV. There is no correlation between permeability and porosity; however, those tuffs with a larger proportion of small pores tend to have lower permeability. The exception is sample G1-3116, which

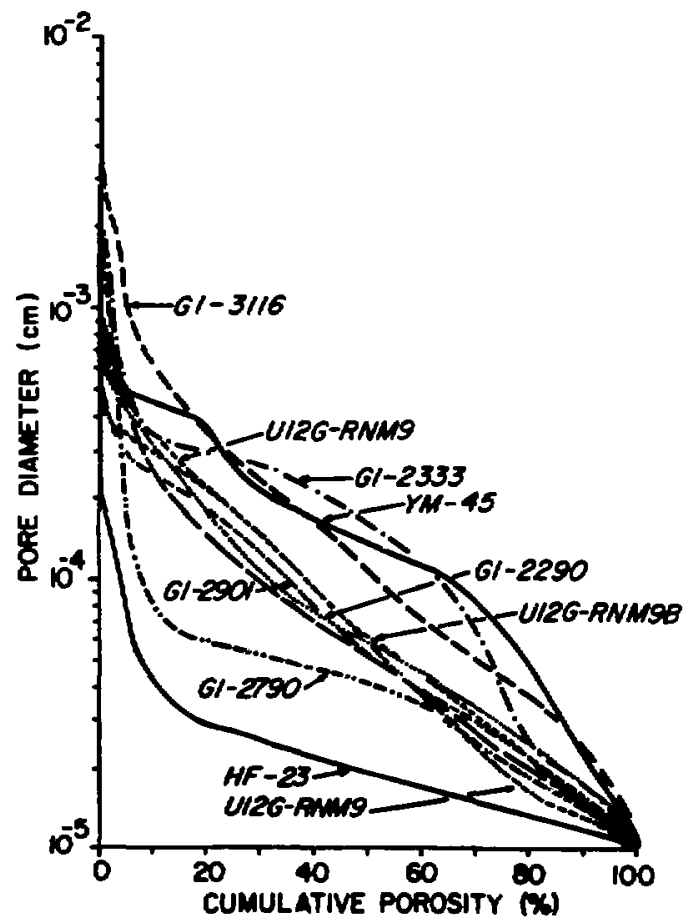

Fig. 50. Cumulative pore-size distributions for NTS tuff samples. 


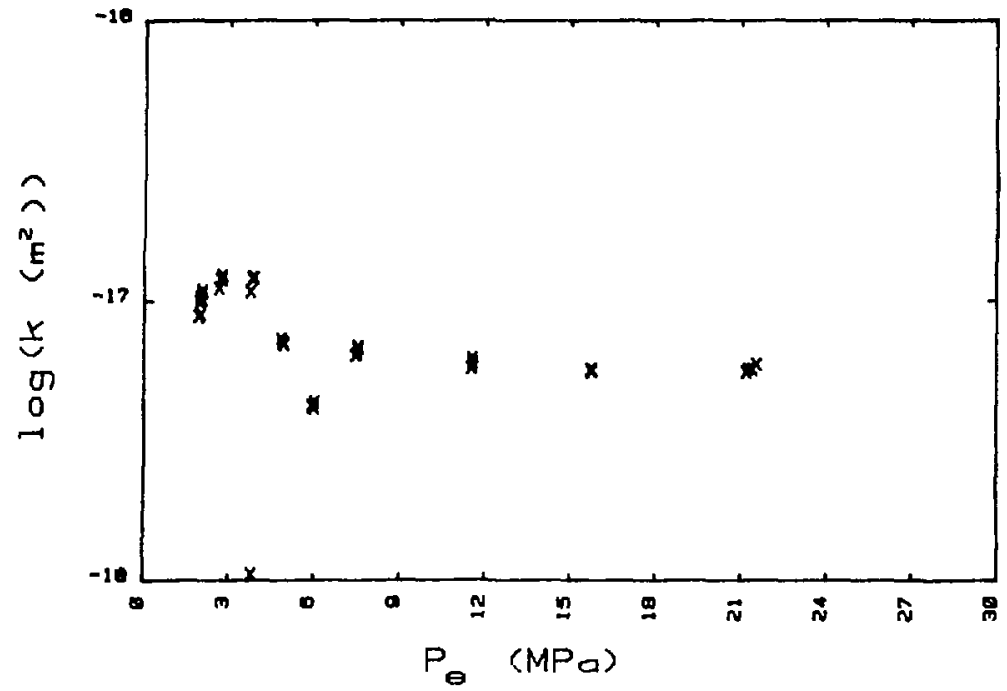

Fig. 51. Permeability (k) of sample G1-2233 (cit parallel to axis of hole) as a function of effective confining pressure $\left(\mathrm{P}_{\mathrm{e}}\right)$.

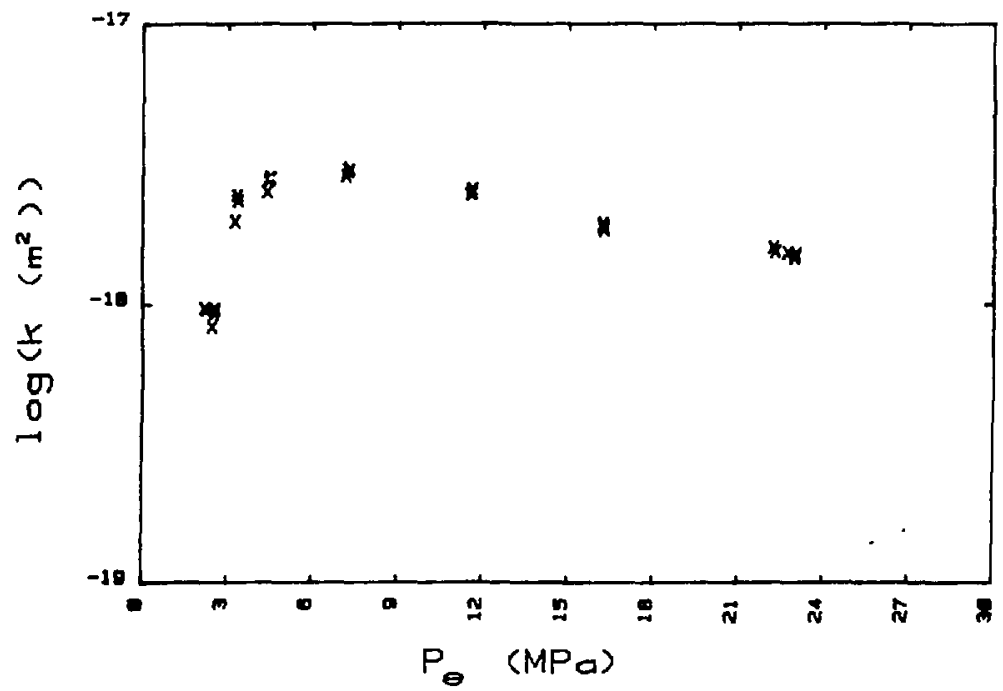

Fig. 52. Permeability (k) of sample G1-2290 (cut perpendicular to axis of hole) as a function of effective confining pressure $\left(\mathrm{P}_{e}\right)$. 


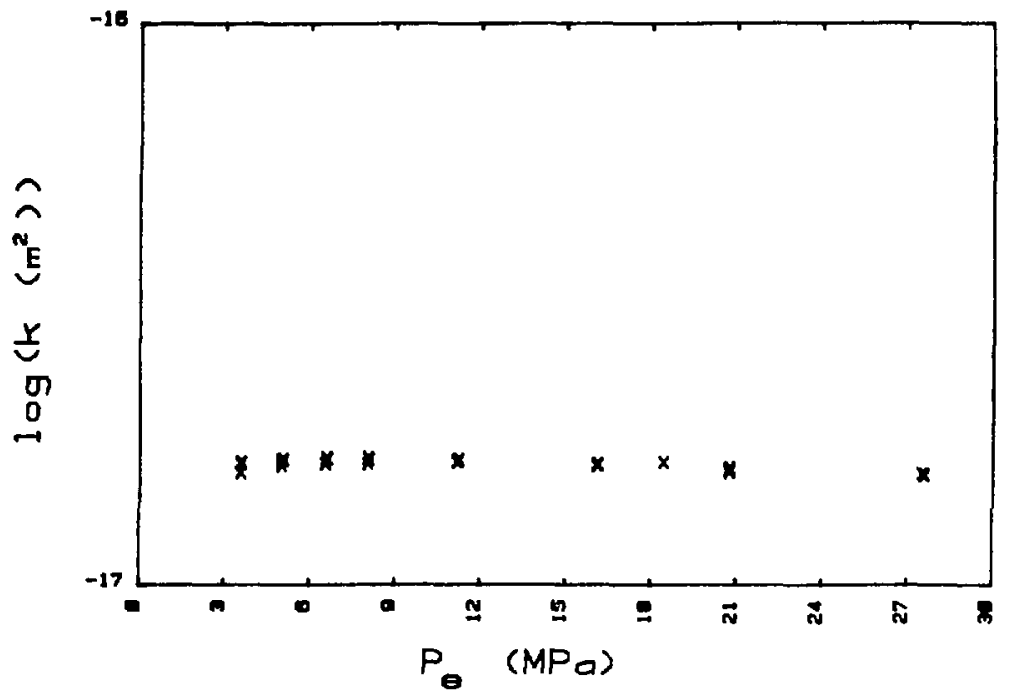

Fig. 53. Permeability (k) of sample G1-2840 (cut parallel to axis of hole) as a function of effective confining pressure $\left(P_{e}\right)$.

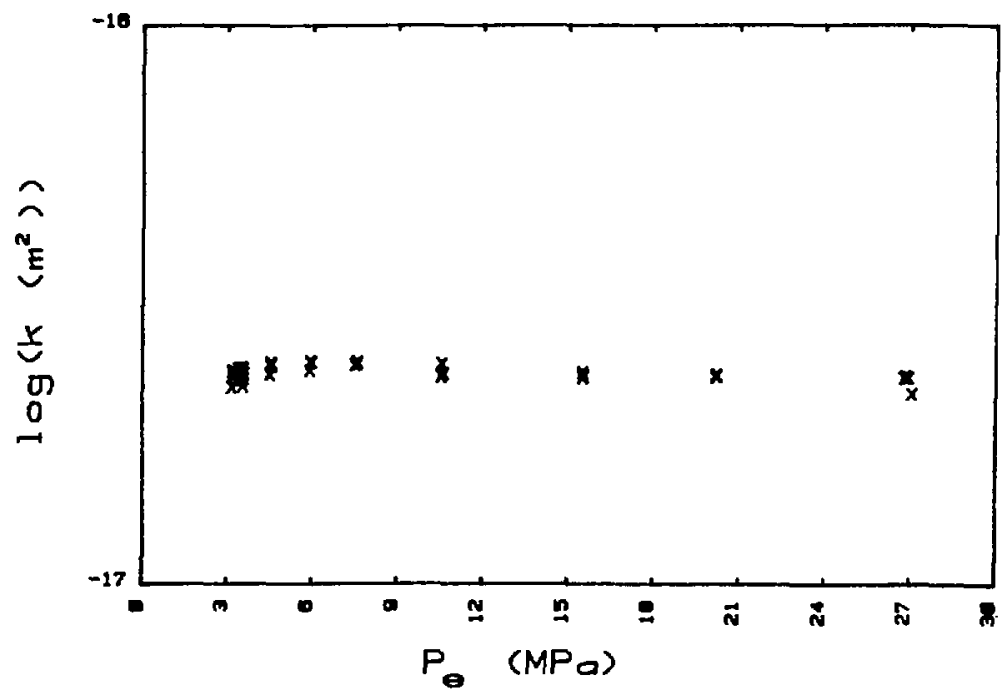

Fig. 54. Permeability (k) of sample G1-2840 (cut perpendicular to axis of hole) as a function of effective confining pressure $\left(\mathrm{P}_{e}\right)$. 


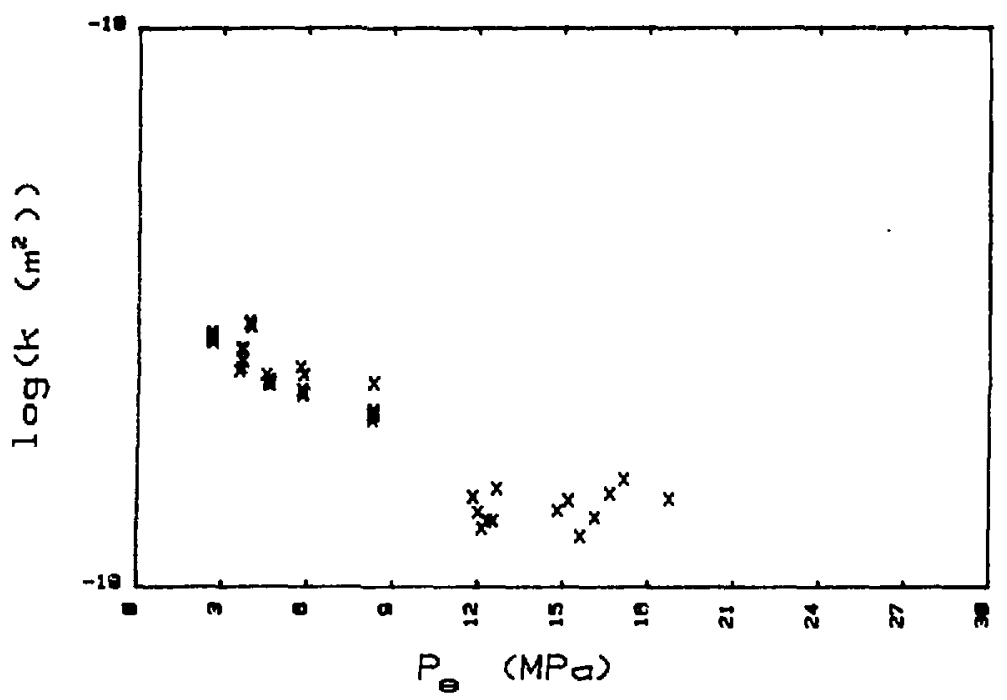

Fig. 55. Permeability (k) of sample G1-3116 as a function of effective confining pressure $\left(\mathrm{P}_{\mathrm{e}}\right)$.

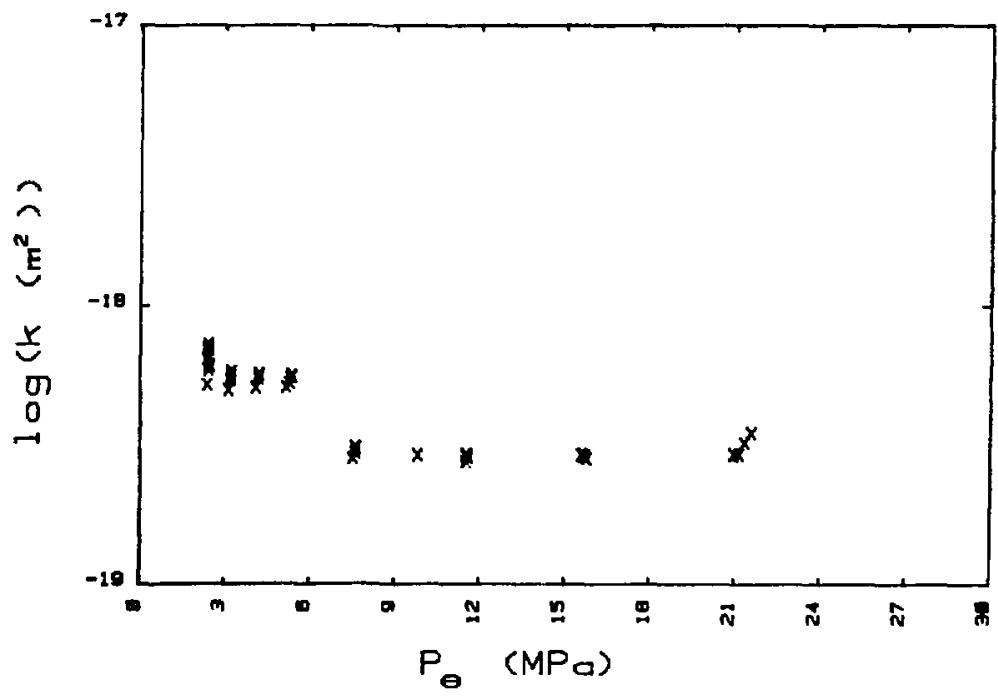

Fig. 56. Permeability (k) of sample U12G-RNM9 (5.9 to $6.4 \mathrm{ft}$, side A, position $A)$ as a function of effective confining pressure $\left(\mathrm{P}_{e}\right)$. 


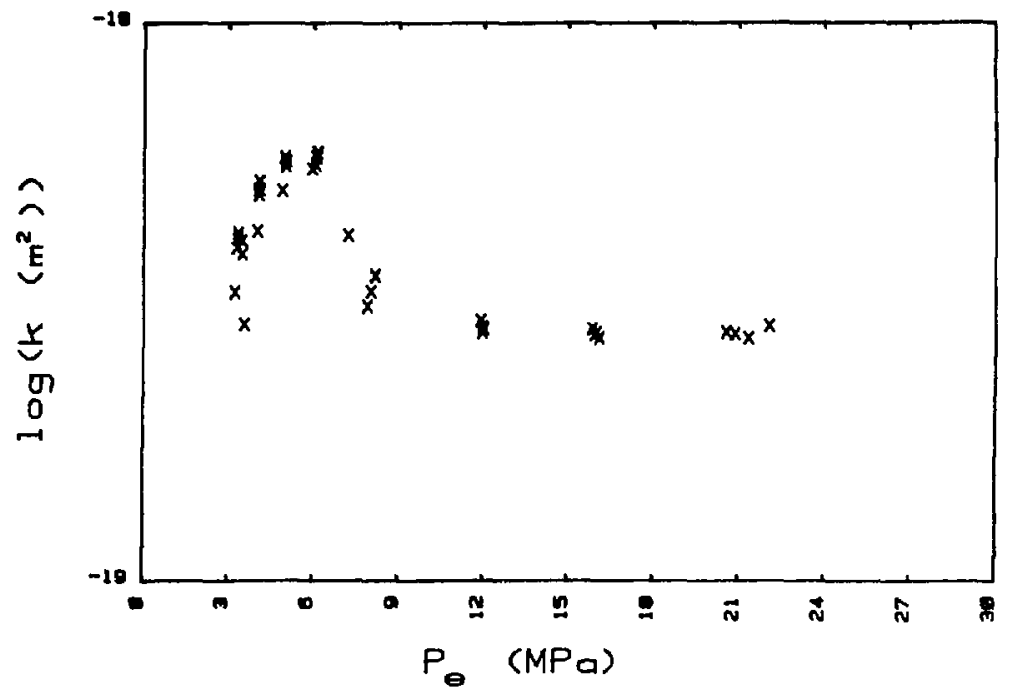

Fig. 57. Permeability (k) of sample U12G-RNM9 (5.9 to $6.4 \mathrm{ft}$, side A, position $B)$ as a function of effective confining pressure $\left(P_{e}\right)$.

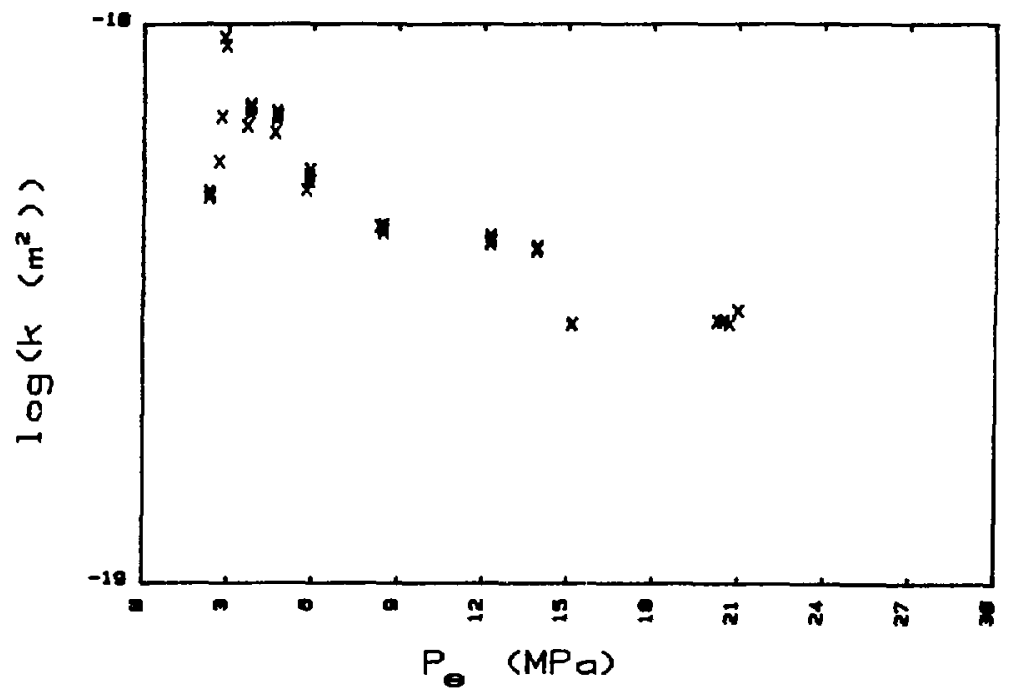

Fig. 58. Permeability (k) of sample U12G-RNM9 (5.9 to $6.4 \mathrm{ft}$, side A, position $C)$ as a function of effective confining pressure $\left(P_{e}\right)$. 


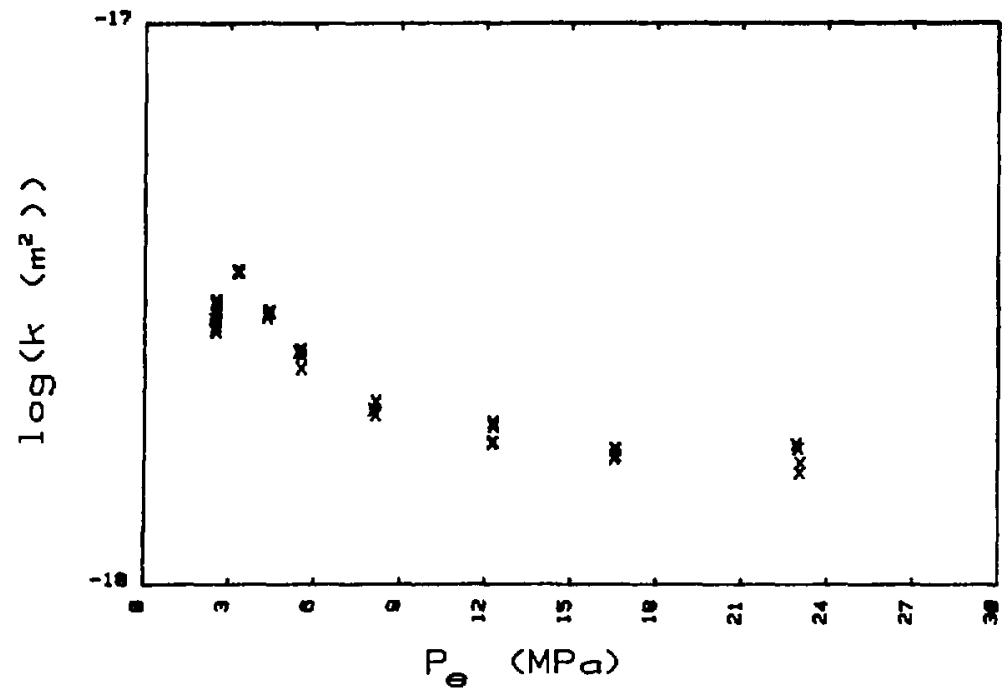

Fig. 59. Permeability (k) of sample U12G-RNM9 (5.9 to $6.4 \mathrm{ft}$, side B, position $A)$ as a function of effective confining pressure $\left(P_{e}\right)$.

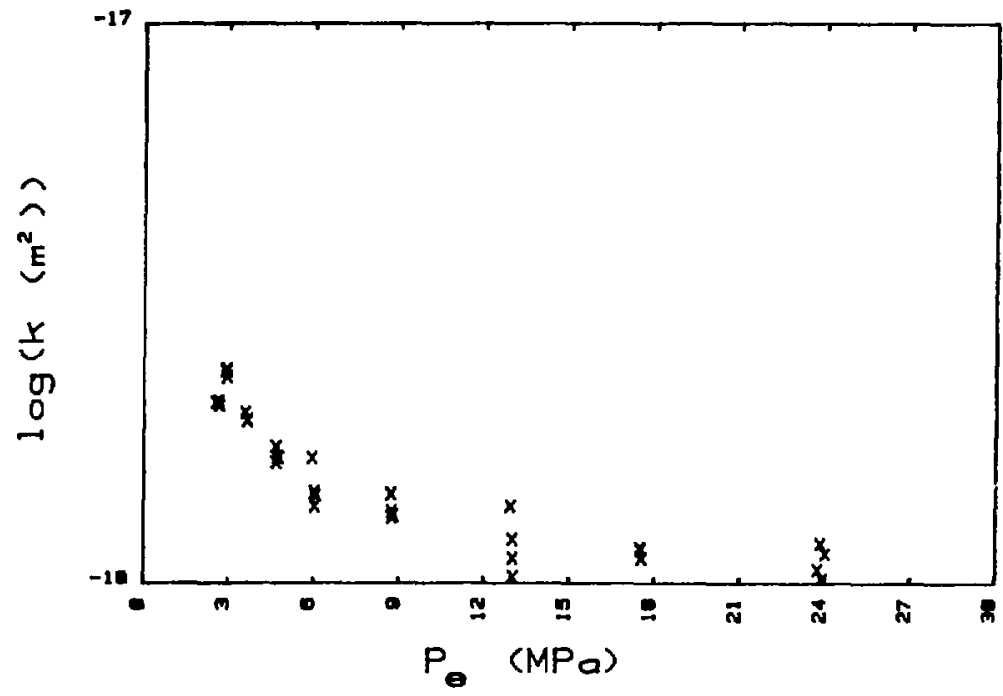

Fig. 60. Permeability (k) of sample U12G-RNM9 (5.9 to $6.4 \mathrm{ft}$, side B, position $B)$ as a function of effective confining pressure $\left(P_{e}\right)$. 


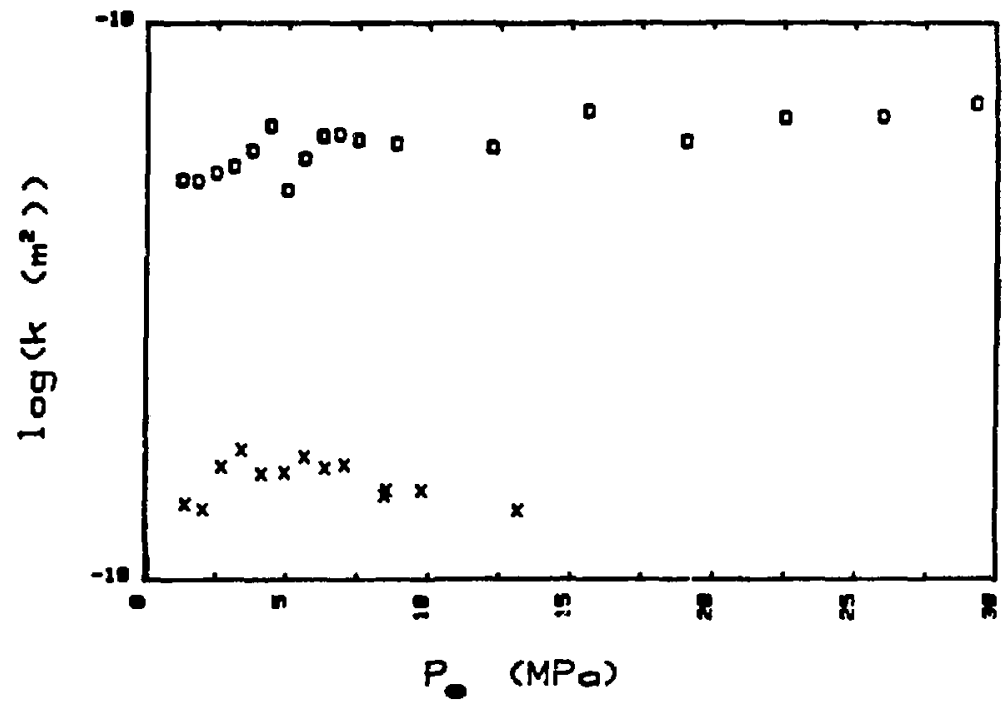

Fig. 61. Permeability (k) of sample 1087.3-1087.8 as a function of effective confining pressure $\left(P_{\mathrm{e}}\right)$. $X$ and $O$ represent the before and after sample, respectively.

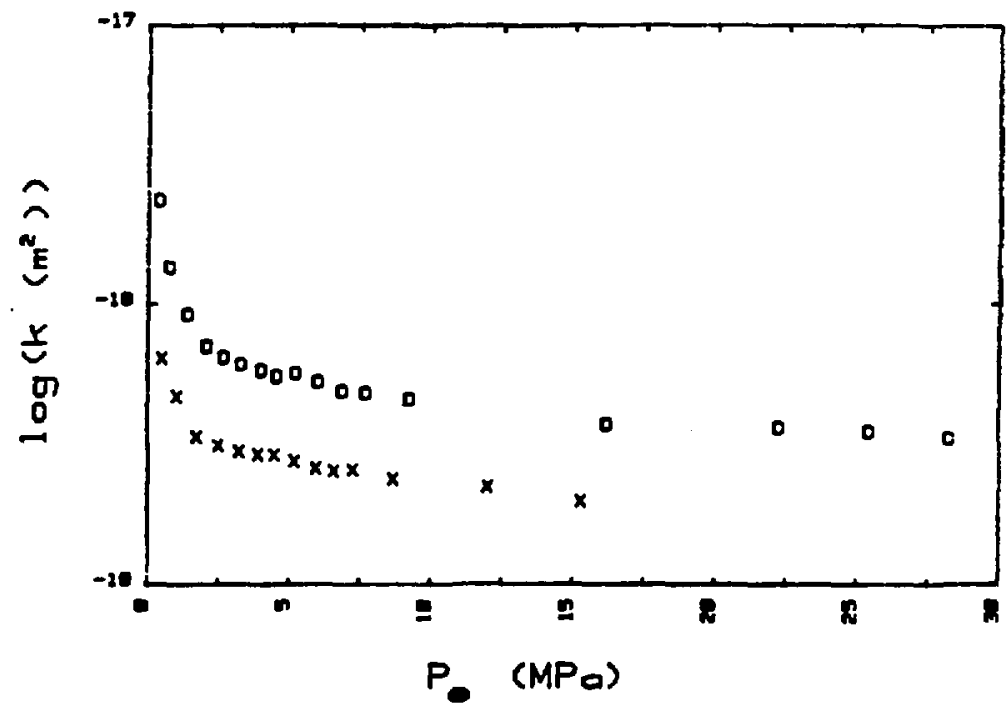

Fig. 62. Permeability (k) of sample 1100.6-1101.6 as a function of effective confining pressure $\left(P_{0}\right)$. $X$ and 0 represent the before and after sample, respectively. 


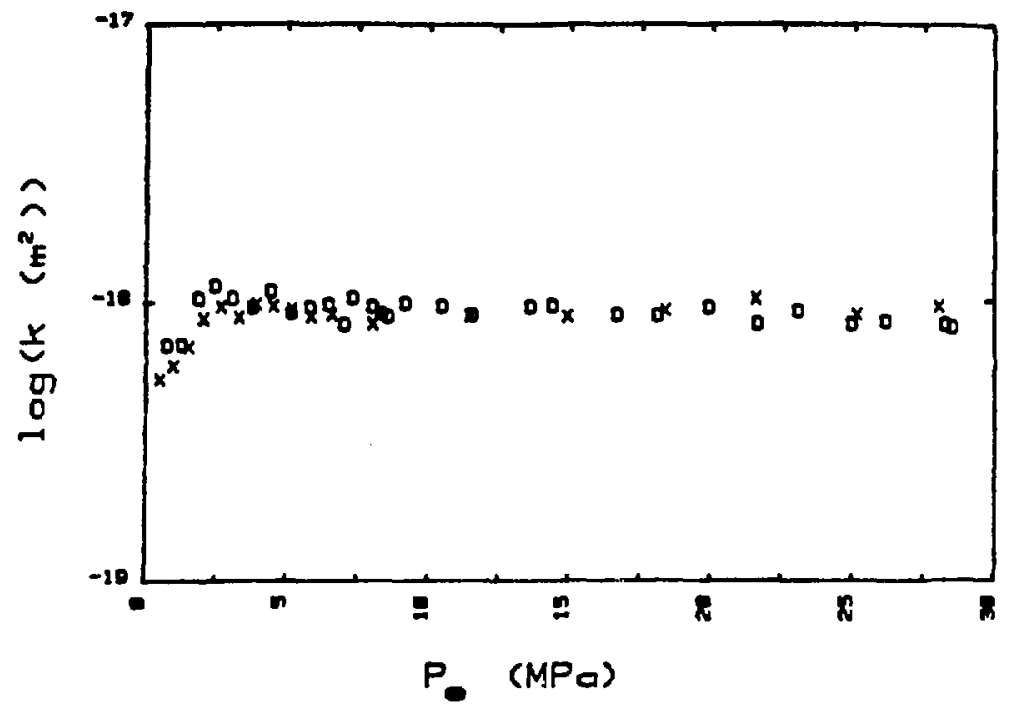

Fig. 63. Permeability (k) of sample 1370.8-1371.4 as a function of effective confining pressure $\left(\mathrm{P}_{\mathrm{e}}\right) . \mathrm{X}$ and 0 represent the before and after sample, respectively.

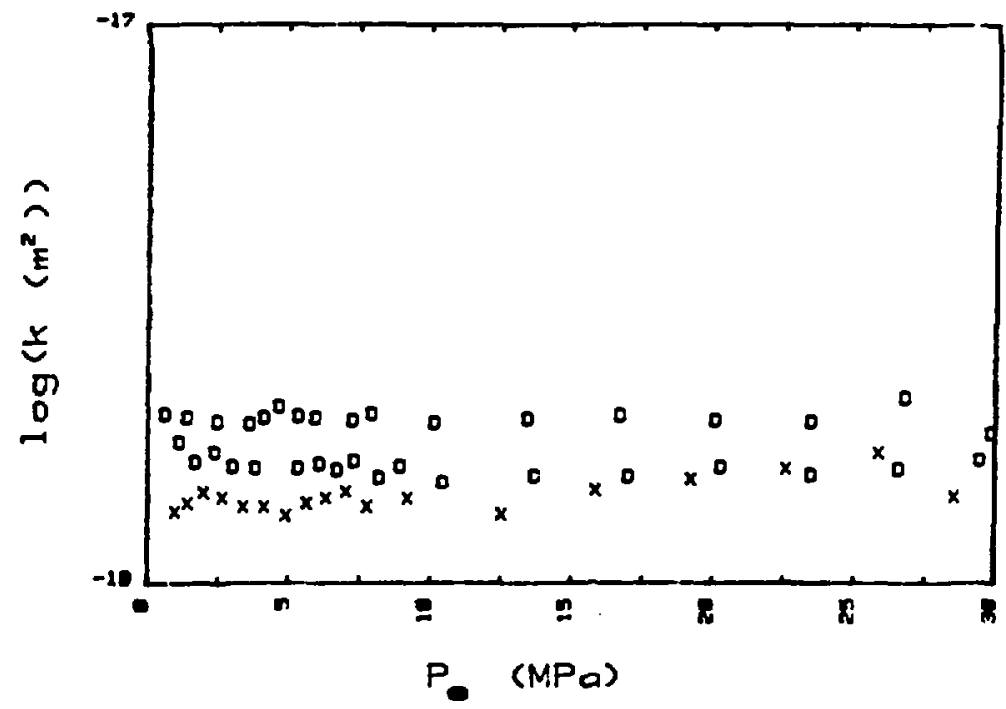

Fig. 64. Permeability (k) of sample 1640.9-1642.1 as a function of effective confining pressure $\left(\mathrm{P}_{\mathrm{e}}\right) . \quad X$ and 0 represent the before and after sample, respectively. 
TABLE XLIV

PERMEABILITY AND POROSITY OF NTS TUFFS

\begin{tabular}{|c|c|c|c|c|c|c|}
\hline Sample & $\begin{array}{c}\text { Permeability } \\
\left(m^{2}\right) \\
\end{array}$ & $\begin{array}{c}\text { Grain Density } \\
\left(\mathrm{g} / \mathrm{cm}^{3}\right) \\
\end{array}$ & $\begin{array}{l}\text { Porosity } \\
>0.1 \mu \mathrm{m} \\
\end{array}$ & $\begin{array}{c}\text { Total } \\
\text { Porosity } \\
\end{array}$ & $\begin{array}{c}\text { Total } \\
\text { Porosity } \\
\end{array}$ & $\begin{array}{c}\text { Porosity } \\
>0.1 \\
(\%) \\
\end{array}$ \\
\hline G1-2233 & $6-10 \times 10^{-18}$ & 2.24 & 0.27 & 0.35 & 0.38 & 22 \\
\hline G1-2901 & $>2.5 \times 10^{-17}$ & 2.49 & 0.15 & 0.19 & 0.19 & 20 \\
\hline $\begin{array}{l}\text { U12G RNM\#9 } \\
5.9-6.4\end{array}$ & $3-30 \times 10-19$ & 2.20 & 0.15 & 0.33 & $0.31-0.39$ & 55 \\
\hline $\begin{array}{l}\text { U12G RNMA9 } \\
16.2-17.5\end{array}$ & -- & 2.20 & 0.23 & 0.40 & -- & 44 \\
\hline G1-2290 & $9-20 \times 10^{-19}$ & -- & $0.19-0.25$ & -- & 0.33 & 33 \\
\hline G1-2790 & -- & 2.54 & 0.14 & 0.20 & - & 33 \\
\hline $\mathrm{YM}-45$ & $>2.5 \times 10^{-17}$ & 2.53 & 0.18 & 0.21 & 0.21 & 16 \\
\hline$G 1-2333$ & $>2.5 \times 10^{-17}$ & 2.68 & 0.29 & 0.37 & 0.35 & 23 \\
\hline G1-3116 & $1-4 \times 10^{-19}$ & 2.48 & 0.23 & 0.27 & 0.26 & 16 \\
\hline
\end{tabular}

${ }_{A}$ greater amount of porosity may be present in $>0.1-\mu m$ pores. These pores would not be measured by nercury porosimetry because the mercury could not pass through $<0.1-\mu m$ pores to reach the larger pores. $b_{\text {Total }}$ porosity calculated from grain density measurements.

Total porosity calculated from weight loss after drying the water-saturated samples. 
differs from the others in that it contains a high proportion of lithic fragments and is 20 to $40 \%$ analcime. The reason for the low permeability, however, is unknown. There is good agreement between the porosity calculated from grain density measurements and those determined from wet and dry weights of the samples. This correlation is important because it indicates that all the porosity is filled with water during the permeability measurements.

Table XLV summarizes data for permeability, porosity, and storage capacity for "before" and "after" samples from a 5.5-month "soak test" at $120^{\circ} \mathrm{C}$. The before and after samples, although closely associated, are physically different samples; therefore, some initial differences are to be expected. The porosity differences that exist between before and after samples are certainly not the result of the soak test, because the physical dimensions of the samples did not change, nor did the mineralogy change appreciably. The only definite change was in sample G1-1087.3-1087.8: the permeability increased by a factor of about 4 over the course of the soak test. A similar but smaller change may

TABLE XLV

PERMEABILITY, STORAGE CAPACITY, AND PORÓSITY

FOR TUFF SOAK TESTS

\begin{tabular}{|c|c|c|c|}
\hline Sample & $\begin{array}{l}\text { Permeability } \\
\left(\mathrm{m}^{2}\right) \\
\end{array}$ & $\begin{array}{c}\text { Storage Capacity } \\
(1 / \mathrm{MPa}) \\
\end{array}$ & Porosity \\
\hline \multicolumn{4}{|l|}{ G1-1087.3-1087.8 } \\
\hline Before & $1.5 \times 10^{-19}$ & $1.1 \times 10^{-4}$ & 0.11 \\
\hline After & $6.0 \times 10^{-19}$ & $1.1 \times 10^{-4}$ & 0.14 \\
\hline
\end{tabular}

G1-1100.6-1101.6

Before

$2.3 \times 10^{-19}$

$1.4 \times 10^{-4}$

0.10

After

$4.5 \times 10^{-19}$

$6.2 \times 10^{-5}$

0.08

G1-1370.8-1371.4

Before $8.6 \times 10^{-19}$

After

$9.1 \times 10^{-19}$

$3.4 \times 10^{-4}$

0.21

$3.0 \times 10^{-4}$

0.26

G1-1640.9-1642.1

Before

$1.4 \times 10^{-18}$

$3.6 \times 10^{-4}$

0.29

After

$1.5 \times 10^{-18}$

$2.7 \times 10^{-18}$

0.29 
have taken place in G1-1100.6-1101.6. The mechanism by which the permeability increases is unclear; however, the mineralogy of the unchanged samples is dorinated by clinoptilolite. Although no changes were noted in the mineralogy of any of the samples, it seems likely that some small change did occur because the samples showing a change in permeability are those with a mineral composition that is unstable under soak-test conditions.

The storage capacity measurements for the soak test samples are shown in Figs. 65-68. Storage capacity is the additional volume of fluid that can be stored in a unit volume of rock by a unit increase in pore fluid pressure; it is a function of both the porosity and the compressibility of the pores. When fluid pressure in the pores is increased, the fluid is compressed and the pores are enlarged, thereby increasing capacity. The porosity and storage capacity numbers taken together indicate that approximately half the storage is the result of fluid compression and half from pore compressibility. Over the range of effective confining pressure from 0 to $30 \mathrm{MPa}$, which should cover conditions in a repository at Yucca Mountain, pore compression results in a negligible change in porosity.

The permeability of tuff is quite low. This contrasts with the relatively high porosity ( 7 to $40 \%$ ) of the samples tested. Porosity clearly does not determine permeability because there is no correlation between the two. Mercury porosimetry indicates the permeability is more closely related to pore size. In tuffs, low permeability is probably the result of small connections between larger pores. This suggests that the constrictivity of tuff may be relatively large.

Permeability shows only a slight variation with effective confining pressure. The storage capacity and porosity values taken together indicate that the pore compressibility of tuff is quite small, and as a consequence, the porosity of tuff also varies little with effective confining pressure. Because the permeability does not show a marked decrease with increasing effective confining pressure, it seems likely that the stiffness of the pores indicated by pore compressibility applies to the small connections as well as the larger pores that make up most of the porosity. Therefore, studies of tuff pore structure made on unpressurized samples should apply well to the rock at depth.

Permeability measurements on samples before and after heating wet at $120^{\circ} \mathrm{C}$ for 5.5 months show no significant change in permeability in tuffs 


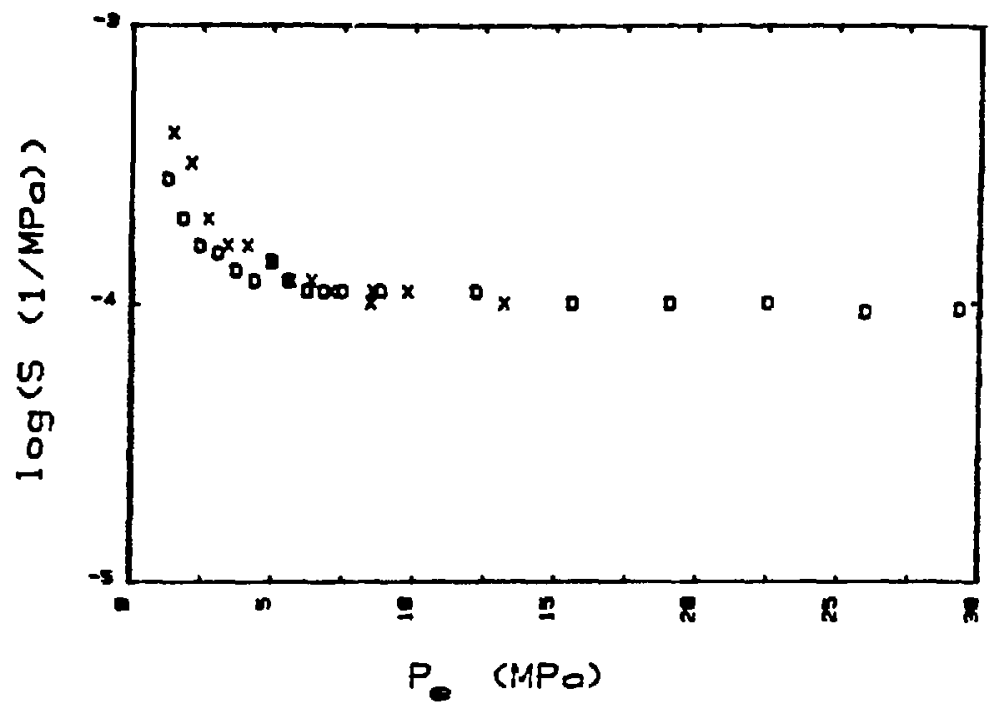

Fig. 65. Storage capacity (S) of sample 1087.3-1087.8 as a function of effective confining pressure $\left(P_{e}\right)$. $X$ and $O$ represent the before and after sample, respectively.

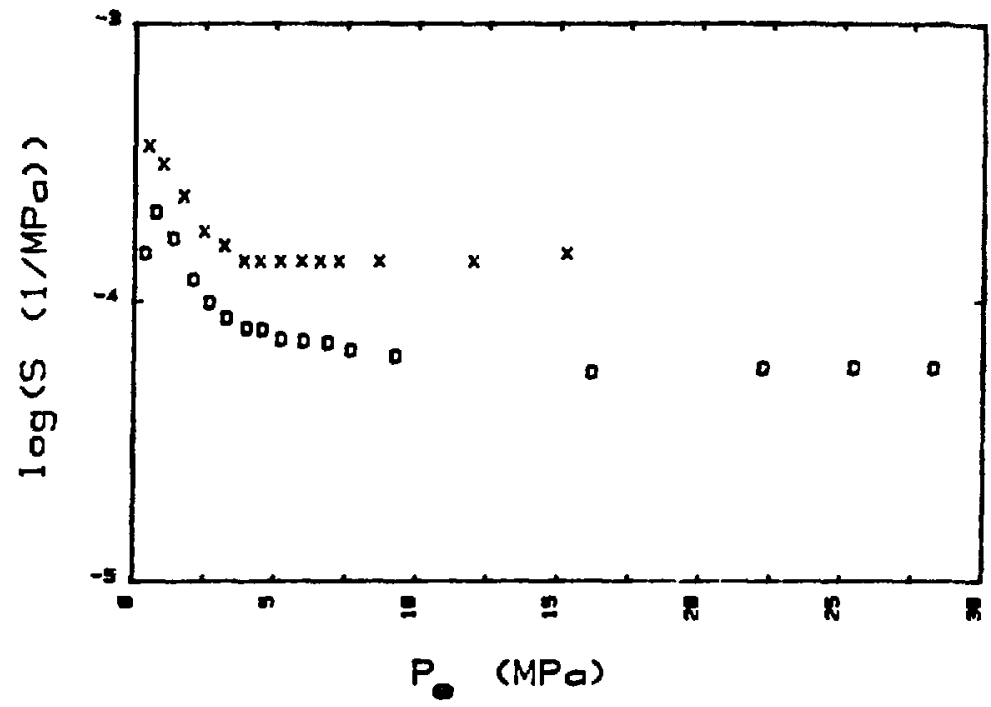

Fig. 66. Storage capacity (S) of sample 1100.6-1101.6 as a function of effective confining pressure $\left(P_{e}\right) . X$ and $O$ represent the before and after sample, respectively. 


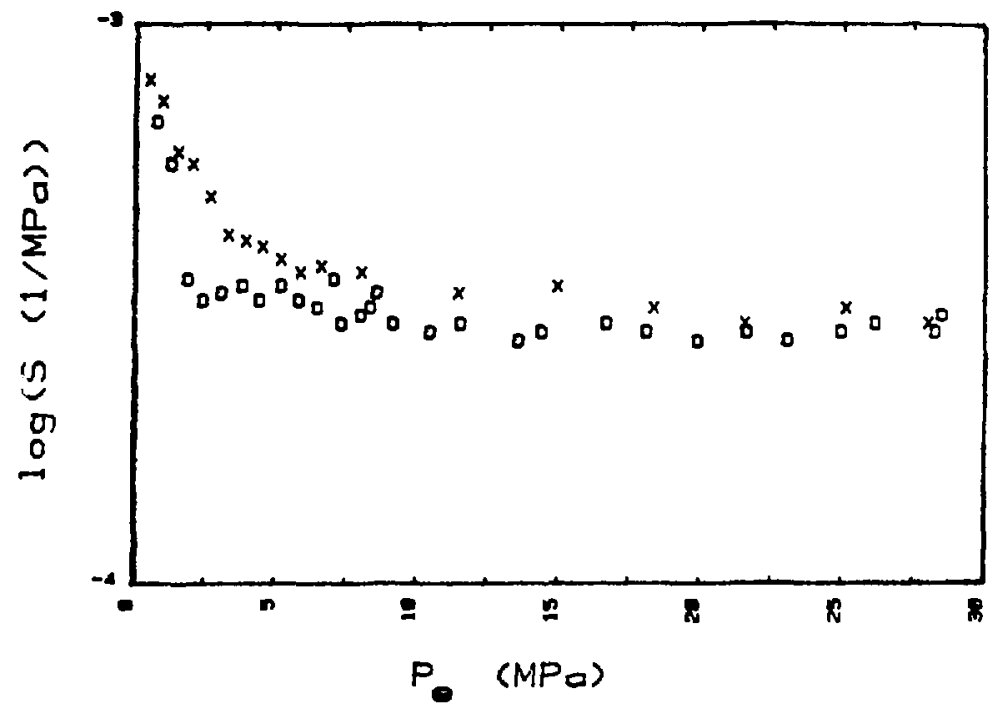

Fig. 67. Storage capacity (S) of sample 1370.8-1371.4 as a function of effective confining pressure $\left(P_{e}\right) . X$ and 0 represent the before and after sample, respectively.

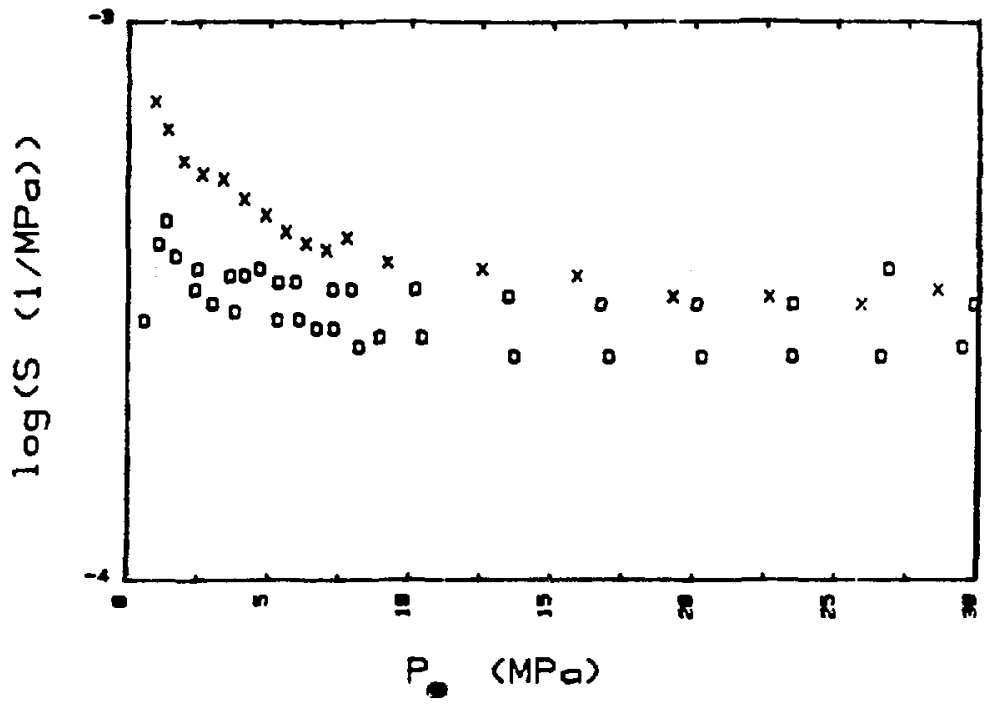

Fig. 68. Storage capacity (S) of sample 1640.9-1642.1 as a function of effective confining pressure $\left(P_{e}\right)$. $X$ and $O$ represent the before and after sample, respectively. 
composed predominantly of zeolites. However, some increase in permeability was observed in samples dominated by quartz, cristobalite, and potassium feldspar.

\section{Diffusion Measurements}

Diaphragm diffusion cells (Fig. 69) were designed and constructed to directly measure diffusion through disks cut from the tuffs. The diffusion experiments are performed by cementing a tuff disk, 1 in. in diameter by $0.25 \mathrm{in}$. thick, into the membrane-holding disk of the cell. A solution with a high concentration of the diffusing species is placed in the lower reservoir, and a solution of lower concentration is placed in the upper reservoir. The resulting concentration gradient causes molecular diffusion through the tuff disk. All solutions used in the tuff diffusion experiments are prepared using water from well $\mathrm{J}-13$ as the solvent.

The concentration of the diffusing species is continuously monitored in the upper reservoir using the pumping and detection system shown in Fig. 70 . The detector consists of a Plexiglas flow-through cell into which a bromide ion-selective electrode and a reference electrode are inserted. The solution from the upper reservoir is pumped through the cell with a peristaltic pump. Originally, a liquid chromatograph pump was used, but the peristaltic pump is simpler to use and more reliable. A pH-millivolt meter measures the output from the ion-selective electrode, and an analog signal is recorded on a chart recorder.

Two diffusion experiments have been performed on sample U12G-RNM9 and one on sample G1-2290 by using solutions of NaBr in J-13 well water. The tuff disks were soaked in the $\mathrm{NaBr}$ solution for several days; then the lower reservoir was filled with the $\mathrm{NaBr}$ solution and the tuff was sealed in place in the diffusion cel1. Vacuum was applied to the upper reservoir to remove air from the disk and to initiate a flow of solution through the disk. Several hours were required to draw a few milliliters of solution through the disk. An additional quantity of $\mathrm{NaBr}$ solution was then poured into the upper reservoir, and the cell was placed in a constant temperature bath at $25.0 \pm 0.1^{\circ} \mathrm{C}$ for several hours before the test. This procedure was designed to ensure that the pure fluid in the tuff disk was in equilibrium with the solution in the lower reservoir at the beginning of the test. The tests were initiated by removing all or part of the solution in the upper reservoir and replacing it with J-13 well water. 


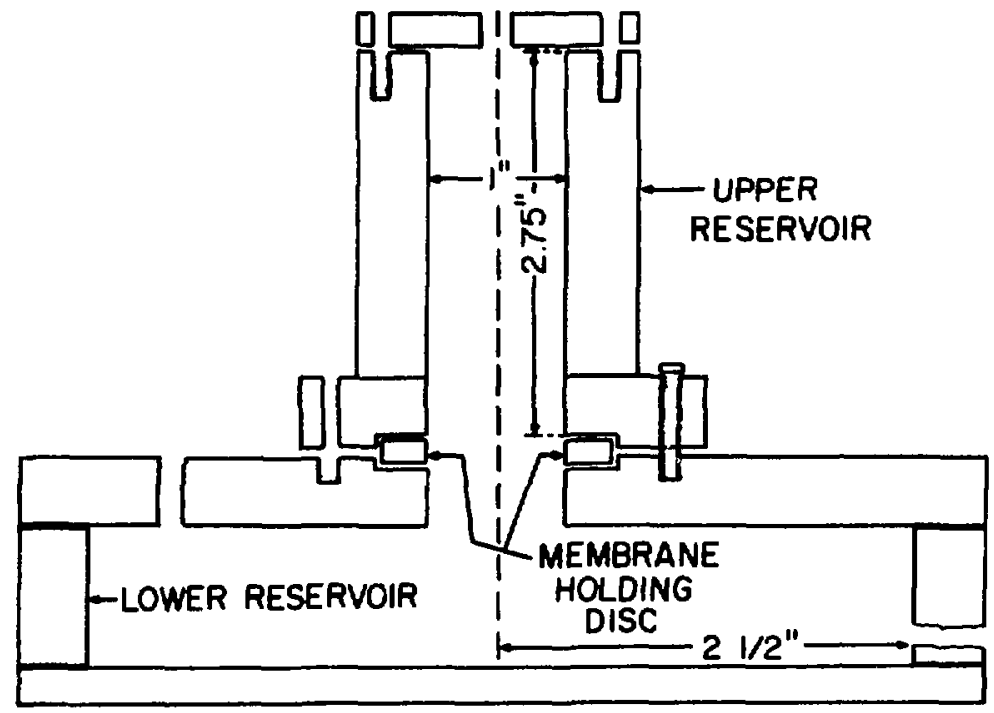

Fig. 69. Plexiglas diaphragm diffusion cell.

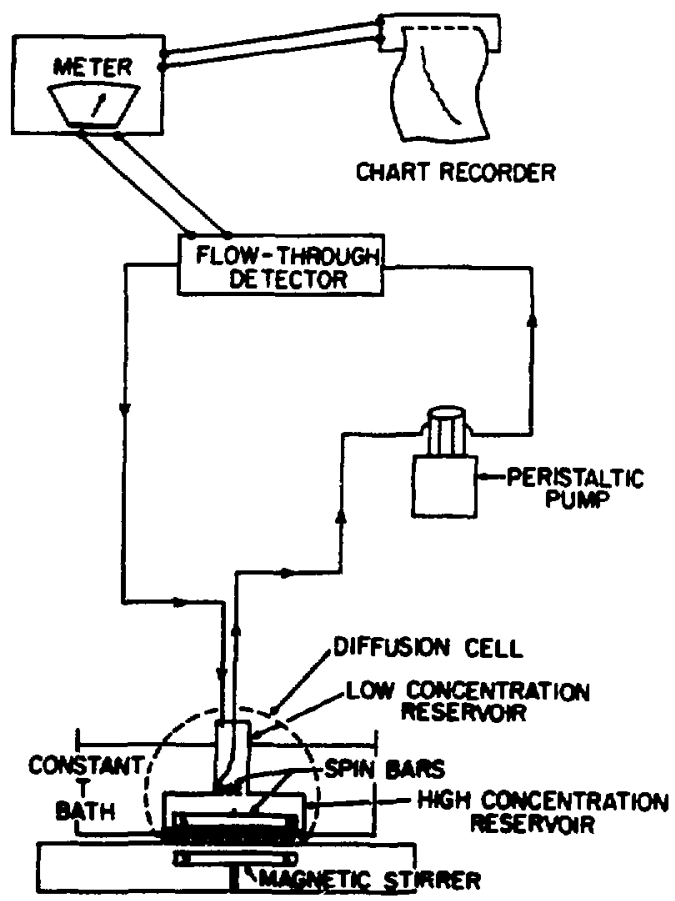

Fig. 70. Schenatic of the pumping and detection system of the diffusion cell. 
The resulting tine vs concentration data are analyzed using the steadystate method, 57 which employs only data taken after a sufficient time has elapsed to establish an approximately linear concentration gradient across the disk. When such a gradient is established, the average diffusion coefficient is given by

$$
\bar{D}_{e}=\frac{1}{\beta t} \ln \Delta C^{\hbar}
$$

where

$$
\begin{aligned}
\vec{D}_{e}= & \text { the average effective diffusion coefficient, } \\
\beta= & \text { a cell constant dependent on the surface area and thickness } \\
& \text { of the membrane and the volumes of the upper and lower reservoirs, } \\
t= & \text { the time, and } \\
\Delta C^{*}= & \text { the ratio of the differences between the reservoir concentrations } \\
& \text { at time } t, \text { when a linear concentration gradient has been established, } \\
& \text { and at time } t .
\end{aligned}
$$

To compute $\Delta C^{\frac{1}{n}}$, both the upper and lower reservoir concentrations must be known. The upper reservoir concentration is measured, and the lower reservoir concentration is computed from the change in upper reservoir concentration. In practice, the lower reservoir concentration changes by $<1 \%$ during the course of the experiments.

To apply the steady-state method, a time must be selected after which a linear concentration gradient is assumed. This time is determined by plotting 1n $\Delta C^{*}$ vs time, as shown in Fig. 71 for sample U12G-RMM9; here the In $\Delta C^{*}$ becomes linear in $t$ after $\sim 400$ minutes. Regression analysis is then used to compute $\mathrm{D}_{e}^{-}$ from the linear portion of the curve.

Eventually, the modified analytical solution of Spacek and Kubin ${ }^{58}$ will be used to analyze the nonlinear portion of the curve. Use of the nonlinear solution requires a special computer program that is not yet complete.

Using the techniques described above, the first diffusion test on sample U12G-RNM9 yielded an effective diffusion coefficient of $(7.6 \pm 0.2) \times$ $10^{-7} \mathrm{~cm}^{2} /$ second. The second test on the same disk, performed 2 weeks later, yielded a value of $(3.70 \pm 0.03) \times 10^{-6} \mathrm{~cm}^{2} /$ second. The first test was performed with $0.02 \mathrm{M} \mathrm{NaBr}$ and the second with $0.05 \mathrm{M} \mathrm{NaBr}$. The higher diffusion coefficient from the second test may be the result of sealant deterioration around the tuff disk or deterioration of the tuff disk itself. 


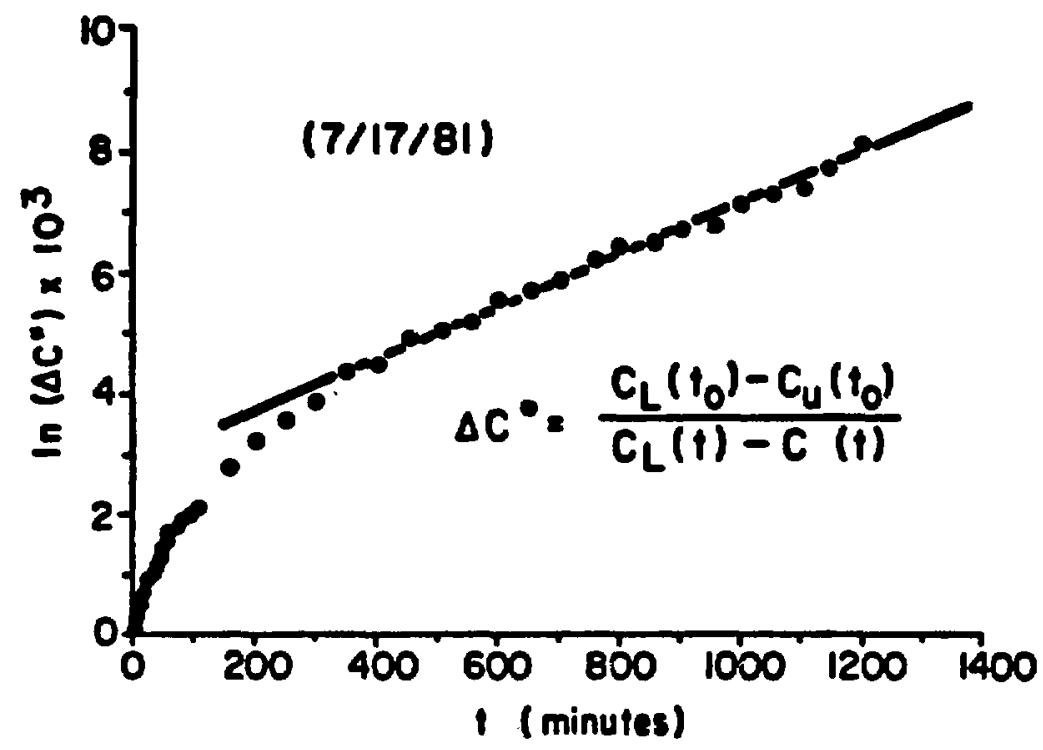

Fig. 71. Time vs concentration curve from a diffusion test on sample U12G-RNM9 .

The diffusion test performed on sample G1-2290 yielded an effective diffusion coefficient of $(4.0 \pm 0.6) \times 10^{-7} \mathrm{~cm}^{2} /$ second. The NaBr concentration used in this experiment was $0.05 \mathrm{M}$. The ratio of the effective diffusion coefficient to the free aqueous diffusion coefficient is $\sim 3 \times 10^{-2}$. Porter et al. 59 have related the effective diffusion coefficient to the free aqueous diffusion coefficient by the equation

where

$$
D_{\text {eff }}=\alpha \varepsilon\left(L / L_{e}\right)^{2} D \text {, }
$$

$D_{\text {eff }}=$ the effective diffusion coefficient,

$\alpha=$ an empirical correction factor, often called the constrictivity,

$\varepsilon \quad=$ the porosity, and

$L / L_{e}=$ the macroscopic diffusion length over the effective diffusion length ( $1 /$ tortuosity).*

$* \mathrm{~L}_{\mathrm{e}} / \mathrm{L}$ is the same as $\tau$ used in Sec. IV.C. 3 of this report. 
Using a porosity of 0.28 and a tortuosity of 0.70 , is $\alpha$ computed to be $\sim 0.2$ for the G1-2290 sample. The factor $\alpha$ has been interpreted as a measure of ionic interactions with the electric double layer on the matrix grains and changes in pore-fluid viscosity in small pores. If $\alpha$ is indeed a measure of ion-surface interactions, then it should be a function of the ionic strength of the test solutions. Double-iayer theory predicts that $\alpha$ should decrease with decreasing ionic strength. At the ionic strength of well $\mathrm{J}-13$ water $\left(\sim 5 \times 10^{-3} \mathrm{M}\right), \alpha$ and the effective diffusion coefficient might be considerably lower.

Measurements have also been made of the effective diffusion coefficient of NaBr through tuff samples U12G-RNM9 (5.9 to $6.4 \mathrm{ft}$, Side B, Position A) and YM-45 by using the steady-state technique and the apparatus described above. Table XLVI shows the measured values, and the diffusion curves from which these values were calculated are shown in Figs. 72 and 73 . The variation of the observed diffusion curve from the regression line in these figures appears to be caused primarily by diurnal temperature variation in the laboratory, which affects the output from the ion-selective electrode. This problem will be corrected by continuously monitoring the solution temperature near the electrodes that are using the data acquisition computer and by applying a temperature correction to millivolt readings. The ratio of the effective diffusion coefficient to the free aqueous diffusion coefficient for $\mathrm{NaBr}$ is also listed in Table XLVI. The difference in the effective diffusion coefficients between these two samples may in part be explained by the differences in their median pore diameters. The median pore diameter of U12G-RNM9B is $\sim 0.62 \mu \mathrm{m}$, whereas that of $\mathrm{YM}-45$ is $\sim 1.7 \mu \mathrm{m}$ (Fig. 50); their measured porosities, however, are nearly the same.

TABLE XLVI

EFFECTIVE DIFFUSION COEFFICIENTS FOR NaBr

\begin{tabular}{|c|c|c|c|}
\hline Sample & $\begin{array}{c}\text { Initial } \\
\text { Concentration (M) } \\
\end{array}$ & $D_{e}\left(\mathrm{~cm}^{2} / \mathrm{s}\right)$ & $\mathrm{D}_{\mathrm{e} / \mathrm{D}_{\mathrm{o}}^{\mathrm{a}}}$ \\
\hline $\begin{array}{l}\text { U12G-RNM9 } \\
(5.9 \text { to } 6.4 \mathrm{ft} .)\end{array}$ & $4.95 \times 10^{-2}$ & $1.15 \times 10^{-6} \pm 1.8 \times 10^{-8}$ & 0.07 \\
\hline YM-45 & $4.89 \times 10^{-2}$ & $1.93 \times 10^{-6} \pm 4.1 \times 10^{-8}$ & 0.12 \\
\hline
\end{tabular}

${ }^{a_{D_{0}}}$ is the free aqueous diffusion coefficient of NaBr. 


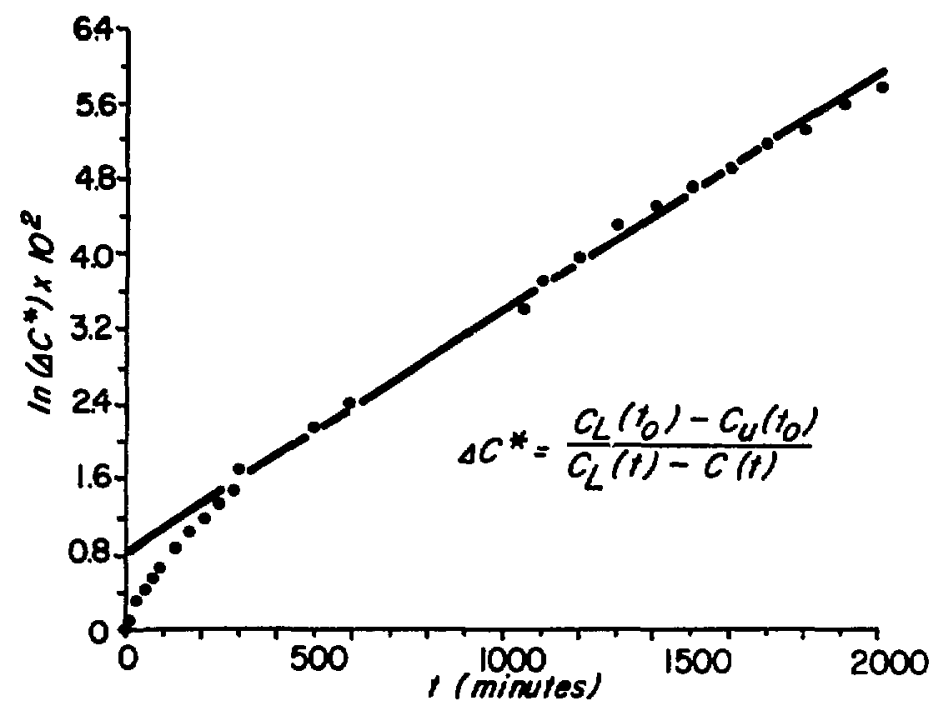

Fig. 72. Time vs concentration curve from a diffusion test on sample U12G-RNM9 (5.9 to $6.4 \mathrm{ft}$, Side B).

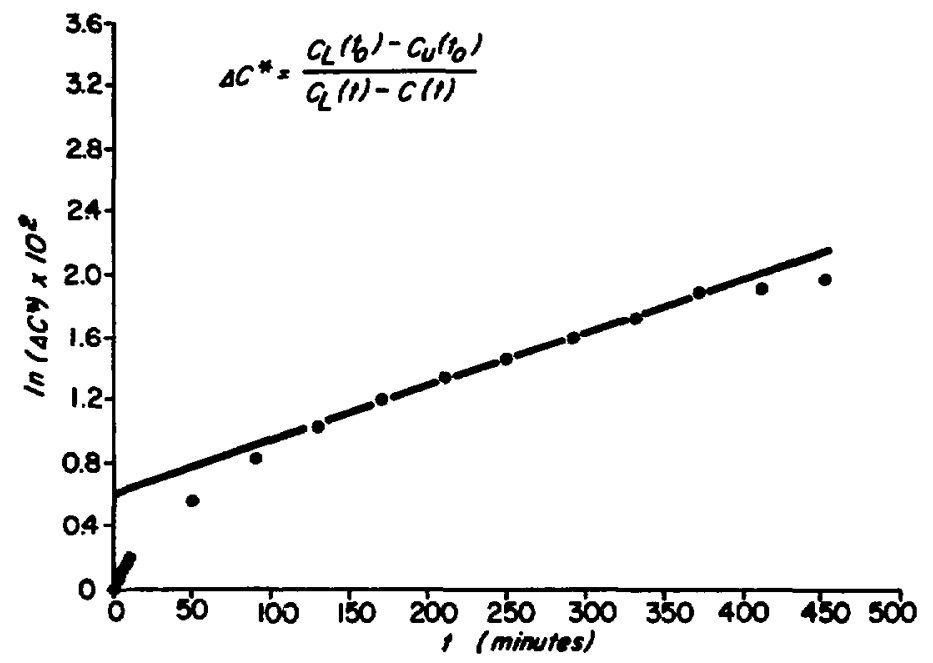

Fig. 73. Time vs concentration curve from a diffusion test on sample MM-45. 
Fluorobenzoate anions are being considered as tracers for field studies. Determination of the free aqueous diffusion coefficients of the fluorobenzorte tracers has been approached in two ways. First, the limiting ionic conductances of the anions have been measured and used to compute the diffusion coefficients at infinite dilution. Second, efforts are underway to directly measure the diffusion coefficients in concentrated solutions, using the diaphragm diffusion cells described earlier. The limiting ionic conductance of an ion is defined as its equilvalent ionic conductance at infinite dilution. These values for the fluorobenzoate anions were determined by measuring the molar conductances of their sodium and potassium salts at various concentrations. For strong electrolytes such as these, the molar conductance is described by the empirical equation 60

$$
\Lambda=\Lambda_{0}^{+}-k_{c} c^{1 / 2}
$$

where

$$
\begin{aligned}
& \Lambda^{+}=\text {the molar conductance, } \\
& \Lambda_{0}^{+}=\text {the molar conductance at infinite dilution, } \\
& \mathbf{k}_{c}=\text { an experimental constant, and } \\
& c=\text { the molar concentration. }
\end{aligned}
$$

The sodium and potassium salts of the fluorobenzoic acids were prepared by titrating the acids with the appropriate base to the equivalence points. The resulting salt solution was then used to prepare more dilute solutions. The conductivities of these solutions were then measured with the apparatus shown in Fig. 74; the temperature bath was set at $25 \pm 0.05^{\circ} \mathrm{C}$. The values of $\wedge_{0}$ for the salt solutions were determined by regression techniques. The limiting ionic conductance of the anion was then computed from the Kohlrausch's Law of the Independent Migration of Ions, 60

$$
\Lambda_{0}=\Lambda_{0}^{+}+\Lambda_{0}^{-}
$$

where $\Lambda_{0}^{+}$values for sodium and potassium ions are known.

The resulting values for the limiting ionic conductances and computed diffusion coefficients are listed in Table XLVII. Based on the differences between the measured and the published values for benzoate and $p$-fluorobenzoate, 


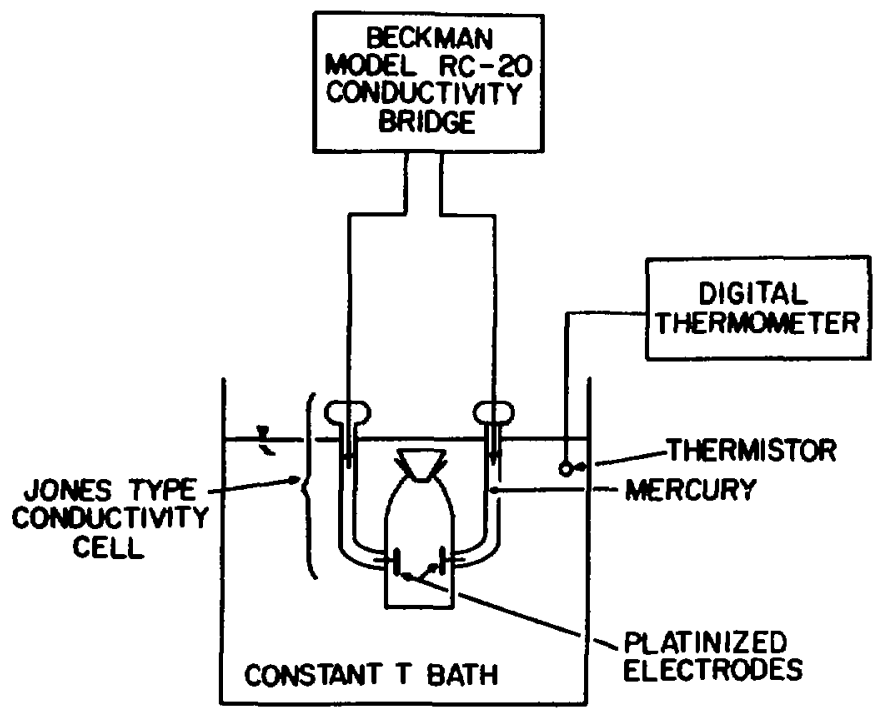

Fig. 74. Apparatus used for electrical conductivity measurements.

TABLE XLVII

LIMITING IONIC CONDUCTANCES OF BENZOATE AND

FLUOROBENZOATE IONS AND COMPUTED DIFFUSION COEFFICIENTS

$\Lambda_{0}\left(\frac{\mathrm{cm}^{2}}{\mathrm{ohm}-\mathrm{eq} \cdot}\right)$

Species

benzoate

p-fluorobenzoate

m-fluorobenzoate

o-fluorobenzoate

m-trifluoromethylbenzoate

pentafluorobenzoate

\section{Published Measured}

30.8

35.0

30.0

30.5

27.9

27.1

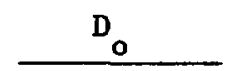

$0.82 \times 10^{-5}$

$0.93 \times 10^{-5}$

$0.80 \times 10^{-5}$

$0.81 \times 10^{-5}$

$0.74 \times 10^{-5}$

$0.72 \times 10^{-5}$ 
the error in the other measured values is estimated to be $\sim 7 \%$. The source of this error is not known, but this level of accuracy seems adequate for these tests.

Considerable effort was devoted to developing procedures that measure diffusion coefficients of the tracers by using the diaphragm diffusion cell. Such experiments are performed by sealing a sintered glass filter into the membrane-holding disk of the diffusion cell. The diffusion experiment is then conducted in the same way as those for tuff diffusion, except that the solvent is distilled water and a flow-through conductivity cell is used for a detector. To measure a diffusion coefficient in this way, diffusion experiments are first done with salts of known diffusion coefficients so that a cell constant can be computed. The cell constant corrects the apparent diffusion coefficient for the area, thickness, tortuosity, and porosity of the glass filter.

In initial experiments with a medium glass filter, an attempt was made to determine a cell constant using $\mathrm{LiCl}, \mathrm{NaBr}$, and $\mathrm{KCl}$ solutions. It was not possible to obtain consistent cell constants using these salts, apparently because of convection through the filter caused by stirring the two reservoirs. Recent experiments using a fine glass filter with $\mathrm{LiCl}$ and $\mathrm{KCl}$ have determined cell constants that agree within $5 \%$. Soon diffusion experiments will begin that use sodium and potassium salts of the fluorobenzoates.

\section{Diffusion into the Rock Matrix. Kinetic sorption experiments} (sorption as a function of time) have been performed on thin tablets of tuff. The uptake of activity has been measured as a function of time for a number of elements on several tuffs. These data should fit the solution for diffusion into a plane sheet if any edge effect is ignored. The diffusion equation for this case is

$$
\frac{\partial C}{\partial t}=D \frac{\partial^{2} c}{\partial x^{2}},
$$

with the initial conditions

$$
c=0,-\ell<x<1 \text {, and } t=0 \text {, }
$$


and with the boundary conditions

a $\frac{\partial C}{\partial t}= \pm D \frac{\partial C}{\partial x} \quad, x= \pm \ell$, and $t>0$,

where

$$
\begin{aligned}
& C=\text { the concentration in solution, } \\
& D=\text { the apparent diffusion coefficient, } \\
& a=\text { the corrected depth of solution, } \\
& l=\text { half the thickness of the tablet (sheet), } \\
& t=\text { the time, and } \\
& x=\text { the position in the sheet. }
\end{aligned}
$$

In other words, the rate of loss of tracer from solution is equal to the rate at which tracer enters the sheet through the surfaces at $\mathrm{x}= \pm l$.

The general solution to this problem is given by Crank: 61

$$
\frac{M_{t}}{M_{\infty}}=1-\sum_{n=1}^{\infty} \frac{2 v(1+v)}{1+v+v^{2} q_{n}^{2}} e^{-D q_{n}^{2} t / l^{2}},
$$

where $M_{t}$ is the amount of solute in the solid phase at time $t$, values of $q_{n}$ are the nonzero positive roots of $\tan q_{n}=-v q_{n}$, and $v=a / k \ell$, the solutionto-solid volume ratio divided by the partition factor $K$. The fractional uptake of activity at equilibrium is given by

$$
\frac{M_{\infty}}{2 \mathrm{aC}_{0}}=\frac{1}{1+\mathrm{V}}
$$

If $V$ is small, $<0.01$, which is the usual case for large $K_{d}$ values, the following approximate relation may be used for early times:

$$
\frac{M_{t}}{M_{\infty}}=1-e^{T / V^{2}} \operatorname{erfc}\left(T / V^{2}\right)^{\frac{1}{2}}
$$

where $\mathrm{T}=\mathrm{Dt} / \mathrm{\ell}^{2}$. A plot of this function, given in Fig. 75 , may be used to calculate the apparent diffusion coefficient, provided the $K_{d}$ value is known. Thus, it should be possible to estimate the constrictivity-tortuosity factor from these experinents. 


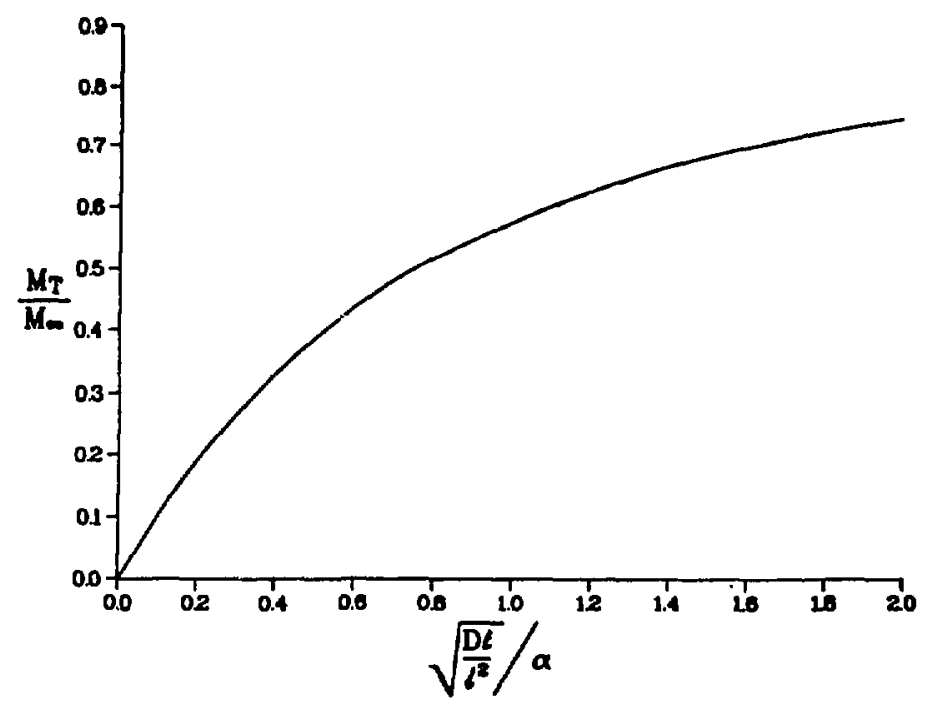

Fig. 75. Uptake of activity by a plane sheet for $V<0.01$.

2. Sorption by Tuff Wafers. For wafers of three tuffs, G1-1436, 61-1883, and G1-1982, the rate of uptake for various radionuclides was studied. The wafers, $0.75 \mathrm{in}$. in diameter, 2 to $3 \mathrm{~mm}$ thick, and 1.1 to $1.4 \mathrm{~g}$ in weight, were suspended on Teflon thread and then placed in $\mathrm{J}-13$ water that had been pretreated as described for batch measurements. After 4 to 27 days, the wafers were removed and added to pretreated groundwater that contained the radionuclides of interest. The rock-to-solution ratio was 1:20. At various times aliquots of solution were removed from the tubes containing the G1-1883 and G1-1982 wafers and counted. The average sorption ratios calculated are given in Tables XLVIII and XLIX; average $R_{d}$ values from batch measurements on washed, crushed fractions of the same tuffs and sorption ratios obtained from elutions are also given in both tables for comparison. The sorption ratios from the wafer experiments and the column results are also in fairly good agreement.

The wafers of G1-1436 tuff were removed, counted, and then returned to the solutions; very few aqueous samples were taken. The sorption data are reported as per cent sorbed in Table $L$. These results, particularly at long 
TABLE XLVIII

SORPTION ON WAFERS OF TUFF G1-1883 ${ }^{\mathrm{a}}$

\begin{tabular}{|c|c|c|c|}
\hline \multirow{2}{*}{$\begin{array}{l}\text { Time } \\
\text { (hours) } \\
\end{array}$} & \multicolumn{3}{|c|}{$R_{d}(m \ell / g)$} \\
\hline & $\mathbf{S} r$ & $C_{s}$ & $\mathrm{Ba}$ \\
\hline 2.55 & 0.85 & 11 & 7.5 \\
\hline 4.53 & 8.8 & 25 & 20 \\
\hline 6.00 & 11 & 32 & 26 \\
\hline 10.0 & 18 & 63 & 50 \\
\hline 15.0 & 22 & 75 & 61 \\
\hline 21.1 & 26 & 100 & 87 \\
\hline 24.0 & 27 & 100 & 93 \\
\hline 120 & 24 & 190 & 130 \\
\hline 144 & 25 & 210 & 140 \\
\hline 192 & 26 & 230 & 140 \\
\hline 305 & 24 & 230 & 150 \\
\hline (504) & (22) & $(190)$ & $(180)$ \\
\hline 869 & 40 & 230 & 210 \\
\hline Column] $]^{b}$ & {$[14 ; 28]$} & {$[129 ; 283]$} & {$[85 ; 162]$} \\
\hline
\end{tabular}

${ }^{a_{a}}$ The numbers in parentheses are the contact time and average $R_{d}$ values from
batch measurements with washed fractions $>38 \mu \mathrm{m}$.

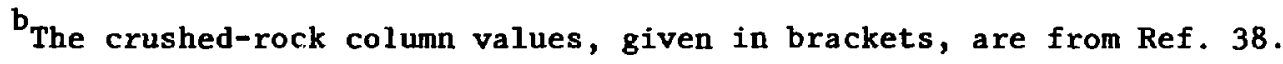

contact times, are subject to considerable error, as is indicated by yields of "120\%." Desorption of the Gl-1436 wafers was monitored by counting aliquots of solution. Table LI gives the sorption ratios for desorption times of 0.083 to 526 hours. Agreement with batch desorption data for strontium and cesium is good; desorption ratios for ${ }^{133} \mathrm{Ba}$ and ${ }^{152}$ Eu were not calculated because neither activity was detected in the 1-m

Technetium and iodine were contacted with other G1-1436 wafers (see Tables LII and LIII). Approximately $0.9 \%$ of the initial untraced solution was taken up by the dry wafers on pretreating, which indicates that if there were complete exchange with the ${ }^{95} \mathrm{Tc} /{ }^{131}$ I-traced water, $\sim 0.9 \%$ of the radionuclides should be found with the wafer. After $\sim 50$ hours, there appeared to be even more iodide than that amount on the wafer, which indicates sorption. However, the 
TABLE XLIX

SORPTION ON WAFERS OF TUFF G1-1982 ${ }^{\mathrm{a}}$

\begin{tabular}{|c|c|c|c|}
\hline \multirow{2}{*}{$\begin{array}{l}\text { Time } \\
\text { (hours) } \\
\end{array}$} & \multicolumn{3}{|c|}{$R_{d}(m \ell / g)$} \\
\hline & $\mathrm{Sr}$ & Cs & $\mathrm{Ba}$ \\
\hline 5.0 & 17 & 65 & 45 \\
\hline 7.0 & 30 & 110 & 78 \\
\hline 12.0 & 36 & 180 & 130 \\
\hline 16.0 & 45 & 190 & 170 \\
\hline 112 & 84 & 620 & 580 \\
\hline 136 & 86 & 670 & 560 \\
\hline 190 & 88 & 760 & 560 \\
\hline 303 & 84 & 750 & 650 \\
\hline (336) & (53) & $(1120)$ & $(670)$ \\
\hline$(504)$ & $(62)$ & $(1200)$ & $(800)$ \\
\hline 860 & 80 & 1000 & 710 \\
\hline [Column] $^{\mathbf{b}}$ & {$[53]$} & {$[1350-1720]$} & [483] \\
\hline
\end{tabular}

${ }^{a}$ The numbers in parentheses are the contact time and average $R_{d}$ values from batch measurements with washed fractions $>38 \mu \mathrm{m}$.

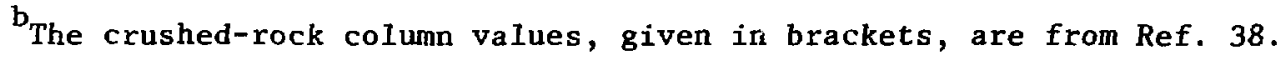

counting statistics were quite poor because of the decay of the ${ }^{131} \mathrm{I}$, so the experiment was repeated with a larger amount of ${ }^{131} \mathrm{I}$. At 721 hours no sorption was observed, and $\sim 98 \%$ of the water in the pretreated (saturated) rock apparently had exchanged with the water that was traced with ${ }^{131_{I}} \mathrm{I}$. The amount of ${ }^{131} \mathrm{I}$ expected on the wafers as a result of exchange with the saturated rock was $0.70 \%$; $0.69 \%$ was observed. A crushed-rock column of $\mathrm{G} 1-1436$, run at $11 \mathrm{~m} /$ year, also gave no indication of ${ }^{131} \mathrm{I}^{-}$sorption; in fact, iodide exclusion was observed: the iodide was eluted before the water front that was monitored with HTO.

3. Diffusion with Nonlinear Sorption. In general, the equations that have been used to describe fracture flow with matrix diffusion and simple diffusion into tuffaceous rock treated sorption as linear with concentration. 62,63 This approach clearly has a serious deficiency because sorption on nonzeolitized 
TABLE L

SORPTION ON STRONTIUM, CESIUM, BARIUM, AND EUROPIUM

USING TUFF G1-1436 WAFERS

Time

(hours)

0.083

0.25

0.50

1.11

1.57

2.00

4.50

6.50

9.00

14.0

38.0

62.0

182
Ainount Sorbed

(\%)

$\begin{array}{rrrr}{ }^{85} \mathrm{Sr} & { }^{137} \mathrm{Cs} & { }^{133} \mathrm{Ba} & { }^{152} \mathrm{Eu} \\ 10.1 & 18.1 & 8.7 & 1.3 \\ 27.9 & 45.9 & 22.5 & 5.3 \\ 50.3 & 75.0 & 38.0 & 9.2 \\ 74.2 & 98.8 & 53.3 & 12.5 \\ 88.7 & 109 & 61.7 & 16.7 \\ 96.3 & 116 & 66.2 & 21.7 \\ 108 & 122 & 79.2 & 37.3 \\ 110 & 122 & 83.8 & 44.5 \\ 118 & 125 & 92.6 & 49.0 \\ 112 & 12.1 & 91.9 & 61.8 \\ 113 & 120 & 95.9 & 93.3 \\ 113 & 119 & 100 & 97.3 \\ 107 & 119 & 104 & 100.6\end{array}$

TABLE LI

DESORPTION OF TUFF G1-1436 WAFERS $^{\mathrm{a}}$

Time

(hours)

0.083

0.25

0.50

2.0

24

526

21-42 days (Batch)

\begin{tabular}{ll}
\multicolumn{2}{c}{$R_{d}(\mathrm{ml} / \mathrm{g})$} \\
\hline${ }^{85} \mathrm{Sr}$ & $\frac{137 \mathrm{Cs}}{30800(2400)}$ \\
$759000(7500)$ & $26700(1400)$ \\
$326000(25000)$ & $19100(300)$ \\
$125000(57900)$ & $15400(5600)$ \\
$153900(6400)$ & $16400(1270)$ \\
$96500(6300)$ & $14900(2960)$ \\
$87000(13000)$ & $24000(2300)$
\end{tabular}

${ }^{a}$ The standard deviations of the means are given in parentheses. 


\section{TABLE LII \\ SORPTION OF IODINE AND TECHNETIUM ON \\ TUFF G1-1436 WAFERS}

\begin{tabular}{l}
$\begin{array}{c}\text { Time } \\
\text { (hours) }\end{array}$ \\
\hline 0.083 \\
0.25 \\
32 \\
54 \\
86 \\
149 \\
309 \\
569 \\
929 \\
1022
\end{tabular}

Average Sorption ${ }^{a}$

(\%)

\begin{tabular}{cl}
\hline Tc & $\frac{1}{0.052(0.010)}$ \\
$0.047(0.010)$ & $0.123(0.005)$ \\
$0.106(0.007)$ & $0.612(0.111)$ \\
$0.417(0.036)$ & $0.915(0.176)$ \\
$0.542(0.050)$ & $1.99(0.461)$ \\
$0.860(0.083)$ & $1.68(0.400) \quad[R d=1.55]$ \\
$0.598(0.028)$ & $3.02(0.649)$ \\
$0.746(0.054)$ & $4.85(0.719)$ \\
$0.844(0.074)$ & activity decayed \\
$0.646(0.147)$ & activity decayed \\
$0.692(0.138)$ &
\end{tabular}

${ }^{a}$ The standard deviations of the means are given in parentheses.

TABLE LIII

REPEAT OF IODINE SORPTION ON TUFF G1-1436 WAFERS

\begin{tabular}{|c|c|}
\hline $\begin{array}{l}\text { Time } \\
\text { (hours) }\end{array}$ & $\begin{array}{c}\text { Sorption } \\
(\%)\end{array}$ \\
\hline 24 & $0.356(0.041)$ \\
\hline 120 & $0.333(0.019)$ \\
\hline 283 & $0.422(0.041)$ \\
\hline 457 & $0.561(0.048)$ \\
\hline 721 & $0.687(0.061)$ \\
\hline
\end{tabular}

${ }^{a}$ The standard deviations of the means are given in parentheses. 
tuff has already been shown nonlinear. The isotherm measurements ${ }^{3}$ on tuff YM-22 show that sorption of simple cations of strontiun, cesium, and barium gives a Freundlich isotherm exponent <1.0. A nonlinear isotherm complicates the equations for matrix diffusion by giving the diffusion coefficient a concentration dependence that renders the differential equations nonlinear.

The formula for the apparent diffusion coefficient

$$
D_{\text {app }}=\frac{D^{i} \varepsilon\left(\alpha / \tau^{2}\right)}{K_{d} \rho},
$$

where

$$
\begin{aligned}
\mathrm{D}^{i} & =\text { the ionic diffusion coefficient, } \\
\varepsilon & =\text { the porosity, } \\
\alpha / \tau^{2} & =\text { the constrictivity-tortuosity factor, } \\
\mathrm{K}_{\mathrm{d}} & =\text { the distribution coefficient, and } \\
\rho & =\text { the density, }
\end{aligned}
$$

shows how the isotherm affects the diffusion coefficient. If the $K_{d}$ is constant, as in the linear isotherm, the apparent diffusion coefficient remains constant. The dependence of the $K_{d}$ on ion concentration for a Freundlich isotherm is shown in Sec. IV.C. The Freundlich isotherm presents special problems for the diffusion equations because for a Freundlich isotherm parameter $<1$ the $\mathrm{K}_{\mathrm{d}}$ is infinity for a concentration of zero and the apparent diffusion coefficient is zero. In a finite-difference analysis of the problem, no diffusion can occur unless the initial concentration in the rock is greater than zero. This means that either an arbitrary cutoff must be given to the Freundlich isotherm, below which it becomes linear, or the initial conditions must be altered to arrive at a solution. This problem does not exist for the Langmuir isotherm, and its applicability to these data is now being investigated.

Another phenomenon that leads to a nonlinear diffusion equation is fixation, where some fraction of the ions are irreversibly fixed in the matrix. This problem is nearly identical to that presented by Crank ${ }^{61}$ for simultaneous diffusion with a bimolecular reaction.

A computer program that uses the finite difference method is being developed to apply some of those mechanisms to matrix diffusion. Eventually, the program 
will be incorporated into a transport model so that a more realistic model can be developed.

\section{Flow Studies}

1. Fracture Flow. Experiments comparing radionuclide transport by fracture flow in tuff and granite have provided a test of the validity of some simple models: in particular, the matrix diffusion model of Neretnieks. ${ }^{62}$ Some simple straight-flow experiments were designed so that the results could be compared with the model. A system based on the permeability apparatus of Brace et al. 64 was employed. Tuff and granite cores $2.54 \mathrm{~cm}$ in diameter were fractured lengthwise and placed in a Teflon sleeve; confining pressure was applied to control the aperture. Two $5-\mathrm{cm}$ cores of welded tuff from the Bullfrog (G1-2335) and Tram (G1-2840) Members were fractured by using a Brazil load test. The G1-2840 core was placed in the permeability apparatus at a confining pressure of 3000 psi. The permeability at this pressure was 3.3 microdarcys, which is somewhat less than that measured for similar cores not containing fractures (Sec. IV.B). Therefore, fracture flow independent of porous flow was not established. The confining pressure was lowered to $1000 \mathrm{psi}$ without a significant increase in permeability, and the Teflon sleeve would not seal at pressures $<1000$ psi.

This particular technique is not suitable for fracture flow experiments with tuff in the laboratory; however, similar experiments have been performed with granite, which is not as plastic as tuff. These experiments are relevant in that they will be used to validate the models that will be applied to the tuff; granite, of course, has a much lower porosity, which decreases the effect of matrix diffusion relative to that of tuff. The elution of ${ }^{85} \mathrm{Sr}$ and ${ }^{137} \mathrm{Cs}$ was observed in flow through fractured Climax Stock (CS) granite. The breakthrough curves were compared with predicted curves for analytic solution flowing through a one-dimensional fracture that is coupled to diffusion into the matrix. 62 The fracture volume and aperture, in particular, were determined by using Darcy's law. The experiments were performed on small cores under confining pressure to simulate depth and to close the fracture. The flow through the fracture was straight flow, which allowed a direct comparison with the one-dimensional calculations.

The flow of fluid through a fracture can be described by the Darcy equation or cubic law 


$$
\frac{Q}{\Delta h}=c(2 b)^{3},
$$

where

$$
\begin{aligned}
& Q=\text { the flow rate in } \mathrm{m}^{3} / \text { second, } \\
& \Delta \mathrm{h}=\text { the hydraulic head in meters of water, } \\
& 2 \mathrm{~b}=\text { the aperture, and } \\
& \mathrm{C}=\mathrm{a} \text { constant for a given geometry. }
\end{aligned}
$$

For straight flow

$$
C=\frac{W}{L} \frac{\rho g}{12 \mu},
$$

where

$$
\begin{aligned}
& \mathrm{L}=\text { the length of the fracture, } \\
& \mathbf{W}=\text { the width, } \\
& \boldsymbol{\rho}=\text { the density of the fluid, } \\
& \mathbf{g}=\text { the gravitational constant, did } \\
& \mu=\text { the viscosity of the fluid. }
\end{aligned}
$$

The validity of Eq. (10) has been demonstrated by Witherspoon et al. 65 Some fractures require a correction factor for the effect of surface roughness on the flow; such a factor $f$ was defined by Witherspoon et al. ${ }^{65}$ and inserted into the Darcy equation:

$$
\frac{Q}{\Delta h}=\frac{C}{f}(2 b)^{3}
$$

Values of $f$ varied from 1.04 to 1.21 in a granite fracture with straight flow. Assuming $f=1.00$ rather than 1.21 , however, would result in only a $7 \%$ error in the aperture.

Two small granite cores $2.54 \mathrm{~cm}$ in diameter by $1.59 \mathrm{~cm}$ long were used in the experiments. Core No, 1 had a natural fracture that appeared to be filled, and it was mechanically opened before the experiment. Core No. 2 contained no jatural fractures but was stressed to induce one. Each core was placed in a 
Teflon sleeve, where it remained throughout the experiment, and was stressed. Each core was then placed in a modified permeability apparatus similar to that of Brace et al. 64 The apertures were determined by measuring the flow rate vs the hydraulic head. Table LIV summarizes the results.

Tl, aperture of core No. 1 (with a natural fracture) was in good agreement with the measurements of Witherspoon et al. ${ }^{65,66}$ and Isherwood. "The stressinduced fracture, however, had an unusually large residual aperture that may have been a result of granite grains being lodged in the fracture, which prevented proper mating of the rock surfaces. Alternatively, there may be fractures in planes other than the principal fracture plane, which were not apparent before the experiment and which extend through the core.

The transport of radionuclides by flow through a single fracture has been solved analytically for a one-dimensional fracture with matrix diffusion. 62 This model does not include velocity dispersion but should serve well as a first approximation to the experiment and as a benchmark for numerical code development. The effect of matrix porosity on the transport of radionuclides was clearly demonstrated by Neretnieks. 62

The following definitions are used in the discussion.

(1) The volumetric sorption ratio $k_{d} \rho$ is given by

$$
K_{d} \rho=\varepsilon_{p}+\left(1-\varepsilon_{p}\right) K_{d}^{\rho} \rho_{s},
$$

where

$$
\begin{aligned}
& K_{d}^{\prime}=\text { the distribution coefficient }\left(K_{d}^{\prime}=0\right. \text { for a nonsorbing } \\
& \text { material }), \\
& \varepsilon_{p}=\text { the matrix porosity, and } \\
& \rho_{s}=\text { the density of the solid. }
\end{aligned}
$$

(2) The effective diffusion coefficient is given by

$$
D_{\text {eff }}=\varepsilon_{p} \frac{\alpha}{\tau} D^{i}
$$

*Information supplied by D. Isherwood, Lawrence Livermore National Laboratory, Livermore, CA, 94550 (June 1981). 
TABLE LIV

APERTURES OF GRANITE FRACTURES UNDER STRESS

CS granite core No. 1

CS granite core No. 2

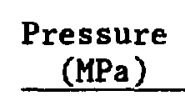

24.8

35.9

27.6

13.8

Isherwood $^{a}$

21

16

10

Witherspoon et al. ${ }^{b}$ run No. 1
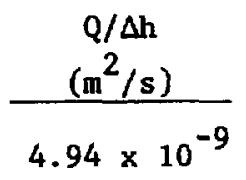

$2.15 \times 10^{-8}$

$2.01 \times 10^{-8}$

$2.47 \times 10^{-8}$

$1.84 \times 10^{-9}$

$3.56 \times 10^{-9}$

$1.23 \times 10^{-9}$
$2 b$

( $\mu \mathrm{m})$

15.6

25.4

24.8

26.6

13.6

17.0

25.6
$4.08 \times 10^{-10}$
$8.3 \times 10^{-10}$
$1.14 \times 10^{-9}$
17.0
7.7
12.5
9.7
8.0
10.8

${ }^{a}$ From information supplied by D. Isherwood, Lawrence Livermore National Laboratory, Livermore, CA 94550 (June 1981).

${ }^{\mathrm{b}} \operatorname{Ref.} 65$.

where

$$
\begin{aligned}
& \mathrm{D}^{i}=\text { the ionic diffusion coefficient, } \\
& \alpha=\text { the constrictivity of the pores, and } \\
& \tau=\text { the torcuosity of the pores. }
\end{aligned}
$$

(3) The apparent diffusion coefficient is given by

$$
D_{\text {app }}=\frac{D_{\text {eff }}}{K_{d} \rho}
$$


Transport of radionuclides through a single fracture can be described mathematically by the following expressions.

(1) Diffusion in the rock matrix is given by

$$
\frac{\partial C_{p}}{\partial t}=D_{\text {app }} \frac{\partial^{2} C_{p}}{\partial z^{2}}-\lambda C_{p}
$$

(2) The sorption and convection are given by

$$
\frac{\partial C_{f}}{\partial t}+U_{f} \frac{\partial C_{f}}{\partial x}=\left.\frac{D_{e f f}}{b} \frac{\partial C_{p}}{\partial z}\right|_{z=0}-\lambda C_{f},
$$

where

$C_{p}=$ aqueous concentration in pores,

$c_{f}=$ aqueous concentration in fissures,

$x$ = distance along the fracture,

$\mathbf{z}=$ distance into the matrix from the fracture surface,

$b$ = half-width of the fracture,

$\mathrm{u}_{\mathrm{f}}=$ water velocity, and

$\lambda=$ decay constant.

The solution to these equations with the appropriate boundary and initial conditions for a concentration step of duration $\Delta t$ is

$$
\begin{aligned}
& \frac{C_{p}}{C_{0}}=e^{-\lambda t} \operatorname{erfc}\left(\frac{G}{\left[t_{w}-\left(t_{0}+t\right)\right]^{1 / 2}}\right) \\
& -\operatorname{erfc}\left(\frac{G}{\left[t-\left(t_{w}+t_{0}+\Delta t\right)\right]^{1 / 2}}\right),
\end{aligned}
$$

where

$$
\begin{aligned}
G & =\left[\left(D_{\text {eff }}+\frac{1}{2} \frac{U_{f}(2 b) z}{x}\right) / 2 b\left(D_{a p p}\right)^{1 / 2}\right] \cdot t_{w}, \\
t_{w} & =\text { the time required for the water to reach } x, \text { and } \\
t_{0} & =\text { the iaitial time. }
\end{aligned}
$$


The breakthrough curve can be calculated by using the first error function only. This solution can also be found in Carslaw and Jaeger. 67 The breakthrough curves for core No. 1 were calculated by using the parameters in Table LV that are based on our earilier data. ${ }^{68}$ The porosity of the matrix in the core that was used was not actually determined, and $\varepsilon_{\rho}=0.005$ was chosen as a nominal value. The range of porosity values determined for CS granite varies from 0.0015 to 0.008 . Another parameter that has not been determined for the particular granite core used is the constrictivity-tortuosity term $\alpha / \tau^{2}$, which was somewhat arbitrarily taken as 0.1. Breakthrough curves calculated for two porosities, 0.001 and 0.005 , illustrate the dramatic dependence on porosity; these curves and the results of the experiment are shown in Figs. 76 and 77 . The CS granite core No. 1, which had an aperture of $15.6 \mathrm{\mu m}$, was injected with a tracer solution containing ${ }^{137} \mathrm{Cs}$ and ${ }^{85} \mathrm{Sr}$. The specific activities were $1.21 \times 10^{4}$ and $4.64 \times 10^{4} \mathrm{cpm} / \mathrm{ml}$, respectively. The $50 \mathrm{~m} \ell$ of traced solution were injected at a rate of $0.2 \mathrm{ml} / \mathrm{hour}$, which corresponds to a fluid velocity of $1.41 \times 10^{-2} \mathrm{~cm} /$ second. This fluid velocity is faster than the fluid velocities used in previous experiments with crushed-rock columns. 38 One CS granite crushed-rock column was run at a comparable velocity. The observed plate height indicated that mass transfer was not a limiting factor, which is borne out by the close agreement between the experimental results and the calculated breakthrough curve.

The considerable scatter in the data in Figs. 76 and 77 was caused primarily by observed faulty and erratic operation of the automatic fraction collector. In spite of experimental difficulties, the agreement between the experimental

TABLE LV

PARAMETER VALUES USED TO CALCULATE BREAKTHROUGH SURVES FOR CS GRANITE

\begin{tabular}{cccc} 
Parameter & \multicolumn{2}{c}{$85 \mathrm{Sr}$} & $137 \mathrm{cs}$ \\
\cline { 2 - 3 } $\mathrm{D}^{\mathrm{i}}$ & $7.75 \times 10^{-6} \mathrm{~cm}^{2} / \mathrm{second}$ & $2.02 \times 10^{-5} \mathrm{~cm}^{2} / \mathrm{second}$ \\
$\mathrm{K}_{\mathrm{d}}$ & $8 \mathrm{~m} \ell / \mathrm{g}$ & $400 \mathrm{m \ell} / \mathrm{g}$ \\
$\rho_{\mathrm{s}}$ & $3.01 \mathrm{~g} / \mathrm{cm}^{3}$ & $3.01 \mathrm{~g} / \mathrm{cm}^{3}$ \\
$\alpha / \tau^{2}$ & 0.1 & 0.1 \\
$\mathrm{U}_{\mathrm{f}}$ & $1.41 \times 10^{-2} \mathrm{~cm} / \mathrm{second}$ & $1.41 \times 10^{-2} \mathrm{~cm} / \mathrm{second}$ \\
$2 \mathrm{~b}$ & $15.6 \mu \mathrm{m}$ & $15.6 \mu \mathrm{m}$ \\
$\times$ & $1.59 \mathrm{~cm}$ & $1.59 \mathrm{~cm}$
\end{tabular}




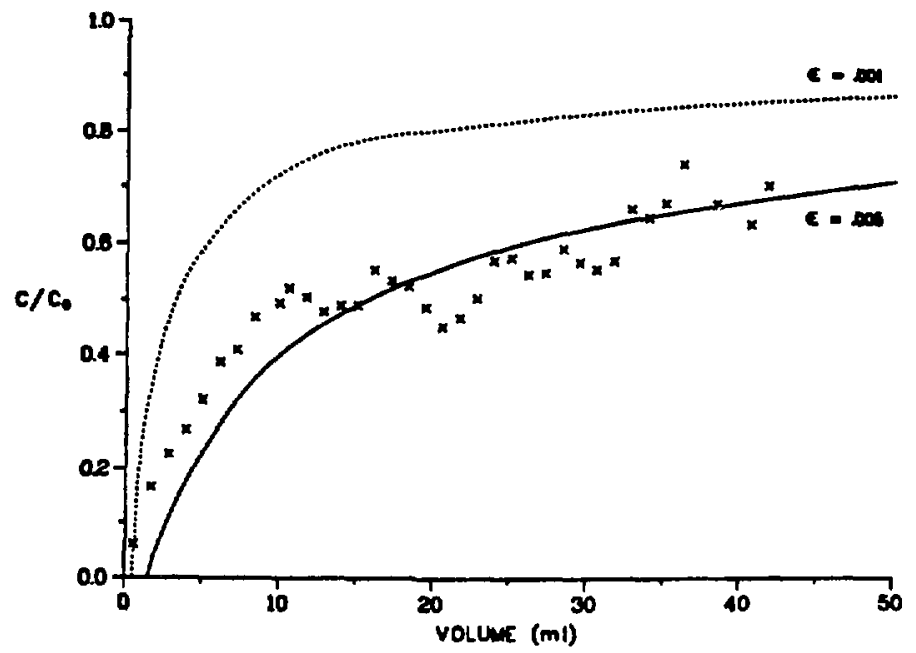

Fig. 76. Breakthrough curves for ${ }^{137} \mathrm{Cs}$ were calculated using porosities $\varepsilon=0.001$ and $\varepsilon=0.005$. The points $(x)$ are experimental data.

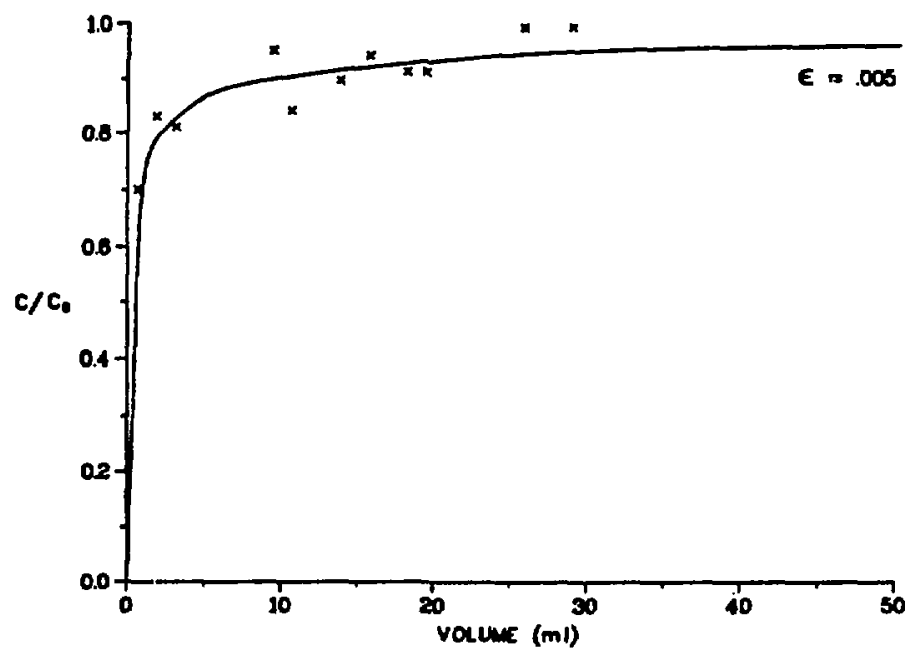

Fig. 77. Breakthrough curves for ${ }^{85} \mathrm{Sr}$ were calculated using a porosity $\varepsilon=0.005$. The points $(x)$ are experimental data. 
results and the calculation is remarkable. The $K_{d}$ values used in the calculation were based on batch sorption data. ${ }^{68}$ Unfortunately, the parameter $\alpha / \tau^{2}$ must be estimated; perhaps $\alpha / \tau^{2}$ eventually could be determined directly from diffusion experiments, and possibly with a larger scale experiment, the matrix porosity could be determined for the particular rock, independent of the fracture-flow experiment.

The tuff samples G2-2335 and G1-2840, described above, were encased in a polyurethane sealant with stainless steel endcaps. The sealant was designed to be sufficiently viscous that there was no intrusion into the fracture. The core was not subjected to confining pressure and water was pumped through the fracture at low pressure. The apertures were determined using.the methou described for the granite samples. The cores were equilibrated by passing pretreated water through them for more than 3 weeks. Forty-milliliter "slugs" of groundwater traced with ${ }^{85} \mathrm{Sr}$ and ${ }^{137} \mathrm{Cs}$ were passed through the fractures, and elution through the tuff fractures of ${ }^{85} \mathrm{Sr}$ and ${ }^{137} \mathrm{Cs}$ was monitored.

The breakthough curves were compared with theoretical curves for the analytic solution flowing through a one-dimensional fracture that is coupled to diffusion into the matrix. 62 The fracture volume and aperture, in particular, were determined using Darcy's law. ${ }^{65}$ The tuff samples were not placed under confining pressure because they sealed under moderate pressure $(\sim 1000 \mathrm{psi})$, whereupon the fracture permeability was reduced to the same magnitude as the matrix permeability.

The flow through the fracture was straight flow, permitting direct comparison with the one-dimensional calculations. The parameters used in the onedimensional calculations are given in Table LVI.

In Figs. 78 to 85 , experimental values are compared with theoretical curves obtained by substituting $R_{d}$ values from batch measurements ("batch $K_{d}$

TABLE LVI

PARAMETER VALUES USED TO CALCULATE ELUTION CURVES

\begin{tabular}{ccc} 
Parameter & Sample G1-2335 & Sample G1-2840 \\
\cline { 2 - 3 }$\rho_{\mathrm{s}}$ & 1.71 & 2.02 \\
$\alpha / \tau^{2}$ & 0.1 & 0.1 \\
$U_{f}$ & $2.85 \times 10^{-2} \mathrm{~cm} /$ second & $2.76 \times 10^{-2} \mathrm{~cm} / \mathrm{second}$ \\
$2 \mathrm{~b}$ & $30.7 \mu \mathrm{m}$ & $31.7 \mu \mathrm{m}$ \\
$\mathrm{x}$ & $4.76 \mathrm{~cm}$ & $4.75 \mathrm{~cm}$ \\
$\varepsilon$ & 0.312 & 0.191
\end{tabular}




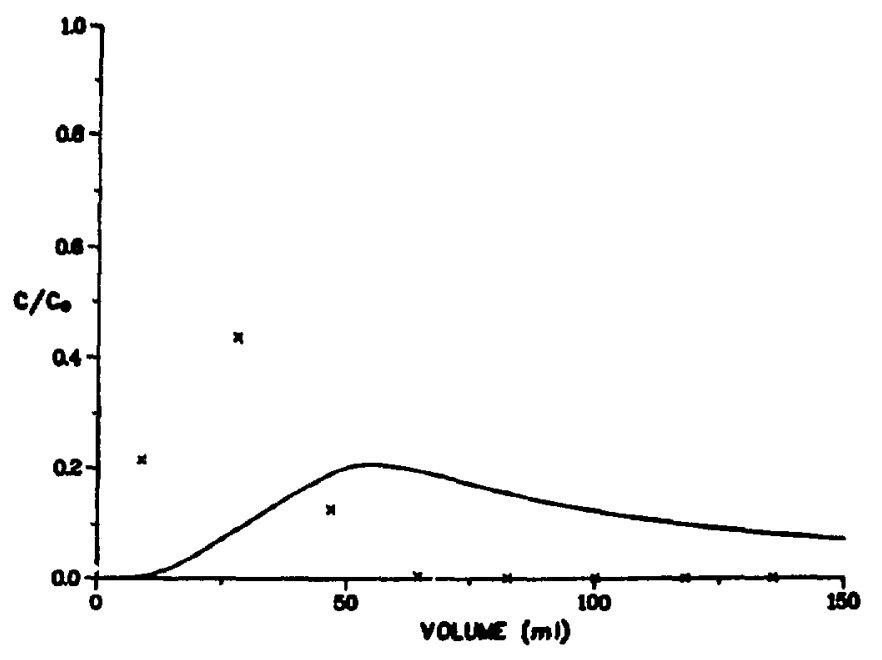

Fig. 78. Elution of a 40-ml slug of groundwater (traced with ${ }^{85} \mathrm{Sr}$ ) through a fractured G1-2335 tuff core. Theoretical curve (solid line) assumes the experimental batch $\mathrm{K}_{\mathrm{d}}(148 \mathrm{ml} / \mathrm{g})$. The points $(x)$ represent experimental data.

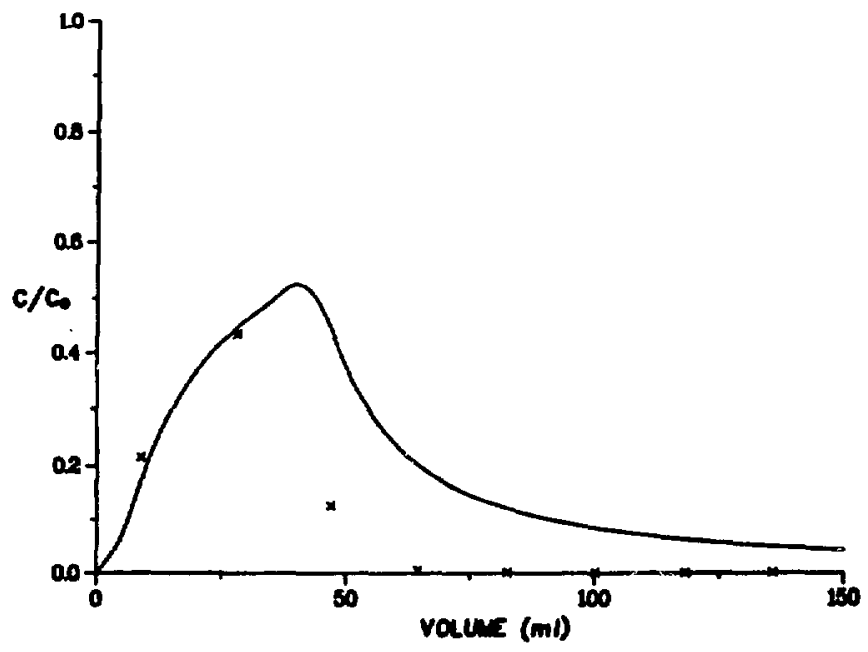

Fig. 79. Elution of a $40-\mathrm{ml}$ slug of groundwater (traced with ${ }^{85} \mathrm{Sr}$ ) through a fractured G1-2335 tuff core. Theoretical curve (solid line) assumes $K_{d}=30 \mathrm{~m} / / \mathrm{g}$. The points $(\mathrm{x})$ represent experimental data. 


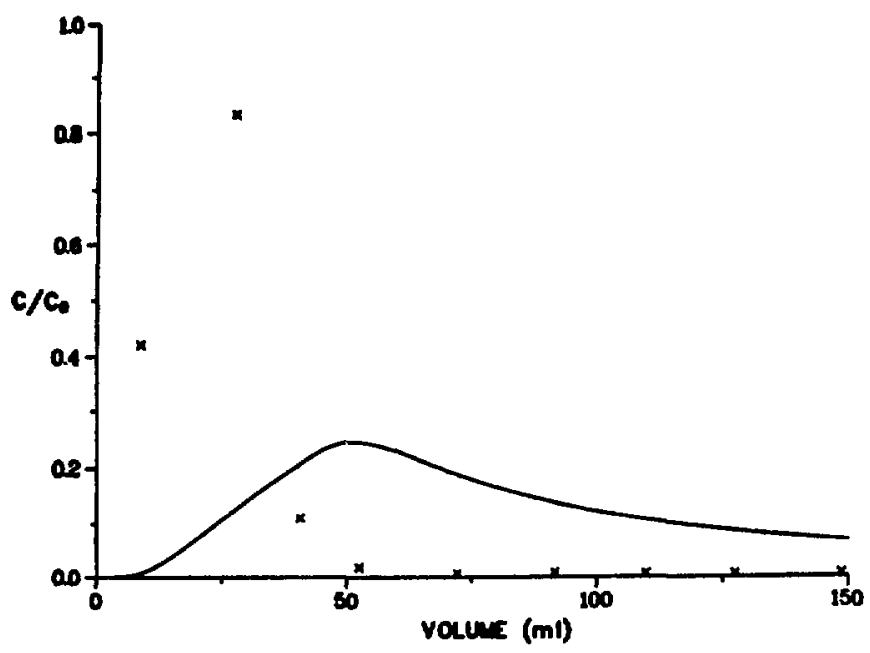

Fig. 80. Elution of a $40-\mathrm{m} \ell$ slug of groundwater (traced with ${ }^{85} \mathrm{Sr}$ ) through a fractured G1-2840 tuff core. Theoretical curve (solid line) assumes the experimental batch $K_{d}(165 \mathrm{ml} / \mathrm{g})$. The points ( $x$ ) represent experimental data.

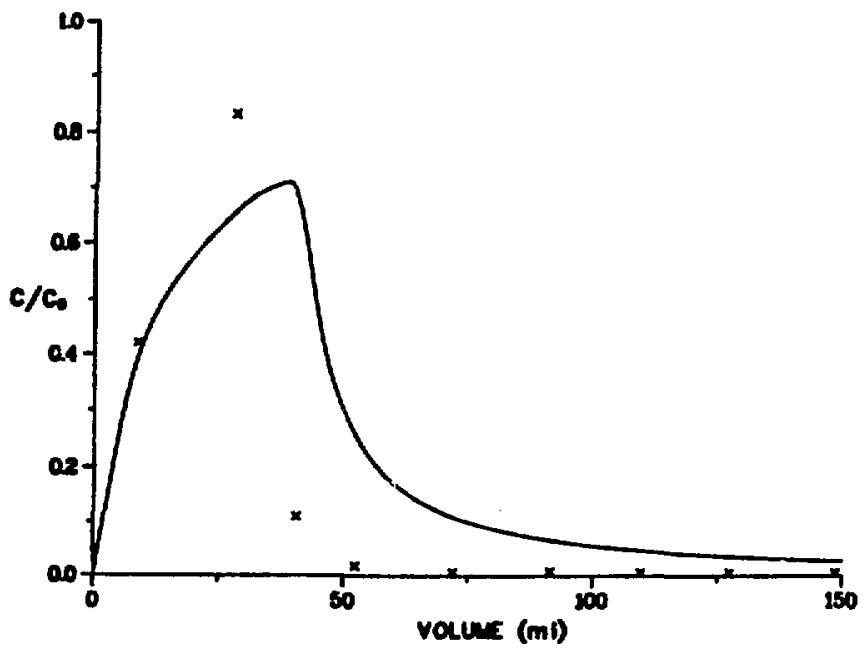

Fig. 81. Elution of a 40 -me slug of groundwater (traced with ${ }^{85} \mathrm{Sr}$ ) throligh a fractured G1-2840 tuff core. Theoretical curve (solid line) assumes $K_{d}=16 \mathrm{~m} / / \mathrm{g}$. The points $(x)$ represent experimental data. 


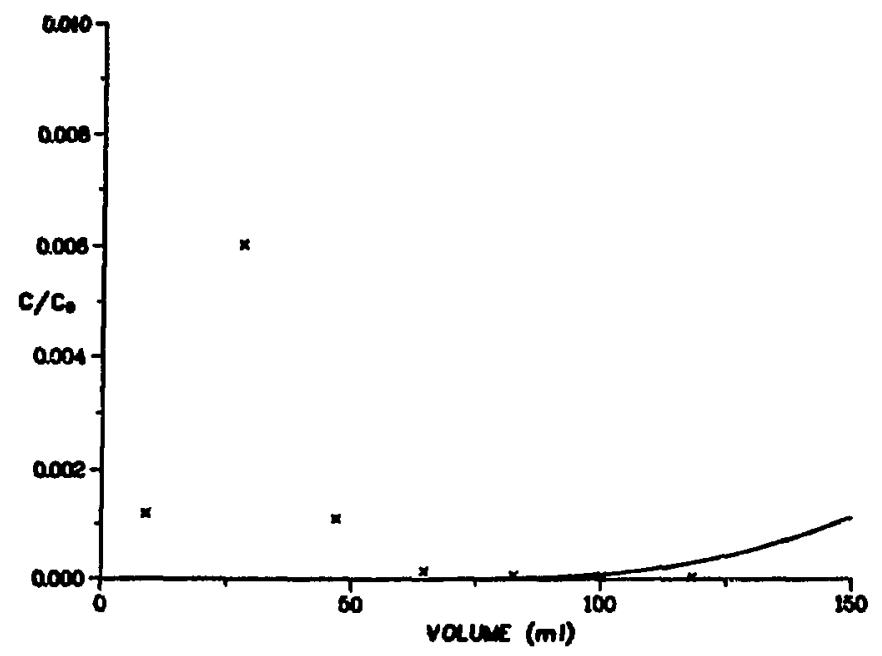

Fig. 82. Elution of a 40-me slug of groundwater (traced with ${ }^{137} \mathrm{Cs}$ ) through a fractured G1-2335 tuff core. Theoretical curve (solid line) assumes the experimental batch $K_{d}(1100 \mathrm{~m} / / \mathrm{g})$. The points $(x)$ represent experimental data.

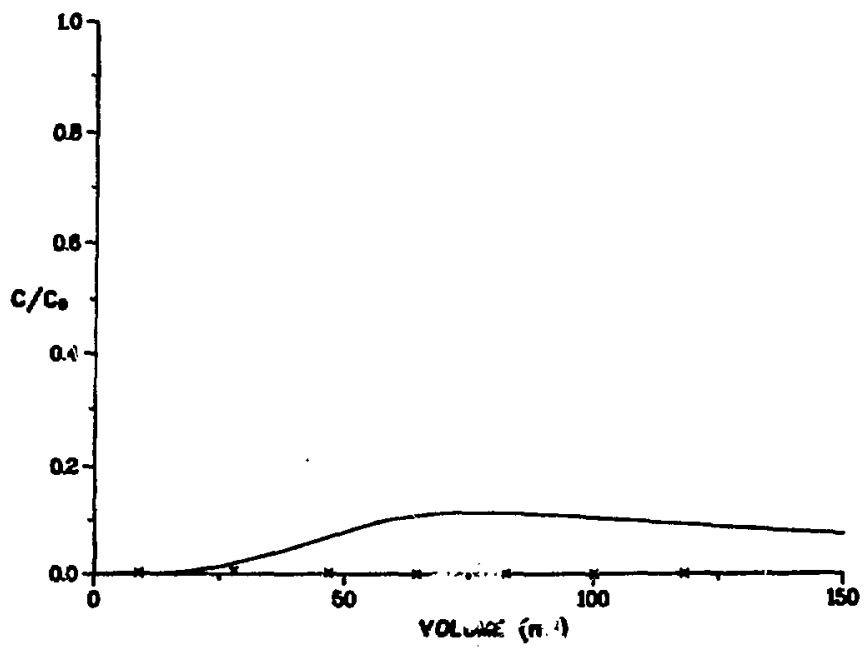

Fig. 83. Elution of a 40-ml slug of groundwater (traced with ${ }^{137} \mathrm{Cs}$ ) through a fractued G1-2335 tuff core. Theoretical curve (solid line) assumes $K_{d}=220 \mathrm{ml} / \mathrm{g}$. The points ( $x$ ) represent experimental data. 


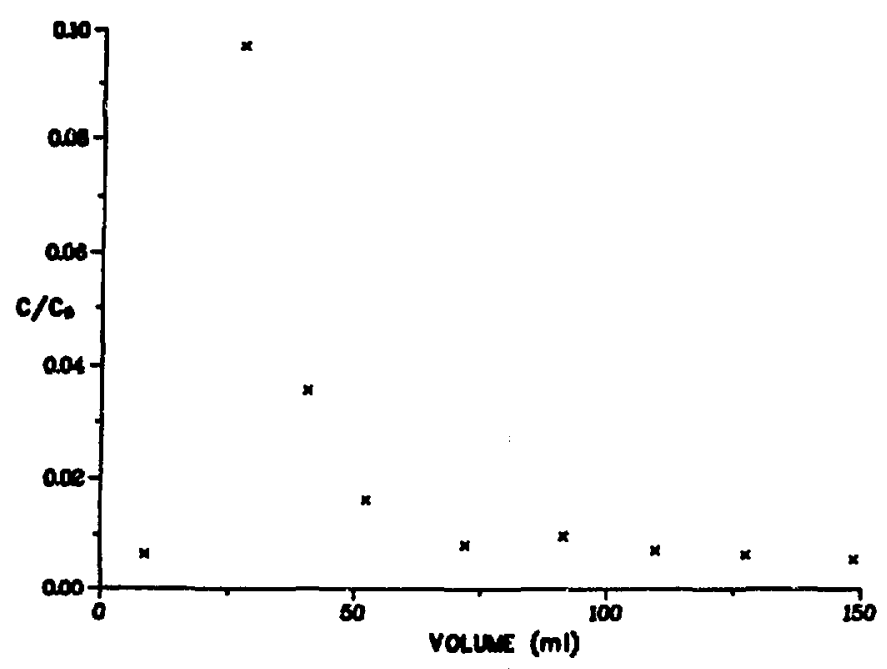

Fig. 84. Elution of a $40-\mathrm{ml}$ slug of groundwater (traced with ${ }^{137} \mathrm{Cs}$ ) through a fractured G1-2840 tuff core. The theoretical curve, which is too low to be visible on the same scale as the experimental data, assumes the experimental batch $\mathrm{K}_{\mathrm{d}}(2200 \mathrm{~m} \ell / \mathrm{g})$. The points $(\mathrm{x})$ represent experimental data.

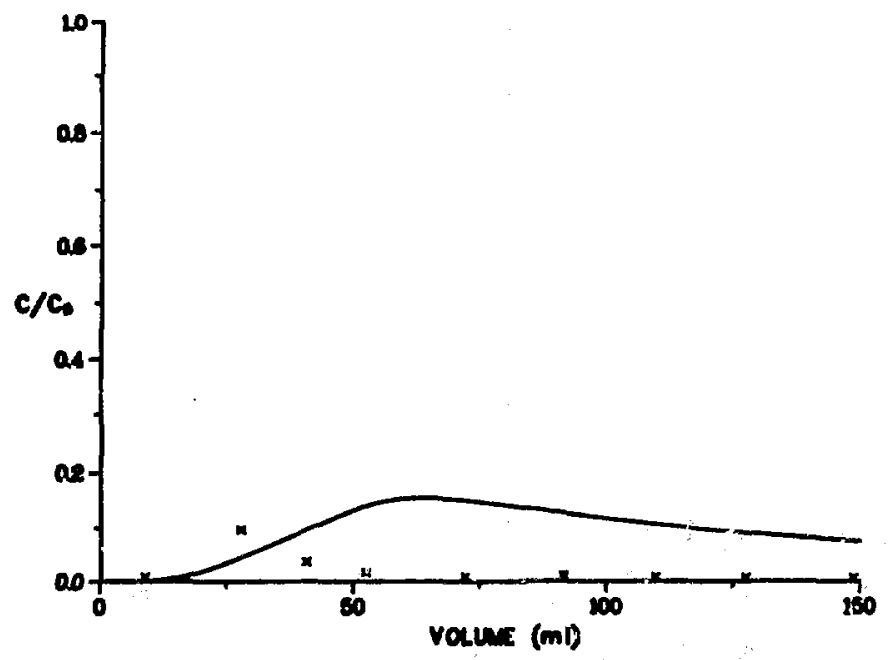

Fig. 85. Elution of a 40-me slug of groundwater (traced with ${ }^{137} \mathrm{Cs}$ ) through a fractured G1-2840 tuft core. Theoretical curve (solid line) assumes $K_{d}=110 \mathrm{~m} / \mathrm{g}$. The points (x) represent experimental data. 
values" in this section) for the $K_{d}$ values in the calculations and by using a lower value for a closer fit to the experimental results.

The results of the fracture-flow experiments with tuff samples from cores G1-2335 and GI-2840 were not in agreement with the calculation obtained when the batch $K_{d}$ values were used (Figs. $78,80,82$, and 84 ). The $K_{d}$ values that gave the best fits (Figs. 79 and 81 ) to the breakthrough portion of the strontium elution were 30 and $16 \mathrm{ml} / \mathrm{g}$ for samples G1-2335 and G1-2840, respectively. The batch measurements yielded 148 and $160 \mathrm{ml} / \mathrm{g}$ for samples G1-2335 and G1-2840, respectively. A general trend observed in sorption experiments on tuff indicates that batch measurements yield $K_{d}$ values 3 to 5 times larger than the $K_{d}$ values determined by column experiments; these fracture-flow experiments were consistent with that trend. In addition, the shape of the elution calculated for the tuffs is not in agreement with observed elution. The activity desorbs more slowly than would be expected for reversible, diffusion-controlled sorption. This observation is also consistent with previous measurements of sorption on tuff. In general, the $K_{d}$ values determined by desorbing activity from tuff are considerably larger than those determined from the sorption process. The values from the cesium runs do not fit even when $K_{d}$ values are lowered. When the batch $K_{d}$ values were used, the expected peak arrival time was off-scale with respect to the experimental peak (Figs. 82 and 84 ). Lowering the $K_{d}$ values, however, brought the peak arrival time closer to the experimental, but the area under the curve became greater than the experimental curve (Figs. 83 and 85 ). A satisfactory fit to the experimental elution curves for cesium could not be achieved using the simple matrix diffusion model.

The lack of agreement between the experimental and theoretical elution curves suggests a more complex sorption mechanism than simple linear sorption. A nonlinear isotherm or an irreversible sorptive process could perhaps be invoked to model these results. Alternatively, severe channeling could cause premature breakthrough, but the similarity of the two samples tends to make this process an unlikely explanation. Sorption and matrix diffusion in tuff (especially welded tuff) appear, therefore, to be more complex than in granite.

A one-dimensional solution to the fracture-flow matrix diffusion equation described above was used to calculate the effect of matrix diffusion that can be expected in a field experiment such as that proposed for G Tunne1. This solution is simplistic and considers only the matrix diffusion effect; more complex models are being developed to include dispersion, porous flow in the 
matrix, and complex flow fields in the fracture. Nonetheless, the results of this simple nodel are useful because matrix diffusion will be a dominant mechanism affecting radionuclide transport in tuff by fracture flow. For several years matrix diffusion has been considered an important factor in element transport through fractures in crystalline rock. ${ }^{62,69,70}$ The effect will be much more dramatic in tuff, even over relatively short distances, because tuff porosity is several orders of magnitude higher than that of granite.

The physical properties of G-Tunnel tuff have been determined in previous measurements. Table LVII contains a list of the nominal parameter values chosen for matrix diffusion calculations. The ionic diffusion coefficient corresponds to that of strontium and is an intermediate value compared to the ionic diffusivities of most monovalent and divalent ions. Fissure apertures from 10 to $100 \mu \mathrm{m}$ and flow velocities $U_{f}$ from 1 to $100 \mathrm{~m} /$ day were used in the calculations.

Figure 86 shows the activity profile for a nonsorbing tracer, $K_{d}^{\prime}=0$, with a flow rate of $1 \mathrm{~m} /$ day after 300 days. Although the water has traveled $300 \mathrm{~m}$, activity has traveled only $9 \mathrm{~cm}$. This result is caused by loss of tracer to the rock matrix; Fig. 87 shows that tracer has penetrated far beyond $1 \mathrm{~cm}$. This apparent retardation occurs because the concentration gradient at the tracer front is the highest and, therefore, diffusion there is the fastest; activity in the leading edge is lost to the matrix until the concentration in the matrix builds up. These results clearly indicate that in the G-Tunnel field experiment a flow rate of $1 \mathrm{~m} /$ day would be too slow because the planned duration of the experiment is 30 to 60 days for both sorbing and nonsorbing tracers.

\section{TABLE LVII}

PHYSICAL PARAMETERS USED FOR MATRIX DIFFUSION

CALCULATIONS WITH G-TUNNEL TUFF

\section{Parameter}

Density

Matrix porosity

Constrictivity/tortuosity

Ionic diffusion coefficient

Effective diffusion coefficient

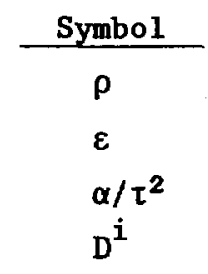

$D_{\text {eff }}$

Value
$1.6 \mathrm{~g} / \mathrm{cm}^{2}$
0.30
0.10
$7.74 \times 10^{-10} \mathrm{v}^{2} /$ second
$2.71 \times 10^{-11} \mathrm{a}^{2} /$ second




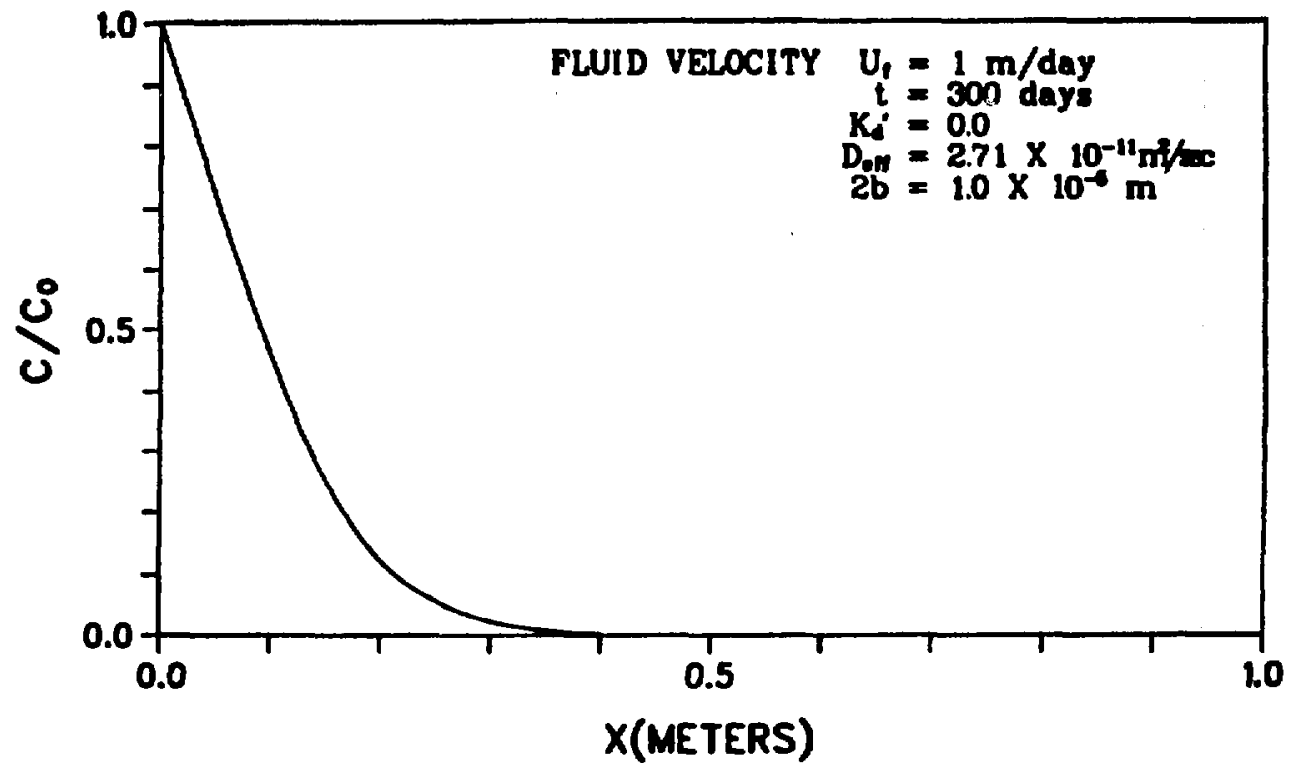

Fig. 86. Concentration profile for the fracture surface $(z=0)$.

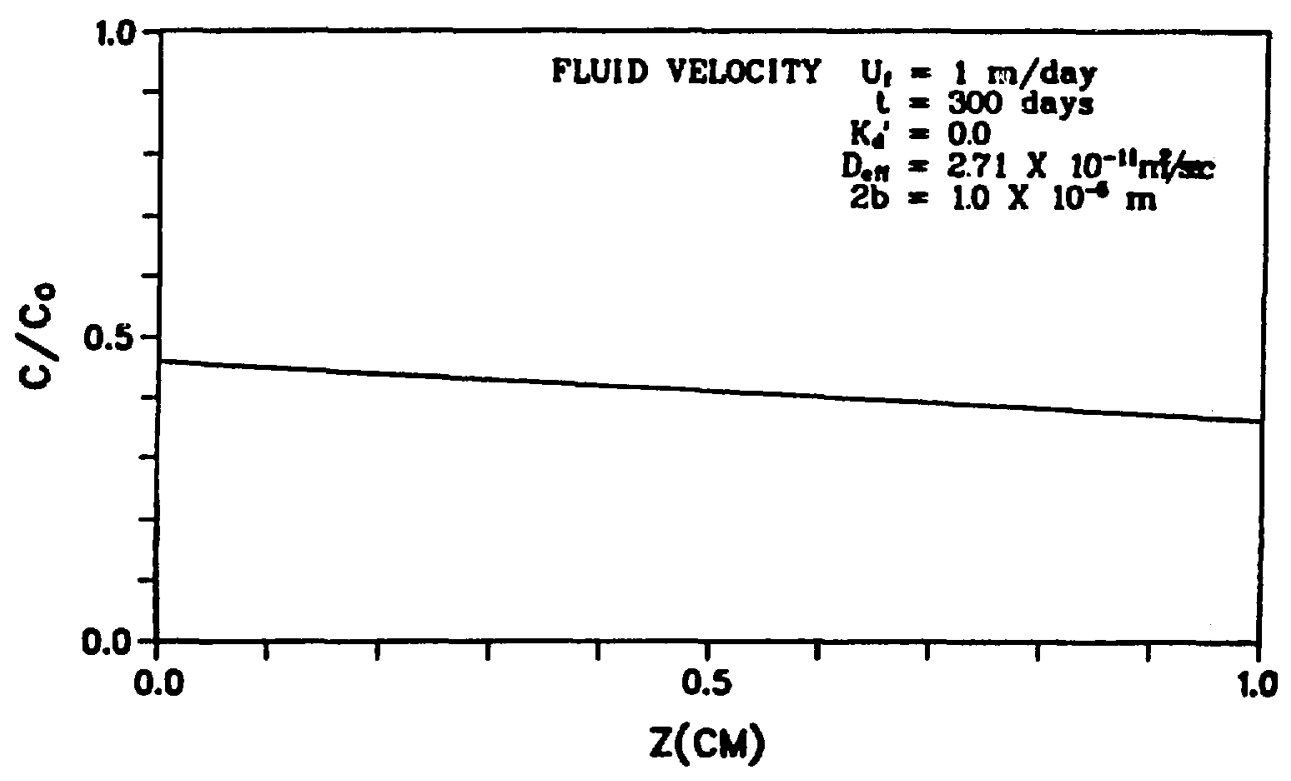

Fig. 87. Concentration profile for the rock matrix $(x=0.1 \mathrm{~m})$. 
Figures 88 to 91 show similar profiles at fluid velocities of 10 and $100 \mathrm{~m} / \mathrm{day}$. At $100 \mathrm{~m} /$ day the tracer begins to exit the $1-\mathrm{m}$ fracture in 0.3 days, and at $10 \mathrm{~m} /$ day the tracer front $\left(\mathrm{C} / \mathrm{C}_{0}=0.5\right)$ moves $10 \mathrm{~cm}$ in 3 days. These velocities, therefore, show movement that is ressonable for the time scale of the experiment.

Figures 92-94 show the surface profile for sorbing tracers with a fluid velocity of $100 \mathrm{~m} /$ day. Tracers with $\mathrm{K}_{\mathrm{d}}$ values $\leq 1000 \mathrm{ml} / \mathrm{g}$ will move more than $10 \mathrm{~cm}$ in 30 days. Figure 95 shows the effect of a 100- $\mu \mathrm{m}$ fissure aperture. A larger aperture would make it possible to use slower fluid velocities or tracers with much higher $K_{d}$ values. However, the former may be the only option available if equipment design limits the flow rates that can be introduced into the fracture.

An interesting and important consequence of these studies is the effect of matrix diffusion of nonsorbing species in fractured media: there is an apparent retardation of that transport as a result of matrix diffusion. To illustrate this effect, the breakthrough can be defined as the point at which the concentration is $50 \%$ of the initial concentration. Inserting $C / C_{0}=0.5$ into Eq. (11) yields

$$
0.5=\operatorname{erfc}\left(\frac{G}{\left(t-t_{w}\right)^{\frac{1}{2}}}\right) \text {, }
$$

and

$$
\frac{G}{\left(t-t_{w}\right)^{\frac{3}{2}}}=0.477 \text {, }
$$

or

$$
\frac{D_{\text {eff }} t_{w}}{2 b\left(D_{\text {app }}\right)^{\frac{3}{2}}\left(t-t_{w}\right)^{\frac{3}{2}}}=0.477,
$$

which can be rewritten in terms of a retardation factor $R_{f}$,

$$
R_{f}=\frac{t_{\frac{1}{k}}}{t_{w}}=1+\frac{\varepsilon^{2} D^{i} \alpha / \tau^{2}}{(2 b)^{2}} t_{w} .
$$




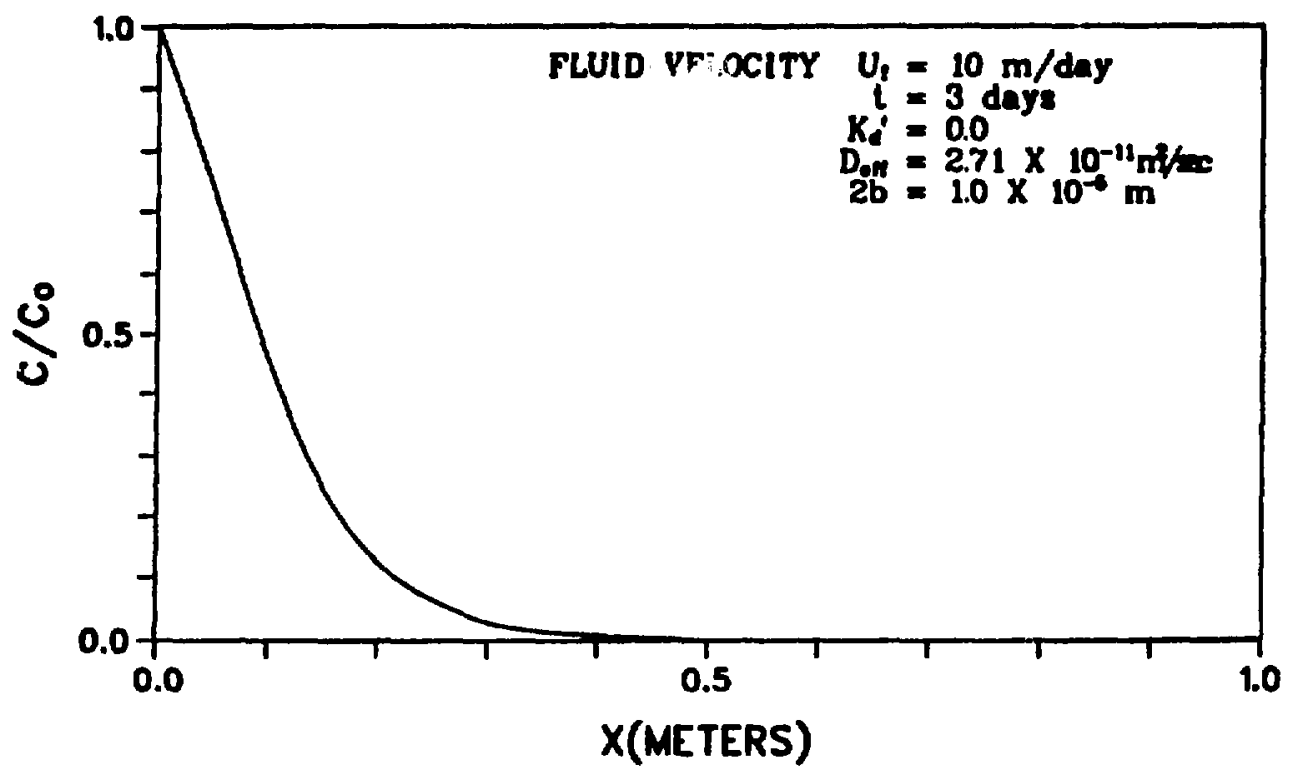

Fig. 88. Concentration profile for the fracture surface $(z=0)$.

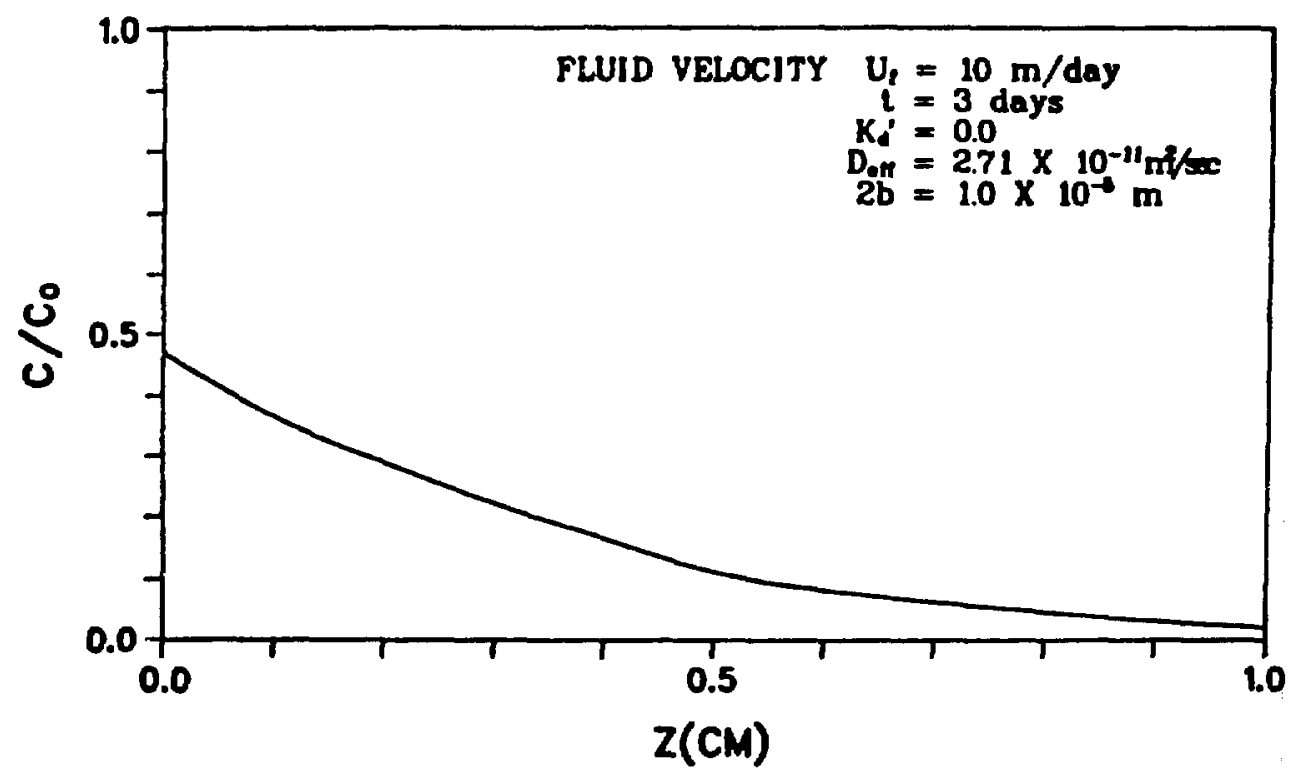

Fig. 89. Concentration profile for the rock matrix $(x=0.1 \mathrm{~m})$. 


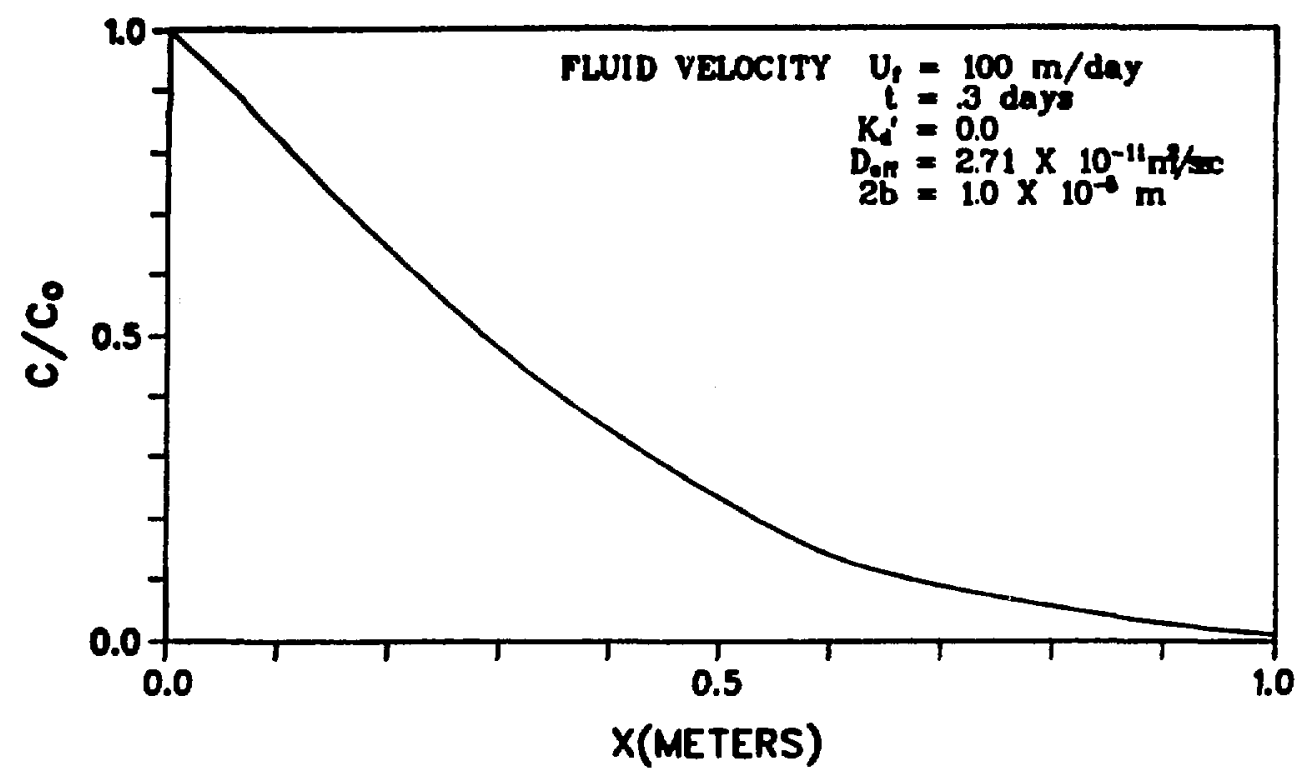

Fig. 90. Concentration profile for the fracture surface $(z=0)$.

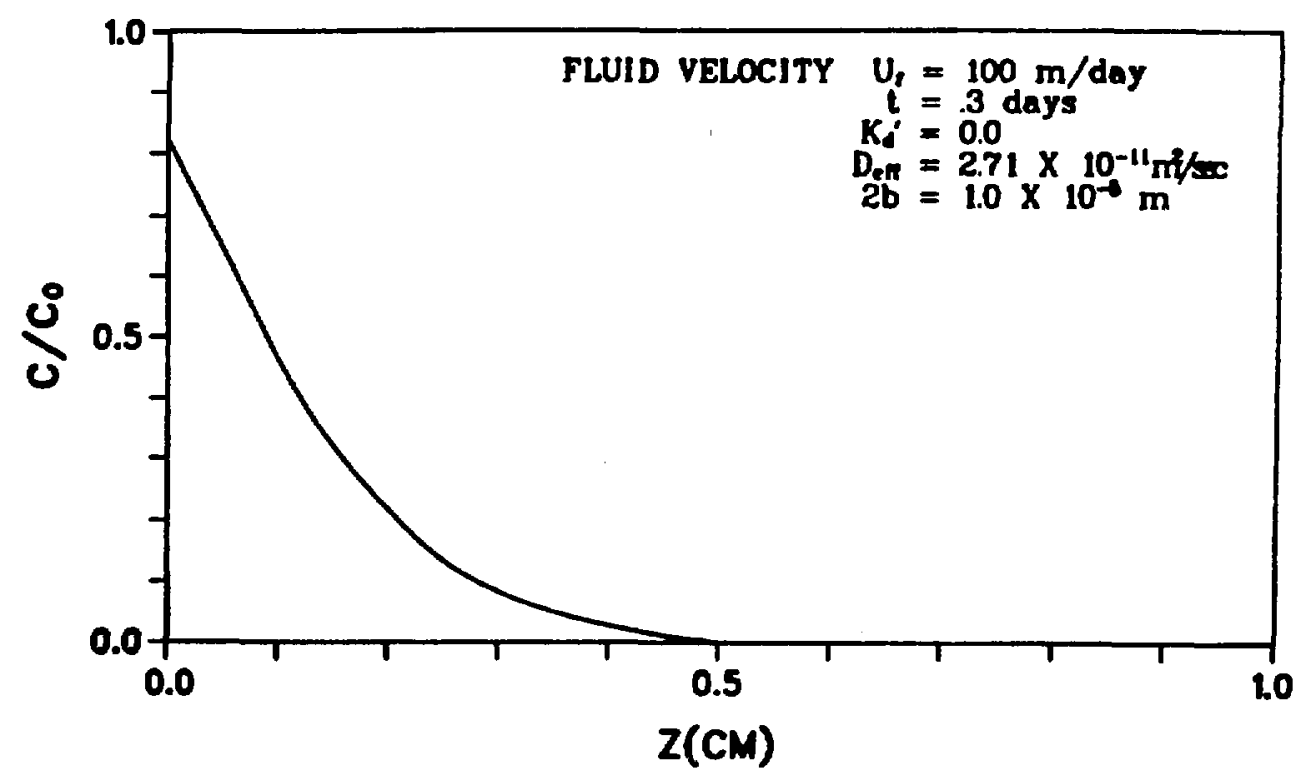

Fig. 91. Concentration profile for the rock matrix $(x=0.1 \mathrm{~m})$. 


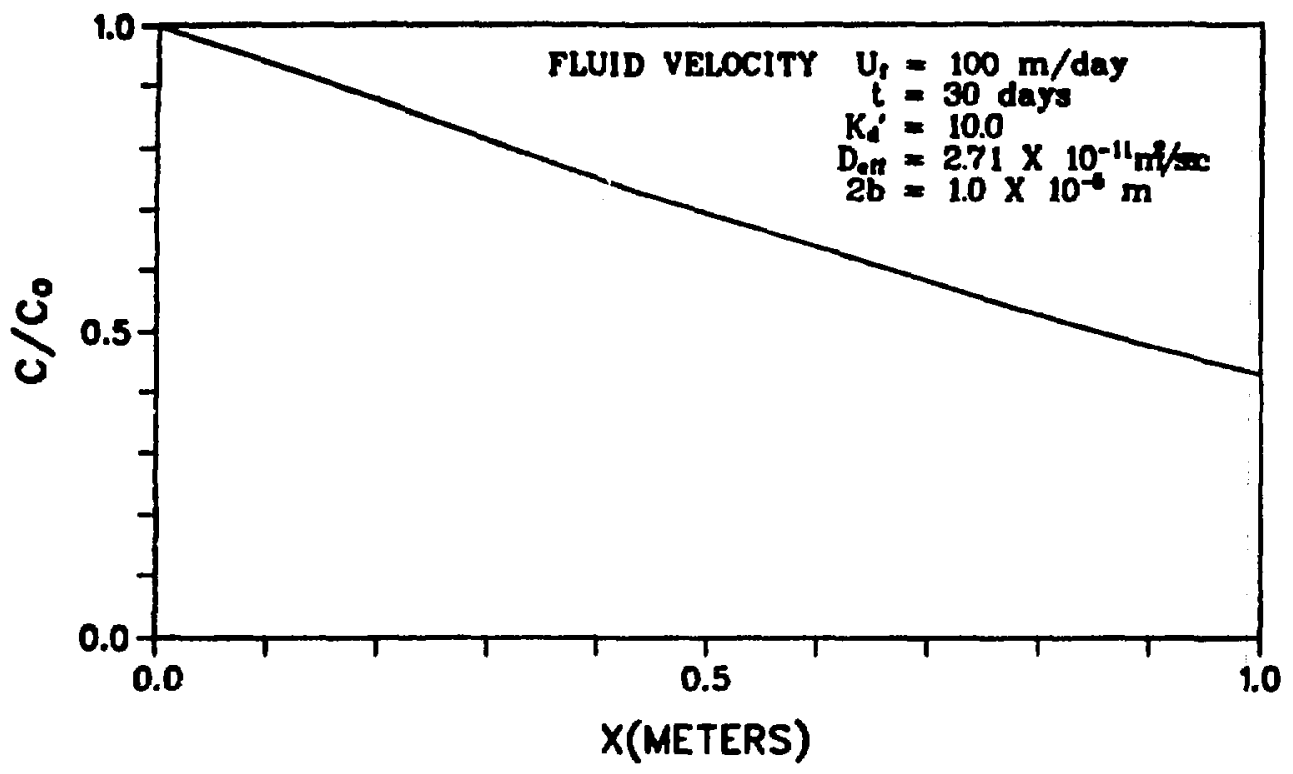

Fig. 92. Concentration profile for the fracture surface $(z=0)$.

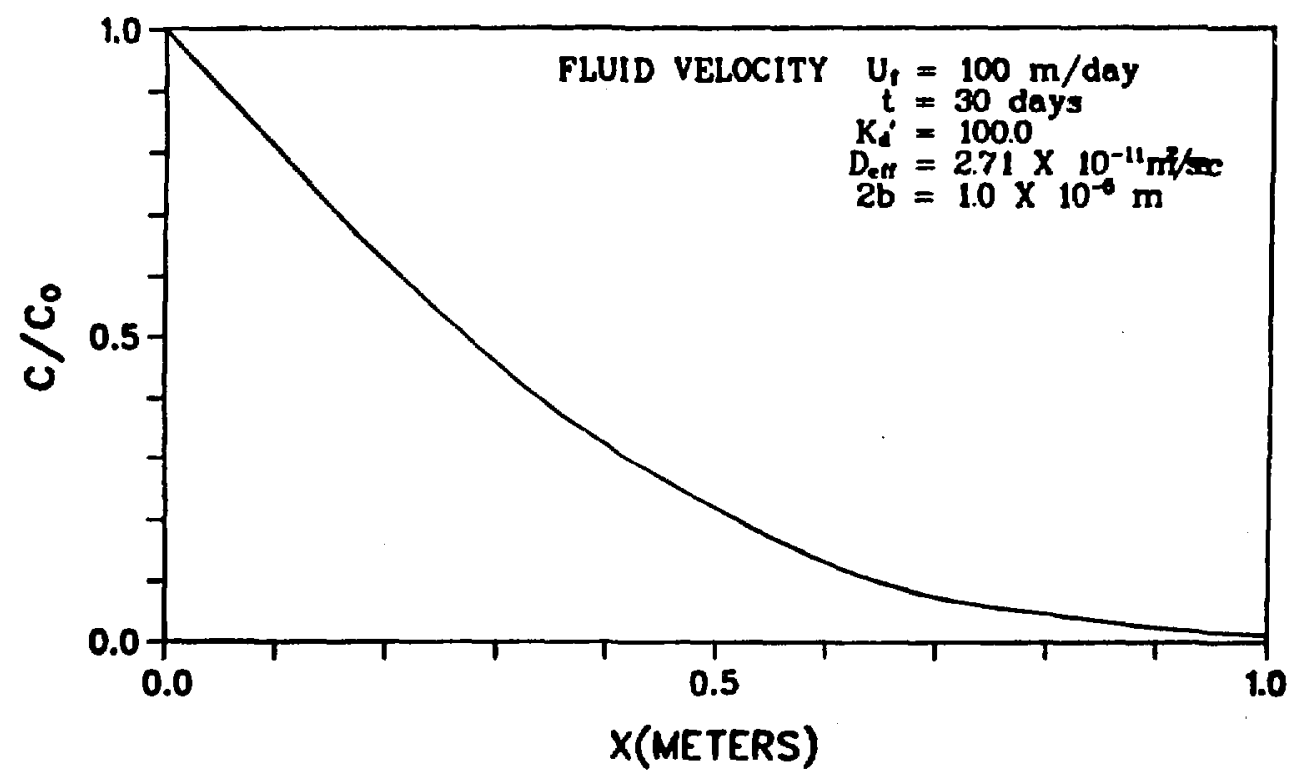

Fig. 93. Concentration profile for the fracture surface $(z=0)$. 


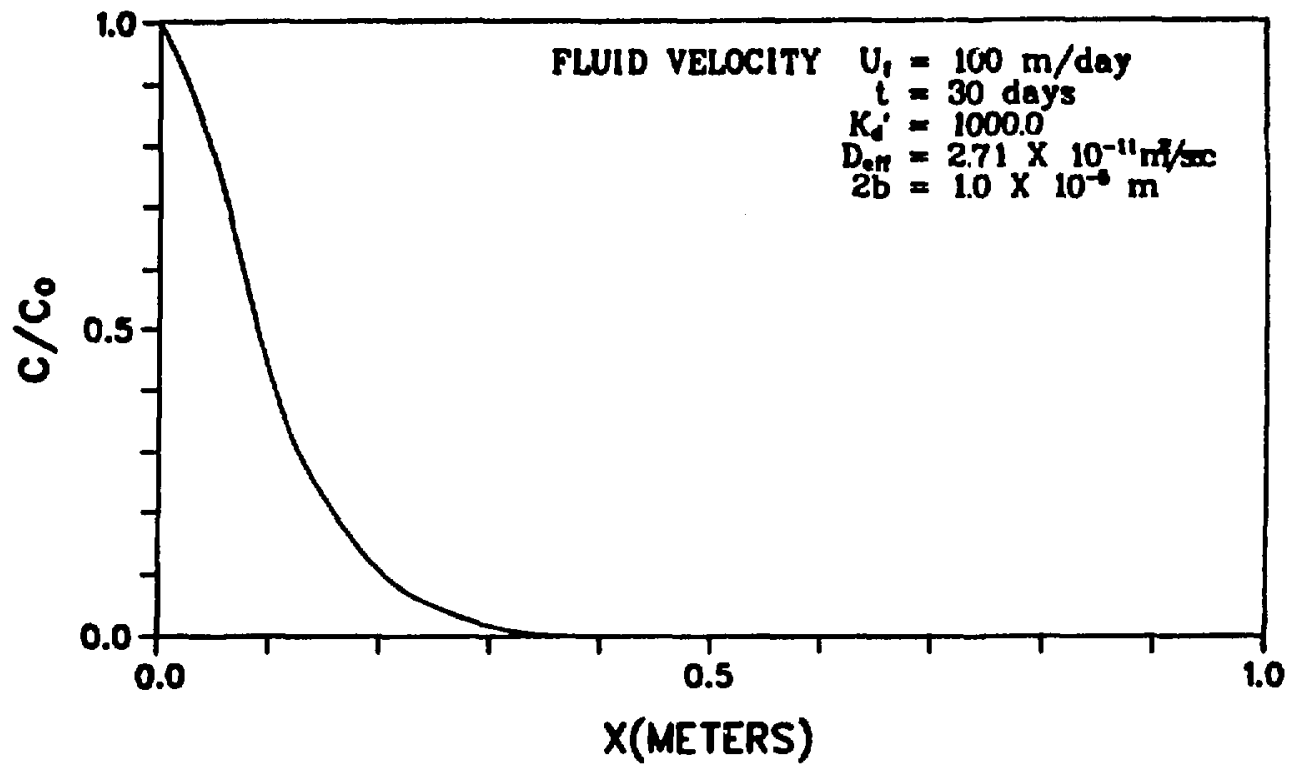

Fig. 94. Concentration profile for the fracture surface $(z=0)$.

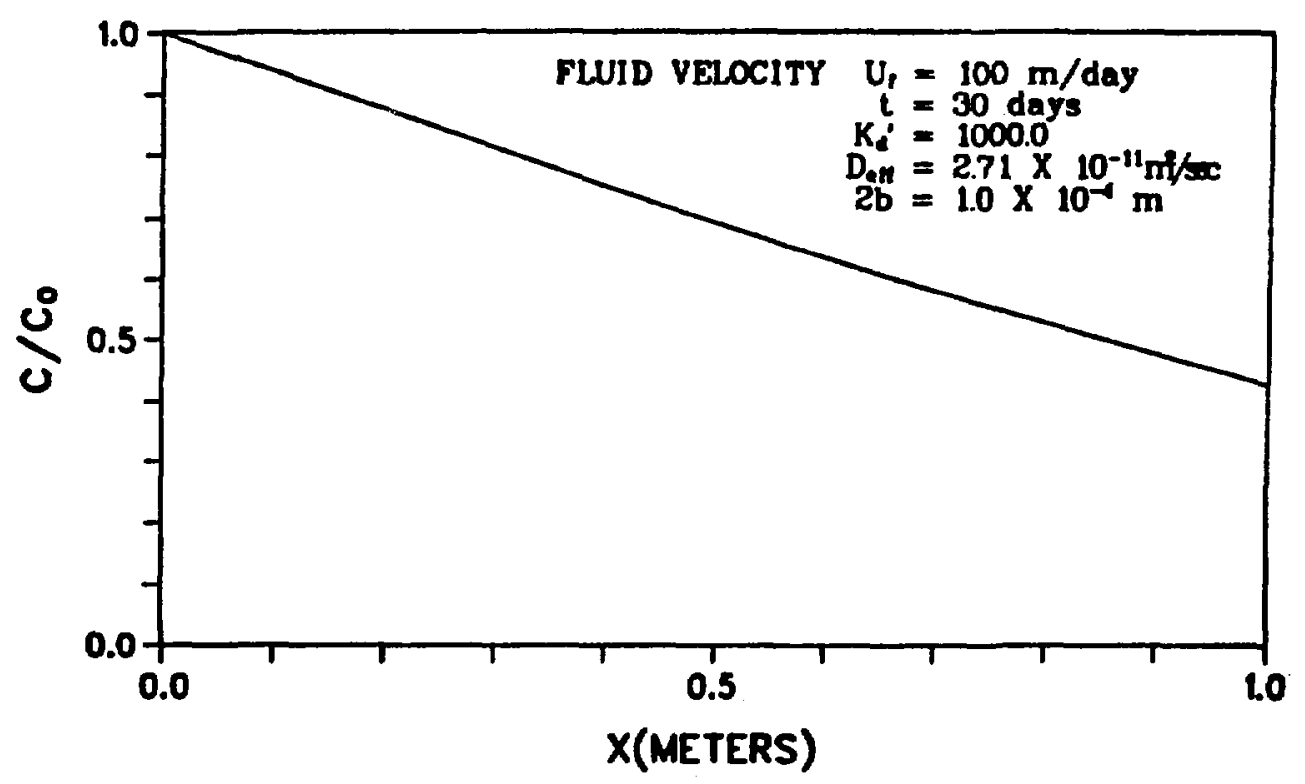

Fig. 95. Concentration profile for the fracture surface $(z=0)$. 
To estimate the magnitude of this effect, some reasonable values for the parameters can be applied, based on laboratory and field measurements. For instance, in well USW-H1 the USGS found a highly permeable zone in the Prow Pass Member with $\sim 10 \mathrm{~m} /$ day hydraulic conductivity. The fracture spacing, as reported in their USW-G1 report, ${ }^{71}$ is an average of $7.6 \mathrm{~m} /$ day for the Prow Pass Member. These data can be used then to estimate an average aperture $2 b$ for the Prow Pass Member. The permeability

$$
\mathbf{k}_{\mathrm{s}}=\frac{2 \mathrm{~b}}{\mathrm{~s}} \mathrm{k}_{\mathrm{f}}
$$

and the fracture permeability

$$
k_{f}=\frac{(2 b)^{2}}{12}
$$

so that

$$
k_{s}=\frac{(2 b)^{3}}{12 s}
$$

where $\mathrm{S}$ is the fracture spacing. The average aperture corresponding to a fracture spacing of $7.6 \mathrm{~m}$ and a hydraulic conductivity of $10 \mathrm{~m} /$ day, or 0.12 darcys, is $2.2 \times 10^{-2} \mathrm{~cm}$. The maximum residence time for the water $t_{w}$ before the rock becomes saturated by the diffusing species is determined from the fracture spacing by using the following formula for the penetration depth $\eta_{0.01}$, at which $\mathrm{C} / \mathrm{Co}=0.01$ :

$$
\eta_{0.01}=4\left(D_{a} t\right)^{\frac{3}{2}}
$$

Setting $\eta_{0.01}=0.5 \mathrm{~S}=3.8 \mathrm{~m}$ yields a time $t=2.86$ years, assuming $D_{a}=$ $10^{-6} \mathrm{~cm}^{2} /$ second. This represents an upper limit for $t_{w}$, and therefore $R_{f}$, where Eq. (12) is still applicable.

If the water flowing through the repository moves at a rate of $100 \mathrm{~m} / \mathrm{year}$, the retardation factor for a nonsorbing soluble species along a 1-km flow path is $4.08 \times 10^{4}$, assuming a porosity $\varepsilon$ of $25 \%$. Porosity is significant in this phenomenon. For instance, the same conditions applied to granite, $\varepsilon=0.005$, would yield a retardation factor of only 17 . 
2. Porous Flow. Experiments examining the transport of radionuclides through porous media have been conducted with the primary aim of determining the radionuclide retardation that will be provided by geochemical processes along flow paths. These experiments, using both crushed-tuff and solid-tuff columns, provided intermediate steps in the laboratory-to-field link. These experiments test whether batch sorption measurements are applicable to dynamic systems and also whether the sorptive properties of the rock have been seriously altered by crushing the tuff samples. Some specific questions have been addressed.

(1) Does crushing rock samples expose minerals that would otherwise be unavailable for interaction with a natural flow of water through the solid rock?

(2) Are the kinetics of sorption fast enough so that, at flow velocities encountered in the field, batch measurements would still contribute to accurate predictions of retardation?

(3) Are there fixation reactions?

(4) Do batch measurements provide accurate data for dynamic flow systems?

E. N. Treher reported on the crushed-rock column experiments and gave a detailed description of the methods used. 38 Chromatographic columns packed with crushed rock have been used for all of the studies. The following radionuclides have been used in these studies: ${ }^{131} \mathrm{I},{ }^{85} \mathrm{Sr},{ }^{137} \mathrm{Cs},{ }^{133} \mathrm{Ba},{ }^{141} \mathrm{Ce}$, ${ }^{152} \mathrm{Eu},{ }^{95} \mathrm{Tc}^{\mathrm{m}}$, and ${ }^{3} \mathrm{H}$. Some of the columns have been run at two flow rates. The crushed-rock columns have begun to provide information on dynamic effects in radionuclide transport through porous media.

Radionuclide sorption on rocks is principally a surface phenomenon; therefore, the surface condition is a critical parameter in experiments involving partitioning of radionuclides between solutions and geologic media. Studies using crushed rock involve mineral surfaces newly exposed to the atmosphere and to water; those using geologic thin sections involve surfaces exposed and possibly altered by grinding and polishing. In an attempt to work with unaltered rock surfaces, we have initiated experinents in which pumped groundwater is used to move radionuclides through rock cores.

The apparatus has been iescribed in Sec. IV.A. The rock cores are 15.9-mlong by 25.4-m-dian right-circular cylinders. The confining pressure is usually 1000 psi. The groundwater used in these experiments is pretreated with the 
appropriate crushed tuff, centrifuged, and filtered through a $0.05-\mu m$ membrane. Before the introduction of radionuclides to the rock cores, pretreated water is forced through the cores to pretreat the rock surfaces. This minimizes alteration of the rock during the experiment and perhaps better simulates the in situ conditions.

a. Strontium-85 in YM-22 Tuff. The movement of ${ }^{85} \mathrm{Sr}$ in water flowing through a core of YM-22 tuff was observed. This is a devitrified and densely welded tuff from the Topopah Spring Member and has relatively poor sorptive properties. The core was loaded with $20 \mu l$ of ${ }^{85} \mathrm{Sr}$ activity. Water was pumped through the core for a 12-month period, with an average flow rate of $0.8 \mathrm{ml} /$ day. About $40 \%$ of the activity was eluted at a fairly constant rate; no peak was observed. The core was sectioned, and the residual activity was found to be relatively low in the top few millimeters of the core; it then increased to a peak in the core midsection and remained high throughout the reminder of the core (Fig. 96). Based on the anticipated volume corresponding to elution of $50 \%$ of the activity, a crude estimate was made of the $R_{d}$ value for strontium on this YM-22 core. The resultant value of $\sim 20 \mathrm{ml} / \mathrm{g}$ is less than the $53 \mathrm{ml} / \mathrm{g}$ value obtained from batch measurements but similar to those obtained with crushed-rock columns. ${ }^{3}$ More elution and distribution profile studies of this type are planned after modifications in the experimental methodology are completed.

b. Anericium-241 in YM-45 Tuff. The movement of ${ }^{241}$ Am through a YM-45 core was observed by monitoring the eluted solution and using microautoradiography on core slices. The column was run for 5 weeks with a flow rate of

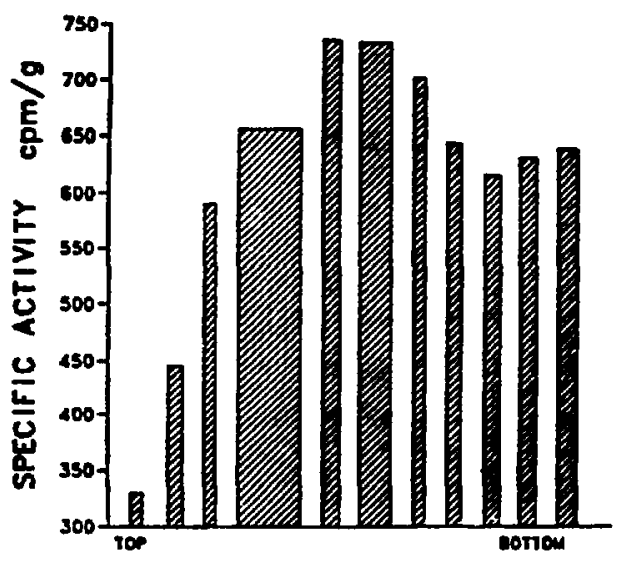

CORE THICKNESS H $=1 \mathrm{~mm}$

Fig. 95. Distribution of ${ }^{85}$ Sr activity in a solid core of $\mathrm{YM-22}$ tuff. 
$0.8 \mathrm{ml} /$ day. Approximately $0.2 \%$ of the activity added to the column was collected.

On disassembly of the pressure apparatus, it was found that the metal end cap in contact with the top surface of the core showed a high level of radioactivity and that the bottom end cap showed almost none. The activity on the core sides and bottom was measured using microautoradiography. Low-to-moderate levels of activity were found on these surfaces. That the ${ }^{241} \mathrm{Am}$ seemed to have been deposited primarily in an aggregated form, as a high ratio of stars to single tracks, was observed in the autoradiographs. One giant colloid was observed on the bottom surface.

The core was then sectioned with a rock saw. Three slices about $1 \mathrm{mr}$ thick were cut from the top of the core, and one slice was cut from the bottom of the core. The activity levels in these slices were measured with a NaI(TI) well crystal and an alpha porportional counter. The activity on the top surface of the core was $10^{3}$ to $10^{4}$ times greater than the activities on the bottom of the top slice and the other interior surfaces. The activities on the exterior surfaces and on the interior slice surfaces were compared using a collimator with the porportional counter. The exterior surfaces had activity levels greater by a factor of 5 than those of the interior surfaces. The activity on the exterior surface was relatively uniform, varying from region to region by only a factor of 2 .

The results of this experiment indicate that almost all of the activity originally placed on the top core face remained there. The small fraction of activity that was mobilized by the water flow appears to have been deposited at higher concentrations on the exterior surfaces than in the interior of the core. This may imply that more flow occurred over these surfaces than through the core or that these surfaces wexe particularly sorptive. The observation that a large fraction of ${ }^{241} \mathrm{Am}$ was in an aggregated form on the exterior surfaces, however, indicates that this portion of the activity probably did not flow through the core.

Tuff samples from the USW-G1 drill hole have been run as colunns in experiments that use a peristaltic puxp. These samples have been equilibrated with appropriate pretreated water; the experiments run for as long as 3 months. Currently, samples are sealed in Plexiglas jackets with Buehler epoxy. This method of potting seens to be adequate to provide a jacket that can withstand stress caused by the swelling of certain mineral phases, for example, montmorillonite, which occurs when the cores are wet. Previously, glass jackets were used and vere found to crack after 4 weeks. 
Samples of the G1-1982 and G1-1883 cores from the Prow Pass Member were loaded with spikes of tritium to determine the dispersivity of the rock and the free column volume. The G1-1982 sample contained a small amount of montmorillonite clay that swelled and disintegrated during equilibration with pretreated water. This left some significant pits ( 1 to $2 \mathrm{~mm}$ deep) in the ends of the core. The sample 01-1982 material also appeared to be more heterogeneous, having some colored zones that appear to be denser than the surrounding material.

The tritium elutions shown in Figs. 97 and 98 can be fit with a simple dispersion-convection model for a one-dimensional flow field. The Peclet numbers determined by fitting the data were 4 and 10 for samples $\mathrm{Gl}-1982$ and G1-1883, respectively. The fluid velocity used in these columns was about $6 \times 10^{-5} \mathrm{~cm} / \mathrm{second}$.

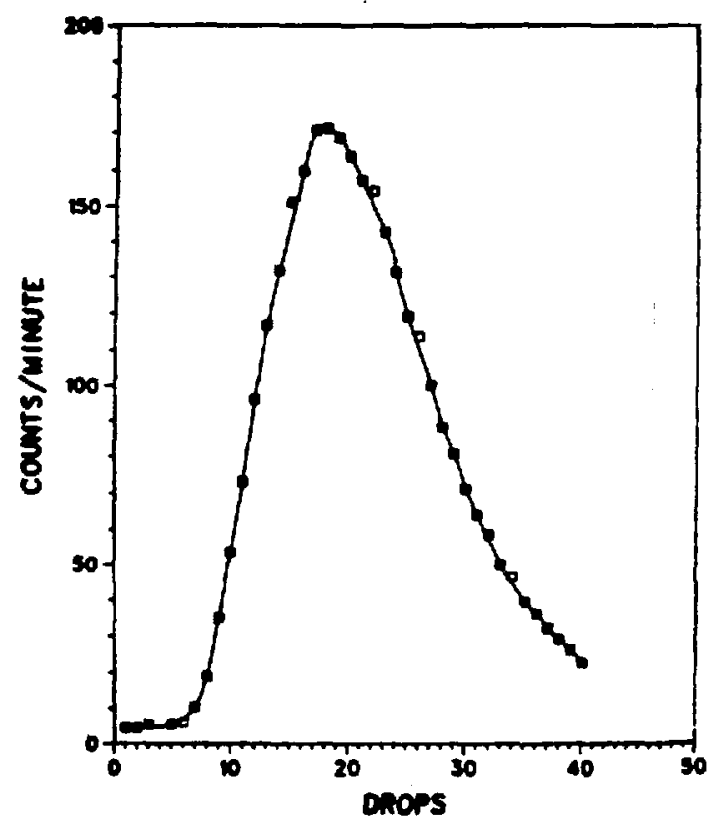

Fig. 97. Tuff G1-1883 tritium elution. 


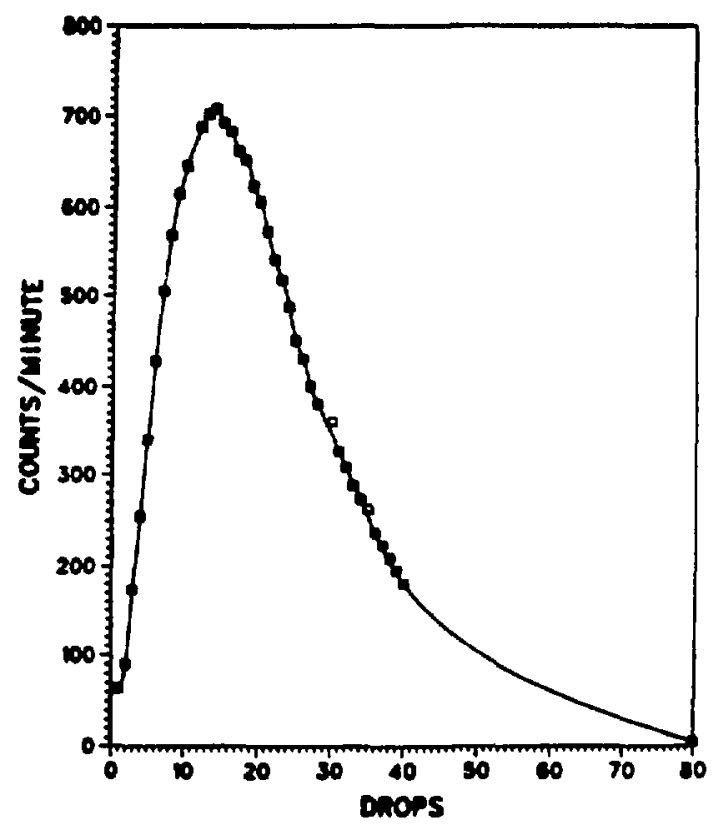

Fig. 98. Tuff G1-1982 tritium elution.

A core of sample $\mathrm{YM}-49$, a zeolitized tuff, was encased in epoxy by using t.echniques described in a previous report ${ }^{3}$ for use in the high-pressure system. After the rock was equilibrated for 4 weeks with pretreated water, the free column volume was determined by tritium elution. (The peak was greatly broadened by diffusion.) The dore was then loaded with $1.6 \times 10^{5}$ gamma counts/minute of ${ }^{237} \mathrm{Pu}$ and about $10^{5} \mathrm{dis} /$ minute of ${ }^{239} \mathrm{Pu}$. The mixture was prepared by drying the tracer and bringing the plutonium into "solution" by shaking with pretreated water. No effort was made to remove particulates from the solution. In order to achieve flow through the core, it was necessary to increase the pressure to 450 psi from the 200 psi used for the pretreatment, which suggests partial clogging of pores by particulates. A flow rate of $0.7 \mathrm{ml} / \mathrm{day}$ was achieved at this pressure, which corresponds to a fluid velocity of $6.4 \times 10^{-6} \mathrm{~cm} / \mathrm{second}$. This velocity is close to the estimate of Blankennagel and Weir 72 of $7 \mathrm{x}$ $10^{-6} \mathrm{~cm} / \mathrm{second}$ for flow predominantly through interstitial permeability beneath Pahute Mesa at the NTS. To date (three free column volumes), no activity above background, that is, $<10$ counts/minute, has been observed in the eluate.

These columns provide data to establish whether there are minerals made available by crushing that are not naturally available. The colums also allow examination for dynanic effects. 
The above studies have resulted in four conclusions.

(1) The sorption ratios determined using column methods agree with those determined by the batch techniques within a factor of 10. Recent studies indicate that much of the disparity between batch and column results may be the result of particle fractionation. When the same particle-size distributions are studied, the results are in reasonable agreement.

(2) At water velocities comparable to regional flow velocities $\left(\sim 10^{-5} \mathrm{~cm} /\right.$ second $)$ the shapes of peak elutions for simple ions are comparable to what would be expected from diffusional broadening alone. This may indicate that at these velocities kinetics is not an important factor.

(3) The anion exclusion effect may have been observed in a highly zeolitized tuff.

(4) Plutonium particulate matter was filtered out by flow through a solid-core column.

These studies also determined the permeabilities, porosities, and dispersivities of solid-tuff samples.

V. NATURAL ANALOGUES

The study of natural analogues to waste repository environments can provide important information about long-term chemical reaction and transport. Such analogues can be used to extrapolate experimental data from laboratory time, days and months, to "geologic time," the hundreds to hundreds of thousands of years that may be required for isolation of waste in a repository.

The study of hot-spring environments in felsic tuffs of Nevada is a potentially important source of information on the long-term behavior of Yucca Mountain tuffs in a hydrothermal gradient. Mineral alteration in these localities can give information about

(1) the response of the near-field repository environment to the thermal pulse that is expected after initial emplacement of waste and

(2) the tuff's response to the normal geothermal gradient, that is, the natural increase of temperature with depth.

Examining the surface hot-spring deposits is much simpler for these purposes 
than studying boreholes in the warm, deeper regions; it also will give nearly the same information because the effect of increasing temperature is much greater than that of the increasing pressure with depth.

A literature search was conducted for location maps and published articles on geothermal systems in Nevada, particularly those located in felsic tuffs similar to the tuffs of the NTS. The published material on geothermal alteration in tuffs of the right chemical and mineralogical composition and in the right general geologic environment proved inadequate for use in the present study. A survey of geothermal occurrences in Nevada tuffs is being made; work at Los Alamos then will characterize rock alteration and element transport at the one or two most appropriate geothermal sites.

Another useful analogue study may be the investigation of rock matrix diffusion of elements near ore bodies. Matrix diffusion has been proposed as a process that would retard element transport in a medium where hydrology is dominated by fracture flow. Recent measurements of lead isotope abundance in a rock core from the Oklo uranium mines suggest that lead may have diffused into a crystalline rock matrix from a fracture that had once been an aqueous transport path. Isotopic analyses can provide information on the time at which the transport took place, the amount of material that moved, and the total distance traveled.

\section{GEOCHEMICAL AND TRANSPORT MODELING}

Geochemical and transport models have been developed and tested both in support of the nuclear waste management programs at Los Alamos and to contribute towards the development of an overall performance model which will meet the requirements as stated in the 10-CFR-60 and 40-CFR-191 objectives. Efforts in geochemical modeling have been concentrated on testing available codes and improving the thermodynamic data base. Transport models have been used to design experiments and to interpret experimental results.

One of the major questions to be addressed in these studies is, can retardation processes be predicted? Geochemical models can be used to predict the chemical species that should occur in a groundwater systen. These models can also predict mineral solubility and solubility linits for the waste element species. These predictions influence the expected retardation of the waste elewents; for instance, an anionic species would not be sorbed by zeolite minerals, whereas cations are strongly sorbed. The data base for these geo- 
chemical models is being updated to include thermodynamic data for the minerals composing Yucca Mountain tuff. Transport models are being updated to include appropriate sorption mechanisms and to account for the dependence of sorption on concentration, that is nonlinear isotherms, and field tests to test the predictive ability of transport codes are being developed.

Another major question to be addressed is, how can performance assessment models be validated? The most obvious method is a direct comparison between the predictions of models and experimental results. The geochemical model EQ3 has been used to calculate uranium and plutonium solubilities in $\mathrm{J}-13$ water; validating experiments are being planned and are described in Chap. 2, Sec. V. The transport code TRACR3D ${ }^{73}$ was used to simulate and help design a field experiment in a single fracture under conditions that would be expected above the water table at the NTS.

A. Geochemical Modeling of Groundwater Interactions

Two aqueous-chemical-equilibrium computer programs are being made operational at Los Alamos to investigate groundwater interactions with stored wastes. A compiler for the PL/I language has been obtained to use with the NOS system of the Los Alamos CDC computers, and attempts were made to compile the WATEQ2 program; ${ }^{74}$ however, that program uses a number of PL/I features that are not available on the CDC compiler. Two options are being investigated: reprogramming WATEQ2 to be compatible with the CDC $\mathrm{PL} / \mathrm{I}$ compiler and using the Los Alamos WX Division IBM computer. The EQ3/6 computer program 75 is now running on the LTSS system of the Los Alamos computers, and test problems obtained with the program have been run.

Concentration data ${ }^{76}$ taken in 1964 on a water sample from well $\mathrm{J}-13$ have been used with four chemical-equilibrium computer programs: GEOCHEM, 77 REDEQL.EPAK, ${ }^{78,79}$ WATEQF, ${ }^{80}$ and EQ3/6. All programs indicated that the water was supersaturated with respect to some minerals; however, the predicted precipitates differed for GEOCHEM, REDEQL.EPAK, and EQ3/6. WATEQF does not predict precipitates; it merely indicates supersaturation. The variation in the results was caused by two differences among the programs. The programs do not consider the same group of solid phases; thus, a solid predicted to precipitate by one program may not even be considered by another program. Also, the thermodynamic data for the solids and aqueous complexes differ among the various programs. The NNWSI program requires a study of groundwater chemistry in the Yucca Mountain vicinity; the study includes the development of chemical-equilibrium 
models for the interaction of groundwater with minerals in the local tuff. To model these systems, thermodynamic data for the local minerals are required in the form of equilibrium constants or free energies of formation. Some of these data are available in thermodynamic data bases or as part of existing computer prograns that model chemical equilibrium. Data for other minerals, such as some of the zeolites found near Yucca Mountain, have not been measured.

This section describes the results of a preliminary search for free-energy data at $25^{\circ} \mathrm{C}$ for some mineral phases found during exploratory drilling at or near Yucca Mountain. The most interesting reactions are those for the formation of minerals from aqueous species. Equilibrium constant or free-energy data are presented for these formation reactions from a particular base set of aqueous species. Data were found for silica (crystalline and amorphous forms), alkali feldspars, some clays, and one zeolite (analcime); no data were found for three other zeolites observed at Yucca Mountain: clinoptilolite, heulandite, and mordenite. Free-energy data were estimated for these minerals by three different techniques. Many of the minerals of interest actually exist as solid solutions; however, few thermodynamic data are available for solid solutions, and the data presented here are all for simple compounds with specific compositions.

1. Minerals of Interest. Exploratory drilling in the vicinity of Yucca Mountain has sampled areas that are being considered for radioactive-waste storage. ${ }^{4,39,81}$ These areas are primarily beds of silicic tuffs that contain large amounts of zeolites. ${ }^{81}$ Table LVIII lists minerals that have been observed in and near these beds. The tuff also contains varying amounts of vitreous material or glass. This glass is not a thermodynamically stable phase; it cannot be assigned thermodynamic data or employed in equilibrium calculations.

Analyses of mineral phases generally yield a range of compositions. 4,39 For chemical modeling, however, specific compositions are usually needed. Table LVIII also includes chemical formulae that were used to develop the thermodynamic data. The silica minerals all have the formula $\mathrm{SiO}_{2}$; amorphous silica may actually be hydrated, but it is usually treated as $\mathrm{SiO}_{2}$ in equilibrium calculations. Sanidine $\left(\mathrm{KAlSi}_{3} \mathrm{O}_{8}\right)$ and albite $\left(\mathrm{NaAlSi}_{3} \mathrm{O}_{8}\right)$ are two alkali feldspars found at Yucca Mountain for which thermodynamic data are available.

'If a chemical equilibrium model considers solid solutions, the solid-solution phase has a variable composition. Only one computer model, EQ3/6, has begun to introduce solid solutions, but that work has not been completed. 
TABLE LVIII

MINERALS OF INTEREST IN THE YUCCA MOUNTAIN AREA

\begin{tabular}{|c|c|c|}
\hline Group & Mineral & Chemical Formula ${ }^{a}$ \\
\hline Silica & $\begin{array}{l}\text { quartz } \\
\text { cristobalite } \\
\text { amorphous silica }\end{array}$ & $\begin{array}{l}\mathrm{SiO}_{2} \\
\mathrm{SiO}_{2} \\
\mathrm{SiO}_{2}\end{array}$ \\
\hline Alkali feldspar & $\begin{array}{l}\text { albite } \\
\text { sanidine } \\
\text { microcline }\end{array}$ & $\begin{array}{l}\mathrm{NaAlSi}_{3} \mathrm{O}_{8} \\
\mathrm{KAlSi}_{3} \mathrm{O}_{8} \\
\mathrm{KAlSi}_{3} \mathrm{O}_{8}\end{array}$ \\
\hline Clay & $\begin{array}{l}\text { montmorillonite } \\
\text { beidellite } \\
\text { beidellite } \\
\text { nontronite } \\
\text { nontronite }\end{array}$ & 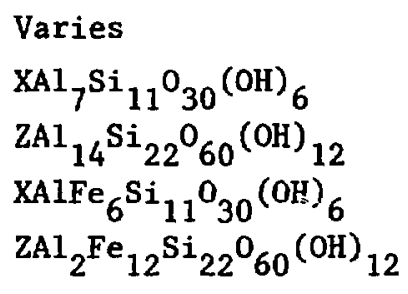 \\
\hline Zeolite & $\begin{array}{l}\text { analcime } \\
\text { clinoptilolite } \\
\text { clinoptilolite } \\
\text { heulandite } \\
\text { mordenite }\end{array}$ & $\begin{array}{l}\mathrm{Na}\left[\mathrm{AlSi}_{2} \mathrm{O}_{6}\right] \cdot \mathrm{H}_{2} \mathrm{O} \\
\mathrm{X}_{2}\left[\mathrm{Al}_{2} \mathrm{Si}_{10} \mathrm{O}_{24}\right] \cdot 8 \mathrm{H}_{2} \mathrm{O} \\
\mathrm{Z}\left[\mathrm{Al}_{2} \mathrm{Si}_{10} \mathrm{O}_{24}\right] \cdot 8 \mathrm{H}_{2} \mathrm{O} \\
\mathrm{Ca}\left[\mathrm{Al}_{2} \mathrm{Si}_{7} \mathrm{O}_{18}\right] \cdot 6 \mathrm{H}_{2} \mathrm{O} \\
\mathrm{X}\left[\mathrm{AlSi}_{5} \mathrm{O}_{12}\right] \cdot 3 \mathrm{H}_{2} \mathrm{O}\end{array}$ \\
\hline
\end{tabular}

The silicon/aluminum atomic ratios measured for the phases identified as alkali feldspars generally range from 3 to 3.4 (Ref. 39). These phases may contain some silica, so the formulae noted above are probably representative (see Table LVIII). All the alkali feldspars observed have both sodium and potassium present. ${ }^{4,39}$ They are probably solid solutions rather than mixtures of pure compounds. Thus, modeling only the pure compounds is an approximation to the actual situation. Polymorphs (minerals with the same chemical formula but different structures) for sanidine and albite have also been observed at 
Yucca Mountain. ${ }^{39}$ Although free-energy data for reactions involving polymorphs are generally similar, significant differences can exist. ${ }^{82}$ Data for microcline, a polymorph of sanidine, are included here for comparison.

Smectite clay identified as montmorillonite has been observed in the Yucca Mountain area, ${ }^{4,39,83}$ and Table LIX lists atomic ratios for a few of the samples. Thermodynamic data were found for a number of smectite clays with the montmorillonite structure (see Table LVIII). The formulae labeled beidellite in Table IVIII have been called montmorillonite in chemical literature. ${ }^{84}$ The observed silicon/aluminum and silicon/iron atomic ratios (see Table LIX) do not match either the beidellite or nontronite formulae in Table LVIII; instead, they appear to represent some intermediate material. Thermodynamic data are also available for a number of true montmorillonite clays with specific alkali metal and alkaline earth compositions (Sec. VI.A.2.b); these include the Aberdeen, Belle Fourche, Colony, Arizona, and Wyoming montmorillonites, and some data are presented for these materials. The alkali metal and alkaline earth cations in clays seem to occupy easily exchanged positions. The actual minerals are more nearly solid solutions, where all the cations may be present. As in the case of the alkali feldspars, the thermodynamic data are for compounds with specific compositions.

TABLE LIX

MONTMORILLONITE DATA FROM YUCCA MOUNTAIN AREA

Atomic Ratios

\begin{tabular}{lcc}
\hline \multicolumn{3}{c}{ Atomic Ratios } \\
Si/Al & Si/Fe & $\begin{array}{c}\text { Alkaline Earth/ } \\
\text { Alkali Metal }\end{array}$ \\
\cline { 3 - 3 } & & \\
4.7 & 28 & 0.003 \\
3.1 & 94 & 0.088 \\
3.5 & 49 & 0.028 \\
& & \\
3.1 & 41 & 0.40 \\
2.4 & 47 & 0.55
\end{tabular}

${ }_{\mathrm{a}}^{\mathrm{Ref} .} 39$.
${ }^{\mathrm{b}}$ Ref. 4.

$\frac{\text { Sample }}{\text { UE25a-1 }}$

YM-43

YM-45

YM-53

$J-13^{b}$

$J A-36-B C$

2.4

47

0.55

JA-36-BC 
Four zeolites have been identified in the Yucca Mountain area; they are analcime, clinoptilolite, heulandite, and mordenite. Table LX lists some atomic ratios for a few samples of these minerals. Zeolites usually show a range of compositions; the formulae for these minerals, as listed in Table LVIII, are generally. accepted. 48,85,86 The predominant cation observed for analcime is sodium. 4 The single formula in Table LVIII represents the observed composition of analcime reasonably well. Clinoptilolite shows varying amounts of sodium, potassium, calcium, and magnesium. ${ }^{4,39}$ These cations are normally exchangeable; thermodynamically, the mineral should probably be considered as a solid solution. However, only the four pure compounds shown in Table LVIII were considered. Heulandite is structuraliy similar to clinoptilolite; the silicon/aluminum plus iron ratio is lower and the exchange cation is predominantly calcium. ${ }^{4,39}$ The single formula in Table LVIII represents the observed composition. Mordenite shows predominantly sodium and potassium as the exchange cations. ${ }^{39}$ The observed silicon/aluminum plus iron ratios (Table LX) are somewhat below the accepted formulae for mordenite (Table LVIII). Free-energy data were found for analcime only, and data were estimated for clinoptilolite, heulandite, and mordenite by three different techniques.

2. Data from Existing Sources. There are many existing compilations of free-energy or equilibrium constant data that include information on mineral species. Helgeson has produced a number of such compilations, 84 the most recent being the SUPCRT computer program. 87 The data collected by Robie and Waldbaum, which represent free energies of mineral formation from the elements in their standard states, are also widely used. ${ }^{88}$ Recently, Benson and Teague have tabulated thermodynamic data that are of interest when studying radioactive waste systems. ${ }^{89}$ Another source of data is the chemical-equilibrium computer programs that include thermodynamic data in their data bases. Four programs available at Los Alamos are EQ $3 / 6,{ }^{75}$ WATEQF, 80 GEOCHEM, 77 and REDEQL.EPAK; 78,79 data from WATEQ2 ${ }^{74}$ are also available, although the program is not currently running here.

In some cases, there is considerable disagreement among the vagious data sources, as is evident in the data presented here from different sources. All data are presented as $\log _{10} K$, where $K$ is the equilibrium constant for the formation reaction of the mineral from aqueous species at $25^{\circ} \mathrm{C}$. The free energy for this reaction $\Delta G^{\circ}$ is related to $K$ as 
TABLE LX

ZEOLITE DATA FROM YUCCA MOUNTAIN AREA

Atomic Ratios

$\frac{\text { Mineral }}{\text { Analcime }}$

$\frac{\text { Sample }}{\mathrm{J}-13^{\mathrm{a}}} \quad$ Si/A

$\mathrm{JA}-26$

2.9

$<0.01$

0.005

JA-31

2.7

$<0.01$

0.007

$\mathrm{JA}-35$

2.3

$<0.01$

0.003

Clinoptilolite

UE25a-1 ${ }^{b}$

YM-32

5.2

$<0.01$

1.61

YM-35

6.1

$<0.01$

0.81

YM-36

5.1

$<0.01$

1.43

YM-38

4.4

$<0.01$

0.85

YM-40

4.8

0.04

0.70

YM- 47

5.5

$<0.01$

1.09

YM-49

5.7

0.05

0.42

YM-5 1

5.0

$<0.01$

1.62

$$
\mathrm{J}-13^{\mathrm{a}}
$$

JA-20

4.6

$\mathrm{NR}^{\mathrm{C}}$

1.49

JA-23

4.7

$<0.01$

1.23

Heulandite

$$
\text { UE } 25 a-1^{b}
$$

$$
\text { YM-30 }
$$

YM-31

3.9

$<0.01$

8.5

YM- 42

3.2

0.04

3.1

YM-42

3.8

$<0.01$

3.7

4.2

$<0.01$

1.5

Mordenite

$$
\begin{array}{r}
\text { UE25a-1 } \\
\text { YM-46 } \\
\text { YM-46 }
\end{array}
$$

3.2

$<0.01$

0.008

3.0

0.15

0.009

\footnotetext{
aef. 4.

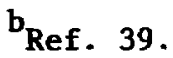

$\mathrm{c}_{\mathrm{NR}}=$ not reported .
} 


$$
\Delta G^{0}=-2.3026 \mathrm{RT} \log _{10} \mathrm{~K}
$$

where $R$ is the gas constant $(1.9872 \mathrm{cal} / \mathrm{mole} K)$ and $T$ is the absolute temperature (K). At $25^{\circ} \mathrm{C}, \mathrm{T}=298.15 \mathrm{~K}$,

$$
\Delta \mathrm{G}_{\mathrm{r}}^{\circ}(\mathrm{kcal} / \mathrm{mole})=-1.364 \log _{10} \mathrm{~K}
$$

Formation reactions for the various minerals from the aqueous species are listed in the following sections.

a. Silica. The formation reaction for solid silica phases is

$$
\mathrm{SiO}_{2}^{\circ}=\mathrm{SiO}_{2}(\mathrm{x})
$$

where $\mathrm{SiO}_{2}{ }^{\circ}$ is the aqueous silica species and $\mathrm{x}$ is $q$, $c$, or a for quartz, cristobalite, or amorphous silica, respectively. (Some data bases use $\mathrm{H}_{2} \mathrm{SiO}_{3}{ }^{\circ}$ or $\mathrm{H}_{4} \mathrm{SiO}_{4}^{\circ}$ as the basic aqueous species.) Table LXI lists values of $\log _{10} \mathrm{~K}$ for $\mathrm{Eq}$. (13). It is evident that many of the compilations or data bases use the same source, and the agreement among the various sources is quite good.

\begin{tabular}{|c|c|c|c|}
\hline \multirow[b]{2}{*}{ Source } & \multicolumn{3}{|c|}{$\mathrm{g}_{10} \mathrm{~K}$} \\
\hline & $\overline{\mathrm{SiO}_{2}[\mathrm{q}]}$ & $\mathrm{SiO}_{2}[\mathrm{c}]$ & $\mathrm{SiO}_{2}[\mathrm{a}]$ \\
\hline SUPCRT (Ref. 87) & +4.00 & +3.45 & +2.71 \\
\hline Benson and Teague (Ref. 89) & +4.00 & +4.18 & +2.71 \\
\hline WATEQF (Ref. 80) & +4.01 & +3.59 & +3.02 \\
\hline EQ3/6 (Ref. 75) & +4.00 & +3.45 & +2.71 \\
\hline REDEQL.EPAK (Ref, 78) & -- & -- & $+2.70^{b}$ \\
\hline GEOCHEM (Ref. 77) & -- & -- & $+2.70^{b}$ \\
\hline
\end{tabular}

TABLE LXI

THERMODYNAMIC DATA FOR SILICA MINERALS 
b. Alkali Feldspar. The formation reactions for the two alkali feldspars are

$$
\begin{aligned}
& \mathrm{Na}^{+}+\mathrm{Al}^{3+}+3 \mathrm{SiO}_{2}^{\circ}+2 \mathrm{H}_{2} \mathrm{O}_{\ell}=\mathrm{NaAlSi}_{3} \mathrm{O}_{8}+4 \mathrm{H}^{+} \text {and } \\
& \mathrm{K}^{+}+\mathrm{Al}^{3+}+3 \mathrm{SiO}_{2}^{\circ}+2 \mathrm{H}_{2} \mathrm{O}_{\ell}=\mathrm{KAlSi}_{3} \mathrm{O}_{8}+4 \mathrm{H}^{+},
\end{aligned}
$$

where $\mathrm{H}_{2} \mathrm{O}_{\ell}$ is liquid water. Table LXII lists values of $\log _{10} \mathrm{~K}$ for these reactions. Three of the sources list data for low and high albite; ${ }^{75,87,89}$ they are all based on SUPCRT. WATEQF lists data for albite only; it does not have data for sanidine or microcline, but the value for adularia ( $\mathrm{KAlSi}{ }_{3} \mathrm{O}_{8}$ ), a polymorph of sanidine, is shown for comparison. The agreement among the various sources is quite good.

c. Clays. The formation reactions for the beidellite clays are

$$
\begin{aligned}
& \mathrm{X}^{+}+7 \mathrm{Al}^{3+}+11 \mathrm{SiO}_{2}{ }^{\circ}+14 \mathrm{H}_{2} \mathrm{O}_{\ell}=\mathrm{XAl}_{7} \mathrm{Si}_{11} \mathrm{O}_{30}(\mathrm{OH})_{6}+22 \mathrm{H}^{+} \text {and } \\
& \mathrm{Z}^{2+}+14 \mathrm{Al}^{3+}+22 \mathrm{SiO}_{2}^{\mathrm{O}}+28 \mathrm{H}_{2} \mathrm{O}_{\ell}=\mathrm{ZAl}_{14} \mathrm{Si}_{22} \mathrm{O}_{60}{ }^{(\mathrm{OH})}{ }_{12}+44 \mathrm{H}^{+},
\end{aligned}
$$

TABLE LXII

THERMODYNAMIC DATA FOR ALKALI FELDSPAR MINERALS

Source

SUPCRT (Ref. 87)

\begin{tabular}{|c|c|c|}
\hline \multicolumn{3}{|c|}{$\log _{10} \mathrm{~K}$} \\
\hline $\begin{array}{l}\text { Albite } \\
\text { Eq. (14) }\end{array}$ & $\begin{array}{l}\text { Sanidine } \\
\text { Eq. (15) } \\
\end{array}$ & $\begin{array}{l}\text { Microcline } \\
\text { Eq. (15) }\end{array}$ \\
\hline$-3.10^{\mathrm{a}}$ & -1.28 & -0.08 \\
\hline$-4.42^{b}$ & & \\
\hline
\end{tabular}

Benson and Teague (Ref. 89)

WATEQF (Ref. 80)

EQ3/6 (Ref. 75)

$\begin{array}{rrr}3.09^{\mathrm{a}} & -1.25 & -0.05 \\ -4.41^{\mathrm{b}} & \end{array}$

$-4.05-1.48^{c}$

$\begin{array}{lll}-3.08^{\mathrm{a}} & -1.37 & -0.17\end{array}$

\footnotetext{
aow albite.

$\mathrm{b}_{\text {High albite. }}$

Data are for adularia; no sanidine data are available.
} 
where $X$ is sodium or potassium, and $Z$ is calcium or magnesium. Table LXIII lists values of $\log _{10} K$ for these reactions. The data from GEOCHEM do not agree with data from other sources, and the difference between WATEQF and WATEQ2 for calcium-beidellite is also puzzling.

The formation reactions for the nontronite clays are

$$
\mathrm{X}^{+}+\mathrm{Al}^{3+}+6 \mathrm{Fe}^{3+}+11 \mathrm{SiO}_{2}^{\circ}+14 \mathrm{H}_{2} \mathrm{O}_{\ell}=\mathrm{XAlFe}_{6} \mathrm{Si}_{11} \mathrm{O}_{30}{ }^{(\mathrm{OH})_{6}}+22 \mathrm{H}^{+}
$$

and

$$
\begin{gathered}
\mathrm{Z}^{2+}+2 \mathrm{Al}^{3+}+12 \mathrm{Fe}^{3+}+22 \mathrm{SiO}_{2}^{\circ}+28 \mathrm{H}_{2} \mathrm{O}_{\ell} \\
=\mathrm{ZAl}_{2} \mathrm{Fe}_{12} \mathrm{Si}_{22} \mathrm{O}_{60}{ }^{(\mathrm{OH})_{12}}+44 \mathrm{H}^{+},
\end{gathered}
$$

where $X$ is sodium or potassium and $Z$ is calcium or magnesium. Table LXIV lists values of $\log _{10} \mathrm{~K}$ for these reactions. The data used in $\mathrm{EQ} 3 / 6$ have been estimated by Wolery. 75

Free-energy data have been measured or estimated for a number of montmorillonite clays with specific compositions. $80,90,91,92$ Except in a few isolated cases, these materials are not available in current chemical-equilibrium computer programs. Table LXV lists a sampling of the data. The quantity $\Delta G^{\circ}{ }_{f}$ is the free energy of formation of the mineral from the elements in their standard states at $25^{\circ} \mathrm{C}$; in some cases, it is the only datum reported. For

TABLE LXIII

THERMODYNAMIC DATA FOR BEIDELLITE CLAYS

\begin{tabular}{cccc}
\multicolumn{4}{c}{$\log _{10} K$} \\
$\mathrm{Xq}=\mathrm{Na}(16)$ & $\mathrm{X}=\mathrm{K}$ & $\mathrm{Z}=\mathrm{Ca}$ & $\mathrm{Z}=\mathrm{Mg}$ \\
-19.06 & $\frac{\mathrm{Eq} \cdot(16)}{-18.32}$ & $\frac{\mathrm{Eq} .(17)}{-37.10}$ & $\frac{\mathrm{Eq} \cdot(17)}{-36.60}$ \\
-- & -- & -38.7 & -- \\
-- & -- & -51.8 & -- \\
-11.7 & -10.5 & -21.6 & -21.7 \\
-20.7 & -- & -- & -- \\
-19.12 & -18.24 & -38.02 & -37.81
\end{tabular}


TABLE LXIV

THERMODYNAMIC DATA FOR NONTRONITE CLAYS

Source

EQ3/6 (Ref. 75)

$\overline{\mathrm{X}=\mathrm{Na}} \frac{\log _{10} K}{\mathrm{X}=\mathrm{K}} \mathrm{Z}=\mathrm{Ca} \quad \mathrm{Z}=\mathrm{Mg}$

Eq. (18) Eq. (18) Eq. (19) Eq. (19)

$\begin{array}{llll}+47.6 & +48.4 & +95.3 & +95.6\end{array}$

these minerals, the equilibrium constant for formation fron aqueous species (K) was calculated as described below, using data from SUPCRT. 87

d. Zeolites. Thermodynamic data were found for only one zeolite that had been detected in the Yucca Mountain area: analcime; its formation reaction is

$$
\mathrm{Na}^{+}+\mathrm{Al}^{3+}+2 \mathrm{SiO}_{2}^{\circ}+3 \mathrm{H}_{2} \mathrm{O}_{2}=\mathrm{NaAlSi}_{2} \mathrm{O}_{6} \cdot \mathrm{H}_{2} \mathrm{O}+4 \mathrm{H}^{+}
$$

Table LXVI lists values of $\log _{10} \mathrm{~K}$ for this reaction. The later, more positive value from SUPCRT and EQ3/6 results from a re-evaluation of Helgeson's experimental data. 87 Data for other zeolites, ${ }^{87}$ not detected in the Yucca Mountain area, are also discussed below.

3. Estimated Data. Free-energy data could not be found for three zeolites observed in the Yucca Mountain area (clinoptilolite, heulandite, and mordenite). Three methods were used to estimate thermodynamic data for these minerals. Two of the methods were developed to estimate free energy of formation of clays 90,91 but have not previously been applied to zeolites; the third method has had more general application. 82 In addition to the calculations for the three zeolites noted above, calculations were also done for three other zeolites (analcime, wairakite, and laumontite) for comparison with measured data.

All three wethods estimate the free energy of formation of a mineral from the elements in their standard states at $25^{\circ} \mathrm{C}\left(\Delta G_{f}^{\circ}\right)$. For use in aqueous chemical-equilibrium calculations, the free energy ( $\Delta G^{\circ}{ }_{r}$ ) or equilibrium constant (K) for the formation reaction frc aqueous species wust be known. This 
THERMODYNAMIC DATA FOR MONTMORILLONITE CLAYS

\section{Formula}

$(\mathrm{H}, \mathrm{Na}, \mathrm{K})_{0.28}{ }^{\mathrm{Mg}} 0.29^{\mathrm{Fe}} 0.23^{\mathrm{Al}}{ }_{1.58^{-S i}} \mathrm{~S}_{3.93^{\circ}{ }_{10}(\mathrm{OH})_{2}}$

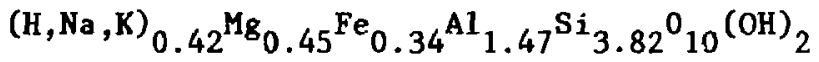
$\left(\mathrm{Ca} 0.19^{\mathrm{Na}} 0.02 \mathrm{~K}_{0.02}\right)\left[\mathrm{Mg}_{0.33} \mathrm{Fe}_{0.14}{ }^{\mathrm{Al}}{ }_{1.59} \mathrm{Si}_{\left.3.93^{0}{ }_{10}\right](\mathrm{OH})}\right.$ $\left.\left(\mathrm{Ca}_{0.1} \mathrm{Na}_{0.27} \mathrm{~K}_{0.02}\right)^{\left[\mathrm{Mg}_{0}\right.} 0.22^{\mathrm{Fe}_{0.19}}{ }^{\mathrm{Al}}{ }_{1.58^{\mathrm{Si}}} 3.94^{0}{ }_{10}\right](\mathrm{OH})_{2}$ $\mathrm{Mg}_{0.225}\left[\mathrm{Mg}_{0.27} \mathrm{Fe}_{0.405} \mathrm{Al}_{1.645} \mathrm{Si}_{\left.3.70^{0}{ }_{10}\right](\mathrm{OH})_{2}}\right.$

$\begin{array}{cc}\begin{array}{c}\Delta \mathrm{G}_{\mathbf{f}}^{\circ} \\ \left(\mathrm{kcal} / \mathrm{mole}^{\mathrm{a}}\right)\end{array} & \frac{\log _{10} K^{b}}{+0.1} \\ -- & -2.6 \\ -- & -11.1 \\ -1252.1 & -7.8 \\ -1246.3 & +1.4\end{array}$

Source

WATEQF (Ref. 80)

WATEQF (Ref. 80)

Nriagu (Ref. 90)

Nriagu (Ref. 90)

Mattigod and

Sposito (Ref. 92)

${ }^{a} \Delta G^{\circ}{ }_{f}$ is the free energy of formation from the elements in their standard states at $25^{\circ} \mathrm{C}$.

$b_{K}$ is the equilibrium constant for formation of the mineral from aqueous species $\mathrm{Na}^{+}, \mathrm{K}^{+}, \mathrm{H}^{+}, \mathrm{Mg}^{2+}, \mathrm{Ca}^{2+}, \mathrm{Fe}^{3+}$, $\mathrm{Al}^{3+}, \mathrm{SiO}_{2}^{\circ}$, and $\mathrm{H}_{2} \mathrm{O}_{\ell}$. 
TABLE LXVI

THERMODYNAMIC DATA FOR ANALCIME

Source

SUPCRT (Ref. 87)

Helgeson (Ref. 84)

WATEQF (Ref. 80$)^{a}$

EQ3/6 (Ref. 75) $\log _{10} \mathrm{~K}$

Eq. (20)

$-7.28$

$-9.37$

$-9.35$

$-7.26$

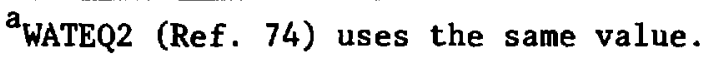

requires a knowledge of values of $\Delta G^{\circ}$ for the aqueous species. With these data, $\Delta G^{\circ}$ for a mineral can be converted into $\Delta G^{\circ}{ }_{r}$ or $K$ for that mineral. The values of $\Delta G^{\circ}$ for the aqueous species should be consistent with the thermodynamic data employed to estimate $\Delta G^{\circ}{ }_{f}$ for that mineral. Values of $\Delta G^{\circ}{ }_{f}$ for aqueous species from three different sources were used in these calculations, $87,88,91$ and the data are shown in Table LXVII. The only significant difference for the zeolite calculations is in $\Delta \mathrm{G}^{\circ}{ }_{f}$ for $\mathrm{Al}^{3+}$; the value used by Tardy and Garrels is 2 to $3 \mathrm{kcal} / \mathrm{mole}$ more negative than from the other sources.

The following sections briefly describe the three methods of estimating $\Delta G^{\circ}{ }_{f}$ of the minerals and present the results.

a. Tardy and Garrels. Tardy and Garrels proposed a simple method of estimating $\Delta G^{\circ}$ of layer silicates. ${ }^{91}$ A reaction that forms the desired mineral is written in terms of the oxides of the elements involved. With analcime, for example,

$$
\frac{1}{2}\left[\mathrm{Na}_{2} \mathrm{O}\right]_{\mathrm{ex}}+\frac{1}{2}\left[\mathrm{Al}_{2} \mathrm{O}_{3}\right]_{\mathrm{sil}}+2\left[\mathrm{SiO}_{2}\right]_{\mathrm{sil}}+\left[\mathrm{H}_{2} \mathrm{O}\right]_{\mathrm{sil}}=\mathrm{NaAlSi}_{2} \mathrm{O}_{6} \cdot \mathrm{H}_{2} \mathrm{O},
$$

where the subscript ex implies that a free energy of formation associated with an exchange reaction should be used for the species, and the subscript sil implies that a free energy of formation within the silicate structure should be used for the species. The alkali metal or alkaline earth cations that normally participate in the ion-exchange reactions have the ex subscript; the 
TABLE LXVII

$\Delta G^{\circ}$ FOR AQUEOUS SPECIES

Aqueous Species

$\mathrm{Na}^{+}$

$\mathrm{Ca}^{2+}$

$\mathrm{Mg}^{2+}$

$\mathrm{Al}^{3+}$

$\mathrm{Fe}^{3+}$

$\mathrm{SiO}_{2}^{\circ}$

$\mathrm{H}_{2} \mathrm{O}_{1}$

$\mathrm{OH}-$

$\mathrm{H}^{+}$

\begin{tabular}{|c|c|c|}
\hline \multicolumn{3}{|c|}{$\underset{(\mathrm{kcal} / \mathrm{fole})}{\Delta \mathrm{G}^{\circ}}$} \\
\hline $\begin{array}{l}\text { Tardy and } \\
\text { Garrels } \\
\end{array}$ & SUPCRT $^{\mathrm{b}}$ & $\begin{array}{l}\text { Robie and } \\
\text { Waldbaum }\end{array}$ \\
\hline-62.5 & -62.62 & -62.54 \\
\hline-67.7 & -67.58 & -67.70 \\
\hline-132.2 & -132.16 & -132.18 \\
\hline-108.9 & -108.70 & -108.90 \\
\hline-119.5 & -116.97 & -116.00 \\
\hline-- & -1.10 & -2.52 \\
\hline-199.12 & -199.19 & $-199.18^{d}$ \\
\hline-56.69 & -56.69 & -56.69 \\
\hline-37.6 & -37.60 & -37.59 \\
\hline 0 & 0 & 0 \\
\hline
\end{tabular}

${ }^{a} \operatorname{Ref.} 91$.

bef. 87 .

C Ref. 88 .

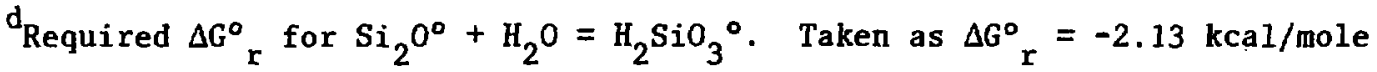
from Ref. 89.

elements in the silicate structure use the sil subscript. The appropriate free-energy data are tabulated by Tardy and Garrels. 91 Table LXVIII lists values of $\Delta G^{\circ}$ calculated for a number of zeolite minerals by this method in the column labeled "TG." For analcime, wairakite, and laumontite, these estimates are $\sim 10$ to $20 \mathrm{kcal} / \mathrm{mole}$ more negative than the experimental results (see Table IXVIII). It was observed that if $\left[\mathrm{Na}_{2}{ }^{0}\right]_{\text {sil }}$ was used in place of $\left[\mathrm{Na}_{2} \mathrm{O}\right]_{\text {ex }}$ in $\mathrm{Eq}$. (21), the estimated value of $\Delta G^{\circ}{ }_{f}$ was closer to the experimental result. The same was true for the other two zeolites, using the experimental data available. Table IXVIII also lists values of $\Delta G^{\circ}{ }_{f}$ calculated from only free-energy data associated with the silicate structure; these results are in the coluan headed "modified TG." Although there is no justification for this 
TABLE LXVIII

THERMODYNAMIC DATA FOR 2EOLITES

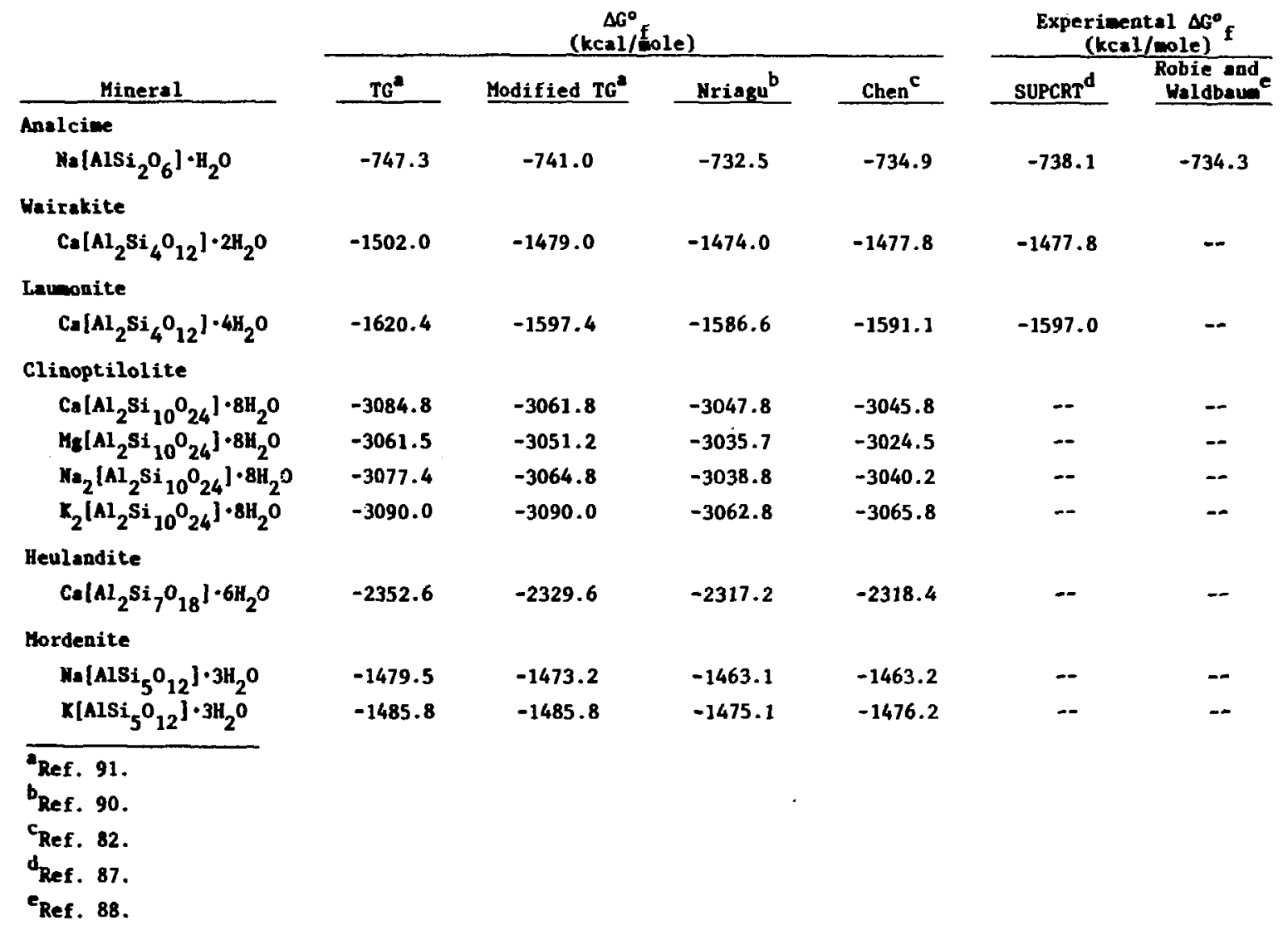


modification, it produces better estimates for the three zeolites for which comparisons can be made.

Tardy and Garrels tabulated values of $\Delta G^{\circ}{ }_{f}$ for aqueous species; ${ }^{91}$ these values are shown in Table LXVII. They were used to convert $\Delta G_{f}^{\circ}$ to $\Delta G^{\circ}{ }_{r}$ for these minerals. Table LXIX shows values of $\Delta G^{\circ}{ }_{r}$ and $\log _{10} K$ for these mincrals, calculated by the modified TG method.

b. Nriagu. Nriagu proposed another simple method for estimating $\Delta G^{\circ}{ }_{f}$ of clay minerals. 90 He also writes a reaction for the formation of the mineral but uses hydroxides of the elements involved. For analcime,

$$
\mathrm{NaOH}+\mathrm{Al}(\mathrm{OH})_{3}+2 \mathrm{Si}(\mathrm{OH})_{4}=\mathrm{NaAlSi}_{2} \mathrm{O}_{6} \cdot \mathrm{H}_{2} \mathrm{O}+5 \mathrm{H}_{2} \mathrm{O} .
$$

The value of $\Delta G^{\circ}{ }_{f}$ for analcime is calculated as

$$
\begin{gathered}
\Delta G_{f}^{\circ} \text { (analcime) }=\Delta G^{\circ}{ }_{f}(\mathrm{NaOH})+\Delta G_{f}^{\circ}\left[\mathrm{Al}(\mathrm{OH})_{3}\right] \\
+2 \Delta G_{f}^{\circ}\left[\mathrm{Si}(\mathrm{OH})_{4}\right]-5\left[\Delta G^{\circ}{ }_{f}\left(\mathrm{H}_{2} \mathrm{O}\right)+\sigma\right] .
\end{gathered}
$$

The parameter $\sigma$ is an empirical correction factor given the value $0.39 \mathrm{kcal} / \mathrm{mole}$. Appropriate values of $\Delta G^{\circ}{ }_{f}$ for the hydroxides are tabulated by Nriagu. Table LXVIII lists values of $\Delta G^{\circ}$ for the zeolites considered in the column headed "Nriagu." For analcime, wairakite, and laumontite, the estimated values are $\sim 3$ to $10 \mathrm{kcal} / \mathrm{mole}$ more positive than the experimental data. No attempt was made to modify this method.

Nriagu did not tabulate values of $\Delta G^{\circ}$ for aqueous species. Data from SUPCRT $^{87}$ (see Table LXVII) were used to convert $\Delta G^{\circ}{ }_{f}$ to $\Delta G^{\circ}{ }_{r}$ for this method. The results are listed in Table LXIX.

c. Chen. Chen has proposed a somewhat more complex method of estimating $\Delta G^{\circ}{ }_{f}$ of silicate minerals. ${ }^{82}$ Whereas the methods of Tardy and Garrels and Nriagu have only been applied to layer silicates or clays, Chen's method has been used for a wider range of silicate minerals, including feldspar and analcime. For this method, a series of reactions is written for forming the mineral of interest from simpler compounds. For analcime, three of the reactions used were 
TABLE LXIX

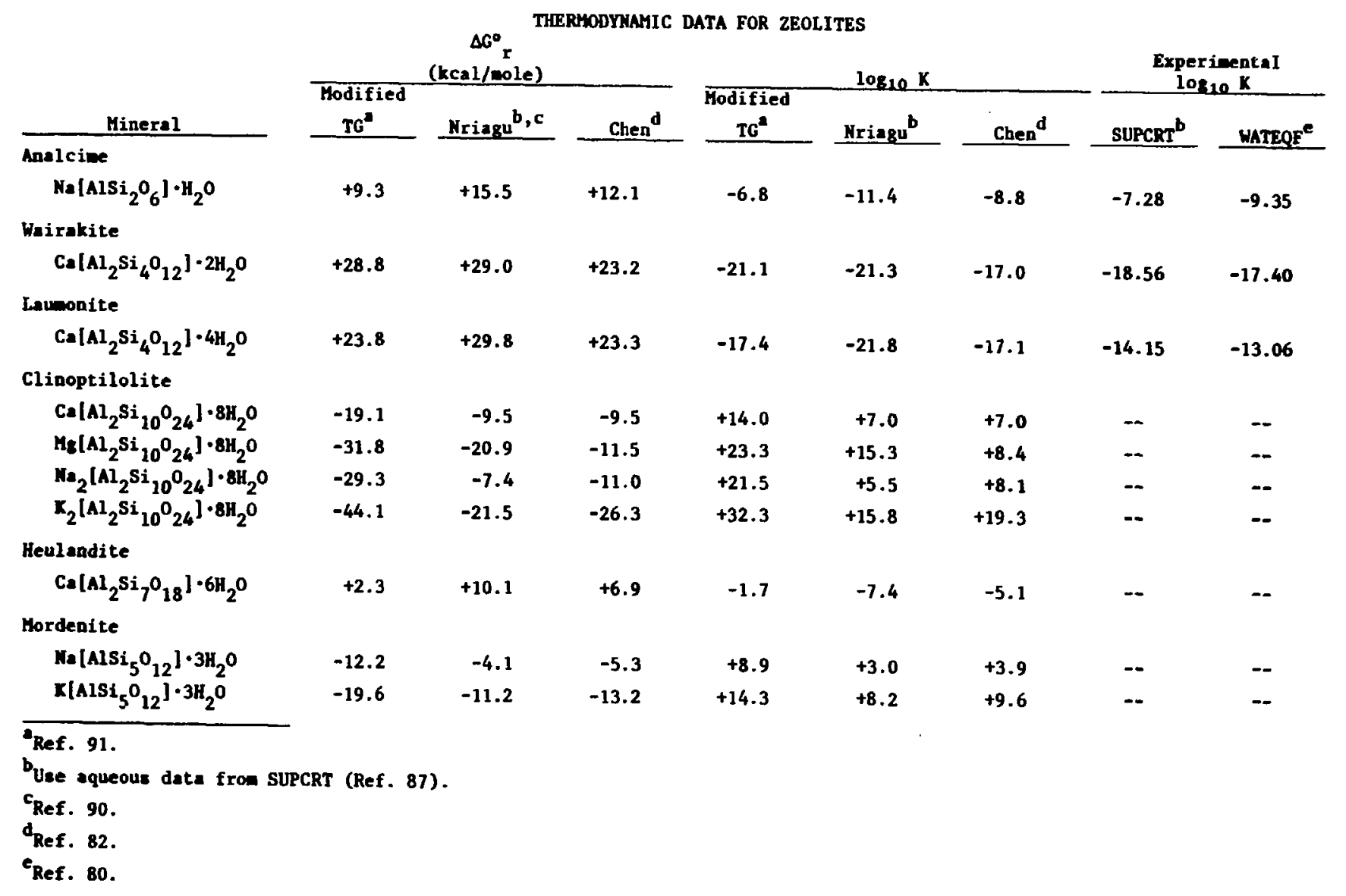




$$
\begin{gathered}
\frac{1}{2} \mathrm{Na}_{2} \mathrm{O}+\frac{1}{2} \mathrm{Al}_{2} \mathrm{O}_{3}+2 \mathrm{SiO}_{2}+\mathrm{H}_{2} \mathrm{O}=\mathrm{NaAlSi}_{2} \mathrm{O}_{6} \cdot \mathrm{H}_{2} \mathrm{O}, \\
\frac{1}{2} \mathrm{Na}_{2} \mathrm{SiO}_{3}+\frac{1}{2} \mathrm{Al}_{2} \mathrm{SiO}_{5}+\mathrm{SiO}_{2}+\mathrm{H}_{2} \mathrm{O}=\mathrm{NaAlSi}_{2} \mathrm{O}_{6} \cdot \mathrm{H}_{2} \mathrm{O} \text {, and } \\
\mathrm{NaAlSiO}_{4}+\mathrm{SiO}_{2}+\mathrm{H}_{2} \mathrm{O}=\mathrm{NaAlSi}_{2} \mathrm{O}_{6} \cdot \mathrm{H}_{2} \mathrm{O} .
\end{gathered}
$$

Using standard values of $\Delta G^{\circ}$ for the reactants, a value of $\Delta G^{\circ} f$ for analcime is calculated for each equation. Chen used $\Delta G^{\circ}{ }_{f}$ data from the tabulation of Robie and Waldbaum ${ }^{88}$ in his analysis; those data were also used here. The values of $\Delta G_{f}^{\circ}$ for the mineral of interest (analcime in this example) will differ for the various reactions. Chen observed that they tend to become more negative as the reactants become more complex and that they seem to approach a limit. He proposed finding the limit by fitting data to the equation,

$$
y_{i}=a \exp \left(b x_{i}\right)+c,
$$

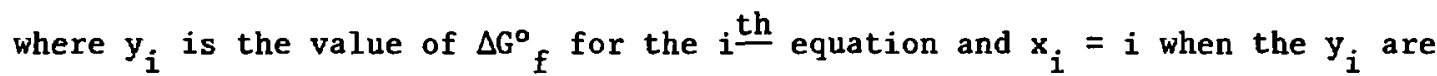
written in order, so that $y_{0}>y_{1}>y_{2} \ldots>y_{n}$. The parameter $c$ is the limit and is taken as $\Delta G_{f}^{\circ}$ for the mineral. Table LXVIII lists values of $\Delta G^{\circ}{ }_{f}$ for the zeolites considered in the column headed "Chen." For analcime, wairakite, and laumontite, the estimates are $\sim 0$ to $6 \mathrm{kcal} / \mathrm{mole}$ more positive than the experimental data.

For calculations by this method, values of $\Delta G^{\circ} f_{\text {for }}$ aqueous species were also taken from the tabulation of Robie and Waldbaum ${ }^{88}$ (Table LXVII). Values of $\Delta G^{0}$ and $\log _{10} \mathrm{~K}$ are listed in Table LXIX.

d. Effect of Composition. Two additional calculations estimated the effect of changing the silicon/aluminum ratio and the amount of water of hydration. The calculations were done for calcium-clinoptilolite $\left[\mathrm{Ca}\left(\mathrm{Al}_{2} \mathrm{Si}_{10} \mathrm{O}_{24}\right) \cdot 8 \mathrm{H}_{2} \mathrm{O}\right]$ only; it has a silicon/aluminum ratio of 5 and has 8 molecules of water of hydration as the formula is written. Table IXX shows values of $\Delta G^{\circ}{ }_{f}$ and $\log _{10} \mathrm{~K}$ for silicon/aluminum ratios of 3,4 , and 5 . These minerals become less stable $\left(\log _{10} \mathrm{~K}\right.$ becomes more negative) as the ratio is reduced. Table LXXI shows values of $\Delta G^{\circ}{ }_{f}$ and $\log _{10} \mathrm{~K}$ as the number of molecules 
TABLE LXX

EFFECT OF SILICON/ALUMINUM RATIO ON $\mathrm{G}_{\mathrm{f}}^{\circ}$ AND LOG ${ }_{10} \mathrm{~K}$

\begin{tabular}{|c|c|c|c|c|c|c|c|}
\hline \multirow[b]{2}{*}{ Formula } & \multirow[b]{2}{*}{$\begin{array}{l}\text { Si/Al } \\
\text { Ratio }\end{array}$} & \multicolumn{3}{|c|}{$\underset{(\mathrm{kcal} / \mathrm{mole})}{\Delta G^{\circ}}$} & \multicolumn{3}{|c|}{$\log _{10} k$} \\
\hline & & $\begin{array}{l}\text { Modified } \\
\mathrm{TG}^{\mathrm{a}}\end{array}$ & Nriagu ${ }^{b}$ & Chen $^{c}$ & $\begin{array}{c}\text { Modified } \\
\text { TG }^{\mathrm{a}}\end{array}$ & Nriagu ${ }^{b}$ & Chen $^{c}$ \\
\hline $\mathrm{Ca}\left[\mathrm{Al}_{2} \mathrm{Si}_{6}{ }_{16}\right] \cdot 8 \mathrm{H}_{2} \mathrm{O}$ & 3 & -2243.4 & -2223.8 & -2227.2 & -2.1 & -13.0 & -9.0 \\
\hline $\mathrm{Ca}\left[\mathrm{Al}_{2} \mathrm{Si}_{8} \mathrm{O}_{20}\right] \cdot 8 \mathrm{H}_{2} \mathrm{O}$ & 4 & -2652.6 & -2635.8 & -2636.5 & +6.0 & -3.0 & -1.0 \\
\hline $\mathrm{Ca}\left[\mathrm{Al}_{2} \mathrm{Si}_{10} \mathrm{O}_{24}\right] \cdot 8 \mathrm{H}_{2} \mathrm{O}$ & 5 & -3061.8 & -3047.8 & -3045.8 & +14.0 & +7.0 & +7.0 \\
\hline
\end{tabular}

${ }_{\text {a Ref. } 91 .}$
$b_{\text {Ref. } 90 .}$
$c_{\text {Ref. } 82 .}$ 


\section{TABLE IXXI}

EFFECT OF WATER OF HYDRATION ON $\Delta G^{\circ}{ }_{f}$ AND $\log _{10} K$

$\Delta \mathrm{G}^{\circ} \mathbf{f}$

(kcal/mole)

Water of

Hydration

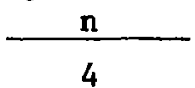

6

8

10

\begin{tabular}{|c|c|c|}
\hline$\underset{T G}{\text { Modifijed }}$ & Nriagu $^{c}$ & Chen $^{d}$ \\
\hline-2825.0 & -2822.6 & -2819.0 \\
\hline-2943.4 & -2935.2 & -2932.4 \\
\hline-3061.8 & -3047.8 & -3045.8 \\
\hline-3180.2 & -3160.4 & -3159.2 \\
\hline
\end{tabular}

\begin{tabular}{|c|c|c|}
\hline$\underset{T G}{M}$ & Nriagu $^{c}$ & Chen $^{d}$ \\
\hline+6.7 & +8.1 & +7.0 \\
\hline+10.3 & +7.6 & +7.0 \\
\hline+14.0 & +7.0 & +7.0 \\
\hline+17.7 & +6.4 & +7.0 \\
\hline
\end{tabular}

${ }_{n}$ is def
$b_{\text {Ref. } 91 .}$
$c_{\text {Ref. } 90 .}$.
$d_{\text {Ref. } 82 .}$ 
of water of hydration varies from 4 to 10 . Chen's method predicts no effect for $\log _{10} \mathrm{~K}$, but the modified Tardy and Garrels' method and Nriagu's method predict opposite effects.

e. Discussion of Estimated Data. The estimated values of $\Delta G_{f}^{\circ}$ for the three zeolites (clinoptilolite, heulandite, and mordenite) are in relatively good agreement. There is less than $1 \%$ difference between the results from the modified Tardy and Garrel's method, Nriagu's method, and Chen's method (Table LXVIII). In most cases, Nriagu's and Chen's methods agree within $0.1 \%$. However, in terms of $\log _{10} \mathrm{~K}$ for the aqueous reaction, large relative differences exist (Table LXIX). The only gauges of accuracy for these estimates are the comparisons for analcime, wairakite, and laumontite. Even these comparisons may not carry much weight because the silicon/aluminum ratio and water of hydration for clinoptilolite, heulandite, and mordenite are generally quite different than for analcime, wairakite, and laumontite. Any inherent errors in the methods that are associated with these variables could lead $t$ o inaccurate estimates.

Another problem with these methods is that they estimate the same free energy for polymorphs. Wairakite and laumontite have different structures, as well as having a different number of waters of hydration. ${ }^{83}$ The only difference in the $\Delta G_{f}^{\circ}$ or $\log _{10} \mathrm{~K}$ estimates, however, is from the water of hydration. The difference in the experimental values of $\log _{10} \mathrm{~K}$ of wairakite and laumontite is 4 ; Chen's method shows no difference in the estimates of the two values, and Nriagu's method shows only $\sim 0.5$. Another method of estimating $\Delta \mathrm{G}^{\circ}{ }_{\mathrm{f}}$, developed by Slaughter, can account for the structure of the mineral, but at the expense of considerable complexity in the calculations. $93-95$

4. Summary and Conclusions. A preliminary search has been conducted for free-energy data at $25^{\circ} \mathrm{C}$ for some of the mineral phases found during exploratory drilling at or near Yucca Mountain. Data were found for silica (quartz, cristobalite, and amorphous silica), alkali feldspars, some clays, and one zeolite (analcime). Data were estimated for three other zeolites (clinoptilolite, heulandite, and mordenite).

When data are available from the SUPCRT compilation, 87 they should be used because they provide a reliable and internally consistent data set. However, there are no data for any clays of interest in SUPCRT and for only one zeolite; resorting to estimation techniques to obtain data for three zeolites adds uncertainty to those data. Differences in $\log _{10} \mathrm{~K}$ for aqueous 
reactions among the three estimation methods are relatively large (Table LXIX). The data estimated by Chen's method ${ }^{82}$ should be used in modeling calculations because his method is more generally applicable than the others. However, a review of the estimated data points out the need for experimental thermodynamic data for these minerals if the results of modeling calculations are to be accurate. This is also true for the clays; although thermodynamic data are available for some clay minerals, the Yucca Mountain clays do not match the composition of these materials.

This review has concentrated on thermodynamic data at $25^{\circ} \mathrm{C}$, but many of the sources used here have additional data for higher temperatures. In principle, Chen's method could also be used to estimate higher temperature data as long as values of $\Delta G^{\circ}$ were available for simpler compounds at the temperature of interest. The other two methods could not be employed easily at other temperatures because they depend on specific data sets that were prepared for $25^{\circ} \mathrm{C}$ only.

Although the data generated in this review are not adequate for accurate chemical-equilibrium calculations, they can be used to learn how to model the Yucca Mountain groundwater system, what modeling codes are most appropriate, and what further effort is needed in areas other than the chemical-equilibrium calculations. Results from equilibrium calculations can be compared with groundwater analyses; these comparisons can be used to test the accuracy of the thermodynamic data and to test as sumptions about the minerals, the groundwater, and how they interact. It may be difficult to uncover the cause of any discrepancies if both thermodynamic data and model assumptions are suspect.

B. Calculated Solubilities of Uranium and Plutonium in Well J-13 Water

The composition of we11 J-13 water may approximate that of water that might ultimately interact with a waste repository at Yucca Mountain. Solubilities of uranium and plutonium in well $\mathrm{J}-13$ water have been calculated using the EQ3 chemical equilibrium computer program. 75 The object of these calculations was to identify the constituents of well J-13 water that most strongly influence the solubility of these actinides. This identification can have two effects on future work: it can indicate where emphasis should be placed in water analyses and where accurate thermodynamic data are required.

Numerous analyses of well $J-13$ water have been made. For these calculations a base-case water composition was defined from analyses on samples 6/81-51 through 6/81-56 (Table II of this report). Table LXXII lists the total 
TABLE LXXII

\begin{tabular}{|c|c|}
\hline $\begin{array}{l}\text { BASE-CASE WELL } \\
\text { Species }\end{array}$ & $\begin{array}{l}J-13 \text { WATER }^{\mathrm{a}} \\
\text { Concentration } \\
(\text { molal) }\end{array}$ \\
\hline $\mathrm{Na}$ & $2.0 \times 10^{-3}$ \\
\hline$K$ & $1.4 \times 10^{-4}$ \\
\hline $\mathrm{Ca}$ & $2.9 \times 10^{-4}$ \\
\hline $\mathrm{Mg}_{8}$ & $7.1 \times 10^{-5}$ \\
\hline $\mathrm{Al}$ & $9.6 \times 10^{-7}$ \\
\hline Si & $1.1 \times 10^{-3}$ \\
\hline $\mathrm{Sr}$ & $4.6 \times 10^{-7}$ \\
\hline $\mathrm{Ba}$ & $1.7 \times 10^{-8}$ \\
\hline Mn & $2.0 \times 10^{-8}$ \\
\hline $\mathrm{Fe}$ & $7.9 \times 10^{-7}$ \\
\hline $\mathrm{V}$ & $6.3 \times 10^{-7}$ \\
\hline $\mathbf{F}$ & $1.1 \times 10^{-4}$ \\
\hline $\mathrm{Cl}$ & $1.8 \times 10^{-4 b}$ \\
\hline$\left(\mathrm{PO}_{4}^{3-}\right)$ & $1.0 \times 10^{-6}$ \\
\hline$\left(\mathrm{NO}_{3}^{-}\right)$ & $1.6 \times 10^{-4}$ \\
\hline$\left(\mathrm{SO}_{4}^{2-}\right)$ & $1.9 \times 10^{-4}$ \\
\hline$\left(\mathrm{Co}_{3}^{2-}\right)$ & $2.9 \times 10^{-3}$ \\
\hline
\end{tabular}

\footnotetext{
${ }_{\mathrm{pH}}=6.9$. Titanium $\left(6 \times 10^{-7} \mathrm{molal}\right)$ and lithium $\left(1 \times 10^{-5}\right.$ molal $)$ were not included in the calculations because they are not available in EQ3.

${ }^{b}$ Amount adjusted to obtain electrical neutrality.
}

amounts of the various species that are assumed present in molal units (mol/kg water). The cation compositions are averages from six samples. Two cations, titanium ( $6 \times 10^{-7}$ molal) and lithium ( $1 \times 10^{-5}$ molal), were not included in the calculations because they are currently not available in the EQ3 program. The anion compositions are from one sample (6/81-51). The water pH was taken as 6.9. The oxidation potential Eh of well $\mathrm{J}-\mathrm{i3}$ water is uncertain; indeed, it is possible that more than one oxidation potential may be required to define the state of the water from different tuff strata. For these reasons, calculations were done at $\mathrm{Eh}=+700,+400,+100$, and $-200 \mathrm{mV}$, which covers the range from a strongly oxidizing condition to a reducing condition. 
Before solubility calculations were done, the programs EQ3 and EQ6 were used to determine the saturation state of the base-case well J-13 water. A speciation calculation with EQ3 indicated that the water was saturated with respect to a large number of silicate minerals. Using EQ6 to calculate actual precipitation resulted in small amounts of quartz $\left(\mathrm{SiO}_{2}\right)$, berlinite $\left(\mathrm{AlPO}_{4}\right)$, fluorapatite $\left[\mathrm{Ca}_{5}\left(\mathrm{PO}_{4}\right)_{3} \mathrm{~F}\right]$, and hematite $\left(\mathrm{Fe}_{2} \mathrm{O}_{3}\right)$ as the predicted precipitates. Equilibrium calculations often indicate that natural waters are supersaturated because of kinetic effects.

Solubility calculations were done with the EQ3 program only. The program was run in a mode in which well $\mathrm{J}-13$ water was assumed to be in contact with a uranium or plutonium solid phase. Only the limiting solid phase, that is, the solid phase that gave the minimum solubility of uranium or plutonium, was assumed present; other solid phases were checked for saturation, however. The minerals with which the well $\mathrm{J}-13$ water is saturated were not allowed to precipitate. Three groups of calculations were done: one for uranium only, one for plutonium only, and one for both uranium and plutonium. For these calculations the program distributed the various species among $\sim 200$ aqueous complexes. About 20 solid uranium phases and 10 solid plutonium phases were checked for saturation.

Uranium alone in well J-13 water shows relatively complex behavior (Table LXXIII). The total uranium in solution, the identity of the limiting solid phase, and the uranium complexes present depend on the solution's Eh. Under oxidizing conditions, schoepite $\left[\mathrm{UO}_{2}(\mathrm{OH})_{2} \cdot \mathrm{H}_{2} \mathrm{O}\right]$ is the limiting solid phase, although both rutherfordine $\left(\mathrm{UO}_{2} \mathrm{CO}_{3}\right)$ and $\beta-\mathrm{UO}_{2}(\mathrm{OH})_{2}(\mathrm{~s})$ are also near saturation. The total uranium in solution, as $U(V I)$, is relatively high and exists as carbonate, phosphate, and hydroxyl complexes. At $\mathrm{Eh}=+100 \mathrm{mV}$, $\mathrm{U}_{4} \mathrm{O}_{9}(\mathrm{~s})$ is the limiting solid phase. The total uranium in solution, still as U(VI), has dropped somewhat; it exists mainly as carbonate and phosphate complexes. At $\mathrm{Eh}=-200 \mathrm{mV}$, uraninite $\left(\mathrm{UO}_{2}\right)$ is the limiting solid phase, but coffinite $\left(\mathrm{USiO}_{4}\right.$ ) is also near saturation. The total uranium in solution, as U(IV), has dropped considerably and exists mainly as one hydroxyl complex.

Plutonium alone in well $\mathrm{J}-13$ water shows relatively simple behavior because the aqueous phase is dominated by one complex: $\mathrm{PuCO}_{3}^{2+}$ (Table IXXIV). Solid $\mathrm{PuO}_{2}$ is the limiting solid phase. The total plutonium in solution is controlled by the amount of carbonate present because the $\mathrm{PuCO}_{3}^{2+}$ complex is so stable. 
TABLE LXXIII

URANIUM SOLUBILITY IN BASE-CASE WELL J-13 WATER

Oxygen fugacity $(\mathrm{atm}) \frac{\mathrm{Eh}(\mathrm{mV})}{$\cline { 2 - 3 }}$\frac{+400}{3 \times 10^{-29}} \frac{+100}{2 \times 10^{-49}} \frac{-200}{9 \times 10^{-70}} }$

Total uranium concentration $(\mathrm{molal}) 3.0 \times 10^{-4} \quad 3.0 \times 10^{-4} \quad 1.4 \times 10^{-6} \quad 1.5 \times 10^{-11}$

$\begin{array}{ccc}\text { Solid uranium phases } & \begin{array}{c}\text { schoepite }^{a} \\ \text { rutherfordine } \\ \beta-\mathrm{UO}_{2}(\mathrm{OH})_{2}\end{array} & \begin{array}{c}\text { schoepite } \\ \text { rutherfordine } \\ \beta-\mathrm{UO}_{2}(\mathrm{OH})_{2}\end{array}\end{array}$

$$
\mathrm{U}_{4} \mathrm{O}_{9}^{\mathrm{a}} \quad \begin{aligned}
& \text { uraninite } \\
& \text { coffinite }
\end{aligned}
$$

Primary uranium aqueous oxidation state

VI

VI

VI

IV

\section{Primary aqueous}

species (molal)
$\mathrm{UO}_{2}\left(\mathrm{CO}_{3}\right)_{2}^{2-}$
$2.5 \times 10^{-4}$
$2.5 \times 10^{-4}$
$8.8 \times 10^{-7}$
$\mathrm{UO}_{2} \mathrm{CO}_{3}^{\circ}$
$3.6 \times 10^{-5}$
$3.6 \times 10^{-5}$
$1.1 \times 10^{-7}$

$$
\mathrm{UO}_{2}\left(\mathrm{HPO}_{4}\right)_{2}^{2-}
$$
$5.1 \times 10^{-7}$
$5.1 \times 10^{-7}$
$3.7 \times 10^{-7}$
$\left(\mathrm{UO}_{2}\right)_{3}(\mathrm{OH})_{5}^{+}$
$6.6 \times 10^{-7}$
$6.6 \times 10^{-7}$

$\mathrm{U}(\mathrm{OH})_{5}^{-}$

$1.5 \times 10^{-11}$

${ }^{a}$ Solid phase controlling solubility; other solid phases also near saturation.

When both uranium and plutonium are present in well $\mathrm{J}-13$ water, the behavior of plutonium is unchanged again because the aqueous $\mathrm{PuCO}_{3}^{2+}$ complex is so stable (Table LXXV). Uranium in the (VI) oxidation state also forms carbonate complexes. Because plutoniu has complexed essentially all the carbonate, there is less uranium in solution as carbonate complexes when plutonium is also present in the Eh range of +700 to $+100 \mathrm{mv}$; thus, the solu- 
TABLE LXXIV

PLUTONIUM SOLUBILITY IN BASE-CASE WELL J-13 WATER

\begin{tabular}{|c|c|c|c|c|}
\hline \multirow[b]{3}{*}{ Oxygen fugacity (atm) } & \multicolumn{4}{|c|}{ Eh $(m V)$} \\
\hline & +700 & +400 & +100 & -200 \\
\hline & $7 \times 10^{-9}$ & $3 \times 10^{-29}$ & $2 \times 10^{-49}$ & $9 \times 10^{-70}$ \\
\hline $\begin{array}{l}\text { Total plutonium } \\
\text { concentration (molal) }\end{array}$ & $2.9 \times 10^{-3}$ & $2.9 \times 10^{-3}$ & $2.9 \times 10^{-3}$ & $2.9 \times 10^{-3}$ \\
\hline Solid plutonium phases & $\mathrm{PuO}_{2}$ & $\mathrm{PuO}_{2}$ & $\mathrm{PuO}_{2}$ & $\mathrm{PuO}_{2}$ \\
\hline $\begin{array}{l}\text { Primary plutonium aqueo } \\
\text { oxidation state }\end{array}$ & IV & IV & IV & IV \\
\hline $\begin{array}{l}\text { Primary aqueous } \\
\text { species (molal) }\end{array}$ & & & & \\
\hline $\mathrm{PuCO}_{3}^{2+}$ & $2.9 \times 10^{-3}$ & $2.9 \times 10^{-3}$ & $2.9 \times 10^{-3}$ & $2.9 \times 10^{-3}$ \\
\hline
\end{tabular}

bility of uranium in well $\mathrm{J}-13$ water is reduced under these conditions (compare Tables LXXIII and LXXV). Rutherfordine $\left(\mathrm{UO}_{2} \mathrm{CO}_{3}\right)$ is no longer near saturation when plutonium is present. At Eh $=-200 \mathrm{mV}$, uranium is in the (IV) oxidation state. The data base for the EQ3 program does not contain any data for U(IV) carbonates; either the complexes do not exist or the thermodynamic properties have not been determined. In either case, the calculation of uranium solubility is unaffected by the presence of plutonium at this Eh value (compare Tables LXXIII and LXXV).

As stated at the beginning of this discussion, the object of these calculations was to identify the constituents of well J-13 water that most strongly influence the solubility of uranium and plutonium. Uranium complexes most often with carbonate, phosphate, and hydroxyl, whereas plutonium complexes with carbonate; thus, carbonate content, phosphate content, and pH are significant variables. These calculations also indicate the important effect of solution Eh on solubilities. The thermodynamic data of uranium and plutonium species in the EQ3 program are generally in agreement with the recent reviews of Langmuir 96 and Lemire and Tremaine. 97 For example, Table LXXVI compares 
TABLE LXXV

URANIUN AND PLUTONIUA SOLUBILITY IN BASE-CASE

WELL J-13 WATER WIEN BOTH ARE PRESENT

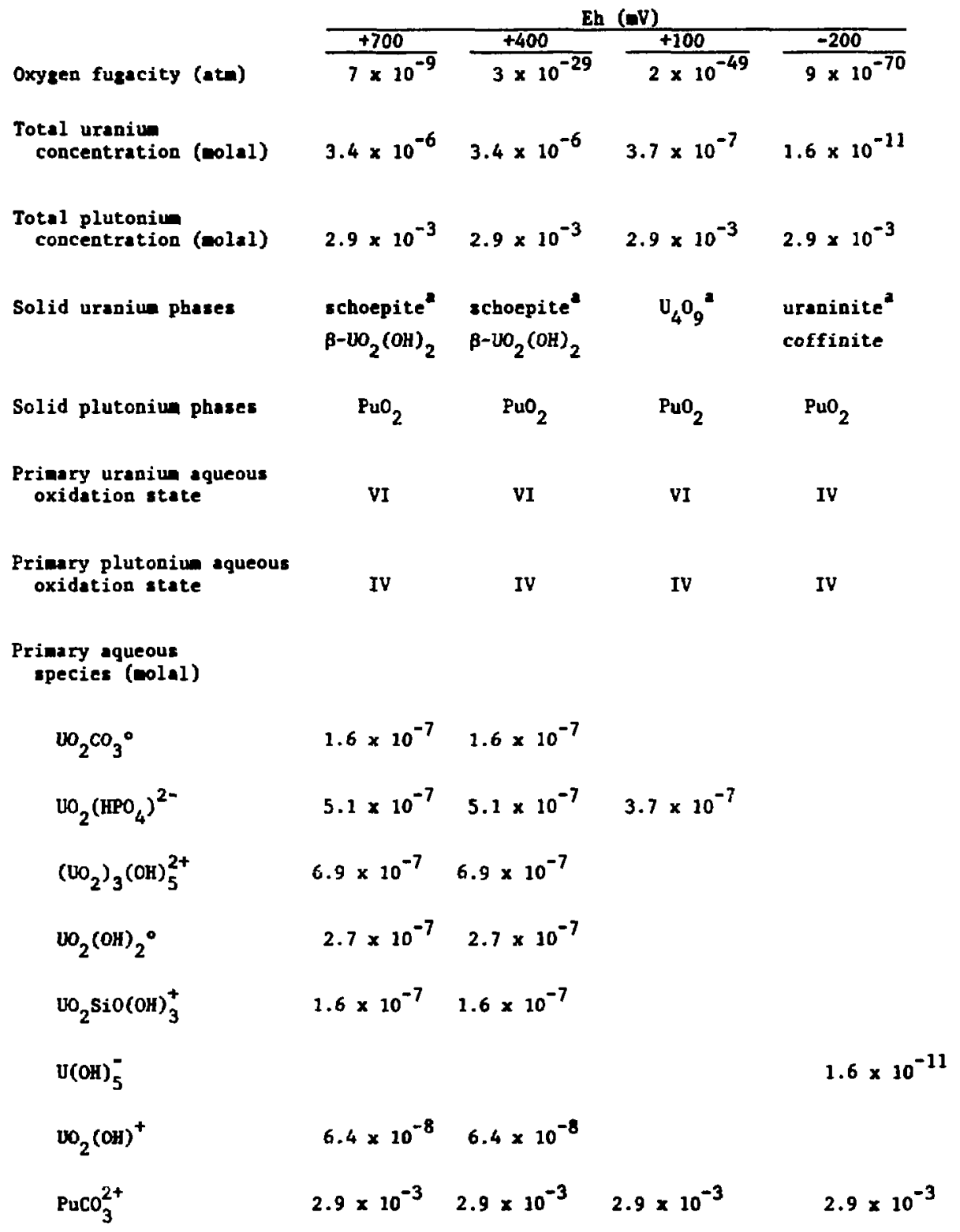

Solid phase controllins solubility; other solid phases also near saturation. 


\begin{tabular}{|c|c|c|c|}
\hline Species & 203 & Lonpeuir & Trenaine $^{b}$ \\
\hline $\mathrm{V}(\mathrm{OH})_{5}^{-}$ & -56.81 & $-56.79^{c}$ & -56.88 \\
\hline $\mathrm{V}(\mathrm{OH})_{4}^{\circ}$ & -47.42 & $-47.41^{c}$ & $-47.47^{c}$ \\
\hline $\mathrm{vO}_{2}(\mathrm{OH})_{2}^{\circ}$ & +12.00 & & \\
\hline $\mathrm{UO}_{2} \mathrm{CO}_{3}$ & -10.10 & -10.06 & -10.10 \\
\hline $\mathrm{UO}_{2}\left(\mathrm{CO}_{3}\right)^{2-}$ & -17.00 & -16.98 & -17.10 \\
\hline $\mathrm{UO}_{2}\left(\mathrm{HPO}_{4}\right)^{\circ}$ & -8.43 & -8.40 & $-8.41^{d}$ \\
\hline $\mathrm{UO}_{2}\left(\mathrm{HPO}_{4}\right)_{2}^{2-}$ & -18.62 & -18.56 & $-18.52^{d}$ \\
\hline $\mathrm{UO}_{2}\left(\mathrm{H}_{2} \mathrm{PO}_{4}\right)^{+}$ & -3.03 & -3.03 & -2.98 \\
\hline$\left(\mathrm{WO}_{2}\right)_{3}(\mathrm{OH})_{5}^{+}$ & -54.31 & -54.30 & -54.39 \\
\hline$\left(\mathrm{UO}_{2}\right)_{2}(\mathrm{OH})_{2}^{+}$ & -22.36 & -22.35 & -22.43 \\
\hline $\mathrm{wO}_{2} \mathrm{SiO}(\mathrm{OH})_{3}^{+}$ & +2.40 & $+2.40^{c}$ & \\
\hline Uraninite & -4.61 & -4.64 & -4.65 \\
\hline $\mathrm{U}_{4} \mathrm{O}_{9}(\mathrm{~s})$ & -44.94 & -44.94 & -45.02 \\
\hline$\beta-\mathrm{WO}_{2}(\mathrm{OH})_{2}(\mathrm{~s})$ & -22.44 & -22.42 & -22.20 \\
\hline Schoepite & +5.44 & +5.40 & +5.65 \\
\hline Coffinite & -7.63 & $-9.18^{c}$ & \\
\hline Rutherfordine & -14.46 & -14.46 & -14.20 \\
\hline $\mathrm{PuCO}_{3}^{2+}$ & -40.70 & & $-40.73^{d}$ \\
\hline $\mathrm{Pu}(\mathrm{OH})_{5}^{-}$ & +14.97 & & $+14.98^{c}$ \\
\hline $\mathrm{PuO}_{2}(\mathrm{~s})$ & -7.35 & & -7.37 \\
\hline $\begin{array}{l}\text { Ref. } 96 . \\
\text { bef. } 97 . \\
c_{\text {Estinated b }}\end{array}$ & & & \\
\hline
\end{tabular}


dissociation-constant data for a number of solid phases and aqueous complexes that proved to be significant in these calculations. However, an examination of these reviews indicates that some of the data are considered estimates or limiting values by the authors (Table LXXVI). Thus, data associated with $\mathrm{UO}_{2}\left(\mathrm{HPO}_{4}\right)_{2}^{2-}, \mathrm{UO}_{2} \mathrm{SiO}(\mathrm{OH})_{3}^{+}, \mathrm{U}(\mathrm{OH})_{5}^{-}$, coffinite, and particularly $\mathrm{PuCO}_{3}^{2+}$ are uncertain. The case of $\mathrm{PuCO}_{3}^{2+}$ is quite important; Lemire and Tremaine indicate that the free energy of formation they report is a lower limit that may overestimate the stability of this complex ${ }^{97}$ and, in these calculations, lead to an overestimation of the solubility of plutonium in the presence of carbonate. Therefore, the stability exhibited by this complex in these calculations may not present an accurate picture of reality. The experimental basis for thermodynamic data for $\mathrm{PuCO}_{3}^{2+}$ and its effect on plutonium solubility in waters with carbonate present are now under review. This analysis highlights the importance of accurate thermodynamic data for a number of aqueous complexes of uranium and plutonium, particularly the carbonate, phosphate, and hydroxyl complexes.

Another aspect of a waste repository's geochemistry that must be understood can be inferred from these calculations. Both carbonate and phosphate complexes are important to the solubility of uranium and plutonium. If sources of these anions are available in the local minerals, the total quantity of available carbonate and phosphate could be greater than is indicated by an analysis of the water alone. A similar situation exists in calculations involving the Eh of the groundwater. It is important to consider not only the Eh of the water but also the oxidation-reduction capacity of the mineralogy. Although these calculations did not consider the local mineralogy, a comprehensive analysis of the solubility of actinides in natural waters must take the local minerals into account.

\section{Transport Modeling}

1. Transport Codes. Several transport codes available at Los Alamos are listed and described in Table LXXVII. These codes can be used to model a variety of problems, including multiphase flow, unsaturated flow, the inverse problem, fracture flow, and three-dimensional systems. TRACR3D has been used to model a field experiment on a single fracture to predict the effect of unsaturation and aid in the design of the experiment. These codes will provide a means of modeling field and laboratory experiments. In the near future it is 
TABLE LXXVII

TRANSPORT CODES AT IOS ALAMOS

Code

TRACRKP

TRACR3D

WAFE

WAFE-OS

KRAK

INVERSE

TRACRI

DIFNS

IVARS
Description

Multicomponent flow in porous/ fractured nedia

Three-disensionsl wass transport in porous/fractured media

Two-phase mass and heat transport in porous aedia

Two-phase mass and heat transport with chemistry in porous media

Fracture growth in a porous nedium

Finds pore-size distributions in porous media from gas diffusion data, NIR data, etc.

Inverts tracer flow data to determine flow path spatial distribution in porous aediun.

Matrix diffusion with nonlinear sorption

One-diwensional flow with atrix diffusion
Details

Transient, two-dinensional flow in fractures and in porous nedis between fractures; up to 5 components, diffusion, diapersion, spatial variation of aterial properties, single phase, and buoyancy; current developwent includes randon fracture networks.

Transient, three-dimensional, nonlinear flow, one or two phases (air and water), cartesian or cylindrical coordiuates, tracers in one phase (liquid or gas), nolecular diffusion, mechanical dispersion tensor, radioactive decay allowed, decay chains, aimle sorption model, capillary pressure, buoyancy, spatial variation of anterial properties, saturated/unsaturated seepage fron/to fractures. Future expansion will concentrate on better rorption model.

Transient, two-dimensional, nonlinear flow, cartesian or cylindrical coordinates, accurate treatnent of water ( $\ell$ and $v$ ) equation of state, spatial variation of material properties, buoyancy, numerically implicit, two phases (air and vapor, water). Future expansion will allow flow of a tracer in a non-isothermal systen.

Transient, two-dimensional, cartesian or cylindrical coordinates, wass and energy transport, nonlinear flow, wulticomponent (9), one or two phases, reactive species, chenical activity in matrix grains, buoyancy, spatial variation in naterial properties (presently structured for oil shale restorting). Extensions will allow dissolution of and precipitation onto matrix particles.

Detailed flow in a crack, nonlinear flow, transient, two-dimensional, ass and energy transport, one or two phases, leakage across crack faces, full coupling between crack shape and extension and fluid dyaanics.

Solves Fredhold integral equation of the first kind.

Two-dinensional, diffusion and dispersion accounted for; uses optinal control approach.

Finite difference solution to one-dinensional diffusion with a concentration-dependent diffusion coefficient, currently Freundlich isothere. Future development will couple one-dimensional convection and include other isother functions.

Analytic solution to one-dinensional flow with atrix diffusion perpendicular to the direction of flow; no flow in the natrix. 
possible that some of these codes can be validated by designing and executing appropriate experiments in the laboratory and field.

2. Calculation of Flow in Fractured Tuff. Various schemes for studying tracer flow in NTS tuff have been considered. One metird would involve injecting water into a fracture from a small-diameter borehole for a long period of time, such as $\sim 100$ days. This pretreatment period will presumably allow the tuff, initially unsaturated, to saturate out to some distance. Then a tracer stream will be injected and the movement of tracer followed by sampling at a number of observation points. Postexperiment drillback could also provide information on where the tracer went and how it dispersed. In another approach to this experiment, water and tracer would be injected into a fracture from a line source and discharged into a line collector one or two meters away. Many variations can be imagined. Unfortunately, features of these experimental designs could make interpretation of tracer observations ambiguous. For example, in the borehole injection version, the flow in a crack will be subject to strong radial divergence. Velocities will be functions of radius, and dispersion will also likely be dependent on distance from the injection point. This spatial dependence in velocity would be reduced in the case of sheet flow (injection and collection from line sources and sinks). However, in both cases, leakage into the tuff matrix will take a long time (if ever) to reach a steady-state condition.

Mathematical models of fluid and tracer flow can be used not only to help interpret results of experiments but also to design experiments by revealing, at least approximately, the implications of a particular design. To provide the best design for water and tracer injection experiments in fractured tuff, a computational parameter study has been outlined. Parameters to be varied are matrix permeability (10 to 100 microdarcys $\mu \mathrm{D}$ ), retardation coefficient ( 1 to $800 \mathrm{ml} / \mathrm{g})$, fracture aperture $(10$ to $100 \mu \mathrm{m})$, flow rates $(0.1$ to $100 \mathrm{~m} /$ day at inlet), and injection strategy (radial flow from a small-diameter borehole or sheet flow from a line source).

The computer code TRACR3D (Ref. 73) is used for these calculations. This code simulates transient air, water, and tracer flow in permeable media for a three-dimensional geometry. Tracer motion can occur in either the air phase or the liquid phase. Transport mechanisms include advection, molecular diffusion, mechanical dispersion, and capillary action. Several equilibrium sorption 
models as well as a nonequilibrium sorption model are included. Material properties such as permeability can vary spatially. The code also can simulate flow in a fracture and transport into or out of a porous material surrounding the fracture.

Computer calculations have been started; Figs. 99-101 display partial results for one calculation. Figure 99 shows the geometry of the problem and the parameter values used. It is assumed that the borehole is packed off just above and below the fracture. Eight regularly spaced sampling holes are assumed at a $2-\mathrm{m}$ radius. Flow is calculated with the $22.5^{\circ}$ wedge shown because of symmetry. Matric potential as a function of saturation is approximated by using that of crushed tuff. ${ }^{98}$ The sampling holes are maintained at $100 \mathrm{kPa}$.

After 90 days of water injection, the pressure distribution in the horizontal fracture and in the tuff above it has evolved to that shown in Fig. 100. Pressure contours are represented as if on a vertical-plane slice through the axis of the borehole and at an angle of $0^{\circ}(y=0)$ from the front face (Fig. 99). Because of the strong radial divergence, pressure drops off rapidly. Figure 101 (shown on same plane as Fig. 97) displays water saturation after 90 days. Water inflow remained virtually constant at $1.1 \mathrm{l} /$ day; water saturation of the matrix is also limited to only about a $1-m$ radius and several centimeters vertically. Capillary action here has smeared the saturation front significantly. Tracer will be injected into the fracture at 100 days. This calculation shows the potentially significant effect of matrix suction on flow in a crack.

VII. SHAFT AND BOREHOLE SEALING

At Los Alamos, the emphasis of this study is to evaluate the geochemical stability of potential shaft and borehole sealing materials in the felsic volcanic tuff environment of Yucca Mountain. The study deals with the chemical compatibility of potential sealing materials with felsic tuff; the two will not be completely compatible because they are not exactly the same chemical phases, nor have they hardened in the same way. The tuff includes highly reactive residual volcanic glass; a number of minerals that were present at relatively high temperatures in the original volcanic unit (quartz, feldspars, a little biotite, etc.); and later, low-temperature, mineral alteration phases (for example, zeolites and clays). The tuff became hard by cooling as a mass of tightly interlocking crystals and glass particles. The sealing material, 


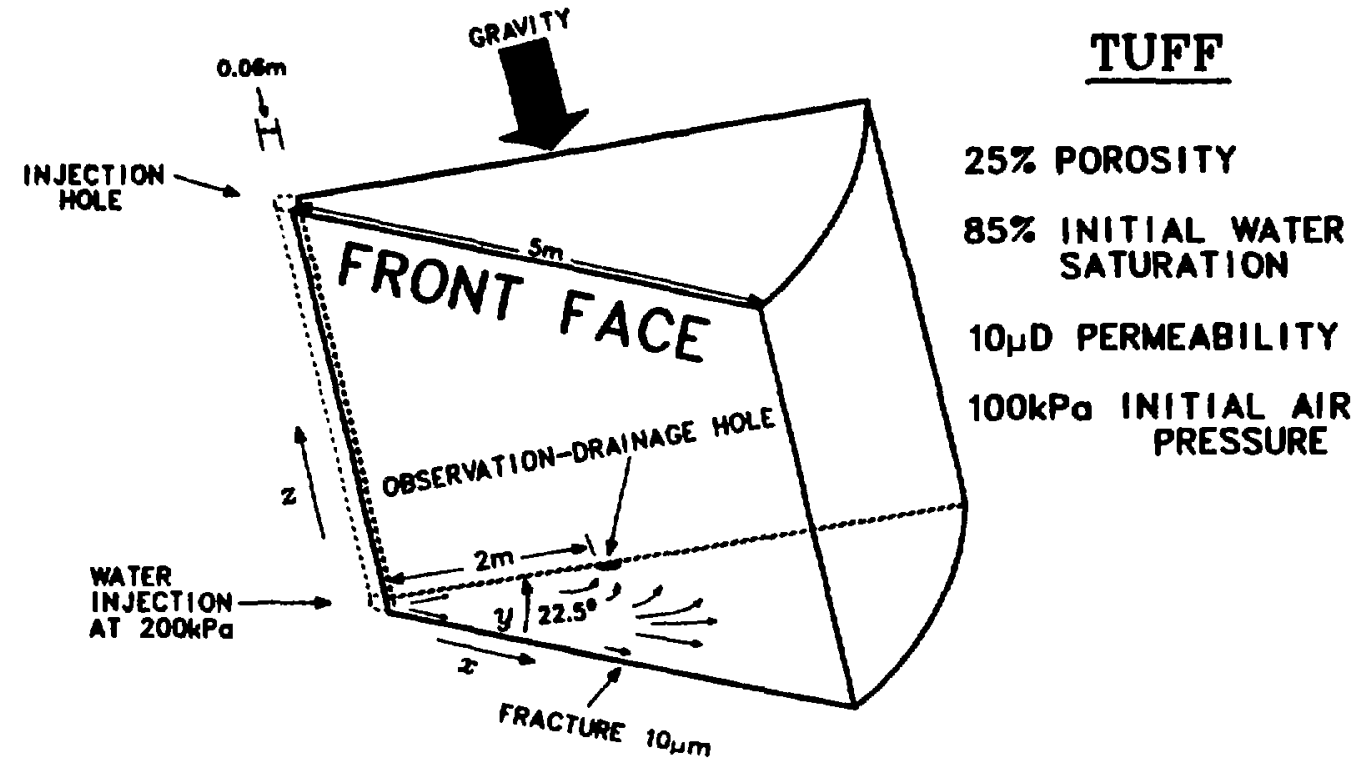

Fig. 99. Cylindrical geometry used in three-dimensional flow calculation.

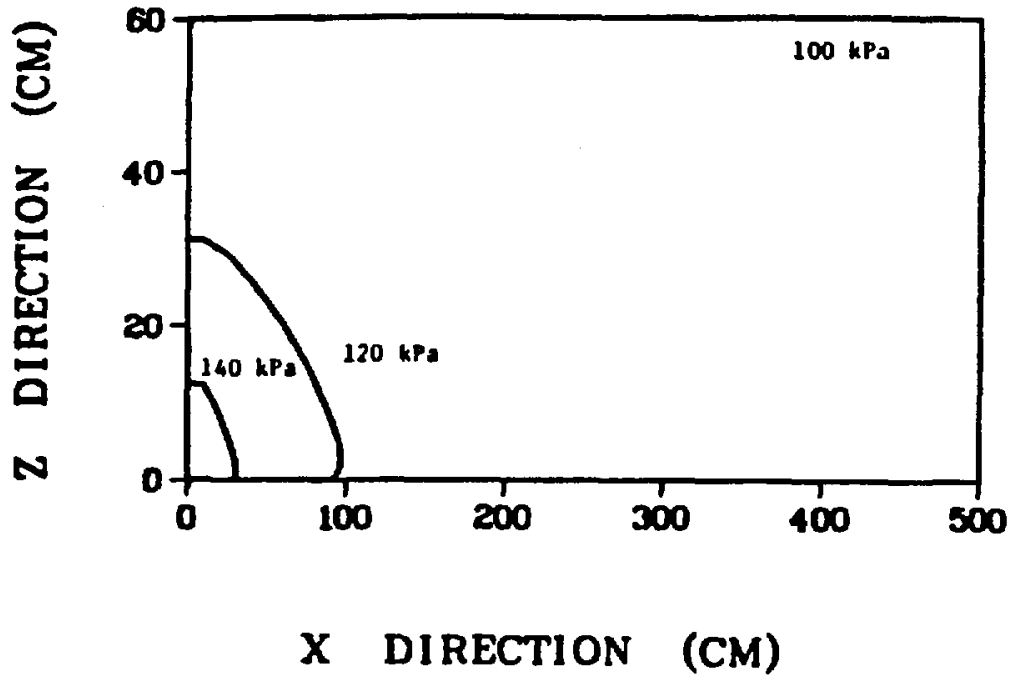

Fig. 100. Pressure contours 90 days after start of water injection. 


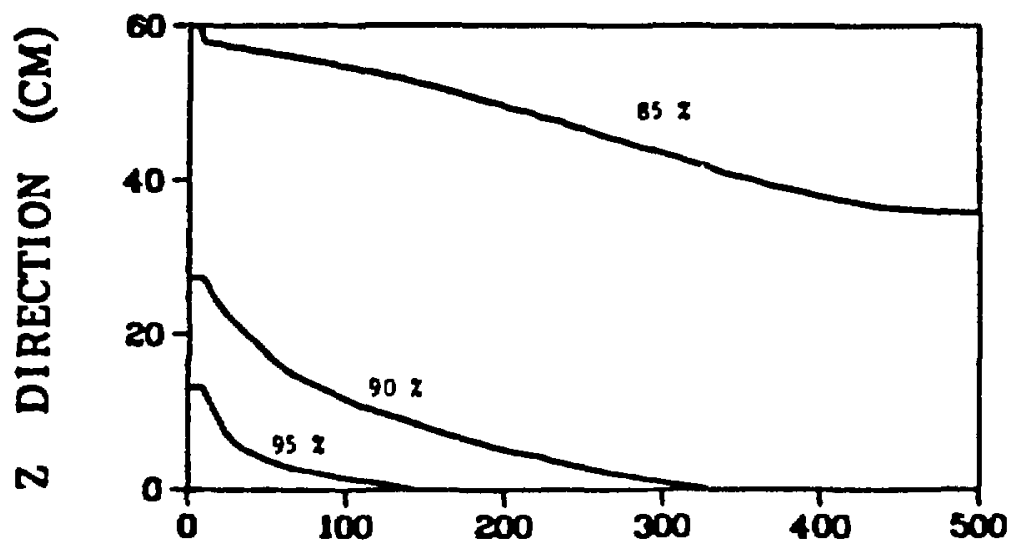

\section{$X$ DIRECTION (CM)}

Fig. 101. Water saturation contours 90 days after start of water injection.

on the other hand, may be a hydraulic cement (hardened by crystallizing or hydrating in place); an inorganic or organic chemical grout (hardened by a chemical reaction after emplacement); a natural material (tuff, clay, zeolite, granite, etc.) held together by a cement or grout; a metal; a ceramic; or various combinations of these. The objectives of the study are to determine

(1) how rapidly the tuff and sealant will react,

(2) what the effect may be of such a reaction on the physical and chemical properties of the sealing material and adjacent rock, and

(3) what might be the added effects of radiolysis or a thermal pulse.

Reports on materials currently under consideration as a sealant for a shaft and borehole in tuff and media other than tuff have been reviewed. A number of organic materials (bitumen, organic grouts, thermoplastics, polymer cements, etc.) have been tentatively ruled out because the effects of long-term bacterial action on their physical and chemical properties are unknown. Bacteria are always present and will be particularly abundant in a facility that has been 
excavated by man. Bacterial action on organic sealants probably could not be simulated adequately bv laboratory study because it would not be possible to know how much degradation would take place over thousands of years.

Incorporating natural materials as a filler (aggregate) in the sealant mixture appears particularly attractive for several reasons. The choice of the proper natural material would add a solid that is already compatible with the rock surrounding the repository: one that would not interact with it any more than the rock is already reacting internally. (All rocks that are candidates for repositories are gradually altering to the clay- and zeolite-bearing phase assemblages that are stable near the earth's surface.) Good natural materials that might be added in the case of a tuff repository include the excavated tuff, granite, or an appropriate clay or zeolite. Very large volumes of sealing material are required to close the shafts, and one of the least expensive filler materials would be local rock. From a chemical stability standpoint, the most attractive group of potential sealants seems to be the inorganic cements or grouts that have already been developed combined with a tuff or granite filler. A testing program will be established to study the possible interaction between these materials and the tuff units of Yucca Mountain that are being considered for the waste repository. 
Chapter 2:

PLANNED STUDIES OF THE GEOCHEMISTRY OF YLCCA MOUNTAIN 


\section{GROUNDWATER GEOCHEMISTRY}

\section{A. Water Chemistry}

There are at least two very important reasons for determining the chemistry of the groundwater [concentrations of dissolved substances, oxygen content, oxidation-reduction potential (Eh), and $\mathrm{pH}]$ : (1) The speciation of nuclear wastes that may become dissolved in the groundwater will largely be controlled by the groundwater chemistry. The speciation, in turn, will affect the precipitation and sorption reactions, complexing, and other retardation mechanisms. For example, solubilities of actinides can vary by up to a factor of $10^{9}$, depending on the oxidation state of the actinide. (2) The corrosion rate of a waste package is also greatly affected by the composition of the groundwater, especially the oxygen content.

Although only a few wells in the vicinity of Yucca Mountain have been tested, many of the cationic and anionic species and their concentrations in the repository area can be stated with a high level of certainty. The groundwater is largely a sodium-potassium-calcium water with sodium being the major cation at a level of $50 \mathrm{mg} / \ell$ (Tables $I I$, IX, and $X$ ). The major anion is the bicarbonate ion at a concentration of $120 \mathrm{mg} / \ell$. These cations, anions, and their concentrations should have little effect on the choice of the waste package, but they are of concern for the choice of backfill.

The portion of the issue that is not resolved and has perhaps the greatest potential impact on groundwater/waste package interaction and speciation is the oxidation-reduction potential of the groundwater.

In many of the wells tested so far, the primary water-producing stratum is the uppermost saturated stratum at or near the standing water level. Because the tuff is vertically fractured in the unsaturated zone above the standing water level, this water has ample opportunity to equilibrate with air and become essentially saturated with oxygen. Even in wells where there are also lower water-producing layers that may not be air saturated, a pumping method in which the pump is located high in the well (at the top water-producing layer) will produce water largely from that layer. To clear the well to formation water and pump water from lower strata, a packer system should be installed below the upper water-producing layer and the water should be pumped from below these packers. 
In addition to determining the oxidation-reduction potential of the groundwater, it is also important to determine what produces it. At Yucca Mountain, the $\mathrm{Fe}^{2+}-\mathrm{Fe}^{3+}$ and $\mathrm{SO}_{4}^{2-}-\mathrm{s}^{2-}$ oxidation-reduction couples are two of the reaction couples most likely to influence the potentials of the groundwater systems. The quantities of these ions in the groundwater for a particular stratum are a measure of the readily available oxidizing or reducing species. Determining the number of these same ions in the tuff itself would lead to an estimate of the total oxidation-reduction capacity of the tuff mass of Yucca Mountain, (See Chap. 1, Sec. II). Many oxidation-reduction processes are mediated or catalyzed by microbiological activity; therefore, to fully understand the oxidation-reduction potential of the area, the influence of microbiological activity must also be considered.

The residual pore water in the tuff itself is a type of groundwater yet to be investigated. Its composition may be different from the normal groundwater, and it is important because under certain circumstances pore water would contact the waste package first.

Experiments to determine the corsequences of thermal effects on groundwater composition have been started. The major changes caused by elevated temperature are an increase in sodium, potassium, and silica content and a decrease in calcium and magnesium content (see Table VII). Although water from wel1 J-13 was used in those experiments, the thermal effects are not expected to be much different for Yucca Mountain groundwater. Additional experiments are certainly needed to fully understand the effect of temperature change on groundwater chemistry.

One issue cannot be resolved until the oxidation-reduction potential of the groundwater is determined: the effect on the groundwater of near-field radiolysis that is induced by the waste package. Radiolysis of water produces both oxidizing and reducing radicals in solution in varying amounts. The quantity of these radicals is altered by reaction with various cationic, anionic, gaseous, or organic getters in the water. Once the chemistry, especially the oxidation-reduction potential, of the groundwater has been established, experiments can be started that will study these effects.

The importance of groundwater chemistry as discussed above also applies to any discussion of retardation processes. The largest single variable that is related to precipitation and/or migration may be the oxidation state of the waste element, and it, in turn, is controlled by the oxidation-reduction potential of the groundwater. 
Because of radioactive decay, the effect of temperature and radiolysis on the groundwater chemistry also changes with time. The effects of temperature and radiation dose need to be studied so that models can include the changes with time.

Microbiological activity in the ground can itself establish an oxidationreduction potential for a system. In addition, this activity can catalyze or mediate geological processes that determine the oxidation-reduction potential of the groundwater. Microbiological entities can also be complexing agents that can alter the transportation or retardation of waste elements in the groundwater. All these reasons make it imperative to study the microbiological processes that could take place in the Yucca Mountain environs. There may be little activity in the tuff at this time, but all drilling and excavating activities will introduce microbiological activity there. A preliminary experiment has shown that the polymer used in drilling holes in Yucca Mountain supports the growth of certain fungi.

To resolve the remaining issues that affect groundwater chemistry, a number of experiments have been designed for completion over the next 2 to 4 years.

1. Chemistry of Formation Groundwater in Yucca Mountain. The most difficult part of determining the chemistry and especially the oxidationreduction potential of groundwaters at Yucca Mountain is not the chemical analysis or Eh measurement technique but the sampling of formation water from the different tuff strata. Air, water, and soap are pumped into the tuff as drilling lubricants when the well is being drilled. These fluids must be cleared from the well and surrounding tuff before the water being pumped is formation water. Lithium is added to all drilling fluids to serve as a tracer; its absence will indicate when the well has been cleaned. However, lithium does have a finite sorption ratio, so it does stay in the tuff longer than the water that is introduced. Perhaps a better tracer method would measure the amount of soap remairing in the water as a function of time. The sorption ratio of these soaps (nondegradable sulfonates) should be extremely small because sulfonates are anions and should not be sorbed by the tuff. As noted earlier, however, there was still evidence of high levels of lithium and soap in wel1 UE25b-1 after pumping more than $1.3 \times 10^{7} \mathrm{gal}$ of water; the well should have been pumped longer. 
In the near future, an experiment must be completed that pumps, samples, and analyzes water from between packers for both a high-and a low-permeability zone. The groundwater in a low-permeability zone will indicate what to expect in a repository area and will also have a strong influence on the waste-package design; the chenistry of the groundwater in the high-permeability zone can be used in modeling one of the more probable transport paths. The effect of the composition of these waters, especially the oxygen and Eh, on the transport properties such as sorption coefficients, speciation, and solubility must be investigated. If the oxidation-seduction potential of the groundwater is something other than fully oxidizing, Eh buffer systems will be necessary to hold and control laboratory experiments at a particular Eh.

2. Oxidation-Reduction Capacity of Tuff. The importance of the oxidationreduction potential of the water has been shown for speciation and solubility of radionuclides and also for transport and retardation processes. The oxidationreduction potential of the water is not only set but also maintained by species in the tuff that can oxidize or reduce other species in the water. Among the species thought to be important in tuff are $\mathrm{Fe}^{2+}-\mathrm{Fe}^{3+}, \mathrm{SO}_{4}^{2-}-\mathrm{s}^{2-}, \mathrm{Mn}^{4+}-$ $\mathrm{Mn}^{2+}$, and also microbiological entities. Analyses for these species in tuff will be developed, and an estimate will be made of the total oxidation-reduction capacity of the tuff. Because recharge water is oxidizing as a result of dissolved air, an estimate must be made of the total capacity of the repository and environs to change that oxidizing quality.

3. Microbiological Activity. A determination must be made of the microbiological activity already present in the tuffs at different depths of Yucca Mountain and what processes can be supported or introduced into Yucca Mountain by the construction of the repository. If care is not taken, some of the fluids put into the tuff during construction of the repository may support the growth of microbiological activity. This could have two possible major effects on the operation of the repository. (1) The oxidation-reduction potential of the groundwater may be altered or maintained at a different value, and (2) the microbiological entities may themselves be organic complexes in the groundwater and may greatly alter radionuclide retardation mechanisms.

4. Radiolysis Effects on Groundwater. The importance of the oxidationreduction potential of the groundwater in the design of the waste package and also in various radionuclide retardation processes has been indicated. Radiol- 
ysis of the groundwater by the waste-package radioactivity could change the Eh of the groundwater. Radiolysis of water produces both oxidizing and reducing radicals; these radicals combine with each other and also with other components in the water. The net effect is that the oxidation-reduction potential of the water can be changed. The water probably becomes more oxidizing, but the experiments to observe radiolysis effects on natural groundwaters have not yet been done. Once the oxidation-reduction potential of Yucca Mountain groundwater has been established, the effect of radiolysis, especially $\alpha$-induced radiolysis, can be studied. It is not a simple experiment and may require the development of Eh moderators or buffers.

4. Temperature Effect on Groundwater Chemistry. Each of the variables affecting the groundwater chemistry can also vary because of the temperature gradient imposed on the repository by radioactive decay of radionuclides in the waste package. Any effect produced by a temperature change from 20 to $200^{\circ} \mathrm{C}$ must be investigated.

\section{B. Behavior of Actinides and Other Multivalent Elements}

There is little information that can be used to predict the behavior of certain multivalent elements in a tuff repository environment. Fundamental data concerning solutions of technetium, iodine, and other multivalent fissionproduct elements, especially actinides, are often lacking. Data such as formation constants (and their dependence on $\mathrm{pH}$ and $\mathrm{Eh}$ ), species stabilities, and rates of reaction are important when predicting the chemical reaction paths followed by elements with multiple oxidation states. A data base of fundamental information must be accumulated, and at the same time, site-specific reaction data must be acquired. These two requirements are reflected in the paragraphs below, where a vital question is asked and is followed by a description of the necessary experimental work to provide the data.

(1) What are the solubilities of $\mathrm{PuO}_{2}$ and related hydrous oxides at values of Eh, $\mathrm{pH}$, and complexing ion concentrations that are likely to be encountered at the repository?

Specific investigations are required to resolve this question.

(a) The solubility of $\mathrm{PuO}_{2}$ and hydrous plutonium oxides at selected values of Eh and $\mathrm{pH}$, in both the presence and absence of complexing anions $\left(\mathrm{HCO}_{3}^{-}, \mathrm{SO}_{4}^{2-}, \mathrm{F}^{-}\right.$, etc.) must be determined. The solids used should be prepared in a variety of ways and charac- 
terized by $x$-ray diffraction and other physical means. Preparation methods include (1) the addition of $\mathrm{Pu}(\mathrm{IV})$ to excess hydroxide, followed by various aging periods, (2) the oxidation of $\mathrm{Pu}$ (III) added to alkaline solutions, (3) the reaction between $\mathrm{Pu}$ (III) and Pu(VI) in slightly alkaline solutions, and (4) the decomposition of $\mathrm{Pu}$ (IV)-carbonate complexes. Observations on the rates of these formation processes also will be important.

(b) Because the Pu(IV) polymer is structurally related to the hydrous oxide, its ionic solubility should be determined at selected Eh and $\mathrm{pH}$ values. In addition, its rates of formation, dissolution, and sedimentation should be determined.

(c) The solubility studies in (a) and (b), above, should use both ${ }^{242} \mathrm{Pu}$ and ${ }^{239} \mathrm{Pu}$ to evaluate possible radiolytic effects. The nature of metastable surface phases probably can be influenced by radiation.

(d) Reactions of plutonium and other actinides should be investigated in complexing media at $\mathrm{pH} 7 \pm 2$. Unfortunately, high concentrations of the complexing ions will be required to minimize solubility difficulties, but the data will be useful for extrapolation to lower concentrations.

(2) What species (dissolved or suspended) are formed by the interaction of the repository groundwater with the waste form?

Experiments pertaining to this question depend upon selection of the waste form and the repository horizon. After these have been identified, it will be possible to set up leaching-type experiments in which the waste form is contacted with groundwater of appropriate composition in a realistic temperature and radiation environment. The leachate may be analyzed for the presence of radionuclides in dissolved or suspended states, and then the speciation of these radionuclides can be determined.

(3) What is the solubility of each radionuclide of concern under conditions present at the respository and along the flow path to the accessible environment?

Many data needed to answer this question are similar to those for question (1), except that all the actinides should be considered, as well as the other multivalent elements of interest. A relatively broad range of temperature, 
Eh, and $\mathrm{pH}$ conditions may exist in the repository environment and along flow paths; therefore, data for a variety of conditions must be obtained. Experimental work will again be complicated by the need to use radionuclide concentrations higher than trace levels to characterize the species (complexes, oxidation states) involved.

(4) What oxidation states and what hydrolysis and complexation products of the actinides and other multivalent elements of concern are stable in the groundwater?

This is really a continuation of question (3), with the emphasis on long-range stability. Experimentation will include identifying the equilibrium states of the chemical species that are involved. Some data are available in the literature, but more detailed information is required, especially in pH ranges above 7 .

(5) What chemical systems can be used to control the Eh in laboratory experiments with multivalent elements?

Experimental work relative to questions (3) and (4) requires improved control of oxidation potentials in solutions under laboratory conditions. The system of using redox indicators as $\mathrm{Eh}$ moderators should be pursued further (Table LXXVIII). Indigo Carmine and Thionine appeared promising in initial experiments, but it must be shown that they react relatively rapidly with the element in question and do not react (or sorb) in other ways.

Some of the possibly useful buffer compounds that cannot be purchased could be synthesized so that a broader choice of Eh values would be available in the proper $\mathrm{pH}$ range.

Some formal potentials and Eh-pH relationships must be determined for those compounds about which not enough specific data are available. Data for Eh indicators have the more readily available published values, hence the emphasis on those compounds.

An Eco Electroprep cell, which is now available, may make it possible to measure, under dynamic conditions, the sorption characteristics of multivalent waste elements during reduction. A platinum-plated titanium electrode and a glassy carbon electrode are used in a flow-through chamber that uses groundwater as electrolyte in a circulating sorption system. An auxiliary Ag-AgCl electrode would be used as the reference, and a potentiostat would supply voltage. Even when the circulation system is operated in a closed mode, 
TABLE LXXVIII

POSSIBLE Eh MODERATORS

\begin{tabular}{|c|c|c|c|}
\hline Organic Couples & $\underline{\operatorname{Em}(V)}$ & $\mathrm{pH}$ & Range \\
\hline Bindschedler's Green & 0.224 & 2 & -9.5 \\
\hline Induline Scarlet & -0.299 & 3.0 & -8.6 \\
\hline Rosinduline $2 \mathrm{G}$ & -0.281 & 4.8 & -11.4 \\
\hline Lissamine Blue BF & -0.253 & 1 & -11 \\
\hline$N, N^{\prime}$-dimethyl viologens & $-0.446^{\mathrm{a}}$ & 8.4 & -13 \\
\hline Indigo Carmine & -0.125 & & $<9^{b}$ \\
\hline Pyocyanine & -0.034 & 1 & -12 \\
\hline Thionine & +0.064 & 1 & -13 \\
\hline
\end{tabular}

experiments that involve potentials $\leq 0.1 \mathrm{~V}$ might have to be done in a controlledatmospheric glove box.

(6) What is the sorption ratio $R_{d}$ value for each hazardous radionuclide species in each type of geologic medium that will be encountered in the flow path from the repository to the accessible environment?

A large amount of data has been accumulated on this subject, and relatively few experiments may be required to fill in missing information. In particular, more information must be acquired about the sorptive behavior of particular chemical species (complexes and verified oxidation states) of some actinides and other multivalent elements. This will require preparation and characterization of solutions at concentrations in the $10^{-3} \mathrm{M}$ range and then dilution before contact with the rock in batch or column experiments. Also, some additional work is required to determine differences caused by aerobic and anaerobic conditions.

(7) What conditions lead to the formation of actinide polymers and colloids? 
Relatively little experimental work has been directed toward answering this question. It has been observed that americium and plutonium leached from borosilicate glass are, in part, in colloidal form. Leaching experiments should be performed with the appropriate waste form and groundwater to examine the leachate for the presence of colloids. Also, there are plans to study the extent to which dissolved actinides and other radioactive elements may sorb on colloidal particles in the solution, thus forming radioactive colloids.

Experimental techniques for identifying and sizing these radiocolloids must be developed; to date, high-speed centrifugation and filtration coupled with microautoradiographic examination of the filters have been used in experiments.

(8) Is particulate transport, including transport of polymers, colloids, and other aggregated species, a significant means for movement of radioactive material through geologic media?

A few experiments have been initiated that seem to indicate particles in the micron-size range can pass through crushed-rock columns and through rock disks (Chap. 1, Sec. II.B.3). These experiments will be extended, initially with commercially prepared colloids tagged with fluorescent dyes or a radioactive label and eventually with "natural" radiocolloids containing actinides and mineral substrates. In this connection the properties of $\mathrm{Pu}(\mathrm{IV})$ colloids are being studied. It will also be necessary to acquire equipment for counting and sizing particles in the submicron-size range.

\section{Isotope Measurements}

Isotope measurements can be used to help interpret the rate of groundwater flow along flow paths between the repository site and the accessible environment. The isotope studies could also help establish the history of climatic and geomorphological processes that might affect a repository or alter radionuclide migration rates.

Los Alamos has not until recently been using isotopic techniques to obtain information about Yucca Mountain, and such techniques used by the USGS in their Yucca Mountain investigations have been limited to ${ }^{14} \mathrm{C}$ and ${ }^{18} \mathrm{O}$ analyses. The information that could be obtained from isotopic analyses includes: (1) origins of the groundwaters and pore waters, (2) flow paths and mixing of aquifers, (3) age and age gradients of the water, (4) paleoclimate information, and (5) natural water/rock interactions and geothermometry. 
Understanding site-specific parameters is important for proper interpretation of the data and correcting for secondary interactions. It is necessary to establish a program for sampling, initial treatment (filtration, nonfiltration; acidifying; precipitating salts), preservation (gas tight, temperature), etc. Analysis and tracing of drilling fluids is very important. The analyses and interpretations should be for specific strata.

Probably the most important isotopes to be determined are ${ }^{3} \mathrm{H},{ }^{14} \mathrm{C},{ }^{2} \mathrm{H}$, ${ }^{18} \mathrm{O},{ }^{13} \mathrm{O}$, and ${ }^{36} \mathrm{Cl}$. Tritium is an indication of recent water, either by contamination or infiltration. The carbon isotopes are most useful for dating. For older waters, ${ }^{36} \mathrm{Cl}$ might prove valuable in determining rates of water movement in the unsaturated zone. Oxygen, carbon, and hydrogen ratios will give information on the thermal history and surface temperatures at the recharge time. Exchange of ${ }^{18} \mathrm{O}$ between $\mathrm{H}_{2} \mathrm{O}$ and $\mathrm{SO}_{4}^{2-}$ is temperature dependent, as is $\mathrm{SO}_{4}^{2-}$ exchange between rocks and water as measured with ${ }^{34} \mathrm{~S}$. Isotopic measurements such as ${ }^{18} 0$ are useful for inferring the origins of pore or bound waters.

The stable noble gases give a good indication of temperature at recharge; they may be useful if their concentrations vary. Radiogenic noble gases $\left({ }^{4} \mathrm{He}\right.$ and $40 \mathrm{Ar}$ ) are good dating tools and also yield information about mixing. Disequilibrium of uranium and of radium isotopes can provide informtion about both age and natural interactions. Protactinium in natural systems may be a useful indicator for the behavior of that element. Isotopic analyses of uranium and thorium in fracture fillings may date those minerals.

Careful interpretation must accompany these measurements. For example, the carbon ratios in the soil, in the tuff, and in the deep rocks must be considered with those in the water. Bacterial action may influpnce sulfur ratios.

A number of state-of-the-art, ultrasensitive isotopic techniques might prove helpful. Iodine-129, produced cosmogenically or by spontaneous fission, could be used to measure migration of that element. Technetium-99 from spontaneous fission is likewise interesting; it should be informative to observe whether this element can be detected in aerated water or in water in a reducing environment. Other potentially useful isotopes include ${ }^{41} \mathrm{Ca}$ and ${ }^{81} \mathrm{Kr}$.

Obviously, isotope techniques can yield a great deal of vital information about how the natural system has behaved over recent and geologic time. Interpretation requires wise selection of the proper models. 


\section{NEAR-FIELD ENVIRONMENT AND PERMEABILITY}

The goal of the continuing hydrothermal stability studies is to determine equilibrium phase assemblages in the tuffs of Yucca Mountain as a function of temperature and fluid pressure. This information is basic to understanding the mechanical properties of the tuff at elevated temperature. It also has a bearing on the questions of water production as the tuff is heated and on changes in sorptive properties that may occur with heating.

Even without mineral dehydration, increased temperature will result in an increase of fluid pressure in the repository, and it will tend to drive water out of the repository. Heating will also decrease the fluid density and tend to drive convective transport of pore fluid. To model fluid movement caused by heating, it will be necessary to understand the permeability and porosity of the rock. These properties can be measured in the laboratory for unfractured tuff, but field tests will also be necessary to understand the effects of fractures and the boundaries of the tuff flows. The tuff flow boundaries may be quite important because the convective transport of fluid will have an appreciable vertical component. To synthesize this information, codes must be acquired that model convective fluid transport in a porous or perhaps a fractured medium. The WAFE code (Table LXXVII) can calculate this convective flow; in fact, the code has already been used for similar applications.

Although there are exceptions, most minerals become more soluble in water as temperature increases, especially in the 25 to $300^{\circ} \mathrm{C}$ range. These variations in solubility with temperature should result in a dissolution of material near the repository and precipitation as the fluid moves away from the repository and cools. This, in turn, may result in increased permeability and porosity and perhaps decreased rock strength near the repository. However, the reverse is probable at greater distances and might contribute considerably to sealing the repository.

Waste elements in the pore fluid at the time of mineral precipitation might be incorporated into the crystal lattices of the precipitating solid phases and thus be effectively imobilized.

Significant changes in waste element solubilities and sorption may result from an increase in temperature and from changes in groundwater chemistry that are caused by the equilibration of tuff and groundwater at elevated temperature.

Changes in the tuff mineralogy can affect several of the issues that are important in assessing the suitability of Yucca Mountain as the location of a 
waste repository. The volume of material that could be affected by changes in mineralogy will extend from the backfill around the canisters (if tuff is used as backfill material) to host rock at a distance where the temperature increase is insufficient to cause mineral changes. Changes in mineralogy in this zone could affect sorptive properties, permeability, groundwater composition, and the mechanical properties of tuff. Two distinct issues require investigation: (1) the thermodynamically stable mineral assemblages must be determined as a function of temperature, pressure, and bulk rock composition; and (2) some estimates must be made of the reaction rates that will produce these assemblages.

To determine the stable mineral assemblages, cold-seal hydrothermal experiments (described in Chap. 1, Sec. III) will continue. These experiments on powdered samples provide reaction rates that are sufficiently rapid to determine the temperature and pressure conditions of chemical reaction between minerals, at least in the 200 to $400^{\circ} \mathrm{C}$ range; from these experiments thermodynamic data can be determined for the minerals involved. The experiments may provide reasonable empirical information on the mineral assemblages to be expected in the higher temperature range. The thermodynamic data will be quite useful for understanding the mineral equilibria at lower temperatures where reaction rates are quite slow on a laboratory time scale but may be relatively fast on the time scale of concern in the repository. These thermodynamic data will also be critical in modeling the groundwater composition in both the near and far field.

Some mineral stability measurements will also be done on whole-rock samples; these solid cylinders of rock will be enclosed in flexible sleeves and subjected to confining stresses similar to those found in Yucca Mountain. The samples will then be heated to the desired temperature, and water will be passed through them. Mineral reaction rates in such experiments should accurately reflect those that can be expected at Yucca Mountain. The rates at which groundwater will equilibrate with the rock can also be determined with these experiments.

The equilibration of groundwater with tuff minerals will also be studied using flexible gold bag vessels ${ }^{99}$ that allow periodic sampling of the fluid in contact with tuff. Although the reaction rates in these experiments will probably not reflect the kinetics of the natural system as well as those in solid-core experiments, quicker equilibration and more certain identification of the equilibrium state can be achieved with this system. 
Just as the rock/groundwater equilibrium is a function of temperature, so are the solubilities of waste elements. Because little is known about uranium and plutonium solubilities at elevated temperature, additional work is needed, especially under alkaline conditions. Here, experiments on pure oxides in the flexible gold bag apparatus would be very valuable; reaction progress could be watched, and the final equilibrium state could be determined. This process would provide valuable solubility information and data against which reaction path codes such as $\mathrm{EQ} 3 / 6$ could be compared. Data are also needed for the temperature effect on waste element sorption, and they can be collected by means of jacketed solid-core experiments. These experiments could be done with an apparatus similar to that now used for permeability measurements.

Noting that mineral solubility generally increases with increasing temperature, it seems likely that tuff will be dissolved in groundwater near the repository and deposited further away as the groundwater cools. This may have a positive effect upon waste containment; because groundwater will flow in zones of the highest permeability, precipitation following groundwater cooling will occur in these pathways and should tend to close them off. Further, waste elements in the groundwater at this time may be coprecipitated in the precipitating minerals and thereby be effectively immobilized. Initial experiments will be run with two cores in tandem. The upstream core will be heated and the downstream core kept at room temperature. The apparatus will be arranged so that permeability and storage capacity can be measured periodically in both cores during the experiment. Later experiments will be run with a single core along which a temperature gradient is imposed. Similar experiments to examine fixation may be run with water containing waste elements.

After the target horizon is more clearly defined, additional permeability, porosity, and storage capacity measurements will also be required in the laboratory. More laboratory measurements will also be needed in conjunction with field permeability tests, but current testing techniques should be sufficient for this purpose.

Repository heating will cause expansion of fluid near the repository and hydrous mineral dehydration will produce free water in and around the repository. Water around the repository thus will be at a higher pressure and less dense than surrounding groundwater. Both these factors will tend to perturb the natural groundwater flow pattern in the vicinity of the repository. To assess the extent of the perturbation, data on repository heat generation must 
be combined with mineral stability data and information on tuff permeability, storage capacity, and porosity.

The possible effects of radiolysis also should be examined. The most important possible effect of radiolysis is the creation of an increased nonequilibrium oxidation state in the groundwater. This problem is important because the waste elements that exist in multiple oxidation states are generaliy more soluble when more highly oxidized. An experimental design to investigate this phenomenon has not yet been formulated.

\section{GEOCHEMICAL RETARDATION}

To predict the geochemical retardation possibilities in the Yucca Mountain area, a data base must be established from which models can be made. Geochemical retardation processes and flow paths between the repository and the accessible environment must be identified. Geochemical retardation will be affected by a number of factors, including (1) sorption processes, (2) the horizon and rock type chosen for the repository, (3) temperature variations, (4) groundwater geochemistry, (5) fixation reactions, (6) diffusion processes, and (7) the effect of mineral precipitation. Information collected through studies of these processes will help predict the rates and concentrations in which the radionuclides could be released into the environment; the studies will also help assess the hazards associated with those potential releases.

\section{A. Sorptive Behavior of Tuff}

The batch sorption technique (Chap. 1, Sec. IV) has been shown to be a powerful tool for obtaining information on sorptive processes and for providing a data base for modeling interactions between waste elements and tuff. This understanding, added to more detailed mineralogic information for Yucca Mountain, should lead to a three-dimensional model of sorptive properties for the hazardous waste elements, emphasizing those properties along potential flow paths from the repository to the biosphere.

The sorptive properties of some types of tuff have yet to be studied to complete the stratigraphic and lithologic correlations. From the stratigraphic viewpoint, tuffs from the Topopah Spring Member and the tuffaceous beds of Calico Hills must be studied in more detail. Care should be taken to select samples that are typical of the mineraisgy of the units and cover major lithologic variations both laterally and horizontally. 
The roles of smectites, illite/muscovite, glass (massive and fine grained), mordenite, and analcime must be quantified further for use in predictive models. Samples that are naturally rich in individual minerals or separated fractions will be studied.

The effects of reducing minerals in tuff, such as pyrite and iron-titanium oxides, that occur below $\sim 3000 \mathrm{ft}$ should be studied for particular waste elements--technetium, plutonium, neptunium, and uranium--that may be reduced and rendered more insoluble or more sorptive. The studies will be done in an inert atmosphere of nitrogen containing carbon dioxide in an abundance that provides approximately the same carbonate concentration as would occur at depth (a concentration that will be determined in the groundwater chemistry program). Knowledge of possible reductive processes along a fiow path are essential because increased retardation may be provided.

If the minerals in rocks do not provide the same redox conditions in the laboratory as are observed in the field, such conditions should be achieved in the laboratory through the use of redox buffers and/or potentiostats. First, however, it must be established that redox buffers do not interfere with natural processes (for example, by complexation).

A number of minerals other than the common tuff minerals considered until recently have been observed in whole-tuff samples and in fractures. The importance of such minerals as chlorite, calcite, albite, sericite, biotite, tridymite, allanite, sphene, and apatite for sorption has to be established. Many of these are not expected to provide a relatively significant number of sorption sites. Factors to be considered, in addition to sorptive properties of a mineral, are the abundance of each mineral and whether it is along a potential flow path to the biosphere.

Should definition of flow paths show possible transport through carbonates or argillites, sorptive phenomena would have to be studied for such horizons. Discharge is often through alluvial detritus. Some work has been done on processes in alluvium, but not for all hazardous nuclides in high-level waste.

The number of samples to be studied for very far-field phenomena should be limited by careful selection of representative samples that will allow averaging of phenomena over large distances.

The minerals that line fractures or lithophysae will be of great influence in sorption. Most of these minerals have been studied, but the detailed study should be completed. 
In conjunction with the experiments under controlled conditions, sorptive studies should be performed with specific oxidation states, complex states, etc. of elements that may exist as several species under the geologic conditions, particularly if the kinetics of transformation among species are slow relative to the duration of the experiment. Column experiments indicate the importance of such considerations.

The interaction of some hazardous waste elements with tuffs has not been studied; the hazard ranking of waste elements will reveal those of importance. Technetium, neptunium, and plutonium have to be studied in more detail. A limited number of experiments with radium will probably establish a regular relationship of the sequence of scrption ratios on tuffs for the alkaline earths as has been observed with cation-exchange resins, so the sorptive behavior of radium may be predictable from the known detailed behavior of strontium and barium. The behavior of curium and actinium should be similar to that of americium; experiments should be performed for confirmation. Samarium should behave as cerium and europium for the expected conditions. Thorium and protactinium are unique, and their behavior has to be established. other elements with potentially hazardous nuclides include nickel, selenium, and iodine.

Kinetic effects and fixation are very important for modeling the interactions of radionuclides and tuffs in flowing systems and for establishing the relative importance of various phenomena. Timed batch experiments and column experiments with varying flow rates will continue.

The effect of temperature changes between $\sim 20$ and $70^{\circ} \mathrm{C}$ has been shown to be minor for sorption for times of a few months. Experiments at higher temperatures, particularly for tuffs containing reducing minerals, should indicate whether enhanced effects (such as increased sorption and fixation or dissolution of the rock and leaching) are possible. Diffusion should be more competitive with sorption at elevated temperatures.

Only limited studies have been done using waters having compositions significantly different from the equilibrium composition. These waters were of greatly different composition, both from each other and from well J-13 water, and large effects were observed. The water sampling program will indicate a more realistic range of compositions for waters that might transport waste elements. Bounding experiments will be performed using such waters . 
Desorption experiments and column experiments give indications of irreversibility of sorption interactions and of possble permanent fixation (Chap. 1 , Sec. IV). Studies of the process should be extended, particularly for poorly sorbing nuclides that may yet be fixed over geologic time.

The role of diffusion processes for retarding the transport of anions such as iodide and pertechnetate through tuff has been established. Anion exclusion processes may act in opposition. Laboratory experiments of anion exclusion and diffusion with long columns or blocks, in addition to field experiments of diffusion, may quantify these concerns. The study of the relative rates of transport of ${ }^{36} \mathrm{C} 1$ (from atmospheric nuclear weapons tests or from cosmic radiation interactions) and HTO can be studied in a selected tuff setting. Knowledge of the relative rates of transport of IITO, ${ }^{36} \mathrm{Cl}$, and ${ }^{129} \mathrm{I}$ from nuclear explosions in tuff would also help elucidate these phenomena.

A systematic program for preserving both rock and water samples for future geochemical study must be established. At this time some cores at Los Alamos are preserved in a nitrogen atmosphere in steel containers. Water samples should be preserved so that analyses of dissolved sulfide, gases, and other components can be performed as future needs dictate.

B. Diffusion

In addition to the geochemical processes of fixation, particulate migration, and sortion, diffusion might be important in retarding radionuclide transport along flow paths. The more permeable horizons in Yucca Mountain are the welded zones that contain cooling joints. It is, therefore, likely that the flow path from a repository to the accessible environment will involve transport by fracture flow. Diffusion is potentially an important mechanism in the retardation of the transport of soluble species in a fracture-flow system. Diffusion can remove nonsorbing species from the rapidly moving fracture fluid, placing them in the stationary matrix fluid, thus causing an effective retardation.

It is necessary to validate models that incorporate matrix diffusion. Diffusion experiments are simpler than fracture-flow experiments because there is no convection and, therefore, there is less ambiguity in the interpretation of the diffusion process coupled with sorption; that is, there is no channeling and mass transfer kinetics are less important. There is a need to develop a diffusion data base so that diffusivities can be predicted for a given chemical 
species in a given tuff. The role of diffusion in inhibiting the transport of soluble species by fracture flow must be demonstrated.

Experiments which have been performed on diffusion include

(1) diffusion through tuff disks, that is, membranes,

(2) diffusivity measurements of various tracers including fluorobenzoic acids,

(3) diffusion into thick sheets of tuff,

(4) fracture-flow studies,

(5) the incorporation of nonlinear isotherms into the diffusion equations, and

(6) pore-shape determinations.

These initial studies (described in Chap. 1, Sec. IV) will be continued in the future and expanded to provide a data base for diffusion in tuff. Parameters that must he measured for a diffusion data base are:

(1) Constrictivity - The ionic diffusion coefficient is reduced by a number of parameters that depend on the geometry of the pores and chemical properties of the pore surfaces. These properties should be rock dependent. In addition to these properties, the constrictivity depends on the size and charge of the diffusing species. Constrictivities must be determined for the various tuff strata and various cations, anions, and neutral species.

(2) Tortuosity - The pores are not straight conduits through the rock, and the effect of the longer path length that chemical species must travel is accounted for by reducing the diffusion coefficient by a tortuosity factor. This parameter should be rock dependent and must be measured for the strata in Yucca Mountain tuff.

(3) Porosity - The diffusivity also depends on the porosity and pore-size distribution. A porosity data base is necessary to predict diffusion in tuff.

(4) Retardation Factors - Diffusion is also slowed by sorption. Retardation factors are determined using other techniques in other studies. Diffusion experiments will provide another check on the reproducibility of retardation factors.

The effect: of sorption processes such as nonlinear isotherms on diffusion can be examined. The studies will also test and validate diffusion models and 
provide data base for modeling. Most important, the efficacy of diffusion in retarding radionuclide transport must be demonstrated.

\section{Flow Studies}

The rate and concentration of radionuclide release into the accessible environment can be predicted, in part through porous-flow studies that measure the permeability and retardation potential of tuffs in the area.

1. Fracture-Flow and Related Sorption Experiments in Tuff. The welded tuff members of Yucca Mountain contain cooling fractures and fault joints; therefore, it is necessary to study the movement of radionuclides in fractured tuff samples, and thereby, assess the importance of matrix diffusion as a mechanism slowing radionuclide transport by fracture flow. This mechanism could have particular importance to the retardation of anionic or neutral species that would not otherwise be sorbed by ion exchange.

Studies to examine retardation in fracture-flow systems will be continued. A fracture-flow system is different from a porous-flow system in several ways. (1) The fluid velocities are typically much greater than in a porous-flow system. (2) It takes a much longer time for dissolved species to penetrate the rock. (3) The sorptive behavior may therefore be quite different from that observed in batch sorption or porous-flow column studies because of slow kinetics or nonlinear isotherms. (4) The sorptive behavior may be different for the minerals on the fracture surface than for the bulk rock. (5) In addition to Fickian dispersion, which occurs in both fracture flow and porous flow, there can be dispersion caused by channeling, which could dominate the hydrodynamic dispersion and could scale with the size of the experiment.

The studies of fracture flow will examine the sorptive behavior of both the fracture-fill material and the tuff matrix. The retardation observed in the fracture-flow systems will be compared with the $R_{d}$ values measured in batch experiments. To eliminate uncertainties in interpretation of the results caused by nonlinear sorptive behavior, the isotherms will also be determined. In addition, experiments will be performed to provide evidence of fixation reactions. All of these experiments will be necessary to complement the fractc:e-flow studies and make possible an unambiguous interpretation of the results. Physical characterization of the fracture fill and tuff atrix must include porosity and effective diffusivities to eliminate the arbitrary assignnent of values to parameters that will influence the modeling of the results. The 
study of retardation in fracture flow requires the complementary studies mentioned above to gain a thorough understanding of the migration of radionuclides in fracture flow.

The elution of radionuclides through tuff fractures in addition to providing a basis for predicting the retardation will provide data on hydrodynamic dispersion in fracture flow. These effects become important in larger systems, such as laboratory experiments with blocks and field studies. Because spreading in the elution curves of chemical species can be caused by either dispersion or reaction (mass transfer) kinetics, it is desirable to study the hydrodynamic dispersion so that there is not this ambiguity in the interpretation of the results.

Probably the most important purpose of these experiments is to validate transport models. These experiments will be used to test the validity of models, primarily TRACR3D, and also to provide an experimental basis for deciding what chemistry or physics needs to be included in the model, or even what processes can be ignored. Another important product of these studies will be a determination of the level of confidence with which the migration of radionuclides in fractured media can be predicted.

2. Porous-Flow Experiments. Several of the tuff strata in Yucca Mountain have low fracture densities. In particular, the bedded tuff of Calico Hills, a potential repository horizon, has few fractures. It is, therefore, important to study porous flow and radionuclide transport in porous systems because some portion of the flow path between the repository and the accessible environment may be dominated by porous flow. Permeabilities of tuff samples from the various tuff strata of Yucca Mountain are needed to model the flow path of water through the repository and the flow rates. Tracer experiments in porous media are needed to simulate the retardation of waste elements in an unfractured system. Tracer experiments are also needed to validate transport models and to confirm laboratory results, such as those from batch sorption measurements.

The majority of available data on the sorptive properties of tuff come from batch or static laboratory measurements. The ability to use these data to predict radionuclide migration in flowing systems depends on the sorptive mechanism and the kinetics of sorption. It is, therefore, useful to perform porous-flow experiments to identify sorption mechanisms that have slow kinetics, which would not be evident in batch measurements. The distribution coefficient $K_{d}$ is a useful means of treating sorption because of its mathematical simplicity 
and because there are few transport models as iilable that can handle a more complex chemical interaction. There have been a number of papers recently that have criticized the $K_{d}$ concept. Some of these criticisms may be justified, but to a large extent the concept has received an ignominious reputation because experiments have been performed under improper conditions. It can be shown that if sorption is caused by an equilibrium process and the groundwater composition is correct, $K_{d}$ values should be reproducible. It is, therefore, useful to verify the validity of the $K_{d}$ concept and to determine the confidence levels that should be assigned to $K_{d}$ values established for Yucca Mountain tuff. Some uncertainty is introduced in the results of crushed-rock column experiments because pulverizing the tuff may alter the sorptive properties by exposing minerals that are unavailable in the solid rock or by increasing the surface area by crushing sorptive minerals. Therefore, solid-core column experiments are needed to verify and clarify the results of the crushed-rock studies.

The majority of the work to date has been with crushed-rock columns. The $K_{d}$ values measured by the column technique are generally a factor of 2 to 3 lower than the corresponding values determined from batch measurements . More recent work has provided evidence that this discrepancy may be caused by a fractionation of fine particles (which are probably rich in montmorillonite grains) in the preparation of crushed rock for batch measurements and crushedrock columns. Therefore, assigning an uncertainty of a factor of 3 to the batch $K_{d}$ values is probably conservative; however, more work is required to determine which value or preparation method is more representative of the bulk rock. An interesting phenomenon that has observed in some crushed-rock column experiments was an apparent "irreversible" sorption of cesium, which was then released at a very slow rate. This may be evidence for fixation in the rock, which, if it occurs in tuff, could have profoundly beneficial consequences for a number of waste elements.

Some experiments have been performed using solid-core columns and have been compared with batch and crushed-rock column measurements (Chap. 1, Sec. IV). Preliminary results show better agreement with crushed-rock columns than with batch measurements. There are, however, advantages and disadvantages to each of these techniques. One disadvantage of solid-core columns is that the sample may not be representative (because of the heterogeneity of tuff) of the larger quantity of rock such as is used for batch and crushed-rock coluans. 
This makes a comparison of results from solid-core columns and the batch and crushed-rock columns less definitive. A number of solid-core columns have been run with nonsorbing tracers. An interesting aspect of these measurements is that dispersion caused by the pore shape and the distribution of flow paths through the rock can be determined. It would be interesting to see if the dispersion in solid rock scales with experiment size. Although dispersion is not considered important in 10-CFR-60, severe dispersion can accelerate the release of radionuclides, especially if levels lower than peak levels are considered.

Permeabilities and porosities have been determined for several tuff samples (Chap. 1, Sec. IV). These measurements are necessary for the interpretation of field tests and for the design $c f$ laboratory experiments. They also provide a data base for modeling flow in fractures and in the near field. The permeabilities of solid-core samples have in general been determined to be near 10 microdarcys, which is an extraordinarily low permeability for rock which has a porosity of about 0.25 . This is a highly favorable property of tuff.

In order to resolve the issues for site characterization, further studies in porous flow are required. Further crushed-rock studies will

(1) continue crushed-rock column studies to further characterize the sorptive potential of Yucca Mountain tuff,

(2) perform column studies using several flow rates to provide data that are sensitive to mass transfer kinetics and to determine if the water velocity will affect the apparent sorption ratio,

(3) perform column studies of more hazardous waste elements with more complex chemical properties, such as plutonium, neptunium, uranium, thorium, etc., and

(4) continue to examine crushed-rock column behavior for evidence of fixation reactions.

Further solid-core column experiments to be performed will

(1) continue solid-core column studies as part of the program to characterize the sorptive potential of Yucca Mountain tuff,

(2) study more highly zeolitized tuff samples because most studies have concentrated on welded and partially welded tuffs, 
(3) porform several column studies with the same tuff but with varying fluid velocity in order to observe kinetic effects,

(4) perform solid-core column experiments with more hazardous and more chemically complex waste elements, in particular the actinides, and

(5) perform experiments with solid-core columns with particulate tracers to determine the filtration potential of tuff.

In addition to the above porous-flow studies, a series of experiments are proposed to study flow through unsaturated tuff. These experiments will

(1) develop a tensiometer for measuring the matric potential and consolidated tuff. Tuff may present special problems because the hydraulic permeability is very low,

(2) develop a laboratory method of determining the degree of saturation of solid tuff samples,

(3) measure the relative permeability of tuff as a function of the degree of saturation,

(4) study the transport of soluble species through tuff columns by an unsaturated flux,

(5) measure the retardation provided by tuff in an unsaturated flow, and

(6) measure the capillarity of tuff and relate this to the pore-size distribution.

\section{Hazard Rank}

High-level nuclear reactor wastes contain a large number of different radionuclides that must be isolated from the accessible environment until they decay to innocuous radiation levels. Some of these will decay rapidly after removal from the reactor and will not require long-term isolation. Others must be isolated for millions of years. The objective of this task is to identify those radionuclides that pose the greatest potential threat to mankind during storage of nuclear wastes in a repository mined in unsaturated tuff in Yucca Mountain. The safety assessment studies required for licensing a repository in Yucca Mountain will then further concentrate on the key radionuclides because these will determine the overall safety of the repository. This has not been done 
heretofore because the fundamental data and comprehensive models needed to perform proper defensible calculations were largely unavailabie.

The TRACR3D and WAFE codes described earlier (Chap. 1, Sec. VI) will be used because they presently include dominant physical and chemical transport processes, particularly for the unsaturated zone. The calculations will also take into account (1) the quantity of the various radionuclides present in reprocessed waste and in spent reactor fuel, (2) the leach rate from the waste form, (3) the thermal field, (4) parent-daughter relationships, and perhaps (5) the presence of a single diffusion-controlled backfill.

\section{NATURAL ANALOGUES}

The study of natural analogs to waste repository environments can give important information on long-term chemical reaction and transport. How do rock properties change and what material moves? Such analogues can be used to extrapolate from laboratory-scale experiments to the time scales of hundreds or thousands of years that may be required for isolation of waste in a repository.

One kind of natural analogue that is particularly relevant to the waste repository environment is the region affected by a warm spring that has been flowing for hundreds of years. The warm spring altered the rock and transported chemical elements as it flowed away from its heat source. The repository will impose a thermal pulse on the local groundwater. Studies of warm springs in felsic tuffs can provide information that will permit prediction of long-term alteration and transport of material by groundwater, which is warmed near the repository, as it cools and moves out towards the accessible envi ronment.

The questions that need to be answered by studies of warm springs in felsic tuffs are:

(1) What is dissolved and precipitated as the warm groundwater flows down the temperature gradient away from the heat source? How does the alteration affect rock properties in the near-field repository envi ronment?

(2) What transport of major, minor, and trace elements takes place? What kinds of trace elements are removed by sorption or as chemical precipitates, both as mineral phases (oxides, silicates, etc.) and as minor solid-solution components in other mineral phases? 
Studies will include a literature search for published information on warm spring localities in Nevada and an initial selection of those that occur in volcanic tuff, acquisition of as much information as possible from workers in the field, a detailed field study and sampling of the two or three most promising localities, laboratory examination of the rock and water samples to determine chemical composition of the water and chemical and physical properties of the rock as a function of temperature at the collection site, and a modeling effort, integrated with other transport and reaction modeling, to describe the transport of chemical elements and the alteration of the rock.

\section{v. GEOCHEMICAL AND TRANSPORT MODELING}

Computer programs must be exercised and developed to provide a bank of codes for performance assessment. These codes will be used to aid in experiment design and interpretation. Through the process of code validation, the geochemical and physical processes that may be unique to the Yucca Mountain site will be included and implemented. To predict the complex mineralogic and geochemical changes that will occur over the times considered for a repository, geochemical modeling must be interfaced to transport models. This interface will at least require a reduction of the number of possible mineralogic and geochemical processes considered. These simplifications can be accomplished by performing sensitivity analyses and appropriate experiments. It is important to establish a feedback loop, whereby models and experiments can interact to help design more effective experiments and improve the codes to meet the needs of a site-specific performance assessment.

Work has already begun, as described in Chap. 1, Sec. VI, to address the modeling needs. The geochemical models WATEQ and EQ3/6 have been used to examine the mineralogic processes associated with Yucca Mountain tuff. Solubilities have been calculated for the actinides in tuffaceous water from well J-13 using EQ3/6. These predictions have already pointed out the need for improvements in the thermodynamic data base, which means new laboratory experiments must be performed.

The transport model TRACR3D has been used to predict fracture flow in a partially saturated medium to aid in the design of a fracture-flow field experiment. This exercise has proved useful in demonstrating that capillary action is an important effect to be considered in performing such a field experiment in the unsaturated zone and in considering an unsaturated horizon for repository use. 
By performing sensitivity studies, geochemical processes that could have a significant impact on the performance of a repository can be identified. This also implies experimental validation of these findings.

Sensitivity studies can also identify needs or elucidate deficiencies in the thermodynamic data base. Validation of codes with field and laboratory findings will aid in the discovery of deficiencies. New experiments that can yield thermodynamic data must be performed to expand the data base. The evidence for nonequilibrium processes in tuff/waste element and tuff/groundwater interactions can only come from experimental findings. These processes, as they are discovered, must be implemented in the geochemical and transport models. Reaction kinetics are presently being implemented in the EQ3/6 model.

As described above, field experiments are being designed with the aid of numerical models. Such experiments will test the ability to extrapolate the results of laboratory experiments to field conditions by using numerical models.

Modeling efforts will be needed to correlate new data from both laboratory and field experiments, and therefore, will continue throughout the experimental program.

In addition to adding new chemistry to the present transport code TRACR3D, the feasibility of combining the geochemical model EQ3/6 with the transport code will be investigated. The combination of these codes will enable the prediction of such mineral processes as precipitation and dissolution of mineral phases along with sorption and fixation of waste elements as they are transported along the flow path to the accessible environment.

VI. SHAFT AND BOREHOLE SEALING

It is important that the shaft and borehole seals of a waste repository maintain their initial properties (such as strength, volume, seal/rock adhesion, permeability, and sorption characteristics) for the length of time that the repository must be effective in isolating its contained waste from the accessible environment. In fact, the efficiency of sealing will become increasingly important through time as protective canisters deteriorate, waste forms are exposed to leaching by groundwater, and hazardous materials begin to migrate. Chemical reaction between sealing material and rock or groundwater can weaken the seals and can also weaken the adjacent rock. The objective of the following tests is to predict the nature and extent of reaction of sealing material with a volcanic tuff environment during the lifetime of the repository. 
Whatever sealing material is ultimately chosen will very probably react with the rock at some rate because the sealant and rock will be chemically different and because volcanic tuff contains tiny, highly reactive mineral and glass particles. The object in choosing an appropriate sealant is to minimize reaction and, especially, to minimize the reaction rate. Tests and experiments are designed to answer the following questions:

(1) What kind of chemical reaction takes place between each potential sealing material and volcanic tuff (and its associated groundwater)?

(2) How rapidly does the sealing material react with the tuff?

(3) What is the effect of the chemical interaction on the physical and chemical properties of the sealing material?

(4) What is the effect of the chemical interaction on the physical and chemical properties of the rock, especially in a possibly disturbed zone immediately adjacent to shafts and boreholes?

(5) What may be the added effects of a thermal pulse or radiolysis?

A. Laboratory Experiments in Agitated Vessels

Initial tests to screen a number of potential sealing materials and aggregates will include agitated vessel studies. The agitated vessels accelerate reaction; this is especially desirable at low temperatures (25 to $200^{\circ} \mathrm{C}$ ) where it is difficult to predict long-term effects from experiments run on laboratory time scales. Agitated runs will be made using tuff fragments alone, cement (or grout) fragments alone, and mixtures of the two, including some fragments containing contacts between the two. The runs will be made with local groundwater and at about 300 bars pressure and approximately 200,100 , and $25^{\circ} \mathrm{C}$. The higher temperature studies accelerate reaction as well as model a possible near-field environment. A few static vessel runs under the same conditions will be made to verify that the more complex agitated vessel conditions do accelerate reaction rates and that they do give the same kinds of run products. Samples will be examined before and after the runs by x-ray diffractometer, optical microscope, scanning electron microscope, and electron microprobe.

\section{B. Laboratory Experiments in Temperature Gradient Circulating Systems}

The most promising candidates for sealing materials will also be tested in circulating systems with controlled temperature gradients. Local groundwater will be circulated very slowly over alternating tuff and cenent samples at about 300 bars pressure through temperature gradients ranging from 25 to $200^{\circ} \mathrm{C}$ 
and from 200 to $25^{\circ} \mathrm{C}$. This will simulate the flow of groundwater into the warm, waste-containing region of a repository and the flow out through the near-field region and into the far-field region.

If much evidence is observed of reactions that affect seal permeability, a second kind of circulating system should be employed in addition to the first. In such a second system, groundwater is forced through 0.75-in.-diam core at controlled temperature and pressure for an extended period of time. The core is then dissected and examined.

C. Field Tests

Field tests of selected materials will include flow tests in which a fluid pressure differential is imposed on a sealed borehole. Such tests will make it possible to observe chemical reaction in the disturbed region immediately adjacent to the borehole in addition to that of the sealed hole. Permeability will be measured during the flow tests. The hole will later be overcored and dissected; the rock and sealing material will be examined in detail for chemical interaction.

\section{Survey of Relevant Thermodynamic Data}

A brief survey will be made of the thermodynamic data that are available on the system $\mathrm{CaO}-\mathrm{SiO}_{2}-\mathrm{Al}_{2} \mathrm{O}_{3}-\mathrm{K}_{2} \mathrm{O}-\mathrm{Na}{ }_{2} \mathrm{O}-\mathrm{MgO}-\mathrm{FeO}_{2}-\mathrm{Fe}_{2} \mathrm{O}_{3}$ at appropriate temperature and pressure (25 to $200^{\circ} \mathrm{C}, 1$ to 300 bars); these data for the combined cement/rock system might be used to verify the experimental results and facilitate modeling.

\section{ACKNOWLEDGEMENTS}

We thank the following people who have contributed their time and effort: D. A. Mann (technical assistance); P. A. Elder, M. E. Lark, and S. Lermuseaux (sample counting and gamma-spectral analyses); and C. E. Gallegos (typing many drafts and the final manuscript). 


\section{REFERENCES}

1. K. Wolfsberg, B. P. Bayhurst, B. M. Crowe, W. R. Daniels, B. R. Erdal, F. 0. Lawrence, A. E. Norris, and J. R. Smyth, "Sorption-Desorption Studies on Tuff. I. Initial Studies with Samples from the J-13 Drill Site, Jackass Flats, Nevada," Los Alamos Scientific Laboratory report LA-7480-MS (April 1979).

2. E. N. Vine, R. D. Aguilar, B. P. Bayhurst, W. R. Daniels, S. J. DeVilliers, B. R. Erda1, F. 0. Lawrence, S. Meastas, P. Q. Oliver, J. L. Thompson, and K. Wolfsberg, "Sorption-Desorption Studies on Tuff. II. A Continuation of Studies with Samples from Jackass Flats, Nevada, and Initial Studies with Samples from Yucca Mountain, Nevada," Los Alamos Scientific Laboratory report LA-8110-MS (January 1980).

3. K. Wolfsberg, R. D. Aguilar, B. P. Bayhurst, W. R. Daniels, S. J. DeVilliers, B. R. Erdal, F. 0. Lawrence, S. Maestas, A. J. Mitchell, P. Q. Oliver, N. A. Raybold, R. S. Rundberg, J. L. Thompson, and E. N. Vine, "SorptionDesorption Studies on Tuff. III. A Continuation of Studies with Samples from Jackass Flats and Yucca Mountain, Nevada," Los Alamos National Laboratory report LA-8747-MS (May 1981).

4. G. H. Heiken and M. L. Bevier, "Petrology of Tuff Units from the J-13 Drill Site, Jackass Flats, Nevada," Los Alamos Scientific Laboratory report LA-7563-MS (February 1979).

5. I. J. Winograd and F. N. Robertson, "Deep Oxygenated Ground Water: Anomaly or Common 0ccurrence?" Science 216, 1227-1230 (1982).

6. D. L. Bish, D. T. Vaniman, F. M. Byers, Jr., and D. E. Broxton, "Summary of the Mineralogy-Petrology of Tuffs of Yucca Mountain and Secondary-Phases of Thermal Stability in Tuffs," Los Alamos National Laboratory report LA-9321-MS (November 1982).

7. A. H. Davis, Ed., "Los Alamos National Laboratory Quality Assurance Plan and Procedures," Los Alamos National Laboratory report LA-9331-MS (in preparation 198?).

8. Code of Federal Regulations, Title 10-Energy. Chap. I - NRC, Part 50: Domestic Licensing of Production and Utilization Facilities, App. B. (January 1, 1982 rev.) [10-CFR-50].

9. R. R. Geoffrion, Ed., "Quality Assurance Manual," Los Alamos National Laboratory report (in preparation 1983).

10. M. Whitfield, "Thermodynamic Limitations on the Use of the Platinum Electrode in Eh Measurements," Limnol. Oceanogr. 19, 857-865 (1974). 
11. J. Boulègue, "Electrochemistry of Reduced Sulfur Species in Natural Waters--I. The $\mathrm{H}_{2} \mathrm{~S}-\mathrm{H}_{2} \mathrm{O}$ System," Geochim. Cosmochim. Acta $\underline{42}$, 1439-1445 (1978).

12. J. Boulègue and G. Michard, "Sulfur Speciations and Redox Processes in Reducing Environments," in Chemical Modeling in Aqueous Systems, E. Jenne, Ed. (American Chemical Society, Washington, D.C., 1979), p. 25.

13. G. F. Lee and W. Stumm, "Determination of Ferrous Iron in the Presence of Ferric Iron with Bathophenanthroline," J. AWWA 52, 1567 (1960).

14. Subrata Banerjee, "Direct Determination of Ferrous Iron in Silicate Racks and Minerals by Iodine Monochloride," Anal. Chem. 46, No. 6 (May 1974).

15. C. F. Baes and R. E Mesmer, The Hydrolysis of Cations (John Wiley and Sons, New York, 1976), p. 190.

16. J. M. Cleveland, The Chemistry of Plutonium (Gordon and Breach Science Publishers, New York, 1970), Chap. 5.

17. J. C. Sullivan, P. A. Bertrand, and G. R. Choppin, "Thermodynamics of Pu(VI) Interaction with Bicarbonate," Radiochimica Acta (in press).

18. B. Allard, H. Kipatsi, and J. O. Liljenzin, "Expected Species of Uranium, Neptunium, and Plutonium in Neutral Aqueous Solutions," J. Inarg. Nucl. Chem. 42, 1015 (1980).

19. V. I. Grebenshchikova and Yu. P. Davydov, "State of Pu(IV) in the Region of $\mathrm{pH}=1.0-12.0$ at Plutonium Concentration of $2 \times 10^{-5} \mathrm{M}$," Radiokhimiya $\underline{7}$, 191 (1965).

20. W. L. Polzer and W. F. Miner, "Plutonium and Americium Behavior in the Soil/Water Environment," Batelle Pacific Northwest Laboratory report BNWL-2117 (1976).

21. W. Stumm and J. J. Morgan, Aquatic Chemistry (Wiley-Interscience, New York, 1970), pp. 300-382.

22. J. M. Ottaway, "Oxidation Reduction Indicators of $E_{0}^{\prime}<0.76$ Volt," in Indicators, E. Bishop, Ed. (Pergamon Press, Oxford, 1972), p. 469.

23. J. C. Harrar, "Techniques, Apparatus, and Analytical Application of Controlled-Potential Coulometry," in Electroanalytical Chemistry, Vol. 8, A. J. Bard, Ed. (Marcel Dekker, New York, 1975), p. 1.

24. D. Rai, R. J. Berne, D. A. Moore, and R. W. Stromatt, "Electron Transfer Method of Controlling Eh during Adsorption of Multivalent Elements by Geologic Media," Battelle Pacific Northwest Laboratories report PNL-SA-6766 (1978). 
25. R. H. Fabian, D. M. Klassen, and R. W. Sonntag, "Synthesis and Spectroscopic Characterization of Ruthenium and Osmium Complexes with Sterically Hindering Ligands," Inorg. Chem. 19 , 1977 (1980)

26. D. A. Buckingham, F. P. I'wyer, H. A. Goodwin, and A. M. Sargeson, "Mono- and Bis-(2,2'-Bypyridine) and (1,10-Phenanthroline) Chelates of Ruthenium and Osmium," Aust. J. Chem. 17, 325 (1964).

27. D. A. Buckingham, F. P. Dwyer, and A. M. Sargeson, "Osmium(III)-Osmium(II) Potentials," Inorg. Chem. $\underline{5}, 1243$ (1966).

28. J. Fuger and F. L. Oetting, The Chemical Thermodynamics of Actinide Elements and Compounds. Part 2. The Actinide Aqueous Ions (IAEA, Vienna, 1976).

29. J. J. Katz and G. T. Seaborg, The Chemistry of the Actinide Elements (Methuen and Co., Ltd., London, 1957), p. 300 and references cited.

30. M. H. Lloyd and R. G. Haire, "The Chemistry of Plutonium in Sol-Gel Processes," Radiochem. Acta 25, 139 (1978).

31. D. Rai and J. L. Swanson, "Properties of Plutonium(IV) Polymer and Environmental Importance," Nucl. Technol. 54, 107 (1981).

32. D. A. Costanzo, R. E. Biggers, and J. T. Bell, "Plutonium Polymerization," J. Inorg. Nuc1. Chem. 35, 609 (1973).

33. S. W. Rabideau, "The Kinetics of the Disproportionation of Plutonium(V)," J. Amer. Chem. Soc. 79, 6350 (1957).

34. L. M. Toth, H. A. Friedman, and M. M. Osborne, "Polymerization of Pu(IV) in Aqueous Nitric Acid Solutions," J. Inorg. Nuc1. Chem. 43 , 2929 (1981).

35. J. L. Thompson and K. Wolfsberg, "Applicability of Microautoradiography to Sorption Studies," Los Alamos National Laboratory report LA-7609-MS (January 1979).

36. J. R. Smyth, J. L. Thompson, and K. Wolfsberg, "Microautoradiographic Studies of the Sorption of U and Am on Natural Rock Samples," Radioact. Waste Manage. 1,13 (1980).

37. J. D. Blacic and R. Anderson, "Methodology for Determining Time-Depeudent Mechanical Properties of Tuff Subjected to Near-Field Reposjtory Crnditions," Los Alamos National Laboratory report LA-9322-MS (January 1983).

38. E. N. Treher and N. A. Raybold, "The Elution of Radionuclides through Columns of Crushed Rock from the Nevada Test Site," Los Alamos National Laboratory report LA-9329-MS (October 1982).

39. M. L. Sykes, G. H. Kaiken, and J. R. Smyth, "Mineralogy and Petrology of Tufí Units from the UE25a-1 Drill Site, Yucca Mountain, Nevada," Los Alamos Scientific Laboratory report LA-8139-MS (November 1979). 
40. D. L. Bish, F. A. Caporuscio, J. F. Copp, B. M. Crowe, J. D. Purson, J. R. Sayth, and R. G. Warren, "Preliminary Stratigraphic and Petrologic Characterization of Core Samples from USW-G1, Yucca Mountain, Nevada," Los Alamos National Laboratory report LA-8840-MS (November 1981).

41. D. L. Bish, "Detailed Mineralogical Characterization of the Bullfrog and Tram Members in USW-G1, with Emphasis on Clay Mineralogy," Los Alamos National Laboratory report IA-9021-MS (October 1981).

42. P. I. Carrol1, F. A. Caporuscio, and D. L. Bish, "Further Description of the Topopah Spring Member of the Paintbrush Tuff in Drill Holes UE25a-1 and USW-G1 and the Lithic Rich Tuff in USW-G1, Yucca Mountain, Nevada," Los Alamos National Laboratory report IA-9000-MS (November 1981).

43. F. Caporuscio, D. Vaniman, D. Bish, D. Broxton, B. Arney, G. Heiken, F. Byers, R. Gooley, E. Semarge, "Petrologic Studies of Drill Cores USW-G2 and UE25b-1H, Yucca Mountain, Nevada," Los Alamos National Laboratory report LA-9255-MS (July 1982).

44. S. E. Haggerty, "Oxidation of Opaque Mineral Oxides in Basalts," in Oxide Minerals, Min. Soc. Am. Short Course Notes 3, Hí-1-H6-100 (1976).

45. R. H. More and R. K. Zeigler, "The Solution of the General Least Squares Problem with Special Reference to High-Speed Computers," Los Alamos National Laboratory report LA-2367 (March 1960).

46. I. L. Ames, Jr., "Some Zeolite Equilibria with Akaline Earth Metal Ions," Am. Mineral. 49, 1099 (1964).

47. L. L. Ames, Jr., "Some Zeolite Equilibria with Alkali Metal Cations," Am. Mineral. 49, 127 (1964).

48. J. R. Smyth and F. A. Caporuscio, "Review of the Thermal Stability and Cation Exchange Properties of the Zeolite Minerals Clinoptilolite, Mordenite, and Analcime: Applications to Radioactive Waste Isolation in Silicic Tuff," Los Alamos National Laboratory report LA-8841-MS (July 1981).

49. R. M. Barrer, "Cation-Exchange Equilibria in Zeolites and Feldspathoids," in Natural Zeolites: Occurrerce, Properties, and Use, L. S. Sand aud F. Mumpton, Eds. (Pergamon, 1978), pp. 385-395.

50. D. E. W. Vaughan, "Properties of Natural Zeolites," in Natural Zeolites Occurence, Properties and Use, L. Sand and F. Mompton, Eds. (Pergamon, 1978), pp. 353-371.

51. J. R. Eliason, "Montmorillonite Exchange Equilibria with Strontium-SodiumCesium," Am. Minera1. 51, 324 (1966). 
52. L. D. Baver, W. H. Gardner, and W. R. Gardner, Soil Physics (John Wiley and Sons, New York, 1972), pp. 20-37.

53. R. E. Gum, Applied Mineralogy (McGraw Hill, New York, 1962).

54. B. Allard, G. W. Beall, and T. Krajewski, "The Sorption of Actinides in Igneous Rocks," Nucl. Technol. 49, 474-480 (1980).

55. B. Allard and G. W. Beall, "Sorption of Americium on Geologic Media," J. Environ. Health, A14(6), 507-518 (1979).

56. G. E. Manger, "The Best Value of Porosity of Lapilli Tuff from the Nevada Test Site," US Geol. Surv. Prof. Paper 525-B (1965), pp. B146-B150.

57. R. A. Robinson and R. H. Stokes, Electrolyte Solutions (Butterworths, London, 1968), pp. 253-257.

58. P. Spacek and M. Kubin, "Diffusion in Gels," J. Polym. Sci. C16, 705 (1967).

59. L. K. Porter, W. D. Kemper, R. D. Jackson, and B. C. Stewart, "Chloride Diffusion in Soils as Influenced oy Moisture Content," Soil Sci. Soc. Am. Proc. 24, 460-463 (1960).

60. W. J. Moore, Physical Chemistry (Prentice Hall, Englewood Cliffs, N.J., 1972), pp. 425-427.

61. J. Crank, The Mathenatics of Diffusion, 2nd ed. (Oxford University Press, London, 1975), p. 52.

62. I. Neretnieks, "Diffusion in the Rock Matrix: An Important Factor in Radionuclide Retardation?" J. Geophys. Res. 85, 4379 (1980).

63. K. L. Erickson and D. R. Fortney, "Preliminary Transport Analyses for Design of the Tuff Radionuclide Migration Field Experiment," Sandia National Laboratories report SAND81-1253 (September 1981).

64. W. R. Brace, J. B. Walsh, and W. T. Frangos, "Permeability of Granite Under High Pressure," J. Geophys. Res. 73, 2225 (1968).

65. P. A. Witherspoon, J. S. Y. Wang, K. Iwai, and J. E. Gale, "Validity of Cubic Law for Fluid Flow in a Deformable Rock Fracture," Lawrence Berkeley National Laboratory report LBL-9557 (October 1979).

66. P. A. Witherspoon, C. H. Amick, J. E. Gale, and K. Iwai, "Observations of a Potential Size-Effect in Experinental Determination of the Hydraulic Properties of Fractures," Lawrence Berkeley National Laboratory report LBL-8571 (May 1979).

67. H. S. Carslaw and J. C. Jaeger, Conduction of Heat in Solids (Oxford University Press, London, 1959), p. 510. 
68. B. R. Erdal, R. D. Aguilar, B. P. Bayhurst, W. R. Daniels, C. J. Duffy, F. 0. Lawrence, S. Maestas, P. Q. Oliver, and K. Wolfsberg, "SorptionDesorption Studies on Granite. I. Initial Studes of Strontium, Technetium, Cesium, Barium, Cerium, Europium, Uranium, Plutonium, and Americium," Los Alamos Scientific Laboratory report LA-7456-MS (February 1979).

69. 1. Neretnieks, "Diffusion in the Rock Matrix--an Important Factor in Radionuclide Migration?" Kärnbränslesäkerhet report KBS-79-19 (May 1979).

70. A. Rasmuson and I. Neretnieks, "Migration of Radionuclides in Fissured Rock--the Influence of Micropore Diffusion and Longitudinal Dispersion," Kärnbränslesäkerhet report KBS-80-24 (December 1979).

71. R. W. Spengler, F. M. Byers, Jr., and J. B. Warner, "Stratigraphy and Structure of Volcanic Rocks in Drill Hole USW-G1, Yucca Mountain, Nye County, Nevada," US Geological Survey open-file report 81-1349.

72. R. K. Blankennagel and J. E. Weir, Jr., "Geohydrology of the Eastern Part of Pahute Mesa, Nevada Test Site, Nye County, Nevada," US Geological Survey professional paper 712-B (1973).

73. B. J. Travis, "TRACR 3D: A Model of Flow and Transport in Porous Fractured Media," Los Alamos National Laboratory report LA-9667-MS (Apri1 1983).

74. J. B. Ball, D. K. Nordstrom, and E. A. Jenne, "Additional and Revised Thermochemical Data and Computer Code for WATEQ2 - A Computerized Chemical Model for Trace and Major Element Speciation and Mineral Equilibria and Natural Water," US Geol. Surv. Water Resources Invest. report WRI 78-116 (January 1980).

75. T. J. Wolery, "Calculations of Chemical Equilibrium Between Aqueous Solution and Minerals: The EQ3/6 Software Package," Lawrence Livermore Laboratory report UCRL-52658 (February 1979).

76. S. L. Schoff and J. E. Moore, "Chemistry and Movement of Ground Water, Nevada Test Site," US Geol. Surv. report TEI-838 (1964).

77. G. Sposito and S. V. Mattigod, "GEOCHEM: A Computer Program for the Calculation of Chemical Equilibria in Soil Solutions and other Natural Water Systems," Department of Soil and Environmental Sciences, University of California, Riverside, California (1979).

78. S. E. Ingle, M. D. Schuldt, and D. W. Schults, "A User's Guide for REDEQL.EPAK," Environ. Prot. Agency report EPA-600/3-78-024 (February 1978).

79. S. E. Ingle, J. A. Keniston, and R. W. Shults, "REDEQL.EPAK, Aqueous Chemical Equilibrium Program," Corvallis Environmental Research Laboratory, Corvallis, Oregon (1979). 
80. L. N. Plummer, B. F. Jones, and A. H. Truesdell, "WATEQF: A FORTRAN IV Version of WATEQ, A Computer Program for Calculating Chemical Equilibrium of Natural Waters, Users Guide," US Geol. Surv. report USGS-WRI-76-13 (September 1976).

81. A. C. Walters and P. R. Carroll, Eds., "Preliminary Stratigraphic and Petrologic Characterization of Core Samples from USW-G1, Yucca Mountain, Nevada," Los Alamos National Laboratory report LA-8840-MS (November 1982).

82. C. H. Chen, "A Method of Estimation of Standard Free Energies of Formation of Silicate Minerals at $298.15 \mathrm{~K}, "$ Am. J. Sci. 275, 801-817 (1975).

83. W. A. Deer, R. A. Howie, and J. Zussman, Rock-Forming Minerals, Vol 3, Sheet Silicates (Longman Group Ltd., London, 1962), pp. 191-245.

84. H. C. Helgeson, "Thermodynamics of Hydrothermal Systems at Elevated Temperatures and Pressures," Am. J. Sci. 267, 729-804 (1969).

85. J. R. Boles, E. M. Flanigen, A. J. Gude, R. L. Hay, F. A. Mumpton, and R. C. Surdam, "Mineralogy and Geology of Natural Zeolites," in Mineralogical Society of America Short Course Notes, Vol. 4, F. A. Mumpton, Ed. (Southern Printing Co., Blacksburg, Virginia, 1978).

86. W. A. Deer, R. A. Howie, and J. Zussman, Rock-Forming Minerals, Vol. 4, Framework Silicates (Longman, Green and Company, Ltd., London, 1963), pp. 351-428.

87. H. C. Helgeson, J. M. De1tny, H. W. Nesbitt, and D. K. Bird, "Summary and Critique of the Thermodynamic Properties of Rock-Forming Minerals," Am. J. Sci. 278A, 1-299 (1978).

88. R. A. Robie and D. R. Waïdbaum, "Thermodynamic Properties of Minerals and Related Substances at $298.15^{\circ} \mathrm{K}\left(25.0^{\circ} \mathrm{C}\right)$ and One Atmosphere $(1.013$ Bars) Pressure and at Higher Temperatures," US Geological Survey Bulletin 1259 (1968).

89. L. V. Benson and L. S. Teaque, "A Tabulation of Thermodynamic Data for Chemical Reactions Involving 58 Elements Common to Radioactive Waste Package Systems," Lawrence Berkeley Laboratory report LBL-11448 (August 1980).

90. J. 0. Nriagu, "Thermochemical Approximations for Clay Minerals," Am. Mineral. 60, 834-839 (1975).

91. Y. Tardy and R. M. Garrels, "A Method of Estimating the Gibbs Energies of Formation of Layer Silicates," Geochim. Cosmochim. Acta 38, 1101-1116 (1974). 
92. S. V. Mattigod and G. Sposito, "Improved Method for Estimating the Standard Free Energies of Formation $\left(\Delta G_{f}^{\circ}, 298.15\right)$ of Smectites," Geochim. Cosmochim. Acta 42, 1753-1762 (1978).

93. M. Slaughter, "Chemical Binding in Silicate Minerals-I. Model for Determining Crystal-Chemical Properties," Geochim. Cosmochim. Acta 30, 299-313 (1966).

94. M. Slaughter, "Chemical Binding in Silicate Minerals-Ir. Computational Methods and Approximations for the Binding Energy of Complex Silicates," Geochim. Cosmochım. Acta 30, 315-322 (1966).

95. M. Slaughter, "Chemical Binding in Silicate Minerals-III. Application of Energy Calculations to the Prediction of Silicate Mineral Stability," Geochim. Cosmochim. Acta 30, 323-339 (1966).

96. D. Langmuir, "Uzanium Solution-Mineral Equilibria at Low Temperatures with Applications to Sedimentary Ore Deposits," Geochim. Cosmochim. Acta 42, 547-569 (1978).

97. R. J. Lemire and P. R. Tremaine, "Uranium and Plutonium Equilibria in Aqueous Solutions to $200^{\circ} \mathrm{C}$," J. Chem. Eng. Data 25, 361-369 (1980).

98. W. R. Abecle, "Determination of Hydraulic Conductivity in Crushed Bandelier Tuff," Los Alamos Scientific Laboratory report LA-8147-MS (November 1979).

99. W. E. Seyfried, Jr., P. C. Gordon, and F. W. Dickson, "A New Reaction Cell for Hydrothermal Solution Equipment," Am. Mineral. 64, 651 (1979). 


\section{APPENDIX A}

SORPTION RATIO DATA FOR TUFFS OF THE YUCCA MOUNTAIN AREA

Data for sorption ratios for tuffs in the Yucca Mountain area, measured since 1978, appear in a number of Los Alamos reports (listed in App. A, Refs. 1-9) and are consolidated here. Individual tables were prepared for each drill core (Tables A-I to A-XXXII). Chapter 1, Sec. IV.A should be consulted for the drill hole, depth, and mineralogic composition of each core; further mineralogic and petrologic descriptions are given in App. B.

For each sorption experiment the tables list $R_{d}$ values and contact times for sorption and desorption, concentration of the element measured, particlesize distribution of the ground tuff, temperature (Amb = ambient), and atmosphere $(\mathrm{CA}=\mathrm{a}$ controlled atmosphere of nitrogen with $\leq 0.2 \mathrm{ppm}$ oxygen and $\leq 20 \mathrm{ppm}$ carbon dioxide). Numbers cited in the reference column refer to the App. A reference list. Any special comments for an experiment are indicated in the footnote column in each table. Entries for sorption and desorption experiments on the same line indicate that the sorption and desorption experiments were performed with the same sample. When appropriate, data in this appendix were averaged for Tables XXI and XXII in Chap. 1, Sec. IV.A. 
TABLE A-I

BATCH SORPTION DATA TOR MUF JA-8

\begin{tabular}{|c|c|c|c|c|c|c|c|c|c|c|}
\hline \multirow[b]{2}{*}{ Element } & \multicolumn{2}{|c|}{ Sorption } & \multicolumn{2}{|c|}{ Desorption } & \multirow{2}{*}{$\begin{array}{l}\text { Element } \\
\text { Concentration } \\
-(M) \\
\end{array}$} & \multirow{2}{*}{$\begin{array}{c}\text { Particle } \\
\text { Size } \\
(\mu \text { ) } \\
\end{array}$} & \multirow[b]{2}{*}{ Tenperature } & \multirow[b]{2}{*}{ Atmosphere } & \multirow[b]{2}{*}{ Ref. } & \multirow[b]{2}{*}{ Note } \\
\hline & $\begin{array}{c}R_{d} \\
(-2 / \rho)\end{array}$ & $\begin{array}{l}\text { Time } \\
\text { (wk) }\end{array}$ & $\begin{array}{c}R_{d} \\
(n e / 2)\end{array}$ & $\begin{array}{l}\text { Time } \\
\text { (wk) }\end{array}$ & & & & & & \\
\hline sr & 260 & 3 & 300 & 12 & $2 \times 10^{-11}$ & $75-500$ & Anb & Air & 1 & 2 \\
\hline$"$ & 270 & 3 & & & $"$ & $"$ & $"$ & $"$ & $"$ & $"$ \\
\hline$"$ & 290 & 6 & 270 & 6 & $"$ & $"$ & $"$ & $"$ & $"$ & $"$ \\
\hline$"$ & 270 & 6 & 320 & 6 & $"$ & $"$ & $"$ & $"$ & $"$ & $"$ \\
\hline$"$ & & & 340 & 3 & $"$ & $"$ & $"$ & $"$ & $"$ & $"$ \\
\hline$"$ & & & 320 & 3 & $"$ & $"$ & $"$ & $"$ & $"$ & $"$ \\
\hline Cs & 2400 & 3 & 3600 & 12 & $6 \times 10^{-9}$ & $"$ & $"$ & $"$ & $"$ & $"$ \\
\hline$"$ & 2200 & 3 & & & $"$ & $"$ & $"$ & $"$ & $"$ & $"$ \\
\hline$"$ & 2600 & 6 & 6200 & 6 & $"$ & $"$ & $"$ & $"$ & $"$ & $"$ \\
\hline$"$ & 3800 & 6 & 4600 & 6 & $"$ & $"$ & $"$ & $"$ & $"$ & $"$ \\
\hline$"$ & & & 4400 & 3 & $"$ & $"$ & $"$ & $"$ & $"$ & $"$ \\
\hline$"$ & & & 4500 & 3 & $"$ & $"$ & $"$ & $"$ & $"$ & $"$ \\
\hline $\mathrm{Ba}$ & 400 & 3 & 520 & 12 & $1 \times 10^{-9}$ & " & ". & $"$ & $"$ & $"$ \\
\hline$"$ & 420 & 3 & & & $"$ & $"$ & " & " & " & $"$ \\
\hline " & 470 & 6 & 270 & 6 & $"$ & $"$ & $"$ & $"$ & $"$ & $"$ \\
\hline$"$ & 450 & 6 & 580 & 6 & $"$ & $"$ & $"$ & $"$ & $"$ & $"$ \\
\hline$"$ & & & 560 & 3 & $"$ & $"$ & " & $"$ & $"$ & $"$ \\
\hline$"$ & & & 520 & 3 & $"$ & $"$ & $"$ & $"$ & $"$ & $"$ \\
\hline Eu & 2100 & 3 & 4000 & 12 & $3 \times 10^{-10}$ & $"$ & $"$ & $"$ & " & $"$ \\
\hline$"$ & 1800 & 3 & & & $"$ & $"$ & $"$ & $"$ & $"$ & $"$ \\
\hline$"$ & 2900 & 6 & 5600 & 6 & $"$ & $"$ & $"$ & $"$ & $"$ & $"$ \\
\hline$"$ & 1700 & 6 & 5600 & 6 & $"$ & $"$ & $"$ & " & $"$ & $"$ \\
\hline$"$ & & & 17000 & 3 & $"$ & $"$ & $"$ & $"$ & $"$ & $"$ \\
\hline$"$ & & & 17000 & 3 & $"$ & $n$ & $"$ & $"$ & $"$ & " \\
\hline
\end{tabular}

a Concentrations are only those added with the tracer; actual concentrations may be higher. 
TABLE A-II

BATCH SORPTION DATA FOR TUFF JA-18

\begin{tabular}{|c|c|c|c|c|c|c|c|c|c|c|}
\hline \multirow[b]{2}{*}{ Element } & \multicolumn{2}{|c|}{ Sorption } & \multicolumn{2}{|c|}{ Desorption } & \multirow{2}{*}{$\begin{array}{c}\text { Element } \\
\text { Concentration } \\
(M) \\
\end{array}$} & \multirow[b]{2}{*}{$\begin{array}{c}\text { Particle } \\
\text { Size } \\
\text { (1) } \\
\end{array}$} & \multirow[b]{2}{*}{ Teuperature } & \multirow[b]{2}{*}{ Atmospheze } & \multirow[b]{2}{*}{ Ref. } & \multirow[b]{2}{*}{ Note } \\
\hline & $\begin{array}{c}R_{d} \\
(-2 / R)\end{array}$ & $\begin{array}{l}\text { Tine } \\
\text { (wt) }\end{array}$ & $\begin{array}{c}R_{d} \\
(n / /)\end{array}$ & $\begin{array}{l}\text { Tine } \\
\text { (wk) }\end{array}$ & & & & & & \\
\hline $\mathbf{S r}$ & 8900 & 2 & 13000 & 3 & $1 \times 10^{-6}$ & $355-500$ & $A \mathbf{b}$ & Air & 2 & \\
\hline " & 6500 & 3 & 5500 & 5 & $"$ & $"$ & $"$ & $"$ & $"$ & \\
\hline$"$ & & & 16000 & 14 & $"$ & $"$ & $"$ & $"$ & 11 & . \\
\hline$"$ & 5800 & 6 & & & $"$ & $"$ & $"$ & $"$ & $" 1$ & \\
\hline$"$ & 16000 & 11 & & & $"$ & $"$ & $"$ & " & $"$ & \\
\hline $\mathrm{Cs}_{8}$ & 10000 & 2 & 16000 & 3 & $1 \times 10^{-8}$ & $n$ & $"$ & $"$ & $"$ & \\
\hline " & 10000 & 3 & 17000 & 5 & " & $"$ & $"$ & $"$ & $"$ & \\
\hline$"$ & & & 15000 & 14 & $"$ & $"$ & $"$ & $"$ & $"$ & $\mathbf{a}$ \\
\hline$"$ & 19000 & 6 & & & $"$ & $"$ & $"$ & $"$ & $"$ & \\
\hline$"$ & 19000 & 11 & & & $"$ & $"$ & $"$ & $"$ & $"$ & \\
\hline $\mathrm{Ba}$ & 2300 & 2 & 8600 & 3 & $2 \times 10^{-7}$ & $"$ & $"$ & $"$ & $"$ & \\
\hline 4 & 3300 & 3 & 23000 & 5 & $"$ & $n$ & " & $"$ & $"$ & \\
\hline$"$ & & & 34000 & 14 & $"$ & $"$ & $"$ & $"$ & $"$ & a \\
\hline$"$ & 7100 & 6 & & & " & $"$ & $"$ & $"$ & $"$ & \\
\hline$"$ & 7100 & 11 & & & $"$ & $"$ & $"$ & $"$ & $"$ & \\
\hline Ce & 20 & 2 & 36 & 3 & $1 \times 10^{-6}$ & $"$ & $"$ & $"$ & $"$ & \\
\hline$"$ & 26 & 3 & 110 & 5 & $"$ & $"$ & $"$ & $"$ & $"$ & \\
\hline$"$ & & & 130 & 14 & " & $"$ & $"$ & $"$ & $"$ & a \\
\hline$"$ & 41 & 6 & & & $"$ & $"$ & $"$ & $"$ & 11 & \\
\hline$"$ & 70 & 11 & & & $"$ & " & $"$ & $"$ & $"$ & \\
\hline Eu & 14 & 2 & 30 & 3 & $6 \times 10^{-7}$ & $"$ & $"$ & $1 t$ & $"$ & \\
\hline " & 18 & 3 & 74 & 5 & $"$ & $"$ & $"$ & " & $"$ & \\
\hline$"$ & & & 140 & 14 & $"$ & $"$ & $"$ & " & " & 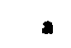 \\
\hline$"$ & 26 & 6 & & & $"$ & $"$ & $"$ & $"$ & $"$ & \\
\hline$"$ & 37 & 11 & & & $"$ & $"$ & $"$ & " & $"$ & \\
\hline $\mathbf{S r}$ & 11000 & 2 & 21000 & 3 & $1 \times 10^{-6}$ & $106-150$ & $"$ & $"$ & $"$ & \\
\hline$"$ & 13000 & 3 & 5800 & 5 & " & $"$ & $"$ & " & $"$ & \\
\hline$"$ & & & 13000 & 14 & $"$ & 11 & $"$ & $"$ & $"$ & a \\
\hline$"$ & 18000 & 6 & & & $"$ & $"$ & $"$ & $"$ & $" 1$ & \\
\hline " & 22000 & 11 & & & $"$ & $"$ & $"$ & $"$ & $"$ & \\
\hline
\end{tabular}

second desorption. 


\begin{tabular}{|c|c|c|c|c|c|c|c|c|c|c|}
\hline \multirow[b]{2}{*}{ Element } & \multicolumn{2}{|c|}{ Sorption } & \multicolumn{2}{|c|}{ Desorption } & \multirow[b]{2}{*}{$\begin{array}{c}\text { Element } \\
\text { Concentration } \\
\text { (H) } \\
\end{array}$} & \multirow[b]{2}{*}{$\begin{array}{c}\text { Particle } \\
\text { Size } \\
(1) \\
\end{array}$} & \multirow[b]{2}{*}{ Tenperature } & \multirow[b]{2}{*}{ Atmosphere } & \multirow[b]{2}{*}{ Ref. } & \multirow[b]{2}{*}{ Note } \\
\hline & $\begin{array}{c}R_{d} \\
(a \ell / 8)\end{array}$ & $\begin{array}{l}\text { Tine } \\
\text { (wk) }\end{array}$ & $\begin{array}{c}R_{d} \\
(\alpha / /)\end{array}$ & $\begin{array}{l}\text { Tine } \\
\text { (wk) }\end{array}$ & & & & & & \\
\hline $\mathrm{Cs}$ & 12000 & 2 & 22000 & 3 & $1 \times 10^{-8}$ & $106-150$ & $A \in b$ & Air & 2 & \\
\hline$"$ & 13000 & 3 & 18000 & 5 & $"$ & $"$ & $"$ & $"$ & $"$ & \\
\hline$"$ & & & 15000 & 14 & $"$ & $"$ & $"$ & $"$ & $n$ & a \\
\hline$"$ & 17000 & 6 & & & 11 & $"$ & $"$ & 1 & $"$ & \\
\hline$n$ & 20000 & 11 & & & $"$ & $"$ & $" 1$ & $"$ & $"$ & \\
\hline $\mathrm{Ba}$ & 3100 & 2 & 24000 & 3 & $2 \times 10^{-7}$ & $"$ & $"$ & $"$ & $"$ & \\
\hline$"$ & 4500 & 3 & 38000 & 5 & $"$ & $n$ & $"$ & " & $n$ & \\
\hline$"$ & & & 40000 & 14 & $"$ & $"$ & $n$ & $"$ & $"$ & a \\
\hline$"$ & 4700 & 6 & & & $"$ & $"$ & $"$ & 11 & $"$ & \\
\hline$"$ & 6600 & 11 & & & $"$ & $"$ & $n$ & 11 & $"$ & \\
\hline $\mathrm{Ce}$ & 26 & 2 & 120 & 3 & $1 \times 10^{-6}$ & $"$ & $"$ & $"$ & $"$ & \\
\hline$"$ & 46 & 3 & 250 & 5 & $"$ & $"$ & $n$ & $"$ & $"$ & \\
\hline$"$ & & & 440 & 14 & $"$ & $" 1$ & $"$ & $"$ & $n$ & a \\
\hline$"$ & 43 & 6 & & & $"$ & $"$ & $"$ & $"$ & $"$ & \\
\hline$"$ & 78 & 11 & & & $"$ & $"$ & $"$ & $"$ & $"$ & \\
\hline Eu & 14 & 2 & 120 & 3 & $6 \times 10^{-7}$ & $"$ & $"$ & $"$ & $"$ & \\
\hline$"$ & 30 & 3 & 210 & 5 & $"$ & $"$ & $"$ & $"$ & $"$ & \\
\hline$"$ & & & 350 & 14 & $"$ & $"$ & $"$ & $"$ & $"$ & $\mathbf{a}$ \\
\hline$"$ & 32 & 6 & & & $"$ & $"$ & $"$ & $"$ & $"$ & \\
\hline$"$ & 66 & 11 & & & $"$ & $"$ & $"$ & $"$ & $"$ & \\
\hline Sr & 16000 & 3 & 17000 & 12 & $8 \times 10^{-11}$ & $75-500$ & $"$ & $"$ & 3 & b \\
\hline$"$ & 45000 & 6 & 16000 & 9 & $"$ & $"$ & $"$ & $"$ & " & $"$ \\
\hline$"$ & 20000 & 12 & 26003 & 3 & $"$ & $"$ & $"$ & $"$ & $"$ & $"$ \\
\hline $\mathrm{Cs}$ & 16000 & 3 & 19000 & 12 & $3 \times 10^{-9}$ & $"$ & " & $"$ & " & $"$ \\
\hline$"$ & 15000 & 6 & 17000 & 9 & $"$ & $"$ & $"$ & $"$ & "1 & $"$ \\
\hline$"$ & 18000 & 12 & 19000 & 3 & $"$ & $"$ & $"$ & $"$ & $"$ & $"$ \\
\hline $\mathrm{Ba}$ & 96000 & 3 & 240000 & 12 & $8 \times 10^{-10}$ & $"$ & $"$ & $"$ & $"$ & $"$ \\
\hline$"$ & 110000 & 6 & 220000 & 9 & $"$ & $"$ & $"$ & $n$ & $"$ & $"$ \\
\hline$"$ & 170000 & 12 & 370000 & 3 & $"$ & $"$ & $"$ & $"$ & $"$ & $"$ \\
\hline $\mathrm{Ce}$ & 1400 & 3 & 2000 & 12 & $8 \times 10^{-13}$ & 11 & $"$ & $"$ & $"$ & $"$ \\
\hline$"$ & 5700 & 6 & 1100 & 9 & $"$ & $"$ & $"$ & " & $"$ & $"$ \\
\hline$"$ & 1300 & 12 & & & $"$ & $"$ & $"$ & $"$ & $"$ & $"$ \\
\hline
\end{tabular}

\footnotetext{
Second desorption.

${ }^{b}$ Concentrations are only those added with the tracer; actual concentrations may be higher.
} 
TABLE A-II (cont)

\begin{tabular}{|c|c|c|c|c|c|c|c|c|c|c|}
\hline \multirow[b]{2}{*}{ Elenent } & \multicolumn{2}{|c|}{ Sorption } & \multicolumn{2}{|c|}{ Desorption } & \multirow{2}{*}{$\begin{array}{c}\text { Elenent } \\
\text { Concentration } \\
\text { (M) } \\
\end{array}$} & \multirow{2}{*}{$\begin{array}{c}\text { Particle } \\
\text { Size } \\
\text { (Hm) } \\
\end{array}$} & \multirow[b]{2}{*}{ Temperature } & \multirow[b]{2}{*}{ Atmosphere } & \multirow[b]{2}{*}{$\underline{\text { Ref. }}$} & \multirow[b]{2}{*}{ Note } \\
\hline & $\begin{array}{c}R_{d} \\
(m \ell / g) \\
\end{array}$ & $\begin{array}{l}\text { Time } \\
\text { (wk) } \\
\end{array}$ & $\begin{array}{c}R_{d} \\
(m \ell / g)\end{array}$ & $\begin{array}{l}\text { Tine } \\
\text { (wk) } \\
\end{array}$ & & & & & & \\
\hline $\mathbf{E u}$ & 1700 & 3 & 3000 & 12 & $1 \times 10^{-10}$ & $75-500$ & Amb & Air & 3 & $a$ \\
\hline$"$ & 1100 & 6 & 2300 & 9 & $"$ & $"$ & " & $"$ & " & $"$ \\
\hline$"$ & 1400 & 12 & 2100 & 3 & $"$ & $"$ & $"$ & $"$ & $"$ & $"$ \\
\hline $\mathrm{Sr}$ & 19000 & 2 & 22000 & 2 & $1 \times 10^{-6}$ & $355-500$ & $70^{\circ} \mathrm{C}$ & $"$ & 2 & \\
\hline$"$ & & & 19000 & 14 & $"$ & 1 & 11 & $"$ & $"$ & b \\
\hline$"$ & 15000 & 3 & 24000 & 3 & $"$ & " & 11 & 12 & $"$ & \\
\hline " & 22000 & 5 & 17000 & 6 & $"$ & $"$ & $"$ & " & $"$ & \\
\hline$"$ & $4300^{C}$ & 9 & & & $"$ & $"$ & $"$ & $"$ & $"$ & c \\
\hline Cs & 21000 & 2 & 22000 & 2 & $1 \times 10^{-8}$ & $"$ & $"$ & $"$ & 11 & \\
\hline$"$ & & & 26000 & 14 & $"$ & $"$ & $"$ & $"$ & $"$ & b \\
\hline$n$ & 18000 & 3 & 21000 & 3 & $"$ & $"$ & $"$ & $"$ & $"$ & \\
\hline$"$ & 19000 & 5 & 21000 & 6 & " & $"$ & $"$ & $"$ & $"$ & \\
\hline$"$ & 19000 & 9 & & & $"$ & $"$ & $"$ & $"$ & $"$ & \\
\hline Ba & 35000 & 2 & 91000 & 2 & $2 \times 10^{-7}$ & $"$ & $"$ & $"$ & $"$ & \\
\hline$"$ & & & 170000 & 14 & $"$ & $"$ & $"$ & $"$ & $" 1$ & $\mathbf{b}$ \\
\hline$"$ & 40000 & 3 & 67000 & 3 & $"$ & $"$ & $"$ & " & " & \\
\hline$"$ & 75000 & 5 & 150000 & 6 & $"$ & $"$ & $"$ & $"$ & $"$ & \\
\hline$"$ & 61000 & 9 & & & $"$ & $"$ & $"$ & $"$ & $"$ & \\
\hline $\mathrm{Ce}$ & 40 & 2 & 240 & 2 & $9 \times 10^{-7}$ & $"$ & " & $"$ & $"$ & \\
\hline$"$ & & & 300 & 14 & $"$ & $"$ & $"$ & $"$ & $"$ & $\mathbf{b}$ \\
\hline$"$ & 45 & 3 & 120 & 3 & $"$ & $"$ & $"$ & $"$ & $"$ & \\
\hline$"$ & 40 & 5 & 220 & 6 & $"$ & $"$ & $"$ & $"$ & $"$ & \\
\hline$"$ & 42 & 9 & & & $"$ & $"$ & $"$ & $"$ & $"$ & \\
\hline $\mathbf{E u}$ & 61 & 2 & 440 & 2 & $3 \times 10^{-7}$ & $"$ & $"$ & $"$ & $"$ & \\
\hline " & & & 840 & 14 & $"$ & $"$ & " & $"$ & $"$ & b \\
\hline$"$ & 62 & 3 & 260 & 3 & $"$ & $"$ & $"$ & $"$ & $"$ & \\
\hline$"$ & 76 & 5 & 500 & 6 & " & $n$ & $"$ & " & $n$ & : \\
\hline " & 89 & 9 & & & $"$ & $"$ & 11 & " & " & \\
\hline
\end{tabular}

\footnotetext{
${ }^{a}$ Concentrations are only those added with the tracer; actual concentrations any be higher. $\mathrm{b}_{\text {second desorption. }}$

Value not included in averages of Table XXI, Chap. 1.
} 
TABLE A-II (cont)

\begin{tabular}{|c|c|c|c|c|c|c|c|c|c|c|}
\hline \multirow[b]{2}{*}{ Elenent } & \multicolumn{2}{|c|}{ Sorption } & \multicolumn{2}{|c|}{ Desorption } & \multirow{2}{*}{$\begin{array}{c}\text { Elenent } \\
\text { Concentration } \\
\text { (M) } \\
\end{array}$} & \multirow[b]{2}{*}{$\begin{array}{c}\text { Particle } \\
\text { Size } \\
(\mu \mathrm{m}) \\
\end{array}$} & \multirow[b]{2}{*}{ Temperature } & \multirow[b]{2}{*}{ Atmosphere } & \multirow[b]{2}{*}{ Ref. } & \multirow[b]{2}{*}{ Note } \\
\hline & $\begin{array}{c}\mathrm{R}_{\mathrm{d}} \\
(\Omega \ell / g) \\
\end{array}$ & $\begin{array}{l}\text { Tine } \\
\text { (wk) } \\
\end{array}$ & $\begin{array}{c}R_{d} \\
(m \ell / g)\end{array}$ & $\begin{array}{l}\text { Tine } \\
\text { (wk) }\end{array}$ & & & & & & \\
\hline $\mathbf{S r}$ & 13000 & 2 & 22000 & 2 & $1 \times 10^{-6}$ & $106-150$ & $70^{\circ} \mathrm{C}$ & Air & 2 & \\
\hline$"$ & & & 17000 & 14 & $"$ & $"$ & $"$ & $"$ & $"$ & a \\
\hline$"$ & 22000 & 3 & 30000 & 3 & $"$ & " & $"$ & $"$ & $"$ & \\
\hline$"$ & 15000 & 5 & 18000 & 6 & $"$ & $"$ & 11 & $n$ & $"$ & \\
\hline " & $4700^{b}$ & 9 & & & $"$ & $"$ & $"$ & $"$ & $"$ & $b$ \\
\hline Cs & 13000 & 2 & 14000 & 2 & $1 \times 10^{-8}$ & $"$ & $"$ & $"$ & $"$ & \\
\hline$"$ & & & 17000 & 14 & $"$ & $"$ & $"$ & $"$ & $"$ & $\mathbf{a}$ \\
\hline$"$ & 21000 & 3 & 17000 & 3 & $"$ & $"$ & $"$ & $"$ & $"$ & \\
\hline$t$ & 16000 & 5 & 17000 & 6 & $"$ & $"$ & $"$ & $"$ & $"$ & \\
\hline$"$ & 17000 & 9 & & . & $"$ & $"$ & $"$ & $"$ & $"$ & \\
\hline $\mathrm{Ba}$ & 23000 & 2 & 70000 & 2 & $2 \times 10^{-7}$ & $"$ & $"$ & $"$ & $"$ & \\
\hline$"$ & & & 110000 & 14 & $"$ & $"$ & " & $"$ & $"$ & $\mathbf{a}$ \\
\hline$"$ & 29000 & 3 & 100000 & 3 & $"$ & $"$ & $"$ & $"$ & $"$ & \\
\hline$"$ & 64000 & 5 & 100000 & 6 & $"$ & $"$ & $"$ & 11 & $"$ & \\
\hline$"$ & 64000 & 9 & & & $"$ & $"$ & $"$ & $"$ & $"$ & \\
\hline $\mathrm{Ce}$ & 40 & 2 & 160 & 2 & $9 \times 10^{-7}$ & " & $"$ & $"$ & $"$ & \\
\hline$"$ & & & 550 & 14 & $"$ & $"$ & $n$ & $"$ & $"$ & a \\
\hline$"$ & 36 & 3 & 350 & 3 & $"$ & $" 1$ & $"$ & $"$ & " & \\
\hline$"$ & 46 & 5 & 230 & 6 & $"$ & $"$ & $"$ & $"$ & $"$ & \\
\hline$"$ & 51 & 9 & & & $"$ & $"$ & $"$ & $"$ & $" 1$ & \\
\hline Eu & 63 & 2 & 280 & 2 & $3 \times 10^{-7}$ & $"$ & $"$ & $"$ & $"$ & \\
\hline " & 76 & 3 & 780 & 3 & 4 & $"$ & $"$ & $"$ & $"$ & \\
\hline$"$ & 110 & 5 & 680 & 6 & $"$ & $"$ & $"$ & $"$ & $"$ & \\
\hline$"$ & 130 & 9 & & & $"$ & 11 & $"$ & $"$ & $"$ & \\
\hline Sr & 24000 & 4 & & & $2 \times 10^{-6}$ & $250-355$ & Amb & $"$ & 4 & $c$ \\
\hline$"$ & 27000 & 9 & & & $"$ & $"$ & $"$ & $"$ & $"$ & $"$ \\
\hline Cs & 11000 & 4 & & & $6 \times 10^{-10}$ & $"$ & $"$ & $"$ & $"$ & $"$ \\
\hline$"$ & 15000 & 9 & & & $"$ & $"$ & $"$ & $"$ & $"$ & $"$ \\
\hline $\mathrm{Ba}$ & 17000 & 4 & & & $1 \times 10^{-6}$ & $"$ & " & $"$ & $"$ & $"$ \\
\hline$"$ & 120000 & 9 & & & $"$ & $"$ & " & $"$ & $"$ & $"$ \\
\hline
\end{tabular}

\footnotetext{
Second desorption.
}

${ }^{b}$ alue not included in averages for Table XXI, Chap. 1 .

${ }^{\mathrm{C}}$ Syrthetic groundwater I; see Ref. 4. 
TABLE A-II (cont)

\begin{tabular}{|c|c|c|c|c|c|c|c|c|c|c|}
\hline \multirow[b]{2}{*}{ Element } & \multicolumn{2}{|c|}{ Sorption } & \multicolumn{2}{|c|}{ Desorpticn } & \multirow{2}{*}{$\begin{array}{c}\text { Element } \\
\text { Concentration } \\
(\mathrm{M})\end{array}$} & \multirow{2}{*}{$\begin{array}{c}\text { Particle } \\
\text { Size } \\
\text { (표) } \\
\end{array}$} & \multirow[b]{2}{*}{ Temperature } & \multirow[b]{2}{*}{ At-osphere } & \multirow[b]{2}{*}{ Ref. } & \multirow[b]{2}{*}{ Note } \\
\hline & $\begin{array}{c}R_{d} \\
(n \ell / 8)\end{array}$ & $\begin{array}{l}\text { Time } \\
\text { (wk) } \\
\end{array}$ & $\begin{array}{c}R_{d} \\
(n \ell / \Omega)\end{array}$ & $\begin{array}{l}\text { Time } \\
\text { (wk) } \\
\end{array}$ & & & & & & \\
\hline $\mathrm{Ce}$ & 950 & 4 & & & $1 \times 10^{-8}$ & $250-355$ & Amb & Air & 4 & a \\
\hline$"$ & 1500 & 9 & & & $"$ & $"$ & $"$ & $"$ & $"$ & $"$ \\
\hline Eu & 130 & 4 & & & $8 \times 10^{-9}$ & $"$ & $"$ & $"$ & $"$ & $"$ \\
\hline$"$ & 1700 & 9 & & & $"$ & $"$ & $"$ & $"$ & $"$ & $"$ \\
\hline$S_{r}$ & 5000 & 4 & & & $2 \times 10^{-6}$ & $"$ & $"$ & $"$ & $"$ & b \\
\hline$"$ & 4900 & 9 & & & $"$ & $"$ & $"$ & $"$ & $"$ & $"$ \\
\hline Cs & 7400 & 4 & & & $1 \times 10^{-9}$ & $"$ & $"$ & $"$ & $"$ & $"$ \\
\hline$"$ & 8400 & 9 & & & $"$ & $"$ & $"$ & $"$ & $"$ & $"$ \\
\hline $\mathrm{Ba}$ & 46000 & 4 & & & $1 \times 10^{-6}$ & $"$ & $"$ & $"$ & $"$ & $"$ \\
\hline$"$ & 82000 & 9 & & & $"$ & $"$ & $"$ & $"$ & $"$ & $"$ \\
\hline Ce & 5200 & 4 & & & $2 \times 10^{-8}$ & $"$ & $"$ & $"$ & $"$ & $"$ \\
\hline$"$ & $>34000$ & 9 & & & $"$ & $"$ & $"$ & $"$ & $"$ & $"$ \\
\hline Eu & 4600 & 4 & & & $3 \times 10^{-8}$ & $"$ & $"$ & $"$ & $"$ & $"$ \\
\hline$"$ & 15000 & 9 & & & $"$ & $"$ & $"$ & $"$ & $"$ & $"$ \\
\hline An & 250 & 1 & & & $4 \times 10^{-7}$ & $106-150$ & $"$ & $"$ & $"$ & \\
\hline$"$ & 200 & 1 & 270 & 2 & $"$ & $"$ & $"$ & $"$ & $"$ & \\
\hline$"$ & 96 & 1 & & & $"$ & $"$ & $"$ & $"$ & $"$ & \\
\hline$"$ & 310 & 1 & 810 & 9 & $"$ & $"$ & $"$ & $"$ & $"$ & \\
\hline$"$ & 86 & 2 & 440 & 5 & " & $"$ & $"$ & $"$ & $"$ & \\
\hline$"$ & 310 & 2 & 710 & 9 & $"$ & $"$ & $"$ & $"$ & $"$ & \\
\hline$"$ & 120 & 4 & & & $"$ & $"$ & $"$ & $"$ & $"$ & \\
\hline$"$ & 200 & 4 & 1200 & 5 & $"$ & $"$ & $"$ & $"$ & $"$ & \\
\hline$"$ & 360 & 4 & 790 & 9 & $"$ & $"$ & $"$ & $"$ & $"$ & \\
\hline$"$ & 220 & 4 & & & $"$ & $"$ & $"$ & $"$ & " & \\
\hline$"$ & 57 & 8 & & & $"$ & $"$ & " & $"$ & $"$ & \\
\hline$"$ & 100 & 8 & 2000 & 5 & $"$ & $"$ & $"$ & " & $"$ & \\
\hline$"$ & 85 & B & 2300 & 9 & $"$ & $"$ & $"$ & $"$ & " & \\
\hline$"$ & 85 & 8 & & & $"$ & $"$ & $"$ & $"$ & $"$ & \\
\hline
\end{tabular}

"Synthetic groundwater I; see Kef. 4.

${ }^{b}$ Syathetic groundwater III; see Ref. 4 . 
TABLE A-II (cont)

\begin{tabular}{|c|c|c|c|c|c|c|c|c|c|c|}
\hline \multirow[b]{2}{*}{ Element } & \multicolumn{2}{|c|}{ Sorption } & \multicolumn{2}{|c|}{ Desorption } & \multirow[b]{2}{*}{$\begin{array}{c}\text { Elenent } \\
\text { Concentration } \\
\text { (M) }\end{array}$} & \multirow[b]{2}{*}{$\begin{array}{c}\text { Particle } \\
\text { Size } \\
(\mu \mathrm{m}) \\
\end{array}$} & \multirow[b]{2}{*}{ Temperature } & \multirow[b]{2}{*}{ Atmosphere } & \multirow[b]{2}{*}{ Ref. } & \multirow[b]{2}{*}{ Note } \\
\hline & $\begin{array}{c}R_{d} \\
(m \ell / g)\end{array}$ & $\begin{array}{l}\text { Tine } \\
\text { (wk) } \\
\end{array}$ & $\begin{array}{c}R_{d} \\
(a \ell / R)\end{array}$ & $\begin{array}{l}\text { Tise } \\
\text { (wk) }\end{array}$ & & & & & & \\
\hline $\mathrm{Pu}$ & 170 & 1 & 160 & 2 & $4 \times 10^{-7}$ & $106-150$ & $A m b$ & Air & 4 & \\
\hline$"$ & 70 & 1 & & & $"$ & $"$ & $"$ & $"$ & $"$ & \\
\hline$"$ & $B:$ & 4 & & & $"$ & $"$ & $"$ & $"$ & $"$ & \\
\hline$"$ & 68 & 8 & & & "1 & $"$ & $"$ & 11 & $\because$ & \\
\hline " & 110 & 2 & 710 & 2 & $1 \times 10^{-13}$ & $"$ & $"$ & $"$ & $"$ & \\
\hline$"$ & 120 & 4 & 450 & 2 & $"$ & $"$ & $"$ & $"$ & $"$ & \\
\hline$"$ & 220 & 3 & 97 & 3 & $n$ & $"$ & $"$ & $1 "$ & $"$ & \\
\hline $\mathbf{U}$ & 4.9 & 1 & 13 & 3 & $1 \times 10^{-?}$ & $\because$ & $"$ & $"$ & $"$ & \\
\hline$"$ & 3.5 & 2 & 12 & 2 & $n$ & $"$ & $"$ & $"$ & $"$ & \\
\hline$"$ & 12 & 3 & 4.4 & 1 & $"$ & $"$ & $"$ & $"$ & $"$ & \\
\hline$"$ & 1.6 & 1 & 9.7 & 3 & $"$ & $355-500$ & $"$ & $"$ & $"$ & \\
\hline$"$ & 1.4 & 2 & 6.4 & 2 & $"$ & $"$ & $"$ & $"$ & " & \\
\hline tr & 1.3 & 3 & 6.3 & 1 & " & $"$ & $"$ & 1 & $"$ & \\
\hline$"$ & 4.2 & 1 & 13 & 3 & $"$ & " & $70^{\circ} \mathrm{C}$ & $"$ & " & \\
\hline " & 3.6 & 2 & 9.5 & 2 & $"$ & $"$ & $"$ & $" 1$ & " & \\
\hline " & 4.3 & 3 & 18 & 1 & $"$ & $"$ & $"$ & $"$ & $"$ & \\
\hline I & -1.5 & 3 & & & $1 \times 10^{-13}$ & iv & Amb & $"$ & 2 & \\
\hline$"$ & -0.5 & 4 & & & $"$ & $"$ & $"$ & $"$ & $"$ & \\
\hline Ho & 4.3 & 3 & & & $1 \times 10^{-14}$ & $"$ & $"$ & $"$ & $"$ & \\
\hline Ru & 67 & 3 & & & $3 \times 10^{-12}$ & $"$ & $"$ & $"$ & $"$ & \\
\hline$"$ & 32 & 4 & & & $"$ & $"$ & $"$ & $"$ & $"$ & \\
\hline $\mathrm{Sb}$ & -0.9 & 3 & & & $6 \times 10^{-12}$ & $"$ & $"$ & " & $"$ & \\
\hline$"$ & 0.2 & 4 & & & $"$ & 11 & $"$ & $"$ & $"$ & \\
\hline
\end{tabular}


TABLE A-III

BATCH SORPTION DATA FOR TUFF JA-26

\begin{tabular}{|c|c|c|c|c|c|c|c|c|c|c|}
\hline \multirow[b]{2}{*}{ E1ement } & \multicolumn{2}{|c|}{ Sorption } & \multicolumn{2}{|c|}{ Desorption } & \multirow{2}{*}{$\begin{array}{c}\begin{array}{c}\text { Element } \\
\text { Concentration } \\
(M) \\
\end{array} \\
\end{array}$} & \multirow{2}{*}{$\begin{array}{c}\text { Particle } \\
\text { Size } \\
\text { ( } \mu \text { m) } \\
\end{array}$} & \multirow[b]{2}{*}{ Tenperature } & \multirow[b]{2}{*}{ Atmosphere } & \multirow[b]{2}{*}{ Ref. } & \multirow[b]{2}{*}{ Note } \\
\hline & $\begin{array}{c}R_{d} \\
(-\ell / R)\end{array}$ & $\begin{array}{l}\text { Time } \\
\text { (wk) } \\
\end{array}$ & $\begin{array}{c}R_{\mathrm{d}} \\
\left(-e_{i}^{\prime}\right)\end{array}$ & $\begin{array}{l}\text { Time } \\
\text { (wk) }\end{array}$ & & & & & & \\
\hline $\mathbf{S r}$ & 36 & 3 & 32 & 12 & $\overline{2 \times 10^{-11}}$ & $75-500$ & Amb & Air & $\overline{1}$ & $\bar{a}$ \\
\hline$"$ & 40 & 3 & 30 & 12 & $"$ & $"$ & $"$ & $"$ & $"$ & $"$ \\
\hline$"$ & 130 & 6 & 38 & 6 & $"$ & $"$ & $"$ & $"$ & $"$ & $"$ \\
\hline$"$ & 170 & 6 & 43 & 6 & $"$ & $"$ & $"$ & $"$ & $"$ & $"$ \\
\hline$"$ & & & 46 & 3 & $"$ & $"$ & $"$ & $"$ & $"$ & $"$ \\
\hline$"$ & & & 46 & 3 & $"$ & $"$ & $"$ & $"$ & $"$ & $"$ \\
\hline Cs & 420 & 3 & 1200 & 12 & $6 \times 10^{-9}$ & $"$ & $"$ & $"$ & $"$ & $"$ \\
\hline$"$ & 720 & 3 & 1500 & 12 & $"$ & $"$ & $"$ & $"$ & $"$ & $"$ \\
\hline$"$ & 2900 & 6 & 1600 & 6 & $"$ & $"$ & $"$ & $"$ & $"$ & $"$ \\
\hline$"$ & 1900 & 6 & 1800 & 6 & $"$ & $"$ & $"$ & $"$ & $"$ & $"$ \\
\hline$"$ & & & 1700 & 3 & $"$ & $"$ & $"$ & $"$ & $"$ & $"$ \\
\hline$"$ & & & 1700 & 3 & $"$ & $"$ & $"$ & $\because$ & $"$ & $"$ \\
\hline $\mathrm{Ba}$ & 210 & 3 & 460 & 12 & $1 \times 10^{-9}$ & $"$ & $"$ & $"$ & $"$ & $"$ \\
\hline$"$ & 270 & 3 & 400 & 12 & $"$ & $"$ & $"$ & $"$ & $"$ & " \\
\hline$"$ & 1200 & 6 & 430 & 6 & $"$ & $"$ & $"$ & $"$ & $"$ & $"$ \\
\hline$"$ & 1400 & 6 & 450 & 6 & $"$ & $"$ & $"$ & $"$ & " & $"$ \\
\hline$"$ & & & 490 & 3 & $"$ & $"$ & $"$ & $"$ & $"$ & $"$ \\
\hline$"$ & & & 480 & 3 & $"$ & $"$ & $"$ & $"$ & $"$ & $"$ \\
\hline Eu & 220 & 3 & 3700 & 12 & $3 \times 10^{-10}$ & $\because$ & $"$ & $"$ & $"$ & $"$ \\
\hline$"$ & 47 & 3 & 3300 & 12 & $"$ & $"$ & $"$ & $"$ & " & $"$ \\
\hline$"$ & 15000 & 6 & 2100 & 6 & $"$ & $"$ & $"$ & $"$ & $"$ & $:$ \\
\hline$"$ & 1200 & 6 & 3100 & 6 & $"$ & $"$ & $"$ & $"$ & $"$ & $"$ \\
\hline$"$ & & & 2400 & 3 & $"$ & $"$ & $"$ & $"$ & $"$ & $\because$ \\
\hline$"$ & & & 3100 & 3 & $"$ & $"$ & $"$ & $"$ & $"$ & $"$ \\
\hline
\end{tabular}

Concentrations are only those added with the tracer; actual concentrations nay be higher. 
TABLE A-IV

BATCH SONPTIOA DATA FOR TUFF JA-2B

\begin{tabular}{|c|c|c|c|c|c|c|c|c|c|c|}
\hline \multirow[b]{2}{*}{ Element } & \multicolumn{2}{|c|}{ Sorption } & \multicolumn{2}{|c|}{ Desorption } & \multirow[b]{2}{*}{$\begin{array}{c}\text { Elenent } \\
\text { Concentration } \\
(\mathrm{H}) \\
\end{array}$} & \multirow[b]{2}{*}{$\begin{array}{l}\text { Particle } \\
\text { Size } \\
(\mu) \\
\end{array}$} & \multirow[b]{2}{*}{ Teaperature } & \multirow[b]{2}{*}{ Atmosphere } & \multirow[b]{2}{*}{ Ref. } & \multirow[b]{2}{*}{ Hote } \\
\hline & $\begin{array}{c}R_{d} \\
(a e / k)\end{array}$ & $\begin{array}{l}\text { Tine } \\
\text { (wh) }\end{array}$ & $\begin{array}{c}R_{d} \\
(x / /)\end{array}$ & $\begin{array}{l}\text { Tive } \\
\text { (wk) }\end{array}$ & & & & & & \\
\hline $\mathbf{S r}$ & 140 & 3 & 120 & 12 & $2 \times 10^{-11}$ & $75-500$ & $A m b$ & Air & 1 & - \\
\hline$"$ & 110 & 3 & 120 & 12 & $"$ & $"$ & " & $"$ & $"$ & $"$ \\
\hline$"$ & 69 & 6 & 110 & 6 & $"$ & $"$ & $"$ & $"$ & $"$ & $"$ \\
\hline$"$ & 54 & 6 & 110 & 6 & $"$ & $"$ & $"$ & $"$ & $"$ & $"$ \\
\hline$"$ & & & 110 & 3 & $"$ & $"$ & $"$ & $"$ & $"$ & $"$ \\
\hline$"$ & & & 110 & 3 & $"$ & $"$ & $"$ & $"$ & $"$ & $"$ \\
\hline Cs & 1700 & 3 & 3100 & 12 & $6 \times 10^{-9}$ & $"$ & $"$ & $"$ & $"$ & $"$ \\
\hline$"$ & 1200 & 3 & 2500 & 12 & " & $"$ & $"$ & $"$ & $" 1$ & $"$ \\
\hline$"$ & 2200 & 6 & 2400 & 6 & $"$ & $"$ & $"$ & $"$ & " & $"$ \\
\hline$"$ & 1500 & 6 & 2200 & 6 & $"$ & $"$ & $"$ & $"$ & $"$ & $"$ \\
\hline$"$ & & & 2300 & 3 & $"$ & $"$ & $"$ & $"$ & $"$ & $"$ \\
\hline$"$ & & & 2700 & 3 & $"$ & $"$ & $"$ & $"$ & $"$ & $"$ \\
\hline $\mathrm{Ba}$ & 900 & 3 & 1200 & 12 & $1 \times 10^{-9}$ & $"$ & $"$ & 11 & $"$ & $"$ \\
\hline$"$ & 760 & 3 & 1200 & 12 & $"$ & $"$ & $"$ & $"$ & $"$ & $"$ \\
\hline$"$ & 720 & 6 & 1200 & 6 & $"$ & $"$ & $"$ & $"$ & $"$ & $"$ \\
\hline$"$ & 900 & 6 & 1200 & 6 & $"$ & $"$ & $"$ & 11 & $"$ & $"$ \\
\hline$"$ & & & 1100 & 3 & $"$ & $"$ & $"$ & $"$ & $"$ & $"$ \\
\hline$"$ & & & 1100 & 3 & $"$ & $"$ & $"$ & $"$ & $"$ & $"$ \\
\hline Eu & 1500 & 3 & 13000 & 12 & $1 \times 10^{-10}$ & $"$ & $"$ & $"$ & $"$ & $"$ \\
\hline$"$ & 1200 & 3 & 13000 & 12 & 14 & $"$ & $"$ & $"$ & $"$ & $"$ \\
\hline$"$ & 5200 & 6 & 11000 & 6 & " & $"$ & $"$ & $"$ & $1 "$ & $"$ \\
\hline$"$ & 600 & 6 & 12000 & 6 & $"$ & $n$ & $"$ & $"$ & $"$ & $"$ \\
\hline$"$ & & & 11000 & 3 & $"$ & $"$ & $"$ & $"$ & $"$ & $"$ \\
\hline$"$ & & & 14000 & 3 & $"$ & $"$ & $"$ & $"$ & $"$ & $"$ \\
\hline
\end{tabular}

\footnotetext{
aconcentrations are only those added with the tracer; actual concentrations may be higher.
} 
TALE A-V

MATCH SORPTION DATA FOR TUFF JA-32

\begin{tabular}{|c|c|c|c|c|c|c|c|c|c|}
\hline \multirow[b]{2}{*}{ Elent } & \multicolumn{2}{|c|}{ Sorption } & \multicolumn{2}{|c|}{ Denorption } & \multirow{2}{*}{$\begin{array}{c}\text { Eleanent } \\
\text { Concentration } \\
\text { (H) }\end{array}$} & \multirow{2}{*}{$\begin{array}{c}\text { Particle } \\
\text { Size } \\
\text { (1) } \\
\end{array}$} & \multirow[b]{2}{*}{ Te-perature } & \multirow[b]{2}{*}{ Atmosphere } & \multirow[b]{2}{*}{ Ref. } \\
\hline & $\begin{array}{c}R_{d} \\
(x / k)\end{array}$ & $\begin{array}{l}\text { Tine } \\
\text { (vk) }\end{array}$ & $\begin{array}{c}k_{d} \\
(n e / n)\end{array}$ & $\begin{array}{l}\text { Tine } \\
\text { (wk) }\end{array}$ & & & & & \\
\hline $\mathbf{S r}$ & 50 & 1 & 55 & 2 & $2 \times 10^{-6}$ & $355-500$ & $A=b$ & Air & 2 \\
\hline$"$ & 52 & 3 & $\$ 2$ & 4 & $"$ & $"$ & " & $"$ & $"$ \\
\hline " & 56 & 5 & 58 & 6 & $"$ & $"$ & $"$ & $"$ & $"$ \\
\hline$"$ & 71 & 8 & 72 & 12 & $1 "$ & $"$ & " & " & $"$ \\
\hline
\end{tabular}

$\begin{array}{llllr}\text { Cs } & 110 & 1 & 200 & 2 \\ " & 120 & 3 & 150 & 4 \\ " & 140 & 5 & 200 & 6 \\ " & 120 & 8 & 180 & 12\end{array}$

$\begin{array}{llll}\text { B. } & 270 & 1 & 360 \\ \text { " } & 310 & 3 & 390 \\ \text { " } & 370 & 5 & 560 \\ \text { " } & 520 & 8 & 700\end{array}$

$\begin{array}{lrrl}\text { Ce } & 66 & 1 & 670 \\ " & 90 & 3 & 490 \\ \text { " } & 90 & 5 & 240 \\ \text { " } & 110 & 8 & 640\end{array}$

$\begin{array}{lrrrr}\text { Eu } & 48 & 1 & 610 \\ " & 92 & 3 & 600 \\ n & 88 & 5 & 570 & 6 \\ " & 120 & 8 & 780 & 12\end{array}$

$48 \quad 1$

$\begin{array}{lr}48 & 1 \\ 41 & 3 \\ 50 & 5 \\ 50 & 11\end{array}$

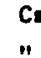

$$
\begin{aligned}
& \text { " } \\
& \text { Ba } \\
& \text { " }
\end{aligned}
$$

"

503

725

55

120

120

170

230

130

150

140

370

370

420

430

560

510

2
4
6
12
$1 \times 10^{-9}$
"
"

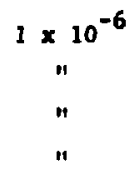
$8 \times 10^{-7}$
1
"

$6 \times 10^{-7}$
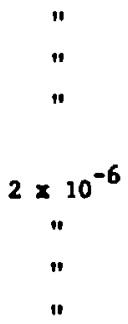

106-150

"

"

"1

$1 \times 10^{-9}$

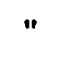

"

"

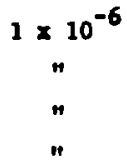

$"$
$"$
1

"

$"$
$"$
$"$

"
"
"

II

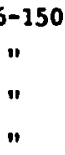

"

n

1)

ฯ 
TADL A-V (cont)

\begin{tabular}{|c|c|c|c|c|c|c|c|c|c|c|}
\hline \multirow[b]{2}{*}{ Element } & \multicolumn{2}{|c|}{ Sorption } & \multicolumn{2}{|c|}{ Desorption } & \multirow{2}{*}{$\begin{array}{c}\text { Elewent } \\
\text { Concentration } \\
\text { (M) } \\
\end{array}$} & \multirow{2}{*}{$\begin{array}{c}\text { Particle } \\
\text { Size } \\
\text { (1) } \\
\end{array}$} & \multirow[b]{2}{*}{ Tesperature } & \multirow[b]{2}{*}{ Atwosphere } & \multirow[b]{2}{*}{ Ref. } & \multirow[b]{2}{*}{ Note } \\
\hline & $\begin{array}{c}R_{d} \\
(n / /)\end{array}$ & $\begin{array}{l}\text { Tine } \\
\text { (wk) }\end{array}$ & $\begin{array}{c}R_{d} \\
(n / L)\end{array}$ & $\begin{array}{l}\text { Tine } \\
(w k)\end{array}$ & & & & & & \\
\hline $\mathrm{Ce}$ & 43 & 1 & 1300 & 1 & $8 \times 10^{-7}$ & $\overline{106-150}$ & Amb & Air & 2 & \\
\hline$"$ & 53 & 3 & 340 & 3 & $"$ & "t & $"$ & $"$ & $"$ & \\
\hline$"$ & 48 & 5 & 310 & 5 & $"$ & $"$ & $"$ & $"$ & $"$ & \\
\hline$"$ & 160 & 8 & 240 & 11 & $"$ & $"$ & $"$ & $"$ & $n$ & \\
\hline Eu & 51 & 1 & 1600 & 1 & $6 \times 10^{-7}$ & $n$ & $"$ & $"$ & $"$ & \\
\hline$"$ & 69 & 3 & 600 & 3 & $"$ & $"$ & " & " & $n$ & \\
\hline$"$ & 73 & 5 & 740 & 5 & $"$ & " & $n$ & 11 & $"$ & \\
\hline$"$ & 190 & 8 & 1300 & 11 & $"$ & $"$ & $"$ & $"$ & $"$ & \\
\hline $\mathbf{S r}$ & 93 & 1 & 80 & 2 & $2 \times 10^{-6}$ & $355-500$ & $70^{\circ} \mathrm{C}$ & " & 19 & \\
\hline$"$ & 110 & 2 & BO & 3 & $"$ & n & $"$ & $"$ & " & \\
\hline 1 & & & 110 & 3 & " & " & $"$ & $"$ & $"$ & • \\
\hline " & 110 & 4 & 120 & 6 & $"$ & $"$ & $"$ & $"$ & " & \\
\hline$"$ & 140 & 8 & 70 & 2 & $"$ & $"$ & $"$ & " & $"$ & \\
\hline Cs & 82 & 1 & 89 & 2 & $2 \times 10^{-9}$ & $"$ & $"$ & $"$ & $"$ & \\
\hline$"$ & 85 & 2 & 96 & 3 & $"$ & $"$ & $"$ & " & $"$ & \\
\hline$"$ & & & 99 & 3 & $"$ & " & " & " & $"$ & a \\
\hline$"$ & 100 & 4 & 110 & 6 & $"$ & $"$ & $"$ & $"$ & $"$ & \\
\hline$"$ & 100 & 8 & 100 & 2 & $"$ & $n$ & $"$ & " & $"$ & \\
\hline Ba & 900 & 1 & 1100 & 2 & $1 \times 10^{-6}$ & $"$ & " & $"$ & $"$ & \\
\hline$"$ & 1000 & 2 & 950 & 3 & " & $"$ & $"$ & " & 14 & \\
\hline$"$ & & & 1600 & 3 & " & $"$ & " & $"$ & $"$ & a \\
\hline$"$ & 1100 & 4 & 1800 & 6 & " & " & " & " & $"$ & \\
\hline$"$ & 1600 & 8 & 1200 & 2 & $"$ & $"$ & $"$ & $"$ & " & \\
\hline Ce & 51 & 1 & 750 & 2 & $"$ & " & " & $"$ & $"$ & \\
\hline$"$ & 61 & 2 & 310 & 3 & $"$ & " & $n$ & " & $"$ & \\
\hline$"$ & & & 1100 & 3 & $n$ & " & $n$ & "1 & " & a \\
\hline$"$ & 130 & 4 & 670 & 6 & $"$ & $"$ & $"$ & $"$ & $"$ & \\
\hline " & 170 & $B$ & 800 & 2 & $"$ & $"$ & $"$ & " & " & \\
\hline Eu & 93 & 1 & 1700 & 2 & $6 \times 10^{-7}$ & $"$ & $"$ & $n$ & $"$ & \\
\hline " & 130 & 2 & 890 & 3 & $"$ & " & " & " & " & \\
\hline " & & & 1100 & 3 & $"$ & $"$ & $"$ & " & $"$ & $a$ \\
\hline$"$ & 380 & 4 & 3200 & 6 & $"$ & $"$ & 1 & " & " & \\
\hline$"$ & 130 & 8 & 4000 & 2 & $"$ & $"$ & $"$ & " & " & \\
\hline
\end{tabular}

Second desorption. 
TABLC A-V (cont)

\begin{tabular}{|c|c|c|c|c|c|c|c|c|c|c|}
\hline \multirow[b]{2}{*}{ Element } & \multicolumn{2}{|c|}{ Sorption } & \multicolumn{2}{|c|}{ Desorption } & \multirow{2}{*}{$\begin{array}{c}\text { Elewent } \\
\text { Concentration } \\
(\mathrm{M}) \\
\end{array}$} & \multirow{2}{*}{$\begin{array}{c}\text { Particle } \\
\text { Size } \\
(10) \\
\end{array}$} & \multirow[b]{2}{*}{ Temperature } & \multirow[b]{2}{*}{ Atmosphere } & \multirow[b]{2}{*}{ Ref. } & \multirow[b]{2}{*}{ Note } \\
\hline & $\begin{array}{c}R_{d} \\
(a / 8)\end{array}$ & $\begin{array}{l}\text { Time } \\
\text { (wk) }\end{array}$ & $\begin{array}{c}\pi_{d} \\
(n / 2)\end{array}$ & $\begin{array}{l}\text { Time } \\
\text { (wk) }\end{array}$ & & & & & & \\
\hline $\mathbf{s r}$ & 82 & 1 & 80 & 2 & $2 \times 10^{-6}$ & $\overline{106-150}$ & $70^{\circ} \mathrm{C}$ & $\overline{\text { Air }}$ & $\overline{2}$ & \\
\hline$"$ & 92 & 2 & 110 & 3 & $"$ & $"$ & $"$ & $"$ & $"$ & \\
\hline$"$ & & & 120 & 3 & $"$ & $"$ & $"$ & $"$ & $"$ &. \\
\hline$"$ & 120 & 4 & 140 & 6 & $"$ & $"$ & $"$ & $"$ & $"$ & \\
\hline$"$ & 160 & 8 & 93 & 2 & $"$ & $"$ & $"$ & $"$ & " & \\
\hline cs & 82 & 1 & 80 & 2 & $2 \times 10^{-9}$ & $"$ & $"$ & $"$ & :" & \\
\hline$"$ & 87 & 2 & 110 & 3 & $"$ & $"$ & $"$ & $"$ & $"$ & \\
\hline$"$ & & & 130 & 3 & $"$ & $"$ & $"$ & $"$ & $"$ & $\mathbf{a}$ \\
\hline$"$ & 120 & 4 & 130 & 6 & $"$ & $"$ & $"$ & $"$ & : & \\
\hline$"$ & 120 & 8 & 120 & 2 & $"$ & $"$ & $"$ & $"$ & $"$ & \\
\hline $\mathrm{Ba}$ & 560 & 1 & 710 & 2 & $1 \times 10^{-6}$ & $"$ & $"$ & $"$ & $"$ & \\
\hline$"$ & 660 & 2 & 940 & 3 & $"$ & $"$ & $"$ & $"$ & $"$ & \\
\hline$"$ & & & 970 & 3 & $"$ & $"$ & $"$ & $"$ & $"$ & a \\
\hline$"$ & 940 & 4 & 1300 & 6 & $"$ & $"$ & $"$ & $"$ & $"$ & \\
\hline$"$ & 1300 & 8 & 1100 & 2 & $"$ & $"$ & $"$ & $"$ & $"$ & \\
\hline Ce & 53 & 1 & 360 & 2 & $"$ & $"$ & $"$ & $"$ & $"$ & \\
\hline$"$ & 64 & 2 & 600 & 3 & $"$ & $"$ & $"$ & $"$ & $"$ & \\
\hline$"$ & & & 710 & 3 & $"$ & $"$ & $"$ & $"$ & $"$ & $a$ \\
\hline$"$ & 64 & 4 & 580 & 6 & $"$ & $"$ & $"$ & $"$ & $"$ & \\
\hline$"$ & 67 & 8 & 460 & 2 & $"$ & $"$ & $"$ & $"$ & $"$ & \\
\hline Eu & 120 & 1 & 880 & 2 & $6 \times 10^{-7}$ & $"$ & $"$ & $"$ & $"$ & \\
\hline$"$ & 180 & 2 & 1500 & 3 & $"$ & $"$ & $"$ & $"$ & " & \\
\hline$"$ & & & 1300 & 3 & $"$ & $"$ & $"$ & $"$ & $"$ & $a$ \\
\hline$"$ & 220 & 4 & 1600 & 6 & $"$ & $"$ & $"$ & $"$ & $"$ & \\
\hline$"$ & 240 & 8 & 2000 & 2 & $"$ & $"$ & $"$ & $"$ & $"$ & \\
\hline Sr & 84 & 4 & & & $2 \times 10^{-6}$ & $250-355$ & bصa & $"$ & 4 & $\mathbf{b}$ \\
\hline$"$ & 85 & 9 & & & $"$ & $"$ & $"$ & $"$ & $"$ & $"$ \\
\hline Cs & 130 & 4 & & & $6 \times 10^{-10}$ & $"$ & $"$ & $"$ & $"$ & $"$ \\
\hline$"$ & 140 & 9 & & & $"$ & $"$ & $"$ & $"$ & $"$ & $"$ \\
\hline Bo & 590 & 4 & & & $1 \times 10^{-6}$ & $"$ & $"$ & $n$ & $"$ & $"$ \\
\hline$"$ & 660 & 9 & & & $"$ & $"$ & $"$ & $"$ & $"$ & $"$ \\
\hline
\end{tabular}

\section{second desorption.}

byntbetic groundwater I; see Ref. 4. 
TABLE A-V (cont)

\begin{tabular}{|c|c|c|c|c|c|c|c|c|c|c|}
\hline \multirow[b]{2}{*}{ Element } & \multicolumn{2}{|c|}{ Sorption } & \multicolumn{2}{|c|}{ Desorption } & \multirow{2}{*}{$\begin{array}{c}\text { Elewent } \\
\text { Concentration } \\
(\mathrm{H}) \\
\end{array}$} & \multirow{2}{*}{ 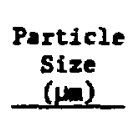 } & \multirow[b]{2}{*}{ Tepperature } & \multirow[b]{2}{*}{ Atwosphere } & \multirow[b]{2}{*}{ Ref. } & \multirow[b]{2}{*}{ Note } \\
\hline & $\begin{array}{c}R_{d} \\
(n e / \ell)\end{array}$ & $\begin{array}{l}\text { Tive } \\
\text { (wk) }\end{array}$ & $\begin{array}{c}R_{d} \\
(n e / d)\end{array}$ & $\begin{array}{l}\text { Tive } \\
\text { (wk) }\end{array}$ & & & & & & \\
\hline $\mathrm{Ce}$ & 2900 & 4 & & & $1 \times 10^{-8}$ & $250-355$ & $A \cap b$ & Air & 4 & $a$ \\
\hline$"$ & $>55000$ & 9 & & & $"$ & " & $"$ & " & " & $"$ \\
\hline $\mathbf{E u}$ & 1700 & 4 & & & $8 \times 10^{-9}$ & $"$ & " & $"$ & $n$ & $"$ \\
\hline$"$ & 4200 & 9 & & & $n$ & $"$ & $n$ & $"$ & $"$ & $"$ \\
\hline $\mathrm{Sr}$ & 18 & 4 & & & $2 \times 10^{-6}$ & $n$ & $"$ & $"$ & $"$ & $\mathbf{b}$ \\
\hline$"$ & 18 & 9 & & & $"$ & $"$ & " & " & 11 & $"$ \\
\hline Cs & 85 & 4 & & & $1 \times 10^{-9}$ & $"$ & $"$ & $"$ & $"$ & $"$ \\
\hline$"$ & 73 & 9 & & & $"$ & $"$ & $"$ & " & $"$ & $"$ \\
\hline $\mathrm{Ba}$ & 160 & 4 & & & $1 \times 10^{-6}$ & $"$ & $"$ & $"$ & $"$ & $"$ \\
\hline$" 1$ & 180 & 9 & & & $"$ & $"$ & $"$ & $"$ & $"$ & 1 \\
\hline Ce & $>30000$ & 4 & & & $1 \times 10^{-8}$ & $"$ & $"$ & $"$ & $"$ & $"$ \\
\hline$"$ & $>45000$ & 9 & & & $"$ & $"$ & $"$ & $"$ & $"$ & $"$ \\
\hline Eu & 24000 & 4 & & & $8 \times 10^{-9}$ & $"$ & $"$ & $"$ & $"$ & $"$ \\
\hline$"$ & $>61000$ & 9 & & & $"$ & $"$ & $"$ & $"$ & $"$ & $"$ \\
\hline Am & 110 & 1 & 2800 & 5 & $1 \times 10^{-6}$ & $106-150$ & $"$ & $"$ & $"$ & \\
\hline$"$ & 110 & 2 & & & $"$ & $"$ & $"$ & $"$ & $"$ & \\
\hline$"$ & 140 & 4 & & & $"$ & $"$ & $"$ & " & $"$ & \\
\hline$"$ & 230 & 4 & 1500 & 5 & $"$ & $"$ & $"$ & $"$ & $"$ & \\
\hline$"$ & 79 & 8 & & & $"$ & " & $"$ & $"$ & $"$ & \\
\hline$"$ & 120 & 1 & & & $"$ & $"$ & $70^{\circ} \mathrm{C}$ & $"$ & $"$ & \\
\hline$"$ & 160 & 2 & & & $"$ & $"$ & $"$ & $"$ & " & \\
\hline$"$ & 130 & 4 & & & $"$ & $"$ & $n$ & $"$ & $"$ & \\
\hline " & 46 & 8 & & & " & " & $"$ & " & $"$ & \\
\hline $\mathbf{P u}$ & 110 & 2 & & & $n$ & " & $A \mathfrak{b}$ & " & $"$ & \\
\hline
\end{tabular}

aynthetic groundwater I; see Ref. 4 .

${ }^{b}$ Synthetic groundwater II; see Ref. 4. 
IADIE $A-V$ (cont)

\begin{tabular}{|c|c|c|c|c|c|c|c|c|c|c|}
\hline \multirow[b]{2}{*}{ Filewent } & \multicolumn{2}{|c|}{ sorption } & \multicolumn{2}{|c|}{ Desorption } & \multirow{2}{*}{$\begin{array}{c}\text { Elewent } \\
\text { Concentration } \\
(\mathrm{H}) \\
\end{array}$} & \multirow[b]{2}{*}{$\begin{array}{c}\text { Particle } \\
\text { Size } \\
\text { (1) } \\
\end{array}$} & \multirow[b]{2}{*}{ Teيperature } & \multirow[b]{2}{*}{ Ateosphere } & \multirow[b]{2}{*}{ Ref. } & \multirow[b]{2}{*}{ Note } \\
\hline & $\begin{array}{c}R_{d} \\
(\mu / /)\end{array}$ & $\begin{array}{l}\text { Time } \\
\text { (wt) }\end{array}$ & $\begin{array}{c}R_{d} \\
(n \ell / \Omega)\end{array}$ & $\begin{array}{l}\text { Tine } \\
\text { (uk) }\end{array}$ & & & & & & \\
\hline $\mathbf{v}$ & 3.5 & 1 & 15 & 3 & $1 \times 10^{-7}$ & $106-150$ & $A=b$ & Air & 4 & \\
\hline$"$ & 2.9 & 2 & 12 & 2 & $"$ & $n$ & " & $"$ & $"$ & \\
\hline " & 2.5 & 3 & 9.6 & 1 & $"$ & $"$ & $"$ & $"$ & $"$ & \\
\hline 11 & 1.4 & 1 & 0.2 & 3 & $"$ & $355-500$ & $"$ & $"$ & 11 & \\
\hline$"$ & 1.3 & 2 & 5.4 & 2 & $"$ & $"$ & $1 "$ & $"$ & $"$ & \\
\hline$"$ & 1.3 & 3 & 5.2 & 1 & $"$ & $"$ & $"$ & $"$ & $"$ & . \\
\hline$"$ & 10 & 1 & 23 & 3 & " & $"$ & $70^{\circ} \mathrm{C}$ & $"$ & $"$ & \\
\hline$"$ & 11 & 2 & 19 & 2 & $"$ & $"$ & $"$ & $"$ & $"$ & \\
\hline$"$ & 15 & 3 & 20 & 1 & $"$ & $"$ & $"$ & $n$ & $"$ & \\
\hline I & 0.35 & 3 & & & $1 \times 10^{-13}$ & $"$ & Aab & $"$ & 2 & \\
\hline$"$ & -0.20 & 4 & & & $"$ & $"$ & $"$ & $"$ & $"$ & \\
\hline Mo & 8.2 & 3 & & & $1 \times 10^{-14}$ & $"$ & $"$ & $"$ & $"$ & \\
\hline $\mathbf{R u}$ & 88 & 3 & & & $3 \times 10^{-12}$ & $"$ & $"$ & $"$ & $n$ & \\
\hline$"$ & 44 & 4 & & & " & 11 & $"$ & $"$ & $"$ & \\
\hline $\mathbf{S b}$ & 0.12 & 3 & & & $6 \times 10^{-12}$ & $"$ & $"$ & " & $"$ & \\
\hline$"$ & -0.88 & 4 & & & $"$ & " & $"$ & $"$ & $n$ & \\
\hline
\end{tabular}


TABLE A-VI

BATCH SORPTION DATA FOR TUFF JA-37

\begin{tabular}{|c|c|c|c|c|c|c|c|c|c|c|}
\hline \multirow[b]{2}{*}{ Eleeent } & \multicolumn{2}{|c|}{ Sorption } & \multicolumn{2}{|c|}{ Desouption } & \multirow[b]{2}{*}{$\begin{array}{c}\text { Elewent } \\
\text { Concentration } \\
(\mathrm{M}) \\
\end{array}$} & \multirow[b]{2}{*}{$\begin{array}{c}\text { Particle } \\
\text { Size } \\
(\mu) \\
\end{array}$} & \multirow[b]{2}{*}{ Temperature } & \multirow[b]{2}{*}{ Atwosphere } & \multirow[b]{2}{*}{ Ref. } & \multirow[b]{2}{*}{ Note } \\
\hline & $\begin{array}{c}R_{d} \\
(m e / g)\end{array}$ & $\begin{array}{l}\text { Time } \\
\text { (wk) }\end{array}$ & $\begin{array}{c}R_{d} \\
(\alpha \ell / \ell)\end{array}$ & $\begin{array}{l}\text { Time } \\
\text { (wk) }\end{array}$ & & & & & & \\
\hline $\mathbf{S r}$ & 260 & 2 & 300 & 2 & $3 \times 10^{-6}$ & $355-500$ & $A \mathbf{b}$ & Air & 2 & \\
\hline$"$ & & & 280 & 4 & $"$ & $"$ & $"$ & $"$ & $"$ & 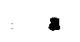 \\
\hline$"$ & 250 & 3 & 320 & 3 & " & $" 1$ & $"$ & $"$ & $"$ & \\
\hline$n$ & & & 320 & 12 & $"$ & $"$ & $n$ & $"$ & $"$ & $\mathbf{a}$ \\
\hline$"$ & 340 & 5 & 350 & 6 & $"$ & $"$ & $"$ & $"$ & $"$ & \\
\hline$"$ & 260 & 10 & & & $"$ & $"$ & $"$ & 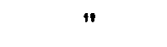 & $"$ & \\
\hline Cs & 700 & 2 & 880 & 2 & $1 \times 10^{-7}$ & $"$ & $"$ & $"$ & $"$ & \\
\hline$"$ & & & 950 & 4 & $"$ & $"$ & $"$ & $"$ & $"$ & $\mathbf{a}$ \\
\hline$"$ & 480 & 3 & 800 & 3 & $"$ & $"$ & 11 & $"$ & $"$ & \\
\hline$"$ & & & 1100 & 12 & $"$ & $"$ & $"$ & $"$ & $n$ & a \\
\hline$"$ & 650 & 5 & 970 & 6 & $"$ & $"$ & $"$ & $"$ & $"$ & \\
\hline$"$ & 750 & 10 & & & $"$ & $"$ & $"$ & $"$ & $"$ & \\
\hline $\mathrm{Ba}$ & 600 & 2 & 840 & 2 & $2 \times 10^{-6}$ & $"$ & " & $"$ & $"$ & \\
\hline$"$ & & & 950 & 4 & $"$ & $"$ & $"$ & $"$ & $"$ & $\mathbf{a}$ \\
\hline$"$ & 660 & 3 & 860 & 3 & $"$ & $"$ & $"$ & $"$ & $"$ & \\
\hline$"$ & & & 990 & 12 & $"$ & $"$ & $"$ & $"$ & $"$ & a \\
\hline 11 & 830 & 5 & 1100 & 6 & $"$ & $"$ & $"$ & $"$ & $"$ & \\
\hline$"$ & 760 & 10 & & & $"$ & $"$ & $"$ & $n$ & 14 & \\
\hline Eu & 4100 & 2 & 6000 & 2 & $3 \times 10^{-7}$ & $"$ & $"$ & $"$ & $"$ & \\
\hline$"$ & & & 6700 & 4 & $"$ & $"$ & $"$ & $"$ & $"$ & $\mathbf{a}$ \\
\hline$"$ & 4000 & 3 & 14000 & 3 & $"$ & $"$ & $"$ & $"$ & $"$ & \\
\hline$"$ & & & 18000 & 12 & $"$ & $"$ & $"$ & $"$ & $"$ & $\mathbf{a}$ \\
\hline$n$ & 6400 & 5 & 18000 & 6 & $"$ & $"$ & $"$ & $"$ & $"$ & \\
\hline$"$ & 6900 & 10 & & & $"$ & $"$ & $"$ & $"$ & $"$ & \\
\hline $\mathbf{S r}$ & 300 & 2 & 320 & 2 & $3 \times 10^{-6}$ & $106-150$ & $"$ & $"$ & $"$ & \\
\hline$"$ & & & 290 & 4 & $"$ & $"$ & $"$ & $"$ & $"$ & a \\
\hline 11 & 280 & 3 & 350 & 3 & $"$ & $"$ & $"$ & " & $"$ & \\
\hline$"$ & & & 320 & 12 & $"$ & $"$ & $"$ & $"$ & $"$ & $\mathbf{a}$ \\
\hline$"$ & 260 & 5 & 260 & 6 & $"$ & $"$ & $"$ & $"$ & $"$ & \\
\hline$"$ & 350 & 10 & & & $"$ & $"$ & $"$ & $"$ & $"$ & \\
\hline
\end{tabular}

Second desorption. 
TABLE A-VI (cont)

\begin{tabular}{|c|c|c|c|c|c|c|c|c|c|c|}
\hline \multirow[b]{2}{*}{ Elenent } & \multicolumn{2}{|c|}{ Sorption } & \multicolumn{2}{|c|}{ Desorption } & \multirow{2}{*}{$\begin{array}{c}\text { Elewent } \\
\text { Concentration } \\
\text { (H) }\end{array}$} & \multirow[b]{2}{*}{$\begin{array}{c}\text { Particle } \\
\text { Size } \\
(1 \mathrm{a}) \\
\end{array}$} & \multirow[b]{2}{*}{ Teuperature } & \multirow[b]{2}{*}{ Atmosphere } & \multirow[b]{2}{*}{ Ref. } & \multirow[b]{2}{*}{ Note } \\
\hline & $\begin{array}{c}R_{d} \\
(\boldsymbol{R} / \boldsymbol{\alpha})\end{array}$ & $\begin{array}{l}\text { Tine } \\
\text { (wl) }\end{array}$ & $\begin{array}{c}R_{d} \\
(\alpha / 2)\end{array}$ & $\begin{array}{l}\text { Tine } \\
\text { (wl) }\end{array}$ & & & & & & \\
\hline $\mathrm{Cs}$ & 530 & 2 & 640 & 2 & $1 \times 10^{-7}$ & $106-150$ & 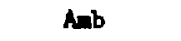 & Air & 2 & \\
\hline$"$ & & & 600 & 4 & $"$ & $"$ & $"$ & $"$ & $"$ & a \\
\hline$"$ & 490 & 3 & 800 & 3 & $"$ & $"$ & $"$ & 11 & $"$ & \\
\hline$"$ & & & 1000 & 12 & $"$ & $"$ & $1 "$ & $"$ & $"$ & $a$ \\
\hline$"$ & 510 & 5 & 720 & 6 & $"$ & $"$ & $"$ & $"$ & $"$ & \\
\hline$"$ & 780 & 10 & & & $"$ & $"$ & $"$ & $"$ & $"$ & \\
\hline $\mathbf{B a}$ & 700 & 2 & 810 & 2 & $2 \times 10^{-6}$ & $"$ & $"$ & it & $"$ & \\
\hline$"$ & & & 750 & 4 & $"$ & $"$ & $"$ & $"$ & $"$ & $\mathbf{a}$ \\
\hline$"$ & 750 & 3 & 1000 & 3 & $n$ & $n$ & $n$ & $"$ & $"$ & \\
\hline$"$ & & & 1100 & 12 & $"$ & $"$ & $"$ & $"$ & $"$ & $\approx$ \\
\hline$"$ & 710 & 5 & 900 & 6 & $"$ & $H$ & $"$ & " & $"$ & 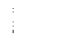 \\
\hline " & 1000 & 10 & & & $"$ & $" 1$ & " & $"$ & $"$ & . \\
\hline Eu & 2100 & 2 & 3900 & 2 & $3 \times 10^{-7}$ & $"$ & $"$ & $"$ & $"$ & : \\
\hline$"$ & & & 16000 & 4 & $"$ & $"$ & $"$ & $"$ & $"$ & $\mathbf{a}$ \\
\hline$"$ & 7600 & 3 & 7700 & 3 & $"$ & $n$ & " & $"$ & " & \\
\hline$"$ & & & 24000 & 12 & $"$ & " & $"$ & $"$ & $"$ & $\mathbf{a}$ \\
\hline$"$ & 7300 & 5 & 17000 & 6 & $"$ & $"$ & " & $"$ & $"$ & \\
\hline " & 9500 & 10 & & & " & $"$ & $"$ & $"$ & " & \\
\hline Sr & 660 & 1 & 900 & 2 & $2 \times 10^{-6}$ & $355-500$ & $70^{\circ} \mathrm{C}$ & t & $"$ & \\
\hline$"$ & & & 820 & 3 & $"$ & $"$ & " & $"$ & $"$ & $\mathbf{a}$ \\
\hline$"$ & 790 & 2 & 1100 & 3 & $"$ & " & $"$ & $"$ & $"$ & \\
\hline$"$ & & . & 1800 & 13 & $"$ & ". & $"$ & $"$ & $"$ & a \\
\hline$"$ & 1000 & 3 & 1500 & 6 & $"$ & $"$ & $"$ & $"$ & $"$ & \\
\hline$"$ & 1500 & 7 & 1500 & 3 & $"$ & $"$ & $"$ & $"$ & $"$ & \\
\hline$C s$ & 1100 & 1 & 1300 & 2 & $4 \times 10^{-8}$ & " & $"$ & $"$ & $"$ & \\
\hline$"$ & & & 1500 & 3 & $"$ & $"$ & $"$ & " & $"$ & a \\
\hline$"$ & 1300 & 2 & 4700 & 3 & $"$ & $"$ & $"$ & $"$ & $"$ & \\
\hline " & & & 4400 & 13 & $"$ & $"$ & $"$ & $"$ & $n$ & 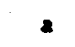 \\
\hline$"$ & 1400 & 3 & 1800 & 6 & " & " & $"$ & " & $"$ & \\
\hline$"$ & 1600 & 7 & 1800 & 3 & $"$ & $"$ & $"$ & $"$ & $"$ & \\
\hline
\end{tabular}

asecond desorption. 
TABLE A-VI (cont)

\begin{tabular}{|c|c|c|c|c|c|c|c|c|c|c|}
\hline \multirow[b]{2}{*}{ Element } & \multicolumn{2}{|c|}{ Sorption } & \multicolumn{2}{|c|}{ Desorption } & \multirow{2}{*}{$\begin{array}{c}\text { Element } \\
\text { Concentration } \\
\text { (M) } \\
\end{array}$} & \multirow[b]{2}{*}{$\begin{array}{c}\text { Particle } \\
\text { Size } \\
\text { (u) } \\
\end{array}$} & \multirow[b]{2}{*}{ Temperature } & \multirow[b]{2}{*}{ Atmosphere } & \multirow[b]{2}{*}{ Ref. } & \multirow[b]{2}{*}{ Note } \\
\hline & $\begin{array}{c}R_{\mathrm{d}} \\
(\boldsymbol{Q} / \mathrm{R})\end{array}$ & $\begin{array}{l}\text { Time } \\
\text { (wk) }\end{array}$ & $\begin{array}{c}R_{\mathrm{a}} \\
(\boldsymbol{e} / \boldsymbol{\alpha})\end{array}$ & $\begin{array}{l}\text { Tine } \\
\text { (wh) }\end{array}$ & & & & & & \\
\hline $\mathrm{Ba}$ & 1700 & 1 & 2500 & 2 & $1 \times 10^{-6}$ & $355-500$ & $70^{\circ} \mathrm{C}$ & Air & 2 & \\
\hline$"$ & & & 3000 & 3 & $"$ & $n$ & $"$ & $"$ & $"$ & - \\
\hline$"$ & 2400 & 2 & 4400 & 3 & $"$ & $"$ & $" 1$ & " & $"$ & \\
\hline$"$ & & & 9900 & 13 & $"$ & $"$ & " & $"$ & $"$ & a \\
\hline " & 3400 & 3 & 6300 & 6 & $"$ & $"$ & $"$ & $"$ & $"$ & \\
\hline$"$ & 6600 & 7 & 5900 & 3 & $"$ & $"$ & $"$ & $"$ & $"$ & \\
\hline Eu & 2100 & 1 & 7600 & 2 & $1 \times 10^{-7}$ & $"$ & $"$ & $"$ & $"$ & \\
\hline " & & & 11000 & 3 & $"$ & $" 1$ & $"$ & $"$ & $"$ & $\mathbf{a}$ \\
\hline$"$ & 4400 & 2 & 12000 & 3 & $"$ & $n$ & $"$ & $"$ & $"$ & \\
\hline$"$ & & & 13000 & 13 & $"$ & $"$ & $"$ & $"$ & $"$ & $\mathbf{a}$ \\
\hline$"$ & 4500 & 3 & 19000 & 6 & $"$ & $"$ & $"$ & $"$ & $"$ & \\
\hline " & 5500 & 7 & 18000 & 3 & $"$ & $"$ & $"$ & $"$ & $"$ & \\
\hline Sr & 770 & 1 & 1000 & 2 & $2 \times 10^{-6}$ & $106-150$ & $"$ & $"$ & $"$ & \\
\hline$"$ & & & 950 & 3 & $"$ & $"$ & $" 1$ & $" 1$ & $"$ & $\mathbf{a}$ \\
\hline$"$ & 820 & 2 & 1200 & 3 & $"$ & $"$ & $"$ & $"$ & $"$ & \\
\hline$"$ & & & 1900 & 13 & $"$ & $"$ & $"$ & $"$ & $"$ & a \\
\hline$"$ & 1200 & 3 & 1900 & 6 & $"$ & $"$ & $"$ & $"$ & $"$ & \\
\hline$"$ & 1600 & 7 & 1400 & 3 & $"$ & $n$ & " & $"$ & $"$ & \\
\hline Cs & 1100 & 1 & 1200 & 2 & $4 \times 10^{-8}$ & $"$ & $"$ & $"$ & $"$ & \\
\hline$"$ & & & 1600 & 3 & $"$ & $"$ & $"$ & $"$ & $"$ & $\mathbf{a}$ \\
\hline$"$ & 1300 & 2 & 4600 & 3 & $n$ & $"$ & $"$ & $"$ & $"$ & \\
\hline$"$ & & & 5800 & 13 & $"$ & $"$ & $"$ & $"$ & $"$ & a \\
\hline$"$ & 1400 & 3 & 1500 & 6 & $"$ & $"$ & $"$ & $"$ & $"$ & \\
\hline$"$ & 1800 & 7 & 1400 & 3 & $"$ & $"$ & $"$ & $"$ & $"$ & \\
\hline $\mathbf{B a}$ & 2000 & 1 & 3300 & 2 & $2 \times 10^{-6}$ & $"$ & " & $"$ & " & \\
\hline$"$ & & & 3500 & 3 & $"$ & $"$ & " & " & ": & $\mathbf{a}$ \\
\hline$n$ & 2500 & 2 & 4800 & 3 & $"$ & $"$ & $"$ & " & $"$ & \\
\hline$"$ & & & 12000 & 13 & $"$ & $"$ & $"$ & $"$ & " : & $a$ \\
\hline$"$ & 4200 & 3 & 7600 & 6 & $"$ & $"$ & $"$ & $"$ & $"$ & \\
\hline " & 6600 & 7 & 7500 & 3 & $"$ & $"$ & $"$ & $"$ & $"$ & \\
\hline
\end{tabular}

\footnotetext{
Second desorption.
} 
TABLE A-VI (cont)

\begin{tabular}{|c|c|c|c|c|c|c|c|c|c|c|}
\hline \multirow[b]{2}{*}{ Elevent } & \multicolumn{2}{|c|}{ Sorption } & \multicolumn{2}{|c|}{ Denorption } & \multirow{2}{*}{$\begin{array}{c}\text { Element } \\
\text { Concentration } \\
(M)\end{array}$} & \multirow{2}{*}{$\begin{array}{l}\text { Particle } \\
\text { Size } \\
\text { (监) } \\
\end{array}$} & \multirow[b]{2}{*}{ Temperature } & \multirow[b]{2}{*}{ Ateosphere } & \multirow[b]{2}{*}{ Ref. } & \multirow[b]{2}{*}{ Note } \\
\hline & $\begin{array}{c}R_{d} \\
(-e / g)\end{array}$ & $\begin{array}{l}\text { Time } \\
\text { (wk) }\end{array}$ & $\begin{array}{c}R_{d} \\
(\alpha / /)\end{array}$ & $\begin{array}{l}\text { Time } \\
\text { (wk) }\end{array}$ & & & & & & \\
\hline Eu & 4000 & 1 & 9700 & 2 & $1 \times 10^{-7}$ & $106-150$ & $70^{\circ} \mathrm{C}$ & Air & 2 & \\
\hline$"$ & & & 19000 & 3 & $"$ & $"$ & $"$ & $"$ & $"$ & $a$ \\
\hline$"$ & 4100 & 2 & 7700 & 3 & $"$ & $"$ & $"$ & $"$ & $"$ & \\
\hline$"$ & & & 24000 & 13 & $"$ & $"$ & $"$ & $n$ & $"$ & 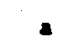 \\
\hline$"$ & 4000 & 3 & 17000 & 6 & $"$ & $"$ & $"$ & $"$ & $"$ & \\
\hline$"$ & 5200 & 7 & 13000 & 3 & $"$ & $"$ & $"$ & $"$ & $"$ & \\
\hline Sr & 420 & 4 & & & $2 \times 10^{-6}$ & $250-355$ & $A=b$ & $"$ & 4 & $\mathbf{b}$ \\
\hline$"$ & 390 & 9 & & & $"$ & $"$ & $"$ & $"$ & $"$ & $"$ \\
\hline Cs & 1300 & 4 & & & $6 \times 10^{-10}$ & $"$ & $"$ & $"$ & $"$ & $"$ \\
\hline$"$ & 1400 & 9 & & & $"$ & $"$ & $"$ & $"$ & $"$ & $"$ \\
\hline Ba & 840 & 4 & & & $1 \times 10^{-6}$ & $"$ & $"$ & $"$ & $"$ & $"$ \\
\hline$"$ & 880 & 9 & & & $"$ & $"$ & $"$ & $"$ & $"$ & $"$ \\
\hline Ce & 27000 & 4 & & & $1 \times 10^{-8}$ & " & $"$ & $"$ & $"$ & $"$ \\
\hline$"$ & $>48000$ & 9 & & & $"$ & $"$ & $"$ & $"$ & $"$ & $"$ \\
\hline Eu & 12000 & 4 & & & $8 \times 10^{-9}$ & $"$ & $"$ & $"$ & $"$ & $"$ \\
\hline$"$ & $>26000$ & 9 & & & $"$ & $"$ & $"$ & $"$ & $"$ & $"$ \\
\hline $\mathbf{S r}$ & 74 & 4 & & & $2 \times 10^{-6}$ & $"$ & $"$ & $"$ & $"$ & c \\
\hline$"$ & 150 & 9 & & & $"$ & $"$ & $"$ & $"$ & $"$ & $"$ \\
\hline $\mathrm{Cs}$ & 840 & 4 & & & $1 \times 10^{-9}$ & $"$ & $"$ & $"$ & $"$ & $"$ \\
\hline$"$ & 760 & 9 & & & $"$ & $"$ & $"$ & $"$ & $"$ & $"$ \\
\hline Ba & 380 & 4 & & & $1 \times 10^{-6}$ & $"$ & $"$ & " & $"$ & $"$ \\
\hline$"$ & 340 & 9 & & & $"$ & $"$ & $"$ & $"$ & $"$ & $"$ \\
\hline Ce & $>36000$ & 4 & & & $2 \times 10^{-8}$ & $"$ & $"$ & $"$ & $"$ & " \\
\hline$"$ & $>33000$ & 9 & & & $"$ & $"$ & $"$ & $"$ & $"$ & $n$ \\
\hline Eu & 13000 & 4 & & & $3 \times 10^{-8}$ & $"$ & $"$ & " & $"$ & $"$ \\
\hline$"$ & 51000 & 9 & & & $"$ & $"$ & $"$ & $"$ & $"$ & $"$ \\
\hline
\end{tabular}

\footnotetext{
second desorption.

bynthetic grounduater I; see Ref. 4.

'Synthetic srousduater II; see Ref. 4.
} 
TABLI A-VI (cont)

\begin{tabular}{|c|c|c|c|c|c|c|c|c|c|c|}
\hline \multirow[b]{2}{*}{ Elesent } & \multicolumn{2}{|c|}{ Sorption } & \multicolumn{2}{|c|}{ Denorption } & \multirow{2}{*}{$\begin{array}{c}\text { Element } \\
\text { Concentrration } \\
(\mathrm{H}) \\
\end{array}$} & \multirow{2}{*}{$\begin{array}{c}\text { Particle } \\
\text { Size } \\
\text { (y- }) \\
\end{array}$} & \multirow[b]{2}{*}{ Tesperature } & \multirow[b]{2}{*}{ Atmosphere } & \multirow[b]{2}{*}{ Ref. } & \multirow[b]{2}{*}{ Note } \\
\hline & $\begin{array}{c}R_{d} \\
(\omega / R)\end{array}$ & $\begin{array}{l}\text { Time } \\
\text { (wk) }\end{array}$ & $\begin{array}{c}R_{d} \\
(\omega e / R)\end{array}$ & $\begin{array}{l}\text { Tine } \\
\text { (wk) }\end{array}$ & & & & & & \\
\hline An & 430 & 1 & & & $1 \times 10^{-6}$ & $106-150$ & $A=b$ & Air & 4 & \\
\hline$"$ & 370 & 2 & 14000 & 5 & $"$ & $"$ & $"$ & $"$ & $"$ & \\
\hline$"$ & 430 & 4 & & & $"$ & $"$ & $"$ & $"$ & $"$ & \\
\hline$"$ & 1500 & 4 & 21000 & 5 & $"$ & $"$ & $"$ & $"$ & $"$ & \\
\hline$"$ & 640 & 8 & & & $"$ & $"$ & $"$ & $"$ & $"$ & \\
\hline$"$ & 520 & 1 & & & $"$ & $"$ & $70^{\circ} \mathrm{C}$ & $"$ & $"$ & \\
\hline$"$ & 680 & 2 & & & $"$ & $"$ & $"$ & $"$ & $"$ & \\
\hline$"$ & 2100 & 2 & & & $"$ & $"$ & $"$ & $"$ & $"$ & \\
\hline$"$ & 960 & 4 & & & $"$ & $"$ & $"$ & $"$ & $"$ & \\
\hline$"$ & 800 & 8 & ' & & $"$ & $"$ & $"$ & $"$ & $"$ & \\
\hline$"$ & 730 & 8 & & & $"$ & $"$ & $"$ & $"$ & $"$ & \\
\hline$"$ & 18000 & 3 & 54000 & 12 & $1 \times 10^{-7}$ & $106-250$ & Amb & $"$ & 5,6 & \\
\hline$"$ & 37000 & 6 & 59000 & 9 & $"$ & $"$ & $"$ & $"$ & $"$ & \\
\hline$"$ & 46000 & 12 & 43000 & 3 & $"$ & $"$ & $"$ & $"$ & $"$ & \\
\hline Pu & 390 & 1 & & & $1 \times 10^{-6}$ & $106-150$ & $"$ & $"$ & 4 & \\
\hline$"$ & 180 & 4 & & & $"$ & $"$ & $"$ & $"$ & $"$ & \\
\hline$"$ & 240 & 1 & & & $"$ & $"$ & $70^{\circ} \mathrm{C}$ & $"$ & $"$ & \\
\hline$"$ & 300 & 3 & 870 & 12 & $4 \times 10^{-12}$ & $106-250$ & $A$ Anb & $"$ & 7 & \\
\hline$"$ & 420 & 6 & 890 & 8 & $"$ & $"$ & $"$ & $"$ & $"$ & \\
\hline$"$ & 560 & 6 & 1700 & 8 & $"$ & $"$ & $"$ & $"$ & $"$ & \\
\hline$"$ & 760 & 13 & 1300 & 3 & $"$ & $"$ & $"$ & $"$ & $"$ & \\
\hline$"$ & 1900 & 13 & 2400 & 3 & $"$ & $"$ & $"$ & $"$ & $"$ & \\
\hline $\mathrm{Np}$ & 20 & 3 & 230 & 12 & $8 \times 10^{-11}$ & $"$ & $"$ & $"$ & $"$ & \\
\hline$"$ & 22 & 6 & 65 & 9 & $"$ & $"$ & $"$ & $"$ & $"$ & \\
\hline$"$ & 42 & 13 & 210 & 3 & $"$ & $"$ & $"$ & $"$ & $"$ & \\
\hline U & 4.0 & 1 & 6.1 & 3 & $1 \times 10^{-7}$ & $106-150$ & $"$ & $"$ & 4 & \\
\hline$"$ & 4.8 & 2 & 9.6 & 2 & $"$ & $"$ & $"$ & $"$ & $"$ & \\
\hline$"$ & 5.1 & 3 & 11 & 1 & $"$ & $"$ & $"$ & $"$ & $"$ & \\
\hline$"$ & 2.6 & 1 & 2.2 & 3 & $"$ & $355-500$ & $"$ & $"$ & $"$ & \\
\hline$"$ & 4.2 & 2 & 9.2 & 2 & $"$ & $"$ & $"$ & $"$ & $"$ & \\
\hline$"$ & 4.7 & 3 & 12 & 1 & $"$ & $"$ & $"$ & $"$ & $"$ & \\
\hline$"$ & 0.5 & 1 & 13 & 1 & $2 \times 10^{-6}$ & $"$ & $"$ & $"$ & 7 & \\
\hline$"$ & 6 & 1 & 13 & 1 & $"$ & $"$ & $"$ & $"$ & $"$ & \\
\hline$"$ & 5 & 1 & 13 & 1 & $"$ & $"$ & $"$ & $"$ & $"$ & \\
\hline$"$ & 13 & 1 & 58 & 3 & $1 \times 10^{-6}$ & $"$ & $70^{\circ} \mathrm{C}$ & $"$ & 4 & \\
\hline$"$ & 17 & 2 & 42 & 2 & $"$ & $"$ & $"$ & $"$ & $"$ & \\
\hline$"$ & 19 & 3 & 40 & 1 & $"$ & $"$ & $"$ & $"$ & $"$ & \\
\hline
\end{tabular}


TAarr A-VI (cont)

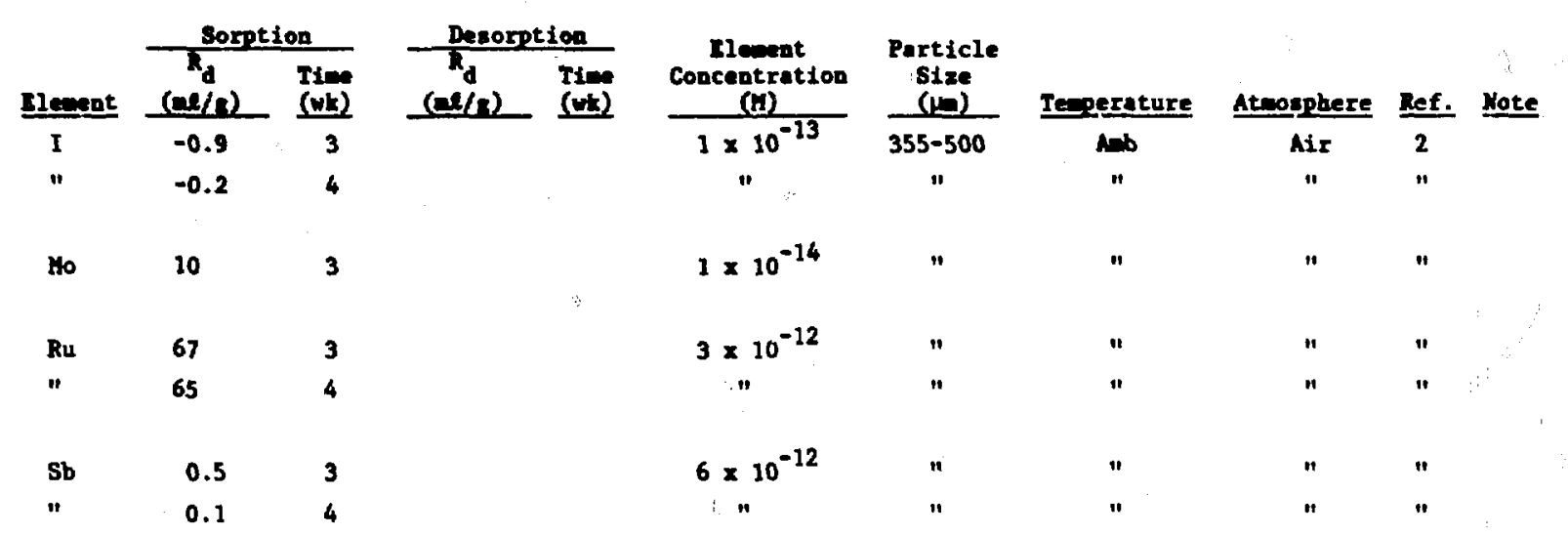

295 
TABLE A-VII

BATCH SORPTION DATA FOR TUFF MM-22

\begin{tabular}{|c|c|c|c|c|c|c|c|c|c|c|}
\hline \multirow[b]{2}{*}{ Element } & \multicolumn{2}{|c|}{ Sorption } & \multicolumn{2}{|c|}{ Desorption } & \multirow{2}{*}{$\begin{array}{c}\text { Elewent } \\
\text { Concentration } \\
(M) \\
\end{array}$} & \multirow[b]{2}{*}{$\begin{array}{c}\text { Particle } \\
\text { Size } \\
\text { (1⿴囗十) } \\
\end{array}$} & \multirow[b]{2}{*}{ Tenperature } & \multirow[b]{2}{*}{ Atwosphere } & \multirow[b]{2}{*}{ Ref. } & \multirow[b]{2}{*}{ Note } \\
\hline & $\begin{array}{c}R_{d} \\
(n / / R)\end{array}$ & $\begin{array}{l}\text { Time } \\
\text { (wk) }\end{array}$ & $\begin{array}{c}R_{d} \\
(\alpha \ell / 8)\end{array}$ & $\begin{array}{l}\text { Time } \\
\text { (wk) }\end{array}$ & & & & & & \\
\hline $\mathbf{S r}$ & 48 & 3 & 59 & 6 & $7 \times 10^{-7}$ & $106-500$ & $A=b$ & Air & 4 & \\
\hline$"$ & 48 & 3 & & & $"$ & $"$ & $"$ & $"$ & $"$ & \\
\hline$"$ & 55 & 6 & 63 & 3 & $"$ & $"$ & $"$ & $"$ & $"$ & \\
\hline$"$ & 62 & 6 & 57 & 3 & $"$ & $"$ & $"$ & $"$ & $"$ & \\
\hline Cs & 260 & 3 & 360 & 6 & $1 \times 10^{-9}$ & $"$ & $"$ & $"$ & $"$ & \\
\hline$"$ & 380 & 3 & & & $"$ & 11 & $"$ & $"$ & $"$ & \\
\hline$"$ & 270 & 6 & 380 & 3 & $"$ & $"$ & $"$ & $"$ & $"$ & \\
\hline$"$ & 240 & 6 & 360 & 3 & $"$ & $"$ & $"$ & $"$ & $"$ & \\
\hline Ba & 910 & 3 & 650 & 6 & $3 \times 10^{-7}$ & $"$ & $"$ & $"$ & $"$ & \\
\hline$"$ & 850 & 3 & & & $"$ & $"$ & $"$ & $"$ & $"$ & \\
\hline$"$ & 980 & 6 & 990 & 3 & $"$ & $"$ & $"$ & $"$ & $"$ & \\
\hline$"$ & 850 & 6 & 830 & 3 & $"$ & $"$ & $"$ & $"$ & $"$ & \\
\hline Ce & 1400 & 3 & 6100 & 6 & $7 \times 10^{-7}$ & $"$ & $"$ & $"$ & $"$ & \\
\hline$"$ & 1200 & 3 & & & $"$ & $"$ & $"$ & $"$ & $"$ & \\
\hline$"$ & 1300 & 6 & 8100 & 3 & $"$ & $"$ & $"$ & $"$ & " & \\
\hline$"$ & 1200 & 6 & 5400 & 3 & $"$ & $"$ & $"$ & $"$ & $"$ & \\
\hline Eu & 1300 & 3 & 3300 & 6 & $5 \times 10^{-8}$ & $"$ & $"$ & $"$ & " & \\
\hline$"$ & 1200 & 3 & & & $"$ & $"$ & " & " & $"$ & \\
\hline$"$ & 1700 & 6 & 3900 & 3 & $"$ & $"$ & $"$ & $"$ & $"$ & \\
\hline$"$ & 1400 & 6 & 3100 & 3 & $"$ & $"$ & $"$ & $"$ & $"$ & \\
\hline Sr & 66 & 6 & 74 & 3 & $7 \times 10^{-7}$ & $<106$ & $"$ & $"$ & $"$ & \\
\hline Cs & 540 & 6 & 500 & 3 & $1 \times 10^{-9}$ & $"$ & $"$ & $"$ & $"$ & \\
\hline Ba & 1300 & 6 & 1600 & 3 & $3 \times 10^{-7}$ & $"$ & $"$ & $"$ & $"$ & \\
\hline Ce & 1600 & 6 & 4900 & 3 & $7 \times 10^{-7}$ & $"$ & $"$ & " & $"$ & \\
\hline Eu & 1500 & 6 & 4000 & 3 & $5 \times 10^{-8}$ & $"$ & $"$ & $"$ & $"$ & \\
\hline Sr & 48 & 3 & 120 & 6 & $7 \times 10^{-7}$ & $75-500$ & $"$ & $\mathrm{CA}$ & 4,8 & \\
\hline$"$ & 53 & 6 & 70 & 3 & $"$ & $n$ & $"$ & $"$ & $"$ & \\
\hline " & 46 & 12 & & & $"$ & $"$ & $"$ & $"$ & $"$ & \\
\hline
\end{tabular}


TABIE A-VII (cont)

\begin{tabular}{|c|c|c|c|c|c|c|c|c|c|c|}
\hline \multirow[b]{2}{*}{ Eleeent } & \multicolumn{2}{|c|}{ Sorption } & \multicolumn{2}{|c|}{ Desorption } & \multirow{2}{*}{$\begin{array}{c}\text { Element } \\
\text { Concentration } \\
\text { (H) } \\
\end{array}$} & \multirow{2}{*}{$\begin{array}{c}\text { Particle } \\
\text { Size } \\
\text { (j) } \\
\end{array}$} & \multirow[b]{2}{*}{ Tepperature } & \multirow[b]{2}{*}{ Atwosphere } & \multirow[b]{2}{*}{ Ref. } & \multirow[b]{2}{*}{ Note } \\
\hline & $\begin{array}{c}R_{d} \\
(n / 2)\end{array}$ & $\begin{array}{l}\text { Tine } \\
\text { (wk) } \\
\end{array}$ & $\begin{array}{c}R_{\mathrm{d}} \\
(\mathrm{n} / \mathrm{k})\end{array}$ & $\begin{array}{l}\text { Tine } \\
\text { (wk) }\end{array}$ & & & & & & \\
\hline CE & 200 & 3 & 340 & 6 & $2 \times 10^{-9}$ & $75-500$ & Amb & $\mathbf{C A}$ & 4,8 & \\
\hline$n$ & 210 & 6 & 280 & 3 & $"$ & $"$ & $"$ & $"$ & $"$ & \\
\hline$"$ & 250 & 12 & & & $"$ & $"$ & $n$ & $"$ & $"$ & \\
\hline Ba & 200 & 3 & 470 & 6 & $3 \times 10^{-7}$ & $"$ & $"$ & $"$ & $"$ & \\
\hline$" 1$ & 300 & 6 & 460 & 3 & $"$ & " & $"$ & $"$ & $"$ & \\
\hline$"$ & 300 & 12 & & & $"$ & $"$ & $"$ & $"$ & $"$ & \\
\hline Ce & 760 & 3 & 2700 & 6 & $2 \times 10^{-8}$ & $1 "$ & $"$ & $" 1$ & $"$ & \\
\hline$n$ & & & 2100 & 3 & $"$ & $n$ & " & $"$ & " & \\
\hline$"$ & 1600 & 12 & & & $"$ & $"$ & $"$ & $"$ & " & \\
\hline Eu & 710 & 3 & 2500 & 6 & $"$ & $"$ & $"$ & " & $n$ & \\
\hline$"$ & & & 3100 & 6 & $"$ & $"$ & $"$ & $"$ & $"$ & \\
\hline$"$ & 1300 & 12 & & & $"$ & $"$ & $"$ & $"$ & $"$ & \\
\hline $\mathbf{S r}$ & 73 & 3 & 120 & 6 & $7 \times 10^{-7}$ & $<75$ & $"$ & $"$ & $"$ & \\
\hline$"$ & 75 & 6 & 100 & 3 & $"$ & $"$ & " & 11 & $"$ & \\
\hline$"$ & 81 & 12 & & & $"$ & $"$ & $"$ & $"$ & " & \\
\hline $\mathrm{Cs}$ & 389 & 3 & 550 & 6 & $2 \times 10^{-9}$ & $"$ & $"$ & $"$ & $"$ & \\
\hline " & 440 & 6 & 490 & 3 & $"$ & $"$ & $"$ & $"$ & " & \\
\hline$"$ & 480 & 12 & & & $"$ & $"$ & $"$ & $"$ & $"$ & \\
\hline Ba & 660 & 3 & 1100 & 6 & $3 \times 10^{-7}$ & $"$ & $"$ & " & $"$ & \\
\hline " & 900 & 6 & 1300 & 3 & n & $"$ & $"$ & $"$ & $"$ & \\
\hline$"$ & 1000 & 12 & & & $"$ & " & " & " & $"$ & \\
\hline Ce & 630 & 3 & 2400 & 6 & $2 \times 10^{-8}$ & It & $n$ & 4 & $"$ & \\
\hline$"$ & 860 & 6 & 2100 & 3 & $"$ & $"$ & $n$ & $"$ & $"$ & \\
\hline$"$ & 760 & 12 & & & " & " & $n$ & $"$ & $"$ & \\
\hline Eu & 800 & 3 & 2200 & 6 & $2 \times 10^{-8}$ & $"$ & n & " & $"$ & \\
\hline$"$ & 990 & 6 & 1900 & 3 & " & 11 & $H$ & $"$ & " & \\
\hline$"$ & 1000 & 12 & & & $"$ & $"$ & $"$ & $"$ & $n$ & \\
\hline
\end{tabular}


TARL A-VII (cont)

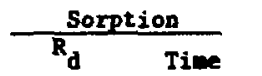

Eleent

"

$\begin{array}{ll}\text { An } & 1500 \\ \text { " } & 1100 \\ \text { " } & 1100 \\ \text { " } & 6500 \\ \text { " } & 6800 \\ \text { " } & 6900 \\ \text { " } & 750 \\ \text { " } & 960 \\ \text { " } & 1200 \\ \text { " } & 21000 \\ & 1800\end{array}$

Pu

$37 \quad 3$

$64 \quad 6$

1300

$100 \quad 12$

1303

$210 \quad 6$

$280 \quad 12$

1203

$400 \quad 6$

$250 \quad 12$

853

2606

$190 \quad 12$

653

$62 \quad 3$

543

413

173

1203

$47 \quad 3$

793

$70 \quad 3$

163

603

$63 \quad 3$

543

$66 \quad 3$

34

\begin{tabular}{cr}
\multicolumn{2}{c}{ Desorption } \\
\hline$R_{d}$ & Tine \\
$(\boldsymbol{R} / /)$ & (wk) \\
\cline { 1 - 1 } 2400 & 12 \\
1900 & 9 \\
3100 & 3 \\
6700 & 12 \\
6500 & 9 \\
7300 & 3 \\
2300 & 12 \\
2300 & 9 \\
4300 & 3 \\
2500 & 12 \\
3100 & 9 \\
7500 & 3
\end{tabular}

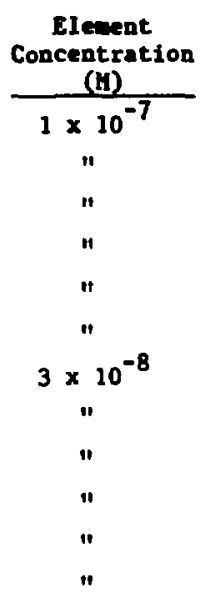

\section{Particle}

(1i) Tenerature Atmosphere Ref. Hote

75-500

Amb

Air 3
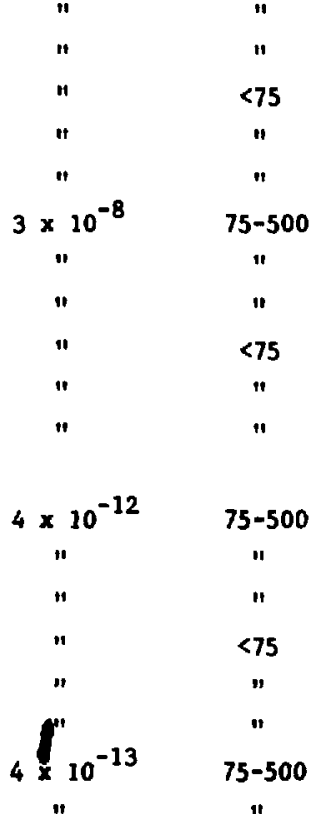

$75-500$

1300

12

1400

1400

1300

1900

970

1800

1200

1000

1500

3100

960

580

470

920

730

2800

1800

1400

1900

910

1100

1200

990

1100

280

9

12

9

3
12

9

3

12

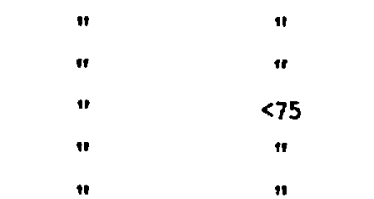

$8 \times 10^{-12}$

\section{5-500}

$3 \times 10^{-11}$

$2 \times 10^{-10}$

$2 \times 10^{-9}$

$1 \times 10^{-8}$

$6 \times 10^{-13}$

$2 \times 10^{-11}$

$1 \times 10^{-10}$

$6 \times 10^{-10}$

$1 \times 10^{-8}$

$8 \times 10^{-12}$

$3 \times 10^{-11}$

$2 \times 10^{-10}$

$2 \times 10^{-9}$

$1 \times 10^{-8}$

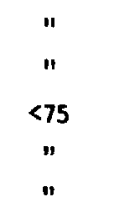

เ

"1

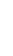

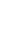

"


TABLE A-VII (cont)

\begin{tabular}{|c|c|c|c|c|c|c|c|c|c|c|}
\hline \multirow[b]{2}{*}{ Element } & \multicolumn{2}{|c|}{ Sorption } & \multicolumn{2}{|c|}{ Deorption } & \multirow{2}{*}{$\begin{array}{c}\text { Element } \\
\text { Concentration } \\
(\mathrm{H}) \\
\end{array}$} & \multirow[b]{2}{*}{$\begin{array}{c}\text { Particle } \\
\text { Size } \\
\text { (1) } \\
\end{array}$} & \multirow[b]{2}{*}{ Te-perature } & \multirow[b]{2}{*}{ Atmonphere } & \multirow[b]{2}{*}{ Ref. } & \multirow[b]{2}{*}{ Note } \\
\hline & $\begin{array}{c}R_{d} \\
(n L / R)\end{array}$ & $\begin{array}{l}\text { Tive } \\
\text { (uls) }\end{array}$ & $\begin{array}{c}k d \\
(n) / 2)\end{array}$ & $\begin{array}{l}\text { Tine } \\
\text { (ut) }\end{array}$ & & & & & & \\
\hline $\boldsymbol{p}_{\mathbf{p}}$ & 5.1 & 3 & 26 & 12 & $1 \times 10^{-10}$ & $75-500$ & $\mathbf{A b}$ & Air & & 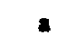 \\
\hline$"$ & 5.8 & 6 & 30 & 9 & $"$ & $"$ & $"$ & $"$ & & $"$ \\
\hline " & 7.0 & 12 & 42 & 3 & " & 11 & $"$ & $"$ & & 4 \\
\hline " & 6.1 & 4 & 18 & 12 & $3 \times 10^{-11}$ & $"$ & $"$ & CA & & $"$ \\
\hline$"$ & 7.0 & 4 & 18 & 12 & $"$ & " & $"$ & " & & $"$ \\
\hline$"$ & 9.5 & 8 & 19 & 7 & $"$ & $"$ & $"$ & " & & \\
\hline " & 9.4 & 8 & 17 & 7 & $"$ & "t & $n$ & $n$ & & $"$ \\
\hline 11 & 9.5 & 12 & 34 & 3 & 11 & $"$ & $n$ & $"$ & & $"$ \\
\hline$"$ & 10 & 12 & 34 & 3 & $"$ & " & $n$ & $"$ & & $"$ \\
\hline $\mathbf{U}$ & 2 & 1 & 15 & 2 & $1 \times 10^{-6}$ & $<106$ & $"$ & Air & 3 & \\
\hline " & 2 & 2 & 8 & 1 & $"$ & " & $"$ & $"$ & $n$ & \\
\hline$"$ & 2 & 3 & & & $"$ & " & " & " & " & \\
\hline " & 1 & 1 & 0 & 2 & $"$ & $106-500$ & $"$ & $"$ & $"$ & \\
\hline " & 2 & 2 & 8 & 1 & $"$ & $"$ & $"$ & $"$ & $"$ & \\
\hline$"$ & 2 & 3 & & & " & $"$ & $"$ & $"$ & " & \\
\hline " & 0 & 1 & & & $n$ & $<75$ & $"$ & CA & 4 & \\
\hline " & 0.9 & 2 & & & " & $"$ & $"$ & $"$ & " & \\
\hline$"$ & 1.2 & 3 & & & " & $"$ & $"$ & " & $"$ & \\
\hline$"$ & 0 & 2 & & & $n$ & $75-500$ & " & " & $"$ & \\
\hline " & 0.8 & 3 & & & " & " & $"$ & " & $"$ & \\
\hline Tc & 0.13 & 6 & 0.47 & 9 & $1 \times 10^{-3}$ & $106-500$ & " & Air & $"$ & \\
\hline " & 0.17 & 6 & 1.2 & 9 & $1 \times 10^{-6}$ & $"$ & $"$ & $"$ & $"$ & \\
\hline$"$ & 0.16 & 6 & 1.9 & 9 & $1 \times 10^{-9}$ & $"$ & $"$ & $"$ & " & \\
\hline$"$ & 0.72 & 6 & 1.2 & 9 & $1 \times 10^{-12}$ & " & $"$ & $"$ & $"$ & \\
\hline$"$ & 0.4 & 3 & 12 & 12 & $"$ & $75-500$ & $"$ & CA & $"$ & \\
\hline$"$ & 3.8 & 3 & 37 & 12 & $"$ & $<75$ & $"$ & $"$ & 11 & \\
\hline$"$ & 6.5 & 6 & 14 & 9 & " & $"$ & " & $"$ & $"$ & \\
\hline " & 3.2 & 12 & 32 & 3 & $"$ & " & " & $"$ & $"$ & \\
\hline " & 0.3 & 6 & 9.5 & 9 & $"$ & $75-500$ & $"$ & " : & $"$ & \\
\hline$"$ & 1.5 & 12 & 5.3 & 3 & $"$ & " & $"$ & 4 & $"$ & \\
\hline
\end{tabular}

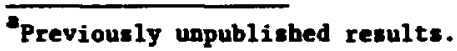


TABLE A-VII (cont)

\begin{tabular}{|c|c|c|c|c|c|c|c|c|c|c|}
\hline \multirow[b]{2}{*}{ Element } & \multicolumn{2}{|c|}{ Sorption } & \multicolumn{2}{|c|}{ Desorption } & \multirow{2}{*}{$\begin{array}{c}\text { Element } \\
\text { Concentration } \\
\text { (H) }\end{array}$} & \multirow{2}{*}{$\begin{array}{c}\text { Particle } \\
\text { Size } \\
\text { (1e) } \\
\end{array}$} & \multirow[b]{2}{*}{ Te-perature } & \multirow[b]{2}{*}{ Atroosphere } & \multirow[b]{2}{*}{ Ref. } & \multirow[b]{2}{*}{ Note } \\
\hline & $\begin{array}{c}R_{d} \\
(\alpha e / g)\end{array}$ & $\begin{array}{l}\text { Time } \\
\text { (wk) }\end{array}$ & $\begin{array}{c}R_{\mathrm{d}} \\
(\omega / \mathrm{s})\end{array}$ & $\begin{array}{l}\text { Time } \\
\text { (wk) }\end{array}$ & & & & & & \\
\hline Sx & 9.4 & 3 & & & $3 \times 10^{-3}$ & $75-500$ & $\mathbf{A} \mathbf{b}$ & Air & 3 & \\
\hline$"$ & 55 & 3 & & & $3 \times 10^{-5}$ & " & $"$ & $"$ & $n$ & \\
\hline$"$ & 71 & 3 & & & $5 \times 10^{-7}$ & $"$ & $"$ & $"$ & $"$ & \\
\hline$"$ & 95 & 3 & & & $6 \times 10^{-7}$ & $"$ & $"$ & $"$ & $"$ & \\
\hline$"$ & 65 & 3 & & & $"$ & $"$ & $"$ & $"$ & $"$ & \\
\hline$"$ & 20 & 3 & & & $3 \times 10^{-3}$ & $<75$ & $"$ & $"$ & $"$ & \\
\hline$"$ & 54 & 3 & & & $3 \times 10^{-5}$ & $"$ & $"$ & $"$ & $"$ & \\
\hline$"$ & 81 & 3 & & & $5 \times 10^{-7}$ & $"$ & $"$ & $"$ & $"$ & \\
\hline$"$ & 70 & 3 & & & $6 \times 10^{-7}$ & $"$ & $"$ & $"$ & $"$ & \\
\hline$"$ & 65 & 3 & & & $"$ & $"$ & $"$ & $"$ & $"$ & \\
\hline Cs & 10 & 3 & & & $2 \times 10^{-3}$ & $75-500$ & $"$ & $"$ & $"$ & \\
\hline$"$ & 110 & 3 & & & $2 \times 10^{-5}$ & $"$ & $"$ & $"$ & $"$ & \\
\hline$"$ & 260 & 3 & & & $2 \times 10^{-7}$ & $"$ & $"$ & $"$ & $"$ & \\
\hline$"$ & 660 & 3 & & & $3 \times 10^{-9}$ & $"$ & $"$ & " & $"$ & \\
\hline$"$ & 550 & 3 & & & $3 \times 10^{-10}$ & $"$ & $"$ & $"$ & $"$ & \\
\hline$"$ & 12 & 3 & & & $2 \times 10^{-3}$ & $<75$ & $"$ & $"$ & $"$ & \\
\hline$"$ & 130 & 3 & & & $2 \times 10^{-5}$ & $"$ & $"$ & $"$ & $"$ & \\
\hline$"$ & 610 & 3 & & & $2 \times 10^{-7}$ & $"$ & $"$ & $"$ & $"$ & \\
\hline$"$ & 380 & 3 & & & $3 \times 10^{-9}$ & $"$ & $"$ & $"$ & $"$ & \\
\hline$"$ & 610 & 3 & & & $3 \times 10^{-10}$ & $"$ & $"$ & $"$ & $"$ & \\
\hline Ba & 210 & 3 & & & $1 \times 10^{-4}$ & $75-500$ & $"$ & $"$ & $"$ & \\
\hline$"$ & 470 & 3 & & & $1 \times 10^{-5}$ & $"$ & $"$ & $"$ & $"$ & \\
\hline$"$ & 790 & 3 & & & $4 \times 10^{-7}$ & $"$ & $"$ & $"$ & $"$ & \\
\hline$"$ & 600 & 3 & & & $3 \times 10^{-7}$ & $"$ & $"$ & $"$ & $"$ & \\
\hline$"$ & 670 & 3 & & & $"$ & $"$ & $"$ & $"$ & $"$ & \\
\hline$"$ & 400 & 3 & & & $1 \times 10^{-4}$ & $<75$ & $"$ & $"$ & $"$ & \\
\hline$"$ & 760 & 3 & & & $1 \times 10^{-5}$ & $"$ & $"$ & $"$ & $"$ & \\
\hline$"$ & 1200 & 3 & & & $4 \times 10^{-7}$ & $"$ & $"$ & $"$ & $"$ & \\
\hline$"$ & 1300 & 3 & & & $3 \times 10^{-7}$ & $"$ & $"$ & $"$ & $"$ & \\
\hline$"$ & 1000 & 3 & & & $"$ & $"$ & $"$ & $"$ & $"$ & \\
\hline Eu & 1100 & 3 & & & $9 \times 10^{-6}$ & $75-500$ & $"$ & $"$ & $"$ & \\
\hline$"$ & 1200 & 3 & & & $3 \times 10^{-6}$ & $"$ & $"$ & $"$ & $"$ & \\
\hline$"$ & 1900 & 3 & & & $4 \times 10^{-8}$ & $"$ & $"$ & $"$ & $"$ & \\
\hline$"$ & 1900 & 3 & & & $1 \times 10^{-8}$ & $"$ & $"$ & " & $"$ & \\
\hline$"$ & 2100 & 3 & & & $3 \times 10^{-9}$ & $"$ & $"$ & $"$ & $"$ & \\
\hline$"$ & 5800 & 3 & & & $9 \times 10^{-6}$ & $<75$ & $"$ & $"$ & $"$ & \\
\hline$"$ & 690 & 3 & & & $3 \times 10^{-6}$ & $"$ & $"$ & $"$ & $"$ & \\
\hline$"$ & 1900 & 3 & & & $4 \times 10^{-8}$ & $"$ & $"$ & $"$ & $"$ & \\
\hline$"$ & 1900 & 3 & & & $1 \times 10^{-8}$ & $"$ & $"$ & $n$ & $"$ & \\
\hline
\end{tabular}


TABLE A-VIII

AATCI SORPTIOW DATA FOR TUFF YM-30

\begin{tabular}{|c|c|c|c|c|c|c|c|c|c|c|}
\hline \multirow[b]{2}{*}{ Elewent } & \multicolumn{2}{|c|}{ Sorption } & \multicolumn{2}{|c|}{ Desorption } & \multirow{2}{*}{$\begin{array}{c}\text { Elewent } \\
\text { Concentration } \\
\text { (M) } \\
\end{array}$} & \multirow{2}{*}{$\begin{array}{l}\text { Particle } \\
\text { Size } \\
\text { (业) } \\
\end{array}$} & \multirow[b]{2}{*}{ Temperature } & \multirow[b]{2}{*}{ Atoosphere } & \multirow[b]{2}{*}{ Ref. } & \multirow[b]{2}{*}{ Note } \\
\hline & $\begin{array}{c}R_{d} \\
(a / 2)\end{array}$ & $\begin{array}{l}\text { Tine } \\
\text { (wk) }\end{array}$ & $\begin{array}{c}R_{d} \\
(a / g)\end{array}$ & $\begin{array}{l}\text { Time } \\
\text { (wk) }\end{array}$ & & & & & & \\
\hline sr & 100 & 3 & 210 & 12 & $8 \times 10^{-11}$ & $75-500$ & $A=b$ & Air & 3 & 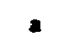 \\
\hline$"$ & 320 & 6 & 260 & 9 & $"$ & $"$ & $"$ & $"$ & $"$ & $"$ \\
\hline$"$ & 360 & 12 & 160 & 3 & $"$ & $"$ & $"$ & $"$ & $"$ & $"$ \\
\hline Cs & 850 & 3 & 1600 & 12 & $3 \times 10^{-9}$ & $"$ & $"$ & $"$ & $"$ & $"$ \\
\hline$"$ & 860 & 6 & 1700 & 9 & $"$ & $"$ & $"$ & $"$ & $"$ & $"$ \\
\hline$"$ & $2000^{b}$ & 12 & 1300 & 3 & $"$ & $"$ & $"$ & $"$ & $"$ & $", b$ \\
\hline Ba & 2000 & 3 & 4200 & 12 & $8 \times 10^{-10}$ & $"$ & $"$ & $"$ & $"$ & $"$ \\
\hline$"$ & 1800 & 6 & 2000 & 9 & $"$ & $"$ & $"$ & $"$ & $"$ & $"$ \\
\hline$"$ & 6400 & 12 & 3100 & 3 & $"$ & $"$ & $"$ & $"$ & $"$ & $"$ \\
\hline Ce & 130000 & 3 & 200000 & 12 & $8 \times 10^{-13}$ & $"$ & $"$ & $"$ & $"$ & $"$ \\
\hline$"$ & $1100000^{b}$ & 6 & 160000 & 9 & $"$ & $"$ & $"$ & $"$ & $"$ & $", b$ \\
\hline$"$ & 330000 & 12 & 150000 & 3 & $"$ & $"$ & $"$ & $"$ & $"$ & $"$ \\
\hline Eu & 94000 & 3 & 10000 & 12 & $1 \times 10^{-10}$ & $"$ & " & $"$ & $"$ & $"$ \\
\hline$"$ & 120000 & 6 & 10000 & 9 & " & $"$ & $"$ & " & $"$ & $"$ \\
\hline$"$ & 260000 & 12 & 12000 & 3 & $"$ & $"$ & $"$ & $"$ & $"$ & $"$ \\
\hline
\end{tabular}

Concentrations axe only those added with the tracer; actual concentrations an be higher.

balue not included in averages for Table XI, Chap. 1. 
TABLE A-IX

BATCH SORPTION DATA FOR TUFF YH-38

\begin{tabular}{|c|c|c|c|c|c|c|c|c|c|c|}
\hline \multirow[b]{2}{*}{ Element } & \multicolumn{2}{|c|}{ Sorption } & \multicolumn{2}{|c|}{ Desorption } & \multirow{2}{*}{$\begin{array}{c}\text { Element } \\
\text { Concentretion } \\
\text { (H) }\end{array}$} & \multirow[b]{2}{*}{$\begin{array}{c}\text { Particle } \\
\text { Size } \\
(1) \\
\end{array}$} & \multirow[b]{2}{*}{ Tewperature } & \multirow[b]{2}{*}{ Atposphere } & \multirow[b]{2}{*}{ Ref. } & \multirow[b]{2}{*}{ Note } \\
\hline & $\begin{array}{c}R_{d} \\
(n e / k) \\
\end{array}$ & $\begin{array}{l}\text { Tine } \\
\text { (wk) }\end{array}$ & $\begin{array}{c}R_{d} \\
(a / z)\end{array}$ & $\begin{array}{l}\text { Tine } \\
\text { (wk) }\end{array}$ & & & & & & \\
\hline $\mathbf{S r}$ & 18000 & 3 & & & $6 \times 10^{-8}$ & $106-500$ & $A \mathrm{Ab}$ & Air & 4 & \\
\hline$" 1$ & 10000 & 6 & 22000 & 3 & $"$ & $"$ & $"$ & $"$ & $"$ & \\
\hline $\mathrm{Cs}$ & 12000 & 3 & & & $1 \times 10^{-9}$ & $"$ & $"$ & $" 1$ & 11 & \\
\hline$"$ & 8200 & 6 & 13000 & 3 & $"$ & " & $"$ & $"$ & $" 1$ & \\
\hline $\mathrm{Ba}$ & 77000 & 3 & & & $4 \times 10^{-7}$ & $"$ & $"$ & $"$ & $"$ & \\
\hline$"$ & 81000 & 6 & 260000 & 3 & $"$ & $"$ & $"$ & $"$ & $"$ & \\
\hline Ce & 900 & 3 & & & $1 \times 10^{-6}$ & $"$ & $n$ & $"$ & $"$ & \\
\hline$"$ & 620 & 6 & 2600 & 3 & $"$ & $"$ & $"$ & $"$ & $"$ & \\
\hline $\mathbf{E u}$ & 2600 & 3 & & & $7 \times 10^{-8}$ & $"$ & $"$ & $"$ & $"$ & \\
\hline$"$ & 1500 & 6 & 7300 & 3 & $"$ & $"$ & $"$ & $"$ & $"$ & \\
\hline $\mathbf{S r}$ & 7400 & 6 & 18000 & 3 & $6 \times 10^{-8}$ & $<106$ & $"$ & $"$ & $"$ & \\
\hline Cs & 5900 & 6 & 13000 & 3 & $1 \times 10^{-9}$ & $"$ & $"$ & $" 1$ & $"$ & \\
\hline $\mathbf{B a}$ & 40000 & 6 & 110000 & 3 & $4 \times 10^{-7}$ & $"$ & $"$ & $"$ & $"$ & \\
\hline Ce & 950 & 6 & 4900 & 3 & $1 \times 10^{-6}$ & $"$ & $"$ & $"$ & $"$ & \\
\hline Eu & 4900 & 6 & 10000 & 3 & $7 \times 10^{-8}$ & $"$ & $"$ & $"$ & $"$ & \\
\hline $\mathbf{S r}$ & 7200 & 3 & 13000 & 6 & $1 \times 10^{-7}$ & $75-500$ & $"$ & $\mathbf{C A}$ & $"$ & \\
\hline$"$ & 8500 & 6 & 14000 & 3 & $"$ & $"$ & " & " & $"$ & \\
\hline$"$ & 7500 & 12 & & & $"$ & $"$ & $"$ & $"$ & $"$ & \\
\hline Cs & 7900 & 3 & 15000 & 6 & $3 \times 10^{-9}$ & $"$ & $"$ & " & $"$ & \\
\hline$"$ & 10000 & 6 & 15000 & 3 & $"$ & $"$ & $"$ & $"$ & $n$ & \\
\hline$"$ & 5400 & 12 & & & $"$ & $"$ & $"$ & $"$ & $n$ & \\
\hline Ba & 34000 & 3 & 58000 & 6 & $4 \times 10^{-7}$ & " & " & " & $"$ & \\
\hline$"$ & 41000 & 6 & 89000 & 3 & $"$ & $"$ & $"$ & $"$ & $"$ & \\
\hline$"$ & 11000 & 12 & & & $"$ & $"$ & $"$ & $"$ & $"$ & \\
\hline $\mathrm{Ce}$ & 560 & 3 & 1300 & 6 & $7 \times 10^{-8}$ & " & $"$ & $"$ & $"$ & \\
\hline$"$ & 850 & 6 & 3200 & 3 & $"$ & $"$ & $"$ & $"$ & $"$ & \\
\hline$"$ & 320 & 12 & & & $"$ & $"$ & $"$ & $"$ & $"$ & \\
\hline
\end{tabular}


TADLE A-IX (cont)

\begin{tabular}{|c|c|c|c|c|c|c|c|c|c|c|}
\hline \multirow[b]{2}{*}{ Elenent } & \multicolumn{2}{|c|}{ Sorption } & \multicolumn{2}{|c|}{ Desorption } & \multirow{2}{*}{$\begin{array}{c}\text { Elewent } \\
\text { Concentration } \\
\text { (M) }\end{array}$} & \multirow{2}{*}{$\begin{array}{c}\text { Particle } \\
\text { Size } \\
\text { (1- } \\
\end{array}$} & \multirow[b]{2}{*}{ Temperature } & \multirow[b]{2}{*}{ Ateosphere } & \multirow[b]{2}{*}{ Ref. } & \multirow[b]{2}{*}{ Note } \\
\hline & $\begin{array}{c}R_{d} \\
(\alpha e / R) \\
\end{array}$ & $\begin{array}{l}\text { Tine } \\
\text { (uk) }\end{array}$ & $\begin{array}{c}R_{d} \\
(n e / s)\end{array}$ & $\begin{array}{l}\text { Time } \\
\text { (uk) }\end{array}$ & & & & & & \\
\hline Eu & 840 & 3 & 1300 & 6 & $9 \times 10^{-8}$ & $75-500$ & $A=b$ & CA & 4 & \\
\hline$"$ & 1000 & 6 & 4300 & 3 & $"$ & $"$ & $"$ & $"$ & $"$ & \\
\hline$"$ & 470 & 12 & & & $"$ & $"$ & $"$ & $"$ & $"$ & \\
\hline Sr & 9100 & 3 & 23000 & 6 & $1 \times 10^{-7}$ & $<75$ & $"$ & $"$ & 4,8 & \\
\hline$"$ & 23000 & 6 & 18000 & 3 & $"$ & $"$ & $"$ & $"$ & $"$ & \\
\hline$"$ & 8000 & 12 & & & $"$ & $"$ & $"$ & $"$ & $"$ & \\
\hline Cs & 9500 & 3 & 12000 & 6 & $3 \times 10^{-9}$ & $"$ & $"$ & $"$ & $"$ & \\
\hline$"$ & 14000 & 6 & 8000 & 3 & $"$ & $"$ & $"$ & $"$ & $"$ & \\
\hline$"$ & 9000 & 12 & & & $n$ & $"$ & $"$ & $"$ & $"$ & \\
\hline Ba & 57000 & 3 & 63000 & 6 & $4 \times 10^{-7}$ & $"$ & $"$ & $"$ & $"$ & \\
\hline$"$ & $>210000$ & 6 & 46000 & 3 & $"$ & $"$ & $"$ & $"$ & $"$ & \\
\hline$"$ & 22000 & 12 & & & $"$ & $"$ & $"$ & $"$ & $"$ & \\
\hline $\mathrm{Ce}$ & 650 & 3 & 21000 & 6 & $7 \times 10^{-8}$ & $"$ & $"$ & $"$ & $"$ & \\
\hline$"$ & 320 & 6 & 660 & 3 & $"$ & $"$ & $"$ & $"$ & $"$ & \\
\hline$"$ & 740 & 12 & & & $"$ & $"$ & $"$ & $"$ & $"$ & \\
\hline Eu & 1100 & 3 & 21000 & 6 & $9 \times 10^{-8}$ & $"$ & $"$ & $"$ & $"$ & \\
\hline$"$ & 560 & 6 & 820 & 3 & $"$ & $"$ & $"$ & $"$ & $"$ & \\
\hline$"$ & 1000 & 12 & & & $"$ & $"$ & $"$ & $"$ & $"$ & \\
\hline $\mathbf{S x}$ & 14000 & 3 & & & $4 \times 10^{-7}$ & $<38$ & $"$ & Air & 7 & \\
\hline$"$ & 20000 & 3 & & & $"$ & $"$ & $"$ & $"$ & $"$ & \\
\hline Cs & 11000 & 3 & & & $4 \times 10^{-9}$ & $"$ & $"$ & $"$ & $"$ & \\
\hline$"$ & 20000 & 3 & & & $"$ & $"$ & $"$ & $"$ & $"$ & \\
\hline Ba & 69000 & 3 & & & $4 \times 10^{-7}$ & $"$ & $"$ & $"$ & $"$ & \\
\hline$"$ & 190000 & 3 & & & " & $"$ & $"$ & $"$ & $"$ & \\
\hline Eu & 2200 & 3 & & & $7 \times 10^{-8}$ & $"$ & $"$ & $"$ & $"$ & \\
\hline$"$ & 3000 & 3 & & & $"$ & $"$ & $"$ & " & $"$ & \\
\hline Sr & 20000 & 3 & & & $4 \times 10^{-7}$ & 38-106 & $"$ & $"$ & $"$ & \\
\hline$"$ & 20000 & 3 & & & $"$ & $"$ & $"$ & $"$ & $"$ & \\
\hline Cs & 17000 & 3 & & & $4 \times 10^{-9}$ & $"$ & $"$ & $"$ & $"$ & \\
\hline$"$ & 15000 & 3 & & & $n$ & $"$ & $"$ & $"$ & $"$ & \\
\hline
\end{tabular}


TABLE A-IX (cont)

\begin{tabular}{|c|c|c|c|c|c|c|c|c|c|c|}
\hline \multirow[b]{2}{*}{ Elewent } & \multicolumn{2}{|c|}{ Sorption } & \multicolumn{2}{|c|}{ Desorption } & \multirow{2}{*}{$\begin{array}{c}\text { Element } \\
\text { Concentration } \\
(K) \\
\end{array}$} & \multirow{2}{*}{$\begin{array}{c}\text { Particle } \\
\text { Size } \\
\text { (je) } \\
\end{array}$} & \multirow[b]{2}{*}{ Teuperature } & \multirow[b]{2}{*}{ Ateosphere } & \multirow[b]{2}{*}{ Ref. } & \multirow[b]{2}{*}{ Note } \\
\hline & $\begin{array}{c}R_{d} \\
(m e / \theta)\end{array}$ & $\begin{array}{l}\text { Tive } \\
\text { (wk) }\end{array}$ & $\begin{array}{c}R_{d} \\
(E / /)\end{array}$ & $\begin{array}{l}\text { Tine } \\
\text { (wt) }\end{array}$ & & & & & & \\
\hline $\mathbf{B a}$ & 120000 & 3 & & & $4 \times 10^{-7}$ & $38-106$ & $A=b$ & Air & 7 & \\
\hline$"$ & 100000 & 3 & & & " & $"$ & $"$ & $"$ & $"$ & \\
\hline Eut & 1300 & 3 & & & $7 \times 10^{-8}$ & $"$ & $"$ & $"$ & $"$ & \\
\hline$"$ & 1300 & 3 & & & $"$ & $"$ & 1 & $"$ & $"$ & \\
\hline $\mathrm{Sr}$ & 17000 & 3 & & & $4 \times 10^{-7}$ & $106-500$ & " & $"$ & $"$ & \\
\hline 11 & 18000 & 3 & & & $"$ & $"$ & $"$ & $"$ & $n$ & \\
\hline Cs & 14000 & 3 & & & $4 \times 10^{-9}$ & $"$ & $"$ & $"$ & $"$ & \\
\hline$"$ & 14000 & 3 & & & $"$ & $"$ & $"$ & $"$ & $n$ & \\
\hline $\mathrm{Ba}$ & 57000 & 3 & & & $4 \times 10^{-7}$ & $"$ & $"$ & $"$ & $n$ & \\
\hline$"$ & 100000 & 3 & & & $"$ & " & $"$ & 11 & " & \\
\hline Eu & 1300 & 3 & & & $7 \times 10^{-8}$ & $"$ & $"$ & $"$ & $"$ & \\
\hline$"$ & 1500 & 3 & & & $"$ & $"$ & $n$ & $"$ & $"$ & \\
\hline An & 9500 & 3 & 9900 & 12 & $1 \times 10^{-7}$ & $<75$ & $1 "$ & $"$ & 3 & \\
\hline$"$ & 7300 & 6 & 14000 & 9 & $" 1$ & $"$ & $"$ & $"$ & $"$ & \\
\hline$"$ & 2600 & 12 & 12000 & 4 & $"$ & $"$ & $"$ & $"$ & $"$ & \\
\hline$"$ & 6100 & 3 & 5900 & 12 & " & $75-500$ & $"$ & $n$ & $"$ & \\
\hline$"$ & 5200 & 6 & 9600 & 9 & $"$ & $"$ & $"$ & $"$ & $"$ & \\
\hline$"$ & 2500 & 12 & 5800 & 4 & $"$ & $"$ & $"$ & $"$ & $"$ & \\
\hline $\mathrm{Pu}$ & 130 & 3 & 1700 & 13 & $4 \times 10^{-12}$ & $<75$ & " & $"$ & $"$ & \\
\hline$"$ & 320 & 6 & 2200 & 9 & $"$ & $"$ & 4 & 1 & 11 & \\
\hline$"$ & 650 & 12 & 4300 & 3 & $"$ & $"$ & $"$ & $"$ & " & \\
\hline$"$ & 58 & 3 & 1100 & 13 & $"$ & $75-500$ & $"$ & $"$ & $"$ & \\
\hline$"$ & 120 & 6 & 1000 & 9 & $"$ & $"$ & " & $"$ & $"$ & \\
\hline$"$ & 240 & 12 & 1800 & 3 & $"$ & $"$ & $"$ & $"$ & $"$ & \\
\hline Am & 4600 & 3 & 18000 & 12 & $3 \times 10^{-8}$ & $<75$ & $"$ & CA & $"$ & \\
\hline$"$ & 8800 & 6 & 10000 & 9 & $"$ & $"$ & $"$ & " & $"$ & \\
\hline$"$ & 8200 & 12 & 19000 & 3 & $"$ & 18 & " & $"$ & $"$ & \\
\hline$"$ & 3400 & 3 & 18000 & 12 & $"$ & $75-500$ & $n$ & $"$ & $"$ & \\
\hline$"$ & 3600 & 6 & 8400 & 9 & $"$ & $"$ & $n$ & " & $"$ & \\
\hline$"$ & 5000 & 12 & 9100 & 3 & $"$ & $"$ & $n$ & " & $"$ & \\
\hline
\end{tabular}




\begin{tabular}{|c|c|c|c|c|c|c|c|c|c|c|}
\hline \multirow[b]{2}{*}{ Element } & \multicolumn{2}{|c|}{ Sorption } & \multicolumn{2}{|c|}{ Desorption } & \multirow{2}{*}{$\begin{array}{c}\text { Elewent } \\
\text { Concentration } \\
(\mathrm{M}) \\
\end{array}$} & \multirow{2}{*}{$\begin{array}{c}\text { Particle } \\
\text { Size } \\
(10) \\
\end{array}$} & \multirow[b]{2}{*}{ Teaperature } & \multirow[b]{2}{*}{ Atnosphere } & \multirow[b]{2}{*}{ Ref. } & \multirow[b]{2}{*}{ Note } \\
\hline & $\begin{array}{c}R_{d} \\
(\mu / \rho) \\
\end{array}$ & $\begin{array}{l}\text { Tine } \\
\text { (wk) }\end{array}$ & $\begin{array}{c}R_{d} \\
(\mu / /) \\
\end{array}$ & $\begin{array}{l}\text { Time } \\
\text { (wk) }\end{array}$ & & & & & & \\
\hline Pu & 500 & 3 & $>2200$ & 12 & $2 \times 10^{-13}$ & $<75$ & $A=b$ & CA & 3 & \\
\hline " & 930 & 7 & $>2000$ & 8 & " & " & $"$ & $"$ & $"$ & \\
\hline$"$ & 1100 & 12 & $>2600$ & 3 & " & $"$ & $"$ & " & $"$ & \\
\hline$"$ & 620 & 3 & $>2400$ & 13 & $n$ & $75-500$ & $"$ & $"$ & $" 1$ & \\
\hline 11 & 830 & 7 & $>2200$ & 8 & $"$ & $"$ & $"$ & $"$ & $"$ & \\
\hline$"$ & 830 & 12 & $>2300$ & 3 & $"$ & $"$ & $"$ & " & $"$ & \\
\hline $\mathrm{Np}$ & 9.3 & 4 & 17 & 12 & $4 \times 10^{-11}$ & $"$ & $"$ & Air & & $\mathbf{a}$ \\
\hline$"$ & 9.4 & 4 & 22 & 12 & $"$ & $"$ & $"$ & " & & $"$ \\
\hline$"$ & 11 & 6 & 23 & 9 & $"$ & $"$ & $"$ & $"$ & & $"$ \\
\hline " & 11 & 6 & 24 & 9 & $"$ & $"$ & $"$ & $"$ & & $"$ \\
\hline$"$ & 12 & 12 & 28 & 3 & $"$ & $"$ & 1 . & $"$ & & $"$ \\
\hline$"$ & 14 & 12 & 33 & 3 & $"$ & $"$ & " & $"$ & & $"$ \\
\hline$"$ & 14 & 4 & 22 & 12 & $2 \times 10^{-11}$ & $"$ & $"$ & $C A$ & & $"$ \\
\hline$"$ & 19 & 4 & 250 & 12 & $"$ & $"$ & $"$ & $"$ & & $" 1$ \\
\hline$"$ & 120 & 8 & 310 & 7 & $"$ & $"$ & $"$ & " & & $"$ \\
\hline " & 160 & 8 & 500 & 7 & $"$ & 1 & $"$ & " & & 11 \\
\hline$"$ & 190 & 12 & $2100^{b}$ & 3 & $"$ & $"$ & $"$ & $"$ & & $", b$ \\
\hline$"$ & 77 & 12 & 250 & 3 & $"$ & $"$ & $"$ & $"$ & & $"$ \\
\hline Tc & 2.6 & 3 & 180 & 12 & $1 \times 10^{-12}$ & $n$ & $"$ & $"$ & 4 & \\
\hline$"$ & 7.3 & 6 & 87 & 9 & $"$ & $"$ & $"$ & " & $"$ & \\
\hline$"$ & 21 & 12 & 73 & 3 & $"$ & $"$ & $"$ & $"$ & $"$ & \\
\hline$"$ & 3.0 & 3 & 160 & 12 & $"$ & $<75$ & $"$ & $"$ & $"$ & \\
\hline$"$ & 17 & 6 & 160 & 9 & $"$ & " & $"$ & 14 & $"$ & \\
\hline$"$ & 29 & 12 & 46 & 3 & $"$ & 11 & $"$ & $"$ & $"$ & \\
\hline $\mathbf{U}$ & 5 & 1 & 16 & 1 & $1 \times 10^{-6}$ & $<106$ & "t & Air & 3 & \\
\hline$"$ & 6 & 2 & 15 & 2 & $"$ & " & $"$ & $"$ & 11 & \\
\hline$"$ & 6 & 3 & & & $"$ & $"$ & $"$ & $"$ & $"$ & \\
\hline$"$ & 5 & 1 & 16 & 1 & $"$ & $106-500$ & $"$ & $"$ & $"$ & \\
\hline$"$ & 5 & 2 & 12 & 2 & $"$ & $"$ & $"$ & $n$ & $"$ & \\
\hline$"$ & 5 & 3 & & & " & $"$ & $"$ & $"$ & $1 "$ & \\
\hline$"$ & 17 & 1 & & & $"$ & $<75$ & $"$ & $\mathrm{CA}$ & 4 & \\
\hline " & 20 & 2 & & & " & $n$ & $"$ & $"$ & $"$ & \\
\hline$"$ & 14 & 3 & & & $"$ & $"$ & " & $"$ & " & \\
\hline$"$ & 11 & 1 & & & $"$ & $75-500$ & $"$ & $"$ & $"$ & \\
\hline$n$ & 14 & 2 & & & $n$ & $"$ & $"$ & $"$ & $"$ & \\
\hline$"$ & 15 & 3 & & & $n$ & " & $n$ & $"$ & $"$ & \\
\hline
\end{tabular}

Previously unpublished results.

balue not included in averages for Table XII, Chap. 1 . 
TABLE A-IX (cont)

\begin{tabular}{|c|c|c|c|c|c|c|c|c|c|c|}
\hline \multirow[b]{2}{*}{ Element } & \multicolumn{2}{|c|}{ Sorption } & \multicolumn{2}{|c|}{ Desorption } & \multirow{2}{*}{$\begin{array}{c}\text { Element } \\
\text { Concentration } \\
\text { (M) }\end{array}$} & \multirow[b]{2}{*}{$\begin{array}{c}\text { Particle } \\
\text { Size } \\
(\mu) \\
\end{array}$} & \multirow[b]{2}{*}{ Temperature } & \multirow[b]{2}{*}{ Atmosphere } & \multirow[b]{2}{*}{ Ref. } & \multirow[b]{2}{*}{ Note } \\
\hline & $\begin{array}{c}R_{d} \\
(n / /) \\
\end{array}$ & $\begin{array}{l}\text { Time } \\
\text { (wk) }\end{array}$ & $\begin{array}{c}R_{d} \\
(a l / g)\end{array}$ & $\begin{array}{l}\text { Time } \\
\text { (wk) }\end{array}$ & & & & & & \\
\hline Sx & 1200 & 3 & & & $2 \times 10^{-3}$ & $75-500$ & $A m b$ & Air & 1 & \\
\hline$"$ & 5700 & 3 & & & $2 \times 10^{-5}$ & $"$ & $"$ & $"$ & $"$ & \\
\hline$"$ & 9000 & 3 & & & $2 \times 10^{-7}$ & $"$ & $"$ & $"$ & $"$ & \\
\hline$"$ & 4100 & 3 & & & $7 \times 10^{-8}$ & $"$ & $"$ & $"$ & $"$ & \\
\hline$"$ & 1500 & 3 & & & $6 \times 10^{-8}$ & $"$ & $"$ & $"$ & $"$ & \\
\hline Cs & 3400 & 3 & & & $3 \times 10^{-3}$ & $"$ & $"$ & $"$ & $"$ & \\
\hline$"$ & 6000 & 3 & & & $3 \times 10^{-5}$ & $"$ & $"$ & $"$ & $"$ & \\
\hline$"$ & 4200 & 3 & & & $3 \times 10^{-7}$ & $"$ & $"$ & $"$ & $"$ & \\
\hline$"$ & 4000 & 3 & & & $2 \times 10^{-9}$ & $"$ & $"$ & $"$ & $"$ & \\
\hline$"$ & 3500 & 3 & & & $2 \times 10^{-10}$ & $"$ & $"$ & $"$ & $"$ & \\
\hline $\mathrm{Ba}$ & 79000 & 3 & & & $1 \times 10^{-4}$ & $"$ & $"$ & $"$ & $"$ & \\
\hline$"$ & 75000 & 3 & & & $1 \times 10^{-5}$ & $"$ & $"$ & $"$ & $"$ & \\
\hline$"$ & 36000 & 3 & & & $2 \times 10^{-6}$ & $"$ & $"$ & $"$ & $"$ & \\
\hline$"$ & 68000 & 3 & & & $6 \times 10^{-7}$ & $"$ & $"$ & $"$ & $"$ & \\
\hline$"$ & 33000 & 3 & & & $4 \times 10^{-7}$ & $"$ & $"$ & $"$ & $"$ & \\
\hline $\mathbf{E} u$ & 4600 & 3 & & & $6 \times 10^{-6}$ & $"$ & $"$ & $"$ & $"$ & \\
\hline$"$ & 1700 & 3 & & & $4 \times 10^{-6}$ & $"$ & $"$ & $"$ & $"$ & \\
\hline$"$ & 1700 & 3 & & & $6 \times 10^{-8}$ & $"$ & $"$ & $"$ & $"$ & \\
\hline$"$ & 2700 & 3 & & & $4 \times 10^{-8}$ & $"$ & $"$ & $"$ & $"$ & \\
\hline$"$ & 3700 & 3 & & & $6 \times 10^{-9}$ & $"$ & $"$ & $"$ & $"$ & \\
\hline $\mathrm{Sr}_{\mathbf{r}}$ & 1500 & 3 & & & $2 \times 10^{-3}$ & $<75$ & $"$ & $"$ & $"$ & \\
\hline$"$ & 13000 & 3 & & & $2 \times 10^{-5}$ & $"$ & $"$ & $"$ & $"$ & \\
\hline$"$ & 10000 & 3 & & & $2 \times 10^{-7}$ & $"$ & $"$ & $"$ & $"$ & \\
\hline$"$ & 10000 & 3 & & & $7 \times 10^{-8}$ & $"$ & $"$ & $"$ & $"$ & \\
\hline$"$ & 3300 & 3 & & & $5 \times 10^{-8}$ & $"$ & $"$ & $"$ & $"$ & \\
\hline Cs & 3500 & 3 & & & $3 \times 10^{-3}$ & $"$ & $"$ & $"$ & $"$ & \\
\hline$"$ & 3700 & 3 & & & $3 \times 10^{-5}$ & $"$ & $"$ & $"$ & $"$ & \\
\hline$"$ & 6300 & 3 & & & $3 \times 10^{-7}$ & $"$ & $"$ & $"$ & $"$ & \\
\hline$"$ & 2700 & 3 & & & $2 \times 10^{-9}$ & $"$ & $"$ & $"$ & $"$ & \\
\hline$"$ & 3200 & 3 & & & $2 \times 10^{-10}$ & $"$ & $"$ & $"$ & $"$ & \\
\hline $\mathbf{B a}$ & 100000 & 3 & & & $1 \times 10^{-4}$ & $"$ & $"$ & $"$ & $"$ & \\
\hline$"$ & 93000 & 3 & & & $1 \times 10^{-5}$ & $"$ & $"$ & $"$ & $"$ & \\
\hline$"$ & 97000 & 3 & & & $2 \times 10^{-6}$ & $"$ & $"$ & $"$ & $"$ & \\
\hline$"$ & 81000 & 3 & & & $6 \times 10^{-7}$ & $"$ & $"$ & $"$ & $"$ & \\
\hline$"$ & 72000 & 3 & & & $4 \times 10^{-7}$ & $"$ & $"$ & $"$ & $"$ & \\
\hline
\end{tabular}


TAIIE A-IX (cont)

\begin{tabular}{|c|c|c|c|c|c|c|c|c|c|c|}
\hline \multirow[b]{2}{*}{ Elenent } & \multicolumn{2}{|c|}{ Sorption } & \multicolumn{2}{|c|}{ Desorption } & \multirow[b]{2}{*}{$\begin{array}{c}\text { Element } \\
\text { Concentration } \\
(\mathrm{H}) \\
\end{array}$} & \multirow[b]{2}{*}{$\begin{array}{c}\text { Particle } \\
\text { Size } \\
\text { (1) } \\
\end{array}$} & \multirow[b]{2}{*}{ Teuperature } & \multirow[b]{2}{*}{ Atrosphere } & \multirow[b]{2}{*}{ Ref. } & \multirow[b]{2}{*}{ Note } \\
\hline & $\begin{array}{c}R_{d} \\
(n) / g)\end{array}$ & $\begin{array}{l}\text { Time } \\
\text { (wk) }\end{array}$ & $\begin{array}{c}R_{d} \\
(n / /)\end{array}$ & $\begin{array}{l}\text { Tine } \\
\text { (wl) }\end{array}$ & & & & & & \\
\hline Eu & 7100 & 3 & & & $6 \times 10^{-6}$ & $<75$ & $A=b$ & Air & 1 & \\
\hline "t & 2600 & 3 & & & $3 \times 10^{-6}$ & $"$ & " & " & $"$ & \\
\hline$"$ & 3700 & 3 & & & $6 \times 10^{-8}$ & $"$ & $"$ & 4 & $"$ & \\
\hline$"$ & 3100 & 3 & & & $4 \times 10^{-8}$ & $"$ & $"$ & " & $"$ & \\
\hline$n$ & 5000 & 3 & & & $6 \times 10^{-9}$ & $"$ & $"$ & $" 1$ & $"$ & \\
\hline
\end{tabular}


TALE A-X

MATCK SORPTION DATA FOR TUFF YM-42

\begin{tabular}{|c|c|c|c|c|c|c|c|c|c|c|}
\hline \multirow[b]{2}{*}{ Elenent } & \multicolumn{2}{|c|}{ Sorption } & \multicolumn{2}{|c|}{ Desorption } & \multirow{2}{*}{$\begin{array}{c}\text { Element } \\
\text { Concentration } \\
\text { (M) }\end{array}$} & \multirow[b]{2}{*}{$\begin{array}{c}\text { Particle } \\
\text { Size } \\
\text { (1- }) \\
\end{array}$} & \multirow[b]{2}{*}{ Temperature } & \multirow[b]{2}{*}{ Atrosphere } & \multirow[b]{2}{*}{ Ref. } & \multirow[b]{2}{*}{ Note } \\
\hline & $\begin{array}{c}R_{d} \\
(n e / g)\end{array}$ & $\begin{array}{l}\text { Tine } \\
\text { (wk) } \\
\end{array}$ & $\begin{array}{c}R_{d} \\
(n e / R)\end{array}$ & $\begin{array}{l}\text { Tine } \\
\text { (wk) }\end{array}$ & & & & & & \\
\hline$S x$ & 2900 & 3 & 3300 & 12 & $8 \times 10^{-11}$ & $75-500$ & $A=b$ & Air & 3 & $\mathbf{a}$ \\
\hline$"$ & 3900 & 6 & 3000 & 9 & $"$ & $"$ & $"$ & $"$ & " & $"$ \\
\hline$"$ & 5000 & 12 & 6000 & 3 & $"$ & $"$ & $"$ & $"$ & $"$ & $"$ \\
\hline Cs & 16000 & 3 & 19000 & 12 & $3 \times 10^{-9}$ & $"$ & $"$ & $"$ & $"$ & $"$ \\
\hline$"$ & 17000 & 6 & 19000 & 9 & $"$ & $"$ & $"$ & $"$ & $"$ & " \\
\hline$"$ & 19000 & 12 & 25000 & 3 & " & $"$ & $"$ & $"$ & $"$ & $"$ \\
\hline $\mathrm{Ba}$ & 72000 & 3 & 42000 & 12 & $8 \times 10^{-10}$ & $"$ & $"$ & $"$ & $"$ & $"$ \\
\hline$"$ & 90000 & 6 & 83000 & 9 & $"$ & $"$ & $"$ & $"$ & $"$ & $"$ \\
\hline$"$ & 120000 & 12 & 150000 & 3 & $"$ & $"$ & $"$ & $"$ & $"$ & $"$ \\
\hline $\mathrm{Ce}$ & 35000 & 3 & 46000 & 12 & $8 \times 10^{-13}$ & $"$ & $"$ & $"$ & $"$ & $"$ \\
\hline$"$ & 47000 & 6 & 35000 & 9 & $"$ & $"$ & $"$ & $"$ & $"$ & $"$ \\
\hline$"$ & 39000 & 12 & 52000 & 3 & $"$ & $"$ & $"$ & $"$ & $"$ & $"$ \\
\hline Eu & 45000 & 3 & 64000 & 12 & $1 \times 10^{-10}$ & $"$ & $"$ & $"$ & $"$ & $"$ \\
\hline$"$ & 58000 & 6 & 59000 & 9 & $"$ & $"$ & $"$ & $"$ & $"$ & $"$ \\
\hline$"$ & 53000 & 12 & 70000 & 3 & $"$ & " & " & " & $"$ & $"$ \\
\hline
\end{tabular}

aconcentrations are only those added with the tracer; actual concentrations may be higher. 
TANLE A-X]

MTCH SOAPTION DATA FOR TUFF M-45

\begin{tabular}{|c|c|c|c|c|c|c|c|c|c|c|}
\hline \multirow[b]{2}{*}{ Element } & \multicolumn{2}{|c|}{ Sorption } & \multicolumn{2}{|c|}{ Desorption } & \multirow{2}{*}{$\begin{array}{c}\text { Element } \\
\text { Concentration } \\
\text { (II) } \\
\end{array}$} & \multirow{2}{*}{$\begin{array}{c}\text { Particle } \\
\text { Sixe } \\
\text { (pe) } \\
\end{array}$} & \multirow[b]{2}{*}{ Te-perature } & \multirow[b]{2}{*}{ Ateosphere } & \multirow[b]{2}{*}{ Ref. } & \multirow[b]{2}{*}{ Note } \\
\hline & $\begin{array}{c}R_{d} \\
(\alpha / 2)\end{array}$ & $\begin{array}{l}\text { Tine } \\
\text { (wt) }\end{array}$ & $\begin{array}{c}x_{\mathrm{d}} \\
(\omega 2 / \mathrm{s})\end{array}$ & $\begin{array}{l}\text { Tine } \\
\text { (wk) }\end{array}$ & & & & & & \\
\hline $5 r$ & 170 & 3 & 190 & 6 & $6 \times 10^{-7}$ & $\overline{106-500}$ & $A=b$ & Air & $\overline{4}$ & \\
\hline$"$ & 170 & 6 & 180 & 3 & $"$ & $"$ & $"$ & $"$ & $"$ & \\
\hline$C=$ & 480 & 3 & 450 & 6 & $1 \times 10^{-9}$ & $"$ & $"$ & $"$ & $"$ & \\
\hline$"$ & 320 & 6 & 430 & 3 & $"$ & $"$ & $"$ & $"$ & $"$ & \\
\hline $\mathbf{B a}$ & 1200 & 3 & $150 c$ & 6 & $3 \times 10^{-7}$ & $"$ & $"$ & $"$ & $"$ & \\
\hline$"$ & 1400 & 6 & 1200 & 3 & $"$ & $"$ & $"$ & $"$ & $"$ & \\
\hline Ce & 1000 & 3 & 7400 & 6 & $9 \times 10^{-7}$ & $"$ & $"$ & $"$ & $"$ & \\
\hline$"$ & 710 & 6 & 4700 & 3 & $"$ & $"$ & $"$ & $"$ & $"$ & \\
\hline Eu & 2100 & 3 & 7100 & 6 & $1 \times 10^{-7}$ & $"$ & $"$ & $"$ & $"$ & \\
\hline$"$ & 1600 & 6 & 6400 & 3 & $"$ & $"$ & $"$ & $"$ & $"$ & \\
\hline Sr & 220 & 3 & 240 & 6 & $6 \times 10^{-7}$ & $<106$ & $"$ & $"$ & $"$ & \\
\hline$"$ & 220 & 6 & 230 & 3 & $"$ & $"$ & $"$ & $"$ & $"$ & \\
\hline Cs & 740 & 3 & 780 & 6 & $1 \times 10^{-9}$ & $"$ & $"$ & $"$ & $"$ & \\
\hline$"$ & 550 & 6 & 840 & 3 & $"$ & $"$ & $"$ & $"$ & $"$ & \\
\hline Ba & 970 & 3 & 1200 & 6 & $3 \times 10^{-7}$ & $"$ & $"$ & $"$ & $"$ & \\
\hline$"$ & 1230 & 6 & 1300 & 3 & $"$ & " & $"$ & $"$ & $"$ & \\
\hline Ce & 540 & 3 & 4100 & 6 & $9 \times 10^{-7}$ & $"$ & $"$ & $"$ & $"$ & \\
\hline$"$ & 670 & 6 & 6800 & 3 & $"$ & " & $"$ & $"$ & $"$ & \\
\hline Eu & 990 & 3 & 5800 & 6 & $1 \times 10^{-7}$ & " & $"$ & $"$ & " & \\
\hline " & 1700 & 6 & 9700 & 3 & $"$ & $"$ & $M$ & $"$ & $n$ & \\
\hline
\end{tabular}


TABLE A-XII

BATCH SOMPTIOI DATA FOR TUIF M-46

\begin{tabular}{|c|c|c|c|c|c|c|c|c|c|c|}
\hline \multirow[b]{2}{*}{ Elevent } & \multicolumn{2}{|c|}{ Sorption } & \multicolumn{2}{|c|}{ Desorption } & \multirow[b]{2}{*}{$\begin{array}{c}\text { Element } \\
\text { Concentration } \\
\text { (M) }\end{array}$} & \multirow[b]{2}{*}{$\begin{array}{c}\text { Particle } \\
\text { Size } \\
(1) \\
\end{array}$} & \multirow[b]{2}{*}{ Teeperature } & \multirow[b]{2}{*}{ Atmosphere } & \multirow[b]{2}{*}{ Ref. } & \multirow[b]{2}{*}{ Note } \\
\hline & $\begin{array}{c}R_{d} \\
(n e / /)\end{array}$ & $\begin{array}{l}\text { Time } \\
\text { (wk) }\end{array}$ & $\begin{array}{c}R_{d} \\
(\boldsymbol{\mu} / \boldsymbol{l})\end{array}$ & $\begin{array}{l}\text { Tine } \\
\text { (wk) }\end{array}$ & & & & & & \\
\hline $\mathbf{S r}$ & 90 & 3 & 280 & 12 & $8 \times 10^{-11}$ & $75-500$ & $\mathbf{A b}$ & Air & 3 & $\approx$ \\
\hline$"$ & 170 & 6 & 290 & 9 & $"$ & $"$ & $"$ & $"$ & $"$ & $"$ \\
\hline$n$ & 300 & 12 & 220 & 3 & $"$ & $"$ & $"$ & $"$ & $"$ & $"$ \\
\hline Cs & 500 & 3 & 1800 & 12 & $3 \times 10^{-9}$ & $"$ & $"$ & $"$ & $"$ & $"$ \\
\hline$"$ & 320 & 6 & 2200 & 9 & " & $"$ & $"$ & $"$ & $"$ & $"$ \\
\hline$"$ & 1700 & 12 & 1300 & 3 & $"$ & $"$ & $"$ & $"$ & $"$ & $"$ \\
\hline $\mathbf{B a}$ & 6500 & 3 & 23000 & 12 & $8 \times 10^{-10}$ & $"$ & 11 & $"$ & $"$ & $"$ \\
\hline$"$ & 11000 & 6 & 25000 & 9 & $"$ & $"$ & $" 1$ & $"$ & $"$ & $"$ \\
\hline$"$ & 25000 & 12 & 16000 & 3 & $"$ & $"$ & $"$ & $"$ & $"$ & $"$ \\
\hline $\mathrm{Ce}$ & 97000 & 3 & 370000 & 12 & $8 \times 10^{-13}$ & $"$ & $"$ & $"$ & $"$ & $"$ \\
\hline$"$ & 360000 & 6 & 400000 & 9 & $"$ & $"$ & $"$ & $"$ & $"$ & $"$ \\
\hline$"$ & 470000 & 12 & 230000 & 3 & $"$ & $"$ & $"$ & $"$ & $"$ & " \\
\hline Eu & 110000 & 3 & 27000 & 12 & $1 \times 10^{-10}$ & $"$ & $"$ & $n$ & $"$ & $"$ \\
\hline$"$ & 320000 & 6 & 35000 & 9 & $"$ & " & $"$ & $"$ & $"$ & $"$ \\
\hline$"$ & 490000 & 12 & 31000 & 3 & $"$ & $1 "$ & $"$ & " & " & $"$ \\
\hline
\end{tabular}

\footnotetext{
a Concentrations are only those added with the tracer; actual concentrations way be higher.
} 
TABLE A-XIII

BATCH SORPTION DATA FOR TUFF MM-4B

\begin{tabular}{|c|c|c|c|c|c|c|c|c|c|c|}
\hline \multirow[b]{2}{*}{ Element } & \multicolumn{2}{|c|}{ Sorption } & \multicolumn{2}{|c|}{ Desorption } & \multirow{2}{*}{$\begin{array}{c}\text { Element } \\
\text { Concentration } \\
\frac{(M)}{} \\
\end{array}$} & \multirow[b]{2}{*}{$\begin{array}{c}\text { Particle } \\
\text { Size } \\
\text { (1ص) } \\
\end{array}$} & \multirow[b]{2}{*}{ Temperature } & \multirow[b]{2}{*}{ Atmosphere } & \multirow[b]{2}{*}{ Ref. } & \multirow[b]{2}{*}{ Note } \\
\hline & $\begin{array}{c}R_{d} \\
(\alpha / 2)\end{array}$ & $\begin{array}{l}\text { Time } \\
\text { (wk) }\end{array}$ & $\begin{array}{c}R_{d} \\
(n e / 2)\end{array}$ & $\begin{array}{l}\text { Time } \\
\text { (uk) }\end{array}$ & & & & & & \\
\hline $\mathbf{S r}$ & 15000 & 3 & & & $7 \times 10^{-8}$ & $106-500$ & $A=b$ & Air & 4 & \\
\hline$"$ & 2500 & 6 & 2900 & 3 & $"$ & $"$ & $"$ & $"$ & $"$ & \\
\hline C: & 15000 & 3 & & & $1 \times 10^{-9}$ & $"$ & $"$ & $"$ & $"$ & \\
\hline$"$ & 22000 & 6 & 30000 & 3 & $"$ & $"$ & $"$ & $"$ & $"$ & \\
\hline Ba & 12000 & 3 & & & $4 \times 10^{-7}$ & $"$ & $"$ & $"$ & $"$ & \\
\hline$"$ & 23000 & 6 & 41000 & 3 & $"$ & $"$ & $"$ & $"$ & $"$ & \\
\hline Ce & 870 & 3 & & & $7 \times 10^{-7}$ & $"$ & $"$ & $"$ & $"$ & \\
\hline$"$ & 1900 & 6 & 12000 & 3 & $"$ & $"$ & $"$ & $"$ & $"$ & \\
\hline Eu & 1700 & 3 & & & $5 \times 10^{-8}$ & $"$ & $"$ & $"$ & $"$ & \\
\hline$"$ & 2700 & 6 & 9300 & 3 & $"$ & $"$ & $"$ & $"$ & $"$ & \\
\hline Sr & 1200 & 6 & 2400 & 3 & $7 \times 10^{-8}$ & $<106$ & $"$ & $"$ & $"$ & \\
\hline Cs & 6900 & 6 & 23000 & 3 & $1 \times 10^{-9}$ & $"$ & $"$ & $"$ & $"$ & \\
\hline Ba & 10000 & 6 & 27000 & 3 & $4 \times 10^{-7}$ & $"$ & $"$ & $"$ & $"$ & \\
\hline Ce & 2900 & 6 & 13000 & 3 & $7 \times 10^{-7}$ & $"$ & $"$ & $"$ & $"$ & \\
\hline $\mathbf{E u}$ & 3100 & 6 & 6800 & 3 & $5 \times 10^{-B}$ & $"$ & $"$ & $"$ & $"$ & \\
\hline Te & 0.16 & 6 & 1.5 & 9 & $1 \times 10^{-3}$ & $106-500$ & $"$ & $"$ & $"$ & \\
\hline$"$ & 0.14 & 6 & 1.2 & 9 & $1 \times 10^{-6}$ & $"$ & $"$ & $"$ & $"$ & \\
\hline$"$ & 0.11 & 6 & 2.3 & 9 & $1 \times 10^{-9}$ & $"$ & $"$ & $"$ & $"$ & \\
\hline$"$ & 0.2 & 6 & 1.6 & 9 & $1 \times 10^{-12}$ & $"$ & $"$ & $"$ & $"$ & \\
\hline
\end{tabular}


TABLE A-XIV

BATCH SORPTION DATA FOR TUEF MA-49

\begin{tabular}{|c|c|c|c|c|c|c|c|c|c|c|}
\hline \multirow[b]{2}{*}{ Elesent } & \multicolumn{2}{|c|}{ Sorption } & \multicolumn{2}{|c|}{ Desorption } & \multirow[b]{2}{*}{$\begin{array}{c}\text { Eleaent } \\
\text { Concentration } \\
\text { (H) }\end{array}$} & \multirow[b]{2}{*}{$\begin{array}{c}\text { Particle } \\
\text { Size } \\
\text { (y) } \\
\end{array}$} & \multirow[b]{2}{*}{ Teuperature } & \multirow[b]{2}{*}{ Atmosphere } & \multirow[b]{2}{*}{ Ref. } & \multirow[b]{2}{*}{ Hote } \\
\hline & $\begin{array}{c}R_{d} \\
(-e / g)\end{array}$ & $\begin{array}{l}\text { Tine } \\
\text { (wk) }\end{array}$ & $\begin{array}{c}R_{d} \\
(\alpha / /)\end{array}$ & $\begin{array}{l}\text { Tine } \\
\text { (wk) }\end{array}$ & & & & & & \\
\hline $\mathbf{S r}$ & 2700 & 3 & 4400 & 6 & $6 \times 10^{-8}$ & $106-500$ & $A=b$ & Air & 4 & \\
\hline$"$ & 2600 & 3 & & & $"$ & $"$ & $"$ & " & $"$ & \\
\hline$"$ & 3800 & 6 & 4400 & 3 & $"$ & $H$ & $"$ & $"$ & $"$ & \\
\hline$"$ & 3800 & 6 & 4300 & 3 & 11 & $"$ & $"$ & $"$ & $"$ & \\
\hline Cs & 29000 & 3 & 38000 & 6 & $1 \times 10^{-9}$ & $"$ & $"$ & $"$ & $"$ & \\
\hline$"$ & 37000 & 3 & & & $"$ & $"$ & " & " & $"$ & \\
\hline " & 36000 & 6 & 40000 & 3 & $"$ & $"$ & $"$ & $"$ & $"$ & \\
\hline " & 43000 & 6 & 40000 & 3 & $"$ & " & $"$ & $"$ & $"$ & \\
\hline $\mathbf{B a}$ & 26000 & 3 & 51000 & 6 & $4 \times 10^{-7}$ & $"$ & $1 "$ & $n$ & $n$ & \\
\hline 11 & 30000 & 3 & & & $n$ & $"$ & $"$ & $n$ & 11 & \\
\hline 1 & 59000 & 6 & 69000 & 3 & $n$ & $"$ & $"$ & " & $"$ & \\
\hline$"$ & 54000 & 6 & 76000 & 3 & $n$ & $"$ & $"$ & $"$ & $n$ & \\
\hline Ce & 560 & 3 & 1000 & 6 & $3 \times 10^{-7}$ & $"$ & $"$ & $"$ & $"$ & \\
\hline$"$ & 490 & 3 & & & $"$ & 14 & " & $"$ & $"$ & \\
\hline 11 & 810 & 6 & 1100 & 3 & $"$ & $"$ & " & $"$ & $"$ & \\
\hline$"$ & 350 & 6 & 970 & 3 & $"$ & $"$ & $"$ & $"$ & $"$ & \\
\hline Eu & 1000 & 3 & 1600 & 6 & $1 \times 10^{-7}$ & $"$ & " & 11 & $"$ & \\
\hline$"$ & 1200 & 3 & & & $"$ & $"$ & " & " & $"$ & \\
\hline$"$ & 1500 & 6 & 3100 & 3 & $"$ & $"$ & " & $"$ & " & \\
\hline$"$ & 1000 & 6 & 1600 & 3 & $"$ & $"$ & $"$ & $"$ & $"$ & \\
\hline $\mathbf{S r}$ & 1500 & 3 & 2400 & 6 & $6 \times 10^{-8}$ & $<106$ & $"$ & $"$ & $"$ & \\
\hline$"$ & 2400 & 6 & & & $"$ & $"$ & $"$ & $"$ & $"$ & \\
\hline Cs & 22000 & 3 & 15000 & 6 & $1 \times 10^{-9}$ & $"$ & $"$ & $"$ & " & \\
\hline$"$ & 8700 & 6 & & & $"$ & $"$ & " & $"$ & " & \\
\hline $\mathbf{B a}$ & 17000 & 3 & 28000 & 6 & $4 \times 10^{-7}$ & " & " & $"$ & $"$ & \\
\hline$"$ & 9200 & 6 & & & $"$ & $"$ & " & $"$ & $"$ & \\
\hline $\mathrm{Ce}$ & 570 & 3 & 1400 & 6 & $3 \times 10^{-7}$ & $"$ & $"$ & $"$ & $"$ & \\
\hline$"$ & 530 & 6 & & & " & $"$ & " & $"$ & " & \\
\hline Eu & 1400 & 3 & 2200 & 6 & $1 \times 10^{-7}$ & " & $"$ & $"$ & $n$ & \\
\hline$"$ & 1300 & 6 & & & " & $"$ & $"$ & " & $"$ & \\
\hline
\end{tabular}


TALE A-XIV (cont)

\begin{tabular}{|c|c|c|c|c|c|c|c|c|c|c|}
\hline \multirow[b]{2}{*}{ Element } & \multicolumn{2}{|c|}{ Sorption } & \multicolumn{2}{|c|}{ Deseorption } & \multirow{2}{*}{$\begin{array}{c}\text { Elewent } \\
\text { Concentration } \\
\text { (H) } \\
\end{array}$} & \multirow{2}{*}{$\begin{array}{c}\text { Parifcle } \\
\text { Size } \\
\text { (1⿴) } \\
\end{array}$} & \multirow[b]{2}{*}{ Teuperature } & \multirow[b]{2}{*}{ Atmosphere } & \multirow[b]{2}{*}{ Ref. } & \multirow[b]{2}{*}{ Note } \\
\hline & $\begin{array}{c}R_{d} \\
(n \ell / \varepsilon)\end{array}$ & $\begin{array}{l}\text { Time } \\
\text { (wk) }\end{array}$ & $\begin{array}{c}R_{d} \\
(n \ell / \Omega)\end{array}$ & $\begin{array}{l}\text { Tine } \\
\text { (wk) }\end{array}$ & & & & & & \\
\hline An & 2900 & 3 & $19000^{\circ}$ & 12 & $1 \times 10^{-7}$ & $106-250$ & Amb & Air & 6,9 & a \\
\hline$"$ & 2800 & 6 & 3800 & 9 & $"$ & $"$ & $"$ & $"$ & $"$ & \\
\hline$"$ & 7100 & 12 & 3000 & 3 & $"$ & $"$ & $"$ & $"$ & $"$ & \\
\hline
\end{tabular}

\begin{tabular}{|c|c|c|c|c|c|c|c|c|c|}
\hline Pu & 170 & 3 & 700 & 3 & $2 \times 10^{-12}$ & $<75$ & $"$ & $"$ & 1 \\
\hline$"$ & 160 & 3 & 840 & 3 & $8 \times 10^{-12}$ & $"$ & " & $"$ & $"$ \\
\hline$"$ & 130 & 3 & 780 & 3 & $1 \times 10^{-10}$ & $"$ & $"$ & $"$ & $"$ \\
\hline$"$ & 180 & 3 & 490 & 3 & $4 \times 10^{-10}$ & $"$ & $"$ & $"$ & $"$ \\
\hline$"$ & 270 & 3 & 690 & 3 & $3 \times 10^{-8}$ & $"$ & $"$ & $"$ & $"$ \\
\hline$"$ & 150 & 3 & 670 & 3 & $2 \times 10^{-12}$ & $75-500$ & " & $"$ & $"$ \\
\hline$"$ & 89 & 3 & 790 & 3 & $8 \times 10^{-12}$ & $"$ & $"$ & $"$ & $"$ \\
\hline$"$ & 140 & 3 & 590 & 3 & $1 \times 10^{-10}$ & $"$ & $"$ & $"$ & $"$ \\
\hline$"$ & 220 & 3 & 620 & 3 & $4 \times 10^{-10}$ & $"$ & $"$ & $"$ & $"$ \\
\hline$"$ & 240 & 3 & 340 & 3 & $3 \times 10^{-8}$ & $"$ & $"$ & $"$ & $"$ \\
\hline$"$ & 130 & 3 & 720 & 3 & $3 \times 10^{-12}$ & $"$ & $"$ & $"$ & 7 \\
\hline$"$ & 240 & 3 & 700 & 3 & $2 \times 10^{-11}$ & $"$ & $"$ & $"$ & $"$ \\
\hline " & 390 & 3 & 1300 & 3 & $1 \times 10^{-10}$ & $"$ & $"$ & $"$ & $"$ \\
\hline$"$ & $2000^{b}$ & 3 & 1700 & 3 & $8 \times 10^{-10}$ & $"$ & $"$ & $"$ & $"$ \\
\hline$"$ & 120 & 3 & 560 & 3 & $1 \times 10^{-8}$ & $"$ & $"$ & $"$ & $"$ \\
\hline$"$ & 140 & 3 & 390 & 12 & $3 \times 10^{-12}$ & $106-250$ & $"$ & $"$ & $"$ \\
\hline$"$ & 160 & 6 & 410 & 9 & $"$ & $"$ & $"$ & $"$ & $"$ \\
\hline$"$ & 200 & 6 & 440 & 9 & $"$ & $"$ & " & $"$ & " \\
\hline " & 210 & 12 & 660 & 3 & " & $"$ & " & $"$ & $"$ \\
\hline$"$ & 820 & 12 & 930 & 3 & $"$ & $"$ & $"$ & $"$ & $"$ \\
\hline
\end{tabular}

\begin{tabular}{|c|c|c|c|c|c|c|c|c|c|}
\hline Np & 15 & 3 & 6.7 & 12 & $7 \times 10^{-11}$ & $"$ & $"$ & $"$ & 6 \\
\hline$"$ & 5.3 & 6 & 9.2 & 9 & $"$ & $"$ & $"$ & $"$ & $"$ \\
\hline$n$ & 6.3 & 12 & 20 & 3 & $"$ & $"$ & $"$ & $"$ & " \\
\hline Tc & 0.16 & 6 & 1.3 & 9 & $1 \times 10^{-3}$ & $106-500$ & " & $"$ & 4 \\
\hline$"$ & 0.20 & 6 & 1.5 & 9 & $1 \times 10^{-6}$ & $"$ & $"$ & $"$ & $"$ \\
\hline$"$ & 0.25 & 6 & 2.7 & 9 & $1 \times 10^{-9}$ & " & $"$ & $"$ & " \\
\hline$n$ & 0.23 & 6 & 2.3 & 9 & $1 \times 10^{-12}$ & $"$ & $"$ & $"$ & " \\
\hline
\end{tabular}

"Value not included in everages of Table XXII, Chap. 1.

byalue not included in averages of Table XXI, Chap. 1 .

Rock pretreated for 4.5 wanths. 


\begin{tabular}{|c|c|c|c|c|c|c|c|c|c|c|}
\hline \multirow[b]{2}{*}{ Elesent } & \multicolumn{2}{|c|}{ Sorption } & \multicolumn{2}{|c|}{ Desorption } & \multirow{2}{*}{$\begin{array}{c}\text { Elewent } \\
\text { Concentration } \\
(\mathrm{M}) \\
\end{array}$} & \multirow[b]{2}{*}{$\begin{array}{c}\text { Particle } \\
\text { Size } \\
(\mu) \\
\end{array}$} & \multirow[b]{2}{*}{ Temperature } & \multirow[b]{2}{*}{ Atwosphere } & \multirow[b]{2}{*}{ Ref. } & \multirow[b]{2}{*}{ Note } \\
\hline & $\begin{array}{c}R_{d} \\
(e / s)\end{array}$ & $\begin{array}{l}\text { Time } \\
\text { (wk) }\end{array}$ & $\begin{array}{c}R_{d} \\
(n \ell / \Omega)\end{array}$ & $\begin{array}{l}\text { Time } \\
\text { (wk) }\end{array}$ & & & & & & \\
\hline Sr & 88 & 3 & 80 & 6 & $6 \times 10^{-7}$ & $106-500$ & Amb & Air & 4 & \\
\hline$"$ & 80 & 6 & 84 & 3 & $"$ & $"$ & $"$ & $"$ & $"$ & \\
\hline Cs & 290 & 3 & 290 & 6 & $2 \times 10^{-9}$ & $"$ & $"$ & $"$ & $"$ & \\
\hline$"$ & 200 & 6 & 350 & 3 & $"$ & $"$ & $"$ & $"$ & $"$ & \\
\hline Ba & 720 & 3 & 620 & 6 & $3 \times 10^{-7}$ & $"$ & $n$ & $"$ & $"$ & \\
\hline$"$ & 590 & 6 & 640 & 3 & $"$ & $"$ & $"$ & $"$ & $"$ & \\
\hline Ce & 180 & 3 & 1500 & 6 & $9 \times 10^{-7}$ & $"$ & $"$ & $"$ & $"$ & \\
\hline$"$ & 110 & 6 & 720 & 3 & $"$ & $"$ & $"$ & $"$ & $"$ & \\
\hline Eu & 580 & 3 & 2100 & 6 & $1 \times 10^{-7}$ & $"$ & $"$ & $"$ & $"$ & \\
\hline$"$ & 370 & 6 & 1700 & 3 & $"$ & $"$ & $"$ & $"$ & $"$ & \\
\hline Sr & 95 & 3 & 120 & 6 & $6 \times 10^{-7}$ & $<106$ & $"$ & $n$ & $"$ & \\
\hline$"$ & 97 & 6 & 110 & 3 & $"$ & $"$ & $"$ & $"$ & $"$ & \\
\hline Cs & 270 & 3 & 270 & 6 & $2 \times 10^{-9}$ & $"$ & $"$ & $"$ & $"$ & \\
\hline$"$ & 230 & 6 & 320 & 3 & $"$ & $"$ & $"$ & $"$ & $"$ & \\
\hline Ba & 520 & 3 & 690 & 6 & $3 \times 10^{-7}$ & $"$ & $"$ & $"$ & $"$ & \\
\hline$"$ & 650 & 6 & 680 & 3 & $"$ & $"$ & $"$ & $"$ & $"$ & \\
\hline Ce & 110 & 3 & 1000 & 6 & $9 \times 10^{-7}$ & $"$ & $"$ & $"$ & $"$ & \\
\hline$"$ & 170 & 6 & 690 & 3 & $"$ & $"$ & $"$ & $"$ & $"$ & \\
\hline Eu & 390 & 3 & 1900 & 6 & $1 \times 10^{-7}$ & $"$ & $"$ & $"$ & $"$ & \\
\hline$"$ & 700 & 6 & 1700 & 3 & $"$ & $"$ & $"$ & $"$ & $"$ & \\
\hline Sr & 76 & 3 & 110 & 6 & $8 \times 10^{-8}$ & $75-500$ & $"$ & $\mathrm{CA}$ & 4,8 & a \\
\hline$"$ & 130 & 6 & 120 & 3 & $"$ & $"$ & $"$ & $"$ & $"$ & $"$ \\
\hline$"$ & 70 & 12 & & & $"$ & $"$ & $"$ & $"$ & $"$ & $"$ \\
\hline Cs & 210 & 3 & 260 & 6 & $3 \times 10^{-9}$ & $"$ & $"$ & $"$ & $"$ & $"$ \\
\hline$"$ & 260 & 6 & 310 & 3 & $"$ & $"$ & $"$ & $"$ & $"$ & " \\
\hline$"$ & 240 & 12 & & & $"$ & $"$ & " & $"$ & $"$ & $"$ \\
\hline Ba & 870 & 3 & 470 & 6 & $1 \times 10^{-7}$ & $"$ & $"$ & $"$ & $"$ & $"$ \\
\hline$"$ & 620 & 6 & 640 & 3 & $"$ & $"$ & $"$ & $"$ & $"$ & $"$ \\
\hline$"$ & 350 & 12 & & & $"$ & $"$ & $"$ & $"$ & $"$ & $"$ \\
\hline
\end{tabular}

Concentrations are only those added with the tracer; actual concentrations may be higher. 


\begin{tabular}{|c|c|c|c|c|c|c|c|c|c|c|}
\hline \multirow[b]{2}{*}{ Element } & \multicolumn{2}{|c|}{ Sorption } & \multicolumn{2}{|c|}{ Desorption } & \multirow{2}{*}{$\begin{array}{c}\text { Ilement } \\
\text { Concentration } \\
(\mathrm{H}) \\
\end{array}$} & \multirow{2}{*}{$\begin{array}{c}\text { Particle } \\
\text { Size } \\
(y) \\
\end{array}$} & \multirow[b]{2}{*}{ Te-perature } & \multirow[b]{2}{*}{ Atmosphere } & \multirow[b]{2}{*}{ Ref. } & \multirow[b]{2}{*}{ Note } \\
\hline & $\begin{array}{c}R_{d} \\
(n e / s)\end{array}$ & $\begin{array}{l}\text { Tive } \\
\text { (wl) }\end{array}$ & $\begin{array}{c}R_{d} \\
(n / /)\end{array}$ & $\begin{array}{l}\text { Time } \\
\text { (wk) }\end{array}$ & & & & & & \\
\hline $\mathrm{Ce}$ & 110 & 3 & 680 & 6 & $1 \times 10^{-7}$ & $75-500$ & $A b$ & $\mathbf{C A}$ & 4,8 & $a$ \\
\hline$"$ & 540 & 6 & 2100 & 3 & $"$ & $"$ & 14 & $"$ & $"$ & $"$ \\
\hline$" 1$ & 490 & 12 & & & $"$ & $"$ & $"$ & 11 & $"$ & $"$ \\
\hline Eu & 330 & 3 & 1500 & 6 & $2 \times 10^{-7}$ & $n$ & $"$ & $"$ & $"$ & $"$ \\
\hline$n$ & 850 & 6 & 2100 & 3 & $"$ & it & $"$ & " & $n$ & $"$ \\
\hline$n$ & 770 & 12 & & & $"$ & $"$ & $"$ & $"$ & $"$ & $"$ \\
\hline $\mathbf{S r}$ & 100 & 3 & 150 & 6 & $8 \times 10^{-8}$ & $<75$ & 11 & $n$ & " & 11 \\
\hline$"$ & 150 & 6 & 120 & 3 & $"$ & $"$ & $"$ & $"$ & $"$ & $"$ \\
\hline$"$ & 130 & 12 & & & $"$ & $"$ & $"$ & $"$ & $"$ & $"$ \\
\hline Cs & 360 & 3 & 460 & 6 & $3 \times 10^{-9}$ & " & " & $"$ & 11 & "s \\
\hline$"$ & 370 & 0 & 420 & 3 & $"$ & $"$ & " & $"$ & " & " \\
\hline$"$ & 390 & 12 & & & " & " & " & $"$ & $"$ & $"$ \\
\hline Ba & 610 & 3 & 630 & 6 & $1 \times 10^{-7}$ & " & " & $"$ & $n$ & $n$ \\
\hline$"$ & 740 & 6 & 660 & 3 & " & " & " & $"$ & $"$ & $"$ \\
\hline$"$ & 660 & 12 & & & " & $"$ & " & $"$ & $" 1$ & $"$ \\
\hline Ce & 150 & 3 & 840 & 6 & $1 \times 10^{-7}$ & $"$ & " & " & " & $"$ \\
\hline$"$ & 860 & 6 & 2400 & 3 & " & $"$ & $"$ & $"$ & $"$ & $"$ \\
\hline$n$ & 970 & 12 & & & " & $"$ & $"$ & " & $"$ & $"$ \\
\hline Eu & 530 & 3 & 1700 & 6 & $2 \times 10^{-7}$ & $"$ & 11 & $"$ & $"$ & $"$ \\
\hline " & 1400 & 6 & 2600 & 3 & " & " & $"$ & " & $"$ & $"$ \\
\hline$"$ & 1500 & 12 & & & " & " & $"$ & " & $"$ & $"$ \\
\hline $\mathbf{S r}$ & 280 & 3 & & & $6 \times 10^{-7}$ & $<38$ & " & Air & & $\mathbf{b}$ \\
\hline$"$ & 270 & 3 & & & " & $"$ & " & " & & $"$ \\
\hline " & 57 & 3 & & & " & $38-106$ & " & $"$ & & $"$ \\
\hline$"$ & 56 & 3 & & & $"$ & $"$ & $"$ & $"$ & & $"$ \\
\hline$"$ & 37 & 3 & & & $"$ & $106-500$ & $n$ & $"$ & & $"$ \\
\hline$"$ & 42 & 3 & & & $"$ & $"$ & $"$ & $"$ & & $"$ \\
\hline $\mathrm{Cs}$ & 940 & 3 & & & $3 \times 10^{-9}$ & $<38$ & " & $"$ & & $"$ \\
\hline$"$ & 890 & 3 & & & " & $"$ & $"$ & $"$ & & $"$ \\
\hline$"$ & 190 & 3 & & & $"$ & $38-106$ & $"$ & $n$ & & $"$ \\
\hline " & 190 & 3 & & & $"$ & $n$ & $"$ & $n$ & & $"$ \\
\hline$"$ & 110 & 3 & & & " & $106-500$ & 11 & $n$ & & $"$ \\
\hline$"$ & 130 & 3 & & & $n$ & $"$ & $"$ & n & & $n$ \\
\hline
\end{tabular}

Concentrations are only those added with the tracer; actual concentrations may be higher.

breviously unpublished results. 
TABLE A-XV (cont)

\begin{tabular}{|c|c|c|c|c|c|c|c|c|c|c|}
\hline \multirow[b]{2}{*}{ Elepent } & \multicolumn{2}{|c|}{ Sorption } & \multicolumn{2}{|c|}{ Desorption } & \multirow{2}{*}{$\begin{array}{c}\text { Element } \\
\text { Concentration } \\
\text { (M) } \\
\end{array}$} & \multirow{2}{*}{$\begin{array}{c}\text { Particle } \\
\text { Size } \\
\text { (1E) } \\
\end{array}$} & \multirow[b]{2}{*}{ Temperature } & \multirow[b]{2}{*}{ Atmosphere } & \multirow[b]{2}{*}{ Ref. } & \multirow[b]{2}{*}{ Note } \\
\hline & $\begin{array}{c}R_{d} \\
(n l / g)\end{array}$ & $\begin{array}{l}\text { Time } \\
\text { (wk) }\end{array}$ & $\begin{array}{c}R_{d} \\
(n \ell / g)\end{array}$ & $\begin{array}{l}\text { Time } \\
\text { (wk) }\end{array}$ & & & & & & \\
\hline $\mathrm{Ba}$ & 1700 & 3 & & & $3 \times 10^{-7}$ & $<38$ & Amb & Air & & 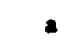 \\
\hline$"$ & 1600 & 3 & & & $"$ & $"$ & $"$ & $"$ & & $"$ \\
\hline$"$ & 480 & 3 & & & $"$ & $38-106$ & $"$ & $"$ & & $"$ \\
\hline$"$ & 470 & 3 & & & $"$ & $"$ & $"$ & $"$ & & $"$ \\
\hline$"$ & 130 & 3 & & & $"$ & $106-500$ & $"$ & $"$ & & $"$ \\
\hline$"$ & 150 & 3 & & & $"$ & $"$ & $"$ & $"$ & & $"$ \\
\hline Eu & 1600 & 3 & & & $4 \times 10^{-8}$ & $<38$ & $"$ & $"$ & & $"$ \\
\hline$"$ & 1600 & 3 & & & $"$ & $"$ & $"$ & $"$ & & $"$ \\
\hline$"$ & 260 & 3 & & & $"$ & $38-106$ & $"$ & $"$ & & $"$ \\
\hline$"$ & 420 & 3 & & & $"$ & $"$ & $"$ & $"$ & & $"$ \\
\hline$"$ & 490 & 3 & & & $"$ & $106-500$ & $"$ & $"$ & & $"$ \\
\hline$"$ & 440 & 3 & & & $"$ & $"$ & $"$ & $"$ & & $"$ \\
\hline
\end{tabular}

\begin{tabular}{|c|c|c|c|c|c|c|c|c|c|}
\hline A & 900 & 3 & 690 & 12 & $1 \times 10^{-7}$ & $<75$ & $"$ & $"$ & 3 \\
\hline$"$ & 1300 & 6 & 670 & 9 & $"$ & $"$ & $"$ & $"$ & $"$ \\
\hline$"$ & 900 & 12 & 560 & 3 & $"$ & $"$ & $"$ & $"$ & " \\
\hline$"$ & 150 & 3 & 400 & 12 & $"$ & $75-500$ & $"$ & $"$ & $"$ \\
\hline " & 150 & 6 & 680 & 9 & $"$ & $"$ & $"$ & $"$ & $"$ \\
\hline$"$ & 160 & 12 & 570 & 3 & $"$ & $"$ & $"$ & $"$ & $"$ \\
\hline$"$ & 1000 & 3 & 2400 & 12 & $"$ & $<75$ & $"$ & $C A$ & $"$ \\
\hline$"$ & 1400 & 6 & 3200 & 9 & $"$ & $"$ & $1 "$ & $"$ & $"$ \\
\hline$"$ & 1900 & 12 & 3800 & 3 & $"$ & $"$ & $"$ & $"$ & $"$ \\
\hline$"$ & 650 & 3 & 1400 & 12 & $"$ & $75-500$ & $"$ & $"$ & $"$ \\
\hline$"$ & 620 & 6 & 1600 & 9 & $"$ & $"$ & $"$ & $"$ & $"$ \\
\hline$"$ & 660 & 12 & 2900 & 3 & $"$ & $"$ & $"$ & $"$ & $"$ \\
\hline $\mathbf{P u}$ & 66 & 3 & 830 & 12 & $5 \times 10^{-12}$ & $<75$ & " & Air & $"$ \\
\hline$"$ & 64 & 6 & 590 & 9 & $"$ & $"$ & $"$ & $"$ & $"$ \\
\hline$"$ & 76 & 12 & 670 & 3 & $"$ & $"$ & $"$ & $"$ & $"$ \\
\hline$"$ & 52 & 3 & 660 & 12 & $"$ & $75-500$ & " & " & $"$ \\
\hline$"$ & 81 & 6 & 600 & 9 & $"$ & $"$ & "े & $"$ & $"$ \\
\hline$"$ & 160 & 12 & 660 & 3 & $"$ & $"$ & $"$ & $"$ & $"$ \\
\hline$"$ & 65 & 3 & 1000 & 12 & $3 \times 10^{-13}$ & $<75$ & $"$ & CA & $"$ \\
\hline$"$ & 100 & 6 & 780 & 9 & $"$ & $"$ & " & $"$ & " \\
\hline$"$ & 120 & 12 & 1900 & 3 & $"$ & $"$ & " & $"$ & $"$ \\
\hline$"$ & 110 & 3 & 850 & 12 & $"$ & $75-500$ & $"$ & $"$ & $"$ \\
\hline$"$ & 205 & 7 & 1600 & 8 & " & $"$ & " & " & $"$ \\
\hline$"$ & 190 & 12 & 1500 & 3 & $"$ & $"$ & " & $"$ & $"$ \\
\hline
\end{tabular}

areviously unpublished results. 
TABLE A-XV (cont)

\begin{tabular}{|c|c|c|c|c|c|c|c|c|c|c|}
\hline \multirow[b]{2}{*}{ Element } & \multicolumn{2}{|c|}{ Sorption } & \multicolumn{2}{|c|}{ Desorption } & \multirow{2}{*}{$\begin{array}{c}\text { Eleaent } \\
\text { Concentration } \\
(\mathrm{M}) \\
\end{array}$} & \multirow[b]{2}{*}{$\begin{array}{c}\text { Particle } \\
\text { Size } \\
\text { (证) } \\
\end{array}$} & \multirow[b]{2}{*}{ Teeperature } & \multirow[b]{2}{*}{ Atwosphere } & \multirow[b]{2}{*}{ Ref. } & \multirow[b]{2}{*}{ Note } \\
\hline & $\begin{array}{c}R_{d} \\
(n e / s)\end{array}$ & $\begin{array}{l}\text { Time } \\
\text { (wk) }\end{array}$ & $\begin{array}{c}R_{d} \\
(\mathrm{me} / \mathrm{s})\end{array}$ & $\begin{array}{l}\text { Time } \\
\text { (uk) }\end{array}$ & & & & & & \\
\hline $\mathbf{u}$ & 2 & 1 & 14 & 2 & $1 \times 10^{-6}$ & $<106$ & $A=b$ & Air & 3 & \\
\hline$"$ & 2 & 2 & 15 & 1 & $"$ & $"$ & $"$ & $"$ & $"$ & \\
\hline$"$ & 2 & 3 & & & $"$ & $"$ & $"$ & $"$ & $"$ & \\
\hline$"$ & 1 & 1 & 4 & 2 & $"$ & $106-500$ & $"$ & $"$ & $"$ & \\
\hline$"$ & 2 & 2 & 19 & 1 & $"$ & $"$ & $"$ & $"$ & $"$ & \\
\hline$"$ & 1 & 3 & & & $"$ & $"$ & $"$ & $"$ & $"$ & \\
\hline$"$ & 1.0 & 1 & & & $"$ & $<75$ & $"$ & $\mathrm{CA}$ & 4 & \\
\hline$"$ & 1.8 & 2 & & & $"$ & $"$ & $"$ & $"$ & $"$ & \\
\hline$"$ & 2.3 & 3 & & & " & $"$ & $"$ & $"$ & $"$ & \\
\hline$"$ & 0.8 & 1 & & & $"$ & $75-500$ & $"$ & $"$ & $"$ & \\
\hline$"$ & 1.4 & 2 & & & $"$ & $"$ & $"$ & $"$ & $"$ & \\
\hline$"$ & 1.7 & 3 & & & $"$ & $"$ & $"$ & $"$ & $"$ & \\
\hline Te & 4.7 & 3 & 140 & 12 & $1 \times 10^{-12}$ & $"$ & " & $"$ & $"$ & \\
\hline$"$ & 3.8 & 6 & 38 & 9 & $"$ & $"$ & $"$ & " & $"$ & \\
\hline$"$ & 33 & 12 & 240 & 3 & $"$ & $"$ & $"$ & $"$ & $"$ & \\
\hline$"$ & 0.7 & 3 & 37 & 12 & $"$ & $<75$ & $"$ & $"$ & $"$ & \\
\hline$"$ & 0.8 & 6 & 13 & 9 & $"$ & $"$ & $"$ & $"$ & $"$ & \\
\hline$"$ & 3.4 & 12 & 5.9 & 3 & " & $"$ & $"$ & $"$ & $"$ & \\
\hline
\end{tabular}


TABI. A-XVI

BATCH SORPTIOI DATA FOR TUFF G1-1292

\begin{tabular}{|c|c|c|c|c|c|c|c|c|c|c|}
\hline \multirow[b]{2}{*}{ Element } & \multicolumn{2}{|c|}{ Sorption } & \multicolumn{2}{|c|}{ Desorption } & \multirow[b]{2}{*}{$\begin{array}{c}\text { Elenent } \\
\text { Concentration } \\
\text { (H) }\end{array}$} & \multirow[b]{2}{*}{$\begin{array}{c}\text { Particle } \\
\text { Size } \\
(\mu \omega) \\
\end{array}$} & \multirow[b]{2}{*}{ Tesperature } & \multirow[b]{2}{*}{ Atmosphere } & \multirow[b]{2}{*}{ Ref. } & \multirow[b]{2}{*}{ Note } \\
\hline & $\begin{array}{c}R_{d} \\
(\boldsymbol{e} / \boldsymbol{e})\end{array}$ & $\begin{array}{l}\text { Tine } \\
\text { (wk) }\end{array}$ & $\begin{array}{c}R_{d} \\
(e / /)\end{array}$ & $\begin{array}{l}\text { Tine } \\
\text { (wk) }\end{array}$ & & & & & & \\
\hline Sr & 200 & 3 & 120 & 6 & $2 \times 10^{-7}$ & $<500$ & $A=b$ & Air & 1 & \\
\hline$"$ & 200 & 3 & 110 & 6 & $"$ & $"$ & $"$ & $"$ & $"$ & \\
\hline$" 1$ & 220 & 6 & 110 & 3 & $"$ & $"$ & $"$ & $"$ & $"$ & \\
\hline$"$ & 190 & 6 & 130 & 3 & $"$ & $"$ & $"$ & $"$ & $"$ & \\
\hline Cs & 390 & 3 & 560 & 6 & $3 \times 10^{-9}$ & $"$ & $"$ & $"$ & $"$ & \\
\hline$"$ & 450 & 3 & 500 & 6 & $"$ & $"$ & $"$ & $"$ & $"$ & \\
\hline$"$ & 500 & 6 & 470 & 3 & $"$ & $"$ & $"$ & $"$ & $"$ & \\
\hline$"$ & 380 & 6 & 520 & 3 & $"$ & $"$ & $"$ & $"$ & $"$ & \\
\hline $\mathbf{B a}$ & 1800 & 3 & 1700 & 6 & $7 \times 10^{-6}$ & $"$ & $"$ & $"$ & $"$ & \\
\hline$"$ & 2200 & 3 & 1400 & 6 & $"$ & $"$ & " & $"$ & $"$ & \\
\hline$"$ & 2800 & 6 & 1400 & 3 & $"$ & $"$ & $"$ & $"$ & $" 1$ & \\
\hline$"$ & 1500 & 6 & $15000^{a}$ & 3 & $"$ & $"$ & $"$ & $"$ & $"$ & $\mathbf{a}$ \\
\hline $\mathrm{Ce}$ & 54 & 3 & 1200 & 6 & $7 \times 10^{-8}$ & " & $"$ & $"$ & $"$ & \\
\hline$"$ & 79 & 3 & 510 & 6 & $"$ & " & $"$ & $"$ & $"$ & \\
\hline$"$ & 82 & 6 & 610 & 3 & " & $"$ & $"$ & " & $"$ & \\
\hline$"$ & 50 & 6 & 94 & 3 & $"$ & $"$ & $"$ & $"$ & $"$ & \\
\hline Eu & 140 & 3 & 780 & 6 & $1 \times 10^{-7}$ & $"$ & $"$ & $"$ & $"$ & \\
\hline$"$ & 160 & 3 & 510 & 6 & " & $"$ & " & $"$ & $"$ & \\
\hline " & 160 & 6 & 490 & 3 & $"$ & $"$ & $"$ & $"$ & $"$ & \\
\hline$"$ & 100 & 6 & 620 & 3 & $"$ & 1 & $"$ & $"$ & $"$ & \\
\hline
\end{tabular}

\footnotetext{
Value not included in averages for Table XXII, Chap. 1 .
} 
TABLE A-XVII

AATCH SORPTION DATA FOR TUFF G1-1436

\begin{tabular}{|c|c|c|c|c|c|c|c|c|c|c|}
\hline \multirow[b]{2}{*}{ Elepent } & \multicolumn{2}{|c|}{ Sorption } & \multicolumn{2}{|c|}{ Desorption } & \multirow{2}{*}{$\begin{array}{c}\text { Elewent } \\
\text { Concentration } \\
\text { (H) } \\
\end{array}$} & \multirow[b]{2}{*}{$\begin{array}{c}\text { Particle } \\
\text { Size } \\
\text { (ر) } \\
\end{array}$} & \multirow[b]{2}{*}{ Tepperature } & \multirow[b]{2}{*}{ Atmosphere } & \multirow[b]{2}{*}{ Ref. } & \multirow[b]{2}{*}{ Note } \\
\hline & $\begin{array}{c}R_{d} \\
(n / /)\end{array}$ & $\begin{array}{l}\text { Tine } \\
\text { (wk) }\end{array}$ & $\begin{array}{c}R_{d} \\
(n / /)\end{array}$ & $\begin{array}{l}\text { Tine } \\
\text { (wlt) }\end{array}$ & & & & & & \\
\hline $\mathbf{s r}$ & 31000 & 3 & 100000 & 6 & $1 \times 10^{-7}$ & $<500$ & $A=b$ & Air & 1 & \\
\hline$"$ & 36000 & 3 & 55000 & 6 & $"$ & $"$ & $"$ & $"$ & 1 & \\
\hline$"$ & 33000 & 6 & 110000 & 3 & $"$ & $n$ & $"$ & $"$ & $n$ & \\
\hline$"$ & 45000 & 6 & 83000 & 3 & $"$ & " & $"$ & " & $"$ & \\
\hline Cs & 7000 & 3 & 29000 & 6 & $3 \times 10^{-9}$ & $"$ & $"$ & $"$ & $"$ & \\
\hline " & 7900 & 3 & 18000 & 6 & $"$ & $"$ & $"$ & $"$ & $"$ & \\
\hline $1 "$ & 7400 & 6 & 26000 & 3 & $"$ & $"$ & $"$ & $"$ & $"$ & \\
\hline " & 9000 & 6 & 23000 & 3 & $"$ & $"$ & $"$ & $"$ & $"$ & \\
\hline Ba & 150000 & 3 & 300000 & 6 & $8 \times 10^{-6}$ & 11 & $"$ & $"$ & $"$ & \\
\hline$"$ & 140000 & 3 & 110000 & 6 & $"$ & $"$ & $"$ & $"$ & " & \\
\hline$"$ & 93000 & 6 & 450000 & 3 & $"$ & $"$ & $"$ & $"$ & " & \\
\hline$"$ & 210000 & 6 & 500000 & 3 & $"$ & " & $"$ & $"$ & $"$ & \\
\hline $\mathrm{Ce}$ & 58000 & 3 & 5900 & 6 & $1 \times 10^{-7}$ & $"$ & $"$ & $"$ & $"$ & \\
\hline$"$ & 62000 & 3 & 5500 & 6 & $"$ & $"$ & $"$ & $"$ & $"$ & \\
\hline$"$ & 41000 & 6 & 8000 & 3 & $"$ & $"$ & $"$ & $" 1$ & $"$ & \\
\hline$"$ & 74000 & 6 & 7200 & 3 & " & $"$ & $n$ & $"$ & $"$ & \\
\hline Eu & 30000 & 3 & 4200 & 6 & $"$ & $n$ & " & $"$ & $"$ & \\
\hline$"$ & 32000 & 3 & 4500 & 6 & $"$ & $"$ & $"$ & $"$ & $"$ & \\
\hline$"$ & 25000 & 6 & 6500 & 3 & $"$ & $n$ & $"$ & $"$ & $"$ & \\
\hline$"$ & 34000 & 6 & 6100 & 3 & $"$ & $"$ & $"$ & $"$ & $"$ & \\
\hline
\end{tabular}


TABLE A-XVIII

BATCH SORPTION DATA FOR TUFF G1-1854

\begin{tabular}{|c|c|c|c|c|c|c|c|c|c|c|}
\hline \multirow[b]{2}{*}{ Element } & \multicolumn{2}{|c|}{ Sorption } & \multicolumn{2}{|c|}{ Desorption } & \multirow{2}{*}{$\begin{array}{c}\text { Element } \\
\text { Concentration } \\
(\mathrm{H})\end{array}$} & \multirow{2}{*}{$\begin{array}{c}\text { Particle } \\
\text { Size } \\
\text { (1⿴囗十) } \\
\end{array}$} & \multirow[b]{2}{*}{ Texperature } & \multirow[b]{2}{*}{ Atmosphere } & \multirow[b]{2}{*}{ Ref. } & \multirow[b]{2}{*}{ Note } \\
\hline & $\begin{array}{c}R_{d} \\
(m \ell / g)\end{array}$ & $\begin{array}{l}\text { Tise } \\
\text { (wk) }\end{array}$ & $\begin{array}{c}R_{d} \\
(n e / e) \\
\end{array}$ & $\begin{array}{l}\text { Tine } \\
\text { (wk) } \\
\end{array}$ & & & & & & \\
\hline $\mathrm{Sr}$ & 32000 & 3 & 95000 & 6 & $3 \times 10^{-8}$ & $75-500$ & $A=b$ & Air & 6 & \\
\hline$"$ & 43000 & 6 & 51000 & 3 & $"$ & $"$ & $"$ & $"$ & $"$ & \\
\hline Cs & 11000 & 3 & 11000 & 6 & $4 \times 10^{-9}$ & $"$ & $"$ & $"$ & $"$ & \\
\hline$"$ & 10000 & 6 & 15000 & 3 & $"$ & $"$ & $"$ & $"$ & $"$ & \\
\hline $\mathrm{Ba}$ & 34000 & 3 & 130000 & 6 & $1 \times 10^{-7}$ & $"$ & $"$ & $"$ & $"$ & \\
\hline$"$ & 34000 & 6 & 87000 & 3 & $"$ & $"$ & $"$ & $"$ & $"$ & \\
\hline Eu & $>120000$ & 3 & 3000 & 6 & $3 \times 10^{-8}$ & $"$ & $"$ & $"$ & $"$ & \\
\hline$"$ & $>14000$ & 6 & 4800 & 3 & $"$ & $"$ & $"$ & $"$ & $"$ & \\
\hline $\mathbf{S r}$ & 71000 & 3 & 71000 & 6 & $"$ & $<75$ & $"$ & $"$ & $"$ & \\
\hline$"$ & 92000 & 6 & $>38000^{\circ}$ & 3 & $"$ & $"$ & $"$ & $"$ & $"$ & a \\
\hline Cs & 15000 & 3 & 14000 & 6 & $4 \times 10^{-9}$ & $"$ & $"$ & $"$ & $"$ & \\
\hline$"$ & 15000 & 6 & 18000 & 3 & $"$ & $"$ & $"$ & $"$ & $"$ & \\
\hline $\mathrm{Ba}$ & 63000 & 3 & 140000 & 6 & $1 \times 10^{-7}$ & $"$ & $"$ & $"$ & $"$ & \\
\hline$"$ & 48000 & 6 & 250000 & 3 & $"$ & $"$ & $"$ & $"$ & $"$ & \\
\hline Eu & $>130000$ & 3 & 4800 & 6 & $3 \times 10^{-8}$ & $"$ & $"$ & $"$ & $"$ & \\
\hline$"$ & $>110000$ & 6 & 6500 & 3 & $"$ & $"$ & $"$ & $"$ & $"$ & \\
\hline
\end{tabular}

${ }^{a}$ Value not included in average for Table XXII, Chap. 1. 
TABLE A-XIX

BATCH SORPTION DATA FOR TUFF G1-1883

\begin{tabular}{|c|c|c|c|c|c|c|c|c|c|c|}
\hline \multirow[b]{2}{*}{ Element } & \multicolumn{2}{|c|}{ Sorption } & \multicolumn{2}{|c|}{ Besorption } & \multirow{2}{*}{$\begin{array}{c}\text { Elewent } \\
\text { Concentration } \\
\text { (M) }\end{array}$} & \multirow{2}{*}{$\begin{array}{c}\text { Particle } \\
\text { Size } \\
\text { (jin) } \\
\end{array}$} & \multirow[b]{2}{*}{ Teaperature } & \multirow[b]{2}{*}{ Atmosphere } & \multirow[b]{2}{*}{ Ref. } & \multirow[b]{2}{*}{ Note } \\
\hline & $\begin{array}{c}R_{d} \\
(n L / /)\end{array}$ & $\begin{array}{l}\text { Tine } \\
\text { (wl) }\end{array}$ & $\begin{array}{c}R_{d} \\
(n \alpha / s)\end{array}$ & $\begin{array}{l}\text { Time } \\
\text { (wh) }\end{array}$ & & & & & & \\
\hline Sr & 58 & 3 & 58 & 6 & $2 \times 10^{-7}$ & $<500$ & $\mathrm{Amb}$ & Air & 1 & \\
\hline$n$ & 59 & 3 & 56 & 6 & $"$ & $n$ & $"$ & $"$ & $"$ & \\
\hline$"$ & 57 & 6 & 60 & 3 & $"$ & $"$ & $"$ & $1 "$ & $"$ & \\
\hline$"$ & 58 & 6 & 62 & 3 & $"$ & $n$ & $"$ & $"$ & it & \\
\hline $\mathrm{Cs}$ & 380 & 3 & 420 & 6 & $3 \times 10^{-9}$ & $"$ & " & $"$ & $"$ & \\
\hline$"$ & 350 & 3 & 430 & 6 & $"$ & $"$ & $"$ & $"$ & $"$ & \\
\hline$"$ & 440 & 6 & 430 & 3 & $"$ & $"$ & $"$ & $"$ & $"$ & \\
\hline$"$ & 440 & 6 & 440 & 3 & $"$ & $"$ & $"$ & $"$ & $"$ & \\
\hline $\mathrm{Ba}$ & $4300^{a}$ & 3 & 440 & 6 & $8 \times 10^{-6}$ & $"$ & $"$ & $"$ & $"$ & $\mathbf{a}$ \\
\hline$"$ & 250 & 3 & 420 & 6 & $"$ & $"$ & $"$ & $"$ & $n$ & \\
\hline$"$ & 480 & 6 & 450 & 3 & $"$ & $"$ & $"$ & $"$ & $n$ & \\
\hline$"$ & 490 & 6 & 460 & 3 & $"$ & $"$ & $"$ & $"$ & $"$ & \\
\hline Ce & 150 & 3 & 2000 & 6 & $7 \times 10^{-8}$ & $"$ & " & $"$ & $"$ & \\
\hline _" & 150 & 3 & 2200 & 6 & " & $" 1$ & $"$ & $"$ & $"$ & \\
\hline$"$ & 300 & 6 & 2200 & 3 & $H$ & $"$ & $"$ & $"$ & $"$ & \\
\hline$"$ & 250 & 6 & 2300 & 3 & $"$ & $"$ & $"$ & $n$ & $"$ & \\
\hline iu & 180 & 3 & 1300 & 6 & $1 \times 10^{-7}$ & $"$ & $"$ & $"$ & $1 "$ & \\
\hline$"$ & 180 & 3 & 1500 & 6 & " & $"$ & $"$ & $"$ & $"$ & \\
\hline$"$ & 330 & 6 & 1300 & 3 & $"$ & $"$ & $"$ & $"$ & $"$ & \\
\hline$"$ & 300 & 6 & 1300 & 3 & " & $"$ & $"$ & $"$ & $n$ & \\
\hline Sr & 26 & 3 & & & $3 \times 10^{-7}$ & $<38$ & $"$ & $"$ & 7 & \\
\hline$"$ & 80 & 3 & & & $"$ & $"$ & $"$ & $"$ & $"$ & \\
\hline $\mathrm{Cs}$ & 310 & 3 & & & $4 \times 10^{-9}$ & $"$ & $"$ & $"$ & $"$ & \\
\hline$"$ & 720. & 3 & & & $"$ & 11 & $"$ & 11 & $"$ & \\
\hline Ba & 230 & 3 & & & $7 \times 10^{-6}$ & $"$ & " & $"$ & " & \\
\hline$n$ & 750 & 3 & & & $"$ & $"$ & $"$ & $"$ & $"$ & \\
\hline Eu & 370 & 3 & & & $3 \times 10^{-8}$ & $"$ & $"$ & $"$ & $"$ & \\
\hline$"$ & 650 & 3 & & & $"$ & $"$ & $"$ & $"$ & $"$ & \\
\hline $\mathbf{S r}$ & 22 & 3 & & & $3 \times 10^{-7}$ & $38-106$ & $"$ & " & $"$ & \\
\hline$"$ & 22 & 3 & & & $"$ & $"$ & $"$ & " & " & \\
\hline
\end{tabular}

Value not used in averages for Table XI, Chap. 1. 
TABIE A-XIX (cont)

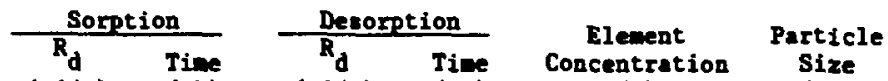

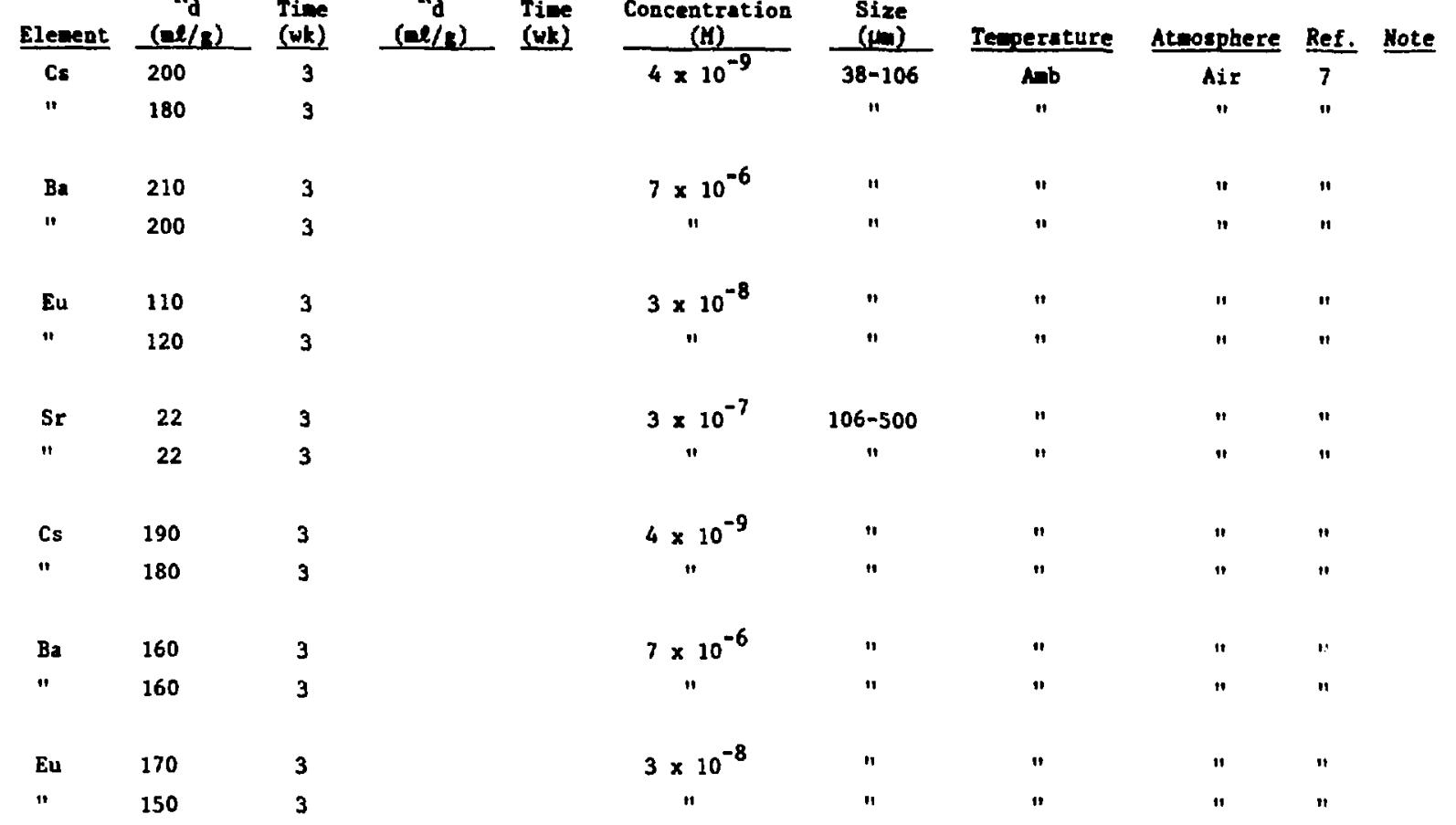

\begin{tabular}{|c|c|c|c|c|c|c|c|c|}
\hline $\mathrm{Na}$ & 0.93 & 2 & 4.2 & 4 & $2 \times 10^{-3}$ & $<500$ & $"$ & $"$ \\
\hline$"$ & 3.0 & 2 & 1.3 & 4 & " & $"$ & $"$ & $" 1$ \\
\hline$"$ & 3.7 & 4 & 3.8 & 2 & $"$ & " & $"$ & 1 \\
\hline " & 1.6 & 4 & 1.7 & 2 & " & " & 11 & $"$ \\
\hline
\end{tabular}

\begin{tabular}{|c|c|c|c|c|c|c|c|c|c|}
\hline Mn & 140 & 2 & 140 & 4 & $1 \times 10^{-11}$ & $"$ & $"$ & " & $"$ \\
\hline " & 120 & 2 & & & $"$ & $"$ & $" 1$ & " & $"$ \\
\hline " & 240 & 4 & 160 & 2 & $"$ & " & " & " & " \\
\hline " & 390 & 4 & 950 & 2 & $"$ & $"$ & $"$ & " & 9 \\
\hline
\end{tabular}

\begin{tabular}{|c|c|c|c|c|c|c|c|c|c|c|}
\hline Se & 1.5 & 2 & 60 & 4 & $1 \times 10^{-9}$ & $"$ & " & $"$ & $"$ & " \\
\hline$"$ & & 2 & 20 & 4 & $n$ & $"$ & $"$ & " & " & " \\
\hline " & 16 & 4 & 72 & 2 & " & $"$ & $"$ & " & $"$ & " \\
\hline$"$ & 3.6 & 4 & 30 & 2 & " & $"$ & $"$ & " & " & " \\
\hline
\end{tabular}

\begin{tabular}{|c|c|c|c|c|c|c|c|c|c|}
\hline Sn & 230 & 2 & 2600 & 4 & $8 \times 10^{-9}$ & $"$ & $"$ & " & $"$ \\
\hline$"$ & & 2 & 2100 & 4 & $"$ & $"$ & " & $"$ & $"$ \\
\hline$"$ & 620 & 4 & 2400 & 2 & $"$ & " & $"$ & " & " \\
\hline$"$ & 290 & 4 & 2700 & 2 & " & $n$ & $"$ & $"$ & " \\
\hline
\end{tabular}

Concentrations are only those added with the tracer; actusl concentrations ary be higher. 
TABLI A-XIX (cont)

\begin{tabular}{|c|c|c|c|c|c|c|c|c|c|c|}
\hline \multirow[b]{2}{*}{ Elesent } & \multicolumn{2}{|c|}{ Sorption } & \multicolumn{2}{|c|}{ Desorption } & \multirow{2}{*}{$\begin{array}{c}\text { Elewent } \\
\text { Concentration } \\
\text { (H) }\end{array}$} & \multirow[b]{2}{*}{$\begin{array}{c}\text { Particle } \\
\text { Size } \\
\text { (1⿴囗十) } \\
\end{array}$} & \multirow[b]{2}{*}{ Te-perature } & \multirow[b]{2}{*}{ Atrosphere } & \multirow[b]{2}{*}{ Ref. } & \multirow[b]{2}{*}{ Note } \\
\hline & $\begin{array}{c}R_{d} \\
(a / R)\end{array}$ & $\begin{array}{l}\text { Tine } \\
\text { (wk) }\end{array}$ & $\begin{array}{c}R_{d} \\
(R / / R)\end{array}$ & $\begin{array}{l}\text { Tine } \\
\text { (wk) } \\
\end{array}$ & & & & & & \\
\hline A & 4200 & 3 & 5900 & 12 & $1 \times 10^{-7}$ & $106-250$ & $A b$ & Air & 6 & \\
\hline$"$ & 4500 & 6 & 6900 & 9 & $"$ & " & " & $"$ & $"$ & \\
\hline$"$ & 5300 & 12 & 8900 & 3 & $"$ & $"$ & $"$ & $"$ & $"$ & \\
\hline $\mathrm{Pu}$ & 51 & 3 & 830 & 12 & $4 \times 10^{-12}$ & $"$ & $"$ & $"$ & 7 & \\
\hline$"$ & 52 & 6 & 740 & 8 & $"$ & $"$ & $"$ & $"$ & $"$ & \\
\hline$"$ & 91 & 6 & 1100 & 8 & $"$ & $"$ & $"$ & $"$ & $"$ & \\
\hline$"$ & 82 & 13 & 960 & 3 & $"$ & $"$ & $"$ & $"$ & $"$ & \\
\hline$"$ & 107 & 13 & 850 & 3 & $"$ & $"$ & $"$ & $"$ & $"$ & \\
\hline Np & 5.3 & 3 & 24 & 12 & $9 \times 10^{-11}$ & $"$ & $"$ & $"$ & $"$ & \\
\hline$"$ & 6.9 & 6 & 29 & 9 & $"$ & $"$ & $"$ & $"$ & $"$ & \\
\hline$"$ & 7.1 & 13 & 56 & 3 & $"$ & $" 1$ & $"$ & $"$ & $"$ & \\
\hline
\end{tabular}




\begin{tabular}{|c|c|c|c|c|c|c|c|c|c|c|}
\hline \multirow[b]{2}{*}{ Elewent } & \multicolumn{2}{|c|}{ Sorption } & \multicolumn{2}{|c|}{ Desorption } & \multirow{2}{*}{$\begin{array}{c}\begin{array}{c}\text { Element } \\
\text { Concentration } \\
(\mathrm{M})\end{array} \\
\end{array}$} & \multirow[b]{2}{*}{$\begin{array}{c}\text { Particle } \\
\text { Size } \\
\text { (监) } \\
\end{array}$} & \multirow[b]{2}{*}{ Temperature } & \multirow[b]{2}{*}{ Atmosphere } & \multirow[b]{2}{*}{ Ref. } & \multirow[b]{2}{*}{ Note } \\
\hline & $\begin{array}{c}R_{d} \\
(n e / s)\end{array}$ & $\begin{array}{l}\text { Time } \\
\text { (wk) }\end{array}$ & $\begin{array}{c}R_{d} \\
(n / / R)\end{array}$ & $\begin{array}{l}\text { Tine } \\
\text { (wk) }\end{array}$ & & & & & & \\
\hline Sr & 430 & 3 & 340 & 6 & $2 \times 10^{-7}$ & $<500$ & $A=b$ & Air & 1 & \\
\hline$"$ & 430 & 3 & 330 & 6 & $"$ & $"$ & $"$ & $"$ & $"$ & \\
\hline$"$ & 450 & 6 & 310 & 3 & " & $"$ & " & " & $"$ & \\
\hline$"$ & 450 & 6 & 310 & 3 & $"$ & $"$ & $"$ & 11 & $"$ & \\
\hline $\mathrm{Cs}$ & 1400 & 3 & 2900 & 6 & $3 \times 10^{-9}$ & $"$ & $"$ & $"$ & " & \\
\hline$"$ & 1400 & 3 & 2000 & 6 & $"$ & 11 & $"$ & $" 1$ & $"$ & \\
\hline$"$ & 1800 & 6 & 2100 & 3 & $"$ & "t & $"$ & $"$ & $" 1$ & \\
\hline$"$ & 1800 & 6 & 2100 & 3 & $"$ & $"$ & $"$ & $"$ & $"$ & \\
\hline $\mathrm{Ba}$ & 2500 & 3 & 2600 & 6 & $4 \times 10^{-6}$ & $"$ & $"$ & $"$ & $"$ & \\
\hline$"$ & 2200 & 3 & 3100 & 6 & " & $" 1$ & $"$ & 11 & $"$ & \\
\hline " & 3100 & 6 & 2800 & 3 & $"$ & $"$ & $"$ & $"$ & $"$ & \\
\hline$"$ & 3200 & 6 & 2600 & 3 & $"$ & $"$ & $"$ & $"$ & $" 1$ & \\
\hline Ce & 520 & 3 & 5900 & 6 & $1 \times 10^{-7}$ & $"$ & $"$ & $"$ & $"$ & \\
\hline$"$ & 450 & 3 & 5600 & 6 & $"$ & $"$ & $"$ & $"$ & $"$ & \\
\hline$"$ & 640 & 6 & 9400 & 3 & $"$ & $"$ & $"$ & $"$ & $"$ & \\
\hline$"$ & 600 & 6 & 7200 & 3 & $"$ & $"$ & $"$ & $"$ & $"$ & \\
\hline Eu & 1500 & 3 & 6100 & 6 & " & $"$ & $"$ & $"$ & $"$ & \\
\hline$"$ & 1300 & 3 & 6500 & 6 & $"$ & $"$ & $"$ & $"$ & $"$ & \\
\hline$"$ & 2000 & 6 & $800^{\circ}$ & 3 & $"$ & $"$ & $"$ & $"$ & $"$ & $\mathbf{a}$ \\
\hline$"$ & 1900 & 6 & 6500 & 3 & $"$ & $"$ & $"$ & " & $"$ & \\
\hline $\mathrm{Sr}$ & 1200 & 2 & & & $2 \times 10^{-7}$ & $<38$ & $"$ & $"$ & 9 & \\
\hline$"$ & 59 & 2 & & & $"$ & $38-75$ & $"$ & $"$ & $"$ & \\
\hline$"$ & 49 & 2 & & & $"$ & $75-250$ & $"$ & $"$ & $"$ & \\
\hline$"$ & 51 & 2 & & & $"$ & $250-500$ & $"$ & $"$ & $"$ & \\
\hline$"$ & 1200 & 3 & & & $"$ & $<38$ & $n$ & $"$ & $"$ & \\
\hline$"$ & 66 & 3 & & & $"$ & $38-75$ & $"$ & $"$ & $"$ & \\
\hline$"$ & 53 & 3 & & & $"$ & $75-250$ & $"$ & $"$ & $"$ & \\
\hline$"$ & 66 & 3 & & & $"$ & $250-500$ & " & $"$ & $"$ & \\
\hline Cs & 3800 & 2 & & & $3 \times 10^{-9}$ & $<38$ & $"$ & $"$ & $"$ & \\
\hline$"$ & 1200 & 2 & & & " & $38-75$ & " & $"$ & " & \\
\hline$"$ & 960 & 2 & & & $"$ & $75-250$ & $"$ & $"$ & $"$ & \\
\hline$"$ & 1200 & 2 & & & $"$ & $250-500$ & $"$ & $"$ & $"$ & \\
\hline " & 3500 & 3 & & & $n$ & $<38$ & " & $"$ & $"$ & \\
\hline$"$ & 1300 & 3 & & & " & $38-75$ & $"$ & $"$ & " & \\
\hline$"$ & 1100 & 3 & & & $"$ & $75-250$ & $"$ & $"$ & $"$ & \\
\hline $1 "$ & 1200 & 3 & & & $"$ & $250-500$ & $"$ & $"$ & $"$ & \\
\hline
\end{tabular}

\footnotetext{
"Value not included in averages for Table XXII, Chap. 1 .
} 
TABLE A-XX (Cont)

\begin{tabular}{|c|c|c|c|c|c|c|c|c|c|c|}
\hline \multirow[b]{2}{*}{ Elewent } & \multicolumn{2}{|c|}{ Sorption } & \multicolumn{2}{|c|}{ Desoxption } & \multirow{2}{*}{$\begin{array}{c}\text { Element } \\
\text { Concentration } \\
(M) \\
\end{array}$} & \multirow{2}{*}{$\begin{array}{c}\text { Particle } \\
\text { Size } \\
\text { (y) } \\
\end{array}$} & \multirow[b]{2}{*}{ Tesperature } & \multirow[b]{2}{*}{ Atmosphere } & \multirow[b]{2}{*}{ Ref. } & \multirow[b]{2}{*}{ Note } \\
\hline & $\begin{array}{c}R_{d} \\
(a) / g)\end{array}$ & $\begin{array}{l}\text { Tine } \\
\text { (wk) } \\
\end{array}$ & $\begin{array}{c}R_{d} \\
(n e / s)\end{array}$ & $\begin{array}{l}\text { Tine } \\
\text { (wk) }\end{array}$ & & & & & & \\
\hline Be & 10000 & 2 & & & $4 \times 10^{-6}$ & $<38$ & $\mathbf{A m b}$ & Air & 9 & \\
\hline$"$ & 670 & 2 & & & $n$ & $38-75$ & $"$ & $"$ & $"$ & \\
\hline$"$ & 570 & 2 & & & $"$ & $75-250$ & " & $"$ & " & \\
\hline$"$ & 690 & 2 & & & $"$ & $250-500$ & $"$ & $"$ & $"$ & \\
\hline 11 & 10000 & 3 & & & $"$ & $<38$ & $n$ & $"$ & $"$ & \\
\hline$"$ & 840 & 3 & & & $"$ & $38-75$ & $"$ & $"$ & $"$ & \\
\hline$"$ & 780 & 3 & & & $"$ & $75-250$ & $"$ & $"$ & $"$ & \\
\hline$"$ & 780 & 3 & & & $"$ & $250-500$ & $"$ & $"$ & $"$ & \\
\hline Eu & 1200 & 2 & & & $1 \times 10^{-7}$ & $<38$ & $"$ & 11 & $"$ & \\
\hline$"$ & 530 & 2 & & & $"$ & $38-75$ & $"$ & $"$ & $"$ & \\
\hline$"$ & 610 & 2 & & & $"$ & $75-250$ & " & $"$ & $"$ & \\
\hline$"$ & 860 & 2 & & & $"$ & $250-500$ & $"$ & $"$ & " & \\
\hline$"$ & 2500 & 3 & & & $"$ & $<38$ & $"$ & $"$ & $"$ & \\
\hline$"$ & 880 & 3 & & & $"$ & $38-75$ & $"$ & $"$ & $"$ & \\
\hline$"$ & 1300 & 3 & & & $"$ & $75-250$ & $n$ & " & $"$ & \\
\hline$"$ & 1100 & 3 & & & $"$ & $250-500$ & $"$ & $"$ & $"$ & \\
\hline
\end{tabular}


TABLE A-XXI

BATCH SORPTION DATA FOR TUFF G1-2233

\begin{tabular}{|c|c|c|c|c|c|c|c|c|c|c|}
\hline \multirow[b]{2}{*}{ Elemeat } & \multicolumn{2}{|c|}{ Sorption } & \multicolumn{2}{|c|}{ Desorption } & \multirow{2}{*}{$\begin{array}{c}\text { Elewent } \\
\text { Concentration } \\
(\mathrm{M}) \\
\end{array}$} & \multirow[b]{2}{*}{$\begin{array}{c}\text { Particle } \\
\text { Size } \\
\text { (je) } \\
\end{array}$} & \multirow[b]{2}{*}{ Tempersture } & \multirow[b]{2}{*}{ Atnosphere } & \multirow[b]{2}{*}{ Ref. } & \multirow[b]{2}{*}{ Note } \\
\hline & $\begin{array}{c}R_{d} \\
(a \ell / R)\end{array}$ & $\begin{array}{l}\text { Time } \\
\text { (wk) }\end{array}$ & $\begin{array}{c}R_{d} \\
(R / / R) \\
\end{array}$ & $\begin{array}{l}\text { Time } \\
\text { (wk) }\end{array}$ & & & & & & \\
\hline Sr & 40000 & 3 & 75000 & 6 & $1 \times 10^{-7}$ & $<500$ & $A=b$ & Air & 1 & \\
\hline$"$ & 55000 & 3 & 190000 & 6 & $"$ & $"$ & $"$ & $"$ & $"$ & \\
\hline$"$ & 49000 & 6 & 13000 & 3 & $"$ & $"$ & $"$ & $"$ & $"$ & \\
\hline$"$ & 50000 & 6 & 82000 & 3 & $"$ & $"$ & $"$ & $"$ & $"$ & \\
\hline Cs & 13000 & 3 & 31000 & 6 & $3 \times 10^{-9}$ & $"$ & $"$ & $"$ & $"$ & \\
\hline$"$ & 11000 & 3 & 29000 & 6 & $"$ & $"$ & $"$ & $"$ & $"$ & \\
\hline$"$ & 15000 & 6 & 6500 & 3 & $"$ & $"$ & $"$ & $"$ & $"$ & \\
\hline$"$ & 14000 & 6 & 25000 & 3 & $"$ & $"$ & $"$ & $"$ & $"$ & \\
\hline Ba & 200000 & 3 & 460000 & 6 & $6 \times 10^{-6}$ & $"$ & $"$ & $"$ & $"$ & \\
\hline$"$ & 210000 & 3 & 100000 & 6 & $"$ & $"$ & $"$ & $"$ & $"$ & \\
\hline$"$ & 330000 & 6 & 160000 & 3 & $"$ & $"$ & $"$ & $"$ & $"$ & \\
\hline$"$ & 250000 & 6 & 220000 & 3 & $"$ & $"$ & $"$ & $"$ & $"$ & \\
\hline $\mathrm{Ce}$ & 840 & 3 & 59000 & 6 & $7 \times 10^{-8}$ & $"$ & $"$ & $"$ & $"$ & \\
\hline$"$ & 1300 & 3 & 14000 & 6 & $"$ & $"$ & $"$ & $"$ & $"$ & \\
\hline$"$ & 2100 & 6 & 2200 & 3 & $"$ & $"$ & $"$ & $"$ & $"$ & \\
\hline$"$ & 1200 & 6 & 4900 & 3 & $"$ & $"$ & $"$ & $"$ & $"$ & \\
\hline Eu & 520 & 3 & 3900 & 6 & $9 \times 10^{-8}$ & $"$ & $"$ & $"$ & $"$ & \\
\hline$"$ & 920 & 3 & 9400 & 6 & $"$ & $"$ & $"$ & $"$ & $"$ & \\
\hline$"$ & 1300 & 6 & 1700 & 3 & $"$ & $"$ & $"$ & $"$ & $"$ & \\
\hline$"$ & 890 & 6 & 3300 & 3 & $"$ & $"$ & $"$ & $"$ & $"$ & \\
\hline $\mathrm{Na}$ & 130 & 2 & 150 & 4 & $2 \times 10^{-3}$ & $"$ & $n$ & $"$ & 6 & \\
\hline$"$ & 140 & 2 & 170 & 4 & $"$ & $"$ & $"$ & $"$ & $"$ & \\
\hline$"$ & 140 & 4 & & & $"$ & $"$ & $"$ & $"$ & $"$ & \\
\hline$"$ & 150 & 4 & & & $"$ & $"$ & $"$ & $"$ & $"$ & \\
\hline Mn & 6800 & 3 & $>10000$ & 4 & $2 \times 10^{-11}$ & $"$ & $"$ & $"$ & $"$ & ^ \\
\hline$"$ & 5800 & 2 & 9300 & 4 & $"$ & $"$ & $"$ & $"$ & $"$ & $"$ \\
\hline$"$ & 6400 & 4 & & & $"$ & $"$ & $"$ & $"$ & $"$ & $"$ \\
\hline$"$ & 5000 & 4 & & & $"$ & $"$ & $"$ & $"$ & $"$ & $"$ \\
\hline Se & 8.5 & 2 & 41 & 4 & $1 \times 10^{-9}$ & $"$ & $"$ & " & $"$ & $"$ \\
\hline$"$ & 16 & 2 & 51 & 4 & $"$ & $"$ & $"$ & $"$ & " & $"$ \\
\hline$"$ & 10 & 4 & & & $"$ & $"$ & $"$ & $"$ & $"$ & $"$ \\
\hline$"$ & 8.7 & 4 & & & " & $"$ & $"$ & $"$ & $"$ & $"$ \\
\hline
\end{tabular}

"Concentrations are only those added with the tracer; actual concentrations may be bigher. 
TABLE A-XXXI (cont)

\begin{tabular}{|c|c|c|c|c|c|c|c|c|c|c|}
\hline \multirow[b]{2}{*}{ Element } & \multicolumn{2}{|c|}{ Sorption } & \multicolumn{2}{|c|}{ Desorption } & \multirow{2}{*}{$\begin{array}{c}\text { Element } \\
\text { Concentration } \\
(\mathrm{M}) \\
\end{array}$} & \multirow{2}{*}{$\begin{array}{c}\text { Particle } \\
\text { Size } \\
(1) \text { ) } \\
\end{array}$} & \multirow[b]{2}{*}{ Temperature } & \multirow[b]{2}{*}{ Atnosphere } & \multirow[b]{2}{*}{ Ref. } & \multirow[b]{2}{*}{ Note } \\
\hline & $\begin{array}{c}R_{d} \\
(-2 / e)\end{array}$ & $\begin{array}{l}\text { Tive } \\
\text { (wk) }\end{array}$ & $\begin{array}{c}R_{d} \\
(n e / E)\end{array}$ & $\begin{array}{l}\text { Tine } \\
\text { (wk) }\end{array}$ & & & & & & \\
\hline $\mathrm{Sn}$ & 480 & 2 & 650 & 4 & $3 \times 10^{-8}$ & $<500$ & $\mathrm{Amb}$ & Air & 1 & $\mathbf{a}$ \\
\hline$n$ & 840 & 2 & 500 & 4 & " & $"$ & $"$ & $"$ & $"$ & $"$ \\
\hline$"$ & 250 & 4 & & & $"$ & $"$ & " & " & $"$ & $"$ \\
\hline$"$ & 280 & 4 & & & $"$ & $"$ & $"$ & 1 & " & " \\
\hline $\mathrm{Na}$ & 140 & 2 & 150 & 4 & $2 \times 10^{-3}$ & $"$ & $"$ & $\mathbf{C A}$ & $"$ & \\
\hline$"$ & 150 & 2 & $1 \%$ & 4 & $"$ & $n$ & $"$ & $"$ & $"$ & \\
\hline$"$ & 140 & 4 & 170 & 2 & " & $"$ & $"$ & $"$ & $"$ & \\
\hline$"$ & 160 & 4 & 130 & 2 & $"$ & $"$ & $"$ & $"$ & $"$ & \\
\hline Mn & 2600 & 2 & 1600 & 4 & $2 \times 10^{-11}$ & $"$ & " & " & " & $\mathbf{a}$ \\
\hline " & 2300 & 2 & 1400 & 4 & $"$ & " & $"$ & $"$ & " & $"$ \\
\hline$"$ & 550 & 4 & 3400 & 2 & " & $"$ & $"$ & $"$ & " & " \\
\hline$"$ & 710 & 4 & 2800 & 2 & $"$ & $"$ & $"$ & $"$ & " & $"$ \\
\hline Se & 6.6 & 2 & 63 & 4 & $1 \times 10^{-9}$ & $"$ & 11 & $"$ & $"$ & 11 \\
\hline$"$ & 21 & 2 & 72 & 4 & $n$ & $"$ & $"$ & $"$ & $"$ & $"$ \\
\hline$"$ & 9.5 & 4 & 200 & 2 & " & $"$ & " & " & $"$ & $"$ \\
\hline " & 18 & 4 & 58 & 2 & $"$ & 11 & $"$ & " & $"$ & $"$ \\
\hline Sn & 290 & 2 & 480 & 4 & $3 \times 10^{-8}$ & $"$ & 4 & $"$ & $"$ & " \\
\hline$"$ & 380 & 2 & 520 & 4 & $"$ & $"$ & $"$ & $"$ & $"$ & $"$ \\
\hline$"$ & 110 & 4 & 1300 & 2 & $n$ & $"$ & $1 t$ & $"$ & " & $"$ \\
\hline$"$ & 170 & 4 & 660 & 2 & $"$ & $"$ & $"$ & $"$ & $"$ & $"$ \\
\hline
\end{tabular}

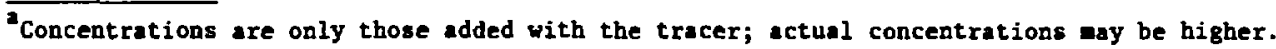


TABLE A-XXII

BATCH SORPTIOH DATA FOR TUFF G1-2289

\begin{tabular}{|c|c|c|c|c|c|c|c|c|c|c|}
\hline \multirow[b]{2}{*}{ Elerent } & \multicolumn{2}{|c|}{ Sarption } & \multicolumn{2}{|c|}{ Desorption } & \multirow{2}{*}{$\begin{array}{c}\text { Element } \\
\text { Concentration } \\
\text { (H) } \\
\end{array}$} & \multirow[b]{2}{*}{$\begin{array}{c}\text { Particle } \\
\text { Size } \\
\text { (1) } \\
\end{array}$} & \multirow[b]{2}{*}{ Tesperature } & \multirow[b]{2}{*}{ Atnosphere } & \multirow[b]{2}{*}{ Ref. } & \multirow[b]{2}{*}{ Note } \\
\hline & $\begin{array}{c}R_{d} \\
(n e / g) \\
\end{array}$ & $\begin{array}{l}\text { Tine } \\
\text { (wk) }\end{array}$ & $\begin{array}{c}R_{d} \\
(n e / s)\end{array}$ & $\begin{array}{l}\text { Tine } \\
\text { (wk) }\end{array}$ & & & & & & \\
\hline $\mathbf{S r}$ & 17000 & 3 & & & $3 \times 10^{-8}$ & $<38$ & $A=b$ & Air & 7 & \\
\hline$"$ & 12000 & 3 & & & $"$ & $"$ & $"$ & " & $"$ & \\
\hline Cs & 43000 & 3 & & & $4 \times 10^{-9}$ & $"$ & $"$ & $n$ & $"$ & \\
\hline$"$ & 27000 & 3 & & & it & $"$ & $"$ & $"$ & $n$ & \\
\hline $\mathrm{Ba}$ & 170000 & 3 & & & $1 \times 10^{-7}$ & $"$ & $"$ & $n$ & $"$ & \\
\hline$"$ & 110000 & 3 & & & $"$ & $"$ & * & $"$ & $"$ & \\
\hline Eu & 1600 & 3 & & & $2 \times 10^{-8}$ & $"$ & 11 & $"$ & $"$ & \\
\hline$"$ & 1400 & 3 & & & $"$ & $"$ & $"$ & $"$ & $"$ & \\
\hline Sr & 6300 & 3 & & & $3 \times 10^{-8}$ & $38-106$ & $"$ & $"$ & $"$ & \\
\hline$"$ & 6400 & 3 & & & $"$ & $"$ & $"$ & $n$ & $"$ & \\
\hline $\mathrm{Cs}$ & 34000 & 3 & & & $4 \times 10^{-9}$ & $"$ & $"$ & $"$ & $"$ & \\
\hline$"$ & 29000 & 3 & & & $"$ & " & $"$ & $"$ & $"$ & \\
\hline $\mathrm{Ba}$ & 54000 & 3 & & & $1 \times 10^{-7}$ & $"$ & $"$ & $"$ & $"$ & \\
\hline$"$ & 48000 & 3 & & & $"$ & $"$ & $"$ & $"$ & $"$ & \\
\hline $\mathrm{Eu}$ & 780 & 3 & & & $2 \times 10^{-8}$ & $"$ & $"$ & $"$ & $"$ & \\
\hline$"$ & 780 & 3 & & & $"$ & $"$ & $"$ & $"$ & $"$ & \\
\hline Sr & 7800 & 3 & & & $3 \times 10^{-8}$ & $106-500$ & $"$ & $"$ & $"$ & \\
\hline$"$ & 8400 & 3 & & & $"$ & $"$ & $"$ & " & " & \\
\hline Cs & 12000 & 3 & & & $4 \times 10^{-9}$ & " & $"$ & " & " & \\
\hline$"$ & 72000 & 3 & & & $"$ & $"$ & $"$ & " & $"$ & \\
\hline $\mathrm{Ba}$ & 90000 & 3 & & & $1 \times 10^{-7}$ & $"$ & $"$ & $"$ & $"$ & \\
\hline$"$ & 70000 & 3 & & & $"$ & $"$ & $"$ & $"$ & $"$ & \\
\hline Eu & 820 & 3 & & & $2 \times 10^{-8}$ & $"$ & " & $"$ & $"$ & \\
\hline$"$ & 810 & 3 & & & 11 & $"$ & $"$ & $"$ & $"$ & \\
\hline $\mathrm{Na}$ & 230 & 2 & 230 & 4 & $3 \times 10^{-3}$ & $<500$ & $"$ & 11 & 6 & \\
\hline$"$ & 220 & 2 & 250 & 4 & $"$ & " & $"$ & $"$ & $"$ & \\
\hline$"$ & 230 & 4 & 260 & 2 & $"$ & $"$ & $H$ & $"$ & $"$ & \\
\hline$n$ & 230 & 4 & 270 & 2 & $"$ & $"$ & " & $"$ & $"$ & \\
\hline
\end{tabular}


TABLE A-XXII (cont)

\begin{tabular}{|c|c|c|c|c|c|c|c|c|c|c|}
\hline \multirow[b]{2}{*}{ Elegent } & \multicolumn{2}{|c|}{ Sorption } & \multicolumn{2}{|c|}{ Desorption } & \multirow[b]{2}{*}{$\begin{array}{c}\text { Blewent } \\
\text { Concentration } \\
\text { (H) }\end{array}$} & \multirow[b]{2}{*}{$\begin{array}{c}\text { Particle } \\
\text { Size } \\
(\mu \mathrm{j}) \\
\end{array}$} & \multirow[b]{2}{*}{ Teuperature } & \multirow[b]{2}{*}{ Atmosphere } & \multirow[b]{2}{*}{ Ref. } & \multirow[b]{2}{*}{ Note } \\
\hline & $\begin{array}{c}R_{d} \\
(\mathbf{R} / \mathbf{R}) \\
\end{array}$ & $\begin{array}{l}\text { Tine } \\
\text { (wk) }\end{array}$ & $\begin{array}{c}R_{d} \\
(n / / g)\end{array}$ & $\begin{array}{l}\text { Tine } \\
\text { (wk) }\end{array}$ & & & & & & \\
\hline Ho & $>10000$ & 2 & $>10000$ & 4 & $1 \times 10^{-11}$ & $<500$ & $A \mathbf{b}$ & Air & 6 & $\mathbf{a}$ \\
\hline$"$ & $>10000$ & 2 & $>10000$ & 4 & $"$ & $"$ & $"$ & $"$ & $"$ & $"$ \\
\hline$"$ & 6000 & 4 & $>10000$ & 2 & $"$ & $"$ & $"$ & " & $n$ & $"$ \\
\hline$"$ & 7300 & 4 & $>10000$ & 2 & $"$ & $"$ & " & $"$ & $"$ & $"$ \\
\hline Se & 24 & 2 & 21 & 4 & $8 \times 10^{-10}$ & $"$ & $"$ & $"$ & $"$ & $"$ \\
\hline$"$ & 8.8 & 2 & 32 & 4 & $"$ & $"$ & $"$ & $"$ & $"$ & $"$ \\
\hline$"$ & 10 & 4 & 58 & 2 & $"$ & $"$ & $"$ & $"$ & $"$ & $"$ \\
\hline$"$ & 12 & 4 & 48 & 2 & $"$ & $"$ & $"$ & $"$ & $"$ & $"$ \\
\hline Sn & 1100 & 2 & 2000 & 4 & $1 \times 10^{-8}$ & $"$ & $"$ & $"$ & $"$ & $"$ \\
\hline$"$ & 560 & 2 & 1000 & 4 & $"$ & $"$ & $"$ & $"$ & $"$ & $"$ \\
\hline$"$ & 550 & 4 & 1400 & 2 & $"$ & $"$ & $"$ & $"$ & $"$ & $"$ \\
\hline$"$ & 640 & 4 & 1500 & 2 & " & $"$ & $"$ & $"$ & $"$ & $"$ \\
\hline
\end{tabular}

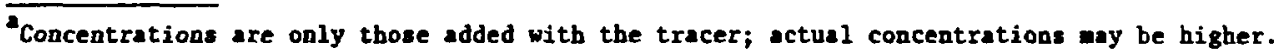


TABLE XXIII

BATCH SORPTION DATA FOR TUFF G1-2333

\begin{tabular}{|c|c|c|c|c|c|c|c|c|c|c|}
\hline \multirow[b]{2}{*}{ Element } & \multicolumn{2}{|c|}{ Sorption } & \multicolumn{2}{|c|}{ Desorption } & \multirow{2}{*}{$\begin{array}{c}\text { Element } \\
\text { Concentration } \\
\text { (N) }\end{array}$} & \multirow[b]{2}{*}{$\begin{array}{c}\text { Particle } \\
\text { Size } \\
\text { (뿌) } \\
\end{array}$} & \multirow[b]{2}{*}{ Temperature } & \multirow[b]{2}{*}{ Ateosphere } & \multirow[b]{2}{*}{ Ref. } & \multirow[b]{2}{*}{ Note } \\
\hline & $\begin{array}{c}R_{d} \\
(n e / s)\end{array}$ & $\begin{array}{l}\text { Time } \\
\text { (wk) }\end{array}$ & $\begin{array}{c}R_{d} \\
(n / / R)\end{array}$ & $\begin{array}{l}\text { Time } \\
\text { (wk) }\end{array}$ & & & & & & \\
\hline sr & 150 & 3 & 130 & 6 & $1 \times 10^{-6}$ & $75-500$ & $A=b$ & Air & 6 & \\
\hline$"$ & 140 & 6 & 110 & 3 & $"$ & $"$ & $"$ & $"$ & $"$ & \\
\hline Cs & 1200 & 3 & 1100 & 6 & $5 \times 10^{-9}$ & $"$ & $"$ & $"$ & $"$ & \\
\hline$"$ & 1100 & 6 & 1000 & 3 & $"$ & $"$ & $"$ & $"$ & $"$ & \\
\hline Ba & 1200 & 3 & 1400 & 6 & $3 \times 10^{-7}$ & $"$ & $"$ & $"$ & $"$ & \\
\hline$"$ & 1100 & 6 & 1100 & 3 & $"$ & $"$ & $"$ & $"$ & $"$ & \\
\hline Eu & 1400 & 3 & 9800 & 6 & $3 \times 10^{-8}$ & $"$ & $"$ & $"$ & $"$ & \\
\hline$"$ & 2900 & 6 & 6800 & 3 & $"$ & $"$ & $"$ & $"$ & $"$ & \\
\hline Sr & 220 & 3 & 160 & 6 & $1 \times 10^{-6}$ & $<75$ & $"$ & $"$ & $"$ & \\
\hline$"$ & 220 & 6 & 160 & 3 & $"$ & $"$ & $"$ & $"$ & $"$ & \\
\hline Cs & 1700 & 3 & 1400 & 6 & $5 \times 10^{-9}$ & $n$ & $"$ & $"$ & $"$ & \\
\hline$"$ & 1500 & 6 & 1300 & 3 & $"$ & $"$ & $"$ & $"$ & $"$ & \\
\hline $\mathrm{Ba}$ & 1900 & 3 & 1700 & 6 & $3 \times 10^{-7}$ & $"$ & $"$ & $"$ & $"$ & \\
\hline$"$ & 1800 & 6 & 1600 & 3 & $"$ & $"$ & $"$ & $"$ & $"$ & \\
\hline Eu & 2000 & 3 & 1200 & 3 & $3 \times 10^{-8}$ & $"$ & $"$ & $"$ & $"$ & \\
\hline " & 3100 & 6 & 11000 & 3 & $"$ & $"$ & $"$ & $"$ & $"$ & \\
\hline
\end{tabular}


TABLI A-XXIV

BATCH SORPTION DATA FOR TUFT G1-2363

\begin{tabular}{|c|c|c|c|c|c|c|c|c|c|c|}
\hline \multirow[b]{2}{*}{ Element } & \multicolumn{2}{|c|}{ Sorption } & \multicolumn{2}{|c|}{ Desorption } & \multirow{2}{*}{$\begin{array}{c}\begin{array}{c}\text { Element } \\
\text { Concentration } \\
(\mathrm{K})\end{array} \\
\end{array}$} & \multirow{2}{*}{$\begin{array}{c}\text { Particle } \\
\text { Size } \\
\text { (1) } \\
\end{array}$} & \multirow[b]{2}{*}{ Tesperature } & \multirow[b]{2}{*}{ Atmosphere } & \multirow[b]{2}{*}{ Ref. } & \multirow[b]{2}{*}{ Note } \\
\hline & $\begin{array}{c}R_{d} \\
(e / 2)\end{array}$ & $\begin{array}{l}\text { Time } \\
\text { (wk) }\end{array}$ & $\begin{array}{c}R_{d} \\
(n e / R)\end{array}$ & $\begin{array}{l}\text { Tine } \\
\text { (wk) }\end{array}$ & & & & & & \\
\hline $\mathbf{S r}$ & 170 & 3 & 140 & 6 & $4 \times 10^{-7}$ & $<500$ & $A=b$ & Air & 1 & \\
\hline$"$ & 180 & 3 & 160 & 6 & $"$ & $"$ & $"$ & $"$ & $"$ & \\
\hline$"$ & 170 & 6 & $1400^{\circ}$ & 3 & $"$ & $"$ & $"$ & $"$ & $"$ & . \\
\hline$"$ & 170 & 6 & 150 & 3 & $"$ & $"$ & $"$ & $"$ & $"$ & \\
\hline CE & 1300 & 3 & 1200 & 6 & $7 \times 10^{-9}$ & $"$ & $"$ & $"$ & $"$ & \\
\hline$"$ & 1200 & 3 & 1300 & 6 & $"$ & $"$ & $"$ & $"$ & $"$ & \\
\hline$"$ & 1400 & 6 & 1200 & 3 & $"$ & $"$ & $"$ & $"$ & $"$ & \\
\hline$"$ & 1400 & 6 & 1200 & 3 & $"$ & $"$ & $"$ & $"$ & $"$ & \\
\hline Ba & 880 & 3 & 800 & 6 & $8 \times 10^{-6}$ & $"$ & $"$ & $"$ & $"$ & \\
\hline$"$ & 830 & 3 & 870 & 6 & $n$ & $"$ & $"$ & $"$ & $"$ & \\
\hline$"$ & 920 & 6 & 780 & 3 & $"$ & $"$ & $"$ & $"$ & $"$ & \\
\hline$"$ & 930 & 6 & 830 & 3 & $"$ & $"$ & $"$ & $"$ & $"$ & \\
\hline Ce & 290 & 3 & 140000 & 6 & $3 \times 10^{-8}$ & $"$ & $"$ & $"$ & $"$ & \\
\hline$"$ & 270 & 3 & 120000 & 6 & $"$ & $"$ & $"$ & $"$ & $"$ & \\
\hline$"$ & 370 & 6 & $1400000^{2}$ & 3 & $"$ & $"$ & $"$ & $"$ & $"$ & 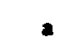 \\
\hline$"$ & 310 & 6 & 130000 & 3 & $"$ & $"$ & $"$ & $"$ & $"$ & \\
\hline Eu & 1300 & 3 & 5700 & 6 & $8 \times 10^{-8}$ & $"$ & $"$ & $"$ & $"$ & \\
\hline$"$ & 1300 & 3 & $450^{2}$ & 6 & $"$ & $"$ & $"$ & $"$ & $"$ & $a$ \\
\hline$"$ & 1900 & 6 & 5900 & 3 & $"$ & $"$ & $"$ & $"$ & $"$ & \\
\hline$"$ & 1600 & 6 & 6700 & 3 & $"$ & $"$ & $"$ & $"$ & $"$ & \\
\hline Sr & 180 & 3 & & & $5 \times 10^{-7}$ & $<38$ & $"$ & $"$ & 7 & \\
\hline$"$ & 170 & 3 & & & $"$ & $"$ & $"$ & $"$ & $"$ & \\
\hline Cs & 1400 & 3 & & & $4 \times 10^{-9}$ & $"$ & $"$ & $"$ & $"$ & \\
\hline$"$ & 1300 & 3 & & & $"$ & $"$ & $"$ & $"$ & $"$ & \\
\hline Ba & 920 & 3 & & & $1 \times 10^{-7}$ & $"$ & $"$ & $"$ & $"$ & \\
\hline$"$ & 860 & 3 & & & $"$ & $"$ & $"$ & $"$ & $"$ & \\
\hline Eu & 5600 & 3 & & & $5 \times 10^{-8}$ & $"$ & $"$ & $"$ & $"$ & \\
\hline$"$ & 5400 & 3 & & & $"$ & $"$ & $"$ & $"$ & $"$ & \\
\hline Sr & 74 & 3 & & & $5 \times 10^{-7}$ & $38-106$ & $"$ & $"$ & $"$ & \\
\hline$"$ & 62 & 3 & & & $"$ & $"$ & $"$ & $"$ & $"$ & \\
\hline
\end{tabular}

value not included in averages for Table XXII, Chap. 1. 
TABLE A-XXXIV (cont)

\begin{tabular}{|c|c|c|c|c|c|c|c|c|c|c|}
\hline \multirow[b]{2}{*}{ Element } & \multicolumn{2}{|c|}{ Sorption } & \multicolumn{2}{|c|}{ Desorption } & \multirow{2}{*}{$\begin{array}{c}\text { Element } \\
\text { Concentration } \\
(\mathrm{M})\end{array}$} & \multirow{2}{*}{$\begin{array}{c}\text { Particle } \\
\text { Size } \\
\text { (m) } \\
\end{array}$} & \multirow[b]{2}{*}{ Tewperature } & \multirow[b]{2}{*}{ Atmosphere } & \multirow[b]{2}{*}{ Ref. } & \multirow[b]{2}{*}{ Note } \\
\hline & $\begin{array}{c}R_{\mathrm{d}} \\
(n / \mathrm{e})\end{array}$ & $\begin{array}{l}\text { Time } \\
\text { (wk) }\end{array}$ & $\begin{array}{c}R_{d} \\
(\alpha e / 2)\end{array}$ & $\begin{array}{l}\text { Time } \\
\text { (wk) }\end{array}$ & & & & & & \\
\hline cs & 550 & 3 & & & $4 \times 10^{-9}$ & $38-106$ & Anb & Air & 7 & \\
\hline$"$ & 520 & 3 & & & $"$ & $"$ & $"$ & $"$ & $"$ & \\
\hline $\mathrm{Ba}$ & 240 & 3 & & & $1 \times 10^{-7}$ & $"$ & $"$ & $"$ & $"$ & \\
\hline$"$ & 250 & 3 & & & $"$ & $"$ & $"$ & $"$ & $"$ & \\
\hline Eu & 780 & 3 & & & $5 \times 10^{-8}$ & $"$ & $"$ & $"$ & $"$ & \\
\hline$"$ & 790 & 3 & & & $"$ & $"$ & $"$ & $"$ & $"$ & \\
\hline $\mathrm{Sr}$ & 58 & 3 & & & $5 \times 10^{-7}$ & $106-500$ & $"$ & $"$ & $"$ & \\
\hline$"$ & 61 & 3 & & & $"$ & $"$ & $"$ & $"$ & $"$ & \\
\hline Cs & 410 & 3 & & & $4 \times 10^{-9}$ & $"$ & $"$ & $"$ & $"$ & \\
\hline$"$ & 380 & 3 & & & $"$ & $"$ & $"$ & $"$ & $"$ & \\
\hline $\mathrm{Ba}$ & 230 & 3 & & & $1 \times 10^{-7}$ & " & $"$ & $"$ & $"$ & \\
\hline$"$ & 210 & 3 & & & $"$ & $"$ & $"$ & $"$ & $"$ & \\
\hline Eu & 780 & 3 & & & $5 \times 10^{-8}$ & $"$ & $"$ & $"$ & $"$ & \\
\hline$"$ & 580 & 3 & & & $"$ & $"$ & $"$ & $"$ & $"$ & \\
\hline
\end{tabular}


TAOLA A-XXXV

WTCH SONPTIOI DATA FOR TUFF G1-2410

\begin{tabular}{|c|c|c|c|c|c|c|c|c|c|c|}
\hline \multirow[b]{2}{*}{ Element } & \multicolumn{2}{|c|}{ Sorption } & \multicolumn{2}{|c|}{ Denorption } & \multirow{2}{*}{$\begin{array}{c}\text { Elenent } \\
\text { Concentration } \\
\text { (H) }\end{array}$} & \multirow[b]{2}{*}{$\begin{array}{c}\text { Particle } \\
\text { Size } \\
(\mu) \\
\end{array}$} & \multirow[b]{2}{*}{ Te-perature } & \multirow[b]{2}{*}{ Atnosphere } & \multirow[b]{2}{*}{ Ref. } & \multirow[b]{2}{*}{ Note } \\
\hline & $\begin{array}{c}R_{d} \\
(-2 / \Omega)\end{array}$ & $\begin{array}{l}\text { Tine } \\
\text { (wk) }\end{array}$ & $\begin{array}{c}R_{d} \\
(-2 / z)\end{array}$ & $\begin{array}{l}\text { Tine } \\
\text { (vk) }\end{array}$ & & & & & & \\
\hline Sr & 170 & 3 & 150 & 6 & $4 \times 10^{-7}$ & $75-500$ & $A=b$ & Air & 6 & \\
\hline$"$ & 170 & 6 & 130 & 3 & $"$ & $"$ & $"$ & $"$ & $"$ & \\
\hline Cs & 1200 & 3 & 1200 & 6 & $4 \times 10^{-9}$ & $"$ & $n$ & " & $"$ & \\
\hline II & 1300 & 6 & 1000 & 3 & $"$ & $"$ & $"$ & $"$ & $"$ & \\
\hline Ba & & 3 & 1900 & 6 & $2 \times 10^{-7}$ & $"$ & $"$ & $"$ & $"$ & \\
\hline$"$ & 1800 & 6 & 1600 & 3 & $"$ & $"$ & $"$ & $"$ & $"$ & \\
\hline Eu & 360 & 3 & 8700 & 6 & $2 \times 10^{-8}$ & $"$ & $"$ & $"$ & $"$ & \\
\hline$"$ & 510 & 6 & 3300 & 3 & $"$ & $"$ & $"$ & $"$ & $"$ & \\
\hline $\mathbf{S r}$ & 280 & 3 & 250 & 6 & $4 \times 10^{-7}$ & $<75$ & $"$ & $"$ & $"$ & \\
\hline$"$ & 280 & 6 & 240 & 3 & $"$ & $"$ & $"$ & $n$ & $"$ & \\
\hline Cs & 2000 & 3 & 1800 & 6 & $4 \times 10^{-9}$ & $"$ & $"$ & $"$ & $"$ & \\
\hline$"$ & 2000 & 6 & 1800 & 3 & " & $"$ & $"$ & $"$ & $"$ & \\
\hline Ba & & 3 & 3000 & 6 & $2 \times 10^{-7}$ & $"$ & $"$ & $"$ & $"$ & \\
\hline " & 3000 & 6 & 3000 & 3 & " & $"$ & $"$ & $"$ & " & \\
\hline Eu & 390 & 3 & 10000 & 6 & $2 \times 10^{-8}$ & " & $"$ & $"$ & $"$ & \\
\hline$"$ & 440 & 6 & 5700 & 3 & $"$ & $"$ & $n$ & $"$ & $"$ & \\
\hline
\end{tabular}


TABLE A-XXVI

BATCY SORPTION DATA FOR TUFF G1-2476

\begin{tabular}{|c|c|c|c|c|c|c|c|c|c|c|}
\hline \multirow[b]{2}{*}{ Element } & \multicolumn{2}{|c|}{ Sorption } & \multicolumn{2}{|c|}{ Desorption } & \multirow{2}{*}{$\begin{array}{c}\text { Element } \\
\text { Coscentration } \\
(\mathrm{M})\end{array}$} & \multirow{2}{*}{$\begin{array}{c}\text { Particle } \\
\text { Size } \\
\left.\text { ( } \boldsymbol{\mu}^{\mathrm{m}}\right) \\
\end{array}$} & \multirow[b]{2}{*}{ Teuperature } & \multirow[b]{2}{*}{ Atwosphere } & \multirow[b]{2}{*}{ Ref. } & \multirow[b]{2}{*}{ Note } \\
\hline & $\begin{array}{c}R_{d} \\
(-\ell / p) \\
\end{array}$ & $\begin{array}{l}\text { Time } \\
\text { (wk) }\end{array}$ & $\begin{array}{c}R_{d} \\
(\mu / / 8)\end{array}$ & $\begin{array}{l}\text { Time } \\
\text { (wk) }\end{array}$ & & & & & & \\
\hline St & 41 & 3 & 210 & 6 & $9 \times 10^{-7}$ & $75-500$ & Anb & Air & 6 & \\
\hline$"$ & 40 & 6 & 200 & 3 & $"$ & $"$ & $"$ & $"$ & $"$ & \\
\hline Cs & 660 & 3 & 1500 & 6 & $5 \times 10^{-9}$ & $"$ & $"$ & $"$ & $"$ & \\
\hline$"$ & 740 & 6 & 1500 & 3 & $"$ & $"$ & $"$ & $"$ & $"$ & \\
\hline Ba & 370 & 3 & 3800 & 6 & $1 \times 10^{-7}$ & $"$ & $"$ & $"$ & $"$ & \\
\hline$"$ & 400 & 6 & 3600 & 3 & $"$ & $"$ & $"$ & $"$ & $"$ & \\
\hline Eu & 3300 & 3 & 15000 & 6 & $6 \times 10^{-8}$ & $"$ & $"$ & $"$ & $"$ & \\
\hline$"$ & 3100 & 6 & 15000 & 3 & $"$ & $"$ & $"$ & $"$ & $"$ & \\
\hline Sr & 49 & 3 & 200 & 6 & $9 \times 10^{-7}$ & $<75$ & $"$ & $"$ & $"$ & \\
\hline$"$ & 51 & 6 & 220 & 3 & $"$ & $"$ & $"$ & $"$ & $"$ & \\
\hline Cs & 810 & 3 & 1600 & 6 & $5 \times 10^{-9}$ & $"$ & $"$ & $"$ & $"$ & \\
\hline$"$ & 920 & 6 & 1800 & 3 & $"$ & $"$ & $"$ & $"$ & $"$ & \\
\hline Ba & 480 & 3 & 3200 & 6 & $1 \times 10^{-7}$ & $"$ & $"$ & $"$ & $"$ & \\
\hline$"$ & 520 & 6 & 3500 & 3 & $"$ & $"$ & $"$ & $"$ & $"$ & \\
\hline Eu & 4600 & 3 & 9300 & 6 & $6 \times 10^{-8}$ & $"$ & $"$ & $"$ & $"$ & \\
\hline$"$ & 5100 & 6 & 12000 & 3 & $"$ & $"$ & $"$ & $"$ & $"$ & \\
\hline
\end{tabular}


TABLE A-XXXVII

BATCH SORPTION DATA FOR TUFF G1-2698

\begin{tabular}{|c|c|c|c|c|c|c|c|c|c|c|}
\hline \multirow[b]{2}{*}{ Eleoent } & \multicolumn{2}{|c|}{ Sorption } & \multicolumn{2}{|c|}{ Derorption } & \multirow[b]{2}{*}{$\begin{array}{c}\text { Element } \\
\begin{array}{c}\text { Concentration } \\
(\mathrm{K})\end{array} \\
\end{array}$} & \multirow[b]{2}{*}{$\begin{array}{c}\text { Particle } \\
\text { Size } \\
\text { (1) } \\
\end{array}$} & \multirow[b]{2}{*}{ Temperature } & \multirow[b]{2}{*}{ Atmosphere } & \multirow[b]{2}{*}{ Ref. } & \multirow[b]{2}{*}{ Note } \\
\hline & $\begin{array}{c}R_{d} \\
(n \ell / g)\end{array}$ & $\begin{array}{l}\text { Tine } \\
\text { (wk) }\end{array}$ & $\begin{array}{c}R_{d} \\
(n / / R)\end{array}$ & $\begin{array}{l}\text { Time } \\
\text { (wk) }\end{array}$ & & & & & & \\
\hline $\mathbf{S r}$ & 49000 & 3 & 200000 & 6 & $2 \times 10^{-7}$ & $<500$ & $A b$ & Air & 1 & \\
\hline " & 39000 & 3 & 170000 & 6 & $"$ & $"$ & $"$ & $"$ & $"$ & \\
\hline$"$ & 43000 & 6 & 350000 & 3 & $"$ & $"$ & $"$ & " & $"$ & \\
\hline$"$ & 37000 & 6 & 130000 & 3 & $"$ & $"$ & " & " & $"$ & \\
\hline Cs & 8200 & 3 & 15000 & 6 & $5 \times 10^{-9}$ & $"$ & $"$ & $"$ & $"$ & \\
\hline$"$ & 7400 & 3 & 16000 & 6 & $"$ & $"$ & " & $"$ & $"$ & \\
\hline$"$ & 8500 & 6 & 20000 & 3 & $"$ & $"$ & $"$ & $"$ & $"$ & \\
\hline$"$ & 6600 & 6 & 17000 & 3 & $"$ & $"$ & $"$ & $"$ & $"$ & \\
\hline $\mathrm{Ba}$ & 61000 & 3 & 47000 & 6 & $8 \times 10^{-6}$ & $"$ & $"$ & $"$ & $"$ & \\
\hline$" 1$ & 56000 & 3 & 390000 & 6 & $"$ & $"$ & $"$ & 11 & $"$ & \\
\hline$"$ & 77000 & 6 & 62000 & 3 & $"$ & $"$ & $"$ & $"$ & " & \\
\hline$"$ & 58000 & 6 & 270000 & 3 & " & $"$ & $"$ & $"$ & $"$ & \\
\hline $\mathrm{Ce}$ & 220 & 3 & 2800 & 6 & $1 \times 10^{-7}$ & $"$ & $"$ & $"$ & $"$ & \\
\hline$"$ & 170 & 3 & 2400 & 6 & $"$ & $"$ & $"$ & $"$ & $"$ & \\
\hline$"$ & 270 & 6 & 1600 & 3 & $"$ & "1 & $n$ & $"$ & $"$ & \\
\hline$"$ & 310 & 6 & 1200 & 3 & $"$ & $"$ & $"$ & $"$ & " & \\
\hline Eu & 180 & 3 & 2100 & 6 & $4 \times 10^{-7}$ & $"$ & $"$ & $"$ & $"$ & \\
\hline$"$ & 130 & 3 & 1900 & 6 & " & " & $"$ & $"$ & $"$ & \\
\hline$"$ & 230 & 6 & 1000 & 3 & $"$ & " & $n$ & $"$ & $"$ & \\
\hline$"$ & 260 & 6 & 850 & 3 & $"$ & $"$ & $"$ & $"$ & $"$ & \\
\hline
\end{tabular}


TABLE A-XOVIII

BATCH SORPTIOY DATA FOR TUET G1-2840

\begin{tabular}{|c|c|c|c|c|c|c|c|c|c|c|}
\hline \multirow[b]{2}{*}{ Element } & \multicolumn{2}{|c|}{ Sorption } & \multicolumn{2}{|c|}{ Desarption } & \multirow{2}{*}{$\begin{array}{c}\text { Element } \\
\text { Concentration } \\
(M) \\
\end{array}$} & \multirow{2}{*}{$\begin{array}{c}\text { Particle } \\
\text { Size } \\
\text { (些) } \\
\end{array}$} & \multirow[b]{2}{*}{ Teuperature } & \multirow[b]{2}{*}{ Atwosphere } & \multirow[b]{2}{*}{ Ref. } & \multirow[b]{2}{*}{ Note } \\
\hline & $\begin{array}{c}R_{d} \\
(A l / 8)\end{array}$ & $\begin{array}{l}\text { Time } \\
\text { (wk) } \\
\end{array}$ & $\begin{array}{c}R_{d} \\
(\Omega e / g)\end{array}$ & $\begin{array}{l}\text { Tise } \\
\text { (wk) } \\
\end{array}$ & & & & & & \\
\hline Sr & 160 & 3 & 130 & 6 & $1 \times 10^{-6}$ & $75-500$ & Anb & Air & 6 & \\
\hline$"$ & 159 & 6 & 130 & 3 & $"$ & $"$ & $"$ & $"$ & $"$ & \\
\hline Cs & 2400 & 3 & 2000 & 6 & $5 \times 10^{-9}$ & $"$ & $"$ & $"$ & $"$ & \\
\hline$"$ & 2000 & 6 & 2100 & 3 & $"$ & $"$ & $n$ & $"$ & $"$ & \\
\hline $\mathrm{Ba}$ & 2000 & 3 & 2300 & 6 & $2 \times 10^{-7}$ & $"$ & $"$ & $"$ & $"$ & \\
\hline$"$ & 2100 & 6 & 2300 & 3 & $"$ & $"$ & $"$ & $"$ & $"$ & \\
\hline Eu & 4500 & 3 & 8000 & 6 & $3 \times 10^{-8}$ & $"$ & $"$ & $"$ & $"$ & \\
\hline$"$ & 5300 & 6 & 7900 & 3 & $"$ & $"$ & $"$ & $"$ & $"$ & \\
\hline Sr & 170 & 3 & 150 & 6 & $1 \times 10^{-6}$ & $<75$ & $"$ & $"$ & $"$ & \\
\hline$"$ & 170 & 6 & 160 & 3 & $"$ & $"$ & $"$ & $"$ & $"$ & \\
\hline Cs & 2800 & 3 & 2500 & 6 & $5 \times 10^{-9}$ & $"$ & $"$ & $"$ & $"$ & \\
\hline$"$ & 2500 & 6 & 2500 & 3 & $"$ & $"$ & $"$ & $"$ & $"$ & \\
\hline $\mathrm{Ba}$ & 2300 & 3 & 2700 & 6 & $2 \times 10^{-7}$ & $"$ & $"$ & $"$ & $"$ & \\
\hline$"$ & 2600 & 6 & 2800 & 3 & " & $"$ & $"$ & $"$ & $"$ & \\
\hline Eu & 5000 & 3 & 12000 & 6 & $3 \times 10^{-8}$ & $"$ & $"$ & " & $"$ & \\
\hline$"$ & 6200 & 6 & 7900 & 3 & $"$ & $"$ & $"$ & $"$ & $"$ & \\
\hline
\end{tabular}


TABLE A-XXXIX

BATCH SORPTION DATA FOR TUFF G1-2854

\begin{tabular}{|c|c|c|c|c|c|c|c|c|c|c|}
\hline \multirow[b]{2}{*}{ Eleeent } & \multicolumn{2}{|c|}{ Sorption } & \multicolumn{2}{|c|}{ Desorption } & \multirow[b]{2}{*}{$\begin{array}{c}\text { Elewent } \\
\text { Concentration } \\
(M)\end{array}$} & \multirow[b]{2}{*}{$\begin{array}{c}\text { Particle } \\
\text { Size } \\
\text { (1) } \\
\end{array}$} & \multirow[b]{2}{*}{ Temperature } & \multirow[b]{2}{*}{ Atmosphere } & \multirow[b]{2}{*}{ Ref. } & \multirow[b]{2}{*}{ Note } \\
\hline & $\begin{array}{c}R_{d} \\
(n e / g)\end{array}$ & $\begin{array}{l}\text { Tine } \\
\text { (wk) }\end{array}$ & $\begin{array}{c}R_{d} \\
(n e / 2)\end{array}$ & $\begin{array}{l}\text { Tine } \\
\text { (wt) }\end{array}$ & & & & & & \\
\hline $\mathbf{s r}$ & 94 & 3 & 96 & 6 & $5 \times 10^{-7}$ & $75-500$ & $A=b$ & Air & 6 & \\
\hline$"$ & 93 & 6 & 97 & 3 & $"$ & $"$ & $"$ & $"$ & $"$ & \\
\hline $\mathrm{Cs}$ & 1200 & 3 & 1100 & 6 & $5 \times 10^{-9}$ & $"$ & $"$ & " & $"$ & \\
\hline$"$ & 950 & 6 & 1200 & 3 & $"$ & $"$ & $"$ & $"$ & $"$ & \\
\hline $\mathrm{Ba}$ & 950 & 3 & 1300 & 6 & $1 \times 10^{-7}$ & $"$ & $"$ & $"$ & " & \\
\hline$n$ & 1000 & 6 & 1300 & 3 & $"$ & $"$ & $"$ & $"$ & $"$ & \\
\hline Eu & 1100 & 3 & 4800 & 6 & $2 \times 10^{-8}$ & $"$ & $"$ & $"$ & $" 1$ & \\
\hline$"$ & 1500 & 6 & 5200 & 3 & $"$ & $"$ & $"$ & $"$ & " & \\
\hline $\mathbf{S r}$ & 120 & 3 & 130 & 6 & $5 \times 10^{-7}$ & $<75$ & $"$ & $"$ & $"$ & \\
\hline$"$ & 60 & 6 & 92 & 3 & $"$ & $"$ & $"$ & $"$ & $"$ & \\
\hline Cs & 1700 & 3 & 1900 & 6 & $5 \times 10^{-9}$ & $"$ & $"$ & $"$ & $"$ & \\
\hline " & 510 & 6 & 1300 & 3 & " & $"$ & $"$ & $"$ & $"$ & \\
\hline Ba & 1600 & 3 & 3700 & 6 & $1 \times 10^{-7}$ & $"$ & $"$ & $"$ & $"$ & \\
\hline$"$ & 6500 & 6 & 1600 & 3 & $"$ & $"$ & $"$ & 11 & $"$ & \\
\hline $\mathbf{E u}$ & 1100 & 3 & 7200 & 6 & $2 \times 10^{-8}$ & $"$ & $"$ & $"$ & $"$ & \\
\hline$n$ & 2600 & 6 & 3100 & 3 & $"$ & $"$ & $"$ & $"$ & $"$ & \\
\hline
\end{tabular}


TABLE A-XOX

BATCH SORPTION DATA FOR TUFF G1-2901

\begin{tabular}{|c|c|c|c|c|c|c|c|c|c|c|}
\hline \multirow[b]{2}{*}{ Elewent } & \multicolumn{2}{|c|}{ Sorption } & \multicolumn{2}{|c|}{ Desorption } & \multirow{2}{*}{$\begin{array}{c}\text { Element } \\
\text { Concentration } \\
(\mathrm{M})\end{array}$} & \multirow{2}{*}{$\begin{array}{l}\text { Particle } \\
\text { Size } \\
(10) \\
\end{array}$} & \multirow[b]{2}{*}{ Temperature } & \multirow[b]{2}{*}{ Atmophere } & \multirow[b]{2}{*}{ Ref. } & \multirow[b]{2}{*}{ Note } \\
\hline & $\begin{array}{c}R_{d} \\
(n / / R) \\
\end{array}$ & $\begin{array}{l}\text { Time } \\
\text { (wk) }\end{array}$ & $\begin{array}{c}R_{d} \\
(a / 2)\end{array}$ & $\begin{array}{l}\text { Time } \\
\text { (uk) }\end{array}$ & & & & & & \\
\hline Sr & 68 & 3 & 67 & 6 & $1 \times 10^{-6}$ & $<500$ & $A a b$ & Air & 1 & \\
\hline$"$ & 66 & 3 & 66 & 6 & $"$ & $"$ & $"$ & $"$ & $"$ & \\
\hline$"$ & 69 & 6 & 69 & 3 &. & $"$ & $"$ & $"$ & $"$ & \\
\hline$"$ & 71 & 6 & $740^{\circ}$ & 3 & $"$ & $"$ & $"$ & $"$ & $"$ & $\mathbf{a}$ \\
\hline$C_{5}$ & 1400 & 3 & 1300 & 6 & $6 \times 10^{-9}$ & $"$ & $"$ & $"$ & $"$ & \\
\hline$"$ & 950 & 3 & 1400 & 6 & $"$ & $"$ & $"$ & $"$ & $"$ & \\
\hline$"$ & 1400 & 6 & 1400 & 3 & $"$ & $"$ & $"$ & $"$ & $"$ & \\
\hline$"$ & 1400 & 6 & 1400 & 3 & $"$ & $"$ & $"$ & $"$ & $"$ & \\
\hline $\mathrm{Ba}$ & 3900 & 3 & 1900 & 6 & $1 \times 10^{-6}$ & $"$ & $"$ & $"$ & $"$ & \\
\hline$"$ & 1200 & 3 & 2000 & 6 & $"$ & $"$ & $"$ & $"$ & $"$ & \\
\hline$"$ & 1900 & 6 & 2000 & 3 & $"$ & $"$ & $"$ & $"$ & $"$ & \\
\hline$"$ & 1900 & 6 & 2000 & 3 & $"$ & $"$ & $"$ & $"$ & $"$ & \\
\hline Ce & 40000 & 3 & 38000 & 6 & $9 \times 10^{-9}$ & $"$ & $"$ & $"$ & $"$ & \\
\hline$"$ & 35000 & 3 & 40000 & 6 & $"$ & $"$ & " & $"$ & $"$ & \\
\hline$"$ & 45000 & 6 & 37000 & 3 & $"$ & $"$ & " & $"$ & $"$ & \\
\hline$"$ & 49000 & 6 & 40000 & 3 & $"$ & $"$ & $"$ & $"$ & $"$ & \\
\hline Eu & 195000 & 3 & 220000 & 6 & $4 \times 10^{-8}$ & $"$ & $"$ & $"$ & $"$ & \\
\hline$"$ & 230000 & 3 & 200000 & 6 & $"$ & $"$ & $"$ & $"$ & $"$ & \\
\hline$"$ & 210000 & 6 & 210000 & 3 & $"$ & $"$ & $"$ & $"$ & $"$ & \\
\hline$"$ & 210000 & 6 & 200000 & 3 & $"$ & " & $"$ & $"$ & $"$ & \\
\hline $\mathrm{Na}$ & 3.5 & 2 & 5.5 & 4 & $2 \times 10^{-3}$ & $"$ & $"$ & $"$ & 6 & \\
\hline$"$ & 1.4 & 2 & 5.5 & 4 & $"$ & $"$ & $"$ & $"$ & $"$ & \\
\hline " & 3.7 & 4 & 6.9 & 2 & $"$ & $"$ & $"$ & $"$ & $"$ & \\
\hline$"$ & 4.5 & 4 & 6.5 & 2 & $"$ & $"$ & $"$ & $"$ & $"$ & \\
\hline Mn & $>10000$ & 2 & $>10000$ & 4 & $1 \times 10^{-11}$ & $"$ & $"$ & $"$ & $"$ & b \\
\hline$"$ & $"$ & 2 & $"$ & 4 & $"$ & $"$ & $"$ & $"$ & $"$ & $"$ \\
\hline$"$ & $"$ & 4 & $"$ & 2 & $"$ & $"$ & $"$ & $"$ & $"$ & $"$ \\
\hline$"$ & $"$ & 4 & $"$ & 2 & $"$ & $"$ & " & $"$ & $"$ & $"$ \\
\hline Se & 7.3 & 2 & 64 & 4 & $8 \times 10^{-10}$ & $"$ & $"$ & $"$ & $"$ & $"$ \\
\hline$"$ & 10 & 2 & 69 & 4 & $"$ & $"$ & $"$ & $"$ & $"$ & $"$ \\
\hline$"$ & 4.0 & 4 & 94 & 2 & $"$ & $"$ & " & $"$ & $"$ & $"$ \\
\hline " & 13 & 4 & 69 & 2 & & $"$ & $"$ & $"$ & " & " \\
\hline
\end{tabular}

value not included in averages for Table XXII, Chap. 1.

boncentrations are only those added with the tracer; actual concentrations may be bigher. 
TABLF $\operatorname{sod}$ (cont)

\begin{tabular}{|c|c|c|c|c|c|c|c|c|c|c|}
\hline \multirow[b]{2}{*}{ Elenent } & \multicolumn{2}{|c|}{ Sorption } & \multicolumn{2}{|c|}{ Desorption } & \multirow[b]{2}{*}{$\begin{array}{c}\text { Elewent } \\
\text { Concentration } \\
(\mathrm{M}) \\
\end{array}$} & \multirow[b]{2}{*}{$\begin{array}{c}\text { Particle } \\
\text { Size } \\
\text { (1) } \\
\end{array}$} & \multirow[b]{2}{*}{ Tewperature } & \multirow[b]{2}{*}{ Atposphere } & \multirow[b]{2}{*}{ Ref. } & \multirow[b]{2}{*}{ Note } \\
\hline & $\begin{array}{c}\bar{R}_{d} \\
(n / /)\end{array}$ & $\begin{array}{l}\text { Tine } \\
\text { (wh) }\end{array}$ & $\begin{array}{c}R_{d} \\
(n / /)\end{array}$ & $\begin{array}{l}\text { Tine } \\
\text { (wh) }\end{array}$ & & & & & & \\
\hline $\mathrm{Sn}$ & $>10000$ & 2 & $>10000$ & 4 & $6 \times 10^{-9}$ & $<500$ & Abb & Air & 6 & $a$ \\
\hline$"$ & $H$ & 2 & $"$ & 4 & $"$ & $"$ & $"$ & $"$ & $"$ & \\
\hline$"$ & $"$ & 4 & $"$ & 2 & $"$ & " & $"$ & " & $" 1$ & \\
\hline$"$ & $"$ & 4 & $"$ & 2 & $"$ & $" 1$ & $"$ & $"$ & $"$ & \\
\hline
\end{tabular}

\footnotetext{
Concentrations are only those added with the tracer; actual concentrations may be higher.
} 
TABLE A-XXXI

BATCH SORPTION DATA FOR TUFF G1-3116

\begin{tabular}{|c|c|c|c|c|c|c|c|c|c|c|}
\hline \multirow[b]{2}{*}{ Element } & \multicolumn{2}{|c|}{ Sorption } & \multicolumn{2}{|c|}{ Desorption } & \multirow{2}{*}{$\begin{array}{c}\text { Elewent } \\
\text { Concentration } \\
(\mathrm{M}) \\
\end{array}$} & \multirow[b]{2}{*}{$\begin{array}{c}\text { Particle } \\
\text { Size } \\
(10) \\
\end{array}$} & \multirow[b]{2}{*}{ Tewperature } & \multirow[b]{2}{*}{ Atmosphere } & \multirow[b]{2}{*}{ Ref. } & \multirow[b]{2}{*}{ Note } \\
\hline & $\begin{array}{c}R_{d} \\
(n e / g)\end{array}$ & $\begin{array}{l}\text { Time } \\
\text { (wk) }\end{array}$ & $\begin{array}{c}R_{d} \\
(R / g) \\
\end{array}$ & $\begin{array}{l}\text { Time } \\
\text { (wk) }\end{array}$ & & & & & & \\
\hline $\mathbf{S r}$ & 2500 & 3 & 3100 & 6 & $2 \times 10^{-7}$ & $<500$ & $A m b$ & Air & 1 & \\
\hline$"$ & 2500 & 3 & 58000 & 6 & $"$ & $"$ & $"$ & $"$ & $"$ & \\
\hline$"$ & 2200 & 6 & 3500 & 3 & $"$ & $"$ & $"$ & $"$ & $"$ & \\
\hline$"$ & 2400 & 6 & 32000 & 3 & $"$ & $"$ & $"$ & $"$ & $"$ & \\
\hline Cs & 7600 & 3 & 6200 & 6 & $5 \times 10^{-9}$ & $"$ & $"$ & $"$ & $"$ & \\
\hline$"$ & 7000 & 3 & 21000 & 6 & $"$ & $"$ & $"$ & $"$ & $"$ & \\
\hline " & 5500 & 6 & 7900 & 3 & $"$ & $"$ & $H$ & $"$ & $"$ & \\
\hline$\because$ & 5200 & 6 & 8500 & 3 & $"$ & $"$ & $"$ & $"$ & $"$ & \\
\hline $\mathrm{Ba}$ & 120000 & 3 & 23000 & 6 & $9 \times 10^{-6}$ & $"$ & $"$ & $"$ & $"$ & \\
\hline$"$ & 130000 & 3 & $7100000^{\circ}$ & 6 & $"$ & $"$ & $"$ & $"$ & $"$ & a \\
\hline$"$ & 120000 & 6 & 280000 & 3 & $"$ & $"$ & $"$ & $"$ & $"$ & \\
\hline$"$ & 130000 & 6 & 190000 & 3 & $"$ & $"$ & $"$ & $"$ & $"$ & \\
\hline Ce & 89 & 3 & 180 & 6 & $1 \times 10^{-7}$ & $"$ & $"$ & $"$ & $"$ & \\
\hline$"$ & 80 & 3 & 5600 & 6 & $"$ & $"$ & " & $"$ & $"$ & \\
\hline$"$ & 100 & 6 & 4200 & 3 & $n$ & $"$ & $"$ & " & $"$ & \\
\hline$"$ & 120 & 6 & 3100 & 3 & $"$ & $"$ & $"$ & $"$ & $"$ & \\
\hline Eu & 680 & 3 & 600 & 6 & $2 \times 10^{-7}$ & $"$ & $"$ & $"$ & $"$ & \\
\hline$"$ & 660 & 3 & 14000 & 6 & $"$ & "t & $"$ & $"$ & $"$ & \\
\hline$"$ & 790 & 6 & 9400 & 3 & $"$ & $"$ & $"$ & $"$ & $"$ & \\
\hline$"$ & 900 & 6 & 8900 & 3 & $"$ & $"$ & $"$ & "1 & $"$ & \\
\hline
\end{tabular}

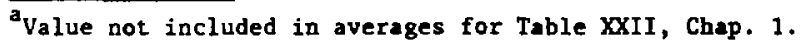


TABLE A-XXXII

BATCH SORPTION DATA FOR TUFF G1-3658

\begin{tabular}{|c|c|c|c|c|c|c|c|c|c|c|}
\hline \multirow[b]{2}{*}{ Element } & \multicolumn{2}{|c|}{ Sorption } & \multicolumn{2}{|c|}{ Desorption } & \multirow{2}{*}{$\begin{array}{c}\text { Element } \\
\text { Concentration } \\
(\mathrm{M}) \\
\end{array}$} & \multirow{2}{*}{$\begin{array}{c}\text { Particle } \\
\text { Size } \\
\text { (1⿴囗十) } \\
\end{array}$} & \multirow[b]{2}{*}{ Tepperature } & \multirow[b]{2}{*}{ At mosphere } & \multirow[b]{2}{*}{ Ref. } & \multirow[b]{2}{*}{ Note } \\
\hline & $\begin{array}{c}R_{d} \\
(n e / \phi)\end{array}$ & $\begin{array}{l}\text { Tine } \\
\text { (wk) }\end{array}$ & $\begin{array}{c}R_{d} \\
(n / /)\end{array}$ & $\begin{array}{l}\text { Time } \\
\text { (wk) }\end{array}$ & & & & & & \\
\hline $\mathbf{s r}$ & 10000 & 3 & 3500 & 6 & $3 \times 10^{-7}$ & $<500$ & $A=b$ & Air & 1 & \\
\hline$"$ & 14000 & 3 & 14000 & 6 & $"$ & $"$ & $"$ & $"$ & $"$ & \\
\hline$n$ & 12000 & 6 & 14000 & 3 & $"$ & $"$ & $"$ & $"$ & $"$ & \\
\hline$"$ & 11000 & 6 & 17000 & 3 & $"$ & $n$ & $"$ & $"$ & $"$ & \\
\hline $\mathrm{Cs}$ & 7800 & 3 & 18000 & 6 & $7 \times 10^{-9}$ & $"$ & $"$ & $"$ & $"$ & \\
\hline$"$ & 8200 & 3 & 10000 & 6 & $"$ & $"$ & $"$ & $"$ & $"$ & \\
\hline$"$ & 8500 & 6 & 10000 & 3 & $"$ & $"$ & $"$ & $"$ & $"$ & \\
\hline$"$ & 7700 & 6 & 11000 & 3 & $"$ & $"$ & $"$ & $:$ & $"$ & \\
\hline $\mathrm{Ba}$ & 8700 & 3 & 3100 & 6 & $9 \times 10^{-6}$ & $"$ & $"$ & $"$ & $"$ & \\
\hline$"$ & 12000 & 3 & 16000 & 6 & $"$ & $"$ & $"$ & $"$ & $"$ & \\
\hline$"$ & 12000 & 6 & 19000 & 3 & $"$ & " & $"$ & $"$ & $"$ & \\
\hline$"$ & 9000 & 6 & 2100 & 3 & $"$ & $"$ & $"$ & $"$ & $"$ & \\
\hline Ce & 640 & 3 & 790 & 6 & $2 \times 10^{-7}$ & $"$ & $"$ & $"$ & $"$ & \\
\hline$"$ & 630 & 3 & 6800 & 6 & $"$ & $"$ & $"$ & $"$ & " & \\
\hline$"$ & 1500 & 6 & 16000 & 3 & $"$ & $"$ & $"$ & $"$ & $"$ & \\
\hline$"$ & 1300 & 6 & 14000 & 3 & $"$ & $"$ & $"$ & $"$ & $"$ & \\
\hline Eu & 510 & 3 & 700 & 6 & $4 \times 10^{-7}$ & $"$ & $"$ & $"$ & $"$ & \\
\hline$"$ & 490 & 3 & 6700 & 6 & $"$ & $"$ & $"$ & $"$ & $"$ & \\
\hline$"$ & 1200 & 6 & 15000 & 3 & $"$ & $"$ & $"$ & $"$ & $"$ & \\
\hline$"$ & 1000 & 6 & 12000 & 3 & " & $"$ & $"$ & $"$ & $"$ & \\
\hline $\mathbf{S r}$ & 98000 & 3 & & & $3 \times 10^{-7}$ & $<38$ & $"$ & $"$ & & - \\
\hline$"$ & 160000 & 3 & & & " & $"$ & $"$ & $"$ & & $"$ \\
\hline$"$ & 120000 & 3 & & & " & $38-106$ & $"$ & $"$ & & $"$ \\
\hline$"$ & 140000 & 3 & & & " & $"$ & $"$ & $"$ & & $"$ \\
\hline$"$ & 130000 & 3 & & & $"$ & $106-500$ & $"$ & " & & $"$ \\
\hline$"$ & 130000 & 3 & & & $"$ & $"$ & $"$ & $n$ & & $1 "$ \\
\hline $\mathrm{Cs}$ & 140000 & 3 & & & $7 \times 10^{-9}$ & $<38$ & $"$ & $"$ & & $"$ \\
\hline 11 & 180000 & 3 & & & $"$ & $"$ & $"$ & $"$ & & $"$ \\
\hline$"$ & 61000 & 3 & & & " & $38-106$ & $"$ & $1 "$ & & 11 \\
\hline$"$ & 70000 & 3 & & & n & $"$ & $"$ & $"$ & & $"$ \\
\hline$"$ & 49000 & 3 & & & $"$ & $106-500$ & $"$ & $"$ & & $"$ \\
\hline$"$ & 50000 & 3 & & & " & $"$ & $"$ & $"$ & & $"$ \\
\hline
\end{tabular}

\footnotetext{
Previously unpublished results.
} 
TABLE A-XXXII (cont)

\begin{tabular}{|c|c|c|c|c|}
\hline \multirow[b]{2}{*}{ Elenent } & \multicolumn{2}{|c|}{ Sorption } & \multicolumn{2}{|c|}{ Desorption } \\
\hline & $\begin{array}{c}\mathrm{R}_{\mathrm{d}} \\
(\mathrm{m} / \mathrm{g} / \mathrm{g})\end{array}$ & $\begin{array}{l}\text { Tiese } \\
\text { (wk) }\end{array}$ & $\begin{array}{c}R_{\mathrm{d}} \\
(\mathrm{e} / \mathrm{s})\end{array}$ & $\begin{array}{l}\text { Tine } \\
\text { (wk) }\end{array}$ \\
\hline $\mathrm{Ba}$ & 120000 & 3 & & \\
\hline$"$ & 190000 & 3 & & \\
\hline$"$ & 90000 & 3 & & \\
\hline$"$ & 100000 & 3 & & \\
\hline$"$ & 130000 & 3 & & \\
\hline$"$ & 140000 & 3 & & \\
\hline Eu & 150000 & 3 & & \\
\hline$"$ & 160000 & 3 & & \\
\hline$"$ & 4100 & 3 & & \\
\hline 11 & 4800 & 3 & & \\
\hline$"$ & 4900 & 3 & & \\
\hline$" 1$ & 5700 & 3 & & \\
\hline
\end{tabular}

$\begin{gathered}\text { Element } \\ \text { Concentration } \\ \text { (H) }\end{gathered}$
$9 \times 10^{-6}$
$" 1 "$
$"$
$"$

Particle

Size (1) $<38$

1

38-106

11

106-500

Temperature Atmosphere Ref. Note

Amb

Air

a

"

"

"

"1

"

"

"

"

"

"

"1

"

"

"

$4 \times 10^{-7}$

"

$<38$

"

"

38-106

"1

"

"

,

"

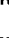

$105-500$

"

"1

"

"

$"$

"

$"$

$"$

"

$"$

"

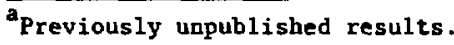




\section{REFERENCES}

1. B. R. Erda1, W. R. Daniels, and K. Wolfsberg, Eds., "Research and Development Related to Nevada Nuclear Waste Storage Investigations, January 1 - March 31, 1981," Los Alamos National Laboratory report LA-8847-PR (July 1981).

2. K. Wolfsberg, B. P. Bayhurst, B. M. Crowe, W. R. Daniels, B. R. Erdal, F. O. Lawrence, A. E. Norris, and J. R. Smyth, "Sorption-Desorption Studies on Tuf. I. Initial Studies with Samples from the J-13 Drill Site, Jackass Flats, Nevada," Los Alamos Scientific Laboratory report LA-7480-MS (April 1979).

3. K. Wolfsberg, R. D. Aguilar, B. P. Bayhurst, W. R. Daniels, S. J. DeVilliers, B. R. Erdal, F. O. Lawrence, S. Maestas, A. J. Mitche1l, P. Q. Oliver, N. A. Raybold, R. S. Rundberg, J. L. Thompson, and E. N. Vine, "SorptionDesorption Studies on Tuff. III. A Continuation of Studies with Samples from Jackass Flats and Yucca Mountain, Nevada," Los Alamos National Laboratory report LA-8747-MS (May 1981).

4. E. N. Vine, R. D. Aguilar, B. P. Bayhurst, W. R. Daniels, S. J. DeVilliers, B. R. Erda1, F. O. Lawrence, S. Maestas, P. Q. Oliver, J. L. Thompson, and K. Wolfsberg, "Sorption-Desorption Studies on Tuff. II. A Continuation of Studies with Samples from Jackass Flats, Nevada, and Initial Studies with Samples from Yucca Mountain, Nevada," Los Alamos Scientific Laboratory report LA-8110-MS (January 1980).

5. K. Wolfsberg and B. R. Erdal, Eds., "Research and Development Related to Nevada Nuclear Waste Storage Investigations, October 1 - December 31, 1980," Los Alamos National Laboratory report LA-8739-PR (April 1981).

6. W. R. Daniels, K. Wolfsberg, D. T. Vaniman, and B. R. Erdal, Eds., "Research and Development Related to Nevada Nuclear Waste Storage Investigations, July 1 - September 30, 1981," Los Alamos National Laboratory report LA-9095-PR (January 1982).

7. K. Wolfsberg, W. R. Daniels, D. T. Vaniman, and B. R. Erdal, Eds., "Research and Development Related to Nevada Nuclear Waste Storage Investigations, October 1 - December 31, 1981," Los Alamos National Laboratory report LA-9225-PR (April 1982).

8. B. M. Crowe, Comp., "Research and Development Related to the Nevada Nuclear Waste Storage Investigations, October 1 - December 31, 1979," Los Alamos Scientific Laboratory report LA-8309-PR (April 1980). 
9. B. R. Erdal, W. R. Daniels, D. T. Vaniman, and K. Wolfsberg, Eds., "Research and Development Related to the Nevada Nuclear Waste Storage Investigations, April 1 - June 30, 1981," Los Alamos National Laboratory report LA-8959-PR (October 1981). 


\section{APPENDIX B}

MINERALOGIC AND PETROLOGIC STUDIES OF TUFFS

FROM YUCCA MOUNTAIN

Appendix B includes sumary core-sample descriptions, ${ }^{\star}$-ray diffraction analyses, and thin section descriptions of samples used in sorption studies. The core-sample descriptions are based on visual examination of the core and drill hole reports published by the USGS. Core specimens are divided into mineralogical assemblages that are based on the textural occurrence and abundance of the mineral phases most likely to contribute to sorption. These include devitrification assemblages where the rock mineralogy is dominated by generally nonsorptive quartz and feldspar; sorptive glass, clay, and zeolite assemblages; and variable mixtures of these phases.

I. G1-1292 TOPOPAH SPRING MEMBER OF THE PAINTBRUSH TUFF

\section{Core Description}

Vitrophyric zone of dense welding, ash-flow tuff; 1 to $2 \%$ phenocrysts; 1 to $2 \%$ rhyolitic lithic fragments. Sample collected about 5 ft below contact with overlying devitrified zone.

X-Ray Diffraction Results (\%)

Alka li

Smectite Illite Clinoptilolite Quartz Cristobalite Feldspar Glass $\operatorname{Tr}$

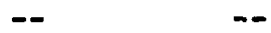

5-10

10-20 80-90

\section{Thin Section Description}

The sample is a densely welded (vitrophyric) ash-flow tuff composed of annealed glass shards, sparse phenocrysts (alkali feldspar, plagioclase, irontitanium oxides, and biotite), and moderately abundant lithic fragments (densely welded, devitrified tuff?). Glass in the sample is largely unaltered with the exception of possible incipient devitrification (suggested by the cristobalite peak, although this could in part be the result of the presence of devitrified lithic fragments) and clay alteration (minor) along hydration fractures (largely perlitic).

* A number in parentheses following the drill hole YM sample numbers indicates the depth (in feet) from which the sample was taken. 


\section{Sorption Category: Glass}

Sorption properties should be dominated by the abundant glass; surface area of crushed glass particles should be lower than for samples of vitric nonwelded tuff because of the complete welding of all glass particles in the former. Clay formed along hydration cracks could be a contributing factor.

II. G1-1436 TUFFACEOUS BEDS OF CALICO HILLS

Core Description

Upper nonwelded, zeolitized ash-flow tuff; 2 to $3 \%$ phenocrysts; sparse volcanic lithic fragments. Sample collected about $10 \mathrm{ft}$ below contact with the overlying Topopah Spring Member.

X-Ray Diffraction Kesulis $(\%)$

$\frac{\text { Smectite }}{--} \frac{\text { Illite }}{\operatorname{Tr}} \frac{\text { Clinoptilolite }}{65-85} \quad \frac{\text { Quartz }}{10-20} \quad \frac{\text { Cristobalite }}{5-10} \quad \frac{\begin{array}{c}\text { Alkali } \\ \text { Feldspar }\end{array}}{5-15} \quad \frac{\text { Glass }}{--}$

\section{Thin Section Description}

Sample is a nonwelded, zeolitized ash-flow tuff. There has been pervasive alteration of all glass fragments to clinoptilolite, including matrix ash, pumice fragments and scattered, originally perlitic rock fragments. Phenocrysts include quartz, alkali feldspar, plagioclase, iron-titanium oxides, and sparse biotite. Lithic fragments include devitrified lava (dacitic or more silicic), devitrified densely welded tuff, and zeolitized perlite fragments.

Sorption Category: Zeolitized

Properties should be dominated by abundant zeolites that replace particles of a wide range of sizes.

III. G1-1854 PROW PASS MEMBER OF THE CRATER FLAT TUFF

Core Description

Partially welded, devitrified, ash-flow tuff with large pumice fragments; 5 to $10 \%$ crystals, conspicuous reddish-brown mudstone lithic fragments. Sample 
collected from the lower, partially welded zone of the upper ash-flow unit, above the vapor phase zone.

X-Ray Diffraction Results (\%)

$\frac{\text { Smectite }}{\operatorname{Tr}} \quad \frac{\text { Illite }}{2-5} \quad \frac{\text { Clinoptilolite }}{25-40} \quad \frac{\text { Quartz }}{2-5} \quad \frac{\text { Cristobalite }}{20-30} \quad \frac{\begin{array}{c}\text { Alkali } \\ \text { Feldspar }\end{array}}{\text { Glass }}$

\section{Thin Section Description}

Nonwelded, zeolitized ash-flow tuff. Slight devitrification of shard and pumice fragments. Moderately crystal rich with alkali feldspar, quartz, plagioclase, iron-titanium oxides, biotite, and pyroxene pseudomorphs. Contains minor siltstone lithic fragments. Moderate degree of zeolitization.

Sorption Category: Zeoitized

Sorption properties should be dominated by zeolites, but sample should be less sorptive than a highly zeolitized sample. Role of illite is unknown.

IV. G1-1883 PROW PASS MEMBER OF THE CRATER FLAT TUFF

Core Description

Partially to moderately welded, devitrified ash-flow tuff; 5 to $10 \%$ phenocrysts; sparse mudstone lithic fragments. Sample collected from the upper part of the most strongly welded zone of the Prow Pass.

X-Ray Diffraction Results (\%) Smectite $\frac{\text { Illite }}{--} \frac{\text { Clinoptilolite }}{--} \frac{\text { Quartz }}{25-40} \frac{\text { Cristobalite }}{5-10} \frac{\begin{array}{c}\text { Alkali } \\ \text { Feldspar }\end{array}}{40-60} \frac{\text { Calcite }}{\operatorname{Tr}} \frac{\text { Glass }}{--}$

\section{Thin Section Description}

Partially welded, devitrified ash-flow tuff with vapor phase. Shards exhibit relatively coarse-grained (granophyric) devitrification; pumice fragments show spherulitic devitrification and contain relatively abundant vapor phase minerals. Phenocrysts include quartz, alkali feldspar, plagioclase, biotite, and iron-titanium oxides with rare pyroxene pseudomorphs. Lithic fragments are relatively sparse and include siltstone and rare, altered, welded tuff. 
Sorption Category: Devitrification and Vapor Phase Assemblage

Sorption properties should reflect the abundance of the assemblage quartz and alkali feldspar and the virtual absence of secondary alteration products (clays and zeolites).

V. G1-1982 PROW PASS MEMBER OF THE CRATER FLAT TUFE

Core Description

Partially welded, devitrified ash-flow tuff with vapor phase; 5 to $15 \%$ phenocrysts; mudstone lithic fragments. Sampie collected from lower, vapor phase bearing, ash-flow tuff.

X-Ray Diffraction Results $(\%)$

$\frac{\text { Smectite }}{\operatorname{Tr}} \frac{\text { Illite }}{--} \frac{\text { Clinoptilolite }}{--} \frac{\text { Quartz }}{25-40} \frac{\text { Cristobalite }}{5-10} \frac{\begin{array}{c}\text { Alkali } \\ \text { Feldspar }\end{array}}{40-60} \frac{\text { Calcite }}{\operatorname{Tr}} \frac{\text { Glass }}{\ldots}$

Thin Section Description

Partially welded, devitrified (spherulitic zone of devitrification) ash-flow tuff with clay alteration in pumice fragments. Pumice fragments and shards are devitrified to radial and fibrous intergrowths of alkali feldspar and cristobalite (quartz). There are at least two stages of devitrification of pumice fragments as indicated by the presence of interior zones of pumice fragments that are composed of coalesced spherulites in turn overgrown by fibrous, nonradial cristobalite and feldspar. Probable relict glass in the pumice fragments has been altered to clays. Sparse vapor phase crystallization is suggested by the presence of inverted tridymite (quartz) with rounded crystal edges suggestive of $\mathrm{SiO}_{2}$ dissolution. Fine-grained ash groundmass shows incipient birefringence suggestive of clay alteration or devitrification.

Sorption Category: Clay and Devitrification Products

Sorption properties should be dominated by the presence of clay and the predominance of the devitrification assemblage quartz and feldspar. $X-r a y$ data indicate a relatively low clay content. 
VI. G-12233 BULIFROG MEMBER OF THE CRATER FLAT TUFF

\section{Core Description}

Nonwelded, devitrified, vapor phase crystallized, slightly zeolitized ashflow tuff; 15 to $20 \%$ phenocrysts, rare volcanic lithic fragments. Sample collected from the nonwelded upper part of the major cooling unit.

X-Ray Diffraction Results (\%)

Alkali

$\frac{\text { Smectite }}{<5} \quad \frac{\text { Illite }}{2-5} \quad \frac{\text { Clinoptilolite }}{20-40} \quad \frac{\text { Mordenite }}{15-30} \quad \frac{\text { Quartz }}{5-10} \quad \frac{\text { Cristobalite }}{10-20} \quad \frac{\text { Feldspar }}{15-25}$

\section{Thin Section Description}

Sample is a crystal-rich, nonwelded zeolitized tuff with only very minor vapor phase mineralization. Phenocrysts include quartz, alkali feldspar, plagioclase, biotite, iron-titanium oxides, and hornblende pseudomorphs. The sample does not contain lithic fragments.

Sorption Category: Zeolitized

The sorption surface area should be high because of the absence of welding, and the sample contains both clinoptilolite and mordenite.

VII. G1-2289 BULLFROG MEMBER OF THE CRATER FLAT TUFF

\section{Core Description}

Partially welded, devitrified ash-flow tuff with zeolitic alteration and vapor phase crystallization, 15 to $20 \%$ phenocrysts; rare volcanic lithic fragments. Sample is from the zeolitized upper part of the Bullfrog cooling unit.

X-Ray Diffraction Results (\%)

Smectite $\frac{\text { Illite }}{\operatorname{Tr}} \frac{\text { Clinoptilolite }}{25-50} \frac{\text { Mordenite }}{30-50} \quad \frac{\text { Quartz }}{\operatorname{Tr}} \quad \frac{\text { Cristobalite }}{5-10} \quad \begin{gathered}\text { Alkali } \\ \text { Feldspar }\end{gathered}$

\section{Thin Section Description}

Partially welded, highly zeolitized ash-flow tuff. Zeolites (at least two textural types) have replaced all original vitric constituents. Small amounts of vapor phase mineralization are present in the punice fragments. Phenocrysts 
include quartz, alkali feldspar, plagioclase, biotite, iron-titanium oxides, and hornblende pseudomorphs. No apparent lithic fragments.

\section{Sorption Category: Zeolitized}

Sorption properties should be controlled by the abundance of zeolites and high surface areas of altered particles.

VIII. G1-2363 BULLFROG MEMBER OF THE CRATER FLAT TUFF

\section{Core Description}

Partially welded ash-flow tuff, zone of vapor phase alteration and granophyric devitrification; 10 to $15 \%$ phenocrysts; sparse siltstone and volcanic lithic fragments. Sample collected from the upper part of the partially welded, devitrified and vapor phase zones.

X-Ray Diffraction Results (\%)

$\frac{\text { Smectite }}{2-5} \quad \frac{\text { Illite }}{2-5} \quad \frac{\text { Clinoptilolite }}{\ldots} \frac{\text { Quartz }}{25-40} \quad \frac{\text { Cristobalite }}{5-10} \quad \frac{\begin{array}{c}\text { Alkali } \\ \text { Feldspar }\end{array}}{40-60} \quad \frac{\text { Glass }}{--}$

\section{Thin Section Description}

Sample is a partially welded, devitrified and vapor phase altered ash-flow tuff. Devitrification assemblages occur as coarse-grained intergrowths within groundmass shards and pumice fragments. In most cases, devitrification products are confined within the shards and pumice fragments. Fibrous devitrification products ranging from elongate sheaths to true spherulites are present within some pumice fragments. Interiors of pumice fragments include vapor phase minerals (quartz). Groundmass ash is devitrified to an extremely fine-grained quartz feldspar assemblage. The rock is relatively crystal rich with alkali feldspar, plagioclase, quartz, biotite, iron-titanium oxides, and rare hornblende. Rare lithic fragments are andesitic lava with pyroxene pseudomorphs.

Sorption Category: Devitxification Assemblage With Clays

The sorptive properties should be dominated by the abundance of devitrification products and the small amounts of smectite and illitic clays. 
IX. G1-2410 BULLFROG MEMBER OF THE CRATER FLAT TUFF

\section{Core Description}

Identical to that for sample G1-2363. Sample collected from lower into the upper partially welded zone, and there is a minor increase in welding in comparison to sample G1-2363.

X-Ray Diffraction Results (\%)

$\frac{\text { Smectite }}{5-10} \quad \frac{\text { Iliite }}{<2} \quad \frac{\text { Clinoptilolite }}{--} \frac{\text { Quartz }}{20-40} \quad \frac{\text { Cristobalite }}{0-10} \quad \frac{\begin{array}{c}\text { Alkali } \\ \text { Feldspar }\end{array}}{30-50} \quad \frac{\text { Glass }}{--}$

Thin Section Description

See description of sample G1-2363.

Sorption Category: Devitrification Assemblage with Clays

See description of sample G1-2363.

X. G1-2476 BULLFROG MEMBER OF THE CRATER FLAT TUFF

Core Description

Moderately welded, devitrified ash-flow tuff with minor vapor phase, 5 to $20 \%$ phenocrysts, sparse mudstone, and volcanic lithic fragments. Sample collected from the upper part of the most strongly welded zone of the Bullfrog (maximum degree of welding is moderate welding).

X-Ray Diffraction Results (\%)

\section{Thin Section Description}

Sample is a moderately welded, devitrified ash-flow tuff with vapor phase products within pumice fregments. Collapsed pumice fragments have devitrification products that consist of plumose to spherulitic intergrowths of quartz and alkali feldspar. The fragments are pocketed with irregular quartz grains. These may represent the initial stages of granophyric recrystallization but more likely are recrystallized zones of vapor phase minerals coriginally tridymite or 
cristobalite that has inverted to quartz). Detailed examination of shards indicates several stages of devitrification. The majority of shards consists of axiolitic intergrowths of quartz and alkali feldspar. They are brown in plain light, suggestive of clay alteration. The shards have thin rim overgrowths of clear devitrification products (axiolitic) that extend into the groundmass. The lack of alteration of these overgrowths and rim occurrence is suggestive of a second stage of devitrification (burial recrystallization). Shards with well-preserved bubble-wall textures have interiors filled with coarse-grained quartz. These fill phases may represent inverted vapor phase products or the initial stages of overprinting by granophyric recrystallization. Phenocrysts include quartz, alkali feldspar, plagioclase, biotite, and altered hornblende. Sparse lithic fragments include siltstone and devitrified welded tuff (?).

\section{Sorption Category: Devitrification Assemblage with Clays}

Devitrification products should dominate sorptive properties with a minor contribution from clays.

XI . G1-2698 TRAM MEMBER OF THE CRATER FLAT TUFF

\section{Core Description}

Partially welded, devitrified ash-flow tuff; 10 to $15 \%$ phenocrysts; sparse volcanic lithic fragments. Sample collected from the upper part of the upper zeolitized zone of the upper ash-flow unit of the Tram member.

X-Ray Diffraction Results (\%)

$\frac{\text { Suectite }}{\operatorname{Tr}} \frac{\text { Illite }}{5-10} \frac{\text { Clinoptilolite }}{30-60} \frac{\text { Quartz }}{10-20} \frac{\text { Cristobalite }}{10-20} \frac{\begin{array}{c}\text { Alkali } \\ \text { Feldspar }\end{array}}{20-30} \frac{\text { Glass }}{--}$

\section{Thin Section Description}

Partially welded, zeolitized ash-flow tuff. Groundmass ash, shards, and pumice fragments (original vitric constituents) are replaced by very fine-grained to fibrous clinoptilolite that preserves the vitroclastic textures. Phenocrysts include quartz, alkali feldspar, plagioclase, biotite, and iron-titanium oxides. Moderately abundant lithic fragments are slightly zeolitized, partially devitrified welded tuff. 


\section{Sorption Category: Zeolitized}

Sorption properties should be controlled by the zeolitization and the high surface areas of zeolitic, original vitric constituents of the partially welded tuff.

XII. G1-2840 TRAM MEMBER OF THE CRATER FLAT TUFF

\section{Core Description}

Partially welded, devitrified, ash-flow tuff. Same as for sample G1-2698, only below upper zeolite zone, and it exhibits a greater degree of welding. More lithic rich than sample G1-2698.

X-Ray Diffraction Results (\%)

$\frac{\text { Saectite }}{2-5} \frac{\text { Illite }}{2-5} \quad \frac{\text { Clinoptilolite }}{--} \underset{40-60}{\frac{\text { Quartz }}{0-10}} \frac{\text { Cristobalite }}{30-50} \frac{\begin{array}{c}\text { Alkali } \\ \text { Feldspar }\end{array}}{--}$

\section{Thin Section Description}

No thin section available. Should be similar to that for sample G1-2854. Sorption Category: Devitrification Assemblage with Clays

XIII. G1-2854 TRAM MEMBER OF THE CRATER FLAT TUFF

\section{Core Description}

Partially welded, devitrified ash-flow tuff. 10 to $15 \%$ phenocrysts; 3 to $5 \%$ volcanic lithic fragnents. Sample collected from the upper ash flow, below zeolitized zone.

\section{X-Ray Diffraction Results (\%)}

$\frac{\text { Sectite }}{2-5} \frac{\text { Illite }}{5-10} \frac{\text { Clinoptilolite }}{--} \frac{\text { Quartz }}{25-40} \frac{\text { Cristobalite }}{5-10} \frac{\begin{array}{c}\text { Alkali } \\ \text { Feldspar }\end{array}}{20-30} \frac{\text { Glass }}{\mathrm{Tr}}$

Thin Section Description

This is a partially welded, devitrified tuff with minor vapor phase. The ash atrix is devitrified to cryptocrystalline phases. The shards show grano- 
phyric devitrification that slightly transects shard boundaries. Some of the shards exhibit axiolitic devitrification with probable clay alteration. They are pale brown in plane light. Pumice fragments show spherulitic to finegrained granophyric devitrification. Minor amounts of coarse-grained, irregularshaped quartz fills punice interiors and probably represents inverted vapor-phase minerals. Phenocrysts include quartz, alkali feldspar, plagioclase, hornblende, biotite, and iron-titanium oxides. There is a diverse assemblage of lithic fragments ranging from devitrified welded tuff to slightly zeolitized welded tuff. The latter fragments if included in $x$-ray analyses, should show zeolites. Sorption Category: Devitrification Assemblage with Clays

XIV. G1-2901 TRAM MEMBER OF THE CRATER FLAT TUFF

Core Description

Same as for sample G1-2854. May show a slightly greater degree of welding. X-Ray Diffraction Results (\%)

$\frac{\text { Smectite }}{\operatorname{Tr}} \frac{\text { Illite }}{2-5} \frac{\text { Clinoptilolite }}{--} \frac{\text { Quartz }}{25-40} \frac{\text { Cristobalite }}{5-10} \frac{\begin{array}{c}\text { Alkali } \\ \text { Feldspar }\end{array}}{40-60} \frac{\text { Calcite }}{2-5} \frac{\text { Glass }}{--}$

Thin Section Description

Identical to that for G1-2854, except for multiple seams of secondary calcite. Sorption Category: Devitrification Assemblage

Sample contains noteworthy amounts of calcite.

XV. G1-3116 TRAM MEMBER OF THE CRATER FLAT TUFF

Core Description

Partially welded, zeolitized ash-flow tuff, 10 to $15 \%$ phenocrysts; 5 to $10 \%$ volcanic lithic fragments. Sample collected from the interior of thin, upper lithic-rich ash flow, within a zone of prominent alteration.

X-Ray Diffraction Results (\%)

Alkali

$\frac{\text { Snectite }}{2-5} \frac{\text { Illite }}{2-5} \frac{\text { Clinoptilolite }}{10-20} \frac{\text { Analcime }}{20-40} \frac{\text { Quartz }}{20-35} \frac{\text { Cristobalite }}{5-10} \frac{\text { Feldspar }}{20-30} \frac{\text { Glass }}{\ldots}$ 
Thin Section Description

No thin section.

Sorption Category: Zeolitized

Sample contains both analcime and clinoptilolite.

XVI. G1-3658 FLOW BRECCIA

\section{Core Description}

Autoclastic flow breccia with devitrified to glassy, poorly sorted breccia fragments. Phenocrysts include plagioclase and hornblende. Sample collected from upper autoclastic zone.

X-Ray Diffraction Results (q)

$\frac{\text { Smectite }}{40-80} \quad \frac{\text { Illite }}{--} \quad \frac{\text { Clinoptilolite }}{--} \quad \frac{\text { Quartz }}{--} \quad \frac{\text { Cristobalite }}{5-10} \quad \frac{\begin{array}{c}\text { Alkali } \\ \text { Feldspar }\end{array}}{30-40}$

Ihin Section Description

Sample is an autoclastic fragment within a breccia sequence. It is porphyritic with isolated to glomeroporphyritic phenocrysts of zoned and commonly inclusion-riddled plagioclase, hornblende, orthopyroxene pseudomorphs, unaltered clinopyroxene, and iron-titanium oxides. The groundmass was originally glassy with aligned microlites of plagioclase and pyroxene. The glass was hydrated, forming perlitic fractures followed by several stages of alteration to clay with the clay minerals concentrated along the hydration fractures. The clay alteration is pervasive throughout the thin section.

Sorption Category: Snectite Clays

The surface area of the altered perlitic groundmass component should be less than a nonwelded ash flow altered to clays.

XVII. JA-8 TOPOPAH SPRING MEMBER OF THE PAINTBRUSH TUFF

\section{Core Description}

Crystal- and lithic-rich, probable air-fall tuff; 9\% phenocrysts, 7\% lithic fragments; from the basal part of the Tiva Canyon Member. 
X-Ray Diffraction Results (\%)

$\frac{\text { Smectite }}{30-60} \quad \frac{\text { Illite }}{--} \quad \frac{\text { Clinoptilolite }}{--} \frac{\text { Quartz }}{<5} \quad \frac{\text { Cristobalite }}{10-20} \quad \frac{\begin{array}{c}\text { Flkali } \\ \operatorname{tr}\end{array}}{30-60}$

\section{Thin Section Description}

The sample is a smectite-altered, crystal- and lithic-rich, probable airfall tuff. Crystals include near-equal amounts of plagioclase and sanidine, iron-titanium oxides, and altered biotite. Abundant lithic fragments include devitrified welded tuff, and moderately to slightly devitrified perlite fragments. Pumice fragments are relatively coarse grained. They have glassy interiors but are fringed along vesicle walls by smectitic clays. Fine ash in the matrix is oxidized (reddish-brown in plane light) and generally altered to slays.

\section{Sorption Category: Clay and Glass}

Sorption properties should be dominated by the abundant glass and smectite clays. The rock should have a high surface exchange area in crushed samples because of the relatively high abundance of clay-altered, fine-grained ash.

XVIII. JA-18 TOPOPAH SPRING MEMBER OF THE PAINTBRUSH TUFF

\section{Core Description}

Partially welded, partially zeolitized, vitric ash-flow tuff; $2 \%$ phenocrysts; $10 \%$ rhyolite lithic fragments. Sample collected from the lower vitric zone immediately below the vitrophyric zone of dense welding.

X-Ray Diffraction Results (\%)

Smectite $\frac{\text { Illite }}{\sim 5} \frac{\text { Clinoptilolite }}{10-20} \underset{\sim 5}{\text { Quartz }} \frac{\text { Cristobalite }}{30-50} \frac{\begin{array}{c}\text { Alkali } \\ \text { Feldspar }\end{array}}{30-50} \frac{\text { Glass }}{\sim 40}$

Thin Section Description

Sample is a partially welded ash-flow tuff with moderate pumice compaction and only slight shard deformation. Groundmass shards exhibit bubble-wail textures, have oxidized but unaltered glass cores, and are fringed by fibrous clintoptilolite with minor intergrown clays. Sone of the shards show minor axilolitic devitrifica- 
tion. Groundmass ash is pervasively zeolitized. Pumice fragments show complex intertonguing devitrification and zeolitization. Crystal fragments include sanidine, subordinate plagioclase, biotite, and iron-titanium oxides. Lithic fragments are uniformly devitrified rhyolite lava and densely welded tuff.

\section{Sorption Category: Zeolite, Clay, and Glass}

The sorption properties should be dominated by the combined assemblage of clays, zeolite, glass, and devitrification products.

XIX. JA-26 PROW PASS MEMBER OF THE CRATER FLAT TUFF

\section{Core Description}

Nonwelded ash-flow and air-fall interval, $11 \%$ phenocrysts; $1 \%$ lithic fragments. Sample collected from the bedded interval between the Prow Pass and Bullfrog Members.

\section{$\underline{X-R a y ~ D i f f r a c t i o n ~ R e s u l t s ~(\%) ~}$}

\begin{tabular}{|c|c|c|c|c|c|c|}
\hline Smectite & Illite & Clinoptilolite & Analcime & Quartz & Cristobalite & $\begin{array}{c}\text { Alkali } \\
\text { Feldspar }\end{array}$ \\
\hline-- & -- & $\operatorname{tr}$ & $30-50$ & $30-50$ & $\operatorname{tr}$ & $10-20$ \\
\hline
\end{tabular}

\section{Thin Section Description}

Crystal-rich, air-fall tuff with pumice fragments and ash altered to analcime. Phenocrysts include sanidine, plagioclase, iron-titanium oxides, and highly embayed quartz. Lithic fragments include devitrified welded tuff and argillite.

Sorption Category: Zeolitized (analcime)

Sorption properties should be dominated by the abundance of secondary analcime and the absence of clay and glass phases.

\section{JA-32 BULLFROG MEMBER OF THE CRATER FLAT TUFF}

\section{Core Description}

Partially welded, devitrified, ash-flow tuff, $12 \%$ phenocrysts; $1 \%$ 1ithic fragents. Sample collected from the lower partially welded zone of the Bullfrog cooling unit. 
$\underline{X-R a y ~ D i f f r a c t i o n ~ R e s u l t s ~(x) ~}$

$\frac{\text { Snectite }}{<5} \frac{\text { Illite }}{5-15} \frac{\text { Clinoptilolite }}{--} \frac{\text { Quartz }}{35-50} \frac{\text { Cristobalite }}{--} \frac{\begin{array}{c}\text { Alkali } \\ \text { Feldspar }\end{array}}{40-60} \frac{\text { Analcine }}{\text { tr }}$

\section{Thin Section Description}

Sample is a partially welded, devitrified ash-flow tuff. Pumice fragments are flattened and devitrified to spherulitic intergrowths of alkali feldspar and quartz; some contain very small amounts of vapor phase minerals. Shard phases are pseudomorphed by coarse-grained granophyric devitrification products that appear to have replaced axiolitic intergrowths. Groundmass ash is devitrified to very fipe-grained quartz and alkali feldspar phases. Phenocrysts include sanidine, plagioclase $\left(\mathrm{An}_{23-27}\right)$, quartz, iron-titanium oxides, and altered biotite. Sparse lithic fragments are devitrified welded tuff.

\section{Sorption Category: Devitrified}

Major potentially sorptive components are devitrification products that are dominated by the assemblage quartz and alkali feldspar. Relatively abundant illitic clays may contribute to sorption properties.

XXI. YM-5 (251) TIVA CANYON MEMBER OF THE PAINTBRUSH TUFF

Core Description

Air-fall tuff; vitric with some clay alteration; 1 to $3 \%$ phenocrysts;

1 to $2 \%$ volcanic lithic fragments.

X-Ray Diffraction Results (\%)

$\frac{\text { Smectite }}{10} \frac{\text { Illite }}{--} \frac{\text { Clinoptilolite }}{--} \frac{\text { Quartz }}{5} \frac{\text { Cristobalite }}{5} \frac{\begin{array}{c}\text { Alkali } \\ \text { Feldspar }\end{array}}{10-20} \frac{\text { Glass }}{70}$

\section{Thin Section Description}

The core sample is a coarse-grained air-fall tuff composed of pumice clasts, crystals and lithic fragments. Pumice fragments are vitric with alteration to clays along fragment edges. Phenocrysts include biotite, clinopyroxene, plagioclase, alkali feldspar, and iron-titanium oxides. 
Lithic fragments are all volcanic, including glassy flow-banded, rhyolite lava, devitrified welded tuff, pyroxene andesite, and a nonwelded, zeolitized tuff.

Sorptive Category: Glass and Clays

Sorptive properties should be dominated by the assemblage of clays and glass. The coating of clays along all surfaces of the pumice clasts should provide an unusually large surface area for sorption.

XXII. YM-22 (868) TOPOPAH SPRING MEMBER OF THE PAINTBUSH TUFF

\section{Core Description}

Densely welded, devitrified, ash-flow tuff; contains sparse amounts of lithophysae; $1 \%$ phenocrysts, abundant rhyolite lithic fragments.

X-Ray Diffraction Results (\%)

Alkali

Smectite $\frac{\text { Illite }}{5-10} \frac{\text { Clinoptilolite }}{<2} \underset{30-50}{-\infty} \frac{\text { Quartz }}{--} \frac{\text { Cristobalite }}{30-50} \frac{\text { Feldspar }}{\ldots}$

\section{Thin Section Description}

Core sample is a densely welded, devitrified ash-flow tuff. Pumice and shard fragments are collapsed and annealed along the complete length of the fragments. Devitrification is primarily granophyric with several textural varieties. The fine-ash matrix has devitrified to extremely fine-grained quartz and feldspar intergrowths; larger shards are composed of coarse-grained quartz-feldspar intergrowths. These, in some cases, are confined within shard boundaries and in other cases cross boundaries. A third type of devitrification consists of relict spherulites that generally surround vapor phase pockets in pumice fragments. They are partially recrystallized to granophyric intergrowths, but their original sperulite outlines are at least partially preserved. These probably represent multiple stages of devitrification: the first being a spherulitic stage probably controlled by the occurrence of vapor phase pockets and the second, a more pervasive granophyric crystallization. Vapor phase minerals consisting of circular to elongate crystals of quartz are present in punice fragments and rarely in the groundnass. Phenocrysts include plagioclase, alkali feldspar, sparse oxidized biotite, and iron-titaniun oxides. Lithic fragments are devitrified welded tuff. 
The sorptive properties should be controlled by the assemblage of quartz and feldspar from this completely devitrified sample with a small contribution from the clays.

XXIII. YM-30 (1264) TOPOPAH SPRING MEMBER OF THE PAINTBRUSH TUFF Core Desciption

Moderate to densely welded, vitric, partially argillized, ash-flow tuff; 2 to $3 \%$ volcanic lithic fragments.

X-Ray Diffraction Results (\%)

$\frac{\text { Smectite }}{5-10} \frac{\text { Illite }}{5} \frac{\text { Clinoptilolite }}{5-10} \frac{\text { Quartz }}{40-60} \frac{\text { Cristobalite }}{5-10} \frac{\begin{array}{c}\text { Alka1i } \\ \text { Feldspar }\end{array} \text { Glass }}{30-50}$

Thin Section Description

This core sample is from the upper part of the vitrophyric zone of dense welding. It is a densely welded, partially devitrified and partially zeolitized ash-flow tuff. Pumice and shard fragments are highly compacted and these originally glass fragments are partially altered to clays and zeolites and partially devitrified. The degree of alteration appears to be controlled by fracturing, and cross cutting alteration seems to indicate multiple episodes of alteration and devitrification. Phenocrysts are sparse and include alkali feldspar, plagioclase, and biotite. Lithic fragments are mostly devitrified welded tuff.

\section{Sorptive Category: Clay-Zeolite Devitrification}

The rock sorptive properties should be controlled by the clays, zeolites, and the devitrification assemblage of quartz and alkali feldspar. The differing textural occurrences of these phases may affect the surface area available for sorption.

XXIV. YM-38 (1504) TUFFACEOUS BEDS OF THE CALICO HILIS

\section{Core Description}

Nonwelded, devitrified to slightly zeolitized ash-flow tuff, less than $1 \%$ phenocrysts; less than $1 \%$ lithic fragnents. 


\section{X-Ray Diffraction Results (y)}

$\frac{\text { Sectite }}{5-15} \quad \frac{\text { Illite }}{<5} \quad \frac{\text { Clinoptilolite }}{40-60} \quad \frac{\text { Quartz }}{15-30} \quad \frac{\text { Cristobalite }}{10-20} \quad \frac{\begin{array}{c}\text { Alkali } \\ \text { Feldspar }\end{array}}{10-20}$

\section{Thin Section Description}

The core sample is a zeolitized, nonwelded ash-flow tuff. Pumice and shard fragments are pseudonorphed by the replacing zeolite, and the alteration is pervasive. Phenocrysts are quartz, alkali feldspar, plagioclase, and both fresh and altered biotite. Lithic fragments are devitrified welded tuff or rhyolite lava and abundant perlite fragments that are altered to zeolite.

\section{Sorptive Category: Zeolite}

The sorptive properties should be doninated by the abundant zeolite that is uniforly distributed through the rock.

XXV. YM-42 (1824) TUFFACEOUS BEDS OF CALICO HILIS

\section{Core Description}

Bedded and reworked tuff; zeolitized and locally silicified; variable lithic fragment content ( 2 to 20\%); stained with iron oxides.

$\underline{X-R a y ~ D i f f r a c t i o n ~ R e s u l t s ~(y) ~}$

$\frac{\text { Smectite }}{\operatorname{tr}} \frac{\text { Illite }}{\operatorname{tr}} \quad \frac{\text { Clinoptilolite }}{20} \quad \frac{\text { Quartz }}{40} \quad \frac{\text { Cristobalite }}{--} \quad \begin{gathered}\text { Alkali } \\ \text { Feldspar }\end{gathered}$

\section{Thin Section Description}

The sample is a crystal-rich reworked tuff containing a great variety of fragments including lithic, pumice, and crystal fragments. The rock matrix, probably composed of fine ash, is zeolitized and highly oxidized.

Sorptive Category: Zeolitized Tuff 
XXVI. MM-45 (1930) PROW PASS MEMBER OF THE CRATER FLAT TUFF

\section{Core Description}

Nonwelded to partially welded, devitrified, vapor phase altered, ash-flow tuff; 7 to $12 \%$ phenocrysts; less than $1 \%$ volcanic and mudstone lithic fragments.

$\underline{X-R a y ~ D i f f r a c t i o n ~ R e s u l t s ~(y) ~}$

$\frac{\text { Smectite }}{1-5} \quad \frac{\text { Clinoptilolite }}{--} \quad \frac{\text { Quartz }}{40-60} \quad \frac{\text { Cristobalite }}{t r} \quad \frac{\begin{array}{c}\text { Alkali } \\ \text { Feldspar }\end{array}}{\text { tr }}$

Thin Section Description

There are no thin sections available for this sample.

Sorption Category: Devitrification Assemblage

XXVII. YM-46 (2002) PROW PASS MEMBER OF THE CRATER FLAT TUFF

Core Description

Moderately welded, devitrified ash-flow tuff; 9 to $10 \%$ phenocrysts; $14 \%$ volcanic and mudstone lithic fragments. (Note: sample was collected within fault zone, faulting Prow Pass against Prow Pass. There is extensive ironoxide staining along surfaces of the fault).

X-Ray Diffraction Results (\%)

$\begin{array}{lllll}\text { Smectite } & \text { Illite } & \text { Clinoptilolite } & \text { Quart.z } & \begin{array}{c}\text { Alkali } \\ \text { Feldspar }\end{array} \\ <5 & -- & 40-60 & 35-45\end{array}$

\section{Thin Section Description}

The core sample is a moderately welded, devitrified ash-flow tuff. Pumice and shard fragments are compacted but not completely annealed along fragment edges. Devitrification phases are axiolitic in most pumice fragments and spherulitic in pumice fragments that contain vapor phase minerals. The groundmass is devitified to fine-grained granophyric to axiolitic intergrowths. Phenocrysts include embayed quartz, alkali feldspar, plagioclase, and biotite. No lithic fragnents were noted in the thin section: 


\section{Sorption Category: Devitrified}

XXVIII. YM-48 (2114) PROW PASS MEMBER OF THE CRATER FLAI TUFF

Core Description

Moderately welded, vitric, ash-flow tuff; 10 to $15 \%$ phenocrysts;

$2 \%$ volcanic and mudstone lithic fragments.

X-Ray Diffraction Results (\%)

$\frac{\text { Smectite }}{<2} \quad \frac{\text { Clinoptilolite }}{20-40} \quad \frac{\text { Quartz }}{5-10} \quad \frac{\text { Cristobalite }}{5-15} \quad \frac{\begin{array}{c}\text { Alkali } \\ \text { Feldspar }\end{array}}{20-40} \quad \frac{\text { Glass }}{40-60}$

Thin Section Description

Partially welded, partially zeolitized, vitric ash-flow tuff. Shard and pumice fragments are only slightly compacted: Shards are still glassy, whereas fine ash and some of the pumice fragments have been altered to zeolite. The sample contains phenocrysts of quartz, alkali feldspar, plagioclase, orthopyroxene, iron-titanium oxides, and trace amounts of hornblende. Lithic fragments are abundant and consist entirely of mudstone.

Sorption Category: Zeolites and Glass

The sorptive properties should be dominated by the abundant zeolite phases with a lesser contribution from glass.

XXIX. YM-49 (2221) PROW PASS MEMBER OF THE CRATER FLAT TUFF

\section{Core Description}

Partially welded, devitrified to zeolitized ash-flow tuff; 10 to $15 \%$ phenocrysts; 2 to $3 \%$ volcanic and mudstone lithic fragments.

$\underline{X \text {-Ray Diffraction Results (\%) }}$

$\frac{\text { Smectite }}{\operatorname{tr}} \frac{\text { Illite }}{\operatorname{tr}} \quad \frac{\text { Clinoptilolite }}{10-20} \quad \frac{\text { Quartz }}{--} \quad \frac{\begin{array}{c}\text { Alkali } \\ \text { Feldspar }\end{array}}{20-30} \quad \frac{\text { Glass }}{40-60}$


Thin Section Description

The core sample is almost identical to sample MM-48, except that the rock is nonwelded.

Sorptive Category: Zeolite and Glass

XXX. YM-54 (2491) BULLFROG MEMBER OF THE CRATER FLAT TUFF

Core Description

Partially to moderately welded, devitrified, vapor phase altered; 10 to $15 \%$ phenocrysts; less than $1 \%$ volcanic lithic fragments.

X-Ray Diffraction Results $(\%)$

$\begin{array}{lllll}\text { Smectite } & \text { Illite } & \text { Quartz } & \text { Cristobalite } & \begin{array}{c}\text { Alkali } \\ \text { Feldspar }\end{array}\end{array}$

\section{Thin Section Description}

The core sample is a moderately welded, devitrified, vapor-phase altered, ash-flow tuff. The rock is uniformly pocketed by vapor phase minerals and shows granophyric devitrification. Shard textures are almost entirely obscured by the crystallization. Phenocrysts include quartz, alkali feldspar, biotite, iron-titanium oxides, and possibly hornblende relicts. Lithic fragments include rare mudstones.

Sorptive Category: Devitrified 